Supplement of Atmos. Chem. Phys., 14, 4617-4641, 2014

http://www.atmos-chem-phys.net/acp-14-4617-2014/

doi:10.5194/acp-14-4617-2014-supplement

(C) Author(s) 2014. CC Attribution 3.0 License.

(c) (1)

Supplement of

\section{Global and regional emissions estimates for $\mathrm{N}_{2} \mathrm{O}$}

\section{E. Saikawa et al.}

Correspondence to: E. Saikawa (eri.saikawa@emory.edu)

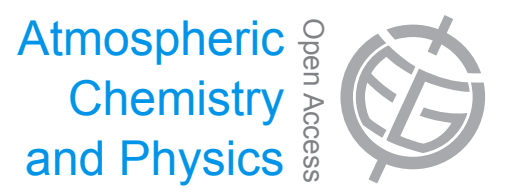



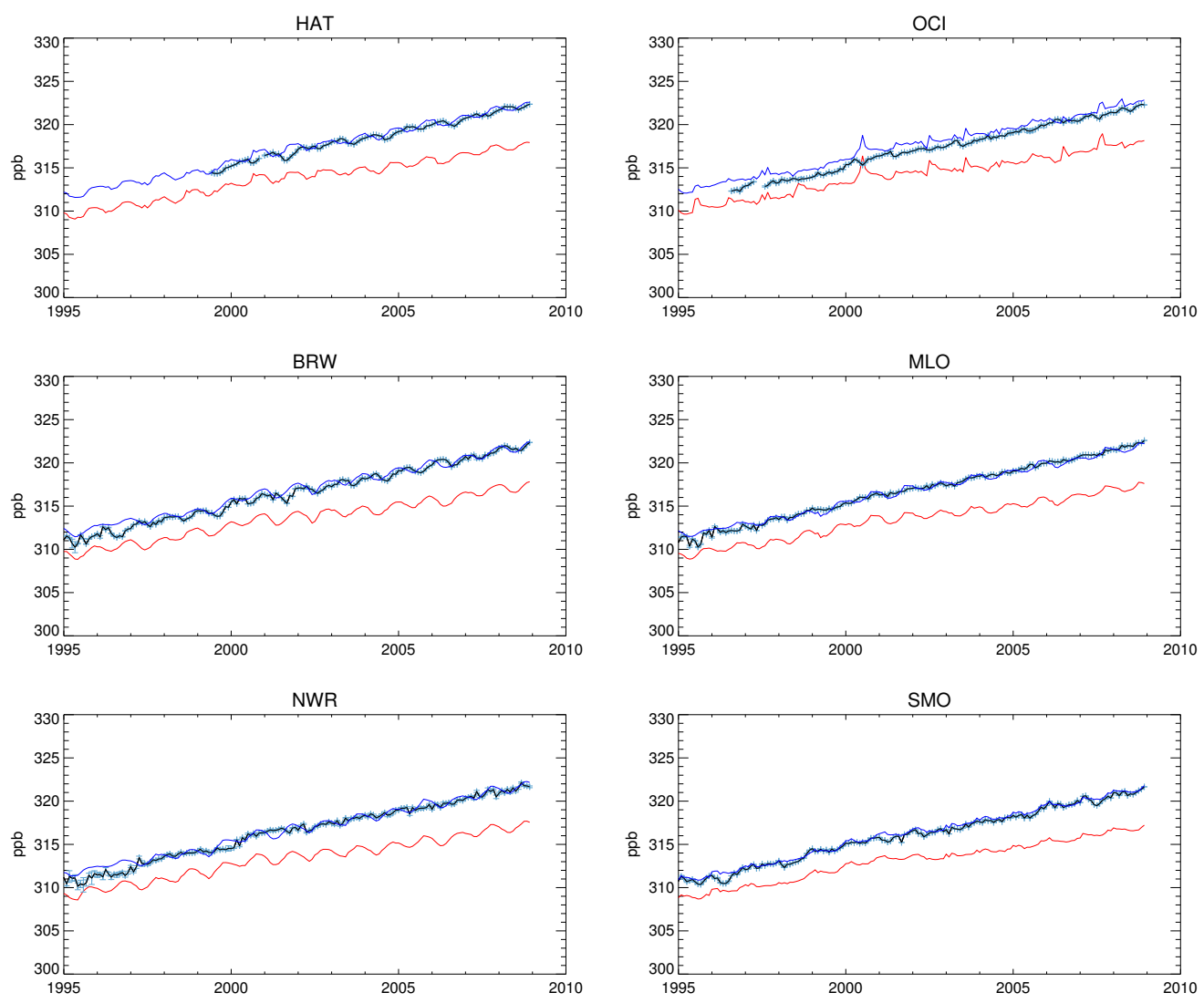

Fig. S1a. Monthly atmospheric mole fractions: measurements (black solid lines with standard deviations), and MOZART v4 model results using prior emissions (red solid lines) and optimized emissions (blue solid lines). 

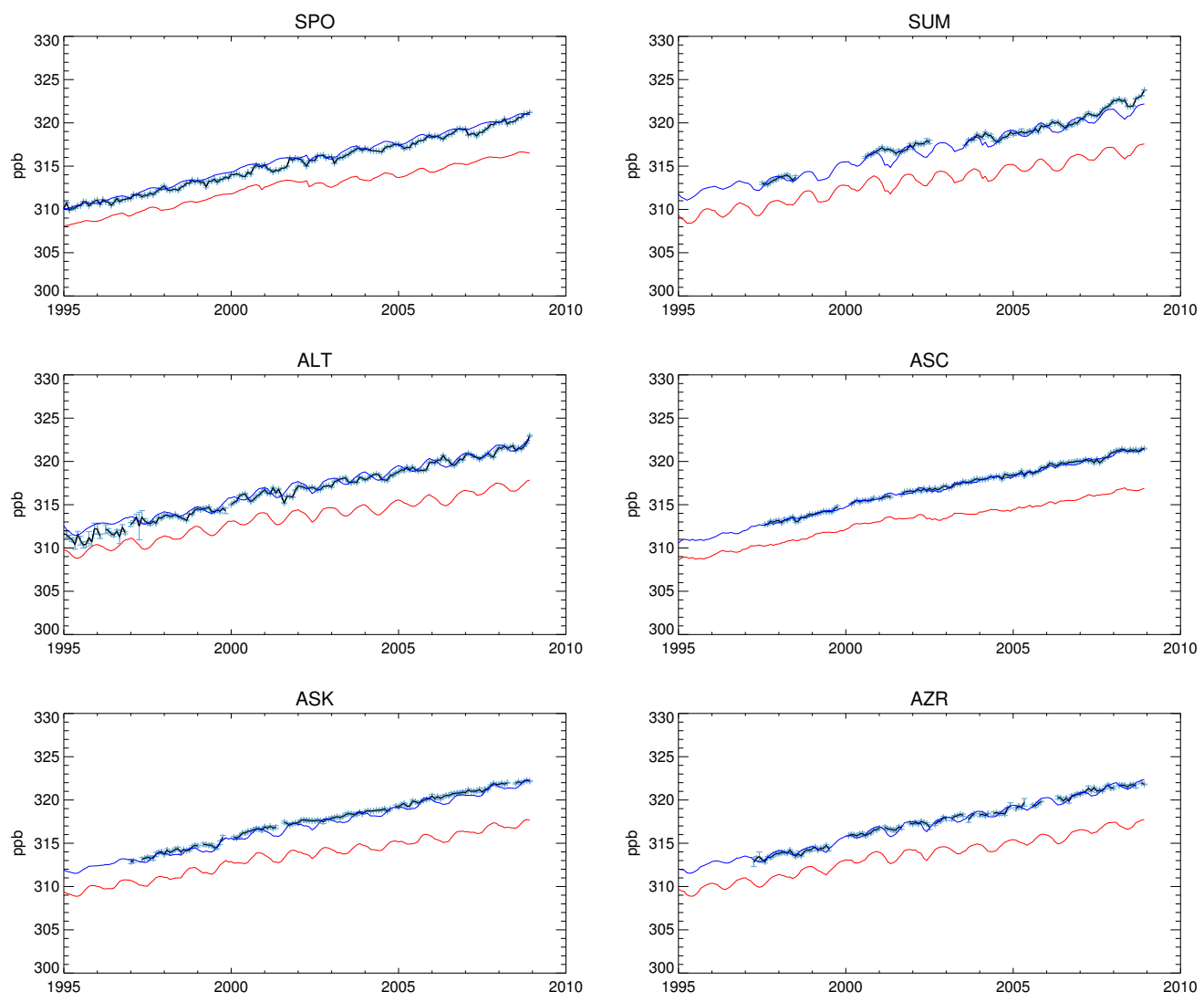

Fig. S1b. Monthly atmospheric mole fractions: measurements (black solid lines with standard deviations), and MOZART v4 model results using prior emissions (red solid lines) and optimized emissions (blue solid lines). 

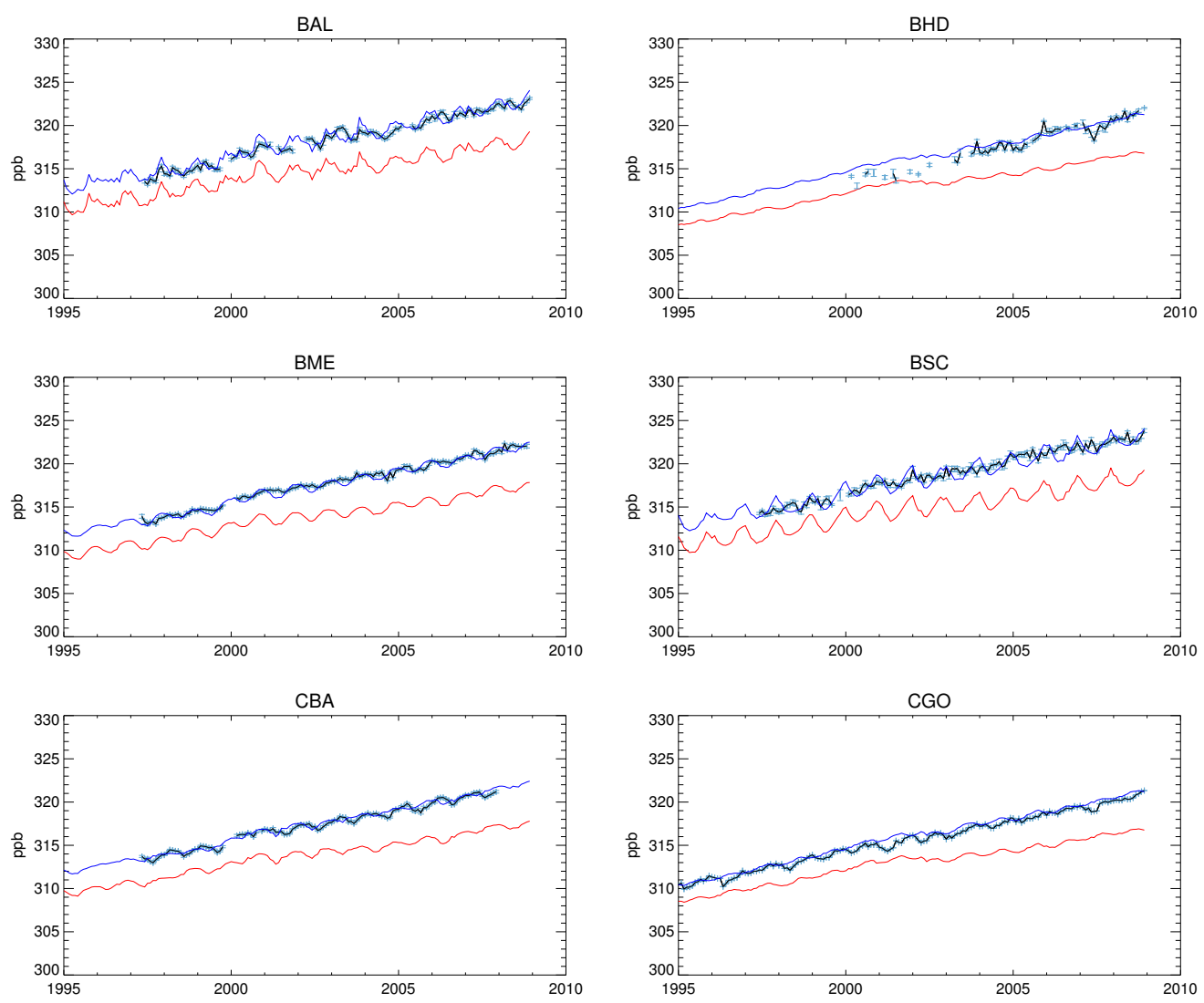

Fig. S1c. Monthly atmospheric mole fractions: measurements (black solid lines with standard deviations), and MOZART v4 model results using prior emissions (red solid lines) and optimized emissions (blue solid lines). 

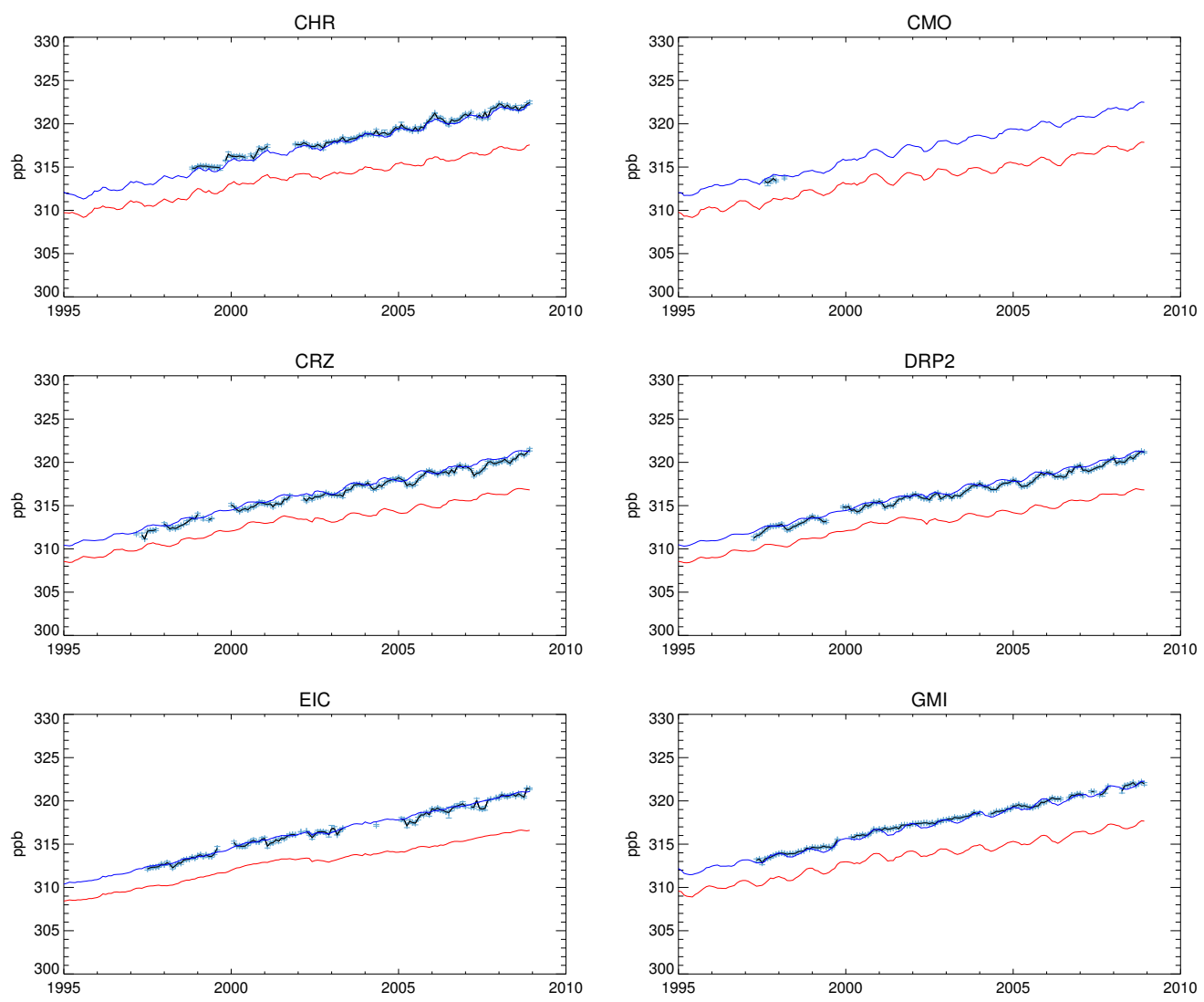

Fig. S1d. Monthly atmospheric mole fractions: measurements (black solid lines with standard deviations), and MOZART v4 model results using prior emissions (red solid lines) and optimized emissions (blue solid lines). 

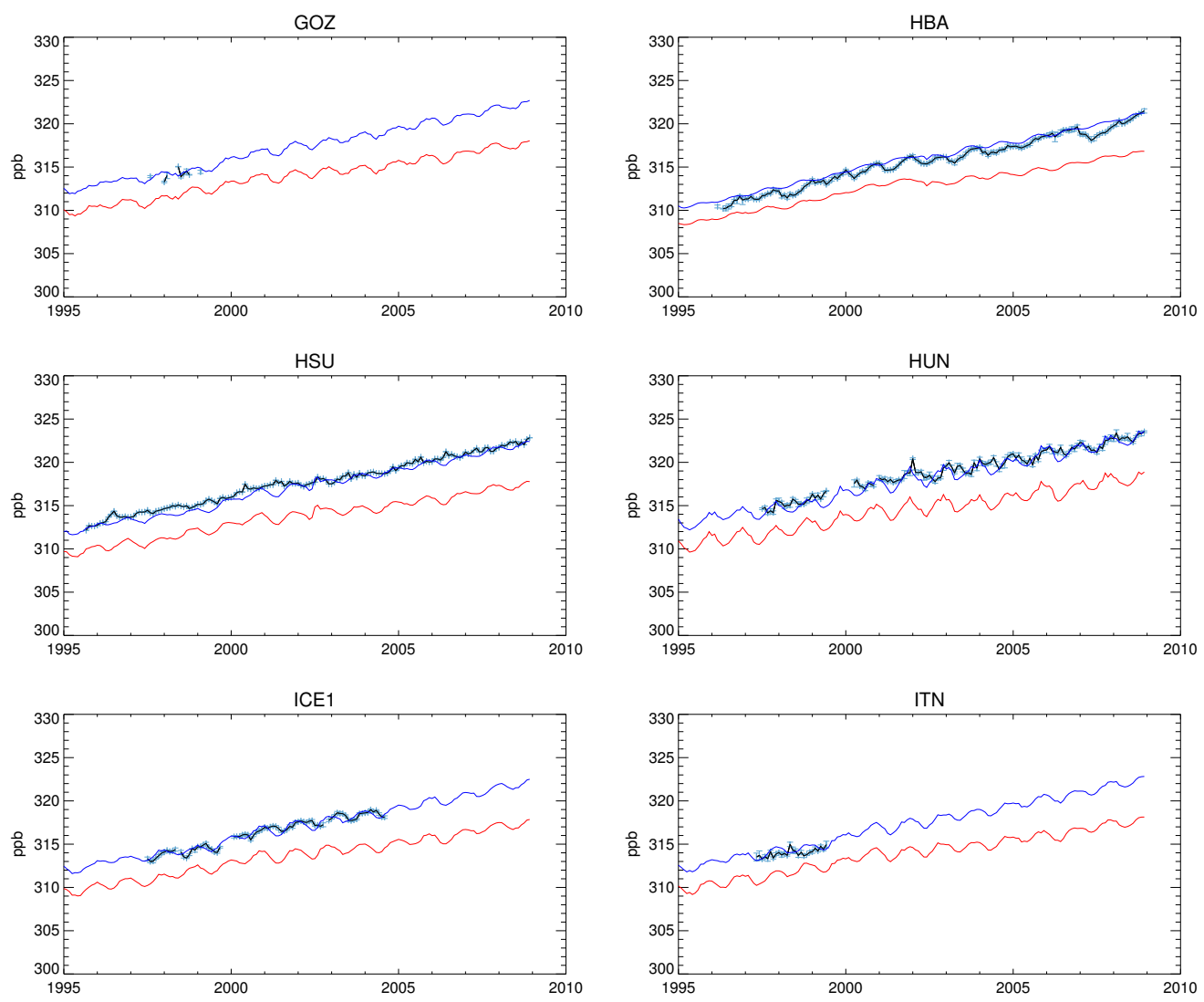

Fig. S1e. Monthly atmospheric mole fractions: measurements (black solid lines with standard deviations), and MOZART v4 model results using prior emissions (red solid lines) and optimized emissions (blue solid lines). 

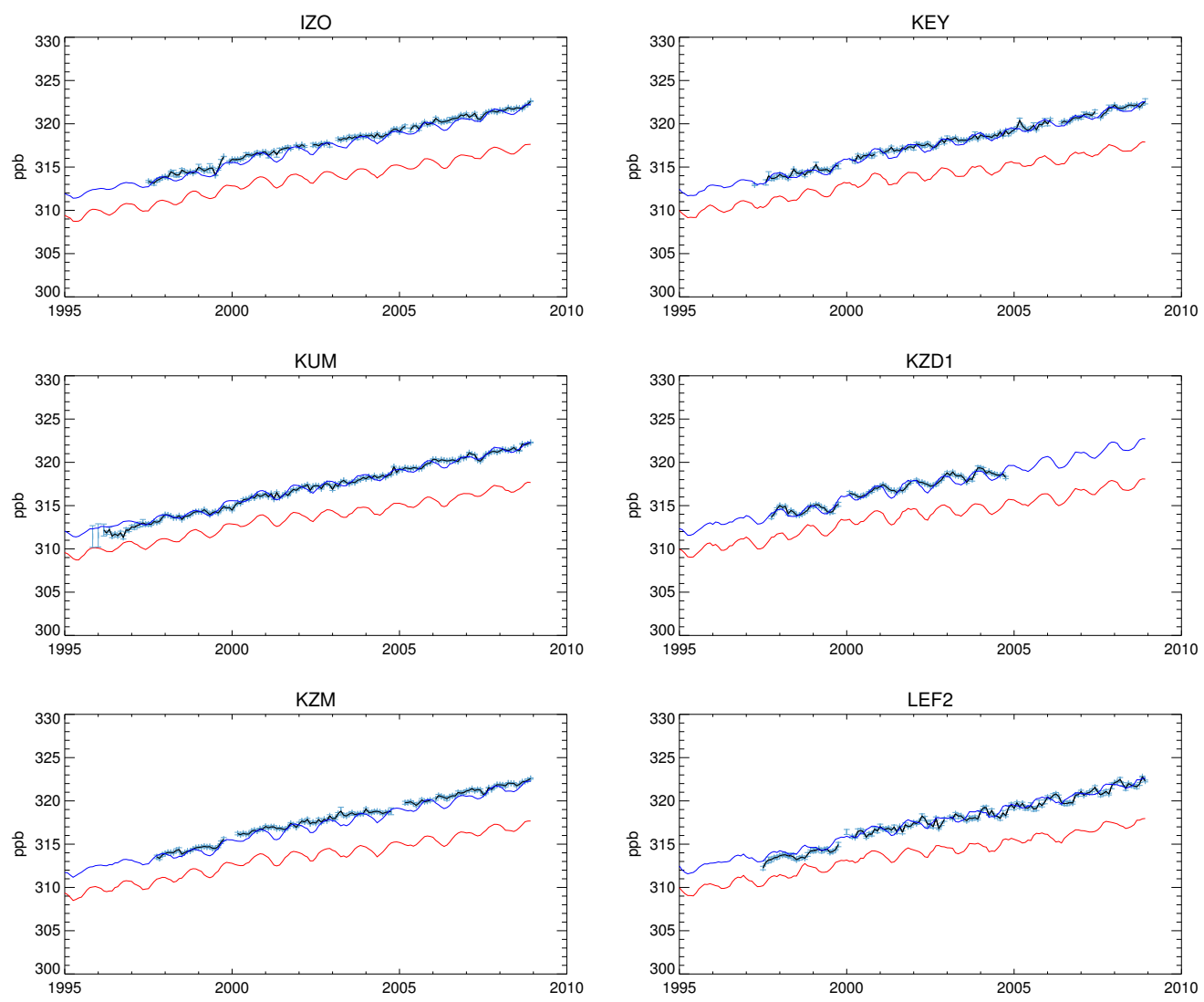

Fig. S1f. Monthly atmospheric mole fractions: measurements (black solid lines with standard deviations), and MOZART v4 model results using prior emissions (red solid lines) and optimized emissions (blue solid lines). 

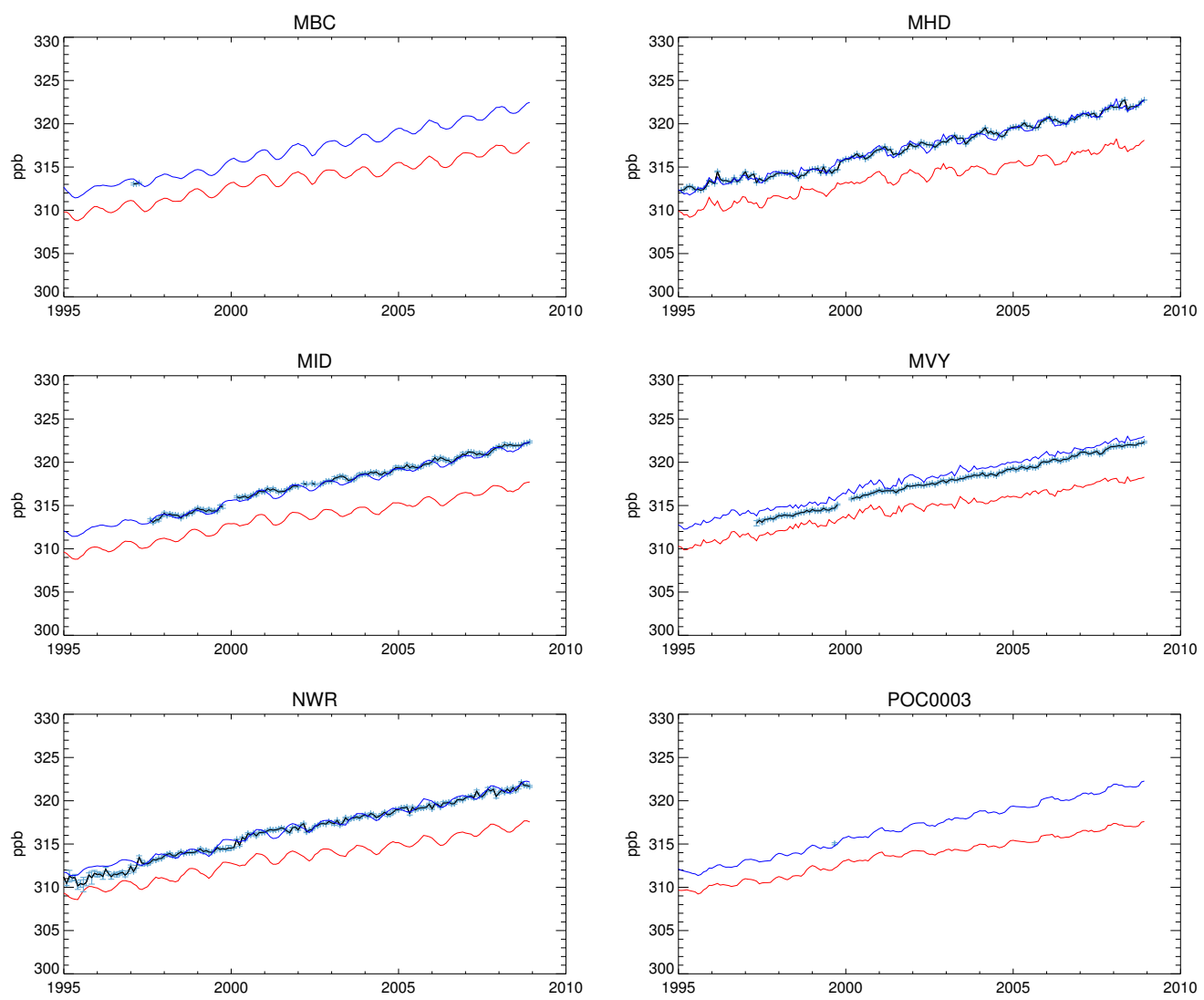

Fig. S1g. Monthly atmospheric mole fractions: measurements (black solid lines with standard deviations), and MOZART v4 model results using prior emissions (red solid lines) and optimized emissions (blue solid lines). 

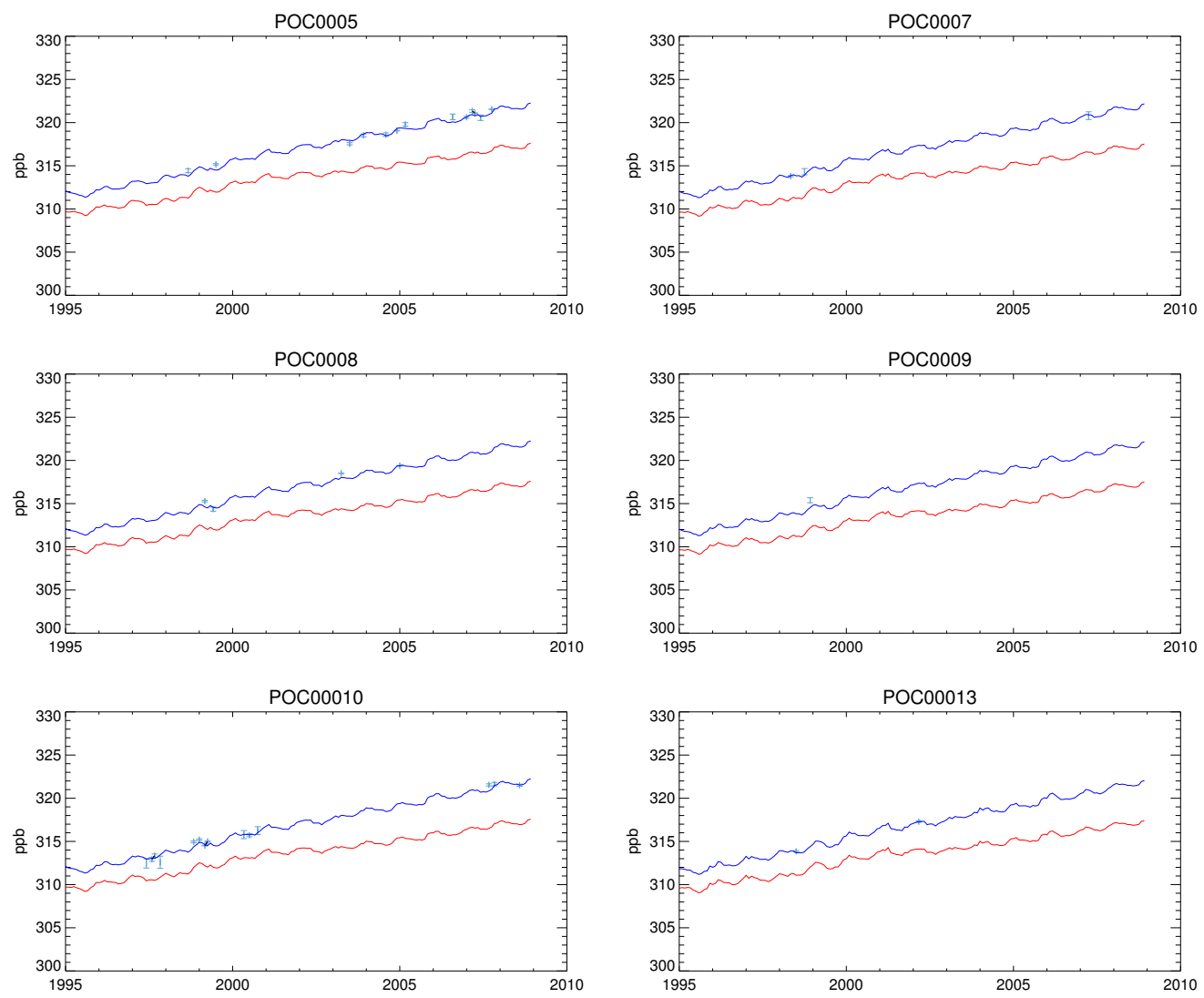

Fig. S1h. Monthly atmospheric mole fractions: measurements (black solid lines with standard deviations), and MOZART v4 model results using prior emissions (red solid lines) and optimized emissions (blue solid lines). 

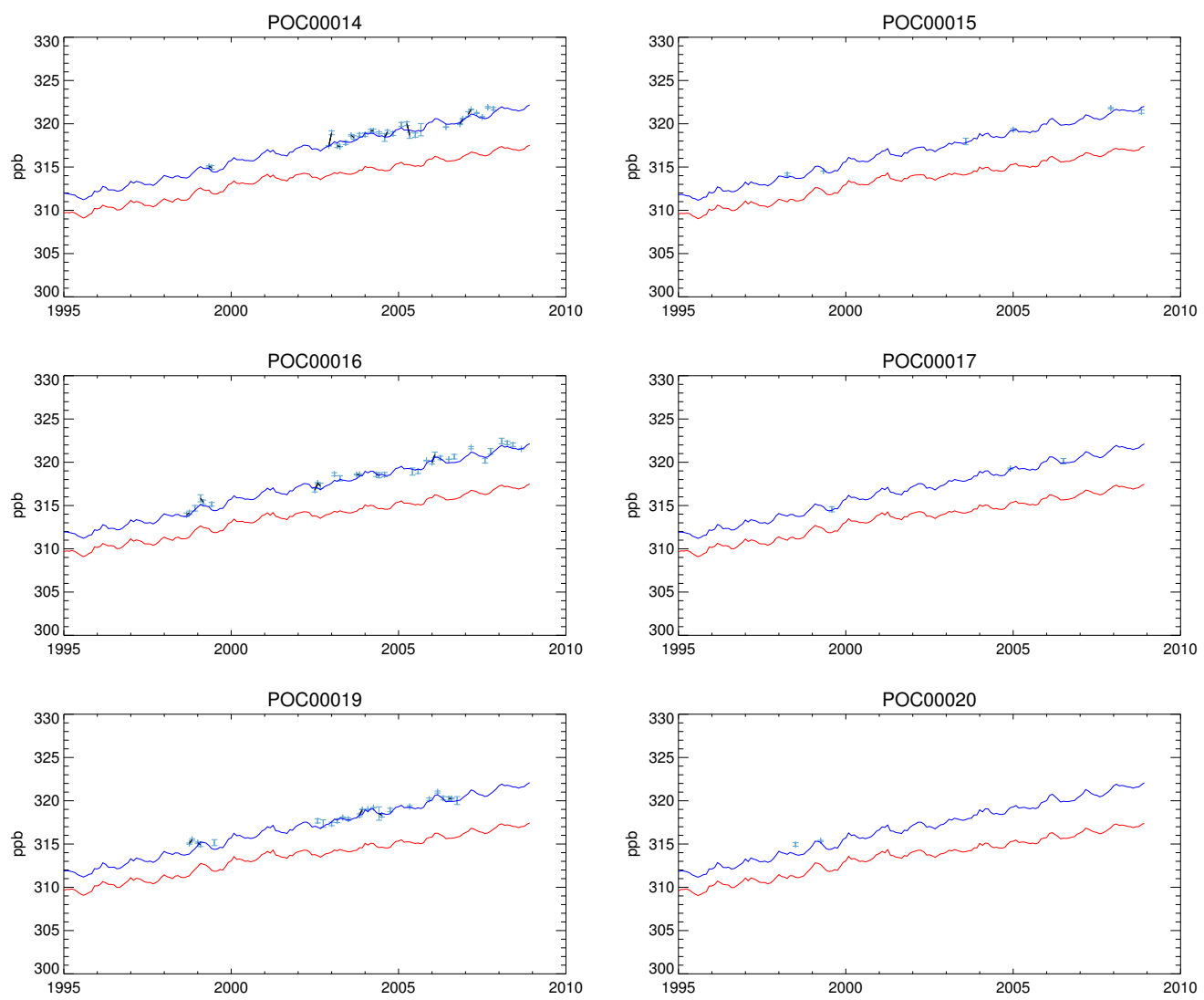

Fig. S1i. Monthly atmospheric mole fractions: measurements (black solid lines with standard deviations), and MOZART v4 model results using prior emissions (red solid lines) and optimized emissions (blue solid lines). 

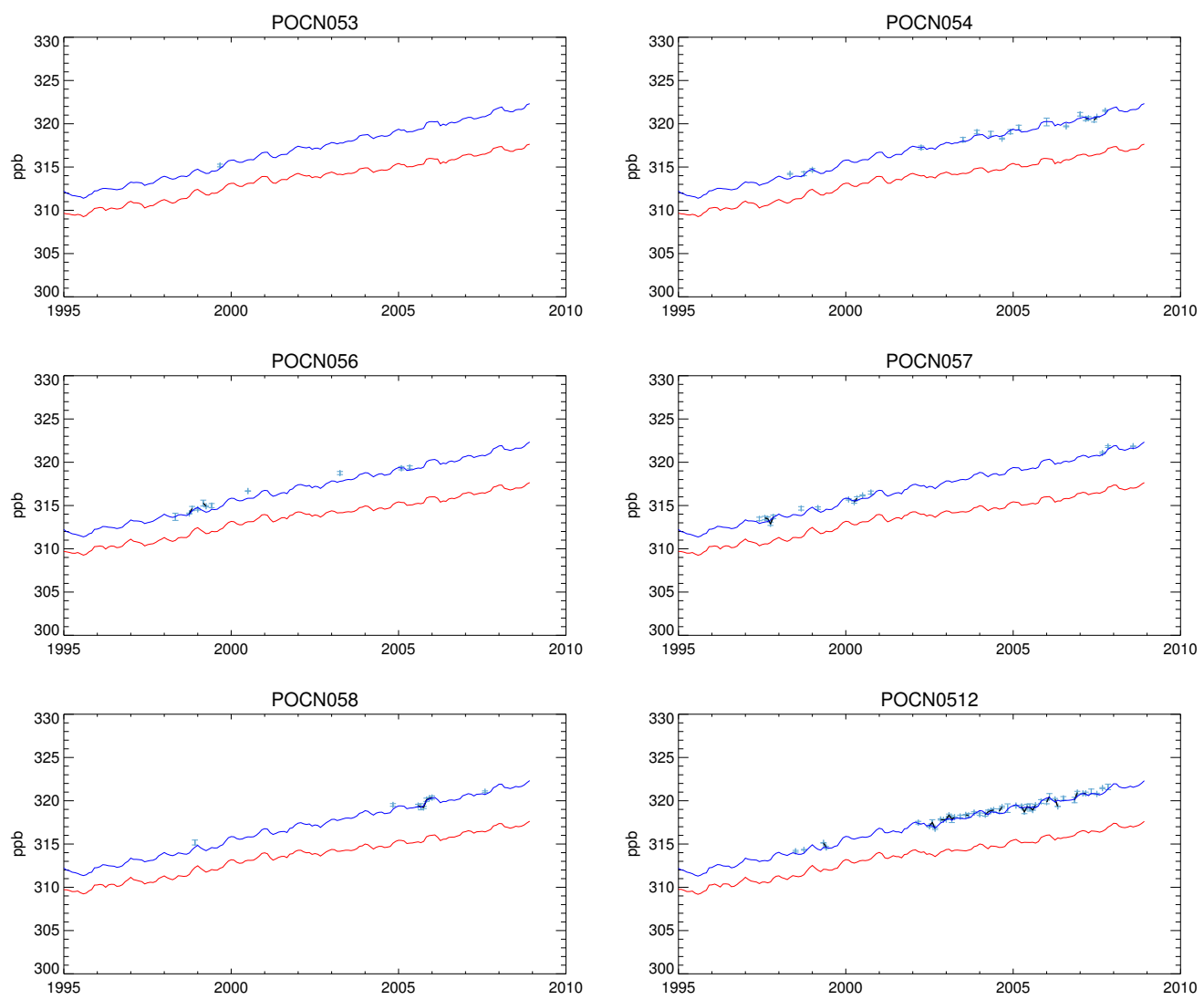

Fig. S1j. Monthly atmospheric mole fractions: measurements (black solid lines with standard deviations), and MOZART v4 model results using prior emissions (red solid lines) and optimized emissions (blue solid lines). 

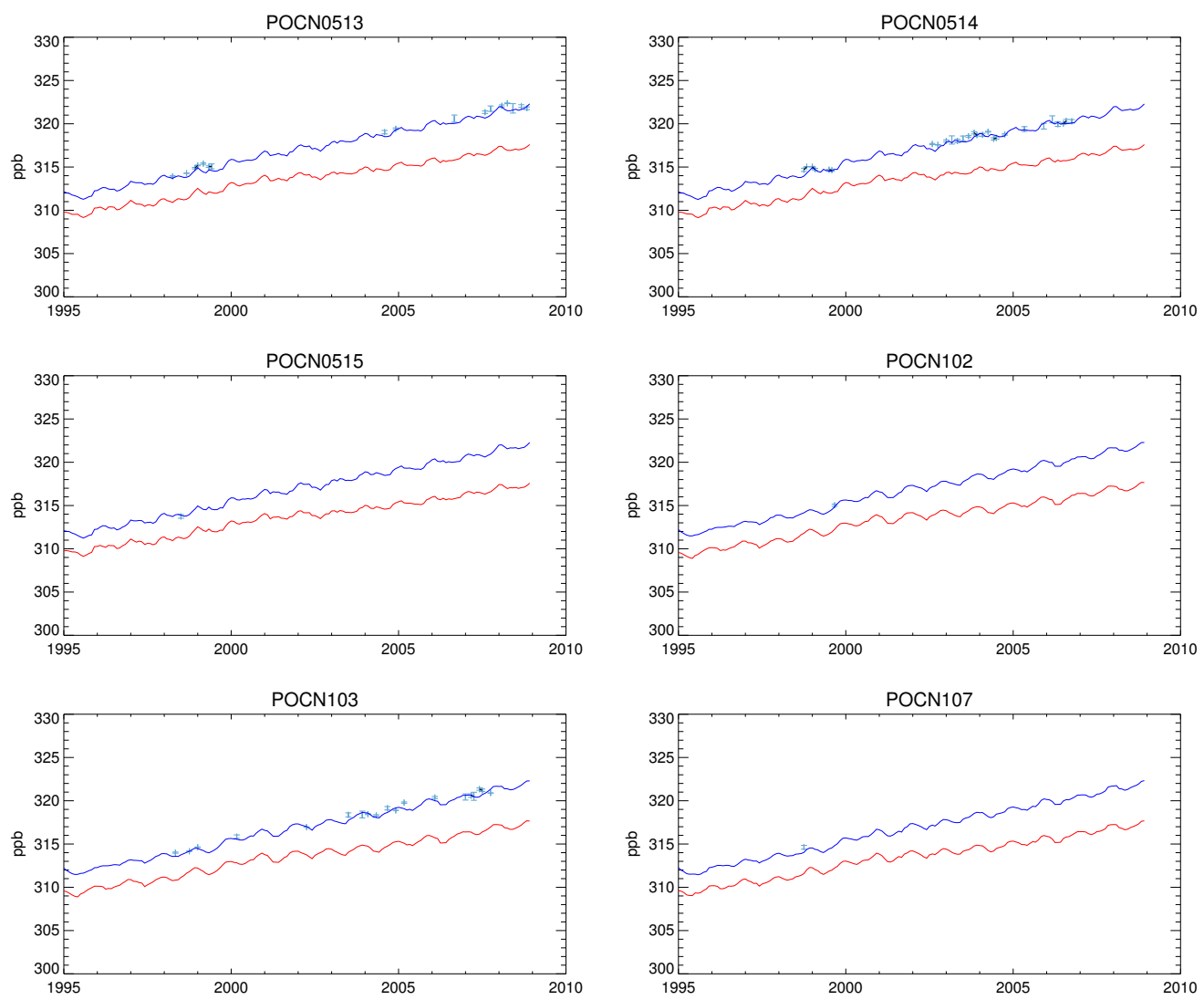

Fig. S1k. Monthly atmospheric mole fractions: measurements (black solid lines with standard deviations), and MOZART v4 model results using prior emissions (red solid lines) and optimized emissions (blue solid lines). 

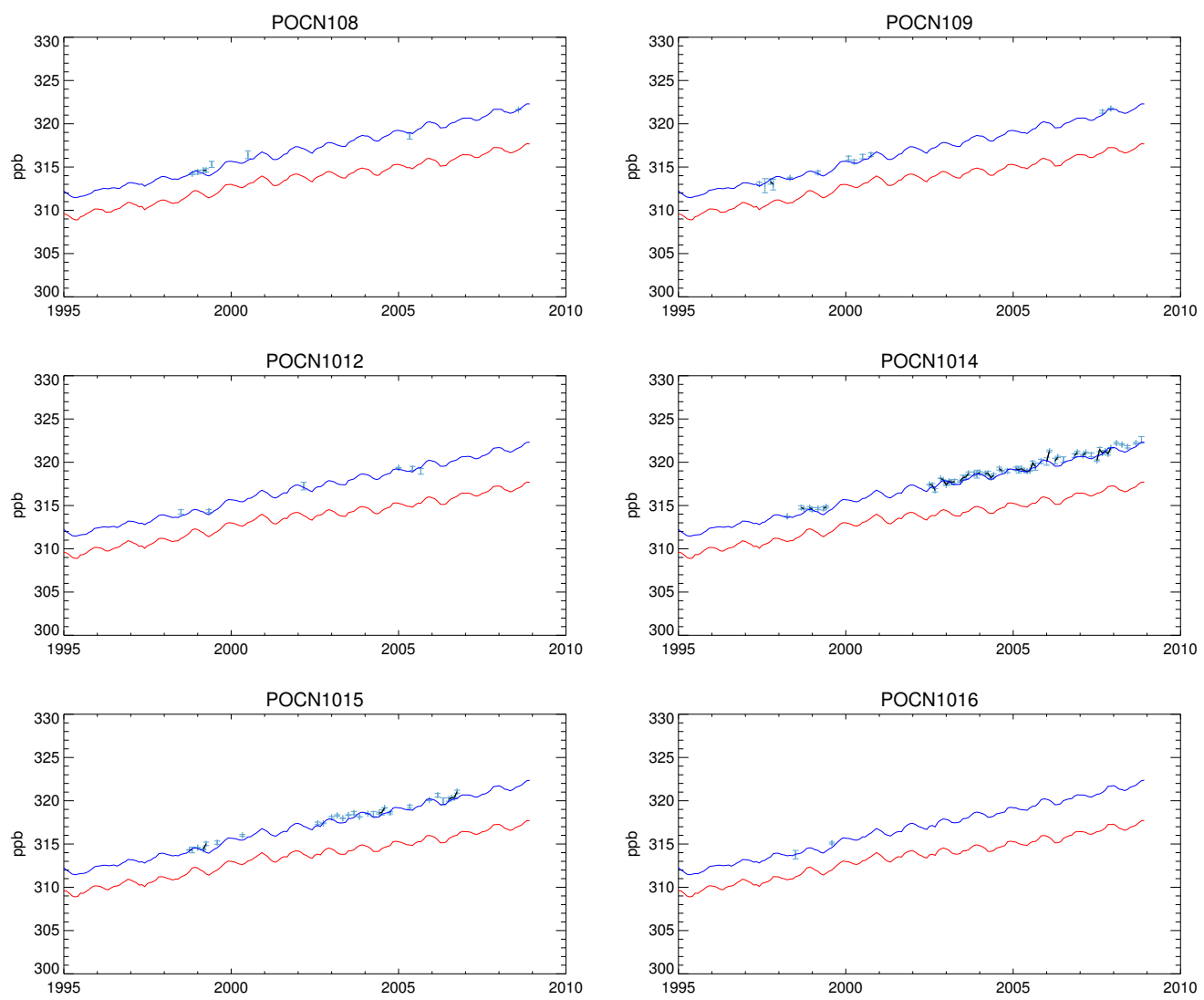

Fig. S1I. Monthly atmospheric mole fractions: measurements (black solid lines with standard deviations), and MOZART v4 model results using prior emissions (red solid lines) and optimized emissions (blue solid lines). 

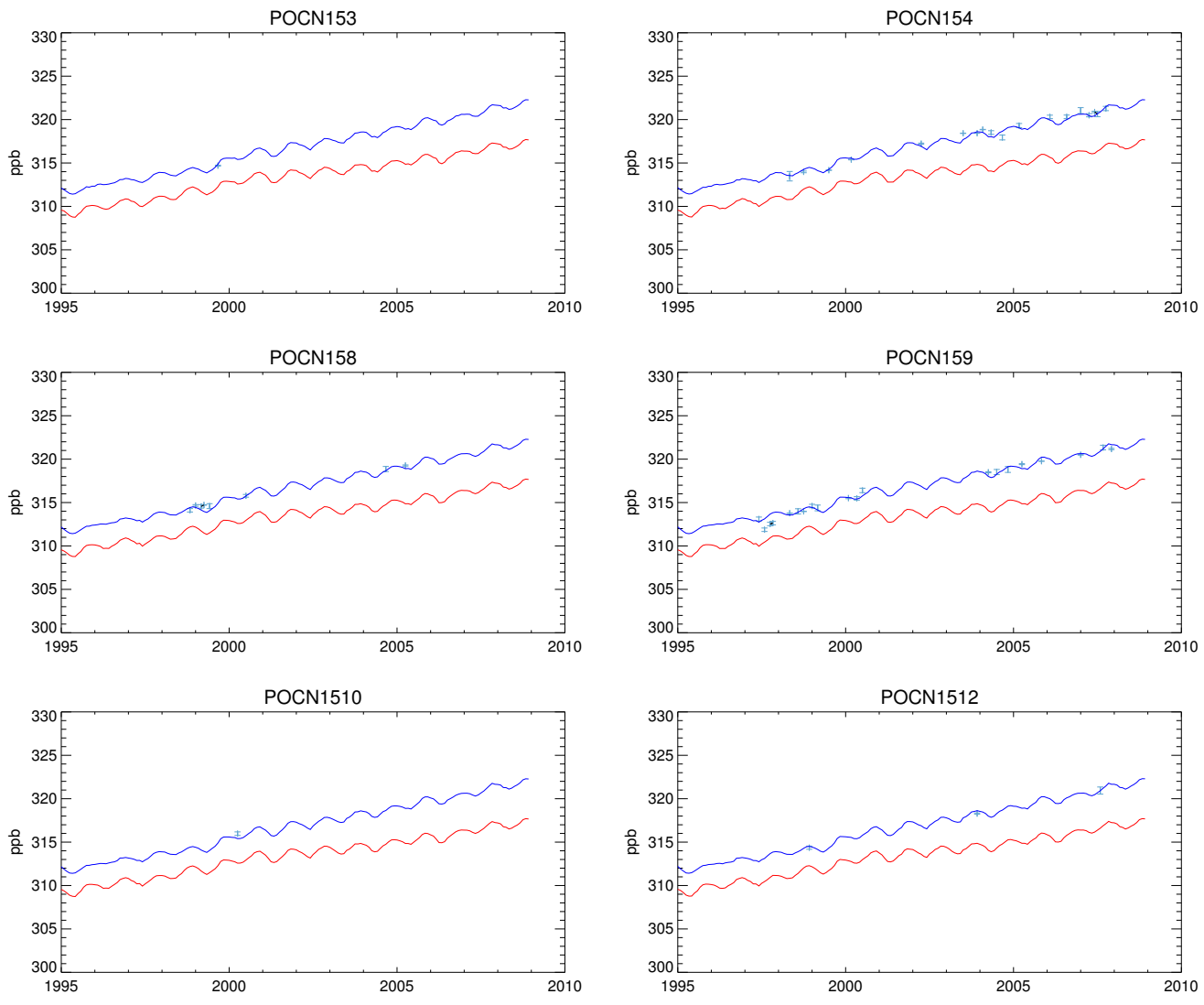

Fig. Sim. Monthly atmospheric mole fractions: measurements (black solid lines with standard deviations), and MOZART v4 model results using prior emissions (red solid lines) and optimized emissions (blue solid lines).

72 

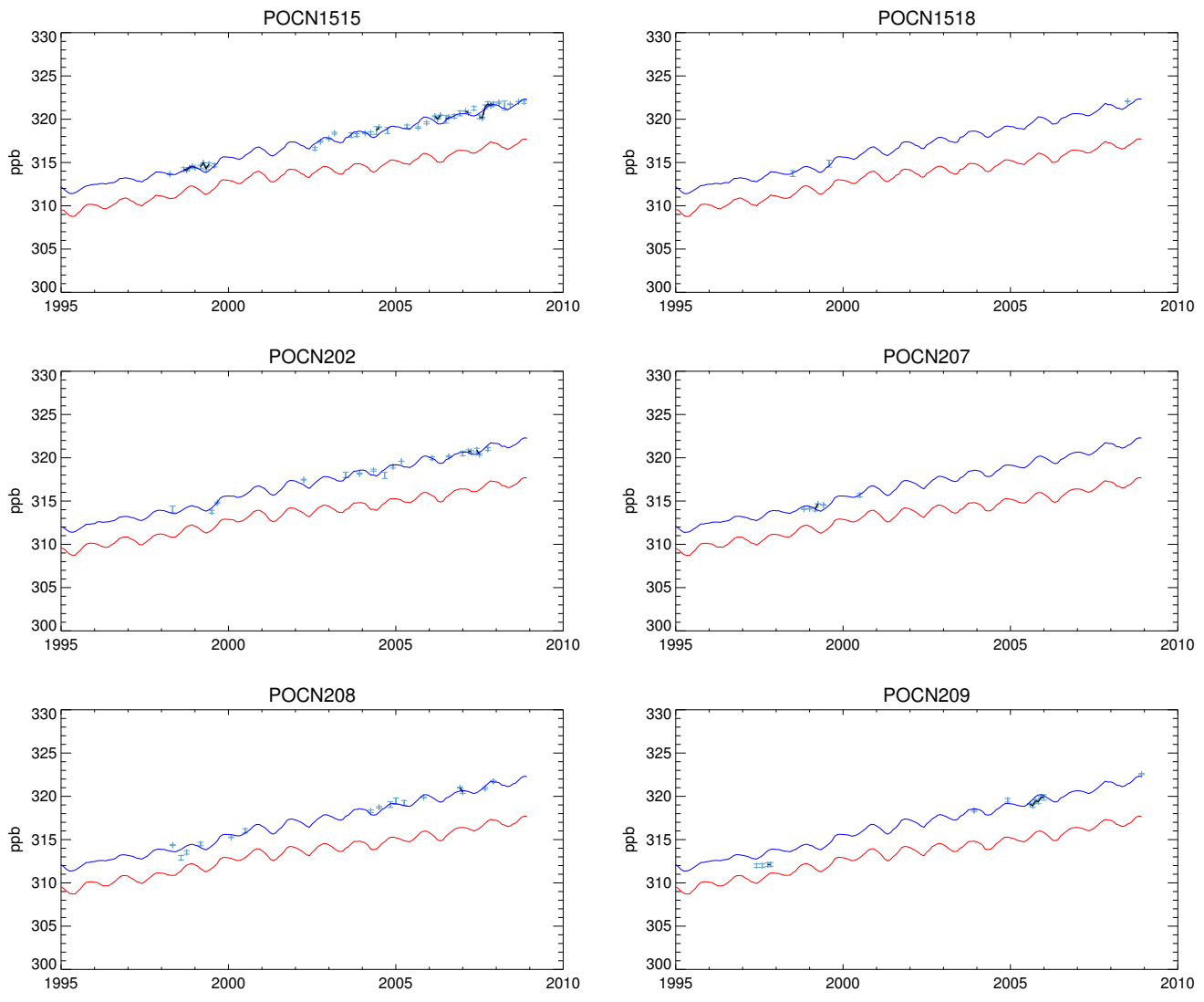

Fig. Sin. Monthly atmospheric mole fractions: measurements (black solid lines with standard deviations), and MOZART vA model results using prior emissions (red solid lines) and optimized emissions (blue solid lines).

73 

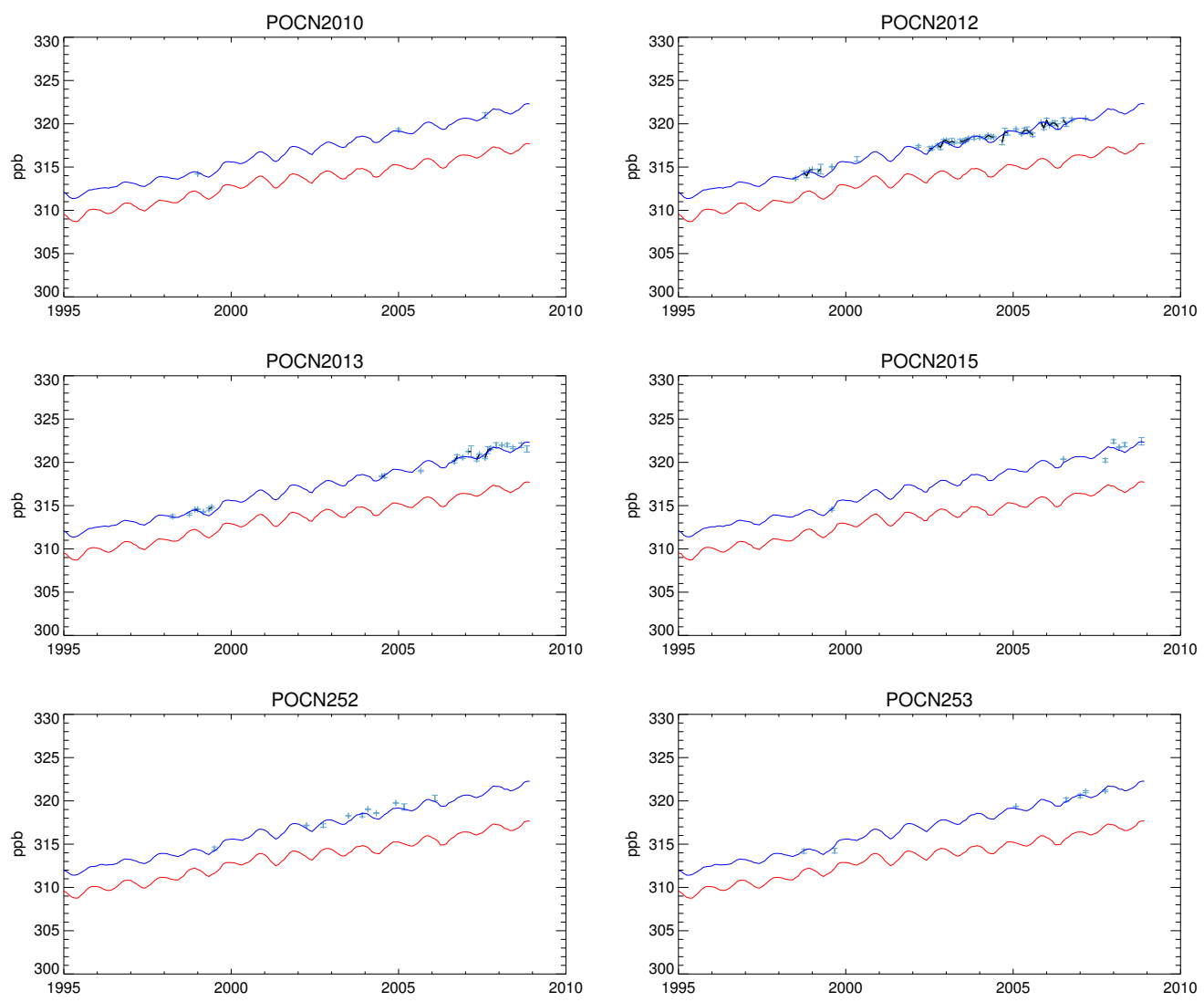

Fig. S10. Monthly atmospheric mole fractions: measurements (black solid lines with standard deviations), and MOZART v4 model results using prior emissions (red solid lines) and optimized emissions (blue solid lines). 

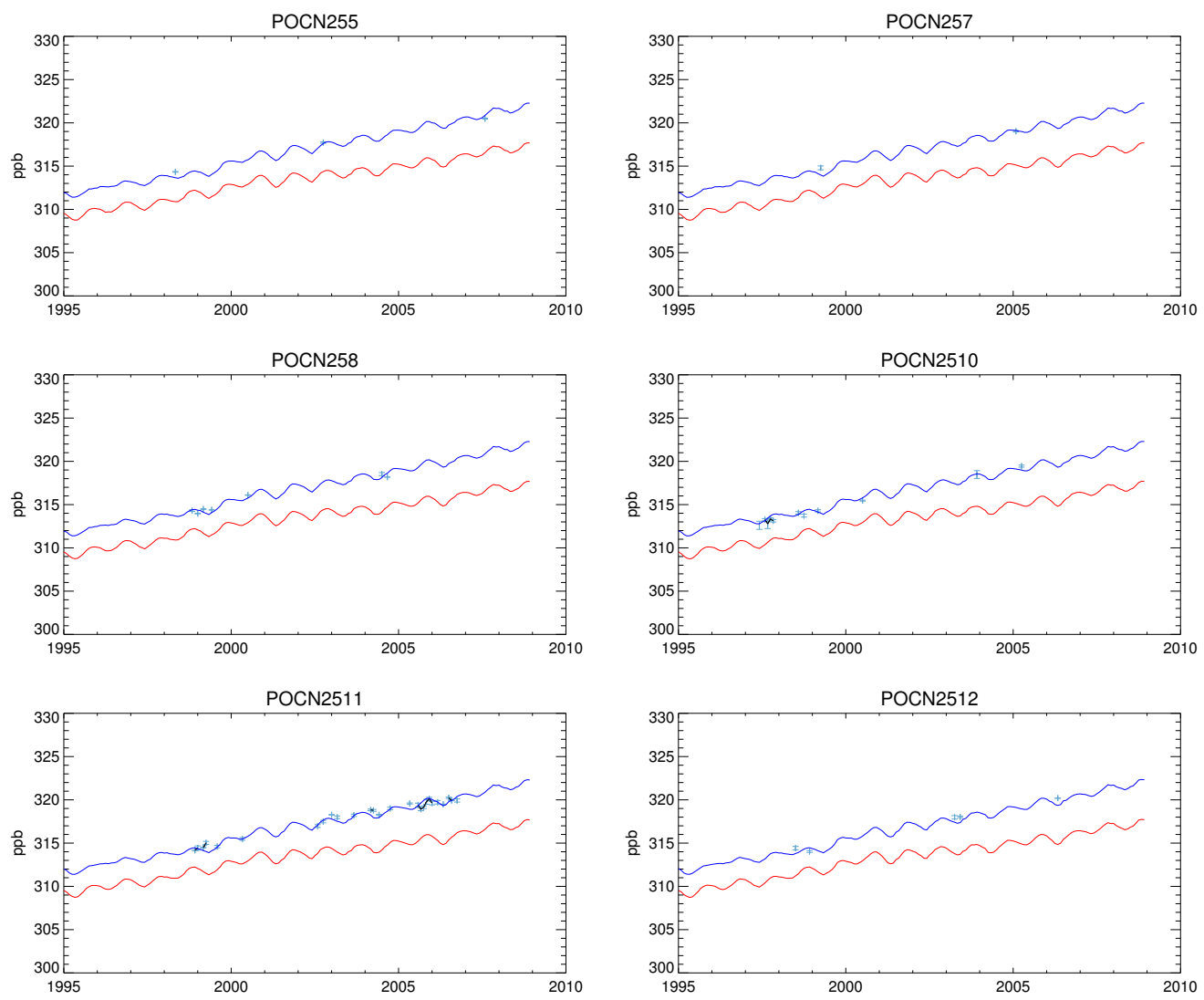

Fig. S1p. Monthly atmospheric mole fractions: measurements (black solid lines with standard deviations), and MOZART v4 model results using prior emissions (red solid lines) and optimized emissions (blue solid lines). 

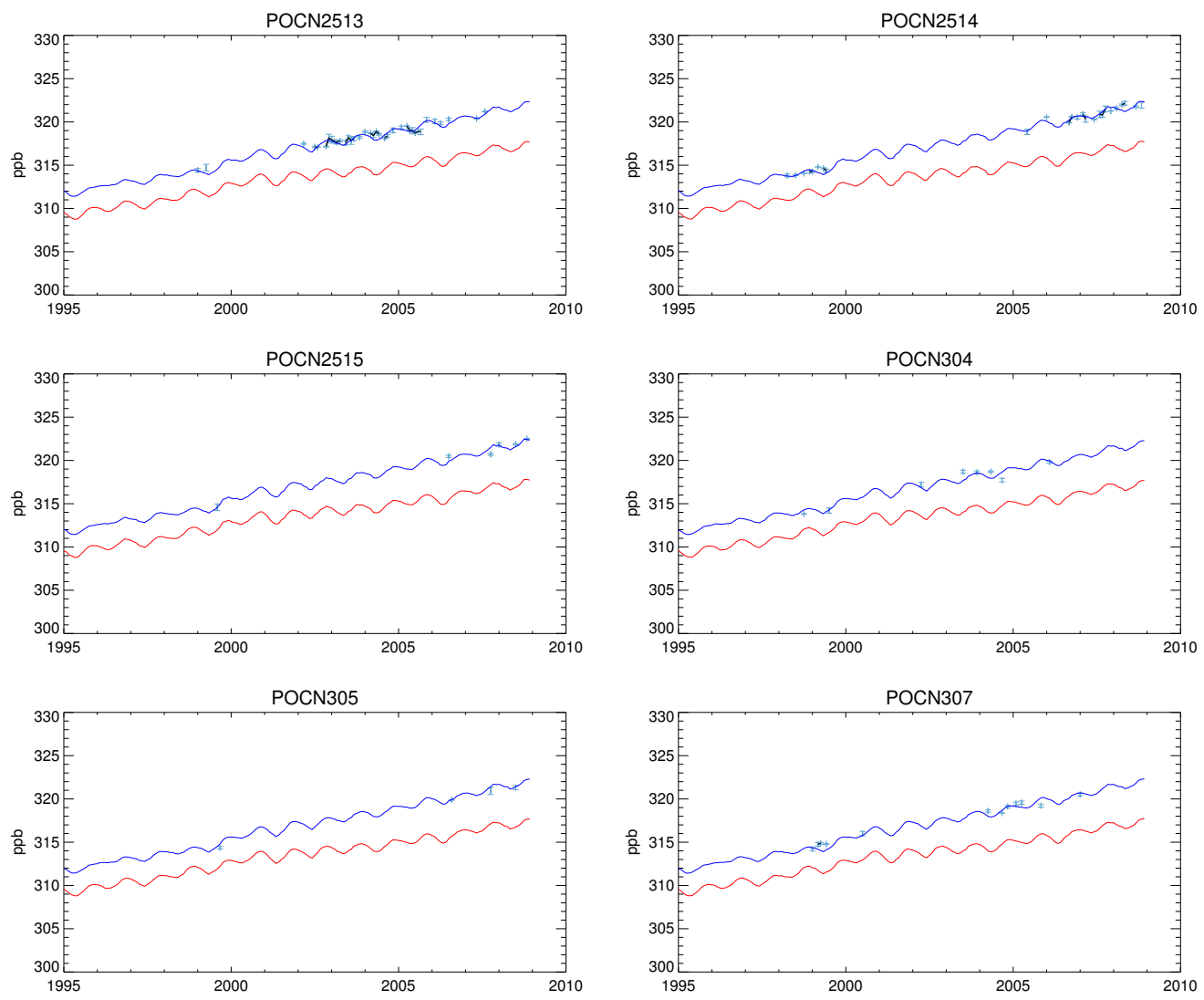

Fig. S1q. Monthly atmospheric mole fractions: measurements (black solid lines with standard deviations), and MOZART v4 model results using prior emissions (red solid lines) and optimized emissions (blue solid lines). 

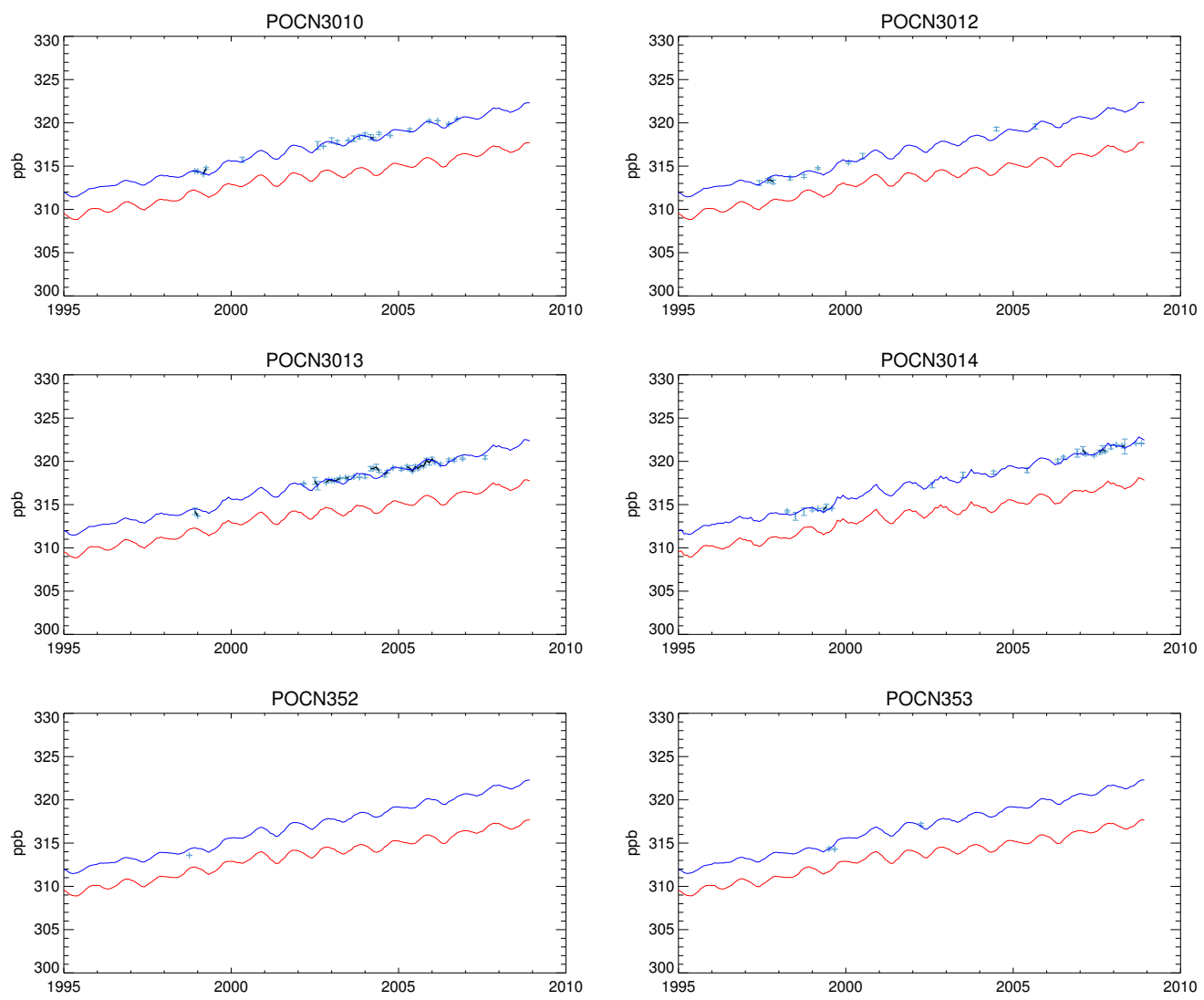

Fig. S1r. Monthly atmospheric mole fractions: measurements (black solid lines with standard deviations), and MOZART v4 model results using prior emissions (red solid lines) and optimized emissions (blue solid lines). 

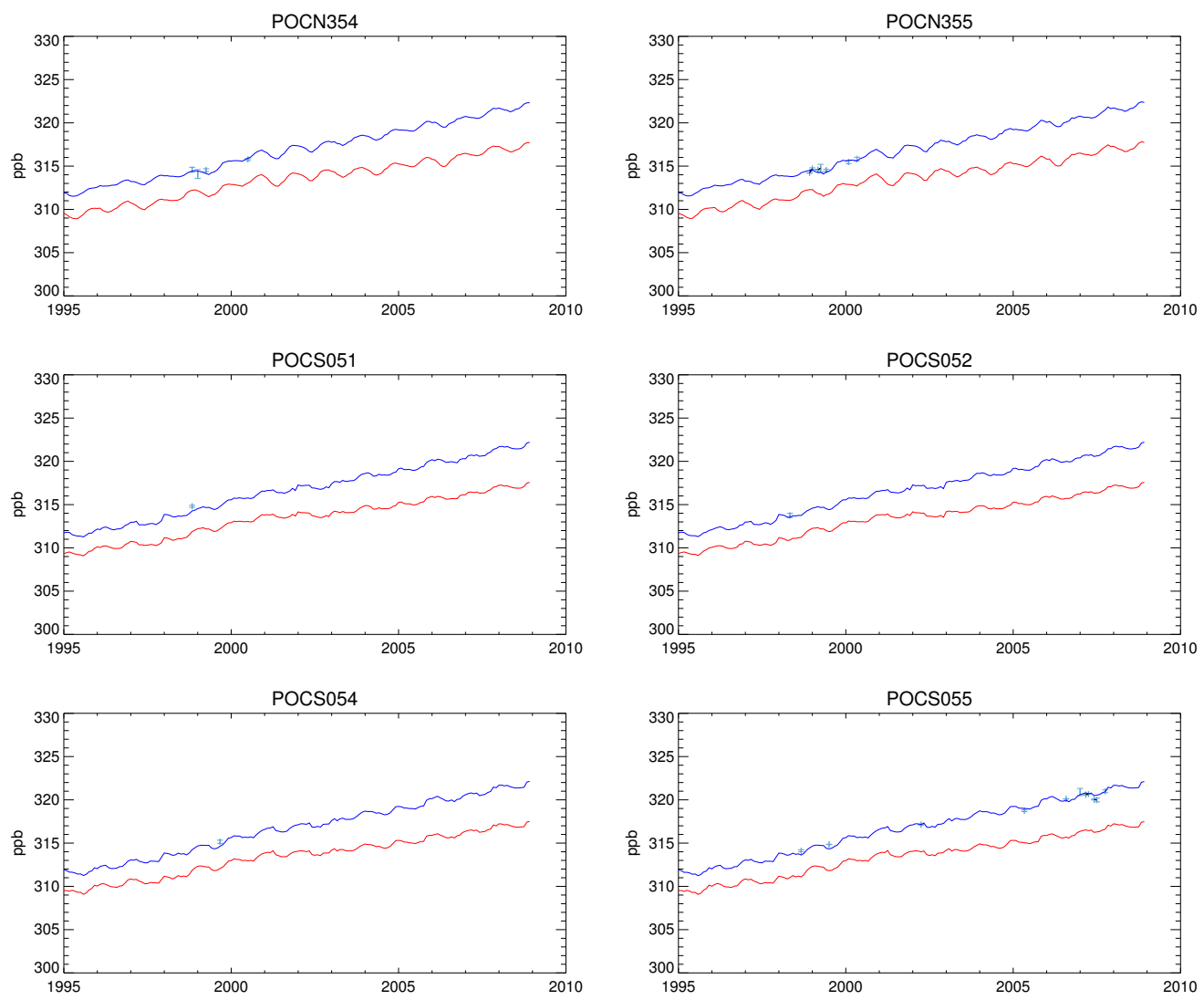

Fig. S1s. Monthly atmospheric mole fractions: measurements (black solid lines with standard deviations), and MOZART v4 model results using prior emissions (red solid lines) and optimized emissions (blue solid lines). 

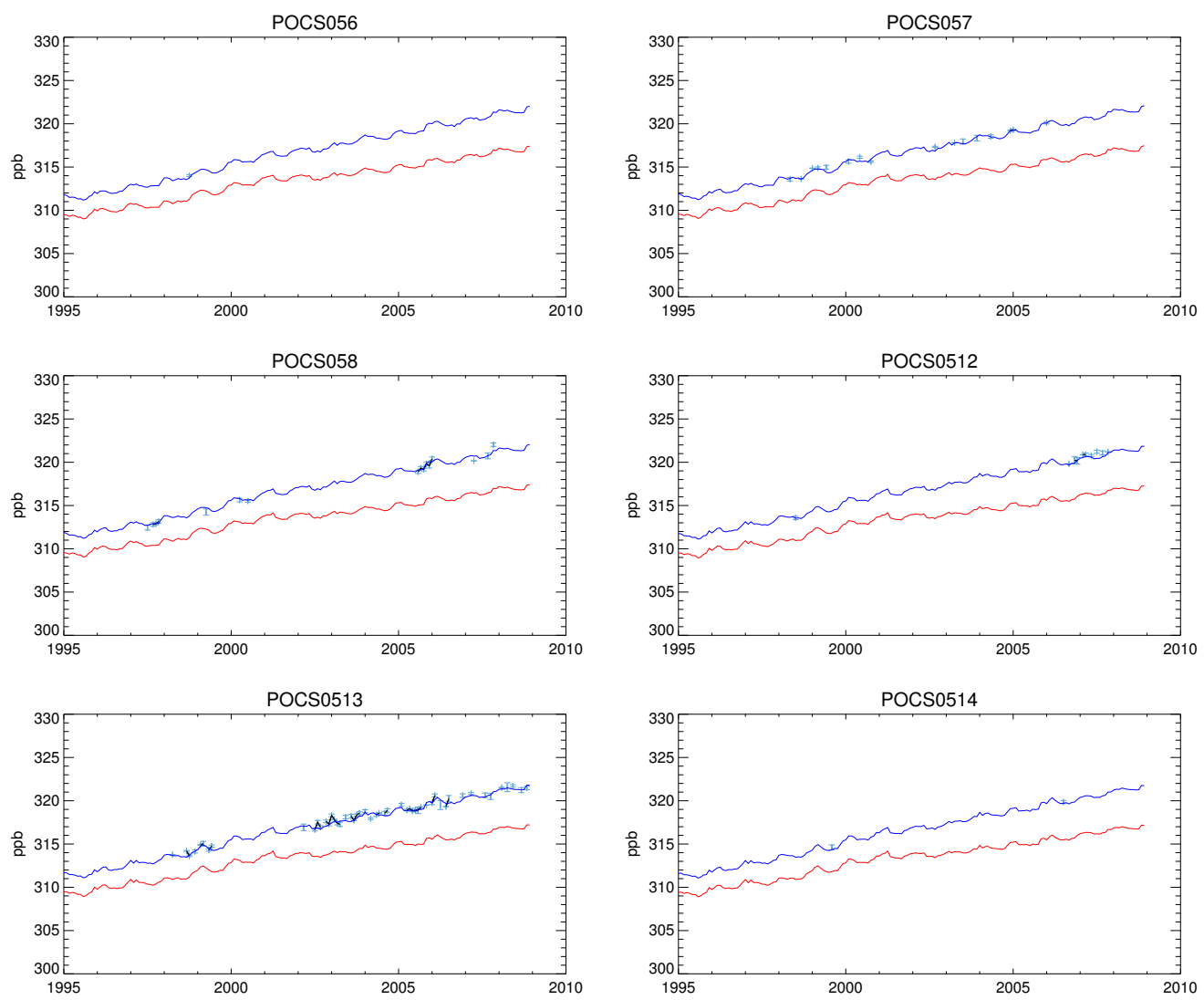

Fig. S1t. Monthly atmospheric mole fractions: measurements (black solid lines with standard deviations), and MOZART v4 model results using prior emissions (red solid lines) and optimized emissions (blue solid lines). 

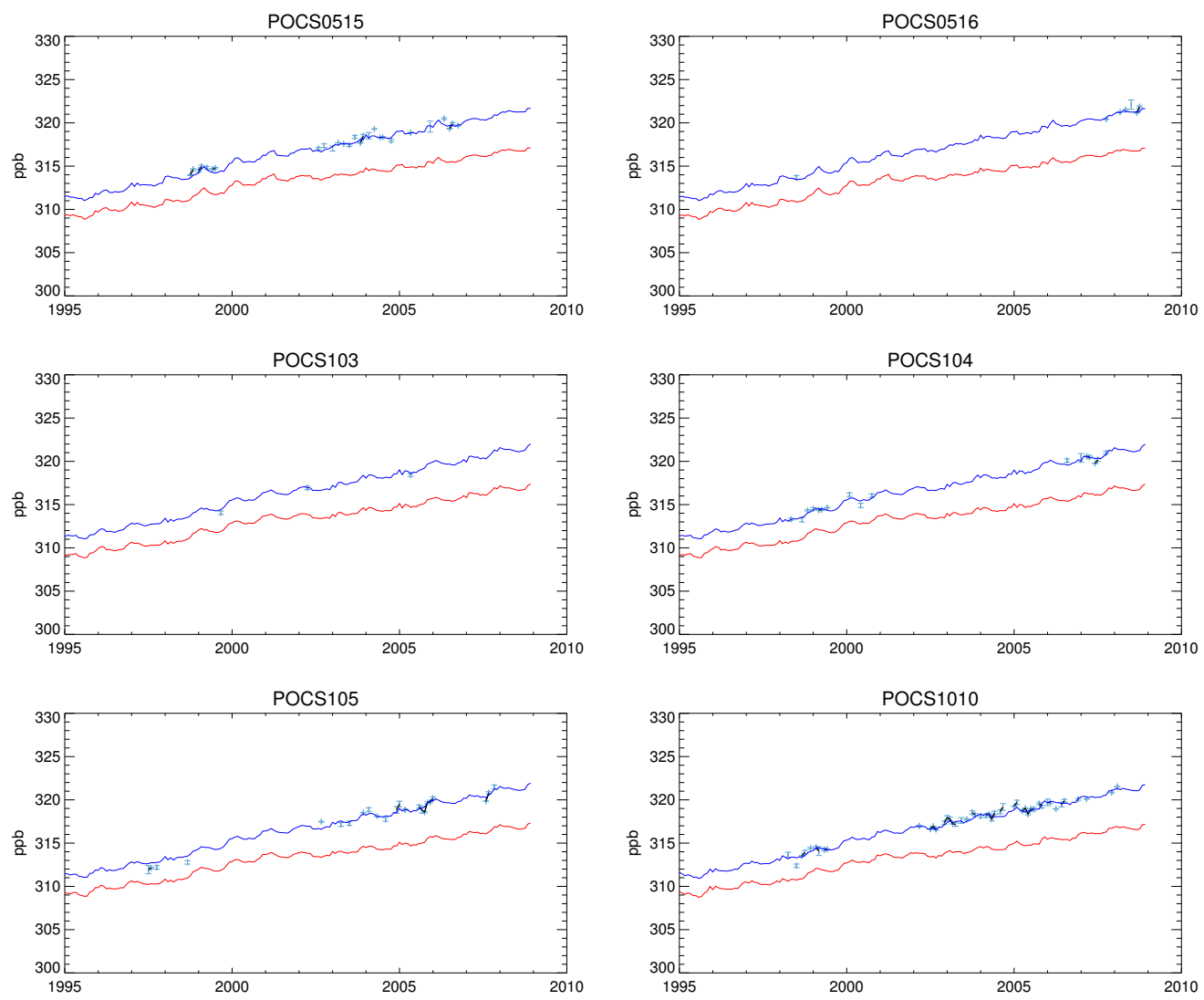

Fig. S1u. Monthly atmospheric mole fractions: measurements (black solid lines with standard deviations), and MOZART v4 model results using prior emissions (red solid lines) and optimized emissions (blue solid lines). 

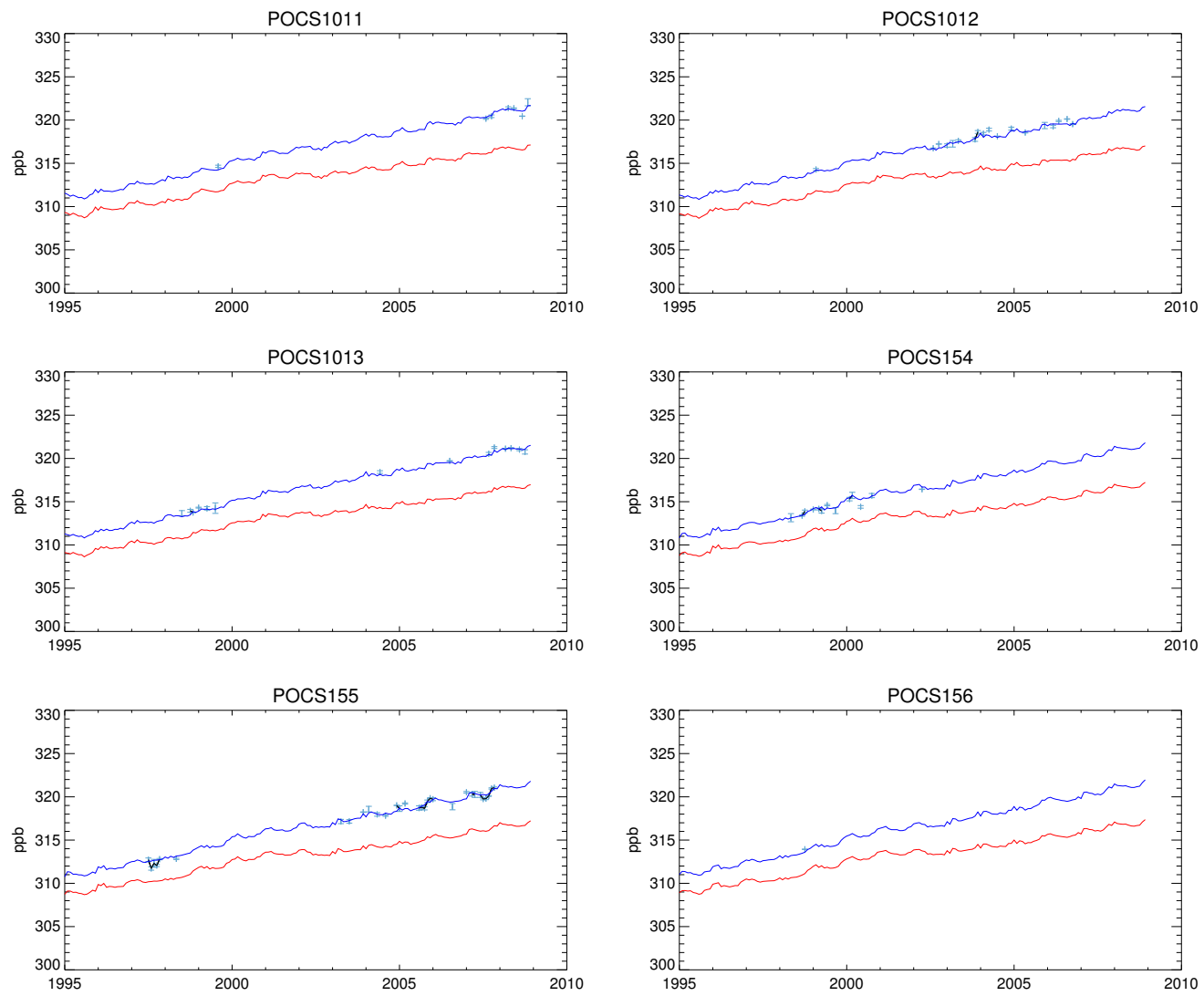

Fig. S1v. Monthly atmospheric mole fractions: measurements (black solid lines with standard deviations), and MOZART v4 model results using prior emissions (red solid lines) and optimized emissions (blue solid lines). 

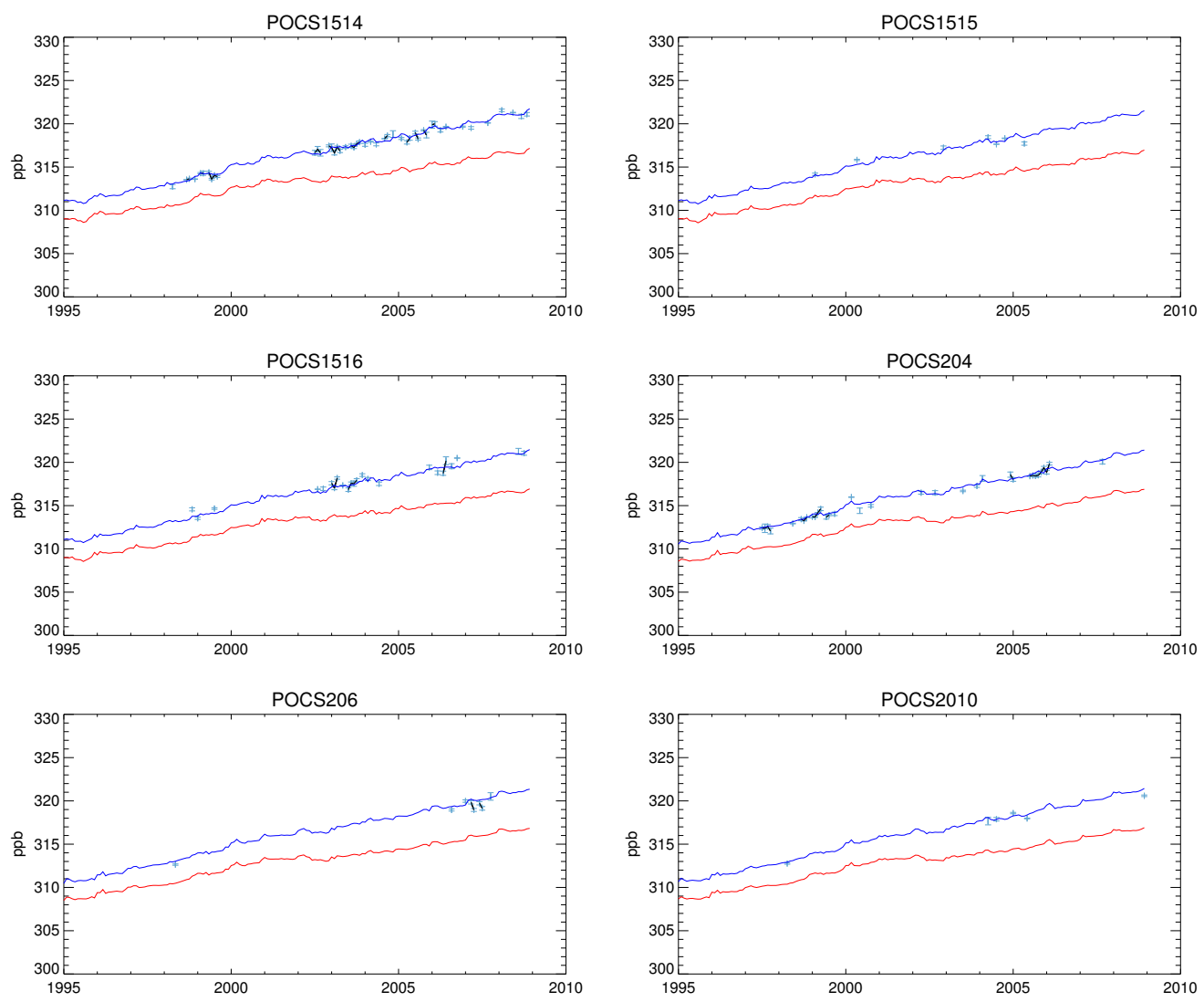

Fig. S1w. Monthly atmospheric mole fractions: measurements (black solid lines with standard deviations), and MOZART v4 model results using prior emissions (red solid lines) and optimized emissions (blue solid lines). 

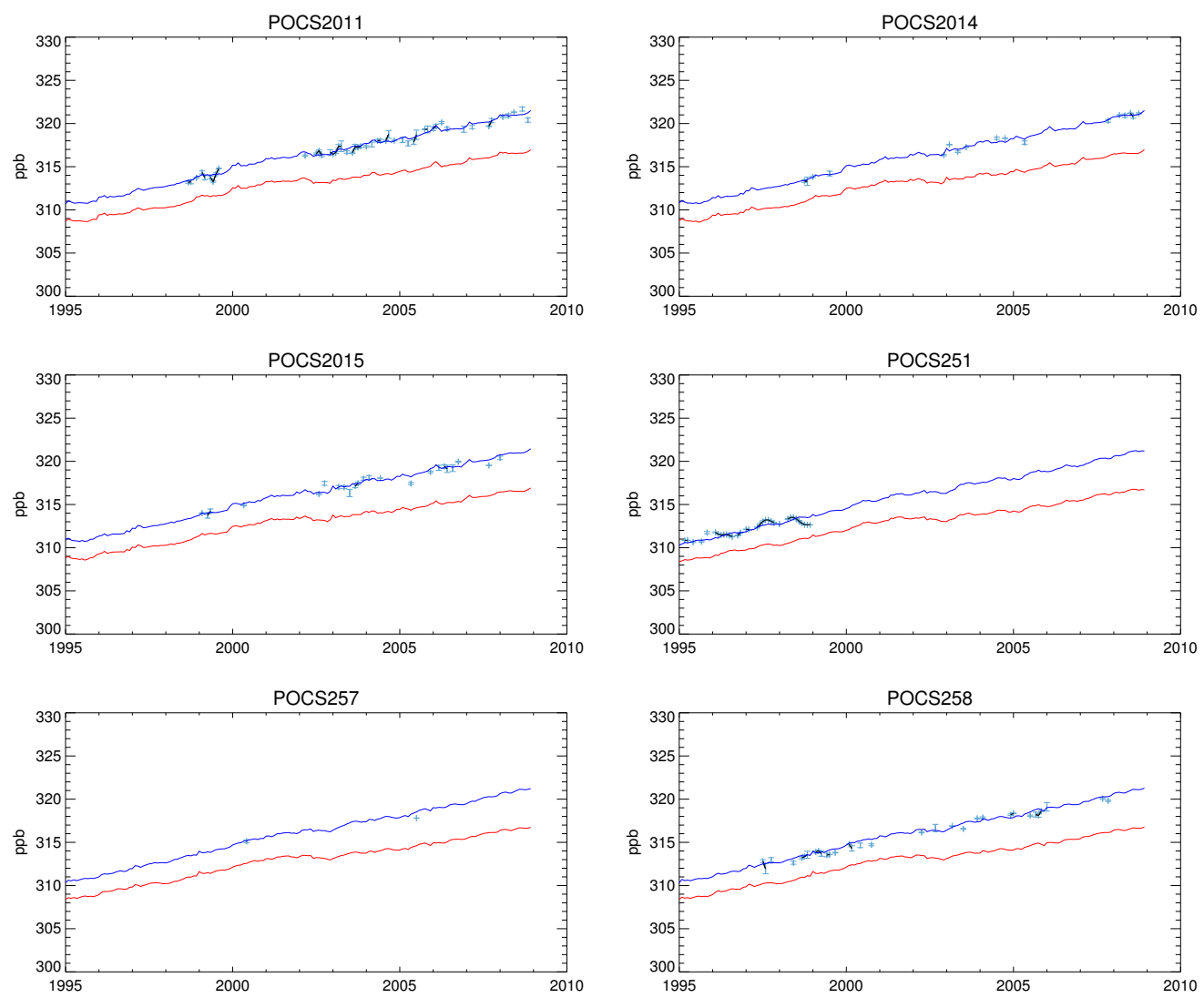

Fig. S1x. Monthly atmospheric mole fractions: measurements (black solid lines with standard deviations), and MOZART v4 model results using prior emissions (red solid lines) and optimized emissions (blue solid lines). 

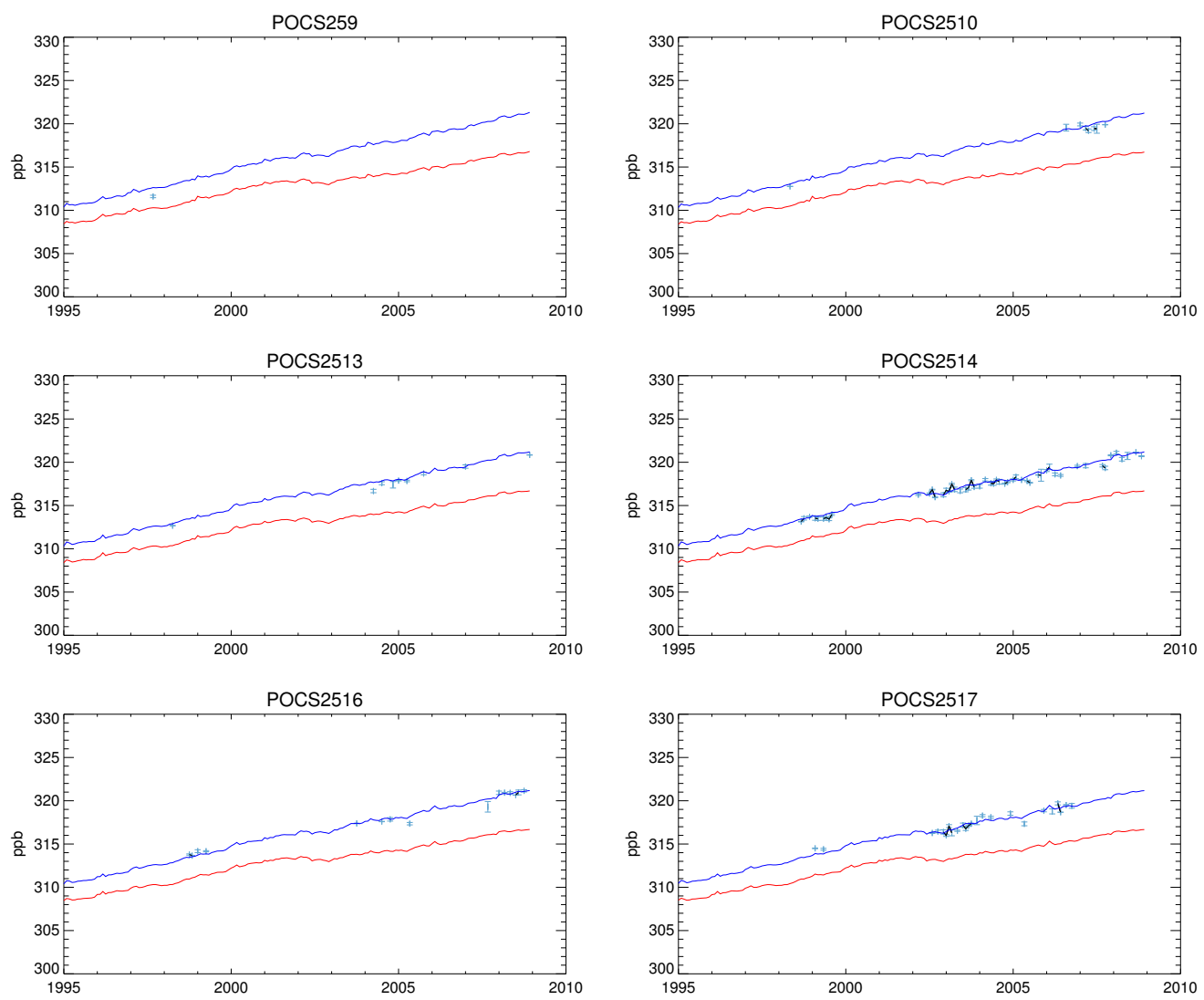

Fig. S1y. Monthly atmospheric mole fractions: measurements (black solid lines with standard deviations), and MOZART v4 model results using prior emissions (red solid lines) and optimized emissions (blue solid lines). 

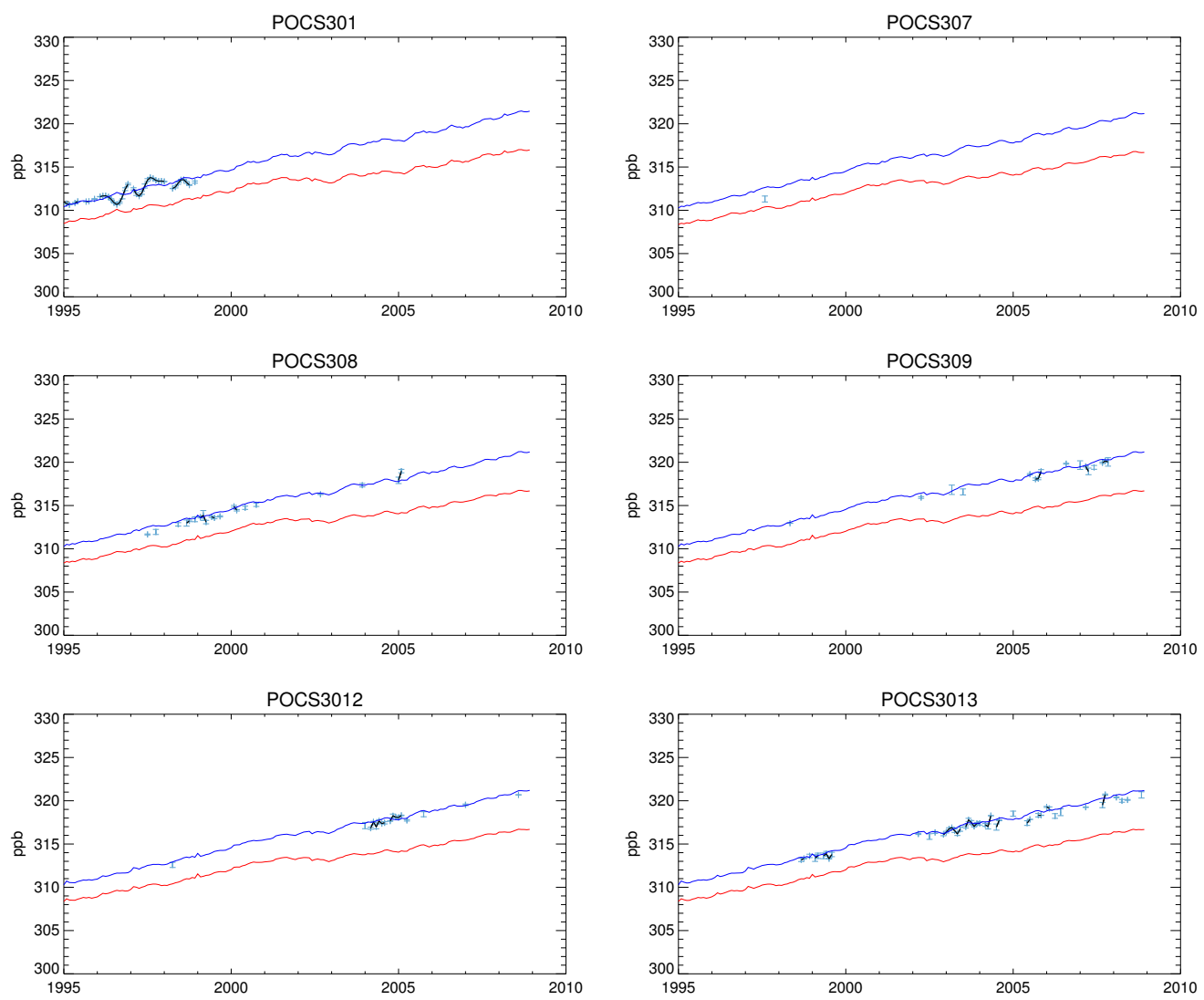

Fig. S1z. Monthly atmospheric mole fractions: measurements (black solid lines with standard deviations), and MOZART v4 model results using prior emissions (red solid lines) and optimized emissions (blue solid lines). 

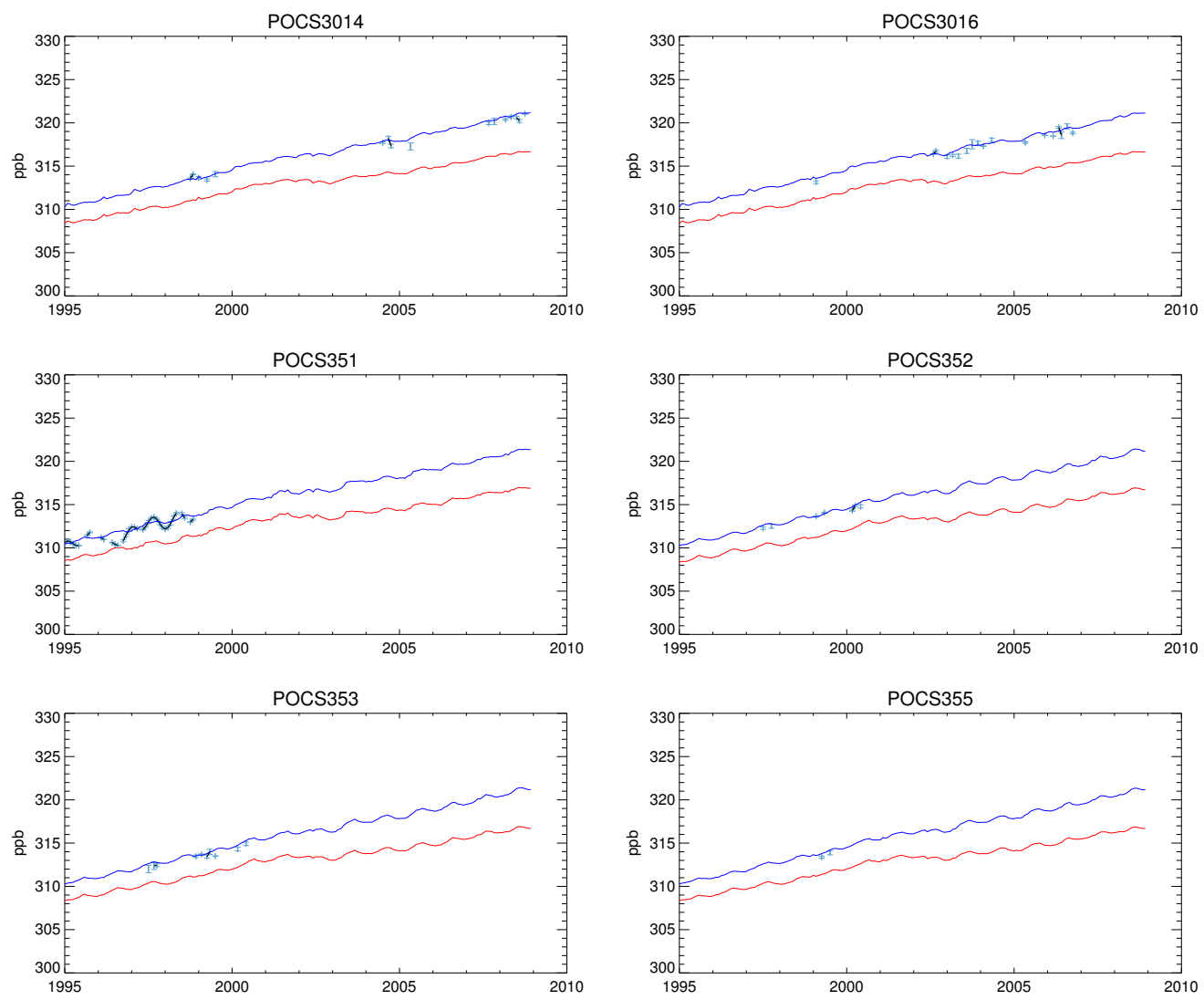

Fig. S1aa. Monthly atmospheric mole fractions: measurements (black solid lines with standard deviations), and MOZART v4 model results using prior emissions (red solid lines) and optimized emissions (blue solid lines). 

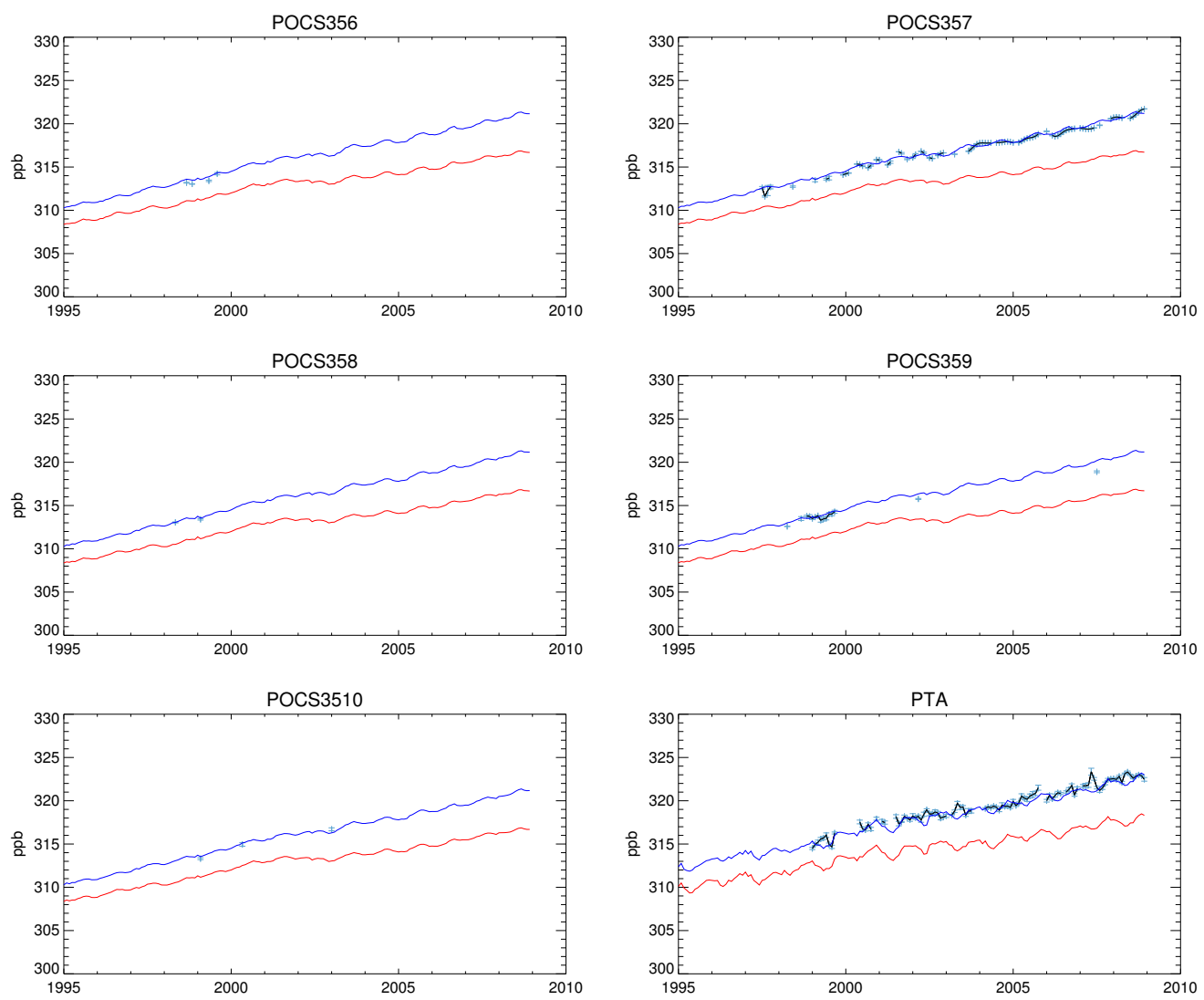

Fig. S1ab. Monthly atmospheric mole fractions: measurements (black solid lines with standard deviations), and MOZART v4 model results using prior emissions (red solid lines) and optimized emissions (blue solid lines). 

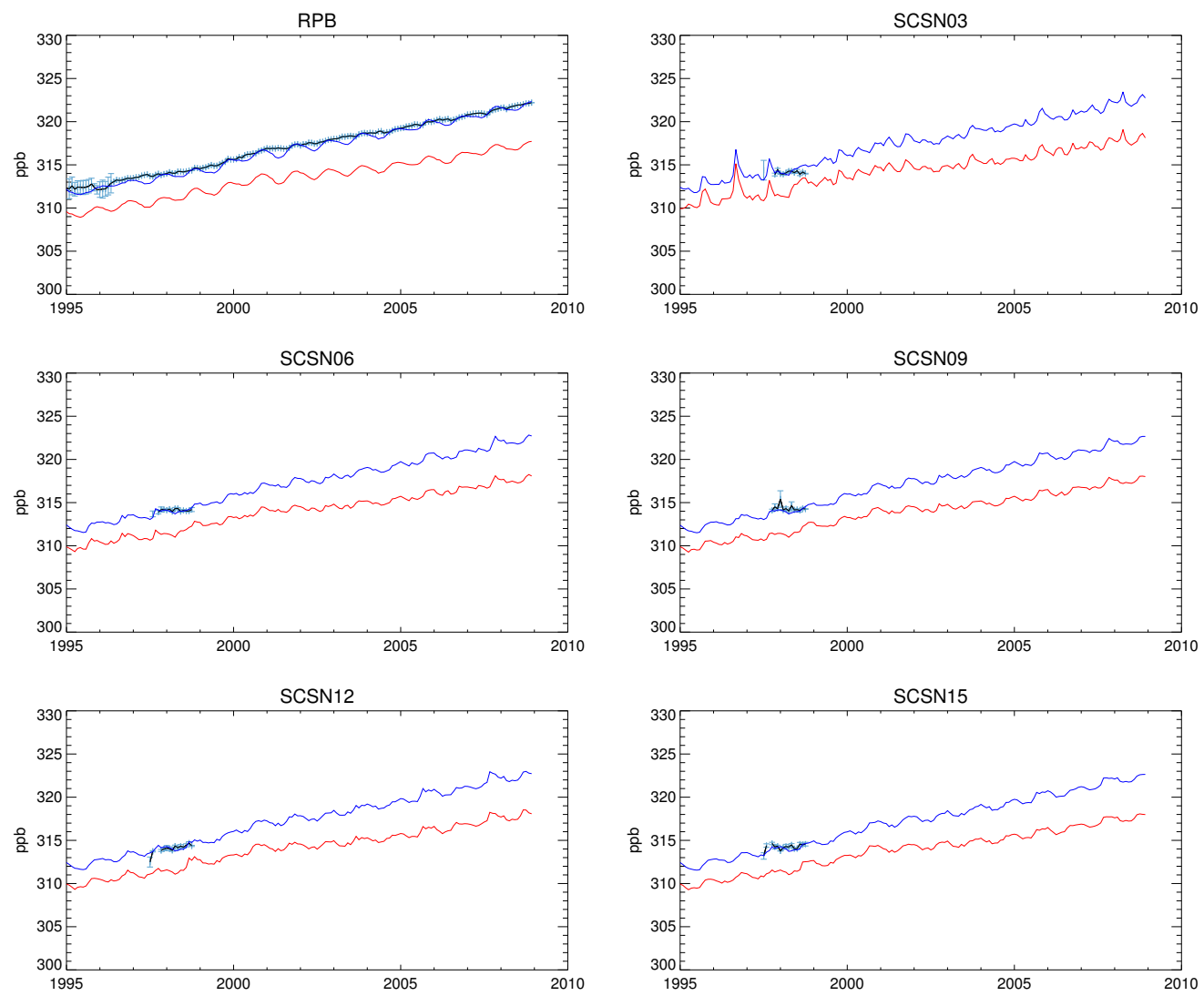

Fig. S1ac. Monthly atmospheric mole fractions: measurements (black solid lines with standard deviations), and MOZART v4 model results using prior emissions (red solid lines) and optimized emissions (blue solid lines). 

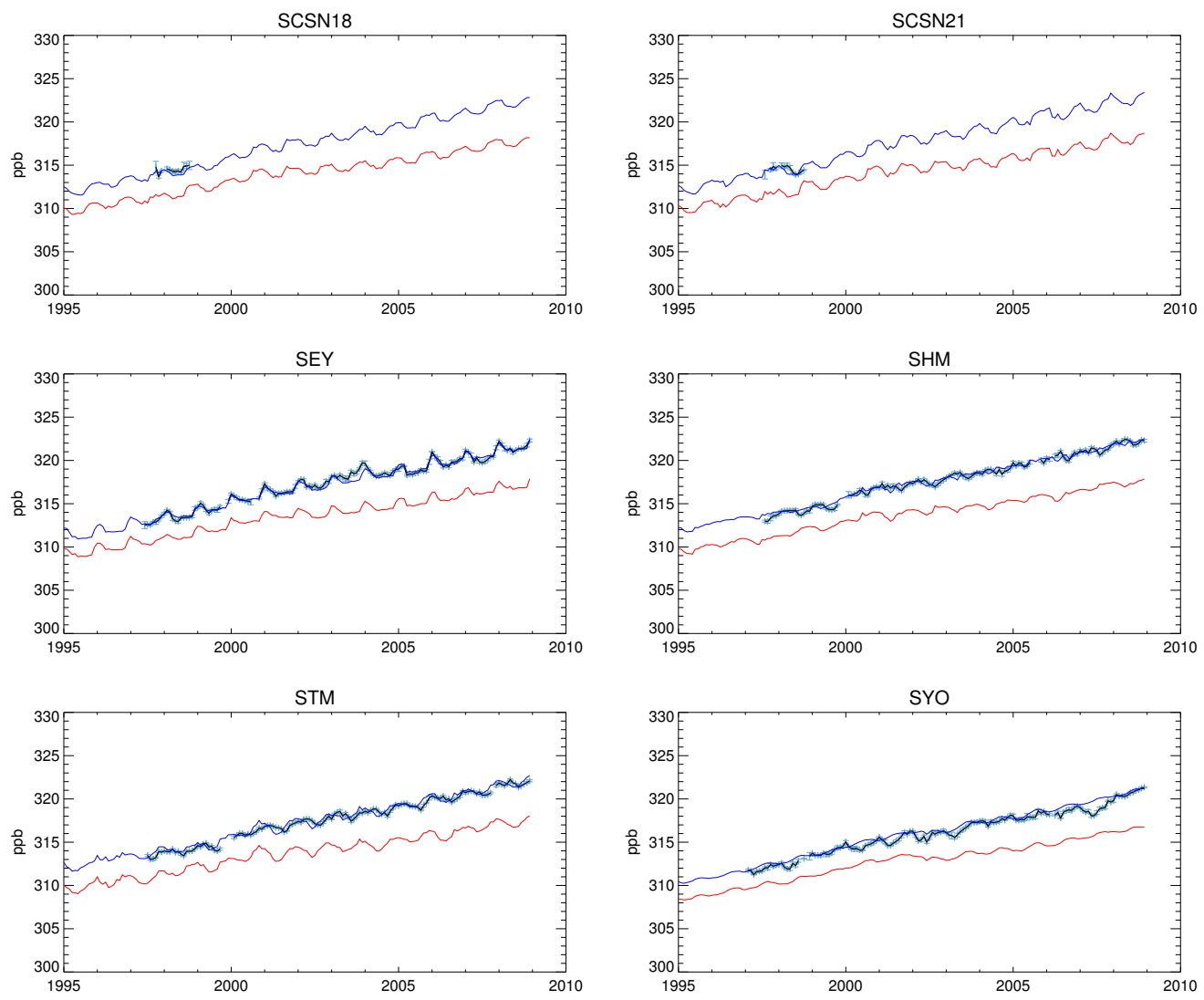

Fig. S1ad. Monthly atmospheric mole fractions: measurements (black solid lines with standard deviations), and MOZART v4 model results using prior emissions (red solid lines) and optimized emissions (blue solid lines). 

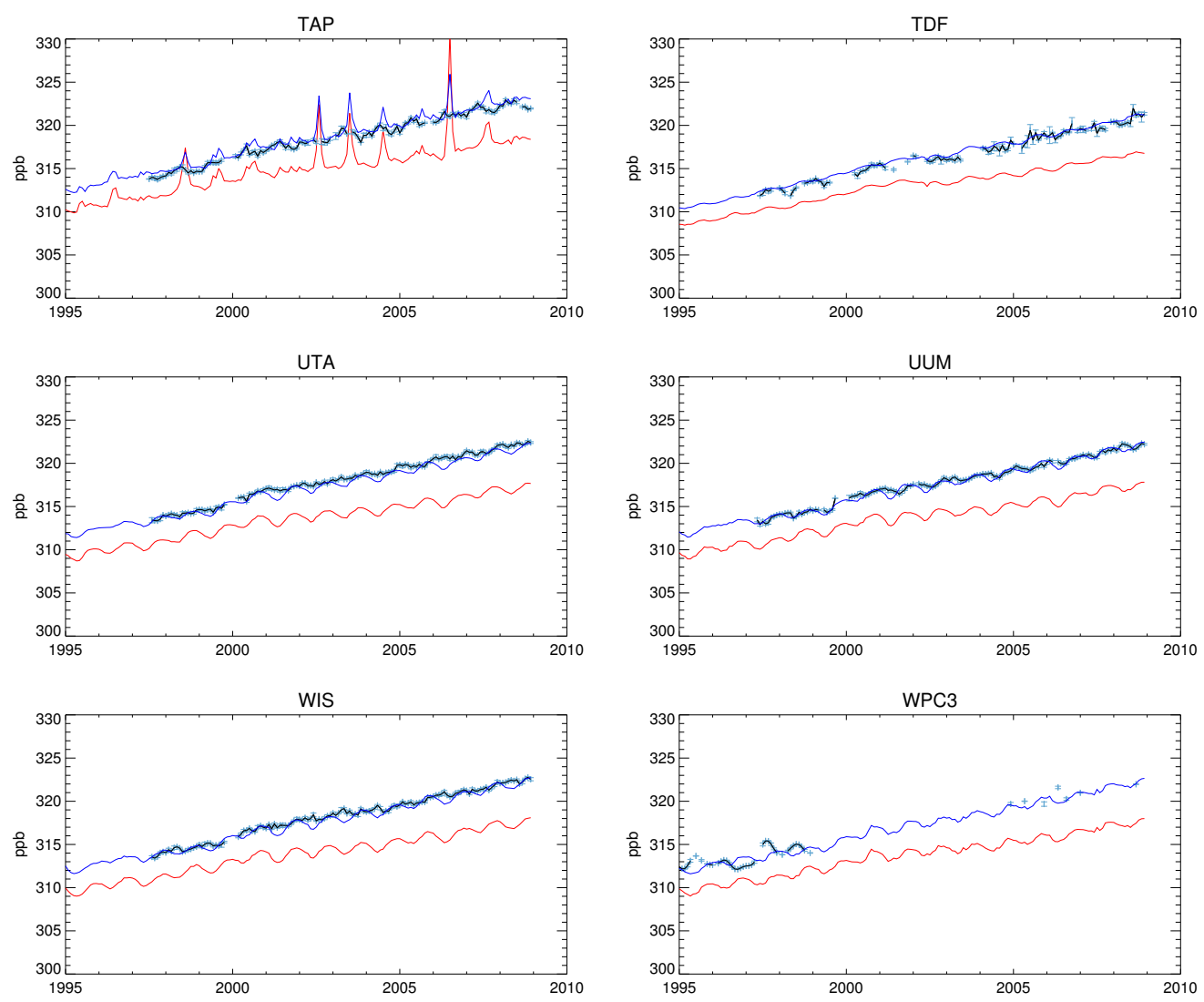

Fig. S1ae. Monthly atmospheric mole fractions: measurements (black solid lines with standard deviations), and MOZART v4 model results using prior emissions (red solid lines) and optimized emissions (blue solid lines). 

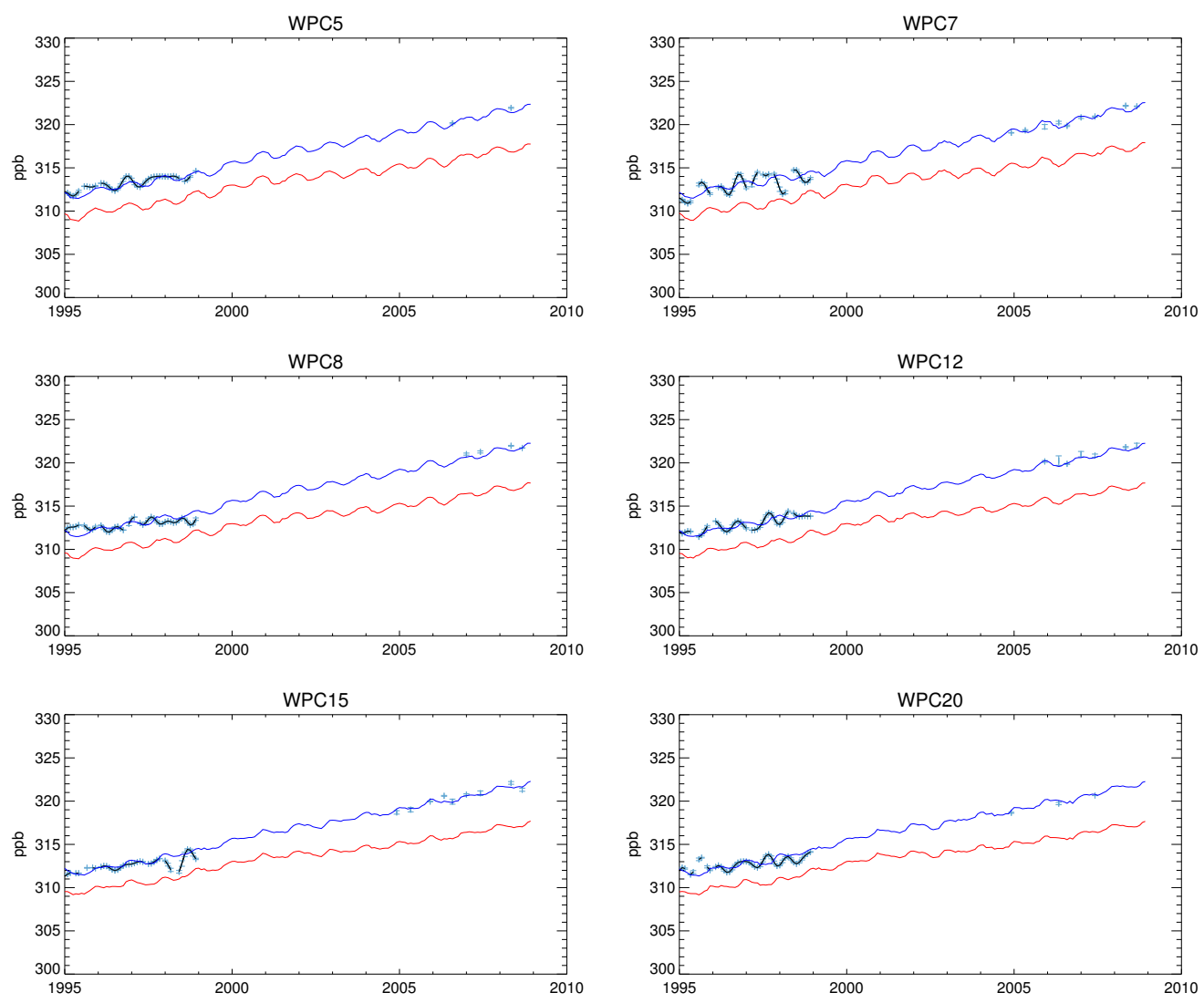

Fig. S1af. Monthly atmospheric mole fractions: measurements (black solid lines with standard deviations), and MOZART v4 model results using prior emissions (red solid lines) and optimized emissions (blue solid lines). 

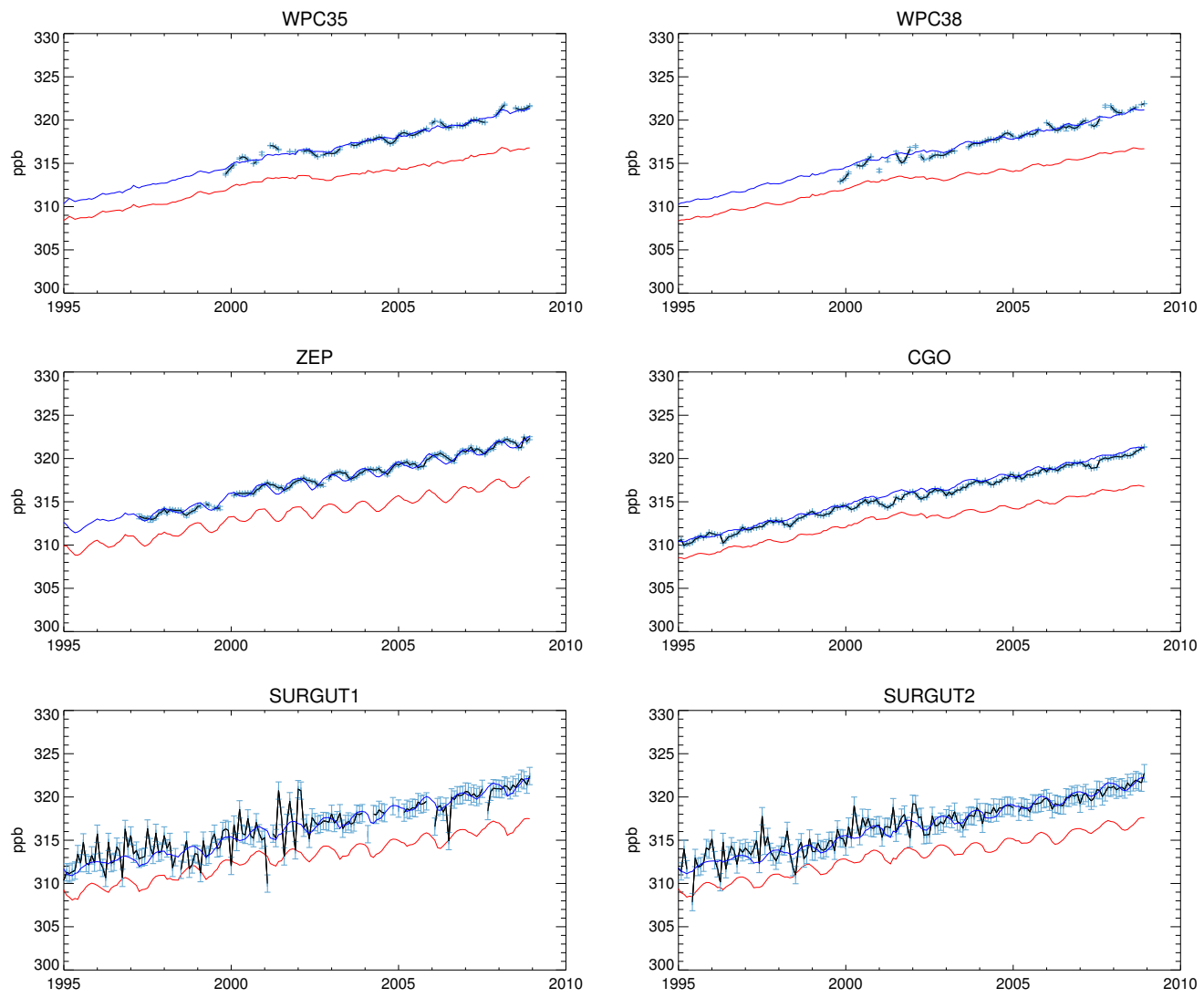

Fig. S1ag. Monthly atmospheric mole fractions: measurements (black solid lines with standard deviations), and MOZART v4 model results using prior emissions (red solid lines) and optimized emissions (blue solid lines). 

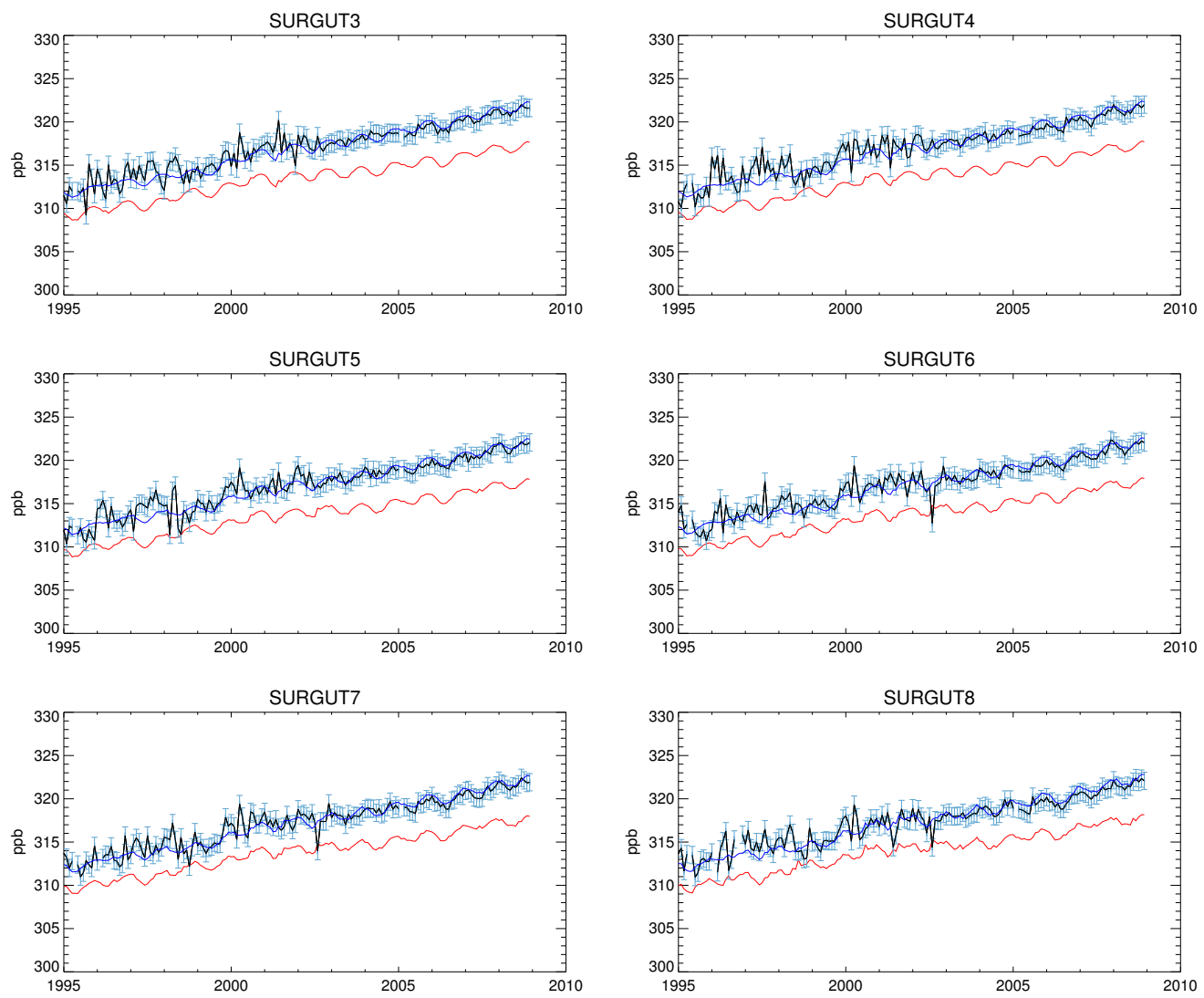

Fig. S1ah. Monthly atmospheric mole fractions: measurements (black solid lines with standard deviations), and MOZART v4 model results using prior emissions (red solid lines) and optimized emissions (blue solid lines). 

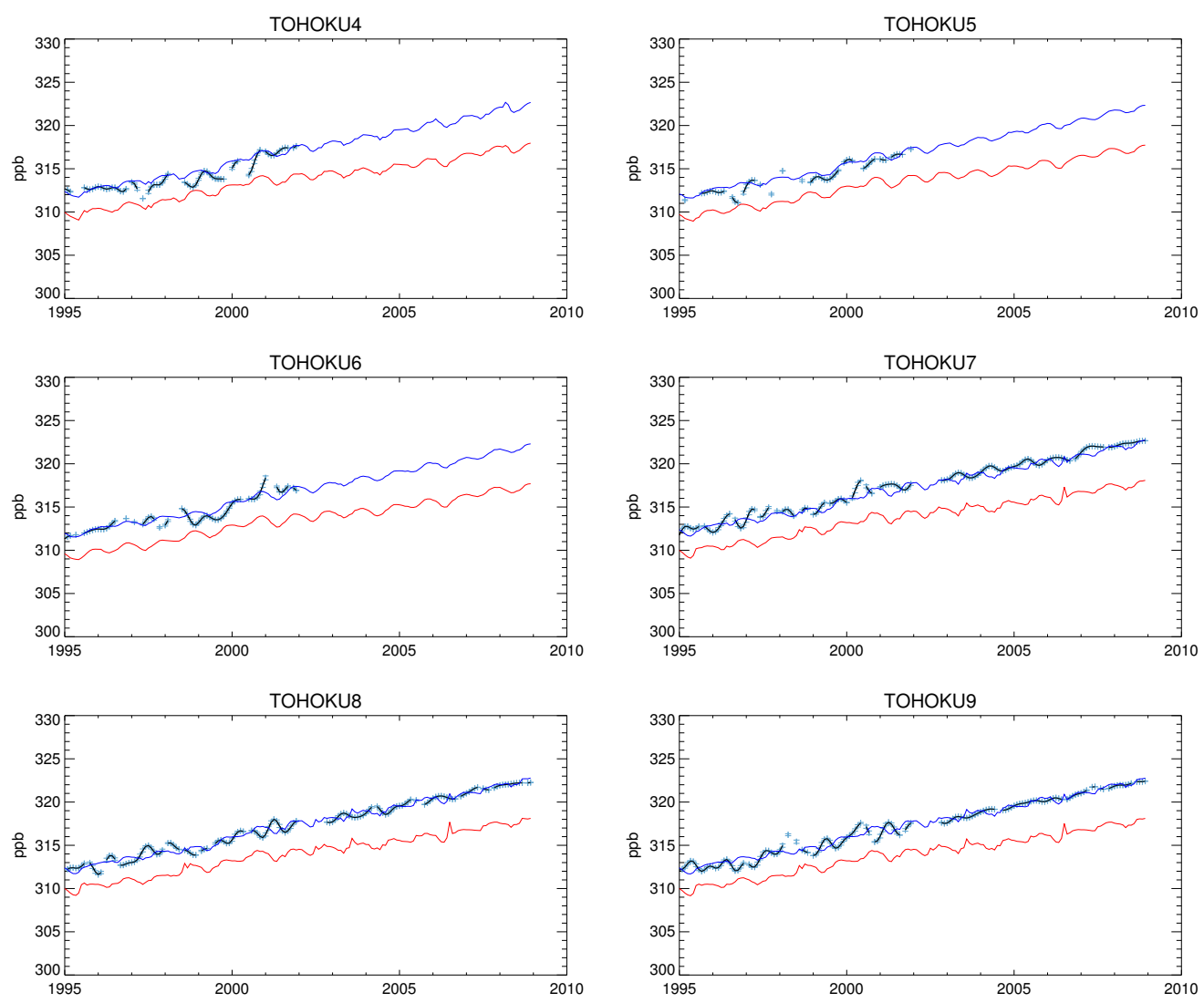

Fig. S1ai. Monthly atmospheric mole fractions: measurements (black solid lines with standard deviations), and MOZART v4 model results using prior emissions (red solid lines) and optimized emissions (blue solid lines). 

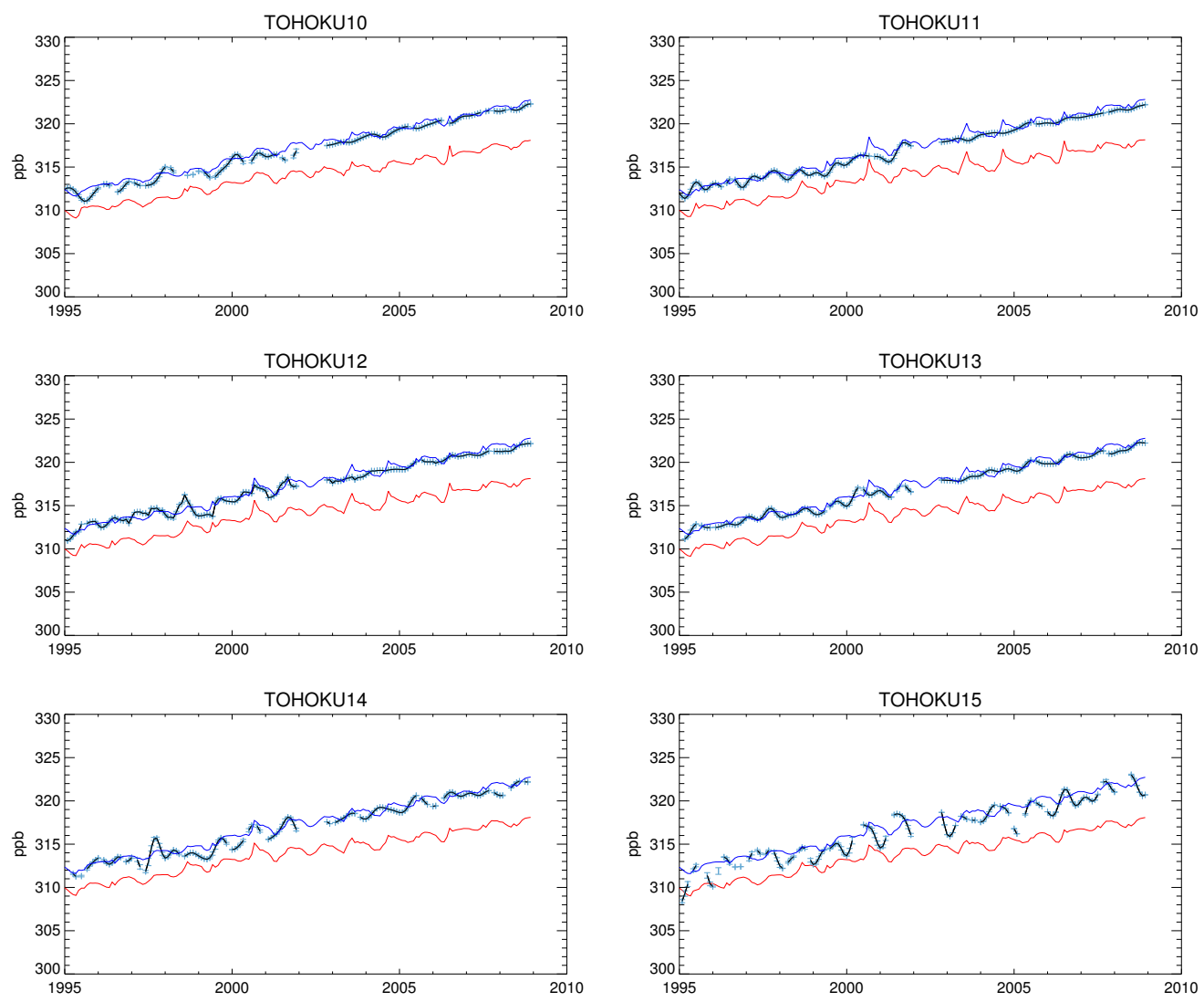

Fig. S1aj. Monthly atmospheric mole fractions: measurements (black solid lines with standard deviations), and MOZART v4 model results using prior emissions (red solid lines) and optimized emissions (blue solid lines). 

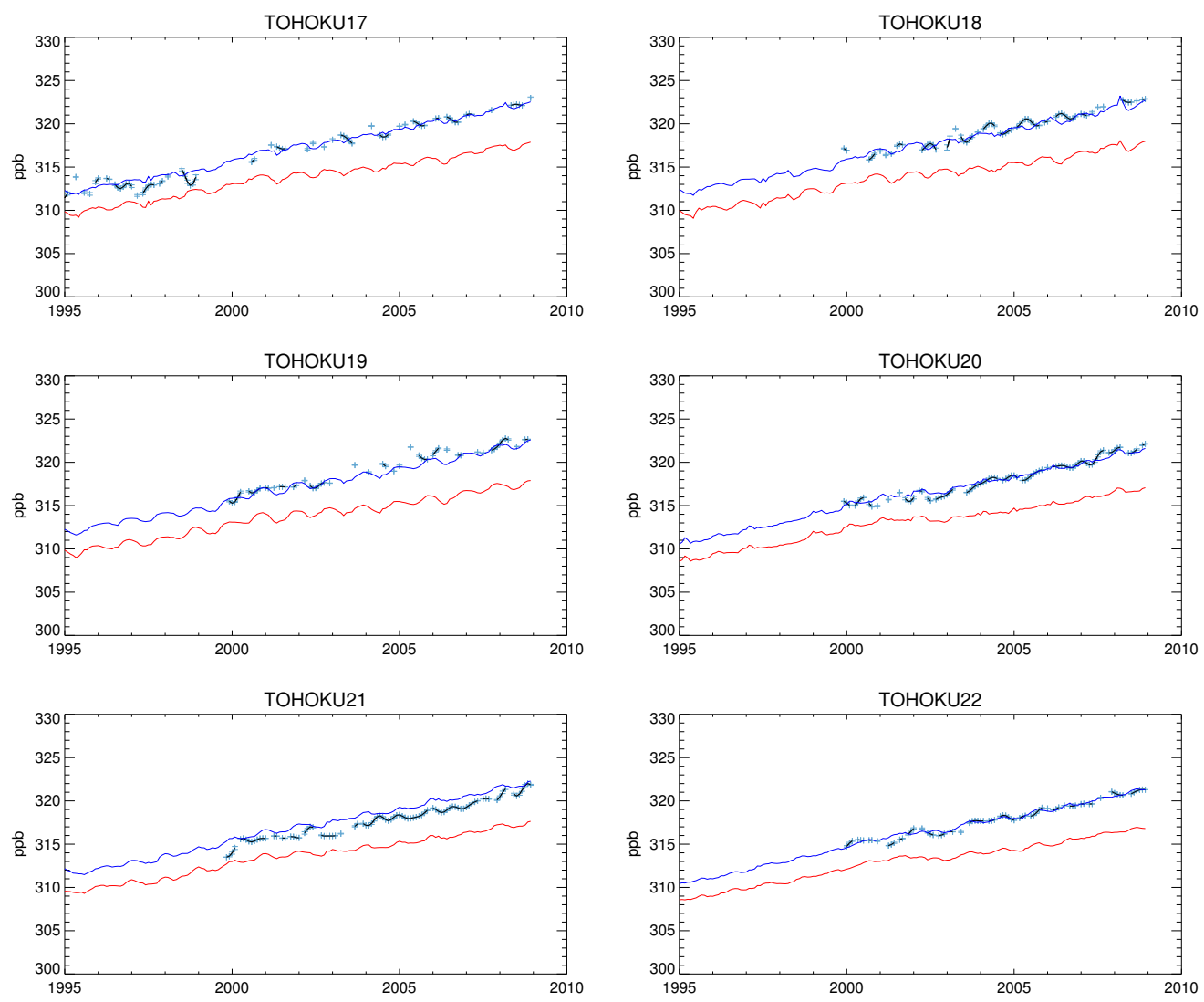

Fig. S1ak. Monthly atmospheric mole fractions: measurements (black solid lines with standard deviations), and MOZART v4 model results using prior emissions (red solid lines) and optimized emissions (blue solid lines). 

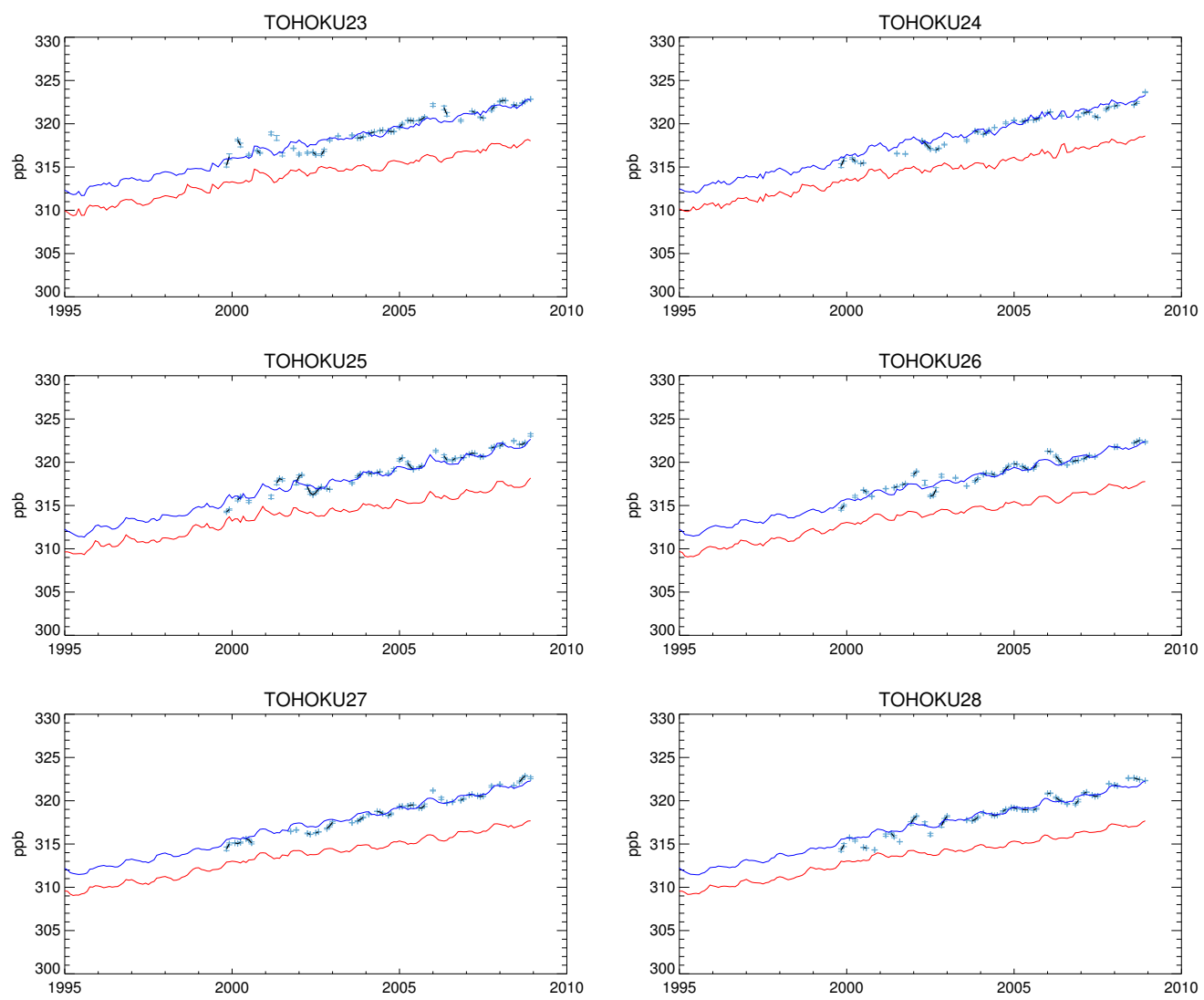

Fig. S1al. Monthly atmospheric mole fractions: measurements (black solid lines with standard deviations), and MOZART v4 model results using prior emissions (red solid lines) and optimized emissions (blue solid lines). 

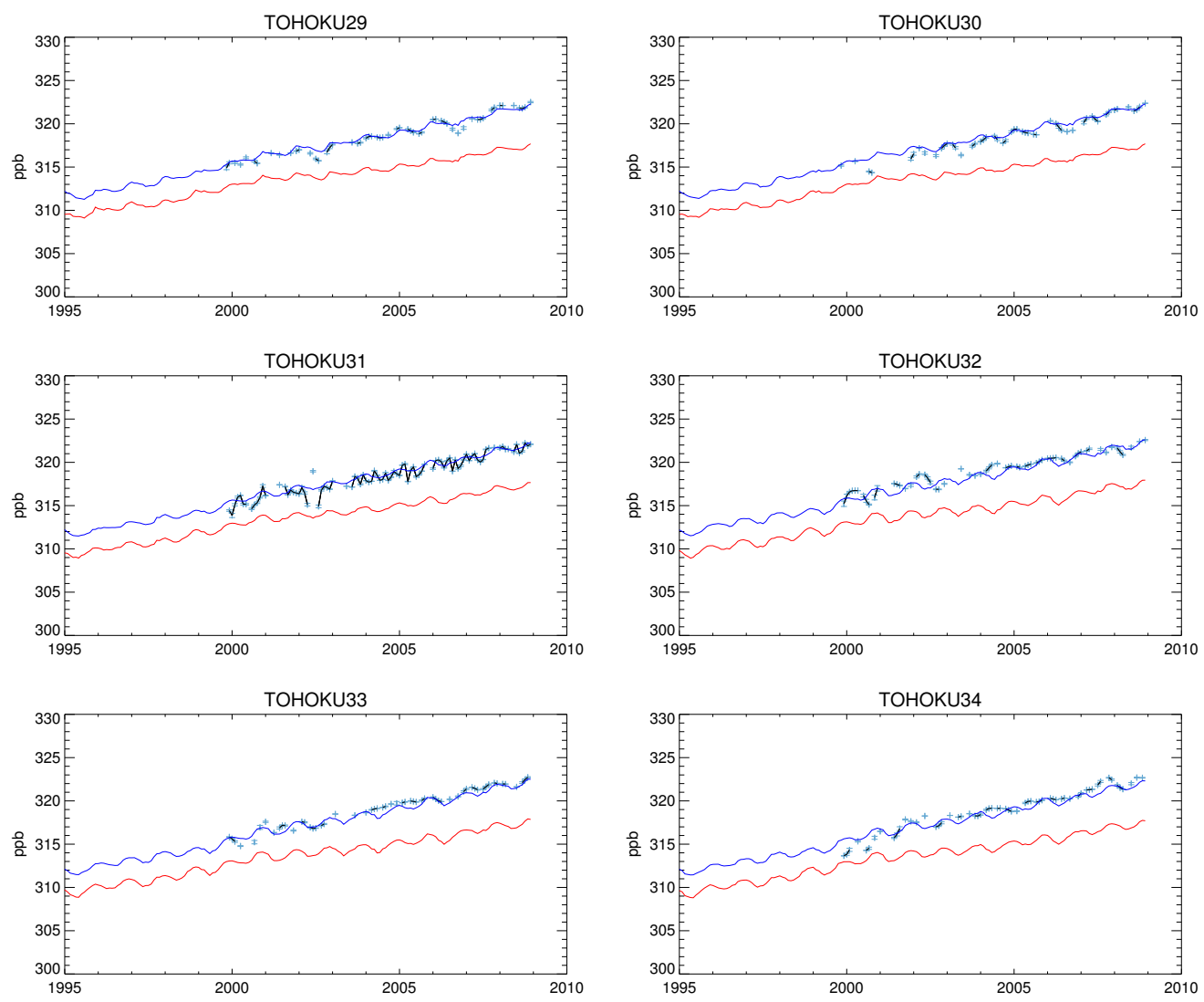

Fig. S1am. Monthly atmospheric mole fractions: measurements (black solid lines with standard deviations), and MOZART v4 model results using prior emissions (red solid lines) and optimized emissions (blue solid lines). 

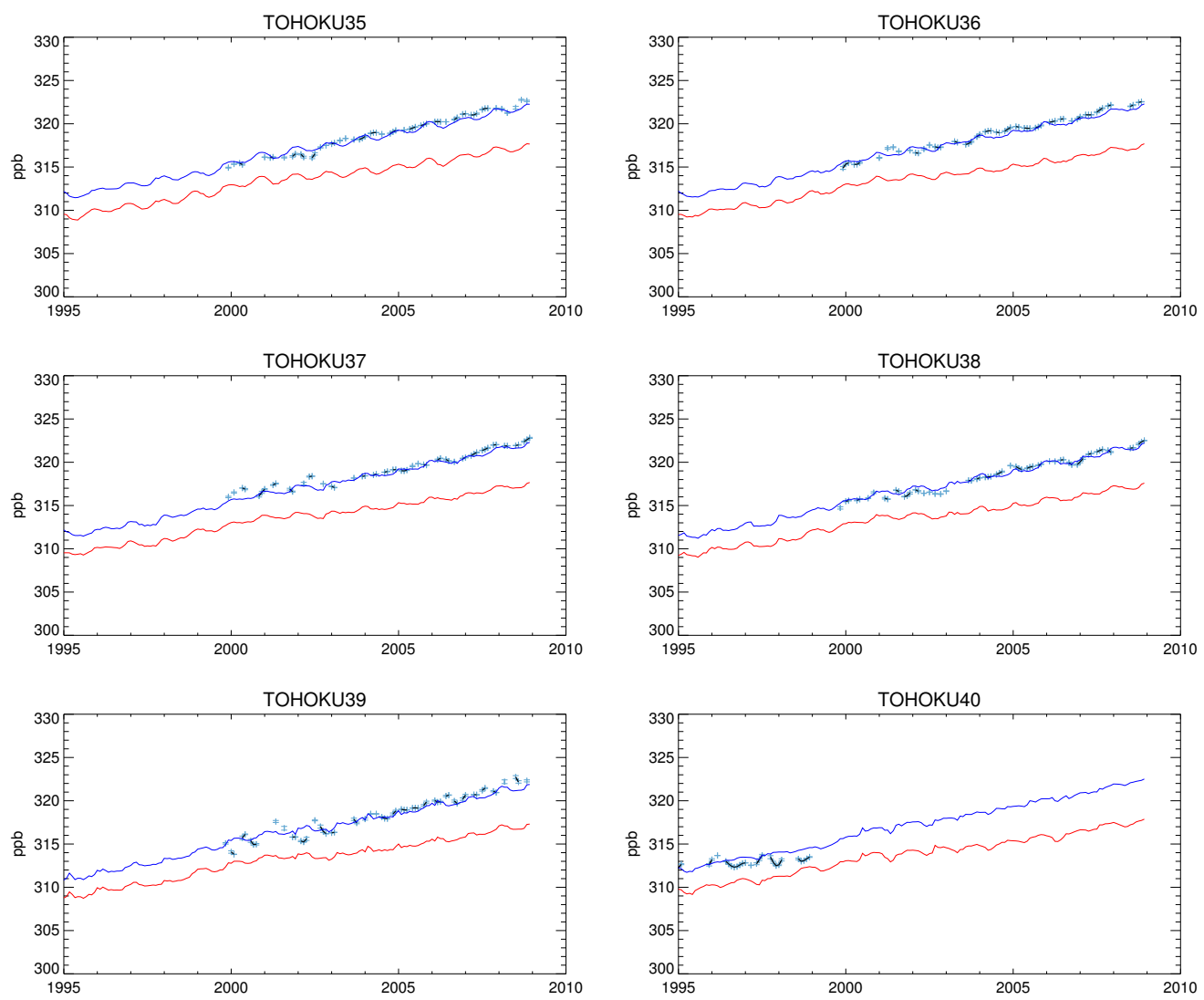

Fig. S1an. Monthly atmospheric mole fractions: measurements (black solid lines with standard deviations), and MOZART v4 model results using prior emissions (red solid lines) and optimized emissions (blue solid lines). 

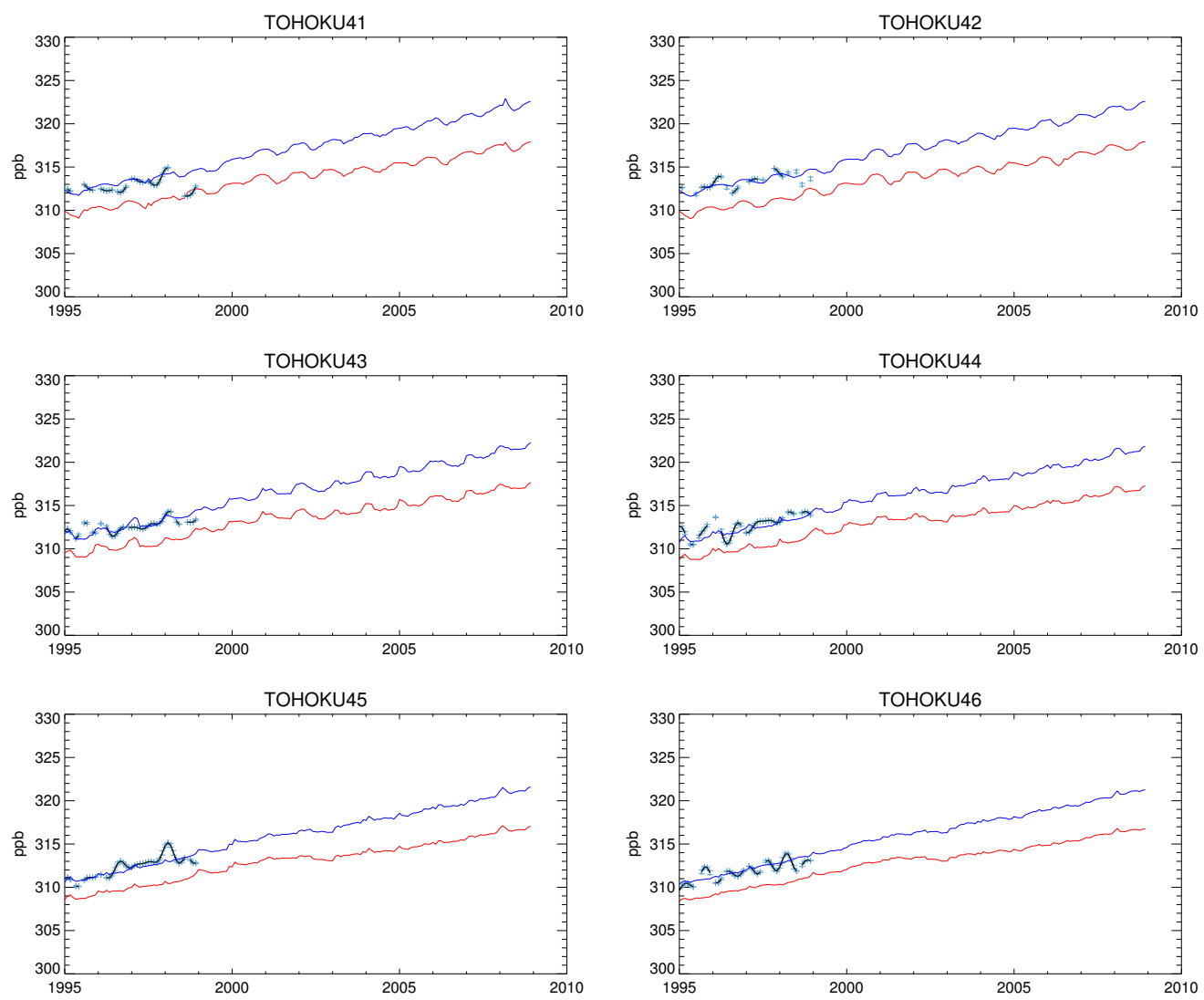

Fig. S1ao. Monthly atmospheric mole fractions: measurements (black solid lines with standard deviations), and MOZART v4 model results using prior emissions (red solid lines) and optimized emissions (blue solid lines). 

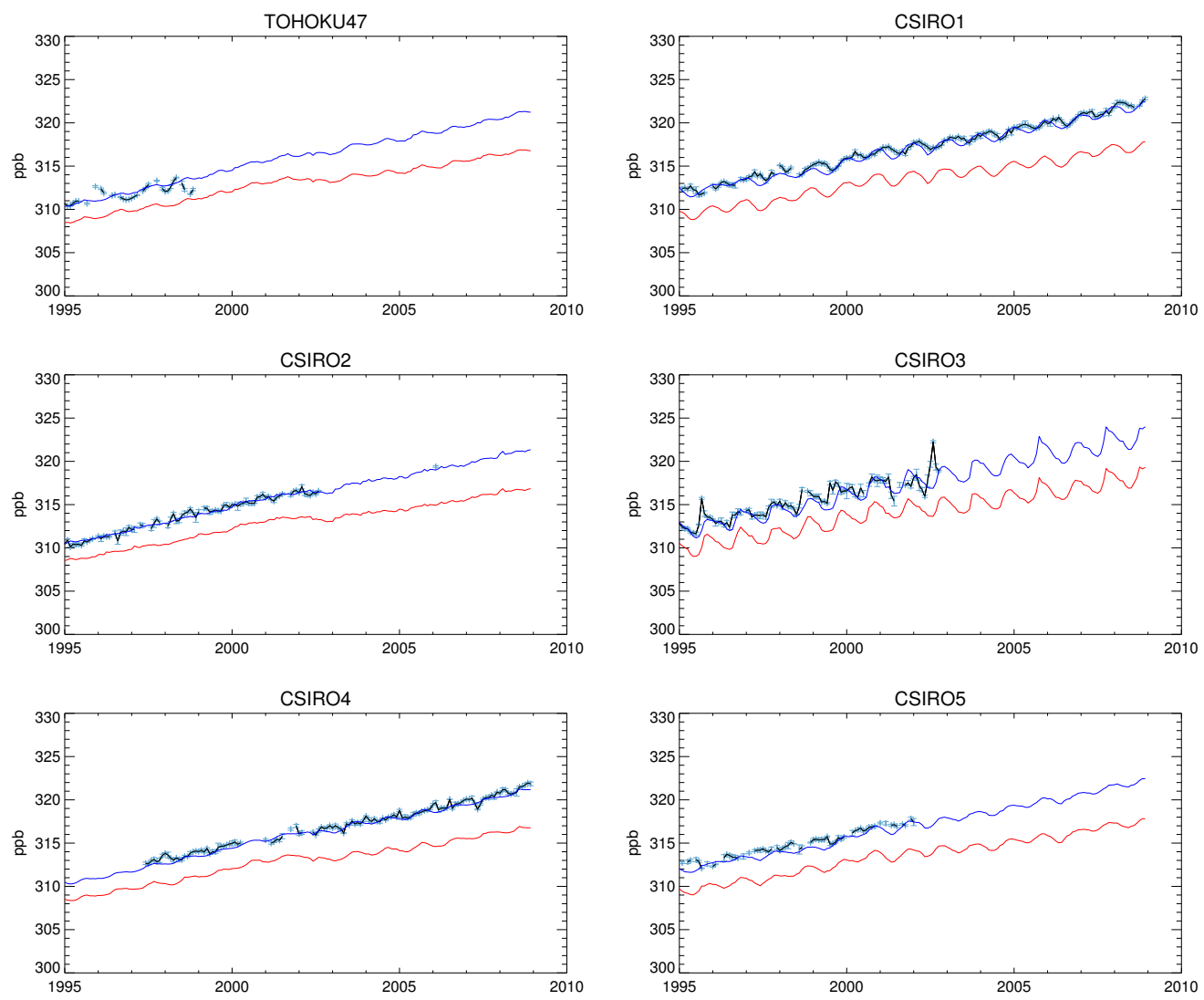

Fig. S1ap. Monthly atmospheric mole fractions: measurements (black solid lines with standard deviations), and MOZART v4 model results using prior emissions (red solid lines) and optimized emissions (blue solid lines). 

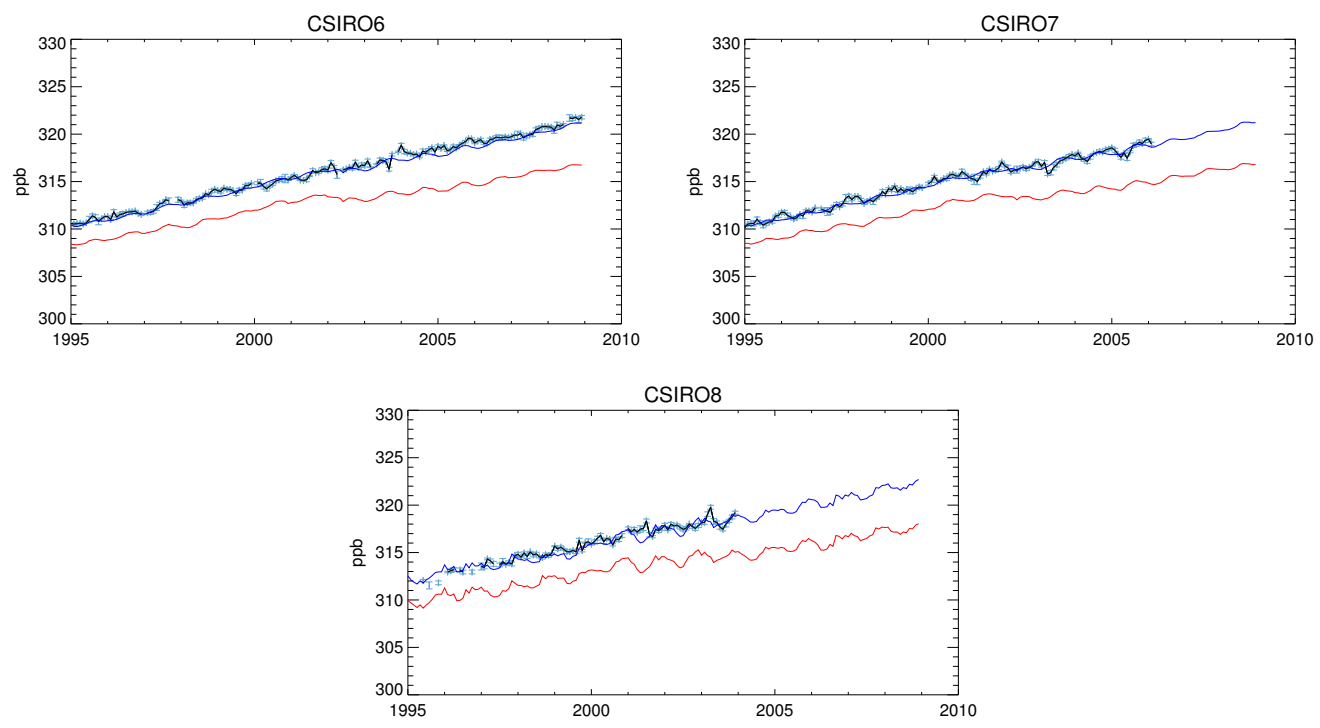

Fig. S1aq. Monthly atmospheric mole fractions: measurements (black solid lines with standard deviations), and MOZART v4 model results using prior emissions (red solid lines) and optimized emissions (blue solid lines). 


\begin{tabular}{|c|c|c|c|c|c|c|c|c|}
\hline \multirow[b]{2}{*}{ Source sector } & \multirow[b]{2}{*}{ Region } & \multicolumn{7}{|c|}{ Agricultural Soil } \\
\hline & & $\begin{array}{l}\text { Africa/M. } \\
\text { East }\end{array}$ & $\begin{array}{l}\text { Southern } \\
\text { Asia }\end{array}$ & $\begin{array}{l}\text { C. /S. } \\
\text { America }\end{array}$ & $\begin{array}{l}\text { Northern } \\
\text { Asia }\end{array}$ & Europe & $\begin{array}{l}\text { North } \\
\text { America }\end{array}$ & Oceania \\
\hline \multirow{7}{*}{$\begin{array}{l}\text { Agricultural } \\
\text { Soil }\end{array}$} & Africa/Middle East & 1 & $-5.30 \mathrm{E}-02$ & $-5.59 \mathrm{E}-03$ & $-2.14 \mathrm{E}-02$ & \begin{tabular}{|l|}
$-1.84 \mathrm{E}-02$ \\
\end{tabular} & $-3.09 \mathrm{E}-02$ & $3.13 \mathrm{E}-03$ \\
\hline & Southern Asia & \begin{tabular}{|l|}
$-5.30 \mathrm{E}-02$ \\
\end{tabular} & 1 & $7.53 \mathrm{E}-03$ & $-9.59 \mathrm{E}-02$ & $1.04 \mathrm{E}-02$ & $-4.49 \mathrm{E}-02$ & $9.10 \mathrm{E}-03$ \\
\hline & Central/South America & $-5.59 \mathrm{E}-03$ & $7.53 \mathrm{E}-03$ & 1 & $-4.50 \mathrm{E}-04$ & $2.61 \mathrm{E}-03$ & $1.39 \mathrm{E}-03$ & $-7.38 \mathrm{E}-03$ \\
\hline & Northern Asia & $-2.14 \mathrm{E}-02$ & $-9.59 \mathrm{E}-02$ & $-4.50 \mathrm{E}-04$ & 1 & $-1.59 \mathrm{E}-01$ & $-4.68 \mathrm{E}-02$ & $-1.09 \mathrm{E}-02$ \\
\hline & Europe & \begin{tabular}{|l|}
$-1.84 \mathrm{E}-02$ \\
\end{tabular} & $1.04 \mathrm{E}-02$ & $2.61 \mathrm{E}-03$ & $-1.59 \mathrm{E}-01$ & 1 & $-6.24 \mathrm{E}-02$ & $7.76 \mathrm{E}-04$ \\
\hline & North America & \begin{tabular}{|l|}
$-3.09 E-02$ \\
\end{tabular} & $-4.49 \mathrm{E}-02$ & $1.39 \mathrm{E}-03$ & $-4.68 \mathrm{E}-02$ & \begin{tabular}{|l|}
$-6.24 \mathrm{E}-02$ \\
\end{tabular} & 1 & $1.93 \mathrm{E}-03$ \\
\hline & Oceania & $3.13 \mathrm{E}-03$ & $9.10 \mathrm{E}-03$ & $-7.38 \mathrm{E}-03$ & $-1.09 \mathrm{E}-02$ & $7.76 \mathrm{E}-04$ & $1.93 \mathrm{E}-03$ & 1 \\
\hline \multirow{7}{*}{ Industrial } & Africa/Middle East & $-5.86 \mathrm{E}-03$ & $-9.86 \mathrm{E}-03$ & $-9.06 \mathrm{E}-04$ & $-6.11 \mathrm{E}-03$ & $-5.35 \mathrm{E}-03$ & $-7.09 \mathrm{E}-03$ & $3.23 \mathrm{E}-04$ \\
\hline & Southern Asia & $8.80 \mathrm{E}-03$ & $-2.52 \mathrm{E}-01$ & $1.97 \mathrm{E}-03$ & $-3.44 \mathrm{E}-02$ & $1.72 \mathrm{E}-02$ & $1.84 \mathrm{E}-02$ & $-4.37 \mathrm{E}-03$ \\
\hline & Central/South America & \begin{tabular}{|l|}
$-5.05 E-04$ \\
\end{tabular} & $6.47 \mathrm{E}-04$ & $-1.33 \mathrm{E}-03$ & $-3.85 \mathrm{E}-05$ & $2.25 \mathrm{E}-04$ & 9.49E-05 & $-6.51 \mathrm{E}-04$ \\
\hline & Northern Asia & $-9.95 \mathrm{E}-03$ & $-4.40 \mathrm{E}-02$ & $1.10 \mathrm{E}-03$ & $-8.19 \mathrm{E}-02$ & \begin{tabular}{|l|}
$-7.72 \mathrm{E}-02$ \\
\end{tabular} & $-2.65 \mathrm{E}-02$ & $-6.61 E-03$ \\
\hline & Europe & \begin{tabular}{|l|}
$-2.45 \mathrm{E}-02$ \\
\end{tabular} & $1.56 \mathrm{E}-03$ & $2.88 \mathrm{E}-03$ & $-1.42 \mathrm{E}-01$ & -3.96E-01 & $-6.79 \mathrm{E}-02$ & $1.37 \mathrm{E}-03$ \\
\hline & North America & \begin{tabular}{|l|}
$-1.56 \mathrm{E}-02$ \\
\end{tabular} & $-2.15 \mathrm{E}-02$ & $7.58 \mathrm{E}-04$ & $-2.30 \mathrm{E}-02$ & \begin{tabular}{|l|}
$-3.31 \mathrm{E}-02$ \\
\end{tabular} & $-6.12 \mathrm{E}-02$ & $9.82 \mathrm{E}-04$ \\
\hline & Oceania & $2.33 \mathrm{E}-04$ & $6.32 \mathrm{E}-04$ & $-4.49 \mathrm{E}-04$ & $-8.24 \mathrm{E}-04$ & $8.22 \mathrm{E}-05$ & $1.19 \mathrm{E}-04$ & $-8.90 \mathrm{E}-03$ \\
\hline \multirow{7}{*}{ Natural Soil } & Africa/Middle East & \begin{tabular}{|l|}
$-6.45 \mathrm{E}-02$ \\
\end{tabular} & $-7.07 \mathrm{E}-02$ & $-2.61 \mathrm{E}-02$ & $-9.84 \mathrm{E}-03$ & \begin{tabular}{|l|}
$-7.65 E-03$ \\
\end{tabular} & $-4.29 \mathrm{E}-02$ & $6.74 \mathrm{E}-03$ \\
\hline & Southern Asia & \begin{tabular}{|l|}
$-3.12 \mathrm{E}-02$ \\
\end{tabular} & $-3.16 \mathrm{E}-01$ & $-1.00 \mathrm{E}-02$ & $-2.34 \mathrm{E}-02$ & $2.08 \mathrm{E}-02$ & $-9.64 \mathrm{E}-03$ & $3.22 \mathrm{E}-02$ \\
\hline & Central/South America & \begin{tabular}{|l|}
$-4.72 \mathrm{E}-02$ \\
\end{tabular} & $3.23 \mathrm{E}-02$ & $-7.88 \mathrm{E}-02$ & $3.71 \mathrm{E}-03$ & $2.23 \mathrm{E}-02$ & $-1.35 \mathrm{E}-02$ & $-4.70 \mathrm{E}-03$ \\
\hline & Northern Asia & $2.92 \mathrm{E}-02$ & $-1.80 \mathrm{E}-02$ & $-4.93 \mathrm{E}-03$ & $-9.46 \mathrm{E}-02$ & $-1.26 \mathrm{E}-02$ & $2.89 \mathrm{E}-02$ & $-2.16 \mathrm{E}-02$ \\
\hline & Europe & $-4.44 \mathrm{E}-03$ & $-1.15 \mathrm{E}-04$ & $1.31 \mathrm{E}-03$ & $-4.06 \mathrm{E}-02$ & $-1.03 \mathrm{E}-01$ & $-2.04 \mathrm{E}-02$ & $2.22 \mathrm{E}-03$ \\
\hline & North America & $-4.13 \mathrm{E}-02$ & $-8.55 \mathrm{E}-02$ & $2.36 \mathrm{E}-03$ & $-4.49 \mathrm{E}-02$ & $-1.01 \mathrm{E}-01$ & $-1.82 \mathrm{E}-01$ & $1.38 \mathrm{E}-02$ \\
\hline & Oceania & $1.62 \mathrm{E}-03$ & $1.38 \mathrm{E}-02$ & $-7.92 \mathrm{E}-03$ & $-8.46 \mathrm{E}-03$ & $2.02 \mathrm{E}-03$ & $3.22 \mathrm{E}-03$ & $-1.00 \mathrm{E}-01$ \\
\hline \multirow{6}{*}{ Ocean } & North Pacific & $6.12 \mathrm{E}-03$ & $-2.34 \mathrm{E}-02$ & $-9.01 \mathrm{E}-04$ & $-2.63 \mathrm{E}-02$ & $1.33 \mathrm{E}-02$ & $-6.44 \mathrm{E}-03$ & $-3.20 \mathrm{E}-03$ \\
\hline & South Pacific & $-1.84 \mathrm{E}-02$ & $6.34 \mathrm{E}-02$ & $-2.19 \mathrm{E}-02$ & $2.01 \mathrm{E}-02$ & $2.63 \mathrm{E}-02$ & $1.49 \mathrm{E}-02$ & $2.61 \mathrm{E}-02$ \\
\hline & Northern Ocean & $1.05 \mathrm{E}-02$ & $3.25 \mathrm{E}-02$ & $-9.60 \mathrm{E}-04$ & $-1.64 \mathrm{E}-02$ & $-5.83 \mathrm{E}-02$ & $-6.06 \mathrm{E}-04$ & $-2.33 \mathrm{E}-03$ \\
\hline & Atlantic & $-3.55 \mathrm{E}-02$ & $-2.90 \mathrm{E}-03$ & $-1.09 \mathrm{E}-02$ & $-3.83 \mathrm{E}-02$ & $-7.79 \mathrm{E}-02$ & $-5.01 \mathrm{E}-02$ & $2.06 \mathrm{E}-03$ \\
\hline & Southern Ocean & $2.24 \mathrm{E}-02$ & $4.63 \mathrm{E}-02$ & $-3.91 \mathrm{E}-02$ & $-9.24 \mathrm{E}-03$ & $-3.20 \mathrm{E}-03$ & $2.15 \mathrm{E}-03$ & $-2.13 \mathrm{E}-02$ \\
\hline & Indian Ocean & $-2.72 \mathrm{E}-02$ & $-1.14 \mathrm{E}-01$ & $-2.66 \mathrm{E}-02$ & $-3.12 \mathrm{E}-03$ & $2.54 \mathrm{E}-03$ & $-5.07 E-03$ & $-3.10 \mathrm{E}-02$ \\
\hline \multirow{7}{*}{$\begin{array}{l}\text { Biomass } \\
\text { burning }\end{array}$} & Africa/Middle East & $-1.96 \mathrm{E}-02$ & $-3.57 \mathrm{E}-02$ & $-8.56 \mathrm{E}-03$ & $4.60 \mathrm{E}-03$ & $4.95 \mathrm{E}-03$ & $-6.89 \mathrm{E}-03$ & $4.23 \mathrm{E}-03$ \\
\hline & Southern Asia & $-1.09 \mathrm{E}-02$ & $-3.43 \mathrm{E}-02$ & $-8.20 \mathrm{E}-03$ & $-6.96 \mathrm{E}-04$ & $3.98 \mathrm{E}-03$ & $-4.09 \mathrm{E}-03$ & $2.98 \mathrm{E}-03$ \\
\hline & Central/South America & $-2.27 \mathrm{E}-03$ & $-3.22 \mathrm{E}-03$ & $-1.53 \mathrm{E}-03$ & $2.12 \mathrm{E}-04$ & $6.19 \mathrm{E}-04$ & $-5.25 \mathrm{E}-04$ & $7.43 \mathrm{E}-04$ \\
\hline & Northern Asia & $1.72 \mathrm{E}-03$ & $-1.45 \mathrm{E}-02$ & $-5.81 \mathrm{E}-04$ & $-7.08 \mathrm{E}-03$ & $1.55 \mathrm{E}-03$ & $3.09 \mathrm{E}-03$ & $-6.05 \mathrm{E}-04$ \\
\hline & Europe & $-1.91 \mathrm{E}-06$ & $1.67 \mathrm{E}-04$ & $6.50 \mathrm{E}-06$ & $-1.77 \mathrm{E}-04$ & $-1.48 \mathrm{E}-04$ & $-1.23 \mathrm{E}-05$ & $1.92 \mathrm{E}-05$ \\
\hline & North America & $6.96 \mathrm{E}-04$ & $2.16 \mathrm{E}-03$ & $-6.89 E-05$ & $1.07 \mathrm{E}-03$ & $-2.04 \mathrm{E}-03$ & $9.03 \mathrm{E}-04$ & $2.05 \mathrm{E}-04$ \\
\hline & Oceania & \begin{tabular}{|l|}
$-9.82 E-04$ \\
\end{tabular} & $-7.69 \mathrm{E}-04$ & $-1.34 \mathrm{E}-03$ & $-1.10 \mathrm{E}-05$ & \begin{tabular}{|l|}
$4.02 \mathrm{E}-04$ \\
\end{tabular} & $2.52 \mathrm{E}-05$ & $-4.27 \mathrm{E}-04$ \\
\hline
\end{tabular}

Fig. S2a. Correlation of the emissions in 1995 for agricultural soil. 


\begin{tabular}{|c|c|c|c|c|c|c|c|c|}
\hline & \multirow{2}{*}{\multicolumn{7}{|c|}{ Industrial }} \\
\hline & & & & & & & & \\
\hline Source sector & Region & $\begin{array}{l}\text { Africa/M. } \\
\text { East }\end{array}$ & $\begin{array}{l}\text { Southern } \\
\text { Asia }\end{array}$ & $\begin{array}{l}\text { C. /S. } \\
\text { America }\end{array}$ & $\begin{array}{l}\text { Northern } \\
\text { Asia }\end{array}$ & Europe & $\begin{array}{l}\text { North } \\
\text { America }\end{array}$ & Oceania \\
\hline \multirow{7}{*}{$\begin{array}{l}\text { Agricultural } \\
\text { Soil }\end{array}$} & Africa/Middle East & $-5.86 \mathrm{E}-03$ & $8.80 \mathrm{E}-03$ & $-5.05 \mathrm{E}-04$ & $-9.95 E-03$ & $-2.45 \mathrm{E}-02$ & $-1.56 \mathrm{E}-02$ & $2.33 \mathrm{E}-04$ \\
\hline & Southern Asia & $-9.86 \mathrm{E}-03$ & $-2.52 \mathrm{E}-01$ & $6.47 \mathrm{E}-04$ & $-4.40 \mathrm{E}-02$ & $1.56 \mathrm{E}-03$ & $-2.15 \mathrm{E}-02$ & $6.32 \mathrm{E}-04$ \\
\hline & Central/South America & $-9.06 \mathrm{E}-04$ & $1.97 \mathrm{E}-03$ & $-1.33 \mathrm{E}-03$ & \begin{tabular}{|c|} 
\\
\end{tabular} & $2.88 \mathrm{E}-03$ & $7.58 \mathrm{E}-04$ & $-4.49 \mathrm{E}-04$ \\
\hline & Northern Asia & $-6.11 \mathrm{E}-03$ & $-3.44 \mathrm{E}-02$ & $-3.85 \mathrm{E}-05$ & \begin{tabular}{|l|}
$-8.19 E-02$ \\
\end{tabular} & $-1.42 \mathrm{E}-01$ & \begin{tabular}{|l|}
$-2.30 \mathrm{E}-02$ \\
\end{tabular} & $-8.24 \mathrm{E}-04$ \\
\hline & Europe & $-5.35 \mathrm{E}-03$ & $1.72 \mathrm{E}-02$ & $2.25 \mathrm{E}-04$ & $-7.72 \mathrm{E}-02$ & $-3.96 \mathrm{E}-01$ & $-3.31 \mathrm{E}-02$ & $8.22 \mathrm{E}-05$ \\
\hline & \begin{tabular}{|l|} 
North America \\
\end{tabular} & $-7.09 \mathrm{E}-03$ & $1.84 \mathrm{E}-02$ & $9.49 \mathrm{E}-05$ & $-2.65 \mathrm{E}-02$ & $-6.79 \mathrm{E}-02$ & $-6.12 \mathrm{E}-02$ & $1.19 \mathrm{E}-04$ \\
\hline & Oceania & $3.23 \mathrm{E}-04$ & $-4.37 \mathrm{E}-03$ & $-6.51 \mathrm{E}-04$ & $-6.61 \mathrm{E}-03$ & $1.37 \mathrm{E}-03$ & $9.82 \mathrm{E}-04$ & $-8.90 \mathrm{E}-03$ \\
\hline \multirow{7}{*}{ Industrial } & Africa/Middle East & 1 & $1.31 \mathrm{E}-03$ & $-8.25 \mathrm{E}-05$ & $-3.13 \mathrm{E}-03$ & $-6.93 \mathrm{E}-03$ & $-3.59 \mathrm{E}-03$ & $2.53 \mathrm{E}-05$ \\
\hline & \begin{tabular}{|l|} 
Southern Asia \\
\end{tabular} & $1.31 \mathrm{E}-03$ & 1 & $1.84 \mathrm{E}-04$ & $-3.67 \mathrm{E}-02$ & $1.55 \mathrm{E}-02$ & $9.51 \mathrm{E}-03$ & $-3.07 \mathrm{E}-04$ \\
\hline & Central/South America & $-8.25 \mathrm{E}-05$ & $1.84 \mathrm{E}-04$ & 1 & $9.33 \mathrm{E}-05$ & $2.43 \mathrm{E}-04$ & $5.35 \mathrm{E}-05$ & $-4.00 \mathrm{E}-05$ \\
\hline & Northern Asia & $-3.13 E-03$ & $-3.67 \mathrm{E}-02$ & $9.33 \mathrm{E}-05$ & 1 & $-7.94 \mathrm{E}-02$ & $-1.30 \mathrm{E}-02$ & $-3.96 \mathrm{E}-04$ \\
\hline & Europe & $-6.93 E-03$ & $1.55 \mathrm{E}-02$ & $2.43 \mathrm{E}-04$ & $-7.94 \mathrm{E}-02$ & 1 & $-3.47 E-02$ & $1.23 \mathrm{E}-04$ \\
\hline & North America & $-3.59 \mathrm{E}-03$ & $9.51 \mathrm{E}-03$ & 5.35E-05 & $-1.30 \mathrm{E}-02$ & $-3.47 E-02$ & 1 & $6.22 \mathrm{E}-05$ \\
\hline & Oceania & $2.53 \mathrm{E}-05$ & $-3.07 E-04$ & $-4.00 \mathrm{E}-05$ & $-3.96 E-04$ & $1.23 \mathrm{E}-04$ & $6.22 \mathrm{E}-05$ & 1 \\
\hline \multirow{7}{*}{ Natural Soil } & Africa/Middle East & $-1.12 \mathrm{E}-02$ & $2.92 \mathrm{E}-02$ & $-2.35 \mathrm{E}-03$ & \begin{tabular}{|l|} 
\\
\end{tabular} & $-1.53 \mathrm{E}-02$ & $-2.16 \mathrm{E}-02$ & 5.84E-04 \\
\hline & Southern Asia & $-5.36 \mathrm{E}-03$ & $-2.60 \mathrm{E}-01$ & $-7.81 \mathrm{E}-04$ & $-4.38 \mathrm{E}-02$ & $2.12 \mathrm{E}-02$ & $-4.06 \mathrm{E}-03$ & $2.32 \mathrm{E}-03$ \\
\hline & Central/South America & $-7.44 \mathrm{E}-03$ & $3.35 \mathrm{E}-02$ & $-6.81 \mathrm{E}-03$ & \begin{tabular}{|l|}
$1.46 \mathrm{E}-02$ \\
\end{tabular} & $2.00 \mathrm{E}-02$ & $-5.85 \mathrm{E}-03$ & $-5.82 \mathrm{E}-05$ \\
\hline & Northern Asia & $6.31 \mathrm{E}-03$ & $-2.27 \mathrm{E}-02$ & $-4.52 \mathrm{E}-04$ & $-5.98 \mathrm{E}-02$ & $-4.17 \mathrm{E}-02$ & $1.02 \mathrm{E}-02$ & $-1.66 \mathrm{E}-03$ \\
\hline & Europe & $-1.18 \mathrm{E}-03$ & $1.36 \mathrm{E}-02$ & $1.16 \mathrm{E}-04$ & $-2.01 \mathrm{E}-02$ & $-8.57 \mathrm{E}-02$ & $-1.04 \mathrm{E}-02$ & 1.93E-04 \\
\hline & North America & $-8.14 \mathrm{E}-03$ & $8.63 E-02$ & $1.42 \mathrm{E}-04$ & $-1.36 \mathrm{E}-02$ & $-9.74 \mathrm{E}-02$ & $-1.03 \mathrm{E}-01$ & 9.94E-04 \\
\hline & Oceania & $1.21 \mathrm{E}-04$ & $-1.02 \mathrm{E}-03$ & $-7.32 \mathrm{E}-04$ & $-6.54 \mathrm{E}-03$ & $3.00 \mathrm{E}-03$ & $1.72 \mathrm{E}-03$ & $-6.82 \mathrm{E}-03$ \\
\hline \multirow{6}{*}{ Ocean } & North Pacific & $1.94 \mathrm{E}-04$ & $-1.05 \mathrm{E}-02$ & $-6.15 \mathrm{E}-05$ & $-1.12 \mathrm{E}-02$ & $1.83 \mathrm{E}-03$ & $-5.82 \mathrm{E}-03$ & $-1.93 \mathrm{E}-04$ \\
\hline & \begin{tabular}{|l} 
South Pacific \\
\end{tabular} & $-2.17 \mathrm{E}-03$ & $2.65 \mathrm{E}-02$ & $-1.96 \mathrm{E}-03$ & \begin{tabular}{|c|} 
\\
\end{tabular} & $3.03 \mathrm{E}-02$ & $7.76 \mathrm{E}-03$ & $1.81 \mathrm{E}-03$ \\
\hline & Northern Ocean & $1.84 \mathrm{E}-03$ & $1.62 \mathrm{E}-03$ & $-7.89 \mathrm{E}-05$ & $-3.35 E-03$ & $-1.79 \mathrm{E}-02$ & $-2.73 \mathrm{E}-03$ & $-1.33 \mathrm{E}-04$ \\
\hline & Atlantic & $-7.09 \mathrm{E}-03$ & $-3.12 E-03$ & $-9.53 \mathrm{E}-04$ & \begin{tabular}{|l|} 
\\
\end{tabular} & $-5.15 \mathrm{E}-02$ & $\begin{array}{ll} & -2.75 \mathrm{E}-02 \\
\end{array}$ & $1.42 \mathrm{E}-04$ \\
\hline & Southern Ocean & $3.01 \mathrm{E}-03$ & $-2.75 \mathrm{E}-02$ & $-3.25 \mathrm{E}-03$ & \begin{tabular}{|l|l|} 
& $-7.55 \mathrm{E}-03$ \\
\end{tabular} & $-4.44 \mathrm{E}-03$ & $1.18 \mathrm{E}-03$ & $-1.72 \mathrm{E}-03$ \\
\hline & Indian Ocean & $-4.52 \mathrm{E}-03$ & $2.67 \mathrm{E}-02$ & $-2.42 E-03$ & \begin{tabular}{l|l|} 
& $1.29 \mathrm{E}-02$ \\
\end{tabular} & $3.34 \mathrm{E}-03$ & $-2.68 \mathrm{E}-03$ & $-2.50 \mathrm{E}-03$ \\
\hline \multirow{7}{*}{$\begin{array}{l}\text { Biomass } \\
\text { burning }\end{array}$} & Africa/Middle East & $-3.06 \mathrm{E}-03$ & $7.63 \mathrm{E}-03$ & $-7.57 \mathrm{E}-04$ & \begin{tabular}{|l|}
$4.66 \mathrm{E}-03$ \\
\end{tabular} & $4.23 E-03$ & \begin{tabular}{|l|}
$-3.19 \mathrm{E}-03$ \\
\end{tabular} & $2.92 \mathrm{E}-04$ \\
\hline & Southern Asia & $-1.91 \mathrm{E}-03$ & $-5.12 \mathrm{E}-04$ & $-7.35 \mathrm{E}-04$ & \begin{tabular}{|l|}
$1.20 \mathrm{E}-03$ \\
\end{tabular} & $3.68 \mathrm{E}-03$ & \begin{tabular}{|l|}
$-2.04 \mathrm{E}-03$ \\
\end{tabular} & $2.37 \mathrm{E}-04$ \\
\hline & Central/South America & $-4.09 \mathrm{E}-04$ & $1.71 \mathrm{E}-03$ & $-1.32 \mathrm{E}-04$ & $\begin{array}{ll}6.29 \mathrm{E}-04 \\
\end{array}$ & $5.51 \mathrm{E}-04$ & $-2.38 \mathrm{E}-04$ & $5.29 \mathrm{E}-05$ \\
\hline & Northern Asia & $2.31 \mathrm{E}-04$ & $-1.74 \mathrm{E}-02$ & $-5.34 \mathrm{E}-05$ & $-6.61 \mathrm{E}-03$ & $-1.73 \mathrm{E}-04$ & $1.54 \mathrm{E}-03$ & $-2.98 \mathrm{E}-05$ \\
\hline & Europe & $-3.19 \mathrm{E}-06$ & $4.65 \mathrm{E}-05$ & $2.31 \mathrm{E}-07$ & $-9.21 \mathrm{E}-05$ & $-1.91 \mathrm{E}-04$ & $-6.03 \mathrm{E}-07$ & $1.15 \mathrm{E}-06$ \\
\hline & North America & $1.93 \mathrm{E}-04$ & $1.72 \mathrm{E}-03$ & $-5.60 \mathrm{E}-06$ & \begin{tabular}{|l|}
$1.03 E-03$ \\
\end{tabular} & $-4.77 \mathrm{E}-05$ & \begin{tabular}{|l|}
$2.26 \mathrm{E}-04$ \\
\end{tabular} & $1.74 \mathrm{E}-05$ \\
\hline & Oceania & $-1.72 \mathrm{E}-04$ & $5.75 \mathrm{E}-04$ & $-1.17 \mathrm{E}-04$ & \begin{tabular}{l|l|} 
& $2.04 \mathrm{E}-04$ \\
\end{tabular} & $4.35 \mathrm{E}-04$ & $3.03 \mathrm{E}-05$ & $-2.09 \mathrm{E}-05$ \\
\hline
\end{tabular}

Fig. S2b. Correlation of the emissions in 1995 for industrial. 


\begin{tabular}{|c|c|c|c|c|c|c|c|c|}
\hline & \multirow{2}{*}{\multicolumn{7}{|c|}{ Natural Soil }} \\
\hline & & & & & & & & \\
\hline Source sector & Region & $\begin{array}{l}\text { Africa/M. } \\
\text { East }\end{array}$ & $\begin{array}{l}\text { Southern } \\
\text { Asia }\end{array}$ & $\begin{array}{l}\text { C. /S. } \\
\text { America }\end{array}$ & $\begin{array}{l}\text { Northern } \\
\text { Asia }\end{array}$ & Europe & $\begin{array}{l}\text { North } \\
\text { America }\end{array}$ & Oceania \\
\hline \multirow{7}{*}{$\begin{array}{l}\text { Agricultural } \\
\text { Soil }\end{array}$} & Africa/Middle East & $-6.45 \mathrm{E}-02$ & $-3.12 \mathrm{E}-02$ & $-4.72 \mathrm{E}-02$ & \begin{tabular}{|l|}
$2.92 \mathrm{E}-02$ \\
\end{tabular} & $-4.44 \mathrm{E}-03$ & $-4.13 \mathrm{E}-02$ & $1.62 \mathrm{E}-03$ \\
\hline & Southern Asia & $-7.07 \mathrm{E}-02$ & $-3.16 \mathrm{E}-01$ & $3.23 \mathrm{E}-02$ & $-1.80 \mathrm{E}-02$ & $-1.15 \mathrm{E}-04$ & $-8.55 \mathrm{E}-02$ & $1.38 \mathrm{E}-02$ \\
\hline & Central/South America & $-2.61 \mathrm{E}-02$ & $-1.00 \mathrm{E}-02$ & $-7.88 \mathrm{E}-02$ & \begin{tabular}{|l|}
$-4.93 \mathrm{E}-03$ \\
\end{tabular} & $1.31 \mathrm{E}-03$ & $2.36 \mathrm{E}-03$ & $-7.92 \mathrm{E}-03$ \\
\hline & Northern Asia & $-9.84 \mathrm{E}-03$ & $-2.34 \mathrm{E}-02$ & $3.71 \mathrm{E}-03$ & \begin{tabular}{|l|}
$-9.46 \mathrm{E}-02$ \\
\end{tabular} & $-4.06 \mathrm{E}-02$ & $-4.49 \mathrm{E}-02$ & $-8.46 \mathrm{E}-03$ \\
\hline & Europe & $-7.65 \mathrm{E}-03$ & $2.08 \mathrm{E}-02$ & $2.23 \mathrm{E}-02$ & $-1.26 \mathrm{E}-02$ & $-1.03 \mathrm{E}-01$ & $-1.01 \mathrm{E}-01$ & $2.02 \mathrm{E}-03$ \\
\hline & \begin{tabular}{|l|} 
North America \\
\end{tabular} & $-4.29 \mathrm{E}-02$ & $-9.64 \mathrm{E}-03$ & $-1.35 \mathrm{E}-02$ & $2.89 \mathrm{E}-02$ & $-2.04 \mathrm{E}-02$ & $-1.82 \mathrm{E}-01$ & $3.22 \mathrm{E}-03$ \\
\hline & Oceania & $6.74 \mathrm{E}-03$ & $3.22 \mathrm{E}-02$ & $-4.70 \mathrm{E}-03$ & $-2.16 \mathrm{E}-02$ & $2.22 \mathrm{E}-03$ & $1.38 \mathrm{E}-02$ & $-1.00 \mathrm{E}-01$ \\
\hline \multirow{7}{*}{ Industrial } & Africa/Middle East & $-1.12 \mathrm{E}-02$ & $-5.36 \mathrm{E}-03$ & $-7.44 \mathrm{E}-03$ & $6.31 \mathrm{E}-03$ & $-1.18 \mathrm{E}-03$ & $-8.14 \mathrm{E}-03$ & $1.21 \mathrm{E}-04$ \\
\hline & \begin{tabular}{|l|} 
Southern Asia \\
\end{tabular} & $2.92 \mathrm{E}-02$ & $-2.60 \mathrm{E}-01$ & $3.35 \mathrm{E}-02$ & $-2.27 \mathrm{E}-02$ & $1.36 \mathrm{E}-02$ & $8.63 \mathrm{E}-02$ & $-1.02 \mathrm{E}-03$ \\
\hline & Central/South America & $-2.35 \mathrm{E}-03$ & $-7.81 \mathrm{E}-04$ & $-6.81 \mathrm{E}-03$ & $-4.52 \mathrm{E}-04$ & $1.16 \mathrm{E}-04$ & $1.42 \mathrm{E}-04$ & $-7.32 \mathrm{E}-04$ \\
\hline & Northern Asia & $2.13 \mathrm{E}-04$ & $-4.38 \mathrm{E}-02$ & $1.46 \mathrm{E}-02$ & \begin{tabular}{|l|}
$-5.98 \mathrm{E}-02$ \\
\end{tabular} & $-2.01 \mathrm{E}-02$ & $-1.36 \mathrm{E}-02$ & $-6.54 \mathrm{E}-03$ \\
\hline & Europe & $-1.53 \mathrm{E}-02$ & $2.12 \mathrm{E}-02$ & $2.00 \mathrm{E}-02$ & \begin{tabular}{|l|}
$-4.17 E-02$ \\
\end{tabular} & $-8.57 \mathrm{E}-02$ & $-9.74 \mathrm{E}-02$ & $3.00 \mathrm{E}-03$ \\
\hline & North America & $-2.16 \mathrm{E}-02$ & $-4.06 \mathrm{E}-03$ & $-5.85 \mathrm{E}-03$ & $1.02 \mathrm{E}-02$ & $-1.04 \mathrm{E}-02$ & $-1.03 \mathrm{E}-01$ & $1.72 \mathrm{E}-03$ \\
\hline & Oceania & 5.84E-04 & $2.32 \mathrm{E}-03$ & $-5.82 \mathrm{E}-05$ & $\mid-1.66 \mathrm{E}-03$ & $1.93 \mathrm{E}-04$ & $9.94 \mathrm{E}-04$ & $-6.82 \mathrm{E}-03$ \\
\hline \multirow{7}{*}{ Natural Soil } & Africa/Middle East & 1 & $-8.00 \mathrm{E}-02$ & $-1.98 \mathrm{E}-01$ & $4.15 \mathrm{E}-02$ & $-4.27 \mathrm{E}-03$ & $-9.98 \mathrm{E}-02$ & 7.89E-04 \\
\hline & Southern Asia & $-8.00 \mathrm{E}-02$ & 1 & $-2.41 \mathrm{E}-01$ & $-1.99 \mathrm{E}-01$ & $2.42 \mathrm{E}-02$ & $-2.10 \mathrm{E}-02$ & $1.63 \mathrm{E}-02$ \\
\hline & Central/South America & $-1.98 \mathrm{E}-01$ & $-2.41 \mathrm{E}-01$ & 1 & $7.29 \mathrm{E}-03$ & $6.57 \mathrm{E}-03$ & $-9.97 \mathrm{E}-02$ & $-1.15 \mathrm{E}-02$ \\
\hline & Northern Asia & $4.15 \mathrm{E}-02$ & $-1.99 \mathrm{E}-01$ & $7.29 \mathrm{E}-03$ & 1 & $-1.29 \mathrm{E}-01$ & $-2.18 \mathrm{E}-01$ & $-3.35 \mathrm{E}-02$ \\
\hline & Europe & $-4.27 \mathrm{E}-03$ & $2.42 \mathrm{E}-02$ & $6.57 \mathrm{E}-03$ & \begin{tabular}{|l|}
$-1.29 \mathrm{E}-01$ \\
\end{tabular} & 1 & $-9.31 \mathrm{E}-02$ & $3.35 \mathrm{E}-03$ \\
\hline & North America & $-9.98 \mathrm{E}-02$ & $-2.10 \mathrm{E}-02$ & $-9.97 \mathrm{E}-02$ & \begin{tabular}{|l|}
$-2.18 \mathrm{E}-01$ \\
\end{tabular} & $-9.31 \mathrm{E}-02$ & 1 & $1.50 \mathrm{E}-02$ \\
\hline & Oceania & $7.89 \mathrm{E}-04$ & $1.63 \mathrm{E}-02$ & $-1.15 \mathrm{E}-02$ & \begin{tabular}{|l|}
$-3.35 \mathrm{E}-02$ \\
\end{tabular} & $3.35 \mathrm{E}-03$ & $1.50 \mathrm{E}-02$ & 1 \\
\hline \multirow{6}{*}{ Ocean } & North Pacific & $3.09 \mathrm{E}-02$ & $-1.76 \mathrm{E}-02$ & $1.06 \mathrm{E}-02$ & $-9.16 \mathrm{E}-02$ & $2.84 \mathrm{E}-02$ & $3.68 \mathrm{E}-02$ & $-4.12 \mathrm{E}-04$ \\
\hline & \begin{tabular}{|l} 
South Pacific \\
\end{tabular} & $-7.54 \mathrm{E}-02$ & $-1.03 \mathrm{E}-01$ & $-3.15 \mathrm{E}-01$ & $-4.24 \mathrm{E}-02$ & $2.45 \mathrm{E}-02$ & $8.21 \mathrm{E}-03$ & $-7.17 \mathrm{E}-03$ \\
\hline & Northern Ocean & $2.07 \mathrm{E}-02$ & $5.38 \mathrm{E}-02$ & $1.96 \mathrm{E}-02$ & $-5.65 \mathrm{E}-02$ & $-8.04 \mathrm{E}-03$ & $1.88 \mathrm{E}-02$ & $-8.56 \mathrm{E}-04$ \\
\hline & Atlantic & $-8.66 \mathrm{E}-02$ & $1.92 \mathrm{E}-02$ & $-4.96 \mathrm{E}-02$ & $5.29 \mathrm{E}-02$ & $-1.03 \mathrm{E}-03$ & $2.10 \mathrm{E}-02$ & $3.08 \mathrm{E}-04$ \\
\hline & Southern Ocean & $1.01 \mathrm{E}-01$ & $1.20 \mathrm{E}-01$ & $7.88 \mathrm{E}-02$ & $-5.36 \mathrm{E}-02$ & $-2.61 \mathrm{E}-03$ & $4.56 \mathrm{E}-02$ & $-6.07 \mathrm{E}-03$ \\
\hline & Indian Ocean & $-6.34 \mathrm{E}-02$ & $-6.25 \mathrm{E}-02$ & $-7.26 \mathrm{E}-02$ & $-6.49 \mathrm{E}-03$ & $3.61 \mathrm{E}-03$ & $-3.62 \mathrm{E}-03$ & $-2.90 \mathrm{E}-02$ \\
\hline \multirow{7}{*}{$\begin{array}{l}\text { Biomass } \\
\text { burning }\end{array}$} & Africa/Middle East & $-6.47 \mathrm{E}-02$ & $2.72 \mathrm{E}-03$ & $-5.97 \mathrm{E}-02$ & $1.66 \mathrm{E}-02$ & $1.74 \mathrm{E}-03$ & $-1.67 \mathrm{E}-02$ & $1.20 \mathrm{E}-03$ \\
\hline & Southern Asia & $-4.11 \mathrm{E}-02$ & $-1.09 \mathrm{E}-02$ & $-2.12 \mathrm{E}-02$ & $1.43 \mathrm{E}-02$ & $7.67 \mathrm{E}-03$ & $3.60 \mathrm{E}-05$ & $-1.44 \mathrm{E}-03$ \\
\hline & Central/South America & $-8.79 \mathrm{E}-03$ & $7.69 \mathrm{E}-04$ & $-1.27 \mathrm{E}-02$ & $4.08 \mathrm{E}-03$ & $1.04 \mathrm{E}-03$ & $-3.76 \mathrm{E}-03$ & $-7.41 \mathrm{E}-04$ \\
\hline & Northern Asia & $1.73 \mathrm{E}-03$ & $8.58 \mathrm{E}-03$ & $4.98 \mathrm{E}-03$ & $-2.61 \mathrm{E}-02$ & $6.98 \mathrm{E}-04$ & $9.36 \mathrm{E}-03$ & $-4.01 \mathrm{E}-04$ \\
\hline & Europe & $5.78 \mathrm{E}-05$ & $3.89 \mathrm{E}-04$ & $9.31 \mathrm{E}-05$ & \begin{tabular}{|l|}
$-1.32 \mathrm{E}-03$ \\
\end{tabular} & $-9.38 \mathrm{E}-05$ & $-8.87 \mathrm{E}-05$ & $3.22 \mathrm{E}-05$ \\
\hline & North America & 7.07E-04 & $5.44 \mathrm{E}-03$ & $-5.07 E-04$ & $-1.39 \mathrm{E}-02$ & $-2.18 \mathrm{E}-03$ & $-6.00 \mathrm{E}-03$ & $2.46 \mathrm{E}-04$ \\
\hline & Oceania & $-4.08 \mathrm{E}-03$ & $-7.06 \mathrm{E}-04$ & $-3.89 E-03$ & $5.27 \mathrm{E}-04$ & $5.19 \mathrm{E}-04$ & $-1.60 \mathrm{E}-04$ & $-1.79 \mathrm{E}-03$ \\
\hline
\end{tabular}

Fig. S2c. Correlation of the emissions in 1995 for natural soil. 


\begin{tabular}{|c|c|c|c|c|c|c|c|}
\hline & & & & & & & \\
\hline & & \multicolumn{6}{|c|}{ Ocean } \\
\hline Source sector & Region & $\begin{array}{l}\text { North } \\
\text { Pacific }\end{array}$ & $\begin{array}{l}\text { South } \\
\text { Pacific }\end{array}$ & $\begin{array}{l}\text { Northern } \\
\text { Ocean }\end{array}$ & Atlantic & $\begin{array}{l}\text { Southern } \\
\text { Ocean }\end{array}$ & $\begin{array}{l}\text { Indian } \\
\text { Ocean }\end{array}$ \\
\hline \multirow{7}{*}{$\begin{array}{l}\text { Agricultural } \\
\text { Soil }\end{array}$} & Africa/Middle East & $6.12 \mathrm{E}-03$ & $-1.84 \mathrm{E}-02$ & $1.05 \mathrm{E}-02$ & $-3.55 E-02$ & $2.24 \mathrm{E}-02$ & $-2.72 \mathrm{E}-02$ \\
\hline & Southern Asia & $-2.34 \mathrm{E}-02$ & $6.34 \mathrm{E}-02$ & $3.25 \mathrm{E}-02$ & $-2.90 \mathrm{E}-03$ & $4.63 \mathrm{E}-02$ & $-1.14 \mathrm{E}-01$ \\
\hline & Central/South America & $-9.01 \mathrm{E}-04$ & $-2.19 \mathrm{E}-02$ & $-9.60 \mathrm{E}-04$ & $-1.09 \mathrm{E}-02$ & $-3.91 \mathrm{E}-02$ & $-2.66 \mathrm{E}-02$ \\
\hline & Northern Asia & $-2.63 \mathrm{E}-02$ & $2.01 \mathrm{E}-02$ & $-1.64 \mathrm{E}-02$ & $-3.83 \mathrm{E}-02$ & $-9.24 \mathrm{E}-03$ & $-3.12 \mathrm{E}-03$ \\
\hline & Europe & $1.33 \mathrm{E}-02$ & $2.63 \mathrm{E}-02$ & $-5.83 \mathrm{E}-02$ & $-7.79 \mathrm{E}-02$ & $-3.20 \mathrm{E}-03$ & $2.54 \mathrm{E}-03$ \\
\hline & \begin{tabular}{|l|} 
North America \\
\end{tabular} & $-6.44 \mathrm{E}-03$ & $1.49 \mathrm{E}-02$ & $-6.06 \mathrm{E}-04$ & $-5.01 E-02$ & $2.15 \mathrm{E}-03$ & $-5.07 E-03$ \\
\hline & Oceania & $-3.20 \mathrm{E}-03$ & $2.61 \mathrm{E}-02$ & $-2.33 \mathrm{E}-03$ & $2.06 \mathrm{E}-03$ & $-2.13 \mathrm{E}-02$ & $-3.10 \mathrm{E}-02$ \\
\hline \multirow{7}{*}{ Industrial } & Africa/Middle East & $1.94 \mathrm{E}-04$ & $-2.17 \mathrm{E}-03$ & $1.84 \mathrm{E}-03$ & \begin{tabular}{|l|}
$-7.09 E-03$ \\
\end{tabular} & $3.01 \mathrm{E}-03$ & $-4.52 \mathrm{E}-03$ \\
\hline & Southern Asia & $-1.05 \mathrm{E}-02$ & $2.65 \mathrm{E}-02$ & $1.62 \mathrm{E}-03$ & $-3.12 \mathrm{E}-03$ & $-2.75 \mathrm{E}-02$ & $2.67 \mathrm{E}-02$ \\
\hline & Central/South America & $-6.15 \mathrm{E}-05$ & $-1.96 \mathrm{E}-03$ & $-7.89 \mathrm{E}-05$ & $-9.53 E-04$ & $-3.25 \mathrm{E}-03$ & $-2.42 \mathrm{E}-03$ \\
\hline & \begin{tabular}{|l} 
Northern Asia \\
\end{tabular} & $-1.12 \mathrm{E}-02$ & $1.96 \mathrm{E}-02$ & $-3.35 \mathrm{E}-03$ & $-2.11 \mathrm{E}-02$ & $-7.55 \mathrm{E}-03$ & $1.29 \mathrm{E}-02$ \\
\hline & Europe & $1.83 \mathrm{E}-03$ & $3.03 \mathrm{E}-02$ & $-1.79 \mathrm{E}-02$ & $-5.15 \mathrm{E}-02$ & $-4.44 \mathrm{E}-03$ & $3.34 \mathrm{E}-03$ \\
\hline & \begin{tabular}{|l} 
North $\mathrm{Am}$ \\
\end{tabular} & $-5.82 \mathrm{E}-03$ & $7.76 \mathrm{E}-03$ & $-2.73 \mathrm{E}-03$ & $-2.75 \mathrm{E}-02$ & $1.18 \mathrm{E}-03$ & E-03 \\
\hline & Oceania & $-1.93 \mathrm{E}-04$ & $1.81 \mathrm{E}-03$ & $-1.33 \mathrm{E}-04$ & $1.42 \mathrm{E}-04$ & $-1.72 \mathrm{E}-03$ & $-2.50 \mathrm{E}-03$ \\
\hline \multirow{7}{*}{ Natural Soil } & Africa/Middle East & $3.09 E-02$ & $-7.54 \mathrm{E}-02$ & $2.07 \mathrm{E}-02$ & $-8.66 \mathrm{E}-02$ & $1.01 \mathrm{E}-01$ & $-6.34 \mathrm{E}-02$ \\
\hline & Southern Asia & $-1.76 \mathrm{E}-02$ & $-1.03 \mathrm{E}-01$ & $5.38 \mathrm{E}-02$ & $1.92 \mathrm{E}-02$ & $1.20 \mathrm{E}-01$ & $-6.25 \mathrm{E}-02$ \\
\hline & Central/South America & $1.06 \mathrm{E}-02$ & $-3.15 \mathrm{E}-01$ & $1.96 \mathrm{E}-02$ & $-4.96 \mathrm{E}-02$ & $7.88 \mathrm{E}-02$ & $-7.26 \mathrm{E}-02$ \\
\hline & Northern Asia & $-9.16 \mathrm{E}-02$ & $-4.24 \mathrm{E}-02$ & $-5.65 E-02$ & $5.29 E-02$ & $-5.36 \mathrm{E}-02$ & $-6.49 \mathrm{E}-03$ \\
\hline & Europe & $2.84 \mathrm{E}-02$ & $2.45 \mathrm{E}-02$ & $-8.04 \mathrm{E}-03$ & $-1.03 E-03$ & $-2.61 \mathrm{E}-03$ & $3.61 \mathrm{E}-03$ \\
\hline & North America & $3.68 \mathrm{E}-02$ & $8.21 \mathrm{E}-03$ & $1.88 \mathrm{E}-02$ & $2.10 \mathrm{E}-02$ & $4.56 \mathrm{E}-02$ & $-3.62 \mathrm{E}-03$ \\
\hline & Oceania & $-4.12 \mathrm{E}-04$ & $-7.17 \mathrm{E}-03$ & $-8.56 \mathrm{E}-04$ & $3.08 \mathrm{E}-04$ & $-6.07 E-03$ & $-2.90 \mathrm{E}-02$ \\
\hline \multirow{6}{*}{ Ocean } & North Pacific & 1 & $2.02 \mathrm{E}-02$ & $-2.14 \mathrm{E}-02$ & $-5.96 \mathrm{E}-02$ & $-1.97 \mathrm{E}-02$ & $2.45 \mathrm{E}-02$ \\
\hline & South Pacific & $2.02 \mathrm{E}-02$ & 1 & $1.37 \mathrm{E}-02$ & $-1.85 \mathrm{E}-02$ & $6.01 \mathrm{E}-02$ & $-2.20 \mathrm{E}-02$ \\
\hline & Northern Ocean & $-2.14 \mathrm{E}-02$ & $1.37 \mathrm{E}-02$ & 1 & $-1.43 \mathrm{E}-01$ & $3.39 E-03$ & $8.13 \mathrm{E}-04$ \\
\hline & Atlantic & $-5.96 \mathrm{E}-02$ & $-1.85 \mathrm{E}-02$ & $-1.43 \mathrm{E}-01$ & 1 & $-4.32 \mathrm{E}-02$ & $-2.95 \mathrm{E}-02$ \\
\hline & Southern Ocean & $-1.97 \mathrm{E}-02$ & $6.01 \mathrm{E}-02$ & $3.39 \mathrm{E}-03$ & $-4.32 \mathrm{E}-02$ & 1 & $-3.89 \mathrm{E}-01$ \\
\hline & Indian Ocean & $2.45 \mathrm{E}-02$ & $-2.20 \mathrm{E}-02$ & $8.13 \mathrm{E}-04$ & $-2.95 \mathrm{E}-02$ & $-3.89 \mathrm{E}-01$ & 1 \\
\hline \multirow{7}{*}{$\begin{array}{l}\text { Biomass } \\
\text { burning }\end{array}$} & Africa/Middle East & $1.25 \mathrm{E}-02$ & $-4.45 \mathrm{E}-02$ & $6.18 \mathrm{E}-03$ & $-3.45 \mathrm{E}-02$ & $2.80 \mathrm{E}-02$ & $-2.12 \mathrm{E}-02$ \\
\hline & Southern Asia & $-3.29 \mathrm{E}-03$ & $-3.48 \mathrm{E}-02$ & $-1.73 \mathrm{E}-03$ & $-1.95 \mathrm{E}-02$ & $4.76 \mathrm{E}-02$ & $-1.18 \mathrm{E}-02$ \\
\hline & Central/South America & $1.06 \mathrm{E}-03$ & $-1.76 \mathrm{E}-02$ & $-7.69 \mathrm{E}-05$ & $-4.14 \mathrm{E}-03$ & $3.76 \mathrm{E}-03$ & $-2.00 \mathrm{E}-03$ \\
\hline & \begin{tabular}{|l} 
Northern Asia \\
\end{tabular} & $-2.26 \mathrm{E}-02$ & $1.92 \mathrm{E}-03$ & $-4.41 \mathrm{E}-04$ & $-1.11 \mathrm{E}-03$ & $-1.21 \mathrm{E}-03$ & 3.37E-03 \\
\hline & Europe & $8.57 \mathrm{E}-05$ & $1.40 \mathrm{E}-04$ & $-2.64 \mathrm{E}-04$ & $-2.52 \mathrm{E}-04$ & $9.58 \mathrm{E}-06$ & $4.60 \mathrm{E}-05$ \\
\hline & North America & $1.12 \mathrm{E}-03$ & $7.13 \mathrm{E}-04$ & $-3.53 \mathrm{E}-03$ & $5.06 \mathrm{E}-04$ & $-1.26 \mathrm{E}-04$ & $1.25 \mathrm{E}-04$ \\
\hline & Oceania & $9.65 \mathrm{E}-05$ & $-4.95 \mathrm{E}-03$ & $-4.05 E-04$ & $-1.90 \mathrm{E}-03$ & $6.60 \mathrm{E}-03$ & $-2.27 \mathrm{E}-03$ \\
\hline
\end{tabular}

Fig. S2d. Correlation of the emissions in 1995 for ocean. 


\begin{tabular}{|c|c|c|c|c|c|c|c|c|}
\hline \multirow[b]{3}{*}{ Source sector } & \multirow[b]{3}{*}{ Region } & \multirow{2}{*}{\multicolumn{7}{|c|}{ Biomass burning }} \\
\hline & & & & & & & & \\
\hline & & $\begin{array}{l}\text { Africa/M. } \\
\text { East }\end{array}$ & $\begin{array}{l}\text { Southern } \\
\text { Asia }\end{array}$ & $\begin{array}{l}\text { C. /S. } \\
\text { America }\end{array}$ & $\begin{array}{l}\text { Northern } \\
\text { Asia }\end{array}$ & Europe & $\begin{array}{l}\text { North } \\
\text { America }\end{array}$ & Oceania \\
\hline \multirow{7}{*}{$\begin{array}{l}\text { Agricultural } \\
\text { Soil }\end{array}$} & Africa/Middle East & $-1.96 \mathrm{E}-02$ & $-1.09 \mathrm{E}-02$ & $-2.27 \mathrm{E}-03$ & $1.72 \mathrm{E}-03$ & $-1.91 \mathrm{E}-06$ & $6.96 \mathrm{E}-04$ & $-9.82 \mathrm{E}-04$ \\
\hline & Southern Asia & $-3.57 \mathrm{E}-02$ & $-3.43 \mathrm{E}-02$ & $-3.22 \mathrm{E}-03$ & $-1.45 \mathrm{E}-02$ & $1.67 \mathrm{E}-04$ & $2.16 \mathrm{E}-03$ & $-7.69 \mathrm{E}-04$ \\
\hline & Central/South America & $-8.56 \mathrm{E}-03$ & $-8.20 \mathrm{E}-03$ & $-1.53 \mathrm{E}-03$ & $-5.81 \mathrm{E}-04$ & $6.50 \mathrm{E}-06$ & $-6.89 \mathrm{E}-05$ & $-1.34 \mathrm{E}-03$ \\
\hline & Northern Asia & $4.60 \mathrm{E}-03$ & $-6.96 \mathrm{E}-04$ & $2.12 \mathrm{E}-04$ & $-7.08 \mathrm{E}-03$ & $-1.77 \mathrm{E}-04$ & $1.07 \mathrm{E}-03$ & $-1.10 \mathrm{E}-05$ \\
\hline & \begin{tabular}{|l} 
Europe \\
\end{tabular} & $4.95 \mathrm{E}-03$ & $3.98 \mathrm{E}-03$ & $6.19 \mathrm{E}-04$ & $1.55 \mathrm{E}-03$ & $-1.48 \mathrm{E}-04$ & $-2.04 \mathrm{E}-03$ & $4.02 \mathrm{E}-04$ \\
\hline & \begin{tabular}{|l} 
North America \\
\end{tabular} & $-6.89 \mathrm{E}-03$ & $-4.09 \mathrm{E}-03$ & $-5.25 \mathrm{E}-04$ & $3.09 \mathrm{E}-03$ & $-1.23 \mathrm{E}-05$ & $9.03 \mathrm{E}-04$ & $2.52 \mathrm{E}-05$ \\
\hline & Oceania & $4.23 \mathrm{E}-03$ & $2.98 \mathrm{E}-03$ & $7.43 \mathrm{E}-04$ & $-6.05 E-04$ & $1.92 \mathrm{E}-05$ & $2.05 \mathrm{E}-04$ & $-4.27 \mathrm{E}-04$ \\
\hline \multirow{7}{*}{ Industrial } & Africa/Middle East & $-3.06 \mathrm{E}-03$ & $-1.91 \mathrm{E}-03$ & $-4.09 \mathrm{E}-04$ & $2.31 \mathrm{E}-04$ & $-3.19 \mathrm{E}-06$ & $1.93 \mathrm{E}-04$ & $-1.72 \mathrm{E}-04$ \\
\hline & Southern Asia & $7.63 \mathrm{E}-03$ & $-5.12 \mathrm{E}-04$ & $1.71 \mathrm{E}-03$ & $-1.74 \mathrm{E}-02$ & $4.65 \mathrm{E}-05$ & $1.72 \mathrm{E}-03$ & $5.75 \mathrm{E}-04$ \\
\hline & Central/South America & $-7.57 \mathrm{E}-04$ & $-7.35 \mathrm{E}-04$ & $-1.32 \mathrm{E}-04$ & $-5.34 \mathrm{E}-05$ & $2.31 \mathrm{E}-07$ & $-5.60 \mathrm{E}-06$ & $-1.17 \mathrm{E}-04$ \\
\hline & Northern Asia & $4.66 \mathrm{E}-03$ & $1.20 \mathrm{E}-03$ & $6.29 \mathrm{E}-04$ & $-6.61 E-03$ & $-9.21 \mathrm{E}-05$ & $1.03 \mathrm{E}-03$ & $2.04 \mathrm{E}-04$ \\
\hline & Europe & $4.23 \mathrm{E}-03$ & $3.68 \mathrm{E}-03$ & $5.51 \mathrm{E}-04$ & $-1.73 \mathrm{E}-04$ & $-1.91 \mathrm{E}-04$ & $-4.77 \mathrm{E}-05$ & $4.35 \mathrm{E}-04$ \\
\hline & North America & $-3.19 \mathrm{E}-03$ & $-2.04 \mathrm{E}-03$ & $-2.38 \mathrm{E}-04$ & $1.54 \mathrm{E}-03$ & $-6.03 E-07$ & $2.26 \mathrm{E}-04$ & $3.03 E-05$ \\
\hline & Oceania & $2.92 \mathrm{E}-04$ & $2.37 \mathrm{E}-04$ & $5.29 \mathrm{E}-05$ & $-2.98 \mathrm{E}-05$ & $1.15 \mathrm{E}-06$ & $1.74 \mathrm{E}-05$ & $-2.09 \mathrm{E}-05$ \\
\hline \multirow{7}{*}{ Natural Soil } & Africa/Middle East & $-6.47 \mathrm{E}-02$ & $-4.11 \mathrm{E}-02$ & $-8.79 \mathrm{E}-03$ & $1.73 \mathrm{E}-03$ & $5.78 \mathrm{E}-05$ & $7.07 E-04$ & $-4.08 \mathrm{E}-03$ \\
\hline & Southern Asia & $2.72 \mathrm{E}-03$ & $-1.09 \mathrm{E}-02$ & $7.69 \mathrm{E}-04$ & $8.58 \mathrm{E}-03$ & $3.89 \mathrm{E}-04$ & $5.44 \mathrm{E}-03$ & $-7.06 \mathrm{E}-04$ \\
\hline & Central/South America & $-5.97 \mathrm{E}-02$ & $-2.12 \mathrm{E}-02$ & $-1.27 \mathrm{E}-02$ & $4.98 \mathrm{E}-03$ & $9.31 \mathrm{E}-05$ & $-5.07 E-04$ & $-3.89 \mathrm{E}-03$ \\
\hline & Northern Asia & $1.66 \mathrm{E}-02$ & $1.43 \mathrm{E}-02$ & $4.08 \mathrm{E}-03$ & $-2.61 \mathrm{E}-02$ & $-1.32 \mathrm{E}-03$ & $-1.39 \mathrm{E}-02$ & $5.27 \mathrm{E}-04$ \\
\hline & Europe & $1.74 \mathrm{E}-03$ & 7.67E-03 & $1.04 \mathrm{E}-03$ & $6.98 \mathrm{E}-04$ & $-9.38 \mathrm{E}-05$ & $-2.18 \mathrm{E}-03$ & $5.19 \mathrm{E}-04$ \\
\hline & North America & $-1.67 \mathrm{E}-02$ & $3.60 \mathrm{E}-05$ & $-3.76 \mathrm{E}-03$ & $9.36 \mathrm{E}-03$ & $-8.87 \mathrm{E}-05$ & $-6.00 \mathrm{E}-03$ & $-1.60 \mathrm{E}-04$ \\
\hline & Oceania & $1.20 \mathrm{E}-03$ & $-1.44 \mathrm{E}-03$ & $-7.41 \mathrm{E}-04$ & $-4.01 \mathrm{E}-04$ & $3.22 \mathrm{E}-05$ & $2.46 \mathrm{E}-04$ & $-1.79 \mathrm{E}-03$ \\
\hline \multirow{6}{*}{ Ocean } & North Pacific & $1.25 \mathrm{E}-02$ & $-3.29 \mathrm{E}-03$ & $1.06 \mathrm{E}-03$ & $-2.26 \mathrm{E}-02$ & $8.57 \mathrm{E}-05$ & $1.12 \mathrm{E}-03$ & $9.65 \mathrm{E}-05$ \\
\hline & South Pacific & $-4.45 \mathrm{E}-02$ & $-3.48 \mathrm{E}-02$ & $-1.76 \mathrm{E}-02$ & $1.92 \mathrm{E}-03$ & $1.40 \mathrm{E}-04$ & $7.13 \mathrm{E}-04$ & $-4.95 \mathrm{E}-03$ \\
\hline & Northern Ocean & $6.18 \mathrm{E}-03$ & $-1.73 \mathrm{E}-03$ & $-7.69 \mathrm{E}-05$ & $-4.41 \mathrm{E}-04$ & $-2.64 \mathrm{E}-04$ & $-3.53 \mathrm{E}-03$ & $-4.05 \mathrm{E}-04$ \\
\hline & Atlantic & $-3.45 \mathrm{E}-02$ & $-1.95 \mathrm{E}-02$ & $-4.14 \mathrm{E}-03$ & $-1.11 \mathrm{E}-03$ & $-2.52 \mathrm{E}-04$ & $5.06 \mathrm{E}-04$ & $-1.90 \mathrm{E}-03$ \\
\hline & Southern Ocean & $2.80 \mathrm{E}-02$ & $4.76 \mathrm{E}-02$ & $3.76 \mathrm{E}-03$ & $-1.21 \mathrm{E}-03$ & $9.58 \mathrm{E}-06$ & $-1.26 \mathrm{E}-04$ & $6.60 \mathrm{E}-03$ \\
\hline & Indian Ocean & $-2.12 \mathrm{E}-02$ & $-1.18 \mathrm{E}-02$ & $-2.00 \mathrm{E}-03$ & $3.37 \mathrm{E}-03$ & $4.60 \mathrm{E}-05$ & $1.25 \mathrm{E}-04$ & $-2.27 \mathrm{E}-03$ \\
\hline \multirow{7}{*}{$\begin{array}{l}\text { Biomass } \\
\text { burning }\end{array}$} & Africa/Middle East & 1 & $-1.66 \mathrm{E}-02$ & $-4.43 \mathrm{E}-03$ & $2.73 \mathrm{E}-04$ & $2.91 \mathrm{E}-05$ & $2.11 \mathrm{E}-04$ & $-2.00 \mathrm{E}-03$ \\
\hline & Southern Asia & $-1.66 \mathrm{E}-02$ & 1 & $-4.81 \mathrm{E}-03$ & $-4.79 \mathrm{E}-03$ & $2.83 E-05$ & $7.57 \mathrm{E}-04$ & $-3.48 \mathrm{E}-03$ \\
\hline & Central/South America & $-4.43 \mathrm{E}-03$ & $-4.81 \mathrm{E}-03$ & 1 & $-2.49 \mathrm{E}-04$ & $3.59 \mathrm{E}-06$ & $8.31 \mathrm{E}-05$ & $-7.74 \mathrm{E}-04$ \\
\hline & Northern Asia & $2.73 \mathrm{E}-04$ & $-4.79 \mathrm{E}-03$ & $-2.49 \mathrm{E}-04$ & 1 & $-1.51 \mathrm{E}-05$ & $4.48 \mathrm{E}-04$ & $-1.89 \mathrm{E}-04$ \\
\hline & Europe & $2.91 \mathrm{E}-05$ & $2.83 \mathrm{E}-05$ & $3.59 \mathrm{E}-06$ & $-1.51 \mathrm{E}-05$ & 1 & $-1.96 \mathrm{E}-06$ & $1.03 \mathrm{E}-06$ \\
\hline & North America & $2.11 \mathrm{E}-04$ & $7.57 \mathrm{E}-04$ & $8.31 \mathrm{E}-05$ & $4.48 \mathrm{E}-04$ & $-1.96 \mathrm{E}-06$ & 1 & $3.70 \mathrm{E}-05$ \\
\hline & Oceania & $-2.00 \mathrm{E}-03$ & $-3.48 \mathrm{E}-03$ & $-7.74 \mathrm{E}-04$ & $-1.89 \mathrm{E}-04$ & $1.03 \mathrm{E}-06$ & $3.70 \mathrm{E}-05$ & 1 \\
\hline
\end{tabular}

Fig. S2e. Correlation of the emissions in 1995 for biomass burning. 


\begin{tabular}{|c|c|c|c|c|c|c|c|c|}
\hline \multirow[b]{2}{*}{ Source sector } & \multirow[b]{2}{*}{ Region } & \multicolumn{7}{|c|}{ Agricultural Soil } \\
\hline & & $\begin{array}{l}\text { Africa/M. } \\
\text { East }\end{array}$ & $\begin{array}{l}\text { Southern } \\
\text { Asia }\end{array}$ & $\begin{array}{l}\text { C. /S. } \\
\text { America }\end{array}$ & $\begin{array}{l}\text { Northern } \\
\text { Asia }\end{array}$ & Europe & $\begin{array}{l}\text { North } \\
\text { America }\end{array}$ & Oceania \\
\hline \multirow{7}{*}{$\begin{array}{c}\text { Agricultural } \\
\text { Soil }\end{array}$} & Africa/Middle East & 1 & $-3.41 \mathrm{E}-02$ & $-7.86 \mathrm{E}-03$ & $-1.87 \mathrm{E}-02$ & $-1.84 \mathrm{E}-02$ & $-3.23 \mathrm{E}-02$ & $1.40 \mathrm{E}-03$ \\
\hline & Southern Asia & $-3.41 \mathrm{E}-02$ & 1 & $1.87 \mathrm{E}-02$ & $-1.07 \mathrm{E}-01$ & $3.33 E-03$ & $-3.28 \mathrm{E}-02$ & $6.14 \mathrm{E}-05$ \\
\hline & Central/South America & $-7.86 \mathrm{E}-03$ & $1.87 \mathrm{E}-02$ & 1 & $-2.76 \mathrm{E}-04$ & $3.60 \mathrm{E}-03$ & $6.68 \mathrm{E}-03$ & $-7.18 \mathrm{E}-03$ \\
\hline & Northern Asia & $-1.87 \mathrm{E}-02$ & $-1.07 \mathrm{E}-01$ & $-2.76 \mathrm{E}-04$ & 1 & $-1.53 \mathrm{E}-01$ & $-5.00 \mathrm{E}-02$ & $-1.55 \mathrm{E}-02$ \\
\hline & Europe & $-1.84 \mathrm{E}-02$ & $3.33 \mathrm{E}-03$ & $3.60 \mathrm{E}-03$ & $-1.53 \mathrm{E}-01$ & 1 & $-7.09 \mathrm{E}-02$ & $-3.21 \mathrm{E}-04$ \\
\hline & North America & $-3.23 E-02$ & $-3.28 \mathrm{E}-02$ & $6.68 \mathrm{E}-03$ & $-5.00 \mathrm{E}-02$ & $-7.09 \mathrm{E}-02$ & 1 & $-1.44 \mathrm{E}-03$ \\
\hline & Oceania & $1.40 \mathrm{E}-03$ & $6.14 \mathrm{E}-05$ & $-7.18 \mathrm{E}-03$ & $-1.55 \mathrm{E}-02$ & $-3.21 \mathrm{E}-04$ & $-1.44 \mathrm{E}-03$ & 1 \\
\hline \multirow{7}{*}{ Industrial } & Africa/Middle East & $-6.22 \mathrm{E}-03$ & $-7.48 \mathrm{E}-03$ & $-1.30 \mathrm{E}-03$ & $-5.56 \mathrm{E}-03$ & $\mid-5.22 \mathrm{E}-03$ & $-7.51 \mathrm{E}-03$ & $-1.89 \mathrm{E}-04$ \\
\hline & \begin{tabular}{|l} 
Southern Asia \\
\end{tabular} & $1.30 \mathrm{E}-02$ & $-2.71 \mathrm{E}-01$ & $5.30 \mathrm{E}-03$ & $-4.21 \mathrm{E}-02$ & $1.76 \mathrm{E}-02$ & $2.54 \mathrm{E}-02$ & $-6.59 \mathrm{E}-03$ \\
\hline & Central/South America & $-7.29 E-04$ & $1.65 \mathrm{E}-03$ & $-2.12 \mathrm{E}-03$ & $-2.26 \mathrm{E}-05$ & $3.11 \mathrm{E}-04$ & $5.51 \mathrm{E}-04$ & $-6.86 \mathrm{E}-04$ \\
\hline & Northern Asia & $-8.18 \mathrm{E}-03$ & $-6.11 \mathrm{E}-02$ & $1.57 \mathrm{E}-03$ & $-7.88 \mathrm{E}-02$ & $-8.30 \mathrm{E}-02$ & $-2.95 \mathrm{E}-02$ & $-1.07 \mathrm{E}-02$ \\
\hline & Europe & $-1.73 E-02$ & $1.52 \mathrm{E}-04$ & $3.43 \mathrm{E}-03$ & $-1.30 \mathrm{E}-01$ & $-3.88 \mathrm{E}-01$ & $-6.50 \mathrm{E}-02$ & $2.75 \mathrm{E}-04$ \\
\hline & North America & $-1.61 \mathrm{E}-02$ & $-1.50 \mathrm{E}-02$ & $3.15 \mathrm{E}-03$ & $-2.34 \mathrm{E}-02$ & $-3.47 \mathrm{E}-02$ & $-7.68 \mathrm{E}-02$ & $-8.24 \mathrm{E}-04$ \\
\hline & Oceania & $1.33 \mathrm{E}-04$ & $4.43 \mathrm{E}-06$ & $-3.80 \mathrm{E}-04$ & $-1.09 \mathrm{E}-03$ & \begin{tabular}{|l|}
$-2.42 \mathrm{E}-05$ \\
\end{tabular} & $-1.52 \mathrm{E}-04$ & $-1.16 \mathrm{E}-02$ \\
\hline \multirow{7}{*}{ Natural Soil } & Africa/Middle East & $-7.31 \mathrm{E}-02$ & $3.25 \mathrm{E}-03$ & $-4.08 \mathrm{E}-02$ & $-8.36 \mathrm{E}-03$ & \begin{tabular}{|l|}
$-1.37 \mathrm{E}-02$ \\
\end{tabular} & $-4.52 \mathrm{E}-02$ & $-3.03 E-03$ \\
\hline & Southern Asia & $-8.43 \mathrm{E}-02$ & $-4.12 \mathrm{E}-01$ & $-2.32 \mathrm{E}-02$ & $-3.92 E-02$ & $3.05 \mathrm{E}-03$ & $-7.60 \mathrm{E}-02$ & $6.42 \mathrm{E}-02$ \\
\hline & Central/South America & $-3.91 E-02$ & $3.99 \mathrm{E}-02$ & $-1.20 \mathrm{E}-01$ & $3.29 \mathrm{E}-03$ & $2.92 \mathrm{E}-02$ & $1.63 \mathrm{E}-02$ & $-8.81 \mathrm{E}-03$ \\
\hline & Northern Asia & $3.66 \mathrm{E}-02$ & $2.08 \mathrm{E}-03$ & $4.14 \mathrm{E}-03$ & $-6.63 \mathrm{E}-02$ & $-9.65 \mathrm{E}-03$ & $8.51 \mathrm{E}-03$ & $-4.55 \mathrm{E}-02$ \\
\hline & Europe & $-8.28 \mathrm{E}-03$ & $-5.84 \mathrm{E}-03$ & $3.09 \mathrm{E}-03$ & $-3.94 \mathrm{E}-02$ & $-9.14 \mathrm{E}-02$ & $-3.92 \mathrm{E}-02$ & $6.07 \mathrm{E}-03$ \\
\hline & North America & $-3.05 E-02$ & $-7.90 \mathrm{E}-03$ & $8.67 \mathrm{E}-03$ & $-4.31 \mathrm{E}-02$ & $-1.01 \mathrm{E}-01$ & $-2.15 \mathrm{E}-01$ & $1.26 \mathrm{E}-02$ \\
\hline & Oceania & \begin{tabular}{|l|}
$-1.51 \mathrm{E}-03$ \\
\end{tabular} & $2.92 \mathrm{E}-02$ & $-1.58 \mathrm{E}-02$ & $-8.91 \mathrm{E}-03$ & $6.79 \mathrm{E}-03$ & $1.08 \mathrm{E}-02$ & $-1.42 \mathrm{E}-01$ \\
\hline \multirow{6}{*}{ Ocean } & North Pacific & $5.80 \mathrm{E}-03$ & $-8.33 \mathrm{E}-02$ & $-6.20 \mathrm{E}-04$ & $-2.48 \mathrm{E}-02$ & $3.10 \mathrm{E}-02$ & $-7.91 \mathrm{E}-03$ & $-7.26 \mathrm{E}-04$ \\
\hline & South Pacific & $-2.43 \mathrm{E}-02$ & $2.85 \mathrm{E}-02$ & $-3.22 \mathrm{E}-02$ & $1.77 \mathrm{E}-02$ & $2.83 \mathrm{E}-02$ & $4.60 \mathrm{E}-03$ & $3.19 \mathrm{E}-02$ \\
\hline & Northern Ocean & $9.65 \mathrm{E}-03$ & $1.32 \mathrm{E}-02$ & $-2.43 \mathrm{E}-03$ & $-2.26 \mathrm{E}-02$ & $-8.40 \mathrm{E}-02$ & $9.75 \mathrm{E}-03$ & $1.33 \mathrm{E}-03$ \\
\hline & Atlantic & $-3.32 \mathrm{E}-02$ & $-2.12 \mathrm{E}-02$ & $-1.58 \mathrm{E}-02$ & $-3.78 \mathrm{E}-02$ & $-7.80 \mathrm{E}-02$ & $-3.85 \mathrm{E}-02$ & $4.47 \mathrm{E}-03$ \\
\hline & Southern Ocean & $6.76 \mathrm{E}-03$ & $4.71 \mathrm{E}-02$ & $-8.04 \mathrm{E}-02$ & $-1.67 \mathrm{E}-02$ & $3.33 \mathrm{E}-03$ & $1.81 \mathrm{E}-02$ & $-2.21 \mathrm{E}-03$ \\
\hline & Indian Ocean & $-3.42 \mathrm{E}-02$ & $-4.76 \mathrm{E}-02$ & $-4.82 \mathrm{E}-02$ & $-5.48 \mathrm{E}-04$ & $7.42 \mathrm{E}-03$ & $1.75 \mathrm{E}-02$ & $-5.92 \mathrm{E}-02$ \\
\hline \multirow{7}{*}{$\begin{array}{l}\text { Biomass } \\
\text { burning }\end{array}$} & Africa/Middle East & $-2.08 \mathrm{E}-02$ & $-1.14 \mathrm{E}-02$ & $-1.42 \mathrm{E}-02$ & $8.23 \mathrm{E}-03$ & $6.89 \mathrm{E}-03$ & $-2.30 \mathrm{E}-03$ & $6.24 \mathrm{E}-03$ \\
\hline & Southern Asia & $-1.27 \mathrm{E}-02$ & $1.16 \mathrm{E}-02$ & $-1.64 \mathrm{E}-02$ & $4.82 \mathrm{E}-03$ & $6.47 \mathrm{E}-03$ & $7.71 \mathrm{E}-03$ & $1.15 \mathrm{E}-02$ \\
\hline & Central/South America & $-2.91 \mathrm{E}-03$ & $7.26 \mathrm{E}-03$ & $-4.79 \mathrm{E}-03$ & $1.11 \mathrm{E}-03$ & $1.20 \mathrm{E}-03$ & $2.47 \mathrm{E}-03$ & $2.67 \mathrm{E}-03$ \\
\hline & \begin{tabular}{|l} 
Northern Asia \\
\end{tabular} & $2.20 \mathrm{E}-03$ & $-1.17 \mathrm{E}-02$ & $-3.26 \mathrm{E}-04$ & $-2.38 \mathrm{E}-03$ & $2.65 \mathrm{E}-03$ & $8.82 \mathrm{E}-04$ & $1.61 \mathrm{E}-03$ \\
\hline & Europe & $2.19 \mathrm{E}-05$ & $-3.06 \mathrm{E}-05$ & $6.82 \mathrm{E}-08$ & $-5.85 \mathrm{E}-05$ & $-1.98 \mathrm{E}-04$ & $-2.68 \mathrm{E}-05$ & $3.86 \mathrm{E}-05$ \\
\hline & North America & $2.65 \mathrm{E}-04$ & $9.61 \mathrm{E}-04$ & $1.42 \mathrm{E}-04$ & $1.26 \mathrm{E}-03$ & $-9.89 \mathrm{E}-04$ & $-7.04 \mathrm{E}-04$ & $1.14 \mathrm{E}-03$ \\
\hline & Oceania & $-1.32 \mathrm{E}-03$ & $5.32 \mathrm{E}-03$ & $-3.26 \mathrm{E}-03$ & $6.60 \mathrm{E}-04$ & $1.03 \mathrm{E}-03$ & $2.56 \mathrm{E}-03$ & $-2.08 \mathrm{E}-05$ \\
\hline
\end{tabular}

Fig. S2f. Correlation of the emissions in 1996 for agricultural soil. 


\begin{tabular}{|c|c|c|c|c|c|c|c|c|}
\hline & \multirow{2}{*}{\multicolumn{7}{|c|}{ Industrial }} \\
\hline & & & & & & & & \\
\hline Source sector & Region & $\begin{array}{l}\text { Africa/M. } \\
\text { East }\end{array}$ & $\begin{array}{l}\text { Southern } \\
\text { Asia }\end{array}$ & $\begin{array}{l}\text { C. /S. } \\
\text { America }\end{array}$ & $\begin{array}{l}\text { Northern } \\
\text { Asia }\end{array}$ & Europe & $\begin{array}{l}\text { North } \\
\text { America }\end{array}$ & Oceania \\
\hline \multirow{7}{*}{$\begin{array}{l}\text { Agricultural } \\
\text { Soil }\end{array}$} & Africa/Middle East & $-6.22 \mathrm{E}-03$ & $1.30 \mathrm{E}-02$ & $-7.29 \mathrm{E}-04$ & $-8.18 \mathrm{E}-03$ & $-1.73 \mathrm{E}-02$ & $-1.61 \mathrm{E}-02$ & $1.33 \mathrm{E}-04$ \\
\hline & Southern Asia & $-7.48 \mathrm{E}-03$ & $-2.71 \mathrm{E}-01$ & $1.65 \mathrm{E}-03$ & \begin{tabular}{|l|} 
\\
\end{tabular} & $1.52 \mathrm{E}-04$ & $-1.50 \mathrm{E}-02$ & $4.43 \mathrm{E}-06$ \\
\hline & Central/South America & $-1.30 \mathrm{E}-03$ & $5.30 \mathrm{E}-03$ & $-2.12 \mathrm{E}-03$ & \begin{tabular}{l|l} 
& $1.57 \mathrm{E}-03$ \\
\end{tabular} & $3.43 \mathrm{E}-03$ & $3.15 \mathrm{E}-03$ & $-3.80 \mathrm{E}-04$ \\
\hline & Northern Asia & $-5.56 \mathrm{E}-03$ & $-4.21 \mathrm{E}-02$ & $-2.26 \mathrm{E}-05$ & \begin{tabular}{|l|} 
\\
\end{tabular} & $-1.30 \mathrm{E}-01$ & \begin{tabular}{|l|}
$-2.34 \mathrm{E}-02$ \\
\end{tabular} & $-1.09 \mathrm{E}-03$ \\
\hline & Europe & $-5.22 \mathrm{E}-03$ & $1.76 \mathrm{E}-02$ & $3.11 \mathrm{E}-04$ & $-8.30 \mathrm{E}-02$ & $-3.88 \mathrm{E}-01$ & $-3.47 \mathrm{E}-02$ & $-2.42 \mathrm{E}-05$ \\
\hline & \begin{tabular}{|l|} 
North America \\
\end{tabular} & $-7.51 \mathrm{E}-03$ & $2.54 \mathrm{E}-02$ & $5.51 \mathrm{E}-04$ & $-2.95 \mathrm{E}-02$ & $-6.50 \mathrm{E}-02$ & $-7.68 \mathrm{E}-02$ & $-1.52 \mathrm{E}-04$ \\
\hline & Oceania & $-1.89 \mathrm{E}-04$ & $-6.59 \mathrm{E}-03$ & $-6.86 \mathrm{E}-04$ & $-1.07 \mathrm{E}-02$ & $2.75 \mathrm{E}-04$ & $-8.24 \mathrm{E}-04$ & $-1.16 \mathrm{E}-02$ \\
\hline \multirow{7}{*}{ Industrial } & Africa/Middle East & 1 & $1.84 \mathrm{E}-03$ & $-1.22 \mathrm{E}-04$ & $-2.83 \mathrm{E}-03$ & $-4.86 \mathrm{E}-03$ & $-3.74 \mathrm{E}-03$ & $-5.14 \mathrm{E}-06$ \\
\hline & \begin{tabular}{|l|} 
Southern Asia \\
\end{tabular} & $1.84 \mathrm{E}-03$ & 1 & $5.02 \mathrm{E}-04$ & $-4.68 \mathrm{E}-02$ & $1.63 \mathrm{E}-02$ & $1.29 \mathrm{E}-02$ & $-4.15 \mathrm{E}-04$ \\
\hline & Central/South America & $-1.22 \mathrm{E}-04$ & $5.02 \mathrm{E}-04$ & 1 & $1.35 \mathrm{E}-04$ & $2.97 \mathrm{E}-04$ & $2.58 \mathrm{E}-04$ & $-3.53 \mathrm{E}-05$ \\
\hline & Northern Asia & $-2.83 \mathrm{E}-03$ & $-4.68 \mathrm{E}-02$ & $1.35 \mathrm{E}-04$ & 1 & $-7.65 \mathrm{E}-02$ & $-1.38 \mathrm{E}-02$ & $-5.88 \mathrm{E}-04$ \\
\hline & Europe & $-4.86 \mathrm{E}-03$ & $1.63 \mathrm{E}-02$ & $2.97 E-04$ & $-7.65 \mathrm{E}-02$ & 1 & $-3.09 \mathrm{E}-02$ & $1.66 \mathrm{E}-05$ \\
\hline & North America & $-3.74 \mathrm{E}-03$ & $1.29 \mathrm{E}-02$ & $2.58 \mathrm{E}-04$ & $-1.38 \mathrm{E}-02$ & $-3.09 \mathrm{E}-02$ & 1 & $-8.05 \mathrm{E}-05$ \\
\hline & Oceania & $-5.14 \mathrm{E}-06$ & $-4.15 \mathrm{E}-04$ & $-3.53 E-05$ & $-5.88 E-04$ & $1.66 \mathrm{E}-05$ & $-8.05 \mathrm{E}-05$ & 1 \\
\hline \multirow{7}{*}{ Natural Soil } & Africa/Middle East & $-1.28 \mathrm{E}-02$ & $5.43 \mathrm{E}-02$ & $-3.68 \mathrm{E}-03$ & \begin{tabular}{|l|}
$3.88 E-03$ \\
\end{tabular} & $-1.38 \mathrm{E}-02$ & $-2.26 \mathrm{E}-02$ & $9.85 \mathrm{E}-07$ \\
\hline & Southern Asia & $-1.45 \mathrm{E}-02$ & $-1.49 \mathrm{E}-01$ & $-2.05 \mathrm{E}-03$ & \begin{tabular}{|l|} 
\\
\end{tabular} & $-1.53 \mathrm{E}-03$ & $-3.40 \mathrm{E}-02$ & $4.10 \mathrm{E}-03$ \\
\hline & Central/South America & $-5.29 \mathrm{E}-03$ & $2.43 \mathrm{E}-03$ & $-1.03 \mathrm{E}-02$ & $1.23 \mathrm{E}-02$ & $2.69 \mathrm{E}-02$ & $1.08 \mathrm{E}-02$ & $7.84 \mathrm{E}-06$ \\
\hline & Northern Asia & $8.73 \mathrm{E}-03$ & $-3.55 \mathrm{E}-02$ & $3.56 \mathrm{E}-04$ & $-5.10 \mathrm{E}-02$ & $-3.08 \mathrm{E}-02$ & $3.48 \mathrm{E}-03$ & $-3.17 \mathrm{E}-03$ \\
\hline & Europe & $-1.96 \mathrm{E}-03$ & $1.56 \mathrm{E}-02$ & $2.68 \mathrm{E}-04$ & $-2.20 \mathrm{E}-02$ & $-7.88 \mathrm{E}-02$ & $-1.77 \mathrm{E}-02$ & 4.09E-04 \\
\hline & North America & $-6.22 \mathrm{E}-03$ & $8.72 \mathrm{E}-02$ & $7.45 \mathrm{E}-04$ & $-1.86 \mathrm{E}-02$ & $-8.62 \mathrm{E}-02$ & $-1.16 \mathrm{E}-01$ & $7.58 \mathrm{E}-04$ \\
\hline & Oceania & $-4.43 E-04$ & $6.78 \mathrm{E}-03$ & $-1.48 \mathrm{E}-03$ & \begin{tabular}{|l|}
$-4.19 E-03$ \\
\end{tabular} & $7.68 \mathrm{E}-03$ & $5.09 \mathrm{E}-03$ & $-8.51 \mathrm{E}-03$ \\
\hline \multirow{6}{*}{ Ocean } & North Pacific & $1.25 \mathrm{E}-04$ & $-1.17 \mathrm{E}-02$ & $1.16 \mathrm{E}-05$ & $-7.61 E-03$ & $2.21 \mathrm{E}-02$ & $-4.44 \mathrm{E}-03$ & $-3.68 \mathrm{E}-05$ \\
\hline & \begin{tabular}{|l} 
South Pacific \\
\end{tabular} & $-3.47 \mathrm{E}-03$ & $2.17 \mathrm{E}-02$ & $-3.05 E-03$ & \begin{tabular}{|c|}
$3.81 \mathrm{E}-02$ \\
\end{tabular} & $2.58 \mathrm{E}-02$ & $3.02 \mathrm{E}-03$ & $2.15 \mathrm{E}-03$ \\
\hline & Northern Ocean & $1.72 \mathrm{E}-03$ & $-3.78 \mathrm{E}-03$ & $-2.01 \mathrm{E}-04$ & \begin{tabular}{l|l|} 
& $-1.20 \mathrm{E}-02$ \\
\end{tabular} & $-5.27 \mathrm{E}-02$ & $2.68 \mathrm{E}-03$ & $1.20 \mathrm{E}-04$ \\
\hline & Atlantic & $-6.50 \mathrm{E}-03$ & $-4.35 \mathrm{E}-03$ & $-1.42 \mathrm{E}-03$ & \begin{tabular}{|l|l|} 
& $-2.32 \mathrm{E}-02$ \\
\end{tabular} & $-6.01 \mathrm{E}-02$ & \begin{tabular}{l|l} 
& $-2.07 \mathrm{E}-02$ \\
\end{tabular} & $2.92 \mathrm{E}-04$ \\
\hline & Southern Ocean & $3.36 \mathrm{E}-05$ & $-2.86 \mathrm{E}-02$ & $-7.01 \mathrm{E}-03$ & \begin{tabular}{|l|l|} 
& $-8.42 \mathrm{E}-03$ \\
\end{tabular} & $2.38 \mathrm{E}-03$ & $8.59 \mathrm{E}-03$ & $-7.93 \mathrm{E}-04$ \\
\hline & Indian Ocean & $-5.72 \mathrm{E}-03$ & $3.94 \mathrm{E}-02$ & $-4.55 \mathrm{E}-03$ & \begin{tabular}{l|l|} 
& $1.57 \mathrm{E}-02$ \\
\end{tabular} & $8.45 \mathrm{E}-03$ & $7.74 \mathrm{E}-03$ & $-3.92 \mathrm{E}-03$ \\
\hline \multirow{7}{*}{$\begin{array}{l}\text { Biomass } \\
\text { burning }\end{array}$} & Africa/Middle East & $-3.22 \mathrm{E}-03$ & $1.76 \mathrm{E}-02$ & $-1.30 \mathrm{E}-03$ & \begin{tabular}{|l|} 
\\
\end{tabular} & $5.90 \mathrm{E}-03$ & \begin{tabular}{|l|}
$-1.03 E-03$ \\
\end{tabular} & $4.73 \mathrm{E}-04$ \\
\hline & Southern Asia & $-2.07 E-03$ & $1.45 \mathrm{E}-02$ & $-1.47 \mathrm{E}-03$ & \begin{tabular}{|l|}
$6.74 E-03$ \\
\end{tabular} & $6.25 \mathrm{E}-03$ & \begin{tabular}{|l|}
$3.21 \mathrm{E}-03$ \\
\end{tabular} & $7.50 \mathrm{E}-04$ \\
\hline & Central/South America & $-4.93 E-04$ & $4.62 \mathrm{E}-03$ & $-4.25 \mathrm{E}-04$ & $\begin{array}{ll}1.60 \mathrm{E}-03 \\
\end{array}$ & $1.20 \mathrm{E}-03$ & $1.08 \mathrm{E}-03$ & $1.74 \mathrm{E}-04$ \\
\hline & Northern Asia & $4.87 \mathrm{E}-04$ & $-1.56 \mathrm{E}-02$ & $-2.96 \mathrm{E}-05$ & $-3.65 \mathrm{E}-03$ & $2.27 \mathrm{E}-03$ & \begin{tabular}{|l|l|}
$8.08 \mathrm{E}-04$ \\
\end{tabular} & $1.14 \mathrm{E}-04$ \\
\hline & Europe & $3.38 \mathrm{E}-06$ & $1.87 \mathrm{E}-05$ & $-3.42 \mathrm{E}-07$ & $-6.46 \mathrm{E}-05$ & $-2.36 \mathrm{E}-04$ & -7.07E-06 & $2.47 \mathrm{E}-06$ \\
\hline & North America & $1.25 \mathrm{E}-04$ & $1.74 \mathrm{E}-03$ & $1.23 \mathrm{E}-05$ & 8.83E-04 & $1.09 \mathrm{E}-04$ & $-3.59 \mathrm{E}-04$ & 7.67E-05 \\
\hline & Oceania & $-2.09 \mathrm{E}-04$ & $2.55 \mathrm{E}-03$ & $-2.91 \mathrm{E}-04$ & $8.93 E-04$ & $1.03 \mathrm{E}-03$ & \begin{tabular}{|l|l|} 
& $1.18 \mathrm{E}-03$ \\
\end{tabular} & $1.46 \mathrm{E}-05$ \\
\hline
\end{tabular}

Fig. S2g. Correlation of the emissions in 1996 for industrial. 


\begin{tabular}{|c|c|c|c|c|c|c|c|c|}
\hline & \multirow{2}{*}{\multicolumn{7}{|c|}{ Natural Soil }} \\
\hline & & & & & & & & \\
\hline Source sector & Region & $\begin{array}{l}\text { Africa/M. } \\
\text { East }\end{array}$ & $\begin{array}{l}\text { Southern } \\
\text { Asia }\end{array}$ & $\begin{array}{l}\text { C. /S. } \\
\text { America }\end{array}$ & $\begin{array}{l}\text { Northern } \\
\text { Asia }\end{array}$ & Europe & $\begin{array}{l}\text { North } \\
\text { America }\end{array}$ & Oceania \\
\hline \multirow{7}{*}{$\begin{array}{l}\text { Agricultural } \\
\text { Soil }\end{array}$} & Africa/Middle East & $-7.31 \mathrm{E}-02$ & $-8.43 \mathrm{E}-02$ & $-3.91 \mathrm{E}-02$ & 3.66E-02 & $-8.28 \mathrm{E}-03$ & $-3.05 \mathrm{E}-02$ & $-1.51 \mathrm{E}-03$ \\
\hline & Southern Asia & $3.25 \mathrm{E}-03$ & $-4.12 \mathrm{E}-01$ & $3.99 \mathrm{E}-02$ & $2.08 \mathrm{E}-03$ & $-5.84 \mathrm{E}-03$ & $-7.90 \mathrm{E}-03$ & $2.92 \mathrm{E}-02$ \\
\hline & Central/South America & $-4.08 \mathrm{E}-02$ & $-2.32 \mathrm{E}-02$ & $-1.20 \mathrm{E}-01$ & $4.14 \mathrm{E}-03$ & $3.09 \mathrm{E}-03$ & $8.67 \mathrm{E}-03$ & $-1.58 \mathrm{E}-02$ \\
\hline & Northern Asia & $-8.36 \mathrm{E}-03$ & $-3.92 \mathrm{E}-02$ & $3.29 \mathrm{E}-03$ & \begin{tabular}{|l|}
$-6.63 \mathrm{E}-02$ \\
\end{tabular} & $-3.94 \mathrm{E}-02$ & $-4.31 \mathrm{E}-02$ & $-8.91 \mathrm{E}-03$ \\
\hline & Europe & $-1.37 \mathrm{E}-02$ & $3.05 \mathrm{E}-03$ & $2.92 \mathrm{E}-02$ & $\mid-9.65 E-03$ & $-9.14 \mathrm{E}-02$ & $-1.01 \mathrm{E}-01$ & $6.79 \mathrm{E}-03$ \\
\hline & North America & $-4.52 \mathrm{E}-02$ & $-7.60 \mathrm{E}-02$ & $1.63 \mathrm{E}-02$ & $8.51 \mathrm{E}-03$ & $-3.92 \mathrm{E}-02$ & $-2.15 \mathrm{E}-01$ & $1.08 \mathrm{E}-02$ \\
\hline & Oceania & $-3.03 E-03$ & $6.42 \mathrm{E}-02$ & $-8.81 \mathrm{E}-03$ & $-4.55 \mathrm{E}-02$ & $6.07 \mathrm{E}-03$ & $1.26 \mathrm{E}-02$ & $-1.42 \mathrm{E}-01$ \\
\hline \multirow{7}{*}{ Industrial } & Africa/Middle East & $-1.28 \mathrm{E}-02$ & $-1.45 \mathrm{E}-02$ & $-5.29 \mathrm{E}-03$ & $8.73 \mathrm{E}-03$ & $-1.96 \mathrm{E}-03$ & $-6.22 \mathrm{E}-03$ & $-4.43 \mathrm{E}-04$ \\
\hline & Southern Asia & $5.43 \mathrm{E}-02$ & $-1.49 \mathrm{E}-01$ & $2.43 \mathrm{E}-03$ & $-3.55 \mathrm{E}-02$ & $1.56 \mathrm{E}-02$ & $8.72 \mathrm{E}-02$ & $6.78 \mathrm{E}-03$ \\
\hline & Central/South America & $-3.68 \mathrm{E}-03$ & $-2.05 \mathrm{E}-03$ & $-1.03 \mathrm{E}-02$ & $3.56 \mathrm{E}-04$ & $2.68 \mathrm{E}-04$ & $7.45 \mathrm{E}-04$ & $-1.48 \mathrm{E}-03$ \\
\hline & Northern Asia & $3.88 \mathrm{E}-03$ & $-3.01 \mathrm{E}-02$ & $1.23 \mathrm{E}-02$ & \begin{tabular}{|l|}
$-5.10 \mathrm{E}-02$ \\
\end{tabular} & $-2.20 \mathrm{E}-02$ & $-1.86 \mathrm{E}-02$ & $-4.19 \mathrm{E}-03$ \\
\hline & Europe & $-1.38 \mathrm{E}-02$ & $-1.53 \mathrm{E}-03$ & $2.69 \mathrm{E}-02$ & \begin{tabular}{|l|}
$-3.08 \mathrm{E}-02$ \\
\end{tabular} & $-7.88 \mathrm{E}-02$ & $-8.62 \mathrm{E}-02$ & $7.68 \mathrm{E}-03$ \\
\hline & North America & $-2.26 \mathrm{E}-02$ & $-3.40 \mathrm{E}-02$ & $1.08 \mathrm{E}-02$ & $3.48 \mathrm{E}-03$ & $-1.77 \mathrm{E}-02$ & $-1.16 \mathrm{E}-01$ & 5.09E-03 \\
\hline & Oceania & $9.85 \mathrm{E}-07$ & $4.10 \mathrm{E}-03$ & $7.84 \mathrm{E}-06$ & -3.17E-03 & 4.09E-04 & $7.58 \mathrm{E}-04$ & $-8.51 \mathrm{E}-03$ \\
\hline \multirow{7}{*}{ Natural Soil } & Africa/Middle East & 1 & $-2.73 \mathrm{E}-01$ & $-2.32 \mathrm{E}-01$ & $6.00 \mathrm{E}-02$ & $-1.66 \mathrm{E}-02$ & $-9.56 \mathrm{E}-02$ & $-1.94 \mathrm{E}-02$ \\
\hline & Southern Asia & $-2.73 \mathrm{E}-01$ & 1 & $-1.20 \mathrm{E}-01$ & $-1.71 \mathrm{E}-01$ & $-1.31 \mathrm{E}-02$ & $-2.07 \mathrm{E}-01$ & $-1.84 \mathrm{E}-03$ \\
\hline & Central/South America & $-2.32 \mathrm{E}-01$ & $-1.20 \mathrm{E}-01$ & 1 & \begin{tabular}{|l|}
$-8.61 \mathrm{E}-02$ \\
\end{tabular} & $3.51 \mathrm{E}-03$ & $-1.47 \mathrm{E}-02$ & $-1.14 \mathrm{E}-02$ \\
\hline & Northern Asia & $6.00 \mathrm{E}-02$ & $-1.71 \mathrm{E}-01$ & $-8.61 \mathrm{E}-02$ & 1 & $-1.51 \mathrm{E}-01$ & $-2.54 \mathrm{E}-01$ & $-5.71 \mathrm{E}-02$ \\
\hline & Europe & $-1.66 \mathrm{E}-02$ & $-1.31 \mathrm{E}-02$ & $3.51 \mathrm{E}-03$ & \begin{tabular}{|l|}
$-1.51 \mathrm{E}-01$ \\
\end{tabular} & 1 & $-1.24 \mathrm{E}-01$ & $1.28 \mathrm{E}-02$ \\
\hline & North America & $-9.56 \mathrm{E}-02$ & $-2.07 \mathrm{E}-01$ & $-1.47 \mathrm{E}-02$ & \begin{tabular}{|l|}
$-2.54 \mathrm{E}-01$ \\
\end{tabular} & $-1.24 \mathrm{E}-01$ & 1 & $3.26 \mathrm{E}-02$ \\
\hline & Oceania & $-1.94 \mathrm{E}-02$ & $-1.84 \mathrm{E}-03$ & $-1.14 \mathrm{E}-02$ & $-5.71 \mathrm{E}-02$ & $1.28 \mathrm{E}-02$ & $3.26 \mathrm{E}-02$ & 1 \\
\hline \multirow{6}{*}{ Ocean } & North Pacific & $4.23 \mathrm{E}-02$ & $9.95 \mathrm{E}-02$ & $-9.29 \mathrm{E}-05$ & $-7.79 \mathrm{E}-02$ & $3.56 \mathrm{E}-02$ & $5.51 \mathrm{E}-02$ & $-3.27 \mathrm{E}-04$ \\
\hline & South Pacific & $-8.39 \mathrm{E}-02$ & $-6.12 \mathrm{E}-02$ & $-5.10 \mathrm{E}-01$ & $1.29 \mathrm{E}-02$ & $2.48 \mathrm{E}-02$ & $5.51 \mathrm{E}-02$ & $-2.37 \mathrm{E}-02$ \\
\hline & \begin{tabular}{|l|} 
Northern Ocean \\
\end{tabular} & $2.66 \mathrm{E}-02$ & $8.89 \mathrm{E}-02$ & $1.41 \mathrm{E}-02$ & $-4.20 \mathrm{E}-02$ & $5.66 \mathrm{E}-03$ & $2.94 \mathrm{E}-02$ & $-3.75 \mathrm{E}-03$ \\
\hline & Atlantic & $-7.08 \mathrm{E}-02$ & $2.96 \mathrm{E}-02$ & $-4.74 \mathrm{E}-02$ & $7.74 \mathrm{E}-02$ & $1.24 \mathrm{E}-02$ & $6.55 \mathrm{E}-02$ & $-1.12 \mathrm{E}-02$ \\
\hline & Southern Ocean & $3.44 \mathrm{E}-02$ & $2.13 \mathrm{E}-01$ & $3.00 \mathrm{E}-02$ & $-3.95 \mathrm{E}-02$ & $1.50 \mathrm{E}-03$ & $2.53 \mathrm{E}-02$ & $-3.28 \mathrm{E}-03$ \\
\hline & Indian Ocean & $-1.02 \mathrm{E}-01$ & $-1.66 \mathrm{E}-01$ & $-1.34 \mathrm{E}-01$ & $1.76 \mathrm{E}-02$ & $1.09 \mathrm{E}-02$ & $4.04 \mathrm{E}-02$ & $-5.62 \mathrm{E}-02$ \\
\hline \multirow{7}{*}{$\begin{array}{l}\text { Biomass } \\
\text { burning }\end{array}$} & Africa/Middle East & $-6.69 \mathrm{E}-02$ & $-5.22 \mathrm{E}-02$ & $-4.03 E-02$ & $2.64 \mathrm{E}-02$ & $2.93 E-03$ & $1.69 \mathrm{E}-03$ & $-9.03 \mathrm{E}-03$ \\
\hline & Southern Asia & $-4.35 \mathrm{E}-02$ & $-9.91 \mathrm{E}-02$ & $6.43 \mathrm{E}-03$ & $3.62 \mathrm{E}-02$ & $8.54 \mathrm{E}-03$ & $2.41 \mathrm{E}-02$ & $-2.04 \mathrm{E}-02$ \\
\hline & Central/South America & $-1.12 \mathrm{E}-02$ & $-1.73 \mathrm{E}-02$ & $-2.53 \mathrm{E}-03$ & $7.86 \mathrm{E}-03$ & $1.23 \mathrm{E}-03$ & $2.43 \mathrm{E}-03$ & $-4.64 \mathrm{E}-03$ \\
\hline & Northern Asia & $3.52 \mathrm{E}-03$ & $-1.96 \mathrm{E}-03$ & $7.46 \mathrm{E}-04$ & $-4.22 \mathrm{E}-02$ & $-2.56 \mathrm{E}-03$ & $-1.96 \mathrm{E}-03$ & $5.51 \mathrm{E}-04$ \\
\hline & \begin{tabular}{|l} 
Europe \\
\end{tabular} & $6.98 \mathrm{E}-05$ & $3.28 \mathrm{E}-04$ & 4.49E-06 & $-9.27 \mathrm{E}-04$ & $-1.40 \mathrm{E}-04$ & $-3.10 \mathrm{E}-04$ & $4.08 \mathrm{E}-05$ \\
\hline & North America & $-4.51 \mathrm{E}-04$ & $4.22 \mathrm{E}-03$ & $-2.29 \mathrm{E}-03$ & $-2.15 \mathrm{E}-02$ & $-2.88 \mathrm{E}-03$ & $-1.08 \mathrm{E}-02$ & $1.28 \mathrm{E}-03$ \\
\hline & Oceania & $-5.41 \mathrm{E}-03$ & $-1.03 \mathrm{E}-02$ & $-1.79 \mathrm{E}-03$ & $3.57 \mathrm{E}-03$ & $1.15 \mathrm{E}-03$ & $3.66 \mathrm{E}-03$ & $-5.64 \mathrm{E}-03$ \\
\hline
\end{tabular}

Fig. S2h. Correlation of the emissions in 1996 for natural soil. 


\begin{tabular}{|c|c|c|c|c|c|c|c|}
\hline & & & & & & & \\
\hline & & \multicolumn{6}{|c|}{ Ocean } \\
\hline Source sector & Region & $\begin{array}{l}\text { North } \\
\text { Pacific }\end{array}$ & $\begin{array}{l}\text { South } \\
\text { Pacific }\end{array}$ & $\begin{array}{l}\text { Northern } \\
\text { Ocean }\end{array}$ & Atlantic & $\begin{array}{l}\text { Southern } \\
\text { Ocean }\end{array}$ & $\begin{array}{l}\text { Indian } \\
\text { Ocean }\end{array}$ \\
\hline \multirow{7}{*}{$\begin{array}{l}\text { Agricultural } \\
\text { Soil }\end{array}$} & Africa/Middle East & $5.80 \mathrm{E}-03$ & $-2.43 \mathrm{E}-02$ & $9.65 \mathrm{E}-03$ & $-3.32 \mathrm{E}-02$ & $6.76 \mathrm{E}-03$ & $-3.42 \mathrm{E}-02$ \\
\hline & Southern Asia & $-8.33 \mathrm{E}-02$ & $2.85 \mathrm{E}-02$ & $1.32 \mathrm{E}-02$ & $-2.12 \mathrm{E}-02$ & $4.71 \mathrm{E}-02$ & $-4.76 \mathrm{E}-02$ \\
\hline & Central/South America & $-6.20 \mathrm{E}-04$ & $-3.22 \mathrm{E}-02$ & $-2.43 \mathrm{E}-03$ & $-1.58 \mathrm{E}-02$ & $-8.04 \mathrm{E}-02$ & $-4.82 \mathrm{E}-02$ \\
\hline & Northern Asia & $-2.48 \mathrm{E}-02$ & $1.77 \mathrm{E}-02$ & $-2.26 \mathrm{E}-02$ & $-3.78 \mathrm{E}-02$ & $-1.67 \mathrm{E}-02$ & $-5.48 \mathrm{E}-04$ \\
\hline & Europe & $3.10 \mathrm{E}-02$ & $2.83 \mathrm{E}-02$ & $-8.40 \mathrm{E}-02$ & $-7.80 \mathrm{E}-02$ & $3.33 E-03$ & $7.42 \mathrm{E}-03$ \\
\hline & \begin{tabular}{|l|} 
North America \\
\end{tabular} & $-7.91 \mathrm{E}-03$ & $4.60 \mathrm{E}-03$ & $9.75 \mathrm{E}-03$ & $-3.85 E-02$ & $1.81 \mathrm{E}-02$ & $1.75 \mathrm{E}-02$ \\
\hline & Oceania & $-7.26 \mathrm{E}-04$ & $3.19 \mathrm{E}-02$ & $1.33 \mathrm{E}-03$ & $4.47 \mathrm{E}-03$ & $-2.21 \mathrm{E}-03$ & $-5.92 \mathrm{E}-02$ \\
\hline \multirow{7}{*}{ Industrial } & Africa/Middle East & $1.25 \mathrm{E}-04$ & $-3.47 \mathrm{E}-03$ & $1.72 \mathrm{E}-03$ & $-6.50 \mathrm{E}-03$ & $3.36 \mathrm{E}-05$ & $-5.72 \mathrm{E}-03$ \\
\hline & Southern Asia & $-1.17 \mathrm{E}-02$ & $2.17 \mathrm{E}-02$ & $-3.78 \mathrm{E}-03$ & $-4.35 \mathrm{E}-03$ & $-2.86 \mathrm{E}-02$ & $3.94 \mathrm{E}-02$ \\
\hline & Central/South America & $1.16 \mathrm{E}-05$ & $-3.05 E-03$ & $-2.01 \mathrm{E}-04$ & $-1.42 \mathrm{E}-03$ & $-7.01 \mathrm{E}-03$ & $-4.55 \mathrm{E}-03$ \\
\hline & \begin{tabular}{|l} 
Northern Asia \\
\end{tabular} & $-7.61 \mathrm{E}-03$ & $1.81 \mathrm{E}-02$ & $-1.20 \mathrm{E}-02$ & $-2.32 \mathrm{E}-02$ & $-8.42 \mathrm{E}-03$ & $1.57 \mathrm{E}-02$ \\
\hline & Europe & $2.21 \mathrm{E}-02$ & $2.58 \mathrm{E}-02$ & $-5.27 \mathrm{E}-02$ & $-6.01 \mathrm{E}-02$ & $2.38 \mathrm{E}-03$ & $8.45 \mathrm{E}-03$ \\
\hline & \begin{tabular}{|l} 
North $\mathrm{Am}$ \\
\end{tabular} & $-4.44 \mathrm{E}-03$ & $3.02 \mathrm{E}-03$ & $2.68 \mathrm{E}-03$ & \begin{tabular}{|l|}
$-2.07 \mathrm{E}-02$ \\
\end{tabular} & $9 \mathrm{E}-03$ & E-03 \\
\hline & Oceania & $-3.68 \mathrm{E}-05$ & $2.15 \mathrm{E}-03$ & $1.20 \mathrm{E}-04$ & $2.92 \mathrm{E}-04$ & $-7.93 \mathrm{E}-04$ & $-3.92 \mathrm{E}-03$ \\
\hline \multirow{7}{*}{ Natural Soil } & Africa/Middle East & $4.23 \mathrm{E}-02$ & $-8.39 \mathrm{E}-02$ & $2.66 \mathrm{E}-02$ & $-7.08 \mathrm{E}-02$ & $3.44 \mathrm{E}-02$ & $-1.02 \mathrm{E}-01$ \\
\hline & Southern Asia & $9.95 \mathrm{E}-02$ & $-6.12 \mathrm{E}-02$ & $8.89 \mathrm{E}-02$ & $2.96 \mathrm{E}-02$ & $2.13 \mathrm{E}-01$ & $-1.66 \mathrm{E}-01$ \\
\hline & Central/South America & $-9.29 \mathrm{E}-05$ & $-5.10 \mathrm{E}-01$ & $1.41 \mathrm{E}-02$ & $-4.74 \mathrm{E}-02$ & $3.00 \mathrm{E}-02$ & $-1.34 \mathrm{E}-01$ \\
\hline & Northern Asia & $-7.79 E-02$ & $1.29 \mathrm{E}-02$ & $-4.20 \mathrm{E}-02$ & $7.74 \mathrm{E}-02$ & $-3.95 \mathrm{E}-02$ & $1.76 \mathrm{E}-02$ \\
\hline & Europe & $3.56 \mathrm{E}-02$ & $2.48 \mathrm{E}-02$ & $5.66 \mathrm{E}-03$ & $1.24 \mathrm{E}-02$ & $1.50 \mathrm{E}-03$ & $1.09 \mathrm{E}-02$ \\
\hline & North America & $5.51 \mathrm{E}-02$ & $5.51 \mathrm{E}-02$ & $2.94 \mathrm{E}-02$ & $6.55 \mathrm{E}-02$ & $2.53 \mathrm{E}-02$ & $4.04 \mathrm{E}-02$ \\
\hline & Oceania & $-3.27 E-04$ & $-2.37 E-02$ & $-3.75 \mathrm{E}-03$ & $-1.12 \mathrm{E}-02$ & $-3.28 \mathrm{E}-03$ & $-5.62 \mathrm{E}-02$ \\
\hline \multirow{6}{*}{ Ocean } & North Pacific & 1 & $3.43 E-02$ & $-2.40 \mathrm{E}-02$ & $-5.81 \mathrm{E}-02$ & $-1.27 \mathrm{E}-02$ & $2.76 \mathrm{E}-02$ \\
\hline & South Pacific & $3.43 E-02$ & 1 & $1.43 \mathrm{E}-02$ & $-4.87 E-02$ & $1.54 \mathrm{E}-02$ & $-3.39 \mathrm{E}-02$ \\
\hline & Northern Ocean & $-2.40 \mathrm{E}-02$ & $1.43 \mathrm{E}-02$ & 1 & $-1.09 \mathrm{E}-01$ & $3.87 \mathrm{E}-04$ & $-3.42 \mathrm{E}-03$ \\
\hline & Atlantic & $-5.81 \mathrm{E}-02$ & $-4.87 \mathrm{E}-02$ & $-1.09 \mathrm{E}-01$ & 1 & $-9.10 \mathrm{E}-02$ & $-3.59 \mathrm{E}-02$ \\
\hline & Southern Ocean & $-1.27 \mathrm{E}-02$ & $1.54 \mathrm{E}-02$ & $3.87 \mathrm{E}-04$ & $-9.10 \mathrm{E}-02$ & 1 & $-6.15 \mathrm{E}-01$ \\
\hline & Indian Ocean & $2.76 \mathrm{E}-02$ & $-3.39 \mathrm{E}-02$ & $-3.42 \mathrm{E}-03$ & $-3.59 \mathrm{E}-02$ & $-6.15 E-01$ & 1 \\
\hline \multirow{7}{*}{$\begin{array}{c}\text { Biomass } \\
\text { burning }\end{array}$} & Africa/Middle East & $1.33 \mathrm{E}-02$ & $-6.22 \mathrm{E}-02$ & $3.34 \mathrm{E}-03$ & $-3.81 \mathrm{E}-02$ & $-1.60 \mathrm{E}-02$ & $-3.74 \mathrm{E}-02$ \\
\hline & Southern Asia & $-1.67 \mathrm{E}-03$ & $-2.23 \mathrm{E}-02$ & $-4.91 \mathrm{E}-03$ & $-2.62 \mathrm{E}-02$ & $-3.65 \mathrm{E}-03$ & $-1.34 \mathrm{E}-02$ \\
\hline & Central/South America & $9.00 \mathrm{E}-04$ & $-1.75 \mathrm{E}-02$ & $-1.15 \mathrm{E}-03$ & $-6.75 \mathrm{E}-03$ & $-1.82 \mathrm{E}-02$ & $-6.52 \mathrm{E}-03$ \\
\hline & \begin{tabular}{|l} 
Northern Asia \\
\end{tabular} & $-3.80 \mathrm{E}-02$ & $2.65 \mathrm{E}-03$ & $-1.63 \mathrm{E}-03$ & $-1.69 \mathrm{E}-04$ & $-4.22 \mathrm{E}-03$ & $6.38 \mathrm{E}-03$ \\
\hline & Europe & $-1.08 \mathrm{E}-05$ & $9.61 \mathrm{E}-05$ & $-2.83 \mathrm{E}-04$ & $-1.55 \mathrm{E}-04$ & $-7.90 \mathrm{E}-06$ & $3.75 \mathrm{E}-05$ \\
\hline & North America & $1.58 \mathrm{E}-04$ & $1.05 \mathrm{E}-03$ & $-3.48 \mathrm{E}-03$ & $1.10 \mathrm{E}-03$ & $9.34 \mathrm{E}-04$ & $7.15 E-04$ \\
\hline & Oceania & $2.34 \mathrm{E}-04$ & $-3.85 \mathrm{E}-03$ & $-1.33 \mathrm{E}-03$ & $-3.82 \mathrm{E}-03$ & $-5.16 \mathrm{E}-03$ & $-5.17 \mathrm{E}-03$ \\
\hline
\end{tabular}

Fig. S2i. Correlation of the emissions in 1996 for ocean. 


\begin{tabular}{|c|c|c|c|c|c|c|c|c|}
\hline & \multirow{2}{*}{\multicolumn{7}{|c|}{ Biomass burning }} \\
\hline & & & & & & & & \\
\hline Source sector & Region & $\begin{array}{l}\text { Africa/M. } \\
\text { East }\end{array}$ & $\begin{array}{l}\text { Southern } \\
\text { Asia }\end{array}$ & $\begin{array}{l}\text { C. /S. } \\
\text { America }\end{array}$ & $\begin{array}{l}\text { Northern } \\
\text { Asia }\end{array}$ & Europe & $\begin{array}{l}\text { North } \\
\text { America }\end{array}$ & Oceania \\
\hline \multirow{7}{*}{$\begin{array}{l}\text { Agricultural } \\
\text { Soil }\end{array}$} & Africa/Middle East & $-2.08 \mathrm{E}-02$ & $-1.27 \mathrm{E}-02$ & $-2.91 \mathrm{E}-03$ & $2.20 \mathrm{E}-03$ & $2.19 \mathrm{E}-05$ & $2.65 \mathrm{E}-04$ & $-1.32 \mathrm{E}-03$ \\
\hline & Southern Asia & $-1.14 \mathrm{E}-02$ & $1.16 \mathrm{E}-02$ & $7.26 \mathrm{E}-03$ & $-1.17 \mathrm{E}-02$ & $-3.06 \mathrm{E}-05$ & $9.61 \mathrm{E}-04$ & $5.32 \mathrm{E}-03$ \\
\hline & Central/South America & $-1.42 \mathrm{E}-02$ & $-1.64 \mathrm{E}-02$ & $-4.79 \mathrm{E}-03$ & $-3.26 \mathrm{E}-04$ & $6.82 \mathrm{E}-08$ & $1.42 \mathrm{E}-04$ & $-3.26 \mathrm{E}-03$ \\
\hline & Northern Asia & $8.23 \mathrm{E}-03$ & $4.82 \mathrm{E}-03$ & $1.11 \mathrm{E}-03$ & $-2.38 \mathrm{E}-03$ & $-5.85 \mathrm{E}-05$ & $1.26 \mathrm{E}-03$ & $6.60 \mathrm{E}-04$ \\
\hline & Europe & $6.89 \mathrm{E}-03$ & $6.47 \mathrm{E}-03$ & $1.20 \mathrm{E}-03$ & $2.65 \mathrm{E}-03$ & $-1.98 \mathrm{E}-04$ & $-9.89 \mathrm{E}-04$ & $1.03 \mathrm{E}-03$ \\
\hline & North America & $-2.30 \mathrm{E}-03$ & $7.71 \mathrm{E}-03$ & $2.47 \mathrm{E}-03$ & $8.82 \mathrm{E}-04$ & $-2.68 \mathrm{E}-05$ & $-7.04 \mathrm{E}-04$ & $2.56 \mathrm{E}-03$ \\
\hline & Oceania & $6.24 \mathrm{E}-03$ & $1.15 \mathrm{E}-02$ & $2.67 \mathrm{E}-03$ & $1.61 \mathrm{E}-03$ & $3.86 \mathrm{E}-05$ & $1.14 \mathrm{E}-03$ & $-2.08 \mathrm{E}-05$ \\
\hline \multirow{7}{*}{ Industrial } & Africa/Middle East & $-3.22 \mathrm{E}-03$ & $-2.07 \mathrm{E}-03$ & $-4.93 \mathrm{E}-04$ & $4.87 \mathrm{E}-04$ & $3.38 \mathrm{E}-06$ & $1.25 \mathrm{E}-04$ & $-2.09 \mathrm{E}-04$ \\
\hline & \begin{tabular}{|l|} 
Southern Asia \\
\end{tabular} & $1.76 \mathrm{E}-02$ & $1.45 \mathrm{E}-02$ & $4.62 \mathrm{E}-03$ & $-1.56 \mathrm{E}-02$ & $1.87 \mathrm{E}-05$ & $1.74 \mathrm{E}-03$ & $2.55 \mathrm{E}-03$ \\
\hline & Central/South America & $-1.30 \mathrm{E}-03$ & $-1.47 \mathrm{E}-03$ & $-4.25 \mathrm{E}-04$ & $-2.96 \mathrm{E}-05$ & $-3.42 \mathrm{E}-07$ & $1.23 \mathrm{E}-05$ & $-2.91 \mathrm{E}-04$ \\
\hline & Northern Asia & 8.49E-03 & $6.74 \mathrm{E}-03$ & $1.60 \mathrm{E}-03$ & $-3.65 \mathrm{E}-03$ & $-6.46 \mathrm{E}-05$ & 8.83E-04 & 8.93E-04 \\
\hline & Europe & $5.90 \mathrm{E}-03$ & $6.25 \mathrm{E}-03$ & $1.20 \mathrm{E}-03$ & $2.27 \mathrm{E}-03$ & $-2.36 \mathrm{E}-04$ & $1.09 \mathrm{E}-04$ & $1.03 \mathrm{E}-03$ \\
\hline & North America & $-1.03 E-03$ & $3.21 \mathrm{E}-03$ & $1.08 \mathrm{E}-03$ & $8.08 \mathrm{E}-04$ & $-7.07 E-06$ & $-3.59 \mathrm{E}-04$ & $1.18 \mathrm{E}-03$ \\
\hline & Oceania & $4.73 \mathrm{E}-04$ & $7.50 \mathrm{E}-04$ & $1.74 \mathrm{E}-04$ & $1.14 \mathrm{E}-04$ & $2.47 \mathrm{E}-06$ & $7.67 \mathrm{E}-05$ & $1.46 \mathrm{E}-05$ \\
\hline \multirow{7}{*}{ Natural Soil } & Africa/Middle East & $-6.69 \mathrm{E}-02$ & $-4.35 \mathrm{E}-02$ & $-1.12 \mathrm{E}-02$ & $3.52 \mathrm{E}-03$ & $6.98 \mathrm{E}-05$ & $-4.51 \mathrm{E}-04$ & $-5.41 \mathrm{E}-03$ \\
\hline & Southern Asia & $-5.22 \mathrm{E}-02$ & $-9.91 \mathrm{E}-02$ & $-1.73 \mathrm{E}-02$ & \begin{tabular}{|l|}
$-1.96 \mathrm{E}-03$ \\
\end{tabular} & $3.28 \mathrm{E}-04$ & $4.22 \mathrm{E}-03$ & $-1.03 \mathrm{E}-02$ \\
\hline & Central/South America & $-4.03 E-02$ & $6.43 \mathrm{E}-03$ & $-2.53 \mathrm{E}-03$ & $7.46 \mathrm{E}-04$ & $4.49 \mathrm{E}-06$ & $-2.29 \mathrm{E}-03$ & $-1.79 \mathrm{E}-03$ \\
\hline & Northern Asia & $2.64 \mathrm{E}-02$ & $3.62 \mathrm{E}-02$ & $7.86 \mathrm{E}-03$ & \begin{tabular}{|l|}
$-4.22 \mathrm{E}-02$ \\
\end{tabular} & $-9.27 E-04$ & $-2.15 \mathrm{E}-02$ & $3.57 \mathrm{E}-03$ \\
\hline & Europe & $2.93 \mathrm{E}-03$ & $8.54 \mathrm{E}-03$ & $1.23 \mathrm{E}-03$ & \begin{tabular}{|l|}
$-2.56 \mathrm{E}-03$ \\
\end{tabular} & $-1.40 \mathrm{E}-04$ & $-2.88 \mathrm{E}-03$ & $1.15 \mathrm{E}-03$ \\
\hline & North America & $1.69 \mathrm{E}-03$ & $2.41 \mathrm{E}-02$ & $2.43 \mathrm{E}-03$ & \begin{tabular}{|l|}
$-1.96 \mathrm{E}-03$ \\
\end{tabular} & $-3.10 \mathrm{E}-04$ & $-1.08 \mathrm{E}-02$ & $3.66 \mathrm{E}-03$ \\
\hline & Oceania & $-9.03 E-03$ & $-2.04 \mathrm{E}-02$ & $-4.64 \mathrm{E}-03$ & $5.51 \mathrm{E}-04$ & $4.08 \mathrm{E}-05$ & $1.28 \mathrm{E}-03$ & $-5.64 \mathrm{E}-03$ \\
\hline \multirow{6}{*}{ Ocean } & North Pacific & $1.33 \mathrm{E}-02$ & $-1.67 \mathrm{E}-03$ & $9.00 \mathrm{E}-04$ & $-3.80 \mathrm{E}-02$ & $-1.08 \mathrm{E}-05$ & $1.58 \mathrm{E}-04$ & $2.34 \mathrm{E}-04$ \\
\hline & South Pacific & $-6.22 \mathrm{E}-02$ & $-2.23 \mathrm{E}-02$ & $-1.75 \mathrm{E}-02$ & $2.65 \mathrm{E}-03$ & $9.61 \mathrm{E}-05$ & $1.05 \mathrm{E}-03$ & $-3.85 \mathrm{E}-03$ \\
\hline & Northern Ocean & $3.34 \mathrm{E}-03$ & $-4.91 \mathrm{E}-03$ & $-1.15 \mathrm{E}-03$ & $-1.63 \mathrm{E}-03$ & $-2.83 \mathrm{E}-04$ & $-3.48 \mathrm{E}-03$ & $-1.33 \mathrm{E}-03$ \\
\hline & Atlantic & $-3.81 \mathrm{E}-02$ & $-2.62 \mathrm{E}-02$ & $-6.75 \mathrm{E}-03$ & $-1.69 \mathrm{E}-04$ & $-1.55 \mathrm{E}-04$ & $\begin{array}{ll}1.10 \mathrm{E}-03 \\
\end{array}$ & $-3.82 \mathrm{E}-03$ \\
\hline & Southern Ocean & $-1.60 \mathrm{E}-02$ & $-3.65 \mathrm{E}-03$ & $-1.82 \mathrm{E}-02$ & $-4.22 \mathrm{E}-03$ & $-7.90 \mathrm{E}-06$ & \begin{tabular}{|l|}
$9.34 \mathrm{E}-04$ \\
\end{tabular} & $-5.16 \mathrm{E}-03$ \\
\hline & Indian Ocean & $-3.74 \mathrm{E}-02$ & $-1.34 \mathrm{E}-02$ & $-6.52 E-03$ & $6.38 \mathrm{E}-03$ & $3.75 \mathrm{E}-05$ & \begin{tabular}{|l|l|}
$5.15 \mathrm{E}-04$ \\
\end{tabular} & $-5.17 \mathrm{E}-03$ \\
\hline \multirow{7}{*}{$\begin{array}{l}\text { Biomass } \\
\text { burning }\end{array}$} & Africa/Middle East & 1 & $-2.76 \mathrm{E}-02$ & $-8.01 \mathrm{E}-03$ & $-3.40 \mathrm{E}-05$ & $2.32 \mathrm{E}-05$ & \begin{tabular}{|l|}
$2.53 \mathrm{E}-04$ \\
\end{tabular} & $-4.14 \mathrm{E}-03$ \\
\hline & Southern Asia & $-2.76 \mathrm{E}-02$ & 1 & $-9.99 \mathrm{E}-03$ & $-4.73 E-03$ & $1.26 \mathrm{E}-05$ & \begin{tabular}{|l|}
$6.32 \mathrm{E}-04$ \\
\end{tabular} & $-7.42 \mathrm{E}-03$ \\
\hline & Central/South America & $-8.01 \mathrm{E}-03$ & $-9.99 \mathrm{E}-03$ & 1 & $-2.09 \mathrm{E}-04$ & $1.69 \mathrm{E}-06$ & \begin{tabular}{|l|}
$5.21 \mathrm{E}-05$ \\
\end{tabular} & $-1.92 \mathrm{E}-03$ \\
\hline & Northern Asia & $-3.40 \mathrm{E}-05$ & $-4.73 \mathrm{E}-03$ & $-2.09 \mathrm{E}-04$ & 1 & $-4.06 \mathrm{E}-05$ & \begin{tabular}{|l|}
$5.75 \mathrm{E}-04$ \\
\end{tabular} & $-1.63 \mathrm{E}-04$ \\
\hline & Europe & $2.32 \mathrm{E}-05$ & $1.26 \mathrm{E}-05$ & $1.69 \mathrm{E}-06$ & \begin{tabular}{|l|}
$-4.06 \mathrm{E}-05$ \\
\end{tabular} & 1 & $-1.41 \mathrm{E}-05$ & $-1.27 \mathrm{E}-07$ \\
\hline & North America & $2.53 \mathrm{E}-04$ & $6.32 \mathrm{E}-04$ & $5.21 \mathrm{E}-05$ & $-5.75 \mathrm{E}-04$ & $-1.41 \mathrm{E}-05$ & 1 & $6.02 \mathrm{E}-05$ \\
\hline & Oceania & $-4.14 \mathrm{E}-03$ & $-7.42 \mathrm{E}-03$ & $-1.92 \mathrm{E}-03$ & $-1.63 \mathrm{E}-04$ & $-1.27 \mathrm{E}-07$ & $6.02 \mathrm{E}-05$ & 1 \\
\hline
\end{tabular}

\section{Fig. S2j. Correlation of the emissions in 1996 for biomass burning.}




\begin{tabular}{|c|c|c|c|c|c|c|c|c|}
\hline \multirow[b]{2}{*}{ Source sector } & \multirow[b]{2}{*}{ Region } & \multicolumn{7}{|c|}{ Agricultural Soil } \\
\hline & & $\begin{array}{l}\text { Africa/M. } \\
\text { East }\end{array}$ & $\begin{array}{l}\text { Southern } \\
\text { Asia }\end{array}$ & $\begin{array}{l}\text { C. /S. } \\
\text { America }\end{array}$ & $\begin{array}{l}\text { Northern } \\
\text { Asia }\end{array}$ & Europe & $\begin{array}{l}\text { North } \\
\text { America }\end{array}$ & Oceania \\
\hline \multirow{7}{*}{$\begin{array}{c}\text { Agricultural } \\
\text { Soil }\end{array}$} & Africa/Middle East & 1 & $-7.03 \mathrm{E}-02$ & $-2.69 \mathrm{E}-03$ & $-3.64 \mathrm{E}-02$ & $-2.69 \mathrm{E}-02$ & $-4.10 \mathrm{E}-02$ & $4.98 \mathrm{E}-03$ \\
\hline & Southern Asia & $-7.03 E-02$ & 1 & $2.67 \mathrm{E}-02$ & $-1.67 \mathrm{E}-01$ & $2.69 \mathrm{E}-02$ & $-7.29 \mathrm{E}-02$ & $1.97 \mathrm{E}-02$ \\
\hline & Central/South America & $-2.69 E-03$ & $2.67 \mathrm{E}-02$ & 1 & $-1.32 \mathrm{E}-03$ & $2.94 \mathrm{E}-03$ & $1.15 \mathrm{E}-02$ & $-1.12 \mathrm{E}-02$ \\
\hline & Northern Asia & $-3.64 \mathrm{E}-02$ & $-1.67 \mathrm{E}-01$ & $-1.32 \mathrm{E}-03$ & 1 & $-1.75 \mathrm{E}-01$ & $-5.11 \mathrm{E}-02$ & $-1.33 \mathrm{E}-02$ \\
\hline & Europe & $-2.69 \mathrm{E}-02$ & $2.69 \mathrm{E}-02$ & $2.94 \mathrm{E}-03$ & $-1.75 \mathrm{E}-01$ & 1 & $-2.34 \mathrm{E}-02$ & $2.55 \mathrm{E}-03$ \\
\hline & North America & $-4.10 \mathrm{E}-02$ & $-7.29 \mathrm{E}-02$ & $1.15 \mathrm{E}-02$ & $-5.11 \mathrm{E}-02$ & $-2.34 \mathrm{E}-02$ & 1 & $3.50 \mathrm{E}-03$ \\
\hline & Oceania & $4.98 \mathrm{E}-03$ & $1.97 \mathrm{E}-02$ & $-1.12 \mathrm{E}-02$ & $-1.33 \mathrm{E}-02$ & $2.55 \mathrm{E}-03$ & $3.50 \mathrm{E}-03$ & 1 \\
\hline \multirow{7}{*}{ Industrial } & Africa/Middle East & $-2.82 \mathrm{E}-02$ & $-1.33 \mathrm{E}-02$ & $-9.82 \mathrm{E}-05$ & $-9.12 \mathrm{E}-03$ & $-9.84 \mathrm{E}-03$ & $-8.73 \mathrm{E}-03$ & $5.04 \mathrm{E}-04$ \\
\hline & \begin{tabular}{|l} 
Southern Asia \\
\end{tabular} & $9.69 \mathrm{E}-03$ & $-3.67 \mathrm{E}-01$ & $3.25 \mathrm{E}-05$ & $-2.60 \mathrm{E}-02$ & $1.52 \mathrm{E}-02$ & $1.27 \mathrm{E}-02$ & $-1.65 \mathrm{E}-03$ \\
\hline & Central/South America & $-2.78 \mathrm{E}-04$ & $2.40 \mathrm{E}-03$ & $-3.44 \mathrm{E}-03$ & $-1.23 \mathrm{E}-04$ & $2.55 \mathrm{E}-04$ & $1.02 \mathrm{E}-03$ & $-1.14 \mathrm{E}-03$ \\
\hline & Northern Asia & $1.67 \mathrm{E}-02$ & $-1.50 \mathrm{E}-02$ & $-4.89 \mathrm{E}-03$ & $-7.50 \mathrm{E}-02$ & $-4.41 \mathrm{E}-02$ & $4.74 \mathrm{E}-02$ & $-1.07 \mathrm{E}-02$ \\
\hline & Europe & $-1.94 \mathrm{E}-02$ & $-1.86 \mathrm{E}-03$ & $2.79 \mathrm{E}-03$ & $-8.79 \mathrm{E}-02$ & $-6.84 \mathrm{E}-01$ & $-2.83 \mathrm{E}-02$ & $2.01 \mathrm{E}-03$ \\
\hline & North America & $-4.47 \mathrm{E}-03$ & $1.08 \mathrm{E}-03$ & $3.44 \mathrm{E}-03$ & $2.68 \mathrm{E}-02$ & $1.36 \mathrm{E}-02$ & $-2.11 \mathrm{E}-01$ & $1.19 \mathrm{E}-03$ \\
\hline & Oceania & $3.35 \mathrm{E}-04$ & $1.25 \mathrm{E}-03$ & $-6.01 \mathrm{E}-04$ & $-9.96 \mathrm{E}-04$ & $1.85 \mathrm{E}-04$ & $1.72 \mathrm{E}-04$ & $-1.74 \mathrm{E}-02$ \\
\hline \multirow{7}{*}{ Natural Soil } & Africa/Middle East & $-1.43 E-01$ & $-6.38 \mathrm{E}-02$ & $-5.53 \mathrm{E}-02$ & $-2.35 \mathrm{E}-02$ & $-1.58 \mathrm{E}-02$ & $-4.80 \mathrm{E}-02$ & $-9.68 \mathrm{E}-05$ \\
\hline & Southern Asia & $-6.19 \mathrm{E}-02$ & $-3.87 \mathrm{E}-01$ & $1.26 \mathrm{E}-02$ & $-2.81 \mathrm{E}-02$ & $3.45 \mathrm{E}-03$ & $-4.48 \mathrm{E}-02$ & $5.52 \mathrm{E}-02$ \\
\hline & Central/South America & $-5.11 \mathrm{E}-02$ & $5.44 \mathrm{E}-02$ & $-1.75 \mathrm{E}-01$ & $1.83 \mathrm{E}-02$ & $2.10 \mathrm{E}-02$ & $1.16 \mathrm{E}-02$ & $-4.06 \mathrm{E}-02$ \\
\hline & Northern Asia & $3.01 \mathrm{E}-02$ & $-7.88 \mathrm{E}-03$ & $5.63 \mathrm{E}-03$ & $-1.69 \mathrm{E}-01$ & $1.47 \mathrm{E}-02$ & $3.23 \mathrm{E}-02$ & $-5.45 \mathrm{E}-02$ \\
\hline & Europe & $-1.12 \mathrm{E}-02$ & $4.20 \mathrm{E}-03$ & $3.22 \mathrm{E}-03$ & $1.29 \mathrm{E}-03$ & $-2.67 \mathrm{E}-01$ & $1.19 \mathrm{E}-02$ & $1.33 \mathrm{E}-02$ \\
\hline & North America & $-3.80 \mathrm{E}-02$ & $-2.96 \mathrm{E}-02$ & $1.15 \mathrm{E}-02$ & $2.44 \mathrm{E}-02$ & $1.31 \mathrm{E}-02$ & $-3.96 \mathrm{E}-01$ & $2.57 \mathrm{E}-02$ \\
\hline & Oceania & $4.66 \mathrm{E}-03$ & $2.80 \mathrm{E}-02$ & $-9.87 \mathrm{E}-03$ & $-6.14 \mathrm{E}-03$ & $4.26 \mathrm{E}-03$ & $9.14 \mathrm{E}-03$ & $-1.46 \mathrm{E}-01$ \\
\hline \multirow{6}{*}{ Ocean } & North Pacific & $-6.26 \mathrm{E}-03$ & $-1.30 \mathrm{E}-01$ & $7.35 \mathrm{E}-03$ & $-7.06 \mathrm{E}-03$ & $1.09 \mathrm{E}-02$ & $-3.16 \mathrm{E}-02$ & $2.33 \mathrm{E}-02$ \\
\hline & South Pacific & $-2.55 \mathrm{E}-02$ & $-7.86 \mathrm{E}-04$ & $-3.84 \mathrm{E}-02$ & $1.83 \mathrm{E}-02$ & $1.19 \mathrm{E}-02$ & $1.32 \mathrm{E}-02$ & $1.41 \mathrm{E}-02$ \\
\hline & Northern Ocean & $1.51 \mathrm{E}-02$ & $-5.23 \mathrm{E}-03$ & $2.30 \mathrm{E}-03$ & $-5.69 \mathrm{E}-02$ & $-3.80 \mathrm{E}-02$ & $-8.57 \mathrm{E}-03$ & $7.72 \mathrm{E}-03$ \\
\hline & Atlantic & $-5.28 \mathrm{E}-02$ & $-1.06 \mathrm{E}-01$ & $-9.89 \mathrm{E}-03$ & $-6.52 \mathrm{E}-02$ & $-4.05 \mathrm{E}-02$ & $-1.25 \mathrm{E}-01$ & $2.50 \mathrm{E}-02$ \\
\hline & Southern Ocean & $2.46 \mathrm{E}-02$ & $8.58 \mathrm{E}-02$ & $-1.26 \mathrm{E}-01$ & $-5.93 \mathrm{E}-03$ & $3.13 \mathrm{E}-03$ & $3.09 \mathrm{E}-02$ & $-1.62 \mathrm{E}-02$ \\
\hline & Indian Ocean & $-1.85 \mathrm{E}-02$ & $-3.75 \mathrm{E}-02$ & $-5.71 \mathrm{E}-02$ & $1.56 \mathrm{E}-03$ & $2.30 \mathrm{E}-03$ & $2.80 \mathrm{E}-02$ & $-4.35 \mathrm{E}-02$ \\
\hline \multirow{7}{*}{$\begin{array}{l}\text { Biomass } \\
\text { burning }\end{array}$} & Africa/Middle East & $-2.46 \mathrm{E}-02$ & $-2.88 \mathrm{E}-02$ & $-1.91 \mathrm{E}-02$ & $9.71 \mathrm{E}-03$ & $2.43 \mathrm{E}-03$ & $-2.59 \mathrm{E}-03$ & $7.91 \mathrm{E}-03$ \\
\hline & Southern Asia & $-1.87 \mathrm{E}-03$ & $-2.38 \mathrm{E}-02$ & $-1.35 \mathrm{E}-02$ & $-1.94 \mathrm{E}-02$ & $9.21 \mathrm{E}-03$ & $2.08 \mathrm{E}-02$ & $1.32 \mathrm{E}-02$ \\
\hline & Central/South America & $-2.28 \mathrm{E}-03$ & $3.47 \mathrm{E}-03$ & $-7.59 \mathrm{E}-03$ & $1.33 \mathrm{E}-03$ & $6.38 \mathrm{E}-04$ & $3.56 \mathrm{E}-03$ & $1.99 \mathrm{E}-03$ \\
\hline & \begin{tabular}{|l} 
Northern Asia \\
\end{tabular} & $4.07 \mathrm{E}-04$ & $-2.00 \mathrm{E}-02$ & $5.47 \mathrm{E}-04$ & $-3.72 \mathrm{E}-03$ & $4.39 \mathrm{E}-03$ & $-7.44 \mathrm{E}-04$ & $4.29 \mathrm{E}-03$ \\
\hline & Europe & $2.88 \mathrm{E}-05$ & $-2.71 \mathrm{E}-05$ & $9.34 \mathrm{E}-06$ & $-2.84 \mathrm{E}-04$ & $5.08 \mathrm{E}-05$ & $-4.16 \mathrm{E}-05$ & $9.24 \mathrm{E}-05$ \\
\hline & North America & $3.07 \mathrm{E}-04$ & $6.42 \mathrm{E}-04$ & $5.44 \mathrm{E}-04$ & $2.55 \mathrm{E}-03$ & $1.06 \mathrm{E}-03$ & $1.19 \mathrm{E}-03$ & $1.58 \mathrm{E}-03$ \\
\hline & Oceania & $2.32 \mathrm{E}-04$ & $3.67 \mathrm{E}-03$ & $-3.62 \mathrm{E}-03$ & $-6.85 \mathrm{E}-04$ & $9.00 \mathrm{E}-04$ & $3.96 \mathrm{E}-03$ & $-5.38 \mathrm{E}-04$ \\
\hline
\end{tabular}

Fig. S2k. Correlation of the emissions in 1997 for agricultural soil. 


\begin{tabular}{|c|c|c|c|c|c|c|c|c|}
\hline & \multirow{2}{*}{\multicolumn{7}{|c|}{ Industrial }} \\
\hline & & & & & & & & \\
\hline Source sector & Region & $\begin{array}{l}\text { Africa/M. } \\
\text { East }\end{array}$ & $\begin{array}{l}\text { Southern } \\
\text { Asia }\end{array}$ & $\begin{array}{l}\text { C. /S. } \\
\text { America }\end{array}$ & $\begin{array}{l}\text { Northern } \\
\text { Asia }\end{array}$ & Europe & $\begin{array}{l}\text { North } \\
\text { America }\end{array}$ & Oceania \\
\hline \multirow{7}{*}{$\begin{array}{l}\text { Agricultural } \\
\text { Soil }\end{array}$} & Africa/Middle East & $-2.82 \mathrm{E}-02$ & $9.69 \mathrm{E}-03$ & $-2.78 \mathrm{E}-04$ & $1.67 \mathrm{E}-02$ & $-1.94 \mathrm{E}-02$ & $-4.47 \mathrm{E}-03$ & $3.35 \mathrm{E}-04$ \\
\hline & Southern Asia & $-1.33 \mathrm{E}-02$ & $-3.67 \mathrm{E}-01$ & $2.40 \mathrm{E}-03$ & 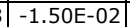 & $-1.86 \mathrm{E}-03$ & $1.08 \mathrm{E}-03$ & $1.25 \mathrm{E}-03$ \\
\hline & Central/South America & $-9.82 \mathrm{E}-05$ & $3.25 \mathrm{E}-05$ & $-3.44 \mathrm{E}-03$ & 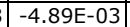 & $2.79 \mathrm{E}-03$ & $3.44 \mathrm{E}-03$ & $-6.01 \mathrm{E}-04$ \\
\hline & \begin{tabular}{|l|} 
Northern Asia \\
\end{tabular} & $-9.12 \mathrm{E}-03$ & $-2.60 \mathrm{E}-02$ & $-1.23 \mathrm{E}-04$ & \begin{tabular}{|l|} 
\\
\end{tabular} & $-8.79 \mathrm{E}-02$ & $2.68 \mathrm{E}-02$ & $-9.96 \mathrm{E}-04$ \\
\hline & Europe & $-9.84 \mathrm{E}-03$ & $1.52 \mathrm{E}-02$ & $2.55 \mathrm{E}-04$ & $-4.41 \mathrm{E}-02$ & $-6.84 \mathrm{E}-01$ & $1.36 \mathrm{E}-02$ & $1.85 \mathrm{E}-04$ \\
\hline & \begin{tabular}{|l|} 
North America \\
\end{tabular} & $-8.73 \mathrm{E}-03$ & $1.27 \mathrm{E}-02$ & $1.02 \mathrm{E}-03$ & \begin{tabular}{|l|}
$4.74 \mathrm{E}-02$ \\
\end{tabular} & $-2.83 \mathrm{E}-02$ & $-2.11 \mathrm{E}-01$ & $1.72 \mathrm{E}-04$ \\
\hline & Oceania & $5.04 \mathrm{E}-04$ & $-1.65 \mathrm{E}-03$ & $-1.14 \mathrm{E}-03$ & 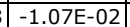 & $2.01 \mathrm{E}-03$ & $1.19 \mathrm{E}-03$ & $-1.74 \mathrm{E}-02$ \\
\hline \multirow{7}{*}{ Industrial } & Africa/Middle East & 1 & $1.38 \mathrm{E}-03$ & $-1.49 \mathrm{E}-05$ & $1.83 \mathrm{E}-03$ & $-5.63 \mathrm{E}-03$ & $-8.85 \mathrm{E}-04$ & $3.29 \mathrm{E}-05$ \\
\hline & \begin{tabular}{|l|} 
Southern Asia \\
\end{tabular} & $1.38 \mathrm{E}-03$ & 1 & $2.64 \mathrm{E}-05$ & $-4.66 \mathrm{E}-02$ & 7.77E-03 & $1.13 \mathrm{E}-02$ & $-1.03 \mathrm{E}-04$ \\
\hline & Central/South America & $-1.49 \mathrm{E}-05$ & $2.64 \mathrm{E}-05$ & 1 & $-4.13 \mathrm{E}-04$ & $2.41 \mathrm{E}-04$ & $3.11 \mathrm{E}-04$ & $-6.47 \mathrm{E}-05$ \\
\hline & Northern Asia & $1.83 \mathrm{E}-03$ & $-4.66 \mathrm{E}-02$ & $-4.13 \mathrm{E}-04$ & 1 & $-2.28 \mathrm{E}-02$ & $-3.10 \mathrm{E}-01$ & $-5.93 \mathrm{E}-04$ \\
\hline & Europe & $-5.63 E-03$ & 7.77E-03 & $2.41 \mathrm{E}-04$ & $-2.28 \mathrm{E}-02$ & 1 & $6.66 \mathrm{E}-03$ & $1.38 \mathrm{E}-04$ \\
\hline & North America & $-8.85 E-04$ & $1.13 \mathrm{E}-02$ & $3.11 \mathrm{E}-04$ & $-3.10 \mathrm{E}-01$ & $6.66 \mathrm{E}-03$ & 1 & $5.36 \mathrm{E}-05$ \\
\hline & Oceania & $3.29 \mathrm{E}-05$ & $-1.03 \mathrm{E}-04$ & $-6.47 E-05$ & $-5.93 E-04$ & $1.38 \mathrm{E}-04$ & $5.36 \mathrm{E}-05$ & 1 \\
\hline \multirow{7}{*}{ Natural Soil } & Africa/Middle East & $-2.45 \mathrm{E}-02$ & $5.04 \mathrm{E}-02$ & $-5.10 \mathrm{E}-03$ & \begin{tabular}{|l|}
$6.33 E-02$ \\
\end{tabular} & $-1.31 \mathrm{E}-02$ & $8.68 \mathrm{E}-04$ & 7.92E-05 \\
\hline & Southern Asia & $-1.04 \mathrm{E}-02$ & $-1.34 \mathrm{E}-01$ & $1.06 \mathrm{E}-03$ & \begin{tabular}{|l|} 
\\
$4.57 E-02$ \\
\end{tabular} & $-5.49 \mathrm{E}-04$ & $5.83 \mathrm{E}-03$ & $3.70 \mathrm{E}-03$ \\
\hline & Central/South America & $-6.24 \mathrm{E}-03$ & $8.30 \mathrm{E}-03$ & $-1.51 \mathrm{E}-02$ & $1.78 \mathrm{E}-02$ & $1.41 \mathrm{E}-02$ & $3.32 \mathrm{E}-03$ & $-1.97 \mathrm{E}-03$ \\
\hline & Northern Asia & $6.62 \mathrm{E}-03$ & $-2.98 \mathrm{E}-02$ & $5.29 \mathrm{E}-04$ & $-4.78 \mathrm{E}-02$ & $1.13 \mathrm{E}-03$ & $3.54 \mathrm{E}-02$ & $-3.51 \mathrm{E}-03$ \\
\hline & Europe & $-4.57 \mathrm{E}-03$ & $1.15 \mathrm{E}-02$ & $2.76 \mathrm{E}-04$ & $2.35 \mathrm{E}-02$ & $-1.92 \mathrm{E}-01$ & $2.09 \mathrm{E}-02$ & $8.41 \mathrm{E}-04$ \\
\hline & North America & $-5.72 \mathrm{E}-03$ & 7.07E-02 & $9.79 E-04$ & $-1.91 \mathrm{E}-01$ & $-1.87 \mathrm{E}-02$ & $-2.51 \mathrm{E}-01$ & $1.51 \mathrm{E}-03$ \\
\hline & Oceania & $7.28 \mathrm{E}-04$ & $1.63 \mathrm{E}-03$ & $-9.85 E-04$ & $-1.08 \mathrm{E}-02$ & $3.67 \mathrm{E}-03$ & $2.60 \mathrm{E}-03$ & $-9.86 \mathrm{E}-03$ \\
\hline \multirow{6}{*}{ Ocean } & North Pacific & $-2.99 \mathrm{E}-03$ & $-3.50 \mathrm{E}-02$ & $7.00 \mathrm{E}-04$ & $1.69 \mathrm{E}-02$ & $-1.10 \mathrm{E}-02$ & $-5.92 \mathrm{E}-03$ & $1.58 \mathrm{E}-03$ \\
\hline & \begin{tabular}{|l} 
South Pacific \\
\end{tabular} & $-2.89 \mathrm{E}-03$ & $1.56 \mathrm{E}-02$ & $-3.89 \mathrm{E}-03$ & \begin{tabular}{|l|} 
\\
\end{tabular} & $1.27 \mathrm{E}-02$ & $-1.27 \mathrm{E}-03$ & $1.12 \mathrm{E}-03$ \\
\hline & Northern Ocean & $3.63 \mathrm{E}-03$ & $-5.19 \mathrm{E}-03$ & $2.45 \mathrm{E}-04$ & $1.24 \mathrm{E}-02$ & $6.82 \mathrm{E}-03$ & $5.90 \mathrm{E}-03$ & $5.65 \mathrm{E}-04$ \\
\hline & Atlantic & $-8.43 \mathrm{E}-03$ & $-2.58 \mathrm{E}-02$ & $-9.32 \mathrm{E}-04$ & \begin{tabular}{|l|}
$-9.37 E-02$ \\
\end{tabular} & $-1.72 \mathrm{E}-02$ & $-6.60 \mathrm{E}-02$ & $1.72 \mathrm{E}-03$ \\
\hline & Southern Ocean & $2.96 \mathrm{E}-03$ & $-2.91 \mathrm{E}-02$ & $-9.66 \mathrm{E}-03$ & 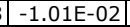 & $5.67 \mathrm{E}-04$ & $1.10 \mathrm{E}-02$ & $-1.66 \mathrm{E}-03$ \\
\hline & Indian Ocean & $-2.50 \mathrm{E}-03$ & $3.06 \mathrm{E}-02$ & $-5.61 \mathrm{E}-03$ & 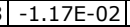 & $5.34 \mathrm{E}-03$ & $1.87 \mathrm{E}-03$ & $-2.97 \mathrm{E}-03$ \\
\hline \multirow{7}{*}{$\begin{array}{l}\text { Biomass } \\
\text { burning }\end{array}$} & Africa/Middle East & $-3.33 \mathrm{E}-03$ & $4.72 \mathrm{E}-03$ & $-1.75 \mathrm{E}-03$ & \begin{tabular}{|c|} 
\\
\end{tabular} & $2.45 \mathrm{E}-03$ & $2.61 \mathrm{E}-04$ & $5.44 \mathrm{E}-04$ \\
\hline & Southern Asia & $6.35 \mathrm{E}-04$ & $1.26 \mathrm{E}-02$ & $-1.21 \mathrm{E}-03$ & \begin{tabular}{|l|} 
\\
\end{tabular} & $9.26 \mathrm{E}-03$ & $5.10 \mathrm{E}-03$ & $1.04 \mathrm{E}-03$ \\
\hline & Central/South America & $-2.20 \mathrm{E}-04$ & $1.37 \mathrm{E}-03$ & $-6.91 \mathrm{E}-04$ & $\begin{array}{ll}1.44 \mathrm{E}-04 \\
\end{array}$ & $7.43 \mathrm{E}-04$ & $1.22 \mathrm{E}-03$ & $1.37 \mathrm{E}-04$ \\
\hline & Northern Asia & $3.55 \mathrm{E}-05$ & $-1.58 \mathrm{E}-02$ & $5.33 \mathrm{E}-05$ & $-1.48 \mathrm{E}-03$ & $8.32 \mathrm{E}-04$ & $2.11 \mathrm{E}-03$ & $2.97 \mathrm{E}-04$ \\
\hline & Europe & $7.26 \mathrm{E}-06$ & $3.14 \mathrm{E}-05$ & $6.86 \mathrm{E}-07$ & 6.98E-05 & $-3.09 \mathrm{E}-05$ & $6.62 \mathrm{E}-05$ & $6.11 \mathrm{E}-06$ \\
\hline & North America & $1.60 \mathrm{E}-04$ & $1.61 \mathrm{E}-03$ & $4.85 \mathrm{E}-05$ & \begin{tabular}{|l|}
$-2.86 \mathrm{E}-04$ \\
\end{tabular} & $7.20 \mathrm{E}-04$ & $3.61 \mathrm{E}-04$ & $1.00 \mathrm{E}-04$ \\
\hline & Oceania & $1.60 \mathrm{E}-04$ & $2.10 \mathrm{E}-03$ & $-3.26 \mathrm{E}-04$ & $-2.18 \mathrm{E}-03$ & $1.02 \mathrm{E}-03$ & $9.98 \mathrm{E}-04$ & $8.73 \mathrm{E}-08$ \\
\hline
\end{tabular}

Fig. S2I. Correlation of the emissions in 1997 for industrial. 


\begin{tabular}{|c|c|c|c|c|c|c|c|c|}
\hline & \multirow{2}{*}{\multicolumn{7}{|c|}{ Natural Soil }} \\
\hline & & & & & & & & \\
\hline Source sector & Region & $\begin{array}{l}\text { Africa/M. } \\
\text { East }\end{array}$ & $\begin{array}{l}\text { Southern } \\
\text { Asia }\end{array}$ & $\begin{array}{l}\text { C. /S. } \\
\text { America }\end{array}$ & $\begin{array}{l}\text { Northern } \\
\text { Asia }\end{array}$ & Europe & $\begin{array}{l}\text { North } \\
\text { America }\end{array}$ & Oceania \\
\hline \multirow{7}{*}{$\begin{array}{l}\text { Agricultural } \\
\text { Soil }\end{array}$} & Africa/Middle East & $-1.43 \mathrm{E}-01$ & $-6.19 \mathrm{E}-02$ & $-5.11 \mathrm{E}-02$ & 3.01E-02 & $-1.12 \mathrm{E}-02$ & $-3.80 \mathrm{E}-02$ & $4.66 \mathrm{E}-03$ \\
\hline & Southern Asia & $-6.38 \mathrm{E}-02$ & $-3.87 \mathrm{E}-01$ & $5.44 \mathrm{E}-02$ & $\mid-7.88 \mathrm{E}-03$ & $4.20 \mathrm{E}-03$ & $-2.96 \mathrm{E}-02$ & $2.80 \mathrm{E}-02$ \\
\hline & Central/South America & $-5.53 \mathrm{E}-02$ & $1.26 \mathrm{E}-02$ & $-1.75 \mathrm{E}-01$ & $5.63 \mathrm{E}-03$ & $3.22 \mathrm{E}-03$ & $1.15 \mathrm{E}-02$ & $-9.87 \mathrm{E}-03$ \\
\hline & Northern Asia & $-2.35 \mathrm{E}-02$ & $-2.81 \mathrm{E}-02$ & $1.83 \mathrm{E}-02$ & \begin{tabular}{|l|}
$-1.69 \mathrm{E}-01$ \\
\end{tabular} & $1.29 \mathrm{E}-03$ & $2.44 \mathrm{E}-02$ & $-6.14 \mathrm{E}-03$ \\
\hline & Europe & $-1.58 \mathrm{E}-02$ & $3.45 \mathrm{E}-03$ & $2.10 \mathrm{E}-02$ & $1.47 \mathrm{E}-02$ & $-2.67 \mathrm{E}-01$ & $1.31 \mathrm{E}-02$ & $4.26 \mathrm{E}-03$ \\
\hline & \begin{tabular}{|l|} 
North America \\
\end{tabular} & $-4.80 \mathrm{E}-02$ & $-4.48 \mathrm{E}-02$ & $1.16 \mathrm{E}-02$ & $3.23 \mathrm{E}-02$ & $1.19 \mathrm{E}-02$ & $-3.96 \mathrm{E}-01$ & $9.14 \mathrm{E}-03$ \\
\hline & Oceania & $-9.68 \mathrm{E}-05$ & $5.52 \mathrm{E}-02$ & $-4.06 \mathrm{E}-02$ & $-5.45 E-02$ & $1.33 \mathrm{E}-02$ & $2.57 \mathrm{E}-02$ & $-1.46 \mathrm{E}-01$ \\
\hline \multirow{7}{*}{ Industrial } & Africa/Middle East & $-2.45 \mathrm{E}-02$ & $-1.04 \mathrm{E}-02$ & $-6.24 \mathrm{E}-03$ & $6.62 \mathrm{E}-03$ & $-4.57 \mathrm{E}-03$ & $-5.72 \mathrm{E}-03$ & $7.28 \mathrm{E}-04$ \\
\hline & \begin{tabular}{|l|} 
Southern Asia \\
\end{tabular} & $5.04 \mathrm{E}-02$ & $-1.34 \mathrm{E}-01$ & $8.30 \mathrm{E}-03$ & $-2.98 \mathrm{E}-02$ & $1.15 \mathrm{E}-02$ & $7.07 \mathrm{E}-02$ & $1.63 \mathrm{E}-03$ \\
\hline & Central/South America & $-5.10 \mathrm{E}-03$ & $1.06 \mathrm{E}-03$ & $-1.51 \mathrm{E}-02$ & $5.29 \mathrm{E}-04$ & $2.76 \mathrm{E}-04$ & $9.79 \mathrm{E}-04$ & $-9.85 \mathrm{E}-04$ \\
\hline & Northern Asia & $6.33 \mathrm{E}-02$ & $4.57 \mathrm{E}-02$ & $1.78 \mathrm{E}-02$ & \begin{tabular}{|l|}
$-4.78 \mathrm{E}-02$ \\
\end{tabular} & $2.35 \mathrm{E}-02$ & $-1.91 \mathrm{E}-01$ & $-1.08 \mathrm{E}-02$ \\
\hline & Europe & $-1.31 \mathrm{E}-02$ & $-5.49 \mathrm{E}-04$ & $1.41 \mathrm{E}-02$ & $1.13 \mathrm{E}-03$ & $-1.92 \mathrm{E}-01$ & $-1.87 \mathrm{E}-02$ & 3.67E-03 \\
\hline & North America & $8.68 \mathrm{E}-04$ & $5.83 E-03$ & $3.32 \mathrm{E}-03$ & $3.54 \mathrm{E}-02$ & $2.09 \mathrm{E}-02$ & $-2.51 \mathrm{E}-01$ & $2.60 \mathrm{E}-03$ \\
\hline & Oceania & 7.92E-05 & $3.70 \mathrm{E}-03$ & $-1.97 \mathrm{E}-03$ & $-3.51 \mathrm{E}-03$ & $8.41 \mathrm{E}-04$ & $1.51 \mathrm{E}-03$ & $-9.86 \mathrm{E}-03$ \\
\hline \multirow{7}{*}{ Natural Soil } & Africa/Middle East & 1 & $-2.31 \mathrm{E}-01$ & $-3.09 \mathrm{E}-01$ & $1.41 \mathrm{E}-01$ & $-1.02 \mathrm{E}-02$ & $-1.31 \mathrm{E}-01$ & 4.04E-04 \\
\hline & Southern Asia & $-2.31 \mathrm{E}-01$ & 1 & $-6.60 \mathrm{E}-02$ & $-3.65 \mathrm{E}-01$ & $4.94 \mathrm{E}-02$ & $-3.07 \mathrm{E}-02$ & $1.86 \mathrm{E}-02$ \\
\hline & Central/South America & $-3.09 \mathrm{E}-01$ & $-6.60 \mathrm{E}-02$ & 1 & \begin{tabular}{|l|}
$-8.91 \mathrm{E}-02$ \\
\end{tabular} & $-1.52 \mathrm{E}-02$ & $-1.80 \mathrm{E}-02$ & $-6.61 \mathrm{E}-02$ \\
\hline & Northern Asia & $1.41 \mathrm{E}-01$ & $-3.65 \mathrm{E}-01$ & $-8.91 \mathrm{E}-02$ & 1 & $-1.87 \mathrm{E}-01$ & $-2.89 \mathrm{E}-01$ & $-5.83 \mathrm{E}-02$ \\
\hline & Europe & $-1.02 \mathrm{E}-02$ & $4.94 \mathrm{E}-02$ & $-1.52 \mathrm{E}-02$ & $-1.87 \mathrm{E}-01$ & 1 & $-7.40 \mathrm{E}-02$ & $9.42 \mathrm{E}-03$ \\
\hline & North America & $-1.31 \mathrm{E}-01$ & $-3.07 E-02$ & $-1.80 \mathrm{E}-02$ & \begin{tabular}{|l|}
$-2.89 \mathrm{E}-01$ \\
\end{tabular} & $-7.40 \mathrm{E}-02$ & 1 & $2.67 \mathrm{E}-02$ \\
\hline & Oceania & $4.04 \mathrm{E}-04$ & $1.86 \mathrm{E}-02$ & $-6.61 \mathrm{E}-02$ & $-5.83 E-02$ & $9.42 \mathrm{E}-03$ & $2.67 \mathrm{E}-02$ & 1 \\
\hline \multirow{6}{*}{ Ocean } & North Pacific & $8.53 \mathrm{E}-02$ & $1.43 \mathrm{E}-01$ & $1.91 \mathrm{E}-02$ & $-2.66 \mathrm{E}-01$ & $7.60 \mathrm{E}-02$ & $9.33 \mathrm{E}-02$ & $2.20 \mathrm{E}-02$ \\
\hline & \begin{tabular}{|l} 
South Pacific \\
\end{tabular} & $-1.06 \mathrm{E}-01$ & $-4.27 \mathrm{E}-02$ & $-4.02 \mathrm{E}-01$ & $\begin{array}{ll}-1.09 \mathrm{E}-02 \\
\end{array}$ & $7.08 \mathrm{E}-03$ & $4.98 \mathrm{E}-02$ & $-3.75 \mathrm{E}-02$ \\
\hline & Northern Ocean & $6.28 \mathrm{E}-02$ & $1.01 \mathrm{E}-01$ & $3.78 \mathrm{E}-02$ & $-8.42 \mathrm{E}-02$ & $7.42 \mathrm{E}-03$ & $-3.63 \mathrm{E}-03$ & $8.94 \mathrm{E}-03$ \\
\hline & Atlantic & $-8.51 \mathrm{E}-02$ & $1.18 \mathrm{E}-01$ & $-4.67 \mathrm{E}-02$ & 4.14E-02 & $4.37 \mathrm{E}-02$ & $2.03 \mathrm{E}-02$ & $1.71 \mathrm{E}-02$ \\
\hline & Southern Ocean & $8.80 \mathrm{E}-02$ & $2.30 \mathrm{E}-01$ & $-1.03 \mathrm{E}-01$ & $-4.27 \mathrm{E}-02$ & $1.19 \mathrm{E}-02$ & $4.27 \mathrm{E}-02$ & $-3.96 \mathrm{E}-03$ \\
\hline & Indian Ocean & $-8.68 \mathrm{E}-02$ & $-1.45 \mathrm{E}-01$ & $-2.05 \mathrm{E}-01$ & $4.27 \mathrm{E}-02$ & $7.61 \mathrm{E}-03$ & $4.75 \mathrm{E}-02$ & $-3.25 \mathrm{E}-02$ \\
\hline \multirow{7}{*}{$\begin{array}{l}\text { Biomass } \\
\text { burning }\end{array}$} & Africa/Middle East & $-1.12 \mathrm{E}-01$ & $3.92 \mathrm{E}-03$ & $-6.51 \mathrm{E}-02$ & $2.12 \mathrm{E}-02$ & $-1.20 \mathrm{E}-03$ & $-3.40 \mathrm{E}-03$ & $8.20 \mathrm{E}-04$ \\
\hline & Southern Asia & $-7.32 \mathrm{E}-02$ & $-8.79 \mathrm{E}-02$ & $6.71 \mathrm{E}-03$ & $5.90 \mathrm{E}-02$ & $3.19 \mathrm{E}-03$ & $3.39 \mathrm{E}-02$ & $-1.42 \mathrm{E}-03$ \\
\hline & Central/South America & $-2.12 \mathrm{E}-02$ & $-9.16 \mathrm{E}-04$ & $-1.84 \mathrm{E}-03$ & $7.45 \mathrm{E}-03$ & $5.30 \mathrm{E}-04$ & $2.02 \mathrm{E}-03$ & $-1.53 \mathrm{E}-03$ \\
\hline & Northern Asia & $8.31 \mathrm{E}-03$ & $1.22 \mathrm{E}-02$ & $-5.16 \mathrm{E}-03$ & $-5.17 \mathrm{E}-02$ & $-5.85 \mathrm{E}-03$ & $-1.01 \mathrm{E}-03$ & $1.76 \mathrm{E}-03$ \\
\hline & Europe & $9.36 \mathrm{E}-05$ & $5.80 \mathrm{E}-04$ & $1.01 \mathrm{E}-04$ & $-1.18 \mathrm{E}-03$ & $-1.49 \mathrm{E}-03$ & $-3.53 \mathrm{E}-05$ & $8.98 \mathrm{E}-05$ \\
\hline & North America & $1.60 \mathrm{E}-03$ & $6.38 \mathrm{E}-03$ & $-2.85 \mathrm{E}-03$ & $-1.98 \mathrm{E}-02$ & $-6.34 \mathrm{E}-03$ & $-1.79 \mathrm{E}-02$ & $1.04 \mathrm{E}-03$ \\
\hline & Oceania & $-8.17 \mathrm{E}-03$ & $-9.15 \mathrm{E}-03$ & $-1.60 \mathrm{E}-03$ & $6.10 \mathrm{E}-03$ & $5.64 \mathrm{E}-04$ & $4.59 \mathrm{E}-03$ & $-3.10 \mathrm{E}-03$ \\
\hline
\end{tabular}

Fig. S2m. Correlation of the emissions in 1997 for natural soil. 


\begin{tabular}{|c|c|c|c|c|c|c|c|}
\hline & & & & & & & \\
\hline & & \multicolumn{6}{|c|}{ Ocean } \\
\hline Source sector & Region & $\begin{array}{l}\text { North } \\
\text { Pacific }\end{array}$ & $\begin{array}{l}\text { South } \\
\text { Pacific }\end{array}$ & $\begin{array}{l}\text { Northern } \\
\text { Ocean }\end{array}$ & Atlantic & $\begin{array}{l}\text { Southern } \\
\text { Ocean }\end{array}$ & $\begin{array}{l}\text { Indian } \\
\text { Ocean }\end{array}$ \\
\hline \multirow{7}{*}{$\begin{array}{l}\text { Agricultural } \\
\text { Soil }\end{array}$} & Africa/Middle East & $-6.26 \mathrm{E}-03$ & $-2.55 \mathrm{E}-02$ & $1.51 \mathrm{E}-02$ & $-5.28 \mathrm{E}-02$ & $2.46 \mathrm{E}-02$ & $-1.85 \mathrm{E}-02$ \\
\hline & Southern Asia & $-1.30 \mathrm{E}-01$ & $-7.86 \mathrm{E}-04$ & $-5.23 E-03$ & $-1.06 \mathrm{E}-01$ & $8.58 \mathrm{E}-02$ & $-3.75 E-02$ \\
\hline & Central/South America & $7.35 \mathrm{E}-03$ & $-3.84 \mathrm{E}-02$ & $2.30 \mathrm{E}-03$ & $-9.89 \mathrm{E}-03$ & $-1.26 \mathrm{E}-01$ & $-5.71 \mathrm{E}-02$ \\
\hline & Northern Asia & $-7.06 \mathrm{E}-03$ & $1.83 \mathrm{E}-02$ & $-5.69 \mathrm{E}-02$ & $-6.52 \mathrm{E}-02$ & $-5.93 \mathrm{E}-03$ & $1.56 \mathrm{E}-03$ \\
\hline & Europe & $1.09 \mathrm{E}-02$ & $1.19 \mathrm{E}-02$ & $-3.80 \mathrm{E}-02$ & $-4.05 E-02$ & $3.13 \mathrm{E}-03$ & $2.30 \mathrm{E}-03$ \\
\hline & \begin{tabular}{|l|} 
North America \\
\end{tabular} & $-3.16 \mathrm{E}-02$ & $1.32 \mathrm{E}-02$ & $-8.57 E-03$ & $-1.25 \mathrm{E}-01$ & $3.09 E-02$ & $2.80 \mathrm{E}-02$ \\
\hline & Oceania & $2.33 \mathrm{E}-02$ & $1.41 \mathrm{E}-02$ & $7.72 \mathrm{E}-03$ & $2.50 \mathrm{E}-02$ & $-1.62 \mathrm{E}-02$ & $-4.35 \mathrm{E}-02$ \\
\hline \multirow{7}{*}{ Industrial } & Africa/Middle East & $-2.99 \mathrm{E}-03$ & $-2.89 \mathrm{E}-03$ & $3.63 \mathrm{E}-03$ & $-8.43 \mathrm{E}-03$ & $2.96 \mathrm{E}-03$ & $-2.50 \mathrm{E}-03$ \\
\hline & Southern Asia & $-3.50 \mathrm{E}-02$ & $1.56 \mathrm{E}-02$ & $-5.19 \mathrm{E}-03$ & $-2.58 \mathrm{E}-02$ & $-2.91 \mathrm{E}-02$ & $3.06 \mathrm{E}-02$ \\
\hline & Central/South America & $7.00 \mathrm{E}-04$ & $-3.89 \mathrm{E}-03$ & $2.45 \mathrm{E}-04$ & $-9.32 \mathrm{E}-04$ & $-9.66 \mathrm{E}-03$ & $-5.61 \mathrm{E}-03$ \\
\hline & \begin{tabular}{|l} 
Northern Asia \\
\end{tabular} & $1.69 \mathrm{E}-02$ & $8.20 \mathrm{E}-04$ & $1.24 \mathrm{E}-02$ & $-9.37 E-02$ & $-1.01 \mathrm{E}-02$ & $-1.17 \mathrm{E}-02$ \\
\hline & Europe & $-1.10 \mathrm{E}-02$ & $1.27 \mathrm{E}-02$ & $6.82 \mathrm{E}-03$ & $-1.72 \mathrm{E}-02$ & $5.67 \mathrm{E}-04$ & $5.34 \mathrm{E}-03$ \\
\hline & \begin{tabular}{|l} 
North $\mathrm{Am}$ \\
\end{tabular} & $-5.92 \mathrm{E}-03$ & $-1.27 \mathrm{E}-03$ & $5.90 \mathrm{E}-03$ & $-6.60 \mathrm{E}-02$ & $0 \mathrm{E}-02$ & $\mathrm{E}-03$ \\
\hline & Oceania & $1.58 \mathrm{E}-03$ & $1.12 \mathrm{E}-03$ & $5.65 \mathrm{E}-04$ & $1.72 \mathrm{E}-03$ & $-1.66 \mathrm{E}-03$ & $-2.97 \mathrm{E}-03$ \\
\hline \multirow{7}{*}{ Natural Soil } & Africa/Middle East & $8.53 \mathrm{E}-02$ & $-1.06 \mathrm{E}-01$ & $6.28 \mathrm{E}-02$ & $-8.51 \mathrm{E}-02$ & $8.80 \mathrm{E}-02$ & $-8.68 \mathrm{E}-02$ \\
\hline & Southern Asia & $1.43 \mathrm{E}-01$ & $-4.27 \mathrm{E}-02$ & $1.01 \mathrm{E}-01$ & $1.18 \mathrm{E}-01$ & $2.30 \mathrm{E}-01$ & $-1.45 \mathrm{E}-01$ \\
\hline & Central/South America & $1.91 \mathrm{E}-02$ & $-4.02 \mathrm{E}-01$ & $3.78 \mathrm{E}-02$ & $-4.67 \mathrm{E}-02$ & $-1.03 \mathrm{E}-01$ & $-2.05 \mathrm{E}-01$ \\
\hline & Northern Asia & $-2.66 \mathrm{E}-01$ & $-1.09 \mathrm{E}-02$ & $-8.42 \mathrm{E}-02$ & $4.14 \mathrm{E}-02$ & $-4.27 \mathrm{E}-02$ & $4.27 \mathrm{E}-02$ \\
\hline & Europe & $7.60 \mathrm{E}-02$ & $7.08 \mathrm{E}-03$ & $7.42 \mathrm{E}-03$ & $4.37 \mathrm{E}-02$ & $1.19 \mathrm{E}-02$ & $7.61 \mathrm{E}-03$ \\
\hline & North America & $9.33 \mathrm{E}-02$ & $4.98 \mathrm{E}-02$ & $-3.63 E-03$ & $2.03 \mathrm{E}-02$ & $4.27 \mathrm{E}-02$ & $4.75 \mathrm{E}-02$ \\
\hline & Oceania & $2.20 \mathrm{E}-02$ & $-3.75 \mathrm{E}-02$ & $8.94 \mathrm{E}-03$ & $1.71 \mathrm{E}-02$ & $-3.96 \mathrm{E}-03$ & $-3.25 \mathrm{E}-02$ \\
\hline \multirow{6}{*}{ Ocean } & North Pacific & 1 & $-3.18 \mathrm{E}-03$ & $-3.75 E-02$ & $-1.83 \mathrm{E}-01$ & $2.64 \mathrm{E}-02$ & 4.17E-02 \\
\hline & South Pacific & $-3.18 \mathrm{E}-03$ & 1 & 3.97E-03 & $-6.36 \mathrm{E}-02$ & $-8.81 \mathrm{E}-03$ & $-9.51 \mathrm{E}-02$ \\
\hline & Northern Ocean & $-3.75 \mathrm{E}-02$ & $3.97 \mathrm{E}-03$ & 1 & $-2.71 \mathrm{E}-01$ & $2.75 \mathrm{E}-02$ & $2.02 \mathrm{E}-03$ \\
\hline & Atlantic & $-1.83 \mathrm{E}-01$ & $-6.36 \mathrm{E}-02$ & $-2.71 \mathrm{E}-01$ & 1 & -7.17E-02 & $-1.14 \mathrm{E}-02$ \\
\hline & Southern Ocean & $2.64 \mathrm{E}-02$ & $-8.81 \mathrm{E}-03$ & $2.75 \mathrm{E}-02$ & $-7.17 \mathrm{E}-02$ & 1 & $-5.71 \mathrm{E}-01$ \\
\hline & Indian Ocean & $4.17 \mathrm{E}-02$ & $-9.51 \mathrm{E}-02$ & $2.02 \mathrm{E}-03$ & $-1.14 \mathrm{E}-02$ & $-5.71 \mathrm{E}-01$ & 1 \\
\hline \multirow{7}{*}{$\begin{array}{c}\text { Biomass } \\
\text { burning }\end{array}$} & Africa/Middle East & $2.54 \mathrm{E}-04$ & $-7.19 \mathrm{E}-02$ & $5.34 \mathrm{E}-03$ & \begin{tabular}{|l|}
$-7.03 E-02$ \\
\end{tabular} & $2.81 \mathrm{E}-03$ & $-2.51 \mathrm{E}-02$ \\
\hline & Southern Asia & $-4.40 \mathrm{E}-03$ & $-3.11 \mathrm{E}-02$ & $-1.22 \mathrm{E}-02$ & $-2.84 \mathrm{E}-02$ & $8.33 \mathrm{E}-02$ & $-7.72 \mathrm{E}-02$ \\
\hline & Central/South America & $1.55 \mathrm{E}-05$ & $-2.06 \mathrm{E}-02$ & $1.06 \mathrm{E}-04$ & $-1.22 \mathrm{E}-02$ & $-2.24 \mathrm{E}-02$ & $-5.29 \mathrm{E}-03$ \\
\hline & \begin{tabular}{|l} 
Northern Asia \\
\end{tabular} & $-3.74 \mathrm{E}-02$ & $-8.38 \mathrm{E}-05$ & $-3.48 \mathrm{E}-03$ & $-5.88 \mathrm{E}-03$ & $5.98 \mathrm{E}-03$ & $4.56 \mathrm{E}-03$ \\
\hline & Europe & $1.27 \mathrm{E}-04$ & $3.49 \mathrm{E}-05$ & $-3.55 \mathrm{E}-04$ & $-4.22 \mathrm{E}-04$ & $9.73 \mathrm{E}-05$ & $-6.93 \mathrm{E}-06$ \\
\hline & North America & $2.03 \mathrm{E}-03$ & $3.31 \mathrm{E}-04$ & $-8.04 \mathrm{E}-03$ & $-1.92 \mathrm{E}-03$ & $2.28 \mathrm{E}-03$ & $1.02 \mathrm{E}-03$ \\
\hline & Oceania & $7.06 \mathrm{E}-04$ & $-1.08 \mathrm{E}-02$ & $-1.53 \mathrm{E}-03$ & $-3.63 \mathrm{E}-03$ & $4.61 \mathrm{E}-03$ & $-8.76 \mathrm{E}-03$ \\
\hline
\end{tabular}

Fig. S2n. Correlation of the emissions in 1997 for ocean. 


\begin{tabular}{|c|c|c|c|c|c|c|c|c|}
\hline \multirow[b]{3}{*}{ Source sector } & \multirow[b]{3}{*}{ Region } & \multirow{2}{*}{\multicolumn{7}{|c|}{ Biomass burning }} \\
\hline & & & & & & & & \\
\hline & & $\begin{array}{l}\text { Africa/M. } \\
\text { East }\end{array}$ & $\begin{array}{l}\text { Southern } \\
\text { Asia }\end{array}$ & $\begin{array}{l}\text { C. /S. } \\
\text { America }\end{array}$ & $\begin{array}{l}\text { Northern } \\
\text { Asia }\end{array}$ & Europe & $\begin{array}{l}\text { North } \\
\text { America }\end{array}$ & Oceania \\
\hline \multirow{7}{*}{$\begin{array}{l}\text { Agricultural } \\
\text { Soil }\end{array}$} & Africa/Middle East & $-2.46 \mathrm{E}-02$ & $-1.87 \mathrm{E}-03$ & $-2.28 \mathrm{E}-03$ & $4.07 \mathrm{E}-04$ & $2.88 \mathrm{E}-05$ & 3.07E-04 & $2.32 \mathrm{E}-04$ \\
\hline & Southern Asia & $-2.88 \mathrm{E}-02$ & $-2.38 \mathrm{E}-02$ & $3.47 \mathrm{E}-03$ & $-2.00 \mathrm{E}-02$ & $-2.71 \mathrm{E}-05$ & $6.42 \mathrm{E}-04$ & $3.67 \mathrm{E}-03$ \\
\hline & Central/South America & $-1.91 \mathrm{E}-02$ & $-1.35 \mathrm{E}-02$ & $-7.59 \mathrm{E}-03$ & $5.47 \mathrm{E}-04$ & $9.34 \mathrm{E}-06$ & $5.44 \mathrm{E}-04$ & $-3.62 \mathrm{E}-03$ \\
\hline & Northern Asia & $9.71 \mathrm{E}-03$ & $-1.94 \mathrm{E}-02$ & $1.33 \mathrm{E}-03$ & $-3.72 \mathrm{E}-03$ & $-2.84 \mathrm{E}-04$ & $2.55 \mathrm{E}-03$ & $-6.85 \mathrm{E}-04$ \\
\hline & \begin{tabular}{|l} 
Europe \\
\end{tabular} & $2.43 \mathrm{E}-03$ & $9.21 \mathrm{E}-03$ & $6.38 \mathrm{E}-04$ & $4.39 \mathrm{E}-03$ & $5.08 \mathrm{E}-05$ & $1.06 \mathrm{E}-03$ & $9.00 \mathrm{E}-04$ \\
\hline & \begin{tabular}{|l} 
North America \\
\end{tabular} & $-2.59 \mathrm{E}-03$ & $2.08 \mathrm{E}-02$ & $3.56 \mathrm{E}-03$ & $-7.44 \mathrm{E}-04$ & $-4.16 \mathrm{E}-05$ & $1.19 \mathrm{E}-03$ & $3.96 \mathrm{E}-03$ \\
\hline & Oceania & $7.91 \mathrm{E}-03$ & $1.32 \mathrm{E}-02$ & $1.99 \mathrm{E}-03$ & $4.29 \mathrm{E}-03$ & $9.24 \mathrm{E}-05$ & $1.58 \mathrm{E}-03$ & $-5.38 \mathrm{E}-04$ \\
\hline \multirow{7}{*}{ Industrial } & Africa/Middle East & $-3.33 \mathrm{E}-03$ & $6.35 \mathrm{E}-04$ & $-2.20 \mathrm{E}-04$ & $3.55 \mathrm{E}-05$ & $7.26 \mathrm{E}-06$ & $1.60 \mathrm{E}-04$ & $1.60 \mathrm{E}-04$ \\
\hline & Southern Asia & $4.72 \mathrm{E}-03$ & $1.26 \mathrm{E}-02$ & $1.37 \mathrm{E}-03$ & $-1.58 \mathrm{E}-02$ & $3.14 \mathrm{E}-05$ & $1.61 \mathrm{E}-03$ & $2.10 \mathrm{E}-03$ \\
\hline & Central/South America & $-1.75 \mathrm{E}-03$ & $-1.21 \mathrm{E}-03$ & $-6.91 \mathrm{E}-04$ & $5.33 \mathrm{E}-05$ & $6.86 \mathrm{E}-07$ & $4.85 \mathrm{E}-05$ & $-3.26 \mathrm{E}-04$ \\
\hline & Northern Asia & $1.06 \mathrm{E}-02$ & $-1.98 \mathrm{E}-02$ & $1.44 \mathrm{E}-04$ & $-1.48 \mathrm{E}-03$ & $6.98 \mathrm{E}-05$ & $-2.86 \mathrm{E}-04$ & $-2.18 \mathrm{E}-03$ \\
\hline & Europe & $2.45 \mathrm{E}-03$ & $9.26 \mathrm{E}-03$ & $7.43 \mathrm{E}-04$ & $8.32 \mathrm{E}-04$ & $-3.09 \mathrm{E}-05$ & $7.20 \mathrm{E}-04$ & $1.02 \mathrm{E}-03$ \\
\hline & North America & $2.61 \mathrm{E}-04$ & $5.10 \mathrm{E}-03$ & $1.22 \mathrm{E}-03$ & $2.11 \mathrm{E}-03$ & $6.62 \mathrm{E}-05$ & $3.61 \mathrm{E}-04$ & $9.98 \mathrm{E}-04$ \\
\hline & Oceania & $5.44 \mathrm{E}-04$ & $1.04 \mathrm{E}-03$ & $1.37 \mathrm{E}-04$ & $2.97 \mathrm{E}-04$ & $6.11 \mathrm{E}-06$ & $1.00 \mathrm{E}-04$ & $8.73 \mathrm{E}-08$ \\
\hline \multirow{7}{*}{ Natural Soil } & Africa/Middle East & $-1.12 \mathrm{E}-01$ & $-7.32 \mathrm{E}-02$ & $-2.12 \mathrm{E}-02$ & $8.31 \mathrm{E}-03$ & $9.36 \mathrm{E}-05$ & $1.60 \mathrm{E}-03$ & $-8.17 \mathrm{E}-03$ \\
\hline & Southern Asia & $3.92 \mathrm{E}-03$ & $-8.79 \mathrm{E}-02$ & $-9.16 \mathrm{E}-04$ & $1.22 \mathrm{E}-02$ & $5.80 \mathrm{E}-04$ & $6.38 \mathrm{E}-03$ & $-9.15 \mathrm{E}-03$ \\
\hline & Central/South America & $-6.51 \mathrm{E}-02$ & $6.71 \mathrm{E}-03$ & $-1.84 \mathrm{E}-03$ & $-5.16 \mathrm{E}-03$ & $1.01 \mathrm{E}-04$ & $-2.85 \mathrm{E}-03$ & $-1.60 \mathrm{E}-03$ \\
\hline & Northern Asia & $2.12 \mathrm{E}-02$ & $5.90 \mathrm{E}-02$ & $7.45 \mathrm{E}-03$ & $-5.17 \mathrm{E}-02$ & $-1.18 \mathrm{E}-03$ & $-1.98 \mathrm{E}-02$ & $6.10 \mathrm{E}-03$ \\
\hline & Europe & $-1.20 \mathrm{E}-03$ & $3.19 \mathrm{E}-03$ & $5.30 \mathrm{E}-04$ & $-5.85 E-03$ & $-1.49 \mathrm{E}-03$ & $-6.34 \mathrm{E}-03$ & 5.64E-04 \\
\hline & North America & $-3.40 \mathrm{E}-03$ & $3.39 \mathrm{E}-02$ & $2.02 \mathrm{E}-03$ & $-1.01 \mathrm{E}-03$ & $-3.53 \mathrm{E}-05$ & $-1.79 \mathrm{E}-02$ & $4.59 \mathrm{E}-03$ \\
\hline & Oceania & $8.20 \mathrm{E}-04$ & $-1.42 \mathrm{E}-03$ & $-1.53 \mathrm{E}-03$ & $1.76 \mathrm{E}-03$ & $8.98 \mathrm{E}-05$ & $1.04 \mathrm{E}-03$ & $-3.10 \mathrm{E}-03$ \\
\hline \multirow{6}{*}{ Ocean } & North Pacific & $2.54 \mathrm{E}-04$ & $-4.40 \mathrm{E}-03$ & $1.55 \mathrm{E}-05$ & $-3.74 \mathrm{E}-02$ & $1.27 \mathrm{E}-04$ & $2.03 \mathrm{E}-03$ & $7.06 \mathrm{E}-04$ \\
\hline & South Pacific & $-7.19 \mathrm{E}-02$ & $-3.11 \mathrm{E}-02$ & $-2.06 \mathrm{E}-02$ & $-8.38 \mathrm{E}-05$ & $3.49 \mathrm{E}-05$ & $3.31 \mathrm{E}-04$ & $-1.08 \mathrm{E}-02$ \\
\hline & Northern Ocean & $5.34 \mathrm{E}-03$ & $-1.22 \mathrm{E}-02$ & $1.06 \mathrm{E}-04$ & $-3.48 \mathrm{E}-03$ & $-3.55 \mathrm{E}-04$ & $-8.04 \mathrm{E}-03$ & $-1.53 \mathrm{E}-03$ \\
\hline & Atlantic & $-7.03 E-02$ & $-2.84 \mathrm{E}-02$ & $-1.22 \mathrm{E}-02$ & $-5.88 \mathrm{E}-03$ & $-4.22 \mathrm{E}-04$ & $-1.92 \mathrm{E}-03$ & $-3.63 \mathrm{E}-03$ \\
\hline & Southern Ocean & $2.81 \mathrm{E}-03$ & $8.33 \mathrm{E}-02$ & $-2.24 \mathrm{E}-02$ & $5.98 \mathrm{E}-03$ & $9.73 \mathrm{E}-05$ & $2.28 \mathrm{E}-03$ & $4.61 \mathrm{E}-03$ \\
\hline & Indian Ocean & $-2.51 \mathrm{E}-02$ & $-7.72 \mathrm{E}-02$ & $-5.29 \mathrm{E}-03$ & $4.56 \mathrm{E}-03$ & $-6.93 \mathrm{E}-06$ & $1.02 \mathrm{E}-03$ & $-8.76 \mathrm{E}-03$ \\
\hline \multirow{7}{*}{$\begin{array}{l}\text { Biomass } \\
\text { burning }\end{array}$} & Africa/Middle East & 1 & $-2.69 \mathrm{E}-02$ & $-1.41 \mathrm{E}-02$ & $-7.75 \mathrm{E}-04$ & $2.71 \mathrm{E}-06$ & $4.52 \mathrm{E}-04$ & $-4.52 \mathrm{E}-03$ \\
\hline & Southern Asia & $-2.69 \mathrm{E}-02$ & 1 & $-1.34 \mathrm{E}-02$ & $-1.91 \mathrm{E}-02$ & $-4.22 \mathrm{E}-05$ & $3.95 \mathrm{E}-04$ & $-1.83 \mathrm{E}-02$ \\
\hline & Central/South America & $-1.41 \mathrm{E}-02$ & $-1.34 \mathrm{E}-02$ & 1 & $-2.51 \mathrm{E}-04$ & $-3.81 \mathrm{E}-06$ & $1.07 \mathrm{E}-04$ & $-2.84 \mathrm{E}-03$ \\
\hline & Northern Asia & $-7.75 \mathrm{E}-04$ & $-1.91 \mathrm{E}-02$ & $-2.51 \mathrm{E}-04$ & 1 & $-1.06 \mathrm{E}-05$ & $-7.48 \mathrm{E}-04$ & $-9.21 \mathrm{E}-04$ \\
\hline & Europe & $2.71 \mathrm{E}-06$ & $-4.22 \mathrm{E}-05$ & $-3.81 \mathrm{E}-06$ & $-1.06 \mathrm{E}-05$ & 1 & $-1.34 \mathrm{E}-05$ & $-5.06 \mathrm{E}-06$ \\
\hline & North America & $4.52 \mathrm{E}-04$ & $3.95 \mathrm{E}-04$ & $1.07 \mathrm{E}-04$ & $-7.48 \mathrm{E}-04$ & $-1.34 \mathrm{E}-05$ & 1 & $7.58 \mathrm{E}-05$ \\
\hline & Oceania & $-4.52 \mathrm{E}-03$ & $-1.83 \mathrm{E}-02$ & $-2.84 \mathrm{E}-03$ & $-9.21 \mathrm{E}-04$ & $-5.06 \mathrm{E}-06$ & $7.58 \mathrm{E}-05$ & 1 \\
\hline
\end{tabular}

Fig. S2o. Correlation of the emissions in 1997 for biomass burning. 


\begin{tabular}{|c|c|c|c|c|c|c|c|c|}
\hline \multirow[b]{2}{*}{ Source sector } & \multirow[b]{2}{*}{ Region } & \multicolumn{7}{|c|}{ Agricultural Soil } \\
\hline & & $\begin{array}{l}\text { Africa/M. } \\
\text { East }\end{array}$ & $\begin{array}{l}\text { Southern } \\
\text { Asia }\end{array}$ & $\begin{array}{l}\text { C. /S. } \\
\text { America }\end{array}$ & $\begin{array}{l}\text { Northern } \\
\text { Asia }\end{array}$ & Europe & $\begin{array}{l}\text { North } \\
\text { America }\end{array}$ & Oceania \\
\hline \multirow{7}{*}{$\begin{array}{l}\text { Agricultural } \\
\text { Soil }\end{array}$} & Africa/Middle East & 1 & $-8.19 \mathrm{E}-02$ & $-1.81 \mathrm{E}-03$ & $-4.30 \mathrm{E}-02$ & $-2.15 \mathrm{E}-02$ & $-3.47 \mathrm{E}-02$ & $1.12 \mathrm{E}-02$ \\
\hline & Southern Asia & $-8.19 \mathrm{E}-02$ & 1 & $2.61 \mathrm{E}-02$ & $-2.41 \mathrm{E}-01$ & $2.88 \mathrm{E}-02$ & $-7.37 \mathrm{E}-02$ & $2.02 \mathrm{E}-02$ \\
\hline & Central/South America & $-1.81 \mathrm{E}-03$ & $2.61 \mathrm{E}-02$ & 1 & $-2.56 \mathrm{E}-03$ & $4.42 \mathrm{E}-03$ & $1.23 \mathrm{E}-02$ & $-1.07 \mathrm{E}-02$ \\
\hline & Northern Asia & $-4.30 \mathrm{E}-02$ & $-2.41 \mathrm{E}-01$ & $-2.56 \mathrm{E}-03$ & 1 & $-1.81 \mathrm{E}-01$ & $-4.90 \mathrm{E}-02$ & $-1.81 \mathrm{E}-02$ \\
\hline & Europe & $-2.15 \mathrm{E}-02$ & $2.88 \mathrm{E}-02$ & $4.42 \mathrm{E}-03$ & $-1.81 \mathrm{E}-01$ & 1 & $-2.29 \mathrm{E}-02$ & $3.54 \mathrm{E}-03$ \\
\hline & North America & $-3.47 \mathrm{E}-02$ & $-7.37 \mathrm{E}-02$ & $1.23 \mathrm{E}-02$ & $-4.90 \mathrm{E}-02$ & $-2.29 \mathrm{E}-02$ & 1 & $3.19 \mathrm{E}-03$ \\
\hline & Oceania & $1.12 \mathrm{E}-02$ & $2.02 \mathrm{E}-02$ & $-1.07 \mathrm{E}-02$ & $-1.81 \mathrm{E}-02$ & $3.54 \mathrm{E}-03$ & $3.19 \mathrm{E}-03$ & 1 \\
\hline \multirow{7}{*}{ Industrial } & Africa/Middle East & $-5.52 \mathrm{E}-02$ & $-1.31 \mathrm{E}-02$ & $6.69 \mathrm{E}-04$ & $-9.83 E-03$ & \begin{tabular}{|l|} 
\\
$-9.23 E-03$ \\
\end{tabular} & $-7.14 \mathrm{E}-03$ & $1.41 \mathrm{E}-03$ \\
\hline & Southern Asia & $6.46 \mathrm{E}-03$ & $-4.41 \mathrm{E}-01$ & $-3.86 \mathrm{E}-04$ & $-3.47 E-02$ & $1.53 \mathrm{E}-02$ & $4.53 \mathrm{E}-03$ & $-2.68 \mathrm{E}-03$ \\
\hline & Central/South America & $-3.02 E-04$ & $2.24 \mathrm{E}-03$ & $-4.07 E-03$ & $-1.73 \mathrm{E}-04$ & $3.84 \mathrm{E}-04$ & $1.07 \mathrm{E}-03$ & $-9.60 \mathrm{E}-04$ \\
\hline & Northern Asia & $2.45 \mathrm{E}-02$ & $6.27 \mathrm{E}-03$ & $-3.07 E-03$ & $-1.50 \mathrm{E}-01$ & $-4.03 E-02$ & $9.66 \mathrm{E}-02$ & $-1.24 \mathrm{E}-02$ \\
\hline & Europe & $-1.35 \mathrm{E}-02$ & $-1.86 \mathrm{E}-03$ & $2.31 \mathrm{E}-03$ & $-5.04 \mathrm{E}-02$ & $-7.17 \mathrm{E}-01$ & $-1.87 \mathrm{E}-02$ & $3.21 \mathrm{E}-04$ \\
\hline & North America & $7.13 \mathrm{E}-03$ & $1.68 \mathrm{E}-02$ & $4.14 \mathrm{E}-03$ & $5.85 \mathrm{E}-02$ & $2.98 \mathrm{E}-02$ & $-2.37 \mathrm{E}-01$ & $-6.45 \mathrm{E}-04$ \\
\hline & Oceania & $7.88 \mathrm{E}-04$ & $1.34 \mathrm{E}-03$ & $-5.67 \mathrm{E}-04$ & $-1.36 \mathrm{E}-03$ & \begin{tabular}{|l|}
$2.68 \mathrm{E}-04$ \\
\end{tabular} & $1.69 \mathrm{E}-04$ & $-2.03 \mathrm{E}-02$ \\
\hline \multirow{7}{*}{ Natural Soil } & Africa/Middle East & $-1.77 \mathrm{E}-01$ & $-1.15 \mathrm{E}-01$ & $-7.21 \mathrm{E}-02$ & $-1.77 \mathrm{E}-03$ & \begin{tabular}{|l|}
$-1.72 \mathrm{E}-02$ \\
\end{tabular} & $-1.63 \mathrm{E}-02$ & $2.25 \mathrm{E}-02$ \\
\hline & Southern Asia & $-2.53 \mathrm{E}-02$ & $-2.48 \mathrm{E}-01$ & $9.52 \mathrm{E}-03$ & $1.09 \mathrm{E}-02$ & $1.30 \mathrm{E}-02$ & $-2.20 \mathrm{E}-02$ & $5.53 \mathrm{E}-02$ \\
\hline & Central/South America & $-3.57 \mathrm{E}-02$ & $8.58 \mathrm{E}-02$ & $-1.99 \mathrm{E}-01$ & $7.60 \mathrm{E}-03$ & $1.56 \mathrm{E}-02$ & 4.97E-03 & $-8.64 \mathrm{E}-02$ \\
\hline & Northern Asia & $2.35 \mathrm{E}-02$ & $4.48 \mathrm{E}-02$ & $3.91 \mathrm{E}-03$ & $-2.03 \mathrm{E}-01$ & $2.17 \mathrm{E}-02$ & $3.06 \mathrm{E}-02$ & $-3.07 \mathrm{E}-02$ \\
\hline & Europe & $-1.90 \mathrm{E}-02$ & $-8.19 \mathrm{E}-03$ & $5.42 \mathrm{E}-03$ & $5.65 \mathrm{E}-03$ & $-2.46 \mathrm{E}-01$ & $1.19 \mathrm{E}-02$ & $9.30 \mathrm{E}-03$ \\
\hline & North America & $-4.21 \mathrm{E}-02$ & $-8.76 \mathrm{E}-02$ & $1.74 \mathrm{E}-02$ & $5.51 \mathrm{E}-02$ & $-1.26 \mathrm{E}-02$ & $-4.65 \mathrm{E}-01$ & $1.60 \mathrm{E}-02$ \\
\hline & Oceania & $5.28 \mathrm{E}-03$ & $2.57 \mathrm{E}-02$ & $-2.69 \mathrm{E}-02$ & $-9.86 \mathrm{E}-03$ & $7.71 \mathrm{E}-03$ & $1.21 \mathrm{E}-02$ & $-1.85 \mathrm{E}-01$ \\
\hline \multirow{6}{*}{ Ocean } & North Pacific & $-1.99 \mathrm{E}-02$ & $-1.56 \mathrm{E}-01$ & $6.11 \mathrm{E}-03$ & $-1.15 \mathrm{E}-03$ & $3.57 \mathrm{E}-03$ & $-5.51 \mathrm{E}-02$ & $2.05 \mathrm{E}-02$ \\
\hline & South Pacific & $-2.00 \mathrm{E}-02$ & $-1.79 \mathrm{E}-02$ & $-2.90 \mathrm{E}-02$ & $1.85 \mathrm{E}-02$ & $1.50 \mathrm{E}-02$ & $2.95 \mathrm{E}-02$ & $3.83 \mathrm{E}-02$ \\
\hline & Northern Ocean & $-3.27 \mathrm{E}-04$ & $-2.07 \mathrm{E}-03$ & $-1.45 \mathrm{E}-03$ & $-6.05 E-02$ & $-6.42 \mathrm{E}-02$ & $-1.65 \mathrm{E}-03$ & $4.51 \mathrm{E}-03$ \\
\hline & Atlantic & $-6.03 E-02$ & $-5.64 \mathrm{E}-02$ & $-2.30 \mathrm{E}-02$ & $-1.60 \mathrm{E}-02$ & $-3.40 \mathrm{E}-02$ & $-1.78 \mathrm{E}-01$ & $2.07 \mathrm{E}-02$ \\
\hline & Southern Ocean & $2.43 \mathrm{E}-02$ & $1.08 \mathrm{E}-01$ & $-1.39 \mathrm{E}-01$ & $-1.94 \mathrm{E}-02$ & $8.49 \mathrm{E}-03$ & $2.98 \mathrm{E}-02$ & $-3.91 \mathrm{E}-02$ \\
\hline & Indian Ocean & $-2.65 \mathrm{E}-02$ & $-6.93 \mathrm{E}-02$ & $-5.69 \mathrm{E}-02$ & $5.45 \mathrm{E}-03$ & $1.86 \mathrm{E}-03$ & $2.25 \mathrm{E}-02$ & $-1.98 \mathrm{E}-02$ \\
\hline \multirow{7}{*}{$\begin{array}{l}\text { Biomass } \\
\text { burning }\end{array}$} & Africa/Middle East & $-2.86 \mathrm{E}-02$ & $-2.46 \mathrm{E}-02$ & $-3.08 \mathrm{E}-02$ & $7.24 \mathrm{E}-03$ & $2.25 \mathrm{E}-03$ & $3.73 \mathrm{E}-03$ & $1.35 \mathrm{E}-02$ \\
\hline & Southern Asia & $-1.35 \mathrm{E}-02$ & $-7.41 \mathrm{E}-02$ & $5.28 \mathrm{E}-03$ & $4.31 \mathrm{E}-03$ & $6.82 \mathrm{E}-03$ & $-1.13 \mathrm{E}-02$ & $8.62 \mathrm{E}-03$ \\
\hline & Central/South America & $-8.04 \mathrm{E}-03$ & $6.33 \mathrm{E}-03$ & $-1.73 \mathrm{E}-02$ & $1.48 \mathrm{E}-03$ & $1.65 \mathrm{E}-03$ & $3.62 \mathrm{E}-03$ & $8.35 \mathrm{E}-03$ \\
\hline & Northern Asia & $3.78 \mathrm{E}-03$ & $-3.39 \mathrm{E}-02$ & $9.01 \mathrm{E}-03$ & $1.88 \mathrm{E}-02$ & $-8.24 \mathrm{E}-03$ & $1.33 \mathrm{E}-02$ & $5.60 \mathrm{E}-03$ \\
\hline & Europe & $-3.11 \mathrm{E}-05$ & $6.75 \mathrm{E}-06$ & $-3.00 \mathrm{E}-05$ & $-6.85 \mathrm{E}-04$ & $-2.88 \mathrm{E}-04$ & $-1.14 \mathrm{E}-04$ & $-9.82 \mathrm{E}-07$ \\
\hline & North America & $3.31 \mathrm{E}-04$ & $-2.76 \mathrm{E}-03$ & $2.03 \mathrm{E}-03$ & $6.65 \mathrm{E}-03$ & $-2.26 \mathrm{E}-03$ & $7.40 \mathrm{E}-03$ & $2.94 \mathrm{E}-03$ \\
\hline & Oceania & $1.70 \mathrm{E}-04$ & $6.45 \mathrm{E}-03$ & $-3.52 \mathrm{E}-03$ & $-1.90 \mathrm{E}-03$ & $5.44 \mathrm{E}-04$ & $1.71 \mathrm{E}-03$ & $-2.71 \mathrm{E}-03$ \\
\hline
\end{tabular}

Fig. S2p. Correlation of the emissions in 1998 for agricultural soil. 


\begin{tabular}{|c|c|c|c|c|c|c|c|c|}
\hline & \multirow{2}{*}{\multicolumn{7}{|c|}{ Industrial }} \\
\hline & & & & & & & & \\
\hline Source sector & Region & $\begin{array}{l}\text { Africa/M. } \\
\text { East }\end{array}$ & $\begin{array}{l}\text { Southern } \\
\text { Asia }\end{array}$ & $\begin{array}{l}\text { C. /S. } \\
\text { America }\end{array}$ & $\begin{array}{l}\text { Northern } \\
\text { Asia }\end{array}$ & Europe & $\begin{array}{l}\text { North } \\
\text { America }\end{array}$ & Oceania \\
\hline \multirow{7}{*}{$\begin{array}{l}\text { Agricultural } \\
\text { Soil }\end{array}$} & Africa/Middle East & $-5.52 \mathrm{E}-02$ & $6.46 \mathrm{E}-03$ & $-3.02 \mathrm{E}-04$ & $2.45 \mathrm{E}-02$ & $-1.35 \mathrm{E}-02$ & $7.13 \mathrm{E}-03$ & $7.88 \mathrm{E}-04$ \\
\hline & Southern Asia & $-1.31 \mathrm{E}-02$ & $-4.41 \mathrm{E}-01$ & $2.24 \mathrm{E}-03$ & 6.27E-03 & $-1.86 \mathrm{E}-03$ & $1.68 \mathrm{E}-02$ & $1.34 \mathrm{E}-03$ \\
\hline & Central/South America & $6.69 \mathrm{E}-04$ & $-3.86 \mathrm{E}-04$ & $-4.07 E-03$ & \begin{tabular}{|l|} 
\\
\end{tabular} & $2.31 \mathrm{E}-03$ & $4.14 \mathrm{E}-03$ & $-5.67 \mathrm{E}-04$ \\
\hline & Northern Asia & $-9.83 E-03$ & $-3.47 \mathrm{E}-02$ & $-1.73 \mathrm{E}-04$ & \begin{tabular}{|l|} 
\\
\end{tabular} & $-5.04 \mathrm{E}-02$ & $5.85 \mathrm{E}-02$ & $-1.36 \mathrm{E}-03$ \\
\hline & Europe & $-9.23 \mathrm{E}-03$ & $1.53 \mathrm{E}-02$ & $3.84 \mathrm{E}-04$ & $-4.03 E-02$ & $-7.17 \mathrm{E}-01$ & $2.98 \mathrm{E}-02$ & $2.68 \mathrm{E}-04$ \\
\hline & \begin{tabular}{|l|} 
North America \\
\end{tabular} & $-7.14 \mathrm{E}-03$ & $4.53 \mathrm{E}-03$ & $1.07 \mathrm{E}-03$ & $9.66 \mathrm{E}-02$ & $-1.87 \mathrm{E}-02$ & $-2.37 \mathrm{E}-01$ & $1.69 \mathrm{E}-04$ \\
\hline & Oceania & $1.41 \mathrm{E}-03$ & $-2.68 \mathrm{E}-03$ & $-9.60 \mathrm{E}-04$ & $-1.24 \mathrm{E}-02$ & $3.21 \mathrm{E}-04$ & $-6.45 \mathrm{E}-04$ & $-2.03 \mathrm{E}-02$ \\
\hline \multirow{7}{*}{ Industrial } & Africa/Middle East & 1 & $8.07 \mathrm{E}-04$ & $3.98 \mathrm{E}-05$ & $2.24 \mathrm{E}-03$ & $-4.43 \mathrm{E}-03$ & $1.40 \mathrm{E}-03$ & $9.80 \mathrm{E}-05$ \\
\hline & \begin{tabular}{|l|} 
Southern Asia \\
\end{tabular} & $8.07 \mathrm{E}-04$ & 1 & $-3.94 \mathrm{E}-05$ & $-3.92 \mathrm{E}-02$ & $3.01 \mathrm{E}-03$ & $1.22 \mathrm{E}-02$ & $-2.04 \mathrm{E}-04$ \\
\hline & Central/South America & $3.98 \mathrm{E}-05$ & $-3.94 \mathrm{E}-05$ & 1 & $-1.92 \mathrm{E}-04$ & $2.00 \mathrm{E}-04$ & $3.93 \mathrm{E}-04$ & $-5.07 \mathrm{E}-05$ \\
\hline & Northern Asia & $2.24 \mathrm{E}-03$ & $-3.92 \mathrm{E}-02$ & $-1.92 \mathrm{E}-04$ & 1 & $-1.94 \mathrm{E}-02$ & $-3.13 \mathrm{E}-01$ & $-7.25 \mathrm{E}-04$ \\
\hline & Europe & $-4.43 E-03$ & $3.01 \mathrm{E}-03$ & $2.00 \mathrm{E}-04$ & $-1.94 \mathrm{E}-02$ & 1 & $9.64 \mathrm{E}-03$ & $2.09 \mathrm{E}-05$ \\
\hline & North America & $1.40 \mathrm{E}-03$ & $1.22 \mathrm{E}-02$ & $3.93 E-04$ & $-3.13 E-01$ & $9.64 \mathrm{E}-03$ & 1 & $-8.64 \mathrm{E}-05$ \\
\hline & Oceania & $9.80 \mathrm{E}-05$ & $-2.04 \mathrm{E}-04$ & $-5.07 E-05$ & $-7.25 E-04$ & $2.09 E-05$ & $-8.64 \mathrm{E}-05$ & 1 \\
\hline \multirow{7}{*}{ Natural Soil } & Africa/Middle East & $-3.09 \mathrm{E}-02$ & $3.36 \mathrm{E}-02$ & $-6.74 \mathrm{E}-03$ & \begin{tabular}{|l|} 
\\
\end{tabular} & $-7.20 \mathrm{E}-03$ & $1.81 \mathrm{E}-02$ & $1.70 \mathrm{E}-03$ \\
\hline & Southern Asia & $-2.84 \mathrm{E}-03$ & $-9.85 \mathrm{E}-02$ & $6.71 \mathrm{E}-04$ & $-1.56 \mathrm{E}-03$ & $-9.62 \mathrm{E}-03$ & $5.09 E-03$ & $4.00 \mathrm{E}-03$ \\
\hline & Central/South America & $-8.21 \mathrm{E}-04$ & $8.61 \mathrm{E}-03$ & $-1.69 \mathrm{E}-02$ & $\begin{array}{l}1.49 \mathrm{E}-02 \\
\end{array}$ & $7.45 \mathrm{E}-03$ & $2.18 \mathrm{E}-03$ & $-5.44 \mathrm{E}-03$ \\
\hline & Northern Asia & $5.29 \mathrm{E}-03$ & $-6.06 \mathrm{E}-03$ & $3.22 \mathrm{E}-04$ & $-6.75 \mathrm{E}-02$ & $2.68 \mathrm{E}-02$ & $2.79 \mathrm{E}-02$ & $-2.32 \mathrm{E}-03$ \\
\hline & Europe & $-6.59 \mathrm{E}-03$ & 8.57E-03 & 4.17E-04 & $2.69 \mathrm{E}-02$ & $-2.01 \mathrm{E}-01$ & $1.67 \mathrm{E}-02$ & $6.36 \mathrm{E}-04$ \\
\hline & North America & $-5.27 \mathrm{E}-03$ & $5.04 \mathrm{E}-02$ & $1.43 \mathrm{E}-03$ & \begin{tabular}{|l|} 
\\
\end{tabular} & $-5.98 \mathrm{E}-05$ & $-2.56 \mathrm{E}-01$ & $9.14 \mathrm{E}-04$ \\
\hline & Oceania & $1.15 \mathrm{E}-03$ & $-5.08 \mathrm{E}-04$ & $-2.56 \mathrm{E}-03$ & \begin{tabular}{|l|} 
\\
\end{tabular} & $2.96 \mathrm{E}-03$ & $3.24 \mathrm{E}-03$ & $-1.23 \mathrm{E}-02$ \\
\hline \multirow{6}{*}{ Ocean } & North Pacific & $-4.21 \mathrm{E}-03$ & $-4.56 \mathrm{E}-02$ & $3.86 \mathrm{E}-04$ & $3.79 \mathrm{E}-02$ & $-5.23 \mathrm{E}-03$ & $1.93 \mathrm{E}-03$ & $1.46 \mathrm{E}-03$ \\
\hline & \begin{tabular}{|l} 
South Pacific \\
\end{tabular} & $-1.03 E-03$ & $4.62 \mathrm{E}-04$ & $-3.61 \mathrm{E}-03$ & \begin{tabular}{|c|} 
\\
\end{tabular} & $8.68 \mathrm{E}-03$ & $9.40 \mathrm{E}-03$ & $3.01 \mathrm{E}-03$ \\
\hline & Northern Ocean & $4.65 \mathrm{E}-04$ & $-3.32 \mathrm{E}-03$ & $-1.40 \mathrm{E}-04$ & $\begin{array}{l}3.69 \mathrm{E}-02 \\
\end{array}$ & $1.15 \mathrm{E}-02$ & $3.73 E-02$ & $4.11 \mathrm{E}-04$ \\
\hline & Atlantic & $-9.56 \mathrm{E}-03$ & $-7.32 \mathrm{E}-03$ & $-2.30 \mathrm{E}-03$ & 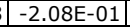 & $-1.13 \mathrm{E}-02$ & $-1.85 \mathrm{E}-01$ & $1.51 \mathrm{E}-03$ \\
\hline & Southern Ocean & $2.54 \mathrm{E}-03$ & $-3.10 \mathrm{E}-02$ & $-1.15 \mathrm{E}-02$ & $2.67 \mathrm{E}-03$ & $3.46 \mathrm{E}-04$ & $1.44 \mathrm{E}-02$ & $-2.72 \mathrm{E}-03$ \\
\hline & Indian Ocean & $-2.39 \mathrm{E}-03$ & $4.02 \mathrm{E}-02$ & $-5.62 E-03$ & 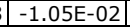 & $2.77 \mathrm{E}-03$ & $3.45 \mathrm{E}-03$ & $-1.62 \mathrm{E}-03$ \\
\hline \multirow{7}{*}{$\begin{array}{l}\text { Biomass } \\
\text { burning }\end{array}$} & Africa/Middle East & $-2.80 \mathrm{E}-03$ & $4.02 \mathrm{E}-03$ & $-2.91 \mathrm{E}-03$ & \begin{tabular}{|c|} 
\\
\end{tabular} & $1.75 \mathrm{E}-03$ & $7.08 \mathrm{E}-03$ & $1.04 \mathrm{E}-03$ \\
\hline & Southern Asia & $-2.24 \mathrm{E}-03$ & $-2.45 \mathrm{E}-02$ & $3.83 E-04$ & $-1.81 E-03$ & $4.05 E-03$ & $5.37 \mathrm{E}-04$ & $6.29 \mathrm{E}-04$ \\
\hline & Central/South America & $-8.84 \mathrm{E}-04$ & $1.22 \mathrm{E}-03$ & $-1.64 \mathrm{E}-03$ & \begin{tabular}{|l|} 
\\
\end{tabular} & $9.28 \mathrm{E}-04$ & $2.57 \mathrm{E}-03$ & $6.87 \mathrm{E}-04$ \\
\hline & Northern Asia & $1.33 \mathrm{E}-03$ & $9.98 \mathrm{E}-03$ & $7.36 \mathrm{E}-04$ & 5.52E-02 & $-3.16 \mathrm{E}-03$ & $1.07 \mathrm{E}-02$ & $3.49 \mathrm{E}-04$ \\
\hline & Europe & $1.77 \mathrm{E}-05$ & $-8.08 \mathrm{E}-05$ & $-2.86 \mathrm{E}-06$ & \begin{tabular}{|l|}
$-2.91 E-04$ \\
\end{tabular} & $-5.85 \mathrm{E}-05$ & $2.79 \mathrm{E}-04$ & $7.55 \mathrm{E}-08$ \\
\hline & North America & $4.59 \mathrm{E}-04$ & $3.11 \mathrm{E}-03$ & $1.63 \mathrm{E}-04$ & $1.36 \mathrm{E}-02$ & $1.72 \mathrm{E}-03$ & $7.78 \mathrm{E}-03$ & $2.04 \mathrm{E}-04$ \\
\hline & Oceania & $9.25 \mathrm{E}-05$ & $2.01 \mathrm{E}-03$ & $-3.36 \mathrm{E}-04$ & $-2.53 E-03$ & $2.00 \mathrm{E}-04$ & $6.78 \mathrm{E}-04$ & $-1.84 \mathrm{E}-04$ \\
\hline
\end{tabular}

Fig. S2p. Correlation of the emissions in 1998 for industrial. 


\begin{tabular}{|c|c|c|c|c|c|c|c|c|}
\hline & \multirow{2}{*}{\multicolumn{7}{|c|}{ Natural Soil }} \\
\hline & & & & & & & & \\
\hline Source sector & Region & $\begin{array}{l}\text { Africa/M. } \\
\text { East }\end{array}$ & $\begin{array}{l}\text { Southern } \\
\text { Asia }\end{array}$ & $\begin{array}{l}\text { C. /S. } \\
\text { America }\end{array}$ & $\begin{array}{l}\text { Northern } \\
\text { Asia }\end{array}$ & Europe & $\begin{array}{l}\text { North } \\
\text { America }\end{array}$ & Oceania \\
\hline \multirow{7}{*}{$\begin{array}{l}\text { Agricultural } \\
\text { Soil }\end{array}$} & Africa/Middle East & $-1.77 \mathrm{E}-01$ & $-2.53 \mathrm{E}-02$ & $-3.57 \mathrm{E}-02$ & \begin{tabular}{|r|}
$2.35 \mathrm{E}-02$ \\
\end{tabular} & $-1.90 \mathrm{E}-02$ & $-4.21 \mathrm{E}-02$ & $5.28 \mathrm{E}-03$ \\
\hline & Southern Asia & $-1.15 \mathrm{E}-01$ & $-2.48 \mathrm{E}-01$ & $8.58 \mathrm{E}-02$ & $4.48 \mathrm{E}-02$ & $-8.19 \mathrm{E}-03$ & $-8.76 \mathrm{E}-02$ & $2.57 \mathrm{E}-02$ \\
\hline & Central/South America & $-7.21 \mathrm{E}-02$ & $9.52 \mathrm{E}-03$ & $-1.99 \mathrm{E}-01$ & $3.91 \mathrm{E}-03$ & $5.42 \mathrm{E}-03$ & $1.74 \mathrm{E}-02$ & $-2.69 \mathrm{E}-02$ \\
\hline & Northern Asia & $-1.77 \mathrm{E}-03$ & $1.09 \mathrm{E}-02$ & $7.60 \mathrm{E}-03$ & \begin{tabular}{|l|}
$-2.03 \mathrm{E}-01$ \\
\end{tabular} & $5.65 \mathrm{E}-03$ & $5.51 \mathrm{E}-02$ & $-9.86 \mathrm{E}-03$ \\
\hline & Europe & $-1.72 \mathrm{E}-02$ & $1.30 \mathrm{E}-02$ & $1.56 \mathrm{E}-02$ & $2.17 \mathrm{E}-02$ & $-2.46 \mathrm{E}-01$ & $-1.26 \mathrm{E}-02$ & $7.71 \mathrm{E}-03$ \\
\hline & \begin{tabular}{|l|} 
North America \\
\end{tabular} & $-1.63 \mathrm{E}-02$ & $-2.20 \mathrm{E}-02$ & $4.97 \mathrm{E}-03$ & $3.06 \mathrm{E}-02$ & $1.19 \mathrm{E}-02$ & $-4.65 \mathrm{E}-01$ & $1.21 \mathrm{E}-02$ \\
\hline & Oceania & $2.25 \mathrm{E}-02$ & $5.53 \mathrm{E}-02$ & $-8.64 \mathrm{E}-02$ & $-3.07 E-02$ & $9.30 \mathrm{E}-03$ & $1.60 \mathrm{E}-02$ & $-1.85 \mathrm{E}-01$ \\
\hline \multirow{7}{*}{ Industrial } & Africa/Middle East & $-3.09 \mathrm{E}-02$ & $-2.84 \mathrm{E}-03$ & $-8.21 \mathrm{E}-04$ & $5.29 \mathrm{E}-03$ & $-6.59 \mathrm{E}-03$ & $-5.27 \mathrm{E}-03$ & $1.15 \mathrm{E}-03$ \\
\hline & \begin{tabular}{|l|} 
Southern Asia \\
\end{tabular} & $3.36 \mathrm{E}-02$ & $-9.85 \mathrm{E}-02$ & $8.61 \mathrm{E}-03$ & $-6.06 \mathrm{E}-03$ & $8.57 \mathrm{E}-03$ & $5.04 \mathrm{E}-02$ & $-5.08 \mathrm{E}-04$ \\
\hline & Central/South America & $-6.74 \mathrm{E}-03$ & $6.71 \mathrm{E}-04$ & $-1.69 \mathrm{E}-02$ & $3.22 \mathrm{E}-04$ & $4.17 \mathrm{E}-04$ & $1.43 \mathrm{E}-03$ & $-2.56 \mathrm{E}-03$ \\
\hline & Northern Asia & $8.19 \mathrm{E}-02$ & $-1.56 \mathrm{E}-03$ & $1.49 \mathrm{E}-02$ & \begin{tabular}{|l|}
$-6.75 \mathrm{E}-02$ \\
\end{tabular} & $2.69 E-02$ & $-1.74 \mathrm{E}-01$ & $-1.72 \mathrm{E}-02$ \\
\hline & Europe & $-7.20 \mathrm{E}-03$ & $-9.62 \mathrm{E}-03$ & $7.45 \mathrm{E}-03$ & $2.68 \mathrm{E}-02$ & $-2.01 \mathrm{E}-01$ & $-5.98 \mathrm{E}-05$ & $2.96 \mathrm{E}-03$ \\
\hline & North America & $1.81 \mathrm{E}-02$ & 5.09E-03 & $2.18 \mathrm{E}-03$ & $2.79 \mathrm{E}-02$ & 1.67E-02 & $-2.56 \mathrm{E}-01$ & $3.24 \mathrm{E}-03$ \\
\hline & Oceania & $1.70 \mathrm{E}-03$ & $4.00 \mathrm{E}-03$ & $-5.44 \mathrm{E}-03$ & $\mid-2.32 \mathrm{E}-03$ & $6.36 \mathrm{E}-04$ & $9.14 \mathrm{E}-04$ & $-1.23 \mathrm{E}-02$ \\
\hline \multirow{7}{*}{ Natural Soil } & Africa/Middle East & 1 & $-1.55 \mathrm{E}-01$ & $-3.67 \mathrm{E}-01$ & $1.03 \mathrm{E}-01$ & $-1.46 \mathrm{E}-02$ & $-1.58 \mathrm{E}-01$ & $-1.68 \mathrm{E}-02$ \\
\hline & Southern Asia & $-1.55 \mathrm{E}-01$ & 1 & $-9.95 \mathrm{E}-02$ & $-5.78 \mathrm{E}-01$ & $1.08 \mathrm{E}-01$ & $3.69 \mathrm{E}-02$ & $4.48 \mathrm{E}-02$ \\
\hline & Central/South America & $-3.67 \mathrm{E}-01$ & $-9.95 \mathrm{E}-02$ & 1 & \begin{tabular}{|l|}
$-5.03 E-02$ \\
\end{tabular} & $-1.42 \mathrm{E}-02$ & $-3.73 \mathrm{E}-02$ & $-1.00 \mathrm{E}-01$ \\
\hline & Northern Asia & $1.03 \mathrm{E}-01$ & $-5.78 \mathrm{E}-01$ & $-5.03 E-02$ & 1 & $-1.83 \mathrm{E}-01$ & $-1.49 \mathrm{E}-01$ & $-4.58 \mathrm{E}-02$ \\
\hline & Europe & $-1.46 \mathrm{E}-02$ & $1.08 \mathrm{E}-01$ & $-1.42 \mathrm{E}-02$ & $-1.83 E-01$ & 1 & $-1.04 \mathrm{E}-01$ & 7.57E-03 \\
\hline & North America & $-1.58 \mathrm{E}-01$ & $3.69 \mathrm{E}-02$ & $-3.73 \mathrm{E}-02$ & \begin{tabular}{|l|}
$-1.49 \mathrm{E}-01$ \\
\end{tabular} & $-1.04 \mathrm{E}-01$ & 1 & $3.06 \mathrm{E}-02$ \\
\hline & Oceania & $-1.68 \mathrm{E}-02$ & $4.48 \mathrm{E}-02$ & $-1.00 \mathrm{E}-01$ & $-4.58 \mathrm{E}-02$ & $7.57 \mathrm{E}-03$ & $3.06 \mathrm{E}-02$ & 1 \\
\hline \multirow{6}{*}{ Ocean } & North Pacific & $7.00 \mathrm{E}-02$ & $1.98 \mathrm{E}-01$ & 2.27E-02 & $-2.28 \mathrm{E}-01$ & $4.50 \mathrm{E}-02$ & $5.58 \mathrm{E}-02$ & $1.06 \mathrm{E}-02$ \\
\hline & South Pacific & $-1.22 \mathrm{E}-01$ & $3.33 \mathrm{E}-03$ & $-4.58 \mathrm{E}-01$ & $-4.26 \mathrm{E}-03$ & $8.71 \mathrm{E}-03$ & $4.98 \mathrm{E}-02$ & $-2.04 \mathrm{E}-02$ \\
\hline & Northern Ocean & $4.68 \mathrm{E}-02$ & $9.63 \mathrm{E}-02$ & $4.98 \mathrm{E}-02$ & $-8.22 \mathrm{E}-02$ & $-1.27 \mathrm{E}-02$ & $-5.86 \mathrm{E}-03$ & $1.33 \mathrm{E}-03$ \\
\hline & Atlantic & $-8.91 \mathrm{E}-02$ & $5.30 \mathrm{E}-02$ & $2.59 \mathrm{E}-02$ & $2.19 \mathrm{E}-02$ & $2.75 \mathrm{E}-02$ & \begin{tabular}{|l|} 
\\
\end{tabular} & $1.85 \mathrm{E}-03$ \\
\hline & Southern Ocean & $1.06 \mathrm{E}-01$ & $2.11 \mathrm{E}-01$ & $-7.48 \mathrm{E}-02$ & $-8.48 \mathrm{E}-02$ & $1.60 \mathrm{E}-02$ & $4.54 \mathrm{E}-02$ & $-2.68 \mathrm{E}-02$ \\
\hline & Indian Ocean & $-8.82 \mathrm{E}-02$ & $-1.38 \mathrm{E}-01$ & $-2.10 \mathrm{E}-01$ & $6.20 \mathrm{E}-02$ & $7.52 \mathrm{E}-03$ & $4.15 \mathrm{E}-02$ & $-6.29 \mathrm{E}-02$ \\
\hline \multirow{7}{*}{$\begin{array}{l}\text { Biomass } \\
\text { burning }\end{array}$} & Africa/Middle East & $-1.43 \mathrm{E}-01$ & $-2.40 \mathrm{E}-02$ & $-1.64 \mathrm{E}-02$ & $2.28 \mathrm{E}-02$ & $4.10 \mathrm{E}-03$ & \begin{tabular}{|l|}
$-8.71 E-04$ \\
\end{tabular} & $-9.39 \mathrm{E}-03$ \\
\hline & Southern Asia & $1.64 \mathrm{E}-02$ & $5.50 \mathrm{E}-02$ & $-1.91 \mathrm{E}-02$ & $-4.07 E-02$ & $-1.44 \mathrm{E}-02$ & \begin{tabular}{|l|} 
\\
\end{tabular} & $-4.67 \mathrm{E}-03$ \\
\hline & Central/South America & $-4.26 \mathrm{E}-02$ & $-6.78 \mathrm{E}-03$ & $1.46 \mathrm{E}-02$ & $3.98 \mathrm{E}-03$ & $-9.86 \mathrm{E}-04$ & $4.54 \mathrm{E}-03$ & $-5.86 \mathrm{E}-03$ \\
\hline & Northern Asia & $1.61 \mathrm{E}-02$ & $2.06 \mathrm{E}-03$ & $-4.80 \mathrm{E}-02$ & $-2.11 \mathrm{E}-01$ & $-4.58 \mathrm{E}-02$ & $-1.70 \mathrm{E}-01$ & $1.30 \mathrm{E}-02$ \\
\hline & Europe & $1.86 \mathrm{E}-04$ & 7.92E-06 & $3.44 \mathrm{E}-04$ & $7.46 \mathrm{E}-04$ & $-3.27 \mathrm{E}-03$ & \begin{tabular}{|l|}
$2.81 \mathrm{E}-04$ \\
\end{tabular} & $-3.08 \mathrm{E}-05$ \\
\hline & North America & $5.02 \mathrm{E}-03$ & $2.72 \mathrm{E}-02$ & $-1.38 \mathrm{E}-02$ & $-5.97 \mathrm{E}-02$ & $-3.41 \mathrm{E}-02$ & \begin{tabular}{|l|}
$-1.11 \mathrm{E}-01$ \\
\end{tabular} & $2.98 \mathrm{E}-03$ \\
\hline & Oceania & $-3.22 \mathrm{E}-03$ & $-8.53 \mathrm{E}-03$ & $-5.29 E-03$ & $3.32 \mathrm{E}-03$ & $8.77 \mathrm{E}-04$ & $4.04 \mathrm{E}-03$ & $-6.14 \mathrm{E}-03$ \\
\hline
\end{tabular}

Fig. S2q. Correlation of the emissions in 1998 for natural soil. 


\begin{tabular}{|c|c|c|c|c|c|c|c|}
\hline & & & & & & & \\
\hline & & \multicolumn{6}{|c|}{ Ocean } \\
\hline Source sector & Region & $\begin{array}{l}\text { North } \\
\text { Pacific }\end{array}$ & $\begin{array}{l}\text { South } \\
\text { Pacific }\end{array}$ & $\begin{array}{l}\text { Northern } \\
\text { Ocean }\end{array}$ & Atlantic & $\begin{array}{l}\text { Southern } \\
\text { Ocean }\end{array}$ & $\begin{array}{l}\text { Indian } \\
\text { Ocean }\end{array}$ \\
\hline \multirow{7}{*}{$\begin{array}{l}\text { Agricultural } \\
\text { Soil }\end{array}$} & Africa/Middle East & $-1.99 E-02$ & $-2.00 \mathrm{E}-02$ & $-3.27 E-04$ & $-6.03 E-02$ & $2.43 E-02$ & $-2.65 \mathrm{E}-02$ \\
\hline & Southern Asia & $-1.56 \mathrm{E}-01$ & $-1.79 \mathrm{E}-02$ & $-2.07 E-03$ & $-5.64 \mathrm{E}-02$ & $1.08 \mathrm{E}-01$ & $-6.93 E-02$ \\
\hline & Central/South America & $6.11 \mathrm{E}-03$ & $-2.90 \mathrm{E}-02$ & $-1.45 \mathrm{E}-03$ & $-2.30 \mathrm{E}-02$ & $-1.39 \mathrm{E}-01$ & $-5.69 \mathrm{E}-02$ \\
\hline & Northern Asia & $-1.15 \mathrm{E}-03$ & $1.85 \mathrm{E}-02$ & $-6.05 \mathrm{E}-02$ & $-1.60 \mathrm{E}-02$ & $-1.94 \mathrm{E}-02$ & $5.45 \mathrm{E}-03$ \\
\hline & Europe & $3.57 \mathrm{E}-03$ & $1.50 \mathrm{E}-02$ & $-6.42 \mathrm{E}-02$ & $-3.40 \mathrm{E}-02$ & $8.49 E-03$ & $1.86 \mathrm{E}-03$ \\
\hline & \begin{tabular}{|l|} 
North America \\
\end{tabular} & $-5.51 \mathrm{E}-02$ & $2.95 \mathrm{E}-02$ & $-1.65 \mathrm{E}-03$ & $-1.78 \mathrm{E}-01$ & $2.98 \mathrm{E}-02$ & $2.25 \mathrm{E}-02$ \\
\hline & Oceania & $2.05 \mathrm{E}-02$ & $3.83 \mathrm{E}-02$ & $4.51 \mathrm{E}-03$ & $2.07 \mathrm{E}-02$ & $-3.91 \mathrm{E}-02$ & $-1.98 \mathrm{E}-02$ \\
\hline \multirow{7}{*}{ Industrial } & Africa/Middle East & $-4.21 \mathrm{E}-03$ & $-1.03 \mathrm{E}-03$ & $4.65 \mathrm{E}-04$ & $-9.56 \mathrm{E}-03$ & $2.54 \mathrm{E}-03$ & $-2.39 \mathrm{E}-03$ \\
\hline & Southern Asia & $-4.56 \mathrm{E}-02$ & $4.62 \mathrm{E}-04$ & $-3.32 \mathrm{E}-03$ & $-7.32 \mathrm{E}-03$ & $-3.10 \mathrm{E}-02$ & $4.02 \mathrm{E}-02$ \\
\hline & Central/South America & $3.86 \mathrm{E}-04$ & $-3.61 \mathrm{E}-03$ & $-1.40 \mathrm{E}-04$ & $-2.30 \mathrm{E}-03$ & $-1.15 \mathrm{E}-02$ & $-5.62 \mathrm{E}-03$ \\
\hline & \begin{tabular}{|l} 
Northern Asia \\
\end{tabular} & 3.79E-02 & $8.61 \mathrm{E}-03$ & $3.69 \mathrm{E}-02$ & $-2.08 \mathrm{E}-01$ & $2.67 E-03$ & $-1.05 \mathrm{E}-02$ \\
\hline & Europe & $-5.23 \mathrm{E}-03$ & $8.68 \mathrm{E}-03$ & $1.15 \mathrm{E}-02$ & $-1.13 \mathrm{E}-02$ & $3.46 \mathrm{E}-04$ & $2.77 \mathrm{E}-03$ \\
\hline & \begin{tabular}{|l} 
North $\mathrm{Am}$ \\
\end{tabular} & $1.93 \mathrm{E}-03$ & $9.40 \mathrm{E}-03$ & $3.73 \mathrm{E}-02$ & $-1.85 \mathrm{E}-01$ & E-02 & E-03 \\
\hline & Oceania & $1.46 \mathrm{E}-03$ & $3.01 \mathrm{E}-03$ & $4.11 \mathrm{E}-04$ & $1.51 \mathrm{E}-03$ & $-2.72 \mathrm{E}-03$ & $-1.62 \mathrm{E}-03$ \\
\hline \multirow{7}{*}{ Natural Soil } & Africa/Middle East & $7.00 \mathrm{E}-02$ & $-1.22 \mathrm{E}-01$ & $4.68 \mathrm{E}-02$ & $-8.91 \mathrm{E}-02$ & $1.06 \mathrm{E}-01$ & $-8.82 \mathrm{E}-02$ \\
\hline & Southern Asia & $1.98 \mathrm{E}-01$ & $3.33 \mathrm{E}-03$ & $9.63 \mathrm{E}-02$ & $5.30 \mathrm{E}-02$ & $2.11 \mathrm{E}-01$ & $-1.38 \mathrm{E}-01$ \\
\hline & Central/South America & $2.27 \mathrm{E}-02$ & $-4.58 \mathrm{E}-01$ & $4.98 \mathrm{E}-02$ & $2.59 \mathrm{E}-02$ & $-7.48 \mathrm{E}-02$ & $-2.10 \mathrm{E}-01$ \\
\hline & Northern Asia & $-2.28 \mathrm{E}-01$ & $-4.26 \mathrm{E}-03$ & $-8.22 \mathrm{E}-02$ & $2.19 \mathrm{E}-02$ & $-8.48 \mathrm{E}-02$ & $6.20 \mathrm{E}-02$ \\
\hline & Europe & $4.50 \mathrm{E}-02$ & $8.71 \mathrm{E}-03$ & $-1.27 \mathrm{E}-02$ & $2.75 \mathrm{E}-02$ & $1.60 \mathrm{E}-02$ & $7.52 \mathrm{E}-03$ \\
\hline & North America & $5.58 \mathrm{E}-02$ & $4.98 \mathrm{E}-02$ & $-5.86 \mathrm{E}-03$ & $1.10 \mathrm{E}-01$ & $4.54 \mathrm{E}-02$ & $4.15 \mathrm{E}-02$ \\
\hline & Oceania & $1.06 \mathrm{E}-02$ & $-2.04 \mathrm{E}-02$ & $1.33 \mathrm{E}-03$ & $1.85 \mathrm{E}-03$ & $-2.68 \mathrm{E}-02$ & $-6.29 E-02$ \\
\hline \multirow{6}{*}{ Ocean } & North Pacific & 1 & $-4.75 \mathrm{E}-02$ & $-1.06 \mathrm{E}-01$ & $-1.87 \mathrm{E}-01$ & $3.88 \mathrm{E}-02$ & $4.18 \mathrm{E}-02$ \\
\hline & South Pacific & $-4.75 \mathrm{E}-02$ & 1 & $-1.03 \mathrm{E}-02$ & $-8.60 \mathrm{E}-02$ & 5.57E-02 & $-3.83 E-02$ \\
\hline & Northern Ocean & $-1.06 \mathrm{E}-01$ & $-1.03 \mathrm{E}-02$ & 1 & $-2.84 \mathrm{E}-01$ & $2.56 \mathrm{E}-02$ & $2.18 \mathrm{E}-03$ \\
\hline & Atlantic & \begin{tabular}{|l|}
$-1.87 \mathrm{E}-01$ \\
\end{tabular} & $-8.60 \mathrm{E}-02$ & $-2.84 \mathrm{E}-01$ & 1 & \begin{tabular}{|l|}
$-7.87 E-02$ \\
\end{tabular} & $-2.77 \mathrm{E}-02$ \\
\hline & Southern Ocean & $3.88 \mathrm{E}-02$ & $5.57 \mathrm{E}-02$ & $2.56 \mathrm{E}-02$ & $-7.87 \mathrm{E}-02$ & 1 & $-6.21 \mathrm{E}-01$ \\
\hline & Indian Ocean & $4.18 \mathrm{E}-02$ & $-3.83 E-02$ & $2.18 \mathrm{E}-03$ & $-2.77 \mathrm{E}-02$ & $-6.21 \mathrm{E}-01$ & 1 \\
\hline \multirow{7}{*}{$\begin{array}{l}\text { Biomass } \\
\text { burning }\end{array}$} & Africa/Middle East & $-2.02 \mathrm{E}-03$ & $-7.37 \mathrm{E}-02$ & $6.39 \mathrm{E}-05$ & $-6.45 \mathrm{E}-02$ & $-1.47 \mathrm{E}-02$ & \begin{tabular}{|c|}
$-6.79 E-02$ \\
\end{tabular} \\
\hline & Southern Asia & $-5.70 \mathrm{E}-02$ & $-4.55 \mathrm{E}-02$ & $-4.71 \mathrm{E}-03$ & $-3.01 \mathrm{E}-02$ & $2.16 \mathrm{E}-02$ & $-4.39 \mathrm{E}-03$ \\
\hline & Central/South America & $-4.70 \mathrm{E}-03$ & $-8.55 \mathrm{E}-02$ & $-2.24 \mathrm{E}-03$ & $-2.39 \mathrm{E}-02$ & $-5.05 E-02$ & $-3.77 \mathrm{E}-02$ \\
\hline & \begin{tabular}{|l} 
Northern Asia \\
\end{tabular} & $-1.46 \mathrm{E}-01$ & $3.48 \mathrm{E}-02$ & $2.11 \mathrm{E}-02$ & $6.11 \mathrm{E}-02$ & $2.37 \mathrm{E}-04$ & $2.75 \mathrm{E}-02$ \\
\hline & Europe & $-1.68 \mathrm{E}-03$ & $-9.26 \mathrm{E}-05$ & $-1.27 \mathrm{E}-03$ & $-1.33 \mathrm{E}-03$ & $5.47 \mathrm{E}-05$ & $3.79 \mathrm{E}-05$ \\
\hline & North America & $1.04 \mathrm{E}-02$ & $7.45 \mathrm{E}-03$ & $-1.28 \mathrm{E}-02$ & $1.54 \mathrm{E}-02$ & $3.17 \mathrm{E}-03$ & $2.90 \mathrm{E}-03$ \\
\hline & Oceania & $9.79 \mathrm{E}-04$ & $-8.61 \mathrm{E}-03$ & $-9.65 E-04$ & $-1.60 \mathrm{E}-03$ & $-6.00 \mathrm{E}-03$ & $-1.64 \mathrm{E}-02$ \\
\hline
\end{tabular}

Fig. S2r. Correlation of the emissions in 1998 for ocean. 


\begin{tabular}{|c|c|c|c|c|c|c|c|c|}
\hline & \multirow{2}{*}{\multicolumn{7}{|c|}{ Biomass burning }} \\
\hline & & & & & & & & \\
\hline Source sector & Region & $\begin{array}{l}\text { Africa/M. } \\
\text { East }\end{array}$ & $\begin{array}{l}\text { Southern } \\
\text { Asia }\end{array}$ & $\begin{array}{l}\text { C. /S. } \\
\text { America }\end{array}$ & $\begin{array}{l}\text { Northern } \\
\text { Asia }\end{array}$ & Europe & $\begin{array}{l}\text { North } \\
\text { America }\end{array}$ & Oceania \\
\hline \multirow{7}{*}{$\begin{array}{l}\text { Agricultural } \\
\text { Soil }\end{array}$} & Africa/Middle East & $-2.86 \mathrm{E}-02$ & $-1.35 \mathrm{E}-02$ & $-8.04 \mathrm{E}-03$ & $3.78 \mathrm{E}-03$ & $-3.11 \mathrm{E}-05$ & $3.31 \mathrm{E}-04$ & $1.70 \mathrm{E}-04$ \\
\hline & Southern Asia & $-2.46 \mathrm{E}-02$ & $-7.41 \mathrm{E}-02$ & $6.33 \mathrm{E}-03$ & $-3.39 \mathrm{E}-02$ & $6.75 \mathrm{E}-06$ & $-2.76 \mathrm{E}-03$ & $6.45 \mathrm{E}-03$ \\
\hline & Central/South America & $-3.08 \mathrm{E}-02$ & $5.28 \mathrm{E}-03$ & $-1.73 \mathrm{E}-02$ & $9.01 \mathrm{E}-03$ & $-3.00 \mathrm{E}-05$ & $2.03 \mathrm{E}-03$ & $-3.52 \mathrm{E}-03$ \\
\hline & Northern Asia & $7.24 \mathrm{E}-03$ & $4.31 \mathrm{E}-03$ & $1.48 \mathrm{E}-03$ & $1.88 \mathrm{E}-02$ & $-6.85 \mathrm{E}-04$ & $6.65 \mathrm{E}-03$ & $-1.90 \mathrm{E}-03$ \\
\hline & Europe & $2.25 \mathrm{E}-03$ & $6.82 \mathrm{E}-03$ & $1.65 \mathrm{E}-03$ & $-8.24 \mathrm{E}-03$ & $-2.88 \mathrm{E}-04$ & $-2.26 \mathrm{E}-03$ & $5.44 \mathrm{E}-04$ \\
\hline & North America & $3.73 \mathrm{E}-03$ & $-1.13 \mathrm{E}-02$ & $3.62 \mathrm{E}-03$ & $1.33 \mathrm{E}-02$ & $-1.14 \mathrm{E}-04$ & $7.40 \mathrm{E}-03$ & $1.71 \mathrm{E}-03$ \\
\hline & Oceania & $1.35 \mathrm{E}-02$ & $8.62 \mathrm{E}-03$ & $8.35 \mathrm{E}-03$ & $5.60 \mathrm{E}-03$ & $-9.82 \mathrm{E}-07$ & $2.94 \mathrm{E}-03$ & $-2.71 \mathrm{E}-03$ \\
\hline \multirow{7}{*}{ Industrial } & Africa/Middle East & $-2.80 \mathrm{E}-03$ & $-2.24 \mathrm{E}-03$ & $-8.84 \mathrm{E}-04$ & $1.33 \mathrm{E}-03$ & $1.77 \mathrm{E}-05$ & $4.59 \mathrm{E}-04$ & $9.25 \mathrm{E}-05$ \\
\hline & Southern Asia & $4.02 \mathrm{E}-03$ & $-2.45 \mathrm{E}-02$ & $1.22 \mathrm{E}-03$ & $9.98 \mathrm{E}-03$ & $-8.08 \mathrm{E}-05$ & $3.11 \mathrm{E}-03$ & $2.01 \mathrm{E}-03$ \\
\hline & Central/South America & $-2.91 \mathrm{E}-03$ & $3.83 \mathrm{E}-04$ & $-1.64 \mathrm{E}-03$ & $7.36 \mathrm{E}-04$ & $-2.86 \mathrm{E}-06$ & $1.63 \mathrm{E}-04$ & $-3.36 \mathrm{E}-04$ \\
\hline & Northern Asia & $1.62 \mathrm{E}-02$ & $-1.81 \mathrm{E}-03$ & $3.28 \mathrm{E}-03$ & $5.52 \mathrm{E}-02$ & $-2.91 \mathrm{E}-04$ & $1.36 \mathrm{E}-02$ & $-2.53 \mathrm{E}-03$ \\
\hline & \begin{tabular}{|l|} 
Europe \\
\end{tabular} & $1.75 \mathrm{E}-03$ & $4.05 \mathrm{E}-03$ & $9.28 \mathrm{E}-04$ & \begin{tabular}{|l|}
$-3.16 \mathrm{E}-03$ \\
\end{tabular} & $-5.85 \mathrm{E}-05$ & $1.72 \mathrm{E}-03$ & $2.00 \mathrm{E}-04$ \\
\hline & North America & $7.08 \mathrm{E}-03$ & $5.37 \mathrm{E}-04$ & $2.57 \mathrm{E}-03$ & 1.07E-02 & $2.79 \mathrm{E}-04$ & $7.78 \mathrm{E}-03$ & $6.78 \mathrm{E}-04$ \\
\hline & Oceania & $1.04 \mathrm{E}-03$ & $6.29 \mathrm{E}-04$ & $6.87 E-04$ & 3.49E-04 & $7.55 \mathrm{E}-08$ & $2.04 \mathrm{E}-04$ & $-1.84 \mathrm{E}-04$ \\
\hline \multirow{7}{*}{ Natural Soil } & Africa/Middle East & $-1.43 \mathrm{E}-01$ & $1.64 \mathrm{E}-02$ & $-4.26 \mathrm{E}-02$ & $1.61 \mathrm{E}-02$ & $1.86 \mathrm{E}-04$ & $5.02 \mathrm{E}-03$ & $-3.22 \mathrm{E}-03$ \\
\hline & Southern Asia & $-2.40 \mathrm{E}-02$ & $5.50 \mathrm{E}-02$ & $-6.78 \mathrm{E}-03$ & $2.06 \mathrm{E}-03$ & $7.92 \mathrm{E}-06$ & $2.72 \mathrm{E}-02$ & $-8.53 \mathrm{E}-03$ \\
\hline & Central/South America & $-1.64 \mathrm{E}-02$ & $-1.91 \mathrm{E}-02$ & $1.46 \mathrm{E}-02$ & $-4.80 \mathrm{E}-02$ & $3.44 \mathrm{E}-04$ & $-1.38 \mathrm{E}-02$ & $-5.29 \mathrm{E}-03$ \\
\hline & Northern Asia & $2.28 \mathrm{E}-02$ & $-4.07 \mathrm{E}-02$ & $3.98 \mathrm{E}-03$ & $-2.11 \mathrm{E}-01$ & $7.46 \mathrm{E}-04$ & $-5.97 \mathrm{E}-02$ & $3.32 \mathrm{E}-03$ \\
\hline & Europe & $4.10 \mathrm{E}-03$ & $-1.44 \mathrm{E}-02$ & $-9.86 \mathrm{E}-04$ & \begin{tabular}{|l|}
$-4.58 \mathrm{E}-02$ \\
\end{tabular} & $-3.27 \mathrm{E}-03$ & $-3.41 \mathrm{E}-02$ & 8.77E-04 \\
\hline & North America & $-8.71 \mathrm{E}-04$ & $1.40 \mathrm{E}-02$ & $4.54 \mathrm{E}-03$ & \begin{tabular}{|l|}
$-1.70 \mathrm{E}-01$ \\
\end{tabular} & $2.81 \mathrm{E}-04$ & $-1.11 \mathrm{E}-01$ & 4.04E-03 \\
\hline & Oceania & $-9.39 \mathrm{E}-03$ & $-4.67 \mathrm{E}-03$ & $-5.86 \mathrm{E}-03$ & $1.30 \mathrm{E}-02$ & $-3.08 \mathrm{E}-05$ & $2.98 \mathrm{E}-03$ & $-6.14 \mathrm{E}-03$ \\
\hline \multirow{6}{*}{ Ocean } & North Pacific & $-2.02 \mathrm{E}-03$ & $-5.70 \mathrm{E}-02$ & $-4.70 \mathrm{E}-03$ & $-1.46 \mathrm{E}-01$ & $-1.68 \mathrm{E}-03$ & $1.04 \mathrm{E}-02$ & $9.79 \mathrm{E}-04$ \\
\hline & \begin{tabular}{|l} 
South Pacific \\
\end{tabular} & $-7.37 \mathrm{E}-02$ & $-4.55 \mathrm{E}-02$ & $-8.55 \mathrm{E}-02$ & $3.48 \mathrm{E}-02$ & $-9.26 \mathrm{E}-05$ & $7.45 \mathrm{E}-03$ & $-8.61 \mathrm{E}-03$ \\
\hline & Northern Ocean & $6.39 \mathrm{E}-05$ & $-4.71 \mathrm{E}-03$ & $-2.24 \mathrm{E}-03$ & $2.11 \mathrm{E}-02$ & $-1.27 \mathrm{E}-03$ & $-1.28 \mathrm{E}-02$ & $-9.65 \mathrm{E}-04$ \\
\hline & Atlantic & $-6.45 \mathrm{E}-02$ & $-3.01 \mathrm{E}-02$ & $-2.39 \mathrm{E}-02$ & $6.11 \mathrm{E}-02$ & $-1.33 \mathrm{E}-03$ & 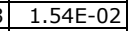 & $-1.60 \mathrm{E}-03$ \\
\hline & Southern Ocean & $-1.47 \mathrm{E}-02$ & $2.16 \mathrm{E}-02$ & $-5.05 E-02$ & $2.37 \mathrm{E}-04$ & $5.47 \mathrm{E}-05$ & 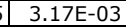 & $-6.00 \mathrm{E}-03$ \\
\hline & Indian Ocean & $-6.79 \mathrm{E}-02$ & $-4.39 E-03$ & $-3.77 \mathrm{E}-02$ & $2.75 \mathrm{E}-02$ & $3.79 \mathrm{E}-05$ & \begin{tabular}{|l|} 
\\
\end{tabular} & $-1.64 \mathrm{E}-02$ \\
\hline \multirow{7}{*}{$\begin{array}{l}\text { Biomass } \\
\text { burning }\end{array}$} & Africa/Middle East & 1 & $-2.74 \mathrm{E}-03$ & $-2.73 \mathrm{E}-02$ & $1.30 \mathrm{E}-02$ & $-6.22 \mathrm{E}-05$ & $3.25 \mathrm{E}-03$ & $-3.46 \mathrm{E}-03$ \\
\hline & Southern Asia & $-2.74 \mathrm{E}-03$ & 1 & $-3.17 \mathrm{E}-04$ & $-1.42 \mathrm{E}-03$ & $-6.79 \mathrm{E}-05$ & \begin{tabular}{|l|}
$-1.13 \mathrm{E}-03$ \\
\end{tabular} & $8.43 \mathrm{E}-04$ \\
\hline & Central/South America & $-2.73 \mathrm{E}-02$ & $-3.17 \mathrm{E}-04$ & 1 & $6.57 \mathrm{E}-03$ & $-7.42 \mathrm{E}-06$ & $5.47 \mathrm{E}-04$ & $-3.27 \mathrm{E}-03$ \\
\hline & Northern Asia & $1.30 \mathrm{E}-02$ & $-1.42 \mathrm{E}-03$ & $6.57 \mathrm{E}-03$ & 1 & $3.76 \mathrm{E}-04$ & $-4.80 \mathrm{E}-02$ & $2.40 \mathrm{E}-03$ \\
\hline & Europe & $-6.22 \mathrm{E}-05$ & $-6.79 \mathrm{E}-05$ & $-7.42 \mathrm{E}-06$ & $3.76 \mathrm{E}-04$ & 1 & $-6.84 \mathrm{E}-05$ & $-4.41 \mathrm{E}-06$ \\
\hline & North America & $3.25 \mathrm{E}-03$ & $-1.13 \mathrm{E}-03$ & 5.47E-04 & \begin{tabular}{|l|}
$-4.80 \mathrm{E}-02$ \\
\end{tabular} & $-6.84 \mathrm{E}-05$ & 1 & $3.20 \mathrm{E}-04$ \\
\hline & Oceania & $-3.46 \mathrm{E}-03$ & $8.43 \mathrm{E}-04$ & $-3.27 \mathrm{E}-03$ & $2.40 \mathrm{E}-03$ & $-4.41 \mathrm{E}-06$ & \begin{tabular}{|l|}
$3.20 \mathrm{E}-04$ \\
\end{tabular} & 1 \\
\hline
\end{tabular}

Fig. S2s. Correlation of the emissions in 1998 for biomass burning. 


\begin{tabular}{|c|c|c|c|c|c|c|c|c|}
\hline \multirow[b]{2}{*}{ Source sector } & \multirow[b]{2}{*}{ Region } & \multicolumn{7}{|c|}{ Agricultural Soil } \\
\hline & & $\begin{array}{l}\text { Africa/M. } \\
\text { East }\end{array}$ & $\begin{array}{l}\text { Southern } \\
\text { Asia }\end{array}$ & $\begin{array}{l}\text { C. /S. } \\
\text { America }\end{array}$ & $\begin{array}{l}\text { Northern } \\
\text { Asia }\end{array}$ & Europe & $\begin{array}{l}\text { North } \\
\text { America }\end{array}$ & Oceania \\
\hline \multirow{7}{*}{$\begin{array}{c}\text { Agricultural } \\
\text { Soil }\end{array}$} & Africa/Middle East & 1 & $-6.62 \mathrm{E}-02$ & $-5.03 \mathrm{E}-03$ & $-3.12 \mathrm{E}-02$ & $-1.52 \mathrm{E}-02$ & $-3.62 \mathrm{E}-02$ & $4.62 \mathrm{E}-03$ \\
\hline & Southern Asia & $-6.62 \mathrm{E}-02$ & 1 & $2.78 \mathrm{E}-02$ & $-2.01 \mathrm{E}-01$ & $2.52 \mathrm{E}-02$ & $-6.80 \mathrm{E}-02$ & $1.76 \mathrm{E}-02$ \\
\hline & Central/South America & $-5.03 E-03$ & $2.78 \mathrm{E}-02$ & 1 & $-2.28 \mathrm{E}-03$ & $5.61 \mathrm{E}-03$ & $1.19 \mathrm{E}-02$ & $-1.65 \mathrm{E}-02$ \\
\hline & Northern Asia & $-3.12 \mathrm{E}-02$ & $-2.01 \mathrm{E}-01$ & $-2.28 \mathrm{E}-03$ & 1 & $-1.82 \mathrm{E}-01$ & $-4.89 \mathrm{E}-02$ & $-1.46 \mathrm{E}-02$ \\
\hline & Europe & $-1.52 \mathrm{E}-02$ & $2.52 \mathrm{E}-02$ & $5.61 \mathrm{E}-03$ & $-1.82 \mathrm{E}-01$ & 1 & $-2.20 \mathrm{E}-02$ & $3.65 \mathrm{E}-03$ \\
\hline & North America & $-3.62 E-02$ & $-6.80 \mathrm{E}-02$ & $1.19 \mathrm{E}-02$ & $-4.89 \mathrm{E}-02$ & $-2.20 \mathrm{E}-02$ & 1 & $4.38 \mathrm{E}-03$ \\
\hline & Oceania & $4.62 \mathrm{E}-03$ & $1.76 \mathrm{E}-02$ & $-1.65 \mathrm{E}-02$ & $-1.46 \mathrm{E}-02$ & $3.65 \mathrm{E}-03$ & $4.38 \mathrm{E}-03$ & 1 \\
\hline \multirow{7}{*}{ Industrial } & Africa/Middle East & $-5.10 \mathrm{E}-02$ & $-1.16 \mathrm{E}-02$ & $3.31 \mathrm{E}-04$ & $-7.43 \mathrm{E}-03$ & $-7.64 \mathrm{E}-03$ & $-7.75 \mathrm{E}-03$ & $7.73 \mathrm{E}-04$ \\
\hline & \begin{tabular}{|l} 
Southern Asia \\
\end{tabular} & $8.17 \mathrm{E}-03$ & $-3.68 \mathrm{E}-01$ & $2.32 \mathrm{E}-03$ & $-5.33 E-02$ & $1.89 \mathrm{E}-02$ & $1.44 \mathrm{E}-03$ & $-1.14 \mathrm{E}-03$ \\
\hline & Central/South America & $-5.44 \mathrm{E}-04$ & $2.51 \mathrm{E}-03$ & $-4.07 E-03$ & $-1.54 \mathrm{E}-04$ & $5.10 \mathrm{E}-04$ & $1.10 \mathrm{E}-03$ & $-1.47 \mathrm{E}-03$ \\
\hline & Northern Asia & $1.53 \mathrm{E}-02$ & $-2.26 \mathrm{E}-02$ & $-6.95 \mathrm{E}-04$ & $-1.17 \mathrm{E}-01$ & $-3.86 \mathrm{E}-02$ & $5.18 \mathrm{E}-02$ & $-5.00 \mathrm{E}-03$ \\
\hline & Europe & $-1.93 E-02$ & $-6.30 \mathrm{E}-06$ & $3.26 \mathrm{E}-03$ & $-5.67 \mathrm{E}-02$ & $-6.72 \mathrm{E}-01$ & $-1.85 \mathrm{E}-02$ & $1.34 \mathrm{E}-03$ \\
\hline & North America & $7.93 \mathrm{E}-03$ & $2.20 \mathrm{E}-02$ & $1.38 \mathrm{E}-03$ & $4.06 \mathrm{E}-02$ & $2.91 \mathrm{E}-02$ & $-2.87 \mathrm{E}-01$ & $8.31 \mathrm{E}-05$ \\
\hline & Oceania & $4.05 \mathrm{E}-04$ & $1.33 \mathrm{E}-03$ & $-1.12 \mathrm{E}-03$ & $-1.26 \mathrm{E}-03$ & $2.84 \mathrm{E}-04$ & $2.41 \mathrm{E}-04$ & $-7.03 E-03$ \\
\hline \multirow{7}{*}{ Natural Soil } & Africa/Middle East & $-1.66 \mathrm{E}-01$ & $-3.89 \mathrm{E}-02$ & $-7.45 \mathrm{E}-02$ & $-9.94 \mathrm{E}-03$ & $-7.61 \mathrm{E}-03$ & $-9.81 \mathrm{E}-03$ & $4.72 \mathrm{E}-04$ \\
\hline & Southern Asia & $-5.07 \mathrm{E}-02$ & $-3.35 \mathrm{E}-01$ & $9.37 \mathrm{E}-04$ & $1.00 \mathrm{E}-02$ & $6.17 \mathrm{E}-03$ & $-1.05 \mathrm{E}-02$ & $3.20 \mathrm{E}-02$ \\
\hline & Central/South America & $-6.17 \mathrm{E}-02$ & $2.98 \mathrm{E}-02$ & $-2.13 \mathrm{E}-01$ & $2.34 \mathrm{E}-02$ & $2.04 \mathrm{E}-02$ & $1.99 \mathrm{E}-02$ & $-5.02 \mathrm{E}-02$ \\
\hline & Northern Asia & $3.01 \mathrm{E}-02$ & $2.27 \mathrm{E}-02$ & $-4.93 \mathrm{E}-03$ & $-2.15 \mathrm{E}-01$ & $1.88 \mathrm{E}-02$ & $2.07 \mathrm{E}-02$ & $-2.37 \mathrm{E}-02$ \\
\hline & Europe & $-3.37 \mathrm{E}-02$ & $-1.67 \mathrm{E}-02$ & $6.59 \mathrm{E}-03$ & $-3.16 \mathrm{E}-02$ & $-2.48 \mathrm{E}-01$ & $-6.75 \mathrm{E}-03$ & $5.61 \mathrm{E}-03$ \\
\hline & North America & $-3.82 \mathrm{E}-02$ & $-6.66 \mathrm{E}-02$ & $1.34 \mathrm{E}-02$ & $7.20 \mathrm{E}-02$ & $-2.22 \mathrm{E}-02$ & $-4.07 \mathrm{E}-01$ & $1.39 \mathrm{E}-02$ \\
\hline & Oceania & \begin{tabular}{|l|}
$-9.23 E-04$ \\
\end{tabular} & $2.18 \mathrm{E}-02$ & $-2.45 \mathrm{E}-02$ & $-4.46 \mathrm{E}-03$ & $5.74 \mathrm{E}-03$ & $7.18 \mathrm{E}-03$ & $-5.76 \mathrm{E}-02$ \\
\hline \multirow{6}{*}{ Ocean } & North Pacific & $-1.73 \mathrm{E}-02$ & $-1.72 \mathrm{E}-01$ & $8.32 \mathrm{E}-03$ & $1.46 \mathrm{E}-02$ & $1.04 \mathrm{E}-02$ & $-9.86 \mathrm{E}-02$ & $1.14 \mathrm{E}-02$ \\
\hline & South Pacific & $1.43 \mathrm{E}-03$ & $2.14 \mathrm{E}-02$ & $-1.24 \mathrm{E}-02$ & $8.23 \mathrm{E}-03$ & $1.03 \mathrm{E}-02$ & $2.46 \mathrm{E}-03$ & $2.36 \mathrm{E}-02$ \\
\hline & Northern Ocean & $4.99 \mathrm{E}-03$ & $-1.05 \mathrm{E}-02$ & $1.82 \mathrm{E}-03$ & $-5.55 \mathrm{E}-02$ & $-9.48 \mathrm{E}-02$ & $-2.23 \mathrm{E}-02$ & $3.27 \mathrm{E}-03$ \\
\hline & Atlantic & $-7.25 \mathrm{E}-02$ & $-7.71 \mathrm{E}-02$ & $-1.46 \mathrm{E}-02$ & $-5.13 \mathrm{E}-02$ & $-6.09 \mathrm{E}-02$ & $-1.98 \mathrm{E}-01$ & $1.20 \mathrm{E}-02$ \\
\hline & Southern Ocean & $1.57 \mathrm{E}-02$ & $1.05 \mathrm{E}-01$ & $-1.59 \mathrm{E}-01$ & $-2.42 \mathrm{E}-02$ & $1.55 \mathrm{E}-02$ & $4.18 \mathrm{E}-02$ & $-5.79 \mathrm{E}-02$ \\
\hline & Indian Ocean & $-3.26 \mathrm{E}-02$ & $-6.51 \mathrm{E}-02$ & $-7.71 \mathrm{E}-02$ & $1.67 \mathrm{E}-02$ & $3.85 \mathrm{E}-03$ & $2.62 \mathrm{E}-02$ & $-5.36 \mathrm{E}-02$ \\
\hline \multirow{7}{*}{$\begin{array}{l}\text { Biomass } \\
\text { burning }\end{array}$} & Africa/Middle East & $-2.58 \mathrm{E}-02$ & $-1.90 \mathrm{E}-02$ & $-1.88 \mathrm{E}-02$ & $3.48 \mathrm{E}-03$ & $2.54 \mathrm{E}-03$ & $-1.42 \mathrm{E}-03$ & $6.22 \mathrm{E}-03$ \\
\hline & Southern Asia & $-9.78 \mathrm{E}-03$ & $-4.11 \mathrm{E}-02$ & $2.38 \mathrm{E}-03$ & $8.84 \mathrm{E}-03$ & $2.77 \mathrm{E}-03$ & $-1.15 \mathrm{E}-02$ & $2.99 \mathrm{E}-03$ \\
\hline & Central/South America & $-3.80 \mathrm{E}-03$ & $4.56 \mathrm{E}-03$ & $-7.67 \mathrm{E}-03$ & $-3.56 \mathrm{E}-04$ & $9.48 \mathrm{E}-04$ & $4.55 \mathrm{E}-03$ & $2.50 \mathrm{E}-03$ \\
\hline & \begin{tabular}{|l} 
Northern Asia \\
\end{tabular} & $2.25 \mathrm{E}-04$ & $-8.32 \mathrm{E}-03$ & $8.16 \mathrm{E}-04$ & $4.21 \mathrm{E}-03$ & $1.38 \mathrm{E}-03$ & $4.82 \mathrm{E}-03$ & $1.28 \mathrm{E}-03$ \\
\hline & Europe & $-3.28 \mathrm{E}-04$ & $-6.04 \mathrm{E}-05$ & $3.36 \mathrm{E}-05$ & $-1.48 \mathrm{E}-06$ & $-4.27 \mathrm{E}-04$ & $1.50 \mathrm{E}-04$ & $2.38 \mathrm{E}-05$ \\
\hline & North America & $1.31 \mathrm{E}-03$ & $-1.53 \mathrm{E}-03$ & $3.77 \mathrm{E}-04$ & $6.95 \mathrm{E}-03$ & $-1.08 \mathrm{E}-03$ & $-3.94 \mathrm{E}-03$ & $6.84 \mathrm{E}-04$ \\
\hline & Oceania & $-9.68 \mathrm{E}-04$ & $5.85 \mathrm{E}-03$ & $-4.63 \mathrm{E}-03$ & $-4.04 \mathrm{E}-04$ & $7.13 \mathrm{E}-04$ & $2.76 \mathrm{E}-03$ & $-4.05 \mathrm{E}-03$ \\
\hline
\end{tabular}

Fig. S2t. Correlation of the emissions in 1999 for agricultural soil. 


\begin{tabular}{|c|c|c|c|c|c|c|c|c|}
\hline & \multirow{2}{*}{\multicolumn{7}{|c|}{ Industrial }} \\
\hline & & & & & & & & \\
\hline Source sector & Region & $\begin{array}{l}\text { Africa/M. } \\
\text { East }\end{array}$ & $\begin{array}{l}\text { Southern } \\
\text { Asia }\end{array}$ & $\begin{array}{l}\text { C. /S. } \\
\text { America }\end{array}$ & $\begin{array}{l}\text { Northern } \\
\text { Asia }\end{array}$ & Europe & $\begin{array}{l}\text { North } \\
\text { America }\end{array}$ & Oceania \\
\hline \multirow{7}{*}{$\begin{array}{l}\text { Agricultural } \\
\text { Soil }\end{array}$} & Africa/Middle East & $-5.10 \mathrm{E}-02$ & $8.17 \mathrm{E}-03$ & $-5.44 \mathrm{E}-04$ & $1.53 \mathrm{E}-02$ & $-1.93 \mathrm{E}-02$ & $7.93 \mathrm{E}-03$ & $4.05 \mathrm{E}-04$ \\
\hline & Southern Asia & $-1.16 \mathrm{E}-02$ & $-3.68 \mathrm{E}-01$ & $2.51 \mathrm{E}-03$ & $-2.26 \mathrm{E}-02$ & $-6.30 \mathrm{E}-06$ & $2.20 \mathrm{E}-02$ & $1.33 \mathrm{E}-03$ \\
\hline & Central/South America & $3.31 \mathrm{E}-04$ & $2.32 \mathrm{E}-03$ & $-4.07 \mathrm{E}-03$ & $-6.95 \mathrm{E}-04$ & $3.26 \mathrm{E}-03$ & $1.38 \mathrm{E}-03$ & $-1.12 \mathrm{E}-03$ \\
\hline & Northern Asia & $-7.43 E-03$ & $-5.33 \mathrm{E}-02$ & $-1.54 \mathrm{E}-04$ & $-1.17 \mathrm{E}-01$ & \begin{tabular}{|l|}
$-5.67 E-02$ \\
\end{tabular} & $4.06 \mathrm{E}-02$ & $-1.26 \mathrm{E}-03$ \\
\hline & Europe & $-7.64 \mathrm{E}-03$ & $1.89 \mathrm{E}-02$ & $5.10 \mathrm{E}-04$ & $-3.86 \mathrm{E}-02$ & $\mid-6.72 \mathrm{E}-01$ & $2.91 \mathrm{E}-02$ & $2.84 \mathrm{E}-04$ \\
\hline & \begin{tabular}{|l|} 
North America \\
\end{tabular} & $-7.75 \mathrm{E}-03$ & $1.44 \mathrm{E}-03$ & $1.10 \mathrm{E}-03$ & $5.18 \mathrm{E}-02$ & $\mid-1.85 \mathrm{E}-02$ & $-2.87 \mathrm{E}-01$ & $2.41 \mathrm{E}-04$ \\
\hline & Oceania & $7.73 \mathrm{E}-04$ & $-1.14 \mathrm{E}-03$ & $-1.47 \mathrm{E}-03$ & $-5.00 \mathrm{E}-03$ & $1.34 \mathrm{E}-03$ & $8.31 \mathrm{E}-05$ & $-7.03 \mathrm{E}-03$ \\
\hline \multirow{7}{*}{ Industrial } & Africa/Middle East & 1 & $7.27 \mathrm{E}-04$ & $1.77 \mathrm{E}-05$ & $1.06 \mathrm{E}-03$ & $-8.18 \mathrm{E}-03$ & $1.37 \mathrm{E}-03$ & $6.05 \mathrm{E}-05$ \\
\hline & \begin{tabular}{|l|} 
Southern Asia \\
\end{tabular} & $7.27 \mathrm{E}-04$ & 1 & $2.29 \mathrm{E}-04$ & $-4.54 \mathrm{E}-02$ & $4.84 \mathrm{E}-03$ & $1.07 \mathrm{E}-02$ & $-1.09 \mathrm{E}-04$ \\
\hline & Central/South America & $1.77 \mathrm{E}-05$ & $2.29 \mathrm{E}-04$ & 1 & $-1.91 \mathrm{E}-05$ & $2.98 \mathrm{E}-04$ & $1.43 \mathrm{E}-04$ & $-9.87 \mathrm{E}-05$ \\
\hline & Northern Asia & $1.06 \mathrm{E}-03$ & $-4.54 \mathrm{E}-02$ & $-1.91 \mathrm{E}-05$ & 1 & \begin{tabular}{|l|}
$-2.94 \mathrm{E}-02$ \\
\end{tabular} & $-2.38 \mathrm{E}-01$ & $-3.73 \mathrm{E}-04$ \\
\hline & Europe & $-8.18 \mathrm{E}-03$ & $4.84 \mathrm{E}-03$ & $2.98 \mathrm{E}-04$ & $-2.94 \mathrm{E}-02$ & 1 & $1.01 \mathrm{E}-02$ & 8.93E-05 \\
\hline & North America & $1.37 \mathrm{E}-03$ & $1.07 \mathrm{E}-02$ & $1.43 \mathrm{E}-04$ & $-2.38 \mathrm{E}-01$ & $1.01 \mathrm{E}-02$ & 1 & $-5.78 \mathrm{E}-06$ \\
\hline & Oceania & $6.05 \mathrm{E}-05$ & $-1.09 \mathrm{E}-04$ & $-9.87 E-05$ & $-3.73 E-04$ & 8.93E-05 & $-5.78 \mathrm{E}-06$ & 1 \\
\hline \multirow{7}{*}{ Natural Soil } & Africa/Middle East & $-2.89 \mathrm{E}-02$ & $5.04 \mathrm{E}-02$ & $-6.96 \mathrm{E}-03$ & $4.96 \mathrm{E}-02$ & \begin{tabular}{|l|}
$-1.08 \mathrm{E}-02$ \\
\end{tabular} & $1.58 \mathrm{E}-02$ & 4.53E-04 \\
\hline & Southern Asia & $-6.21 \mathrm{E}-03$ & $-1.36 \mathrm{E}-01$ & $-8.84 \mathrm{E}-05$ & $2.53 \mathrm{E}-02$ & \begin{tabular}{|l|}
$-1.33 \mathrm{E}-02$ \\
\end{tabular} & $3.05 \mathrm{E}-02$ & $3.02 \mathrm{E}-03$ \\
\hline & Central/South America & $-3.92 \mathrm{E}-03$ & $1.17 \mathrm{E}-02$ & $-1.88 \mathrm{E}-02$ & $2.73 \mathrm{E}-02$ & $1.43 \mathrm{E}-02$ & $1.21 \mathrm{E}-02$ & $-3.52 \mathrm{E}-03$ \\
\hline & Northern Asia & $6.52 \mathrm{E}-03$ & $-3.82 \mathrm{E}-02$ & $-4.49 \mathrm{E}-04$ & $-6.21 \mathrm{E}-02$ & $2.02 \mathrm{E}-02$ & $1.10 \mathrm{E}-03$ & $-2.27 \mathrm{E}-03$ \\
\hline & Europe & $-9.08 \mathrm{E}-03$ & $1.26 \mathrm{E}-02$ & $5.38 \mathrm{E}-04$ & $2.16 \mathrm{E}-02$ & \begin{tabular}{|l|}
$-1.22 \mathrm{E}-01$ \\
\end{tabular} & $3.16 \mathrm{E}-02$ & 4.93E-04 \\
\hline & North America & $-3.65 \mathrm{E}-03$ & $6.85 \mathrm{E}-02$ & $1.05 \mathrm{E}-03$ & $-1.51 \mathrm{E}-01$ & $7.24 \mathrm{E}-03$ & $-2.02 \mathrm{E}-01$ & $1.10 \mathrm{E}-03$ \\
\hline & Oceania & $2.47 \mathrm{E}-04$ & $-8.47 \mathrm{E}-04$ & $-2.24 \mathrm{E}-03$ & $-3.76 \mathrm{E}-03$ & $3.08 \mathrm{E}-03$ & $1.99 \mathrm{E}-03$ & $-5.04 \mathrm{E}-03$ \\
\hline \multirow{6}{*}{ Ocean } & North Pacific & $-4.47 \mathrm{E}-03$ & $-2.90 \mathrm{E}-02$ & $6.85 \mathrm{E}-04$ & $6.87 \mathrm{E}-02$ & $-1.01 \mathrm{E}-02$ & $-3.30 \mathrm{E}-02$ & $1.03 \mathrm{E}-03$ \\
\hline & South Pacific & $7.46 \mathrm{E}-04$ & $7.90 \mathrm{E}-03$ & $-2.19 \mathrm{E}-03$ & $1.32 \mathrm{E}-02$ & $6.60 \mathrm{E}-03$ & $3.11 \mathrm{E}-03$ & $2.11 \mathrm{E}-03$ \\
\hline & Northern Ocean & $1.44 \mathrm{E}-03$ & $-6.59 \mathrm{E}-03$ & $1.70 \mathrm{E}-04$ & $2.89 \mathrm{E}-02$ & $-4.56 \mathrm{E}-03$ & $2.68 \mathrm{E}-02$ & $3.31 \mathrm{E}-04$ \\
\hline & Atlantic & $-1.22 \mathrm{E}-02$ & $-1.67 \mathrm{E}-02$ & $-1.50 \mathrm{E}-03$ & $-2.20 \mathrm{E}-01$ & $-2.10 \mathrm{E}-02$ & $-1.11 \mathrm{E}-01$ & $1.08 \mathrm{E}-03$ \\
\hline & Southern Ocean & $2.64 \mathrm{E}-03$ & $-1.42 \mathrm{E}-02$ & $-1.33 \mathrm{E}-02$ & $-4.56 \mathrm{E}-03$ & $5.68 \mathrm{E}-03$ & $1.03 \mathrm{E}-02$ & $-4.16 \mathrm{E}-03$ \\
\hline & Indian Ocean & $-2.87 \mathrm{E}-03$ & $2.92 \mathrm{E}-02$ & $-7.46 \mathrm{E}-03$ & $1.20 \mathrm{E}-02$ & $5.30 \mathrm{E}-03$ & $-3.54 \mathrm{E}-03$ & $-4.73 \mathrm{E}-03$ \\
\hline \multirow{7}{*}{$\begin{array}{l}\text { Biomass } \\
\text { burning }\end{array}$} & Africa/Middle East & $-2.90 \mathrm{E}-03$ & $1.02 \mathrm{E}-02$ & $-1.83 \mathrm{E}-03$ & $1.37 \mathrm{E}-02$ & $1.36 \mathrm{E}-03$ & $2.03 \mathrm{E}-03$ & $6.17 \mathrm{E}-04$ \\
\hline & Southern Asia & $-1.89 \mathrm{E}-03$ & $-6.59 \mathrm{E}-03$ & $1.85 \mathrm{E}-04$ & $6.53 \mathrm{E}-03$ & $1.85 \mathrm{E}-03$ & $1.50 \mathrm{E}-03$ & $2.53 \mathrm{E}-04$ \\
\hline & Central/South America & $-2.34 \mathrm{E}-04$ & $4.50 \mathrm{E}-03$ & $-7.97 \mathrm{E}-04$ & $3.26 \mathrm{E}-03$ & $3.79 \mathrm{E}-04$ & $1.89 \mathrm{E}-04$ & $2.79 \mathrm{E}-04$ \\
\hline & Northern Asia & $1.85 \mathrm{E}-04$ & $-4.08 \mathrm{E}-03$ & $5.88 \mathrm{E}-05$ & 8.87E-03 & $1.15 \mathrm{E}-03$ & $8.63 \mathrm{E}-03$ & $1.16 \mathrm{E}-04$ \\
\hline & Europe & $-8.00 \mathrm{E}-05$ & $1.46 \mathrm{E}-04$ & $2.48 \mathrm{E}-06$ & $4.03 \mathrm{E}-04$ & $1.06 \mathrm{E}-05$ & $2.83 \mathrm{E}-04$ & $2.11 \mathrm{E}-06$ \\
\hline & North America & $4.48 \mathrm{E}-04$ & $3.05 \mathrm{E}-03$ & $2.56 \mathrm{E}-05$ & $1.21 \mathrm{E}-02$ & $3.99 \mathrm{E}-04$ & $1.18 \mathrm{E}-03$ & $6.45 \mathrm{E}-05$ \\
\hline & Oceania & $-4.78 \mathrm{E}-06$ & $1.61 \mathrm{E}-03$ & $-4.67 \mathrm{E}-04$ & $-1.85 \mathrm{E}-04$ & $3.79 \mathrm{E}-04$ & $2.36 \mathrm{E}-04$ & $-2.77 \mathrm{E}-04$ \\
\hline
\end{tabular}

Fig. S2u. Correlation of the emissions in 1999 for industrial. 


\begin{tabular}{|c|c|c|c|c|c|c|c|c|}
\hline & \multirow{2}{*}{\multicolumn{7}{|c|}{ Natural Soil }} \\
\hline & & & & & & & & \\
\hline Source sector & Region & $\begin{array}{l}\text { Africa/M. } \\
\text { East }\end{array}$ & $\begin{array}{l}\text { Southern } \\
\text { Asia }\end{array}$ & $\begin{array}{l}\text { C. /S. } \\
\text { America }\end{array}$ & $\begin{array}{l}\text { Northern } \\
\text { Asia }\end{array}$ & Europe & $\begin{array}{l}\text { North } \\
\text { America }\end{array}$ & Oceania \\
\hline \multirow{7}{*}{$\begin{array}{l}\text { Agricultural } \\
\text { Soil }\end{array}$} & Africa/Middle East & $-1.66 \mathrm{E}-01$ & $-5.07 \mathrm{E}-02$ & $-6.17 \mathrm{E}-02$ & 3.01E-02 & $-3.37 \mathrm{E}-02$ & $-3.82 \mathrm{E}-02$ & $-9.23 \mathrm{E}-04$ \\
\hline & Southern Asia & $-3.89 \mathrm{E}-02$ & $-3.35 \mathrm{E}-01$ & $2.98 \mathrm{E}-02$ & $2.27 \mathrm{E}-02$ & $-1.67 \mathrm{E}-02$ & $-6.66 \mathrm{E}-02$ & $2.18 \mathrm{E}-02$ \\
\hline & Central/South America & $-7.45 \mathrm{E}-02$ & $9.37 \mathrm{E}-04$ & $-2.13 \mathrm{E}-01$ & \begin{tabular}{|l|}
$-4.93 \mathrm{E}-03$ \\
\end{tabular} & $6.59 \mathrm{E}-03$ & $1.34 \mathrm{E}-02$ & $-2.45 \mathrm{E}-02$ \\
\hline & Northern Asia & $-9.94 \mathrm{E}-03$ & $1.00 \mathrm{E}-02$ & $2.34 \mathrm{E}-02$ & \begin{tabular}{|l|}
$-2.15 \mathrm{E}-01$ \\
\end{tabular} & $-3.16 \mathrm{E}-02$ & $7.20 \mathrm{E}-02$ & $-4.46 \mathrm{E}-03$ \\
\hline & Europe & $-7.61 \mathrm{E}-03$ & $6.17 \mathrm{E}-03$ & $2.04 \mathrm{E}-02$ & $1.88 \mathrm{E}-02$ & $-2.48 \mathrm{E}-01$ & $-2.22 \mathrm{E}-02$ & $5.74 \mathrm{E}-03$ \\
\hline & \begin{tabular}{|l|} 
North America \\
\end{tabular} & $-9.81 \mathrm{E}-03$ & $-1.05 \mathrm{E}-02$ & $1.99 \mathrm{E}-02$ & $2.07 \mathrm{E}-02$ & $-6.75 \mathrm{E}-03$ & $-4.07 E-01$ & $7.18 \mathrm{E}-03$ \\
\hline & Oceania & $4.72 \mathrm{E}-04$ & $3.20 \mathrm{E}-02$ & $-5.02 \mathrm{E}-02$ & $-2.37 \mathrm{E}-02$ & $5.61 \mathrm{E}-03$ & $1.39 \mathrm{E}-02$ & $-5.76 \mathrm{E}-02$ \\
\hline \multirow{7}{*}{ Industrial } & Africa/Middle East & $-2.89 \mathrm{E}-02$ & $-6.21 \mathrm{E}-03$ & $-3.92 \mathrm{E}-03$ & $6.52 \mathrm{E}-03$ & $-9.08 \mathrm{E}-03$ & $-3.65 \mathrm{E}-03$ & $2.47 \mathrm{E}-04$ \\
\hline & \begin{tabular}{|l|} 
Southern Asia \\
\end{tabular} & $5.04 \mathrm{E}-02$ & $-1.36 \mathrm{E}-01$ & $1.17 \mathrm{E}-02$ & $-3.82 \mathrm{E}-02$ & $1.26 \mathrm{E}-02$ & $6.85 \mathrm{E}-02$ & $-8.47 \mathrm{E}-04$ \\
\hline & Central/South America & $-6.96 \mathrm{E}-03$ & $-8.84 \mathrm{E}-05$ & $-1.88 \mathrm{E}-02$ & $-4.49 \mathrm{E}-04$ & $5.38 \mathrm{E}-04$ & $1.05 \mathrm{E}-03$ & $-2.24 \mathrm{E}-03$ \\
\hline & Northern Asia & $4.96 \mathrm{E}-02$ & $2.53 \mathrm{E}-02$ & $2.73 \mathrm{E}-02$ & \begin{tabular}{|l|}
$-6.21 \mathrm{E}-02$ \\
\end{tabular} & $2.16 \mathrm{E}-02$ & $-1.51 \mathrm{E}-01$ & $-3.76 \mathrm{E}-03$ \\
\hline & Europe & $-1.08 \mathrm{E}-02$ & $-1.33 \mathrm{E}-02$ & $1.43 \mathrm{E}-02$ & $2.02 \mathrm{E}-02$ & $-1.22 \mathrm{E}-01$ & $7.24 \mathrm{E}-03$ & $3.08 \mathrm{E}-03$ \\
\hline & North America & $1.58 \mathrm{E}-02$ & $3.05 \mathrm{E}-02$ & $1.21 \mathrm{E}-02$ & $1.10 \mathrm{E}-03$ & $3.16 \mathrm{E}-02$ & $-2.02 \mathrm{E}-01$ & $1.99 \mathrm{E}-03$ \\
\hline & Oceania & $4.53 \mathrm{E}-04$ & $3.02 \mathrm{E}-03$ & $-3.52 \mathrm{E}-03$ & $\mid-2.27 \mathrm{E}-03$ & 4.93E-04 & $1.10 \mathrm{E}-03$ & $-5.04 \mathrm{E}-03$ \\
\hline \multirow{7}{*}{ Natural Soil } & Africa/Middle East & 1 & $-2.60 \mathrm{E}-01$ & $-3.32 \mathrm{E}-01$ & $1.32 \mathrm{E}-01$ & $-1.82 \mathrm{E}-02$ & $-1.33 \mathrm{E}-01$ & $-1.99 \mathrm{E}-02$ \\
\hline & Southern Asia & $-2.60 \mathrm{E}-01$ & 1 & $-5.71 \mathrm{E}-02$ & $-4.75 \mathrm{E}-01$ & $7.53 \mathrm{E}-02$ & $-4.35 \mathrm{E}-02$ & $2.19 \mathrm{E}-02$ \\
\hline & Central/South America & $-3.32 \mathrm{E}-01$ & $-5.71 \mathrm{E}-02$ & 1 & \begin{tabular}{|l|}
$-9.32 E-02$ \\
\end{tabular} & $2.57 \mathrm{E}-03$ & $-1.62 \mathrm{E}-02$ & $-1.30 \mathrm{E}-01$ \\
\hline & Northern Asia & $1.32 \mathrm{E}-01$ & $-4.75 \mathrm{E}-01$ & $-9.32 \mathrm{E}-02$ & 1 & $-1.67 \mathrm{E}-01$ & $-2.49 \mathrm{E}-01$ & $-4.20 \mathrm{E}-02$ \\
\hline & Europe & $-1.82 \mathrm{E}-02$ & $7.53 \mathrm{E}-02$ & $2.57 \mathrm{E}-03$ & \begin{tabular}{|l|}
$-1.67 E-01$ \\
\end{tabular} & 1 & $-1.32 \mathrm{E}-01$ & $2.70 \mathrm{E}-03$ \\
\hline & North America & $-1.33 \mathrm{E}-01$ & $-4.35 \mathrm{E}-02$ & $-1.62 \mathrm{E}-02$ & \begin{tabular}{|l|}
$-2.49 \mathrm{E}-01$ \\
\end{tabular} & $-1.32 \mathrm{E}-01$ & 1 & $1.42 \mathrm{E}-02$ \\
\hline & Oceania & $-1.99 \mathrm{E}-02$ & $2.19 \mathrm{E}-02$ & $-1.30 \mathrm{E}-01$ & $-4.20 \mathrm{E}-02$ & $2.70 \mathrm{E}-03$ & $1.42 \mathrm{E}-02$ & 1 \\
\hline \multirow{6}{*}{ Ocean } & North Pacific & $5.30 \mathrm{E}-02$ & $2.14 \mathrm{E}-01$ & $4.06 \mathrm{E}-02$ & $-3.08 \mathrm{E}-01$ & $6.01 \mathrm{E}-02$ & $9.47 \mathrm{E}-02$ & $4.26 \mathrm{E}-03$ \\
\hline & South Pacific & $9.49 \mathrm{E}-03$ & $-2.41 \mathrm{E}-02$ & $-5.03 \mathrm{E}-01$ & $7.85 \mathrm{E}-03$ & $7.56 \mathrm{E}-03$ & $1.13 \mathrm{E}-02$ & $2.78 \mathrm{E}-02$ \\
\hline & Northern Ocean & $4.25 \mathrm{E}-02$ & $1.17 \mathrm{E}-01$ & $4.59 \mathrm{E}-02$ & $-9.09 \mathrm{E}-02$ & $-7.31 \mathrm{E}-04$ & $-3.02 \mathrm{E}-03$ & $2.12 \mathrm{E}-03$ \\
\hline & Atlantic & $-1.09 \mathrm{E}-01$ & $7.42 \mathrm{E}-02$ & $-4.65 \mathrm{E}-02$ & $4.90 \mathrm{E}-02$ & $2.33 \mathrm{E}-02$ & \begin{tabular}{l|l|} 
& $1.11 \mathrm{E}-01$ \\
\end{tabular} & $9.98 \mathrm{E}-04$ \\
\hline & Southern Ocean & $2.47 \mathrm{E}-02$ & $1.74 \mathrm{E}-01$ & $-1.11 \mathrm{E}-02$ & $-5.93 \mathrm{E}-02$ & $1.47 \mathrm{E}-02$ & $6.51 \mathrm{E}-02$ & $-4.80 \mathrm{E}-02$ \\
\hline & Indian Ocean & $-1.50 \mathrm{E}-01$ & $-1.37 \mathrm{E}-01$ & $-1.88 \mathrm{E}-01$ & $2.19 \mathrm{E}-02$ & $1.02 \mathrm{E}-02$ & $3.13 \mathrm{E}-02$ & $-6.57 \mathrm{E}-02$ \\
\hline \multirow{7}{*}{$\begin{array}{l}\text { Biomass } \\
\text { burning }\end{array}$} & Africa/Middle East & $-1.32 \mathrm{E}-01$ & $-3.72 \mathrm{E}-02$ & $-3.74 \mathrm{E}-02$ & $2.85 \mathrm{E}-02$ & $2.89 E-03$ & \begin{tabular}{|l|}
$-5.56 \mathrm{E}-03$ \\
\end{tabular} & $5.68 \mathrm{E}-04$ \\
\hline & Southern Asia & $8.43 \mathrm{E}-03$ & $3.29 \mathrm{E}-02$ & $-1.69 \mathrm{E}-02$ & $-3.53 \mathrm{E}-02$ & $-9.50 \mathrm{E}-03$ & \begin{tabular}{|l|} 
\\
\end{tabular} & $-1.45 \mathrm{E}-03$ \\
\hline & Central/South America & $-3.68 \mathrm{E}-02$ & $-1.97 \mathrm{E}-02$ & $1.17 \mathrm{E}-02$ & $1.54 \mathrm{E}-02$ & $3.04 \mathrm{E}-03$ & $1.29 \mathrm{E}-03$ & $1.04 \mathrm{E}-03$ \\
\hline & Northern Asia & $2.38 \mathrm{E}-03$ & $1.78 \mathrm{E}-02$ & $-2.21 \mathrm{E}-03$ & $-8.17 \mathrm{E}-02$ & $-1.24 \mathrm{E}-02$ & $-3.77 \mathrm{E}-02$ & $-2.16 \mathrm{E}-05$ \\
\hline & Europe & $-1.83 \mathrm{E}-04$ & $-7.87 E-05$ & $-3.85 \mathrm{E}-06$ & $-1.18 \mathrm{E}-03$ & $-3.30 \mathrm{E}-03$ & \begin{tabular}{|l|}
$-1.98 \mathrm{E}-03$ \\
\end{tabular} & $2.84 \mathrm{E}-05$ \\
\hline & North America & $4.37 \mathrm{E}-03$ & $1.32 \mathrm{E}-02$ & $-3.61 \mathrm{E}-03$ & $-3.60 \mathrm{E}-02$ & $-8.63 \mathrm{E}-03$ & \begin{tabular}{|l|}
$-5.07 E-02$ \\
\end{tabular} & $-1.33 \mathrm{E}-04$ \\
\hline & Oceania & $-1.23 \mathrm{E}-02$ & $-1.19 \mathrm{E}-02$ & $2.09 \mathrm{E}-03$ & $2.99 \mathrm{E}-03$ & $1.49 \mathrm{E}-03$ & $2.95 \mathrm{E}-03$ & $-6.93 \mathrm{E}-03$ \\
\hline
\end{tabular}

Fig. S2v. Correlation of the emissions in 1999 for natural soil. 


\begin{tabular}{|c|c|c|c|c|c|c|c|}
\hline \multirow[b]{2}{*}{ Source sector } & \multirow[b]{2}{*}{ Region } & \multicolumn{6}{|c|}{ Ocean } \\
\hline & & $\begin{array}{l}\text { North } \\
\text { Pacific }\end{array}$ & $\begin{array}{l}\text { South } \\
\text { Pacific }\end{array}$ & $\begin{array}{l}\text { Northern } \\
\text { Ocean }\end{array}$ & Atlantic & $\begin{array}{l}\text { Southern } \\
\text { Ocean }\end{array}$ & $\begin{array}{l}\text { Indian } \\
\text { Ocean }\end{array}$ \\
\hline \multirow{7}{*}{$\begin{array}{l}\text { Agricultural } \\
\text { Soil }\end{array}$} & Africa/Middle East & $-1.73 \mathrm{E}-02$ & $1.43 \mathrm{E}-03$ & 4.99E-03 & $-7.25 \mathrm{E}-02$ & $1.57 \mathrm{E}-02$ & $-3.26 \mathrm{E}-02$ \\
\hline & Southern Asia & $-1.72 \mathrm{E}-01$ & $2.14 \mathrm{E}-02$ & $-1.05 \mathrm{E}-02$ & $-7.71 \mathrm{E}-02$ & $1.05 \mathrm{E}-01$ & $-6.51 \mathrm{E}-02$ \\
\hline & Central/South America & $8.32 \mathrm{E}-03$ & $-1.24 \mathrm{E}-02$ & $1.82 \mathrm{E}-03$ & $-1.46 \mathrm{E}-02$ & $-1.59 \mathrm{E}-01$ & $-7.71 \mathrm{E}-02$ \\
\hline & \begin{tabular}{|l} 
Northern Asia \\
\end{tabular} & $1.46 \mathrm{E}-02$ & $8.23 \mathrm{E}-03$ & $-5.55 \mathrm{E}-02$ & $-5.13 \mathrm{E}-02$ & $-2.42 \mathrm{E}-02$ & $1.67 \mathrm{E}-02$ \\
\hline & Europe & $1.04 \mathrm{E}-02$ & $1.03 \mathrm{E}-02$ & $-9.48 \mathrm{E}-02$ & $-6.09 \mathrm{E}-02$ & $1.55 \mathrm{E}-02$ & $3.85 \mathrm{E}-03$ \\
\hline & \begin{tabular}{|l|} 
North America \\
\end{tabular} & $-9.86 \mathrm{E}-02$ & $2.46 \mathrm{E}-03$ & $-2.23 \mathrm{E}-02$ & $-1.98 \mathrm{E}-01$ & $4.18 \mathrm{E}-02$ & $2.62 \mathrm{E}-02$ \\
\hline & Oceania & $1.14 \mathrm{E}-02$ & $2.36 \mathrm{E}-02$ & $3.27 \mathrm{E}-03$ & $1.20 \mathrm{E}-02$ & $-5.79 \mathrm{E}-02$ & $-5.36 \mathrm{E}-02$ \\
\hline \multirow{7}{*}{ Industrial } & Africa/Middle East & $-4.47 \mathrm{E}-03$ & $7.46 \mathrm{E}-04$ & $1.44 \mathrm{E}-03$ & $-1.22 \mathrm{E}-02$ & $2.64 \mathrm{E}-03$ & $-2.87 \mathrm{E}-03$ \\
\hline & Southern Asia & $-2.90 \mathrm{E}-02$ & $7.90 \mathrm{E}-03$ & $-6.59 \mathrm{E}-03$ & $-1.67 \mathrm{E}-02$ & $-1.42 \mathrm{E}-02$ & $2.92 \mathrm{E}-02$ \\
\hline & Central/South America & $6.85 \mathrm{E}-04$ & $-2.19 \mathrm{E}-03$ & $1.70 \mathrm{E}-04$ & $-1.50 \mathrm{E}-03$ & $-1.33 \mathrm{E}-02$ & $-7.46 \mathrm{E}-03$ \\
\hline & \begin{tabular}{|l|} 
Northern Asia \\
\end{tabular} & $6.87 \mathrm{E}-02$ & $1.32 \mathrm{E}-02$ & $2.89 \mathrm{E}-02$ & $-2.20 \mathrm{E}-01$ & $-4.56 \mathrm{E}-03$ & $1.20 \mathrm{E}-02$ \\
\hline & Europe & $-1.01 \mathrm{E}-02$ & $6.60 \mathrm{E}-03$ & $-4.56 \mathrm{E}-03$ & $-2.10 \mathrm{E}-02$ & $8 \mathrm{E}-03$ & $0 \mathrm{E}-03$ \\
\hline & North America & $-3.30 \mathrm{E}-02$ & $3.11 \mathrm{E}-03$ & $2.68 \mathrm{E}-02$ & $-1.11 \mathrm{E}-01$ & $1.03 \mathrm{E}-02$ & $-3.54 \mathrm{E}-03$ \\
\hline & Oceania & $1.03 \mathrm{E}-03$ & $2.11 \mathrm{E}-03$ & $3.31 \mathrm{E}-04$ & $1.08 \mathrm{E}-03$ & $-4.16 \mathrm{E}-03$ & $-4.73 \mathrm{E}-03$ \\
\hline \multirow{7}{*}{ Natural Soil } & Africa/Middle East & $5.30 \mathrm{E}-02$ & $9.49 \mathrm{E}-03$ & $4.25 \mathrm{E}-02$ & $-1.09 \mathrm{E}-01$ & $2.47 \mathrm{E}-02$ & $-1.50 \mathrm{E}-01$ \\
\hline & Southern Asia & $2.14 \mathrm{E}-01$ & $-2.41 \mathrm{E}-02$ & $1.17 \mathrm{E}-01$ & $7.42 \mathrm{E}-02$ & $1.74 \mathrm{E}-01$ & $-1.37 \mathrm{E}-01$ \\
\hline & \begin{tabular}{|l|} 
Central/South America \\
\end{tabular} & $4.06 \mathrm{E}-02$ & $-5.03 E-01$ & $4.59 \mathrm{E}-02$ & $-4.65 \mathrm{E}-02$ & $-1.11 \mathrm{E}-02$ & $-1.88 \mathrm{E}-01$ \\
\hline & \begin{tabular}{|l|} 
Northern Asia \\
\end{tabular} & $-3.08 \mathrm{E}-01$ & $7.85 \mathrm{E}-03$ & $-9.09 E-02$ & $4.90 \mathrm{E}-02$ & $-5.93 E-02$ & $2.19 \mathrm{E}-02$ \\
\hline & Europe & $\mathrm{E}-02$ & $7.56 \mathrm{E}-03$ & $-7.31 \mathrm{E}-04$ & $3 \mathrm{E}-02$ & $\mathrm{E}-02$ & $2 \mathrm{E}-02$ \\
\hline & \begin{tabular}{|l} 
North America \\
\end{tabular} & $9.47 \mathrm{E}-02$ & $1.13 \mathrm{E}-02$ & $-3.02 \mathrm{E}-03$ & $1.11 \mathrm{E}-01$ & $6.51 \mathrm{E}-02$ & $3.13 \mathrm{E}-02$ \\
\hline & Oceania & $4.26 \mathrm{E}-03$ & $2.78 \mathrm{E}-02$ & $2.12 \mathrm{E}-03$ & $9.98 \mathrm{E}-04$ & $-4.80 \mathrm{E}-02$ & $-6.57 E-02$ \\
\hline \multirow{6}{*}{ Ocean } & North Pacific & 1 & $-3.49 \mathrm{E}-03$ & $-7.19 \mathrm{E}-02$ & $-2.16 \mathrm{E}-01$ & $3.95 \mathrm{E}-02$ & $3.28 \mathrm{E}-02$ \\
\hline & South Pacific & $-3.49 \mathrm{E}-03$ & 1 & $3.00 \mathrm{E}-03$ & $-2.95 \mathrm{E}-02$ & $3.38 \mathrm{E}-03$ & $3.09 \mathrm{E}-02$ \\
\hline & \begin{tabular}{|l|} 
Northern Ocean \\
\end{tabular} & $-7.19 \mathrm{E}-02$ & $3.00 \mathrm{E}-03$ & 1 & $-2.76 \mathrm{E}-01$ & $2.26 \mathrm{E}-02$ & $3.42 \mathrm{E}-03$ \\
\hline & Atlantic & $-2.16 \mathrm{E}-01$ & $-2.95 \mathrm{E}-02$ & $-2.76 \mathrm{E}-01$ & 1 & $-8.16 \mathrm{E}-02$ & $-2.63 \mathrm{E}-02$ \\
\hline & Southern Ocean & $3.95 \mathrm{E}-02$ & $3.38 \mathrm{E}-03$ & $2.26 \mathrm{E}-02$ & $-8.16 \mathrm{E}-02$ & 1 & $-6.12 \mathrm{E}-01$ \\
\hline & Indian Ocean & $3.28 \mathrm{E}-02$ & $3.09 \mathrm{E}-02$ & $3.42 \mathrm{E}-03$ & $-2.63 \mathrm{E}-02$ & $-6.12 \mathrm{E}-01$ & \\
\hline \multirow{7}{*}{$\begin{array}{l}\text { Biomass } \\
\text { burning }\end{array}$} & Africa/Middle East & $-2.97 \mathrm{E}-03$ & $-2.86 \mathrm{E}-02$ & $1.85 \mathrm{E}-03$ & $-5.54 \mathrm{E}-02$ & $-1.96 \mathrm{E}-02$ & $-4.52 \mathrm{E}-02$ \\
\hline & Southern Asia & $-3.43 \mathrm{E}-02$ & $-3.62 \mathrm{E}-03$ & $-6.39 \mathrm{E}-03$ & $-3.40 \mathrm{E}-02$ & $1.12 \mathrm{E}-02$ & $-1.21 \mathrm{E}-03$ \\
\hline & Central/South America & $-6.07 E-04$ & $-2.70 \mathrm{E}-02$ & $-1.08 \mathrm{E}-03$ & $-1.57 \mathrm{E}-02$ & $-5.88 \mathrm{E}-02$ & $-2.25 \mathrm{E}-02$ \\
\hline & Northern Asia & $-5.34 \mathrm{E}-02$ & $3.91 \mathrm{E}-03$ & $-3.37 E-03$ & $1.04 \mathrm{E}-02$ & $1.31 \mathrm{E}-03$ & $4.62 \mathrm{E}-03$ \\
\hline & Europe & $8.06 \mathrm{E}-04$ & $1.46 \mathrm{E}-04$ & $4.36 \mathrm{E}-04$ & 7.04E-04 & $3.94 \mathrm{E}-05$ & $1.22 \mathrm{E}-04$ \\
\hline & North America & $-8.87 \mathrm{E}-03$ & $1.87 \mathrm{E}-03$ & $-7.41 \mathrm{E}-03$ & 8.07E-03 & $1.54 \mathrm{E}-03$ & $1.28 \mathrm{E}-03$ \\
\hline & Oceania & $3.70 \mathrm{E}-05$ & $-5.74 \mathrm{E}-03$ & $-8.00 \mathrm{E}-04$ & $-4.23 \mathrm{E}-03$ & $-1.29 \mathrm{E}-02$ & $-1.67 \mathrm{E}-02$ \\
\hline
\end{tabular}

Fig. S2w. Correlation of the emissions in 1999 for ocean. 


\begin{tabular}{|c|c|c|c|c|c|c|c|c|}
\hline \multirow[b]{3}{*}{ Source sector } & \multirow[b]{3}{*}{ Region } & \multirow{2}{*}{\multicolumn{7}{|c|}{ Biomass burning }} \\
\hline & & & & & & & & \\
\hline & & $\begin{array}{l}\text { Africa/M. } \\
\text { East }\end{array}$ & $\begin{array}{l}\text { Southern } \\
\text { Asia }\end{array}$ & $\begin{array}{l}\text { C. /S. } \\
\text { America }\end{array}$ & $\begin{array}{l}\text { Northern } \\
\text { Asia }\end{array}$ & Europe & $\begin{array}{l}\text { North } \\
\text { America }\end{array}$ & Oceania \\
\hline \multirow{7}{*}{$\begin{array}{l}\text { Agricultural } \\
\text { Soil }\end{array}$} & Africa/Middle East & $-2.58 \mathrm{E}-02$ & $-9.78 \mathrm{E}-03$ & $-3.80 \mathrm{E}-03$ & $2.25 \mathrm{E}-04$ & $-3.28 \mathrm{E}-04$ & $1.31 \mathrm{E}-03$ & $-9.68 \mathrm{E}-04$ \\
\hline & Southern Asia & $-1.90 \mathrm{E}-02$ & $-4.11 \mathrm{E}-02$ & $4.56 \mathrm{E}-03$ & $-8.32 \mathrm{E}-03$ & $-6.04 \mathrm{E}-05$ & $-1.53 \mathrm{E}-03$ & $5.85 \mathrm{E}-03$ \\
\hline & Central/South America & $-1.88 \mathrm{E}-02$ & $2.38 \mathrm{E}-03$ & $-7.67 \mathrm{E}-03$ & $8.16 \mathrm{E}-04$ & $3.36 \mathrm{E}-05$ & $3.77 \mathrm{E}-04$ & $-4.63 \mathrm{E}-03$ \\
\hline & Northern Asia & $3.48 \mathrm{E}-03$ & $8.84 \mathrm{E}-03$ & $-3.56 \mathrm{E}-04$ & $4.21 \mathrm{E}-03$ & $-1.48 \mathrm{E}-06$ & $6.95 \mathrm{E}-03$ & $-4.04 \mathrm{E}-04$ \\
\hline & \begin{tabular}{|l} 
Europe \\
\end{tabular} & $2.54 \mathrm{E}-03$ & $2.77 \mathrm{E}-03$ & $9.48 \mathrm{E}-04$ & $1.38 \mathrm{E}-03$ & $-4.27 \mathrm{E}-04$ & $-1.08 \mathrm{E}-03$ & $7.13 \mathrm{E}-04$ \\
\hline & \begin{tabular}{|l} 
North America \\
\end{tabular} & $-1.42 \mathrm{E}-03$ & $-1.15 \mathrm{E}-02$ & $4.55 \mathrm{E}-03$ & $4.82 \mathrm{E}-03$ & $1.50 \mathrm{E}-04$ & $-3.94 \mathrm{E}-03$ & $2.76 \mathrm{E}-03$ \\
\hline & Oceania & $6.22 \mathrm{E}-03$ & $2.99 \mathrm{E}-03$ & $2.50 \mathrm{E}-03$ & $1.28 \mathrm{E}-03$ & $2.38 \mathrm{E}-05$ & $6.84 \mathrm{E}-04$ & $-4.05 \mathrm{E}-03$ \\
\hline \multirow{7}{*}{ Industrial } & Africa/Middle East & $-2.90 \mathrm{E}-03$ & $-1.89 \mathrm{E}-03$ & $-2.34 \mathrm{E}-04$ & $1.85 \mathrm{E}-04$ & $-8.00 \mathrm{E}-05$ & $4.48 \mathrm{E}-04$ & $-4.78 \mathrm{E}-06$ \\
\hline & Southern Asia & $1.02 \mathrm{E}-02$ & $-6.59 \mathrm{E}-03$ & $4.50 \mathrm{E}-03$ & $-4.08 \mathrm{E}-03$ & $1.46 \mathrm{E}-04$ & $3.05 \mathrm{E}-03$ & $1.61 \mathrm{E}-03$ \\
\hline & Central/South America & $-1.83 \mathrm{E}-03$ & $1.85 \mathrm{E}-04$ & $-7.97 \mathrm{E}-04$ & $5.88 \mathrm{E}-05$ & $2.48 \mathrm{E}-06$ & $2.56 \mathrm{E}-05$ & $-4.67 \mathrm{E}-04$ \\
\hline & Northern Asia & $1.37 \mathrm{E}-02$ & $6.53 \mathrm{E}-03$ & $3.26 \mathrm{E}-03$ & $8.87 \mathrm{E}-03$ & $4.03 E-04$ & $1.21 \mathrm{E}-02$ & $-1.85 \mathrm{E}-04$ \\
\hline & Europe & $1.36 \mathrm{E}-03$ & $1.85 \mathrm{E}-03$ & $3.79 \mathrm{E}-04$ & $1.15 \mathrm{E}-03$ & $1.06 \mathrm{E}-05$ & $3.99 \mathrm{E}-04$ & $3.79 \mathrm{E}-04$ \\
\hline & North America & $2.03 \mathrm{E}-03$ & $1.50 \mathrm{E}-03$ & $1.89 \mathrm{E}-04$ & $8.63 \mathrm{E}-03$ & $2.83 E-04$ & $1.18 \mathrm{E}-03$ & $2.36 \mathrm{E}-04$ \\
\hline & Oceania & $6.17 \mathrm{E}-04$ & $2.53 \mathrm{E}-04$ & $2.79 \mathrm{E}-04$ & $1.16 \mathrm{E}-04$ & $2.11 \mathrm{E}-06$ & $6.45 \mathrm{E}-05$ & $-2.77 \mathrm{E}-04$ \\
\hline \multirow{7}{*}{ Natural Soil } & Africa/Middle East & $-1.32 \mathrm{E}-01$ & $8.43 \mathrm{E}-03$ & $-3.68 \mathrm{E}-02$ & $2.38 \mathrm{E}-03$ & $-1.83 \mathrm{E}-04$ & $4.37 \mathrm{E}-03$ & $-1.23 \mathrm{E}-02$ \\
\hline & Southern Asia & $-3.72 \mathrm{E}-02$ & $3.29 \mathrm{E}-02$ & $-1.97 \mathrm{E}-02$ & $1.78 \mathrm{E}-02$ & $-7.87 \mathrm{E}-05$ & $1.32 \mathrm{E}-02$ & $-1.19 \mathrm{E}-02$ \\
\hline & Central/South America & $-3.74 \mathrm{E}-02$ & $-1.69 \mathrm{E}-02$ & $1.17 \mathrm{E}-02$ & $-2.21 \mathrm{E}-03$ & $-3.85 \mathrm{E}-06$ & $-3.61 \mathrm{E}-03$ & $2.09 \mathrm{E}-03$ \\
\hline & Northern Asia & $2.85 \mathrm{E}-02$ & $-3.53 \mathrm{E}-02$ & $1.54 \mathrm{E}-02$ & $-8.17 \mathrm{E}-02$ & $-1.18 \mathrm{E}-03$ & $-3.60 \mathrm{E}-02$ & $2.99 \mathrm{E}-03$ \\
\hline & Europe & $2.89 \mathrm{E}-03$ & $-9.50 \mathrm{E}-03$ & $3.04 \mathrm{E}-03$ & $-1.24 \mathrm{E}-02$ & $-3.30 \mathrm{E}-03$ & $-8.63 \mathrm{E}-03$ & $1.49 \mathrm{E}-03$ \\
\hline & North America & $-5.56 \mathrm{E}-03$ & $1.33 \mathrm{E}-03$ & $1.29 \mathrm{E}-03$ & $-3.77 \mathrm{E}-02$ & $-1.98 \mathrm{E}-03$ & $-5.07 E-02$ & $2.95 \mathrm{E}-03$ \\
\hline & Oceania & $5.68 \mathrm{E}-04$ & $-1.45 \mathrm{E}-03$ & $1.04 \mathrm{E}-03$ & $-2.16 \mathrm{E}-05$ & $2.84 \mathrm{E}-05$ & $-1.33 \mathrm{E}-04$ & $-6.93 \mathrm{E}-03$ \\
\hline \multirow{6}{*}{ Ocean } & North Pacific & $-2.97 \mathrm{E}-03$ & $-3.43 \mathrm{E}-02$ & $-6.07 E-04$ & $-5.34 \mathrm{E}-02$ & $8.06 \mathrm{E}-04$ & $-8.87 \mathrm{E}-03$ & $3.70 \mathrm{E}-05$ \\
\hline & South Pacific & $-2.86 \mathrm{E}-02$ & $-3.62 \mathrm{E}-03$ & $-2.70 \mathrm{E}-02$ & $3.91 \mathrm{E}-03$ & $1.46 \mathrm{E}-04$ & $1.87 \mathrm{E}-03$ & $-5.74 \mathrm{E}-03$ \\
\hline & Northern Ocean & $1.85 \mathrm{E}-03$ & $-6.39 \mathrm{E}-03$ & $-1.08 \mathrm{E}-03$ & $-3.37 \mathrm{E}-03$ & $4.36 \mathrm{E}-04$ & $-7.41 \mathrm{E}-03$ & $-8.00 \mathrm{E}-04$ \\
\hline & Atlantic & $-5.54 \mathrm{E}-02$ & $-3.40 \mathrm{E}-02$ & $-1.57 \mathrm{E}-02$ & $1.04 \mathrm{E}-02$ & $7.04 \mathrm{E}-04$ & 8.07E-03 & $-4.23 \mathrm{E}-03$ \\
\hline & Southern Ocean & $-1.96 \mathrm{E}-02$ & $1.12 \mathrm{E}-02$ & $-5.88 \mathrm{E}-02$ & $1.31 \mathrm{E}-03$ & $3.94 \mathrm{E}-05$ & \begin{tabular}{|l|}
$1.54 \mathrm{E}-03$ \\
\end{tabular} & $-1.29 \mathrm{E}-02$ \\
\hline & Indian Ocean & $-4.52 \mathrm{E}-02$ & $-1.21 \mathrm{E}-03$ & $-2.25 \mathrm{E}-02$ & $4.62 \mathrm{E}-03$ & $1.22 \mathrm{E}-04$ & $1.28 \mathrm{E}-03$ & $-1.67 \mathrm{E}-02$ \\
\hline \multirow{7}{*}{$\begin{array}{l}\text { Biomass } \\
\text { burning }\end{array}$} & Africa/Middle East & 1 & $-3.14 \mathrm{E}-03$ & $-1.85 \mathrm{E}-02$ & $1.65 \mathrm{E}-03$ & $8.69 \mathrm{E}-05$ & $1.63 \mathrm{E}-03$ & $-5.31 \mathrm{E}-03$ \\
\hline & Southern Asia & $-3.14 \mathrm{E}-03$ & 1 & $9.92 \mathrm{E}-04$ & $-1.99 \mathrm{E}-03$ & $9.13 \mathrm{E}-05$ & 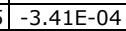 & $5.20 \mathrm{E}-04$ \\
\hline & Central/South America & $-1.85 \mathrm{E}-02$ & $9.92 \mathrm{E}-04$ & 1 & $7.76 \mathrm{E}-04$ & $4.52 \mathrm{E}-05$ & $8.27 \mathrm{E}-04$ & $-4.57 \mathrm{E}-03$ \\
\hline & Northern Asia & $1.65 \mathrm{E}-03$ & $-1.99 \mathrm{E}-03$ & $7.76 \mathrm{E}-04$ & 1 & $-1.57 \mathrm{E}-04$ & $-5.50 \mathrm{E}-03$ & $3.47 \mathrm{E}-04$ \\
\hline & Europe & $8.69 \mathrm{E}-05$ & $9.13 \mathrm{E}-05$ & $4.52 \mathrm{E}-05$ & $-1.57 \mathrm{E}-04$ & 1 & $-1.51 \mathrm{E}-04$ & $1.29 \mathrm{E}-05$ \\
\hline & North America & $1.63 \mathrm{E}-03$ & $-3.41 \mathrm{E}-04$ & $8.27 \mathrm{E}-04$ & $-5.50 \mathrm{E}-03$ & $-1.51 \mathrm{E}-04$ & 1 & $2.52 \mathrm{E}-04$ \\
\hline & Oceania & $-5.31 \mathrm{E}-03$ & $5.20 \mathrm{E}-04$ & $-4.57 \mathrm{E}-03$ & $3.47 \mathrm{E}-04$ & $1.29 \mathrm{E}-05$ & \begin{tabular}{|l|}
$2.52 \mathrm{E}-04$ \\
\end{tabular} & 1 \\
\hline
\end{tabular}

Fig. S2x. Correlation of the emissions in 1999 for biomass burning. 


\begin{tabular}{|c|c|c|c|c|c|c|c|c|}
\hline \multirow[b]{2}{*}{ Source sector } & \multirow[b]{2}{*}{ Region } & \multicolumn{7}{|c|}{ Agricultural Soil } \\
\hline & & $\begin{array}{l}\text { Africa/M. } \\
\text { East }\end{array}$ & $\begin{array}{l}\text { Southern } \\
\text { Asia }\end{array}$ & $\begin{array}{l}\text { C. /S. } \\
\text { America }\end{array}$ & $\begin{array}{l}\text { Northern } \\
\text { Asia }\end{array}$ & Europe & $\begin{array}{l}\text { North } \\
\text { America }\end{array}$ & Oceania \\
\hline \multirow{7}{*}{$\begin{array}{c}\text { Agricultural } \\
\text { Soil }\end{array}$} & Africa/Middle East & 1 & $-7.12 \mathrm{E}-02$ & $-2.19 \mathrm{E}-03$ & $-3.60 \mathrm{E}-02$ & $-1.53 \mathrm{E}-02$ & $-4.27 \mathrm{E}-02$ & $7.14 \mathrm{E}-03$ \\
\hline & Southern Asia & $-7.12 \mathrm{E}-02$ & 1 & $2.59 \mathrm{E}-02$ & $-2.29 \mathrm{E}-01$ & $2.41 \mathrm{E}-02$ & $-9.89 \mathrm{E}-02$ & $2.32 \mathrm{E}-02$ \\
\hline & Central/South America & $-2.19 E-03$ & $2.59 \mathrm{E}-02$ & 1 & $-3.67 \mathrm{E}-03$ & $4.97 \mathrm{E}-03$ & $1.20 \mathrm{E}-02$ & $-1.85 \mathrm{E}-02$ \\
\hline & Northern Asia & $-3.60 \mathrm{E}-02$ & $-2.29 \mathrm{E}-01$ & $-3.67 \mathrm{E}-03$ & 1 & $-1.79 \mathrm{E}-01$ & $-5.25 \mathrm{E}-02$ & $-1.55 \mathrm{E}-02$ \\
\hline & Europe & $-1.53 \mathrm{E}-02$ & $2.41 \mathrm{E}-02$ & 4.97E-03 & $-1.79 \mathrm{E}-01$ & 1 & $-3.76 \mathrm{E}-02$ & $3.15 \mathrm{E}-03$ \\
\hline & North America & $-4.27 \mathrm{E}-02$ & $-9.89 \mathrm{E}-02$ & $1.20 \mathrm{E}-02$ & $-5.25 \mathrm{E}-02$ & $-3.76 \mathrm{E}-02$ & 1 & $6.98 \mathrm{E}-03$ \\
\hline & Oceania & $7.14 \mathrm{E}-03$ & $2.32 \mathrm{E}-02$ & $-1.85 \mathrm{E}-02$ & $-1.55 \mathrm{E}-02$ & $3.15 \mathrm{E}-03$ & $6.98 \mathrm{E}-03$ & 1 \\
\hline \multirow{7}{*}{ Industrial } & Africa/Middle East & $-6.67 \mathrm{E}-02$ & $-9.16 \mathrm{E}-03$ & $1.30 \mathrm{E}-03$ & $-1.11 \mathrm{E}-02$ & $-7.04 \mathrm{E}-03$ & $-7.83 \mathrm{E}-03$ & $1.24 \mathrm{E}-03$ \\
\hline & \begin{tabular}{|l} 
Southern Asia \\
\end{tabular} & $1.23 \mathrm{E}-02$ & $-4.09 \mathrm{E}-01$ & $1.72 \mathrm{E}-03$ & $-6.06 \mathrm{E}-02$ & $2.00 \mathrm{E}-02$ & $8.00 \mathrm{E}-03$ & $-6.01 \mathrm{E}-04$ \\
\hline & Central/South America & $-2.72 \mathrm{E}-04$ & $2.37 \mathrm{E}-03$ & $-4.38 \mathrm{E}-03$ & $-3.15 \mathrm{E}-04$ & $4.42 \mathrm{E}-04$ & $1.09 \mathrm{E}-03$ & $-1.75 \mathrm{E}-03$ \\
\hline & Northern Asia & $8.81 \mathrm{E}-03$ & $-2.29 \mathrm{E}-02$ & $-3.49 \mathrm{E}-03$ & $-1.41 \mathrm{E}-01$ & $-2.86 \mathrm{E}-02$ & $6.68 \mathrm{E}-02$ & $-5.20 \mathrm{E}-03$ \\
\hline & Europe & $-2.06 \mathrm{E}-02$ & $-3.26 \mathrm{E}-03$ & $2.54 \mathrm{E}-03$ & $-4.51 \mathrm{E}-02$ & $-6.26 \mathrm{E}-01$ & $-2.14 \mathrm{E}-02$ & $8.61 \mathrm{E}-04$ \\
\hline & North America & $6.09 \mathrm{E}-03$ & $1.27 \mathrm{E}-02$ & $1.37 \mathrm{E}-03$ & $3.93 \mathrm{E}-02$ & $2.37 \mathrm{E}-02$ & $-2.95 \mathrm{E}-01$ & $-8.37 E-04$ \\
\hline & Oceania & $5.33 \mathrm{E}-04$ & $1.71 \mathrm{E}-03$ & $-1.32 \mathrm{E}-03$ & $-1.09 \mathrm{E}-03$ & $2.29 \mathrm{E}-04$ & $4.66 \mathrm{E}-04$ & $-6.92 \mathrm{E}-03$ \\
\hline \multirow{7}{*}{ Natural Soil } & Africa/Middle East & $\begin{array}{l}-1.97 \mathrm{E}-01 \\
\end{array}$ & $-7.97 \mathrm{E}-02$ & $-6.96 \mathrm{E}-02$ & $-1.49 \mathrm{E}-02$ & $-1.38 \mathrm{E}-02$ & $-4.33 \mathrm{E}-02$ & $3.00 \mathrm{E}-03$ \\
\hline & Southern Asia & $-3.65 \mathrm{E}-02$ & $-2.92 \mathrm{E}-01$ & $1.63 \mathrm{E}-03$ & $1.87 \mathrm{E}-02$ & $6.44 \mathrm{E}-03$ & $-2.03 \mathrm{E}-02$ & $2.16 \mathrm{E}-02$ \\
\hline & Central/South America & $-5.06 \mathrm{E}-02$ & $5.81 \mathrm{E}-02$ & $-2.02 \mathrm{E}-01$ & $1.17 \mathrm{E}-02$ & $2.45 \mathrm{E}-02$ & $2.11 \mathrm{E}-02$ & $-4.93 E-02$ \\
\hline & Northern Asia & $2.10 \mathrm{E}-02$ & $-1.14 \mathrm{E}-02$ & $2.28 \mathrm{E}-03$ & $-2.01 \mathrm{E}-01$ & $1.64 \mathrm{E}-02$ & $2.14 \mathrm{E}-02$ & $-1.55 \mathrm{E}-02$ \\
\hline & Europe & $-2.71 \mathrm{E}-02$ & $4.56 \mathrm{E}-03$ & $5.97 \mathrm{E}-03$ & $-7.53 \mathrm{E}-03$ & $-3.27 \mathrm{E}-01$ & $-4.37 \mathrm{E}-03$ & $4.24 \mathrm{E}-03$ \\
\hline & North America & $-2.49 \mathrm{E}-02$ & $-2.27 \mathrm{E}-02$ & $1.27 \mathrm{E}-02$ & $8.37 \mathrm{E}-02$ & $-1.40 \mathrm{E}-02$ & $-4.14 \mathrm{E}-01$ & $1.10 \mathrm{E}-02$ \\
\hline & Oceania & $5.09 \mathrm{E}-03$ & $2.51 \mathrm{E}-02$ & $-2.41 \mathrm{E}-02$ & \begin{tabular}{|l|}
$-9.53 E-03$ \\
\end{tabular} & $5.03 \mathrm{E}-03$ & $1.07 \mathrm{E}-02$ & $-7.86 \mathrm{E}-02$ \\
\hline \multirow{6}{*}{ Ocean } & North Pacific & $-7.38 \mathrm{E}-03$ & $-1.23 \mathrm{E}-01$ & $1.04 \mathrm{E}-02$ & $2.56 \mathrm{E}-02$ & $4.58 \mathrm{E}-03$ & $-7.06 \mathrm{E}-02$ & $1.19 \mathrm{E}-02$ \\
\hline & South Pacific & $-9.45 \mathrm{E}-03$ & $6.36 \mathrm{E}-03$ & $-1.98 \mathrm{E}-02$ & $1.25 \mathrm{E}-02$ & $1.61 \mathrm{E}-02$ & $5.77 \mathrm{E}-03$ & $1.51 \mathrm{E}-02$ \\
\hline & Northern Ocean & $6.07 \mathrm{E}-03$ & $2.30 \mathrm{E}-03$ & $1.32 \mathrm{E}-03$ & $-5.88 \mathrm{E}-02$ & $-7.41 \mathrm{E}-02$ & $-1.38 \mathrm{E}-02$ & $3.12 \mathrm{E}-03$ \\
\hline & Atlantic & $-6.84 \mathrm{E}-02$ & $-1.09 \mathrm{E}-01$ & $-1.24 \mathrm{E}-02$ & $-4.04 \mathrm{E}-02$ & $-6.04 \mathrm{E}-02$ & $-1.73 \mathrm{E}-01$ & $1.74 \mathrm{E}-02$ \\
\hline & Southern Ocean & $3.46 \mathrm{E}-02$ & $1.35 \mathrm{E}-01$ & $-1.47 \mathrm{E}-01$ & $-2.49 \mathrm{E}-02$ & $1.11 \mathrm{E}-02$ & $4.23 \mathrm{E}-02$ & $-7.17 E-02$ \\
\hline & Indian Ocean & $-3.41 \mathrm{E}-02$ & $-9.65 E-02$ & $-7.33 \mathrm{E}-02$ & $2.16 \mathrm{E}-02$ & $1.13 \mathrm{E}-03$ & $2.42 \mathrm{E}-02$ & $-2.98 \mathrm{E}-02$ \\
\hline \multirow{7}{*}{$\begin{array}{l}\text { Biomass } \\
\text { burning }\end{array}$} & Africa/Middle East & $-2.36 \mathrm{E}-02$ & $-2.57 \mathrm{E}-02$ & $-2.18 \mathrm{E}-02$ & $6.94 \mathrm{E}-03$ & $3.13 \mathrm{E}-03$ & $-2.24 \mathrm{E}-03$ & $5.54 \mathrm{E}-03$ \\
\hline & Southern Asia & $-2.13 \mathrm{E}-03$ & $-1.21 \mathrm{E}-02$ & $1.57 \mathrm{E}-03$ & $2.89 \mathrm{E}-03$ & $1.75 \mathrm{E}-03$ & $-1.06 \mathrm{E}-03$ & $1.44 \mathrm{E}-03$ \\
\hline & Central/South America & $-1.76 \mathrm{E}-03$ & $6.40 \mathrm{E}-04$ & $-5.03 \mathrm{E}-03$ & $6.19 \mathrm{E}-04$ & $8.43 \mathrm{E}-04$ & $2.05 \mathrm{E}-03$ & $9.02 \mathrm{E}-04$ \\
\hline & \begin{tabular}{|l} 
Northern Asia \\
\end{tabular} & $-4.30 \mathrm{E}-04$ & $-1.28 \mathrm{E}-02$ & $3.26 \mathrm{E}-03$ & $2.56 \mathrm{E}-02$ & $6.91 \mathrm{E}-03$ & $1.59 \mathrm{E}-03$ & $2.69 \mathrm{E}-03$ \\
\hline & Europe & $-4.20 \mathrm{E}-04$ & $-5.16 \mathrm{E}-05$ & $3.27 \mathrm{E}-05$ & $9.74 \mathrm{E}-04$ & $3.34 \mathrm{E}-04$ & $4.20 \mathrm{E}-04$ & $1.24 \mathrm{E}-05$ \\
\hline & North America & $6.63 \mathrm{E}-04$ & $1.73 \mathrm{E}-03$ & $4.64 \mathrm{E}-04$ & $-3.19 \mathrm{E}-04$ & $1.05 \mathrm{E}-03$ & $-2.00 \mathrm{E}-02$ & $3.81 \mathrm{E}-04$ \\
\hline & Oceania & $-1.13 \mathrm{E}-03$ & $2.52 \mathrm{E}-03$ & $-4.46 \mathrm{E}-03$ & $-7.31 \mathrm{E}-04$ & $5.59 \mathrm{E}-04$ & $2.43 \mathrm{E}-03$ & $-1.59 \mathrm{E}-03$ \\
\hline
\end{tabular}

Fig. S2y. Correlation of the emissions in $\mathbf{2 0 0 0}$ for agricultural soil. 


\begin{tabular}{|c|c|c|c|c|c|c|c|c|}
\hline & \multirow{2}{*}{\multicolumn{7}{|c|}{ Industrial }} \\
\hline & & & & & & & & \\
\hline Source sector & Region & $\begin{array}{l}\text { Africa/M. } \\
\text { East }\end{array}$ & $\begin{array}{l}\text { Southern } \\
\text { Asia }\end{array}$ & $\begin{array}{l}\text { C. /S. } \\
\text { America }\end{array}$ & $\begin{array}{l}\text { Northern } \\
\text { Asia }\end{array}$ & Europe & $\begin{array}{l}\text { North } \\
\text { America }\end{array}$ & Oceania \\
\hline \multirow{7}{*}{$\begin{array}{l}\text { Agricultural } \\
\text { Soil }\end{array}$} & Africa/Middle East & $-6.67 \mathrm{E}-02$ & $1.23 \mathrm{E}-02$ & $-2.72 \mathrm{E}-04$ & $8.81 \mathrm{E}-03$ & $-2.06 \mathrm{E}-02$ & $6.09 \mathrm{E}-03$ & $5.33 \mathrm{E}-04$ \\
\hline & Southern Asia & $-9.16 \mathrm{E}-03$ & $-4.09 \mathrm{E}-01$ & $2.37 \mathrm{E}-03$ & \begin{tabular}{|l|} 
\\
\end{tabular} & $-3.26 \mathrm{E}-03$ & $1.27 \mathrm{E}-02$ & $1.71 \mathrm{E}-03$ \\
\hline & Central/South America & $1.30 \mathrm{E}-03$ & $1.72 \mathrm{E}-03$ & $-4.38 \mathrm{E}-03$ & \begin{tabular}{|l|} 
\\
\end{tabular} & $2.54 \mathrm{E}-03$ & $1.37 \mathrm{E}-03$ & $-1.32 \mathrm{E}-03$ \\
\hline & Northern Asia & $-1.11 \mathrm{E}-02$ & $-6.06 \mathrm{E}-02$ & $-3.15 \mathrm{E}-04$ & \begin{tabular}{|l|} 
\\
\end{tabular} & $-4.51 \mathrm{E}-02$ & $3.93 \mathrm{E}-02$ & $-1.09 \mathrm{E}-03$ \\
\hline & Europe & $-7.04 \mathrm{E}-03$ & $2.00 \mathrm{E}-02$ & $4.42 \mathrm{E}-04$ & $-2.86 \mathrm{E}-02$ & $-6.26 \mathrm{E}-01$ & $2.37 \mathrm{E}-02$ & $2.29 \mathrm{E}-04$ \\
\hline & \begin{tabular}{|l|} 
North America \\
\end{tabular} & $-7.83 \mathrm{E}-03$ & $8.00 \mathrm{E}-03$ & $1.09 \mathrm{E}-03$ & \begin{tabular}{l|l}
$6.68 \mathrm{E}-02$ \\
\end{tabular} & $-2.14 \mathrm{E}-02$ & $-2.95 \mathrm{E}-01$ & $4.66 \mathrm{E}-04$ \\
\hline & Oceania & $1.24 \mathrm{E}-03$ & $-6.01 \mathrm{E}-04$ & $-1.75 \mathrm{E}-03$ & 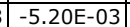 & $8.61 \mathrm{E}-04$ & $-8.37 \mathrm{E}-04$ & $-6.92 \mathrm{E}-03$ \\
\hline \multirow{7}{*}{ Industrial } & Africa/Middle East & 1 & $1.86 \mathrm{E}-03$ & $1.12 \mathrm{E}-04$ & $-4.55 \mathrm{E}-03$ & $-9.71 \mathrm{E}-03$ & $2.15 \mathrm{E}-03$ & $8.94 \mathrm{E}-05$ \\
\hline & \begin{tabular}{|l|} 
Southern Asia \\
\end{tabular} & $1.86 \mathrm{E}-03$ & 1 & $1.90 \mathrm{E}-04$ & $-5.58 \mathrm{E}-02$ & $3.33 \mathrm{E}-03$ & $1.28 \mathrm{E}-02$ & $-4.78 \mathrm{E}-05$ \\
\hline & Central/South America & $1.12 \mathrm{E}-04$ & $1.90 \mathrm{E}-04$ & 1 & $-2.68 \mathrm{E}-04$ & $2.21 \mathrm{E}-04$ & $1.32 \mathrm{E}-04$ & $-1.24 \mathrm{E}-04$ \\
\hline & Northern Asia & $-4.55 \mathrm{E}-03$ & $-5.58 \mathrm{E}-02$ & $-2.68 \mathrm{E}-04$ & 1 & $-3.51 \mathrm{E}-02$ & $-2.78 \mathrm{E}-01$ & $-3.79 \mathrm{E}-04$ \\
\hline & Europe & $-9.71 \mathrm{E}-03$ & $3.33 \mathrm{E}-03$ & $2.21 \mathrm{E}-04$ & $-3.51 E-02$ & 1 & $9.33 \mathrm{E}-03$ & $5.31 \mathrm{E}-05$ \\
\hline & North America & $2.15 \mathrm{E}-03$ & $1.28 \mathrm{E}-02$ & $1.32 \mathrm{E}-04$ & $-2.78 \mathrm{E}-01$ & 9.33E-03 & 1 & $-7.01 \mathrm{E}-05$ \\
\hline & Oceania & $8.94 \mathrm{E}-05$ & $-4.78 \mathrm{E}-05$ & $-1.24 \mathrm{E}-04$ & $-3.79 E-04$ & $5.31 \mathrm{E}-05$ & $-7.01 \mathrm{E}-05$ & 1 \\
\hline \multirow{7}{*}{ Natural Soil } & Africa/Middle East & $-3.73 E-02$ & $4.63 \mathrm{E}-02$ & $-6.63 \mathrm{E}-03$ & \begin{tabular}{|l|} 
\\
$5.04 \mathrm{E}-02$ \\
\end{tabular} & $-1.21 \mathrm{E}-02$ & $1.13 \mathrm{E}-02$ & $3.10 \mathrm{E}-04$ \\
\hline & Southern Asia & $-3.14 \mathrm{E}-03$ & $-1.30 \mathrm{E}-01$ & $-6.35 \mathrm{E}-05$ & \begin{tabular}{|l|} 
\\
$2.36 \mathrm{E}-02$ \\
\end{tabular} & $-4.83 \mathrm{E}-03$ & $9.95 \mathrm{E}-03$ & $1.82 \mathrm{E}-03$ \\
\hline & Central/South America & $5.38 \mathrm{E}-04$ & $1.59 \mathrm{E}-03$ & $-1.80 \mathrm{E}-02$ & $2.31 \mathrm{E}-02$ & $1.12 \mathrm{E}-02$ & $3.87 \mathrm{E}-03$ & $-3.66 \mathrm{E}-03$ \\
\hline & Northern Asia & 5.77E-03 & $-1.42 \mathrm{E}-02$ & $2.36 \mathrm{E}-04$ & $-3.75 \mathrm{E}-02$ & $2.51 \mathrm{E}-02$ & $4.46 \mathrm{E}-02$ & $-1.39 \mathrm{E}-03$ \\
\hline & Europe & $-8.92 \mathrm{E}-03$ & $1.05 \mathrm{E}-02$ & $5.12 \mathrm{E}-04$ & 3.27E-02 & $-2.20 \mathrm{E}-01$ & $2.03 \mathrm{E}-02$ & $3.42 \mathrm{E}-04$ \\
\hline & North America & $8.23 \mathrm{E}-05$ & $6.27 \mathrm{E}-02$ & $9.81 \mathrm{E}-04$ & $-2.05 \mathrm{E}-01$ & $1.51 \mathrm{E}-02$ & $-2.11 \mathrm{E}-01$ & $8.82 \mathrm{E}-04$ \\
\hline & Oceania & $1.34 \mathrm{E}-03$ & $-8.08 \mathrm{E}-04$ & $-2.28 \mathrm{E}-03$ & \begin{tabular}{|l|}
$-8.09 E-03$ \\
\end{tabular} & $1.95 \mathrm{E}-03$ & $3.38 \mathrm{E}-04$ & $-6.12 \mathrm{E}-03$ \\
\hline \multirow{6}{*}{ Ocean } & North Pacific & $-3.53 \mathrm{E}-03$ & $-3.12 \mathrm{E}-02$ & $9.57 \mathrm{E}-04$ & $3.77 \mathrm{E}-02$ & $-1.46 \mathrm{E}-02$ & $-3.89 \mathrm{E}-02$ & $9.23 \mathrm{E}-04$ \\
\hline & \begin{tabular}{|l} 
South Pacific \\
\end{tabular} & $2.76 \mathrm{E}-04$ & $1.01 \mathrm{E}-02$ & $-2.79 \mathrm{E}-03$ & \begin{tabular}{|c|} 
\\
\end{tabular} & $7.66 \mathrm{E}-03$ & $1.60 \mathrm{E}-04$ & $1.75 \mathrm{E}-03$ \\
\hline & Northern Ocean & $1.20 \mathrm{E}-03$ & $-7.39 \mathrm{E}-03$ & $1.47 \mathrm{E}-04$ & \begin{tabular}{l|} 
\\
\end{tabular} & $2.10 \mathrm{E}-02$ & $1.83 \mathrm{E}-02$ & $2.49 \mathrm{E}-04$ \\
\hline & Atlantic & $-9.81 E-03$ & $-1.43 \mathrm{E}-02$ & $-1.20 \mathrm{E}-03$ & \begin{tabular}{|l|} 
\\
\end{tabular} & $-1.02 \mathrm{E}-02$ & $-7.89 \mathrm{E}-02$ & $1.26 \mathrm{E}-03$ \\
\hline & Southern Ocean & $4.39 \mathrm{E}-03$ & $-2.00 \mathrm{E}-02$ & $-1.17 \mathrm{E}-02$ & $-1.91 \mathrm{E}-02$ & $9.49 \mathrm{E}-04$ & $6.68 \mathrm{E}-03$ & $-5.12 \mathrm{E}-03$ \\
\hline & Indian Ocean & $-2.12 \mathrm{E}-03$ & $2.66 \mathrm{E}-02$ & $-7.20 \mathrm{E}-03$ & \begin{tabular}{|l|l|} 
& $9.47 \mathrm{E}-03$ \\
\end{tabular} & $3.61 \mathrm{E}-03$ & $-2.45 \mathrm{E}-03$ & $-2.63 \mathrm{E}-03$ \\
\hline \multirow{7}{*}{$\begin{array}{l}\text { Biomass } \\
\text { burning }\end{array}$} & Africa/Middle East & $-2.03 E-03$ & $5.77 \mathrm{E}-03$ & $-2.07 E-03$ & \begin{tabular}{|c|} 
\\
\end{tabular} & $1.93 \mathrm{E}-03$ & $1.21 \mathrm{E}-03$ & $4.26 \mathrm{E}-04$ \\
\hline & Southern Asia & $-3.87 E-04$ & $-3.62 \mathrm{E}-03$ & $1.43 \mathrm{E}-04$ & $2.21 \mathrm{E}-03$ & $7.02 E-04$ & $9.66 \mathrm{E}-05$ & $1.08 \mathrm{E}-04$ \\
\hline & Central/South America & $-6.24 \mathrm{E}-06$ & $9.10 \mathrm{E}-04$ & $-4.77 \mathrm{E}-04$ & $\begin{array}{ll} & 2.99 \mathrm{E}-04 \\
\end{array}$ & 4.57E-04 & $3.73 \mathrm{E}-04$ & $7.72 \mathrm{E}-05$ \\
\hline & Northern Asia & $2.49 \mathrm{E}-04$ & $-6.34 \mathrm{E}-03$ & $2.71 \mathrm{E}-04$ & $3.36 \mathrm{E}-02$ & $1.01 \mathrm{E}-03$ & $8.49 \mathrm{E}-03$ & $1.87 \mathrm{E}-04$ \\
\hline & Europe & $-5.99 E-05$ & $1.60 \mathrm{E}-05$ & $2.12 \mathrm{E}-06$ & \begin{tabular}{|l|}
$5.16 \mathrm{E}-04$ \\
\end{tabular} & $-1.38 \mathrm{E}-03$ & $3.01 \mathrm{E}-04$ & $7.13 \mathrm{E}-07$ \\
\hline & North America & $7.93 \mathrm{E}-05$ & $-1.33 \mathrm{E}-05$ & $3.52 \mathrm{E}-05$ & \begin{tabular}{|l|}
$1.83 \mathrm{E}-02$ \\
\end{tabular} & $2.91 \mathrm{E}-04$ & $-3.02 \mathrm{E}-02$ & $2.80 \mathrm{E}-05$ \\
\hline & Oceania & $6.12 \mathrm{E}-05$ & $8.22 \mathrm{E}-04$ & $-4.25 \mathrm{E}-04$ & $-6.08 \mathrm{E}-04$ & $2.67 \mathrm{E}-04$ & $3.61 \mathrm{E}-04$ & $-1.21 \mathrm{E}-04$ \\
\hline
\end{tabular}

Fig. S2z. Correlation of the emissions in 2000 for industrial. 


\begin{tabular}{|c|c|c|c|c|c|c|c|c|}
\hline & \multirow{2}{*}{\multicolumn{7}{|c|}{ Natural Soil }} \\
\hline & & & & & & & & \\
\hline Source sector & Region & $\begin{array}{l}\text { Africa/M. } \\
\text { East }\end{array}$ & $\begin{array}{l}\text { Southern } \\
\text { Asia }\end{array}$ & $\begin{array}{l}\text { C. /S. } \\
\text { America }\end{array}$ & $\begin{array}{l}\text { Northern } \\
\text { Asia }\end{array}$ & Europe & $\begin{array}{l}\text { North } \\
\text { America }\end{array}$ & Oceania \\
\hline \multirow{7}{*}{$\begin{array}{l}\text { Agricultural } \\
\text { Soil }\end{array}$} & Africa/Middle East & $-1.97 \mathrm{E}-01$ & $-3.65 \mathrm{E}-02$ & $-5.06 \mathrm{E}-02$ & \begin{tabular}{|l|}
$2.10 \mathrm{E}-02$ \\
\end{tabular} & $-2.71 \mathrm{E}-02$ & $-2.49 \mathrm{E}-02$ & $5.09 \mathrm{E}-03$ \\
\hline & Southern Asia & $-7.97 \mathrm{E}-02$ & $-2.92 \mathrm{E}-01$ & $5.81 \mathrm{E}-02$ & $\mid-1.14 \mathrm{E}-02$ & $4.56 \mathrm{E}-03$ & $-2.27 \mathrm{E}-02$ & $2.51 \mathrm{E}-02$ \\
\hline & Central/South America & $-6.96 \mathrm{E}-02$ & $1.63 \mathrm{E}-03$ & $-2.02 \mathrm{E}-01$ & $2.28 \mathrm{E}-03$ & 5.97E-03 & $1.27 \mathrm{E}-02$ & $-2.41 \mathrm{E}-02$ \\
\hline & Northern Asia & $-1.49 \mathrm{E}-02$ & $1.87 \mathrm{E}-02$ & $1.17 \mathrm{E}-02$ & \begin{tabular}{|l|}
$-2.01 \mathrm{E}-01$ \\
\end{tabular} & $-7.53 \mathrm{E}-03$ & $8.37 \mathrm{E}-02$ & $-9.53 \mathrm{E}-03$ \\
\hline & Europe & $-1.38 \mathrm{E}-02$ & $6.44 \mathrm{E}-03$ & $2.45 \mathrm{E}-02$ & $1.64 \mathrm{E}-02$ & $-3.27 \mathrm{E}-01$ & $-1.40 \mathrm{E}-02$ & $5.03 \mathrm{E}-03$ \\
\hline & \begin{tabular}{|l|} 
North America \\
\end{tabular} & $-4.33 \mathrm{E}-02$ & $-2.03 \mathrm{E}-02$ & $2.11 \mathrm{E}-02$ & $2.14 \mathrm{E}-02$ & $-4.37 \mathrm{E}-03$ & $-4.14 \mathrm{E}-01$ & $1.07 \mathrm{E}-02$ \\
\hline & Oceania & $3.00 \mathrm{E}-03$ & $2.16 \mathrm{E}-02$ & $-4.93 \mathrm{E}-02$ & $-1.55 \mathrm{E}-02$ & $4.24 \mathrm{E}-03$ & $1.10 \mathrm{E}-02$ & $-7.86 \mathrm{E}-02$ \\
\hline \multirow{7}{*}{ Industrial } & Africa/Middle East & $-3.73 \mathrm{E}-02$ & $-3.14 \mathrm{E}-03$ & $5.38 \mathrm{E}-04$ & $5.77 \mathrm{E}-03$ & $-8.92 \mathrm{E}-03$ & $8.23 \mathrm{E}-05$ & $1.34 \mathrm{E}-03$ \\
\hline & \begin{tabular}{|l|} 
Southern Asia \\
\end{tabular} & $4.63 \mathrm{E}-02$ & $-1.30 \mathrm{E}-01$ & $1.59 \mathrm{E}-03$ & $-1.42 \mathrm{E}-02$ & $1.05 \mathrm{E}-02$ & $6.27 \mathrm{E}-02$ & $-8.08 \mathrm{E}-04$ \\
\hline & Central/South America & $-6.63 \mathrm{E}-03$ & $-6.35 \mathrm{E}-05$ & $-1.80 \mathrm{E}-02$ & $2.36 \mathrm{E}-04$ & $5.12 \mathrm{E}-04$ & $9.81 \mathrm{E}-04$ & $-2.28 \mathrm{E}-03$ \\
\hline & Northern Asia & $5.04 \mathrm{E}-02$ & $2.36 \mathrm{E}-02$ & $2.31 \mathrm{E}-02$ & \begin{tabular}{|l|}
$-3.75 \mathrm{E}-02$ \\
\end{tabular} & $3.27 \mathrm{E}-02$ & $-2.05 \mathrm{E}-01$ & $-8.09 \mathrm{E}-03$ \\
\hline & Europe & $-1.21 \mathrm{E}-02$ & $-4.83 \mathrm{E}-03$ & $1.12 \mathrm{E}-02$ & $2.51 \mathrm{E}-02$ & $-2.20 \mathrm{E}-01$ & $1.51 \mathrm{E}-02$ & $1.95 \mathrm{E}-03$ \\
\hline & North America & $1.13 \mathrm{E}-02$ & 9.95E-03 & 3.87E-03 & $4.46 \mathrm{E}-02$ & $2.03 \mathrm{E}-02$ & $-2.11 \mathrm{E}-01$ & $3.38 \mathrm{E}-04$ \\
\hline & Oceania & $3.10 \mathrm{E}-04$ & $1.82 \mathrm{E}-03$ & $-3.66 \mathrm{E}-03$ & \begin{tabular}{|l|}
$-1.39 E-03$ \\
\end{tabular} & $3.42 \mathrm{E}-04$ & $8.82 \mathrm{E}-04$ & $-6.12 \mathrm{E}-03$ \\
\hline \multirow{7}{*}{ Natural Soil } & Africa/Middle East & 1 & $-1.90 \mathrm{E}-01$ & $-3.62 \mathrm{E}-01$ & $8.28 \mathrm{E}-02$ & $-1.65 \mathrm{E}-02$ & $-1.07 \mathrm{E}-01$ & $-1.21 \mathrm{E}-02$ \\
\hline & Southern Asia & $-1.90 \mathrm{E}-01$ & 1 & $-5.41 \mathrm{E}-02$ & $-4.20 \mathrm{E}-01$ & $4.53 \mathrm{E}-02$ & $-2.61 \mathrm{E}-03$ & $1.49 \mathrm{E}-02$ \\
\hline & Central/South America & $-3.62 \mathrm{E}-01$ & $-5.41 \mathrm{E}-02$ & 1 & \begin{tabular}{|l|}
$-1.01 \mathrm{E}-01$ \\
\end{tabular} & $-1.06 \mathrm{E}-02$ & $-2.37 \mathrm{E}-02$ & $-1.23 \mathrm{E}-01$ \\
\hline & Northern Asia & $8.28 \mathrm{E}-02$ & $-4.20 \mathrm{E}-01$ & $-1.01 \mathrm{E}-01$ & 1 & $-1.44 \mathrm{E}-01$ & $-2.85 \mathrm{E}-01$ & $-2.42 \mathrm{E}-02$ \\
\hline & Europe & $-1.65 \mathrm{E}-02$ & $4.53 \mathrm{E}-02$ & $-1.06 \mathrm{E}-02$ & $-1.44 \mathrm{E}-01$ & 1 & $-1.08 \mathrm{E}-01$ & $3.91 \mathrm{E}-03$ \\
\hline & North America & $-1.07 \mathrm{E}-01$ & $-2.61 \mathrm{E}-03$ & $-2.37 \mathrm{E}-02$ & \begin{tabular}{|l|}
$-2.85 \mathrm{E}-01$ \\
\end{tabular} & $-1.08 \mathrm{E}-01$ & 1 & $1.52 \mathrm{E}-02$ \\
\hline & Oceania & $-1.21 \mathrm{E}-02$ & $1.49 \mathrm{E}-02$ & $-1.23 \mathrm{E}-01$ & $-2.42 \mathrm{E}-02$ & $3.91 \mathrm{E}-03$ & $1.52 \mathrm{E}-02$ & 1 \\
\hline \multirow{6}{*}{ Ocean } & North Pacific & $8.67 \mathrm{E}-02$ & $1.37 \mathrm{E}-01$ & $6.66 \mathrm{E}-03$ & $-2.81 \mathrm{E}-01$ & $4.24 \mathrm{E}-02$ & $8.02 \mathrm{E}-02$ & $1.04 \mathrm{E}-02$ \\
\hline & South Pacific & $-4.44 \mathrm{E}-02$ & $-1.60 \mathrm{E}-02$ & $-5.05 \mathrm{E}-01$ & $7.81 \mathrm{E}-03$ & $1.01 \mathrm{E}-02$ & $3.95 \mathrm{E}-02$ & $2.92 \mathrm{E}-02$ \\
\hline & Northern Ocean & $5.64 \mathrm{E}-02$ & $9.11 \mathrm{E}-02$ & $4.61 \mathrm{E}-02$ & $-7.13 \mathrm{E}-02$ & $1.66 \mathrm{E}-02$ & $-8.53 \mathrm{E}-04$ & $3.84 \mathrm{E}-03$ \\
\hline & Atlantic & $-7.98 \mathrm{E}-02$ & $8.16 \mathrm{E}-02$ & $-4.77 E-02$ & $3.41 \mathrm{E}-02$ & $3.92 \mathrm{E}-02$ & $9.79 \mathrm{E}-02$ & $1.24 \mathrm{E}-02$ \\
\hline & Southern Ocean & $1.00 \mathrm{E}-01$ & $1.62 \mathrm{E}-01$ & $-7.49 \mathrm{E}-02$ & $-5.09 \mathrm{E}-02$ & $1.16 \mathrm{E}-02$ & $5.50 \mathrm{E}-02$ & $-3.47 \mathrm{E}-02$ \\
\hline & Indian Ocean & $-1.55 \mathrm{E}-01$ & $-9.91 E-02$ & $-2.19 \mathrm{E}-01$ & $3.10 \mathrm{E}-02$ & $8.93 E-03$ & $1.94 \mathrm{E}-02$ & $-3.40 \mathrm{E}-02$ \\
\hline \multirow{7}{*}{$\begin{array}{l}\text { Biomass } \\
\text { burning }\end{array}$} & Africa/Middle East & $-1.04 \mathrm{E}-01$ & $-7.49 \mathrm{E}-03$ & $-5.90 \mathrm{E}-02$ & $9.18 \mathrm{E}-03$ & $1.07 E-03$ & $3.10 \mathrm{E}-03$ & $-4.00 \mathrm{E}-04$ \\
\hline & Southern Asia & $4.36 \mathrm{E}-03$ & $6.08 \mathrm{E}-03$ & $-8.64 \mathrm{E}-03$ & $-1.65 \mathrm{E}-02$ & $-3.55 \mathrm{E}-03$ & $-3.79 \mathrm{E}-03$ & $7.06 \mathrm{E}-04$ \\
\hline & Central/South America & $-1.31 \mathrm{E}-02$ & $-1.08 \mathrm{E}-03$ & $-9.74 \mathrm{E}-04$ & $2.27 \mathrm{E}-03$ & $1.06 \mathrm{E}-03$ & $2.49 \mathrm{E}-03$ & $-1.75 \mathrm{E}-04$ \\
\hline & Northern Asia & $2.83 \mathrm{E}-03$ & $7.80 \mathrm{E}-02$ & $-1.00 \mathrm{E}-04$ & $-2.54 \mathrm{E}-01$ & $-1.33 \mathrm{E}-02$ & $-5.93 \mathrm{E}-02$ & $3.36 \mathrm{E}-03$ \\
\hline & Europe & $-7.44 \mathrm{E}-04$ & $-6.99 \mathrm{E}-04$ & $5.57 \mathrm{E}-05$ & $1.14 \mathrm{E}-04$ & $-6.16 \mathrm{E}-03$ & $-1.83 \mathrm{E}-03$ & $1.31 \mathrm{E}-05$ \\
\hline & North America & $2.17 \mathrm{E}-03$ & $6.21 \mathrm{E}-05$ & $-6.79 \mathrm{E}-03$ & $-5.21 \mathrm{E}-03$ & $-1.85 \mathrm{E}-03$ & $-9.91 \mathrm{E}-03$ & $7.71 \mathrm{E}-05$ \\
\hline & Oceania & $-1.07 \mathrm{E}-02$ & $-5.85 \mathrm{E}-03$ & $-3.14 \mathrm{E}-04$ & $2.47 \mathrm{E}-03$ & $1.50 \mathrm{E}-03$ & $3.13 \mathrm{E}-03$ & $-4.64 \mathrm{E}-03$ \\
\hline
\end{tabular}

Fig. S2aa. Correlation of the emissions in 2000 for natural soil. 


\begin{tabular}{|c|c|c|c|c|c|c|c|}
\hline & \multirow{2}{*}{\multicolumn{6}{|c|}{ Ocean }} \\
\hline & & & & & & & \\
\hline Source sector & Region & $\begin{array}{l}\text { North } \\
\text { Pacific }\end{array}$ & $\begin{array}{l}\text { South } \\
\text { Pacific }\end{array}$ & $\begin{array}{l}\text { Northern } \\
\text { Ocean }\end{array}$ & Atlantic & $\begin{array}{l}\text { Southern } \\
\text { Ocean }\end{array}$ & $\begin{array}{l}\text { Indian } \\
\text { Ocean }\end{array}$ \\
\hline \multirow{7}{*}{$\begin{array}{l}\text { Agricultural } \\
\text { Soil }\end{array}$} & Africa/Middle East & $-7.38 \mathrm{E}-03$ & $-9.45 \mathrm{E}-03$ & 6.07E-03 & $-6.84 \mathrm{E}-02$ & $3.46 \mathrm{E}-02$ & $-3.41 \mathrm{E}-02$ \\
\hline & Southern Asia & $-1.23 \mathrm{E}-01$ & $6.36 \mathrm{E}-03$ & $2.30 \mathrm{E}-03$ & $-1.09 \mathrm{E}-01$ & $1.35 \mathrm{E}-01$ & $-9.65 \mathrm{E}-02$ \\
\hline & Central/South America & $1.04 \mathrm{E}-02$ & $-1.98 \mathrm{E}-02$ & $1.32 \mathrm{E}-03$ & $-1.24 \mathrm{E}-02$ & $-1.47 \mathrm{E}-01$ & $-7.33 \mathrm{E}-02$ \\
\hline & \begin{tabular}{|l|} 
Northern Asia \\
\end{tabular} & $2.56 \mathrm{E}-02$ & $1.25 \mathrm{E}-02$ & $-5.88 \mathrm{E}-02$ & $-4.04 \mathrm{E}-02$ & $-2.49 \mathrm{E}-02$ & $2.16 \mathrm{E}-02$ \\
\hline & Europe & $4.58 \mathrm{E}-03$ & $1.61 \mathrm{E}-02$ & $\mid-7.41 \mathrm{E}-02$ & $-6.04 \mathrm{E}-02$ & $1.11 \mathrm{E}-02$ & $1.13 \mathrm{E}-03$ \\
\hline & North America & $-7.06 \mathrm{E}-02$ & $5.77 \mathrm{E}-03$ & $-1.38 \mathrm{E}-02$ & $-1.73 \mathrm{E}-01$ & $4.23 \mathrm{E}-02$ & $2.42 \mathrm{E}-02$ \\
\hline & Oceania & $1.19 \mathrm{E}-02$ & $1.51 \mathrm{E}-02$ & $3.12 \mathrm{E}-03$ & $1.74 \mathrm{E}-02$ & $-7.17 \mathrm{E}-02$ & $-2.98 \mathrm{E}-02$ \\
\hline \multirow{7}{*}{ Industrial } & Africa/Middle East & $-3.53 \mathrm{E}-03$ & $2.76 \mathrm{E}-04$ & $1.20 \mathrm{E}-03$ & $-9.81 \mathrm{E}-03$ & $4.39 \mathrm{E}-03$ & $-2.12 \mathrm{E}-03$ \\
\hline & Southern Asia & $-3.12 \mathrm{E}-02$ & $1.01 \mathrm{E}-02$ & $-7.39 \mathrm{E}-03$ & $-1.43 \mathrm{E}-02$ & $-2.00 \mathrm{E}-02$ & $2.66 \mathrm{E}-02$ \\
\hline & Central/South America & $9.57 \mathrm{E}-04$ & $-2.79 \mathrm{E}-03$ & $1.47 \mathrm{E}-04$ & $-1.20 \mathrm{E}-03$ & $-1.17 \mathrm{E}-02$ & $-7.20 \mathrm{E}-03$ \\
\hline & Northern Asia & $3.77 \mathrm{E}-02$ & $1.06 \mathrm{E}-02$ & $2.37 \mathrm{E}-02$ & $-1.58 \mathrm{E}-01$ & $-1.91 \mathrm{E}-02$ & $9.47 \mathrm{E}-03$ \\
\hline & Europe & $-1.46 \mathrm{E}-02$ & $7.66 \mathrm{E}-03$ & $2.10 \mathrm{E}-02$ & $-1.02 \mathrm{E}-02$ & $9.49 \mathrm{E}-04$ & $3.61 \mathrm{E}-03$ \\
\hline & North America & $-3.89 \mathrm{E}-02$ & $1.60 \mathrm{E}-04$ & $1.83 \mathrm{E}-02$ & $-7.89 \mathrm{E}-02$ & $6.68 \mathrm{E}-03$ & $-2.45 \mathrm{E}-03$ \\
\hline & Oceania & $9.23 \mathrm{E}-04$ & $1.75 \mathrm{E}-03$ & $2.49 \mathrm{E}-04$ & $1.26 \mathrm{E}-03$ & $-5.12 \mathrm{E}-03$ & $-2.63 \mathrm{E}-03$ \\
\hline \multirow{7}{*}{ Natural Soil } & Africa/Middle East & $8.67 \mathrm{E}-02$ & $-4.44 \mathrm{E}-02$ & $5.64 \mathrm{E}-02$ & $-7.98 \mathrm{E}-02$ & $1.00 \mathrm{E}-01$ & $-1.55 \mathrm{E}-01$ \\
\hline & Southern Asia & $1.37 \mathrm{E}-01$ & $-1.60 \mathrm{E}-02$ & $9.11 \mathrm{E}-02$ & $8.16 \mathrm{E}-02$ & $1.62 \mathrm{E}-01$ & $-9.91 \mathrm{E}-02$ \\
\hline & Central/South America & $6.66 \mathrm{E}-03$ & $-5.05 \mathrm{E}-01$ & $4.61 \mathrm{E}-02$ & $-4.77 \mathrm{E}-02$ & $-7.49 \mathrm{E}-02$ & $-2.19 \mathrm{E}-01$ \\
\hline & Northern Asia & $-2.81 \mathrm{E}-01$ & $7.81 \mathrm{E}-03$ & \begin{tabular}{|l|}
$-7.13 \mathrm{E}-02$ \\
\end{tabular} & $3.41 \mathrm{E}-02$ & $-5.09 \mathrm{E}-02$ & $3.10 \mathrm{E}-02$ \\
\hline & Europe & $4.24 \mathrm{E}-02$ & $1.01 \mathrm{E}-02$ & $1.66 \mathrm{E}-02$ & $3.92 \mathrm{E}-02$ & $1.16 \mathrm{E}-02$ & $8.93 E-03$ \\
\hline & North America & $8.02 \mathrm{E}-02$ & $3.95 \mathrm{E}-02$ & \begin{tabular}{|l|}
$-8.53 \mathrm{E}-04$ \\
\end{tabular} & $9.79 \mathrm{E}-02$ & $5.50 \mathrm{E}-02$ & $1.94 \mathrm{E}-02$ \\
\hline & Oceania & $1.04 \mathrm{E}-02$ & $2.92 \mathrm{E}-02$ & $3.84 \mathrm{E}-03$ & $1.24 \mathrm{E}-02$ & $-3.47 \mathrm{E}-02$ & $-3.40 \mathrm{E}-02$ \\
\hline \multirow{6}{*}{ Ocean } & North Pacific & 1 & $1.07 \mathrm{E}-02$ & \begin{tabular}{|l|}
$-7.68 \mathrm{E}-02$ \\
\end{tabular} & $-2.00 \mathrm{E}-01$ & $1.90 \mathrm{E}-02$ & $4.55 \mathrm{E}-02$ \\
\hline & South Pacific & $1.07 \mathrm{E}-02$ & 1 & $2.05 \mathrm{E}-02$ & $-4.47 \mathrm{E}-02$ & $3.58 \mathrm{E}-02$ & $1.47 \mathrm{E}-02$ \\
\hline & Northern Ocean & $-7.68 \mathrm{E}-02$ & $2.05 \mathrm{E}-02$ & 1 & $-3.11 \mathrm{E}-01$ & $9.33 \mathrm{E}-03$ & $1.24 \mathrm{E}-02$ \\
\hline & Atlantic & $-2.00 \mathrm{E}-01$ & $-4.47 \mathrm{E}-02$ & $-3.11 \mathrm{E}-01$ & 1 & $-7.18 \mathrm{E}-02$ & $-2.24 \mathrm{E}-02$ \\
\hline & Southern Ocean & $1.90 \mathrm{E}-02$ & $3.58 \mathrm{E}-02$ & $9.33 \mathrm{E}-03$ & $-7.18 \mathrm{E}-02$ & 1 & $-6.26 \mathrm{E}-01$ \\
\hline & Indian Ocean & $4.55 \mathrm{E}-02$ & $1.47 \mathrm{E}-02$ & $1.24 \mathrm{E}-02$ & $-2.24 \mathrm{E}-02$ & $-6.26 \mathrm{E}-01$ & \\
\hline \multirow{7}{*}{$\begin{array}{l}\text { Biomass } \\
\text { burning }\end{array}$} & Africa/Middle East & $1.29 \mathrm{E}-03$ & $-4.73 \mathrm{E}-02$ & $2.61 \mathrm{E}-03$ & $-5.71 \mathrm{E}-02$ & $4.74 \mathrm{E}-03$ & $-4.66 \mathrm{E}-02$ \\
\hline & Southern Asia & $-1.43 \mathrm{E}-02$ & $-2.99 \mathrm{E}-03$ & \begin{tabular}{|l|}
$-9.87 E-04$ \\
\end{tabular} & $-5.33 E-03$ & $3.74 \mathrm{E}-03$ & $1.77 \mathrm{E}-03$ \\
\hline & Central/South America & $3.76 \mathrm{E}-04$ & $-1.68 \mathrm{E}-02$ & $-5.59 \mathrm{E}-04$ & $-7.87 \mathrm{E}-03$ & $-1.44 \mathrm{E}-02$ & $-9.69 \mathrm{E}-03$ \\
\hline & Northern Asia & $-2.96 \mathrm{E}-02$ & $1.05 \mathrm{E}-02$ & \begin{tabular}{|l|}
$-1.04 \mathrm{E}-02$ \\
\end{tabular} & $1.08 \mathrm{E}-02$ & $1.03 \mathrm{E}-02$ & $8.29 \mathrm{E}-03$ \\
\hline & Europe & $7.42 \mathrm{E}-04$ & $1.72 \mathrm{E}-04$ & $1.18 \mathrm{E}-04$ & $-9.17 \mathrm{E}-05$ & $1.32 \mathrm{E}-04$ & $1.15 \mathrm{E}-04$ \\
\hline & North America & $-1.48 \mathrm{E}-02$ & $-3.64 \mathrm{E}-04$ & $1.51 \mathrm{E}-03$ & $9.55 \mathrm{E}-03$ & $4.51 \mathrm{E}-04$ & $1.34 \mathrm{E}-03$ \\
\hline & Oceania & $1.18 \mathrm{E}-03$ & $-8.09 \mathrm{E}-03$ & \begin{tabular}{|l|}
$-4.69 \mathrm{E}-04$ \\
\end{tabular} & $-4.55 \mathrm{E}-03$ & $-8.23 \mathrm{E}-03$ & $-1.45 \mathrm{E}-02$ \\
\hline
\end{tabular}

Fig. S2ab. Correlation of the emissions in 2000 for ocean. 


\begin{tabular}{|c|c|c|c|c|c|c|c|c|}
\hline \multirow[b]{3}{*}{ Source sector } & \multirow[b]{3}{*}{ Region } & \multirow{2}{*}{\multicolumn{7}{|c|}{ Biomass burning }} \\
\hline & & & & & & & & \\
\hline & & $\begin{array}{l}\text { Africa/M. } \\
\text { East }\end{array}$ & $\begin{array}{l}\text { Southern } \\
\text { Asia }\end{array}$ & $\begin{array}{l}\text { C. /S. } \\
\text { America }\end{array}$ & $\begin{array}{l}\text { Northern } \\
\text { Asia }\end{array}$ & Europe & $\begin{array}{l}\text { North } \\
\text { America }\end{array}$ & Oceania \\
\hline \multirow{7}{*}{$\begin{array}{l}\text { Agricultural } \\
\text { Soil }\end{array}$} & Africa/Middle East & $-2.36 \mathrm{E}-02$ & $-2.13 \mathrm{E}-03$ & $-1.76 \mathrm{E}-03$ & $-4.30 \mathrm{E}-04$ & $-4.20 \mathrm{E}-04$ & $6.63 \mathrm{E}-04$ & $-1.13 \mathrm{E}-03$ \\
\hline & Southern Asia & $-2.57 \mathrm{E}-02$ & $-1.21 \mathrm{E}-02$ & $6.40 \mathrm{E}-04$ & $-1.28 \mathrm{E}-02$ & $-5.16 \mathrm{E}-05$ & $1.73 \mathrm{E}-03$ & $2.52 \mathrm{E}-03$ \\
\hline & Central/South America & $-2.18 \mathrm{E}-02$ & $1.57 \mathrm{E}-03$ & $-5.03 \mathrm{E}-03$ & $3.26 \mathrm{E}-03$ & $3.27 \mathrm{E}-05$ & $4.64 \mathrm{E}-04$ & $-4.46 \mathrm{E}-03$ \\
\hline & Northern Asia & $6.94 \mathrm{E}-03$ & $2.89 \mathrm{E}-03$ & $6.19 \mathrm{E}-04$ & $2.56 \mathrm{E}-02$ & $9.74 \mathrm{E}-04$ & $-3.19 E-04$ & $-7.31 \mathrm{E}-04$ \\
\hline & \begin{tabular}{|l} 
Europe \\
\end{tabular} & $3.13 \mathrm{E}-03$ & $1.75 \mathrm{E}-03$ & $8.43 E-04$ & $6.91 \mathrm{E}-03$ & $3.34 \mathrm{E}-04$ & $1.05 \mathrm{E}-03$ & $5.59 \mathrm{E}-04$ \\
\hline & \begin{tabular}{|l} 
North America \\
\end{tabular} & $-2.24 \mathrm{E}-03$ & $-1.06 \mathrm{E}-03$ & $2.05 \mathrm{E}-03$ & $1.59 \mathrm{E}-03$ & $4.20 \mathrm{E}-04$ & $-2.00 \mathrm{E}-02$ & $2.43 \mathrm{E}-03$ \\
\hline & Oceania & $5.54 \mathrm{E}-03$ & $1.44 \mathrm{E}-03$ & $9.02 \mathrm{E}-04$ & $2.69 \mathrm{E}-03$ & $1.24 \mathrm{E}-05$ & $3.81 \mathrm{E}-04$ & $-1.59 \mathrm{E}-03$ \\
\hline \multirow{7}{*}{ Industrial } & Africa/Middle East & $-2.03 \mathrm{E}-03$ & $-3.87 \mathrm{E}-04$ & $-6.24 \mathrm{E}-06$ & $2.49 \mathrm{E}-04$ & $-5.99 \mathrm{E}-05$ & $7.93 \mathrm{E}-05$ & $6.12 \mathrm{E}-05$ \\
\hline & Southern Asia & $5.77 \mathrm{E}-03$ & $-3.62 \mathrm{E}-03$ & $9.10 \mathrm{E}-04$ & $-6.34 \mathrm{E}-03$ & $1.60 \mathrm{E}-05$ & $-1.33 \mathrm{E}-05$ & $8.22 \mathrm{E}-04$ \\
\hline & Central/South America & $-2.07 \mathrm{E}-03$ & $1.43 \mathrm{E}-04$ & $-4.77 \mathrm{E}-04$ & $2.71 \mathrm{E}-04$ & $2.12 \mathrm{E}-06$ & $3.52 \mathrm{E}-05$ & $-4.25 \mathrm{E}-04$ \\
\hline & Northern Asia & $8.87 \mathrm{E}-03$ & $2.21 \mathrm{E}-03$ & $2.99 \mathrm{E}-04$ & $3.36 \mathrm{E}-02$ & $5.16 \mathrm{E}-04$ & $1.83 \mathrm{E}-02$ & $-6.08 \mathrm{E}-04$ \\
\hline & Europe & $1.93 \mathrm{E}-03$ & $7.02 \mathrm{E}-04$ & $4.57 \mathrm{E}-04$ & $1.01 \mathrm{E}-03$ & $-1.38 \mathrm{E}-03$ & $2.91 \mathrm{E}-04$ & $2.67 \mathrm{E}-04$ \\
\hline & North America & $1.21 \mathrm{E}-03$ & $9.66 \mathrm{E}-05$ & $3.73 \mathrm{E}-04$ & $8.49 \mathrm{E}-03$ & $3.01 \mathrm{E}-04$ & $-3.02 \mathrm{E}-02$ & $3.61 \mathrm{E}-04$ \\
\hline & Oceania & $4.26 \mathrm{E}-04$ & $1.08 \mathrm{E}-04$ & $7.72 \mathrm{E}-05$ & $1.87 \mathrm{E}-04$ & $7.13 \mathrm{E}-07$ & $2.80 \mathrm{E}-05$ & $-1.21 \mathrm{E}-04$ \\
\hline \multirow{7}{*}{ Natural Soil } & Africa/Middle East & $-1.04 \mathrm{E}-01$ & $4.36 \mathrm{E}-03$ & $-1.31 \mathrm{E}-02$ & $2.83 \mathrm{E}-03$ & $-7.44 \mathrm{E}-04$ & $2.17 \mathrm{E}-03$ & $-1.07 \mathrm{E}-02$ \\
\hline & Southern Asia & $-7.49 \mathrm{E}-03$ & $6.08 \mathrm{E}-03$ & $-1.08 \mathrm{E}-03$ & $7.80 \mathrm{E}-02$ & $-6.99 \mathrm{E}-04$ & $6.21 \mathrm{E}-05$ & $-5.85 \mathrm{E}-03$ \\
\hline & Central/South America & $-5.90 \mathrm{E}-02$ & $-8.64 \mathrm{E}-03$ & $-9.74 \mathrm{E}-04$ & $-1.00 \mathrm{E}-04$ & 5.57E-05 & $-6.79 \mathrm{E}-03$ & $-3.14 \mathrm{E}-04$ \\
\hline & Northern Asia & $9.18 \mathrm{E}-03$ & $-1.65 \mathrm{E}-02$ & $2.27 \mathrm{E}-03$ & $-2.54 \mathrm{E}-01$ & $1.14 \mathrm{E}-04$ & $-5.21 \mathrm{E}-03$ & $2.47 \mathrm{E}-03$ \\
\hline & Europe & $1.07 \mathrm{E}-03$ & $-3.55 \mathrm{E}-03$ & $1.06 \mathrm{E}-03$ & $-1.33 \mathrm{E}-02$ & $-6.16 \mathrm{E}-03$ & $-1.85 \mathrm{E}-03$ & $1.50 \mathrm{E}-03$ \\
\hline & North America & $3.10 \mathrm{E}-03$ & $-3.79 \mathrm{E}-03$ & $2.49 \mathrm{E}-03$ & $-5.93 E-02$ & $-1.83 \mathrm{E}-03$ & $-9.91 \mathrm{E}-03$ & $3.13 \mathrm{E}-03$ \\
\hline & Oceania & $-4.00 \mathrm{E}-04$ & $7.06 \mathrm{E}-04$ & $-1.75 \mathrm{E}-04$ & $3.36 \mathrm{E}-03$ & $1.31 \mathrm{E}-05$ & $7.71 \mathrm{E}-05$ & $-4.64 \mathrm{E}-03$ \\
\hline \multirow{6}{*}{ Ocean } & North Pacific & $1.29 \mathrm{E}-03$ & $-1.43 \mathrm{E}-02$ & $3.76 \mathrm{E}-04$ & $-2.96 \mathrm{E}-02$ & $7.42 \mathrm{E}-04$ & $-1.48 \mathrm{E}-02$ & $1.18 \mathrm{E}-03$ \\
\hline & South Pacific & $-4.73 \mathrm{E}-02$ & $-2.99 \mathrm{E}-03$ & $-1.68 \mathrm{E}-02$ & $1.05 \mathrm{E}-02$ & $1.72 \mathrm{E}-04$ & $-3.64 \mathrm{E}-04$ & $-8.09 \mathrm{E}-03$ \\
\hline & Northern Ocean & $2.61 \mathrm{E}-03$ & $-9.87 \mathrm{E}-04$ & $-5.59 \mathrm{E}-04$ & $-1.04 \mathrm{E}-02$ & $1.18 \mathrm{E}-04$ & $1.51 \mathrm{E}-03$ & $-4.69 \mathrm{E}-04$ \\
\hline & Atlantic & $-5.71 \mathrm{E}-02$ & $-5.33 \mathrm{E}-03$ & $-7.87 \mathrm{E}-03$ & $1.08 \mathrm{E}-02$ & $-9.17 \mathrm{E}-05$ & $9.55 \mathrm{E}-03$ & $-4.55 \mathrm{E}-03$ \\
\hline & Southern Ocean & $4.74 \mathrm{E}-03$ & $3.74 \mathrm{E}-03$ & $-1.44 \mathrm{E}-02$ & $1.03 \mathrm{E}-02$ & $1.32 \mathrm{E}-04$ & $4.51 \mathrm{E}-04$ & $-8.23 \mathrm{E}-03$ \\
\hline & Indian Ocean & $-4.66 \mathrm{E}-02$ & $1.77 \mathrm{E}-03$ & $-9.69 \mathrm{E}-03$ & $8.29 \mathrm{E}-03$ & $1.15 \mathrm{E}-04$ & $1.34 \mathrm{E}-03$ & $-1.45 \mathrm{E}-02$ \\
\hline \multirow{7}{*}{$\begin{array}{l}\text { Biomass } \\
\text { burning }\end{array}$} & Africa/Middle East & 1 & $-5.41 \mathrm{E}-04$ & $-7.15 \mathrm{E}-03$ & $2.62 \mathrm{E}-03$ & $-3.51 \mathrm{E}-05$ & \begin{tabular}{|l|}
$7.82 \mathrm{E}-04$ \\
\end{tabular} & $-4.76 \mathrm{E}-03$ \\
\hline & Southern Asia & $-5.41 \mathrm{E}-04$ & 1 & $1.48 \mathrm{E}-04$ & $-3.52 \mathrm{E}-03$ & $-3.15 \mathrm{E}-05$ & \begin{tabular}{|l|}
$-4.81 \mathrm{E}-04$ \\
\end{tabular} & $2.79 \mathrm{E}-04$ \\
\hline & Central/South America & $-7.15 \mathrm{E}-03$ & $1.48 \mathrm{E}-04$ & 1 & $4.02 \mathrm{E}-04$ & $2.89 \mathrm{E}-06$ & $1.55 \mathrm{E}-04$ & $-1.76 \mathrm{E}-03$ \\
\hline & Northern Asia & $2.62 \mathrm{E}-03$ & $-3.52 \mathrm{E}-03$ & $4.02 \mathrm{E}-04$ & 1 & $-2.51 \mathrm{E}-04$ & $-3.62 \mathrm{E}-03$ & $7.43 \mathrm{E}-04$ \\
\hline & Europe & $-3.51 \mathrm{E}-05$ & $-3.15 \mathrm{E}-05$ & $2.89 \mathrm{E}-06$ & $-2.51 \mathrm{E}-04$ & 1 & $-1.35 \mathrm{E}-05$ & $1.02 \mathrm{E}-05$ \\
\hline & North America & $7.82 \mathrm{E}-04$ & $-4.81 \mathrm{E}-04$ & $1.55 \mathrm{E}-04$ & $-3.62 \mathrm{E}-03$ & $-1.35 \mathrm{E}-05$ & 1 & $1.57 \mathrm{E}-04$ \\
\hline & Oceania & $-4.76 \mathrm{E}-03$ & $2.79 \mathrm{E}-04$ & $-1.76 \mathrm{E}-03$ & $7.43 \mathrm{E}-04$ & $1.02 \mathrm{E}-05$ & \begin{tabular}{|l|l|} 
& $1.57 \mathrm{E}-04$ \\
\end{tabular} & 1 \\
\hline
\end{tabular}

Fig. S2ac. Correlation of the emissions in 2000 for biomass burning. 


\begin{tabular}{|c|c|c|c|c|c|c|c|c|}
\hline \multirow[b]{2}{*}{ Source sector } & \multirow[b]{2}{*}{ Region } & \multicolumn{7}{|c|}{ Agricultural Soil } \\
\hline & & $\begin{array}{l}\text { Africa/M. } \\
\text { East }\end{array}$ & $\begin{array}{l}\text { Southern } \\
\text { Asia }\end{array}$ & $\begin{array}{l}\text { C. /S. } \\
\text { America }\end{array}$ & $\begin{array}{l}\text { Northern } \\
\text { Asia }\end{array}$ & Europe & $\begin{array}{l}\text { North } \\
\text { America }\end{array}$ & Oceania \\
\hline \multirow{7}{*}{$\begin{array}{c}\text { Agricultural } \\
\text { Soil }\end{array}$} & Africa/Middle East & 1 & $-4.87 \mathrm{E}-02$ & $-5.23 \mathrm{E}-03$ & $-4.11 \mathrm{E}-02$ & $-1.32 \mathrm{E}-02$ & $-2.64 \mathrm{E}-02$ & $6.99 \mathrm{E}-03$ \\
\hline & Southern Asia & $-4.87 \mathrm{E}-02$ & 1 & $2.91 \mathrm{E}-02$ & $-2.00 \mathrm{E}-01$ & $3.30 \mathrm{E}-02$ & $-4.99 \mathrm{E}-02$ & $1.45 \mathrm{E}-02$ \\
\hline & Central/South America & $-5.23 E-03$ & $2.91 \mathrm{E}-02$ & 1 & $-1.24 \mathrm{E}-03$ & $4.60 \mathrm{E}-03$ & $9.56 \mathrm{E}-03$ & $-1.80 \mathrm{E}-02$ \\
\hline & Northern Asia & $-4.11 \mathrm{E}-02$ & $-2.00 \mathrm{E}-01$ & $-1.24 \mathrm{E}-03$ & 1 & $-1.71 \mathrm{E}-01$ & $-4.08 \mathrm{E}-02$ & $-1.75 \mathrm{E}-02$ \\
\hline & Europe & $-1.32 \mathrm{E}-02$ & $3.30 \mathrm{E}-02$ & $4.60 \mathrm{E}-03$ & $-1.71 \mathrm{E}-01$ & 1 & $-1.14 \mathrm{E}-02$ & $2.26 \mathrm{E}-03$ \\
\hline & North America & $-2.64 \mathrm{E}-02$ & $-4.99 \mathrm{E}-02$ & $9.56 \mathrm{E}-03$ & $-4.08 \mathrm{E}-02$ & $-1.14 \mathrm{E}-02$ & 1 & $3.00 \mathrm{E}-03$ \\
\hline & Oceania & $6.99 \mathrm{E}-03$ & $1.45 \mathrm{E}-02$ & $-1.80 \mathrm{E}-02$ & $-1.75 \mathrm{E}-02$ & $2.26 \mathrm{E}-03$ & $3.00 \mathrm{E}-03$ & 1 \\
\hline \multirow{7}{*}{ Industrial } & Africa/Middle East & $-6.51 \mathrm{E}-02$ & $-7.78 \mathrm{E}-03$ & $3.62 \mathrm{E}-04$ & $-1.04 \mathrm{E}-02$ & $-6.58 \mathrm{E}-03$ & $-5.73 \mathrm{E}-03$ & $9.35 \mathrm{E}-04$ \\
\hline & \begin{tabular}{|l} 
Southern Asia \\
\end{tabular} & $1.74 \mathrm{E}-02$ & $-3.72 \mathrm{E}-01$ & $4.52 \mathrm{E}-03$ & $-5.54 \mathrm{E}-02$ & $2.08 \mathrm{E}-02$ & $6.80 \mathrm{E}-03$ & $-2.79 \mathrm{E}-03$ \\
\hline & Central/South America & $-6.20 \mathrm{E}-04$ & $2.43 \mathrm{E}-03$ & $-4.59 \mathrm{E}-03$ & $-3.40 \mathrm{E}-05$ & $4.19 \mathrm{E}-04$ & $7.86 \mathrm{E}-04$ & $-1.46 \mathrm{E}-03$ \\
\hline & Northern Asia & $1.31 \mathrm{E}-02$ & $-3.14 \mathrm{E}-02$ & $-9.54 \mathrm{E}-04$ & $-1.29 \mathrm{E}-01$ & $-3.50 \mathrm{E}-02$ & 7.57E-02 & $-5.84 \mathrm{E}-03$ \\
\hline & Europe & $-7.24 \mathrm{E}-03$ & $3.93 \mathrm{E}-03$ & $1.52 \mathrm{E}-03$ & $-2.92 \mathrm{E}-02$ & $-7.04 \mathrm{E}-01$ & $-1.02 \mathrm{E}-02$ & $-1.42 \mathrm{E}-04$ \\
\hline & North America & $8.51 \mathrm{E}-03$ & $1.88 \mathrm{E}-02$ & $1.81 \mathrm{E}-03$ & $4.35 \mathrm{E}-02$ & $2.43 \mathrm{E}-02$ & $-3.28 \mathrm{E}-01$ & $-9.23 \mathrm{E}-04$ \\
\hline & Oceania & $4.99 \mathrm{E}-04$ & $1.03 \mathrm{E}-03$ & $-1.17 \mathrm{E}-03$ & $-1.28 \mathrm{E}-03$ & $1.65 \mathrm{E}-04$ & $1.77 \mathrm{E}-04$ & $-6.52 \mathrm{E}-03$ \\
\hline \multirow{7}{*}{ Natural Soil } & Africa/Middle East & $-1.58 \mathrm{E}-01$ & $-3.39 \mathrm{E}-03$ & $-6.81 \mathrm{E}-02$ & $8.62 \mathrm{E}-03$ & $-4.32 \mathrm{E}-03$ & $1.28 \mathrm{E}-03$ & $2.22 \mathrm{E}-03$ \\
\hline & Southern Asia & $-8.46 \mathrm{E}-02$ & $-4.07 \mathrm{E}-01$ & $-1.59 \mathrm{E}-02$ & $-4.69 \mathrm{E}-02$ & $-1.39 \mathrm{E}-02$ & $-4.37 \mathrm{E}-02$ & $3.53 \mathrm{E}-02$ \\
\hline & Central/South America & $-2.31 \mathrm{E}-02$ & $6.65 \mathrm{E}-02$ & $-1.89 \mathrm{E}-01$ & $2.03 \mathrm{E}-03$ & $2.17 \mathrm{E}-02$ & $2.12 \mathrm{E}-02$ & $-8.07 \mathrm{E}-02$ \\
\hline & Northern Asia & $2.64 \mathrm{E}-02$ & $-4.10 \mathrm{E}-02$ & $1.16 \mathrm{E}-02$ & $-1.70 \mathrm{E}-01$ & $3.27 \mathrm{E}-02$ & $6.89 \mathrm{E}-02$ & $-6.87 \mathrm{E}-03$ \\
\hline & Europe & $-2.26 \mathrm{E}-02$ & $-4.94 \mathrm{E}-03$ & $4.15 \mathrm{E}-03$ & $-1.87 \mathrm{E}-02$ & $-2.69 \mathrm{E}-01$ & $1.74 \mathrm{E}-02$ & $4.23 \mathrm{E}-03$ \\
\hline & North America & $-3.29 \mathrm{E}-02$ & $-1.09 \mathrm{E}-02$ & $1.38 \mathrm{E}-02$ & $4.17 \mathrm{E}-02$ & $-2.34 \mathrm{E}-02$ & $-4.39 \mathrm{E}-01$ & $1.22 \mathrm{E}-02$ \\
\hline & Oceania & $3.65 \mathrm{E}-03$ & $2.02 \mathrm{E}-02$ & $-2.14 \mathrm{E}-02$ & $-1.19 \mathrm{E}-02$ & $4.39 \mathrm{E}-03$ & $6.58 \mathrm{E}-03$ & $-6.07 \mathrm{E}-02$ \\
\hline \multirow{6}{*}{ Ocean } & North Pacific & $-4.62 \mathrm{E}-03$ & $-1.63 \mathrm{E}-01$ & $8.02 \mathrm{E}-03$ & $-2.42 \mathrm{E}-02$ & $6.51 \mathrm{E}-03$ & $-5.39 \mathrm{E}-02$ & $8.08 \mathrm{E}-03$ \\
\hline & South Pacific & $-3.43 \mathrm{E}-02$ & $2.75 \mathrm{E}-03$ & $-3.51 \mathrm{E}-02$ & $1.86 \mathrm{E}-02$ & $1.46 \mathrm{E}-02$ & $2.04 \mathrm{E}-03$ & $1.01 \mathrm{E}-02$ \\
\hline & Northern Ocean & $7.38 \mathrm{E}-04$ & $-2.84 \mathrm{E}-02$ & $1.22 \mathrm{E}-03$ & $-6.97 \mathrm{E}-02$ & $-8.33 \mathrm{E}-02$ & $-1.23 \mathrm{E}-04$ & $2.78 \mathrm{E}-03$ \\
\hline & Atlantic & $-8.37 \mathrm{E}-02$ & $-1.15 \mathrm{E}-01$ & $-2.12 \mathrm{E}-02$ & $-5.35 \mathrm{E}-02$ & $-4.99 \mathrm{E}-02$ & $-1.80 \mathrm{E}-01$ & $1.64 \mathrm{E}-02$ \\
\hline & Southern Ocean & $2.10 \mathrm{E}-02$ & $7.49 \mathrm{E}-02$ & $-1.53 \mathrm{E}-01$ & $-2.59 \mathrm{E}-02$ & $6.19 \mathrm{E}-03$ & $2.45 \mathrm{E}-02$ & $-5.27 \mathrm{E}-02$ \\
\hline & Indian Ocean & $-2.91 \mathrm{E}-02$ & $-4.98 \mathrm{E}-02$ & $-7.96 \mathrm{E}-02$ & $1.90 \mathrm{E}-02$ & $1.62 \mathrm{E}-03$ & $2.00 \mathrm{E}-02$ & $-2.66 \mathrm{E}-02$ \\
\hline \multirow{7}{*}{$\begin{array}{l}\text { Biomass } \\
\text { burning }\end{array}$} & Africa/Middle East & $-2.19 \mathrm{E}-02$ & $-2.35 \mathrm{E}-03$ & $-2.60 \mathrm{E}-02$ & $8.63 \mathrm{E}-03$ & $2.32 \mathrm{E}-03$ & $3.73 \mathrm{E}-03$ & $3.03 E-03$ \\
\hline & Southern Asia & $-7.75 \mathrm{E}-04$ & $-1.77 \mathrm{E}-02$ & $-4.88 \mathrm{E}-04$ & $-7.61 \mathrm{E}-03$ & $2.02 \mathrm{E}-03$ & $-1.12 \mathrm{E}-03$ & $9.99 \mathrm{E}-04$ \\
\hline & Central/South America & $-1.69 \mathrm{E}-03$ & $1.92 \mathrm{E}-03$ & $-4.62 \mathrm{E}-03$ & $3.57 \mathrm{E}-04$ & $4.67 \mathrm{E}-04$ & $7.83 \mathrm{E}-04$ & $-4.52 \mathrm{E}-04$ \\
\hline & \begin{tabular}{|l} 
Northern Asia \\
\end{tabular} & $3.11 \mathrm{E}-03$ & $-4.37 \mathrm{E}-03$ & $3.09 \mathrm{E}-04$ & $-2.45 \mathrm{E}-03$ & $2.18 \mathrm{E}-03$ & $1.43 \mathrm{E}-02$ & $5.36 \mathrm{E}-04$ \\
\hline & Europe & $-8.51 \mathrm{E}-04$ & $1.62 \mathrm{E}-04$ & $-2.85 \mathrm{E}-05$ & $2.67 \mathrm{E}-04$ & $8.98 \mathrm{E}-04$ & $4.18 \mathrm{E}-04$ & $2.89 \mathrm{E}-05$ \\
\hline & North America & $-6.58 \mathrm{E}-05$ & $-2.60 \mathrm{E}-04$ & $1.23 \mathrm{E}-04$ & $8.45 \mathrm{E}-04$ & $1.12 \mathrm{E}-04$ & $-6.92 \mathrm{E}-04$ & $1.91 \mathrm{E}-04$ \\
\hline & Oceania & $-9.79 E-04$ & $7.84 \mathrm{E}-03$ & $-9.02 \mathrm{E}-03$ & $-8.44 \mathrm{E}-04$ & $1.10 \mathrm{E}-03$ & $3.25 \mathrm{E}-03$ & $-5.59 \mathrm{E}-03$ \\
\hline
\end{tabular}

Fig. S2ad. Correlation of the emissions in 2001 for agricultural soil. 


\begin{tabular}{|c|c|c|c|c|c|c|c|c|}
\hline & \multirow{2}{*}{\multicolumn{7}{|c|}{ Industrial }} \\
\hline & & & & & & & & \\
\hline Source sector & Region & $\begin{array}{l}\text { Africa/M. } \\
\text { East }\end{array}$ & $\begin{array}{l}\text { Southern } \\
\text { Asia }\end{array}$ & $\begin{array}{l}\text { C. /S. } \\
\text { America }\end{array}$ & $\begin{array}{l}\text { Northern } \\
\text { Asia }\end{array}$ & Europe & $\begin{array}{l}\text { North } \\
\text { America }\end{array}$ & Oceania \\
\hline \multirow{7}{*}{$\begin{array}{l}\text { Agricultural } \\
\text { Soil }\end{array}$} & Africa/Middle East & $-6.51 \mathrm{E}-02$ & $1.74 \mathrm{E}-02$ & $-6.20 \mathrm{E}-04$ & $1.31 \mathrm{E}-02$ & $-7.24 \mathrm{E}-03$ & $8.51 \mathrm{E}-03$ & $4.99 \mathrm{E}-04$ \\
\hline & Southern Asia & $-7.78 \mathrm{E}-03$ & $-3.72 \mathrm{E}-01$ & $2.43 \mathrm{E}-03$ & \begin{tabular}{|l|} 
\\
\end{tabular} & $3.93 \mathrm{E}-03$ & $1.88 \mathrm{E}-02$ & $1.03 \mathrm{E}-03$ \\
\hline & Central/South America & $3.62 \mathrm{E}-04$ & $4.52 \mathrm{E}-03$ & $-4.59 \mathrm{E}-03$ & \begin{tabular}{|l|} 
\\
\end{tabular} & $1.52 \mathrm{E}-03$ & $1.81 \mathrm{E}-03$ & $-1.17 \mathrm{E}-03$ \\
\hline & Northern Asia & $-1.04 \mathrm{E}-02$ & $-5.54 \mathrm{E}-02$ & $-3.40 \mathrm{E}-05$ & \begin{tabular}{|l|} 
\\
\end{tabular} & $-2.92 \mathrm{E}-02$ & $4.35 \mathrm{E}-02$ & $-1.28 \mathrm{E}-03$ \\
\hline & Europe & $-6.58 \mathrm{E}-03$ & $2.08 \mathrm{E}-02$ & $4.19 \mathrm{E}-04$ & $-3.50 \mathrm{E}-02$ & $-7.04 \mathrm{E}-01$ & $2.43 \mathrm{E}-02$ & $1.65 \mathrm{E}-04$ \\
\hline & \begin{tabular}{|l|} 
North America \\
\end{tabular} & $-5.73 \mathrm{E}-03$ & $6.80 \mathrm{E}-03$ & $7.86 \mathrm{E}-04$ & $7.57 \mathrm{E}-02$ & $-1.02 \mathrm{E}-02$ & $-3.28 \mathrm{E}-01$ & $1.77 \mathrm{E}-04$ \\
\hline & Oceania & $9.35 \mathrm{E}-04$ & $-2.79 \mathrm{E}-03$ & $-1.46 \mathrm{E}-03$ & 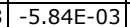 & $-1.42 \mathrm{E}-04$ & $-9.23 \mathrm{E}-04$ & $-6.52 \mathrm{E}-03$ \\
\hline \multirow{7}{*}{ Industrial } & Africa/Middle East & 1 & $2.24 \mathrm{E}-03$ & $1.07 \mathrm{E}-05$ & 2.37E-05 & $-2.41 \mathrm{E}-03$ & $1.66 \mathrm{E}-03$ & $6.52 \mathrm{E}-05$ \\
\hline & \begin{tabular}{|l|} 
Southern Asia \\
\end{tabular} & $2.24 \mathrm{E}-03$ & 1 & $4.31 \mathrm{E}-04$ & $-5.16 \mathrm{E}-02$ & $2.67 \mathrm{E}-03$ & $1.47 \mathrm{E}-02$ & $-1.84 \mathrm{E}-04$ \\
\hline & Central/South America & $1.07 \mathrm{E}-05$ & $4.31 \mathrm{E}-04$ & 1 & $4.76 \mathrm{E}-06$ & $1.32 \mathrm{E}-04$ & $1.80 \mathrm{E}-04$ & $-9.56 \mathrm{E}-05$ \\
\hline & Northern Asia & $2.37 \mathrm{E}-05$ & $-5.16 \mathrm{E}-02$ & $4.76 \mathrm{E}-06$ & 1 & $-1.77 \mathrm{E}-02$ & $-2.77 \mathrm{E}-01$ & $-3.93 \mathrm{E}-04$ \\
\hline & Europe & $-2.41 \mathrm{E}-03$ & $2.67 \mathrm{E}-03$ & $1.32 \mathrm{E}-04$ & $-1.77 \mathrm{E}-02$ & 1 & $4.85 \mathrm{E}-03$ & $-9.44 \mathrm{E}-06$ \\
\hline & North America & $1.66 \mathrm{E}-03$ & $1.47 \mathrm{E}-02$ & $1.80 \mathrm{E}-04$ & $-2.77 \mathrm{E}-01$ & 4.85E-03 & 1 & $-6.85 \mathrm{E}-05$ \\
\hline & Oceania & $6.52 \mathrm{E}-05$ & $-1.84 \mathrm{E}-04$ & $-9.56 \mathrm{E}-05$ & $-3.93 E-04$ & $-9.44 \mathrm{E}-06$ & $-6.85 \mathrm{E}-05$ & 1 \\
\hline \multirow{7}{*}{ Natural Soil } & Africa/Middle East & $-2.65 \mathrm{E}-02$ & $5.16 \mathrm{E}-02$ & $-6.26 \mathrm{E}-03$ & \begin{tabular}{|l|} 
\\
\end{tabular} & $-4.59 \mathrm{E}-04$ & $1.40 \mathrm{E}-02$ & $2.55 \mathrm{E}-04$ \\
\hline & Southern Asia & $-9.57 \mathrm{E}-03$ & $-1.34 \mathrm{E}-01$ & $-1.68 \mathrm{E}-03$ & \begin{tabular}{|l|}
$2.16 \mathrm{E}-02$ \\
\end{tabular} & $-1.74 \mathrm{E}-02$ & $9.76 \mathrm{E}-03$ & $2.59 \mathrm{E}-03$ \\
\hline & Central/South America & $1.48 \mathrm{E}-03$ & $5.39 \mathrm{E}-03$ & $-1.60 \mathrm{E}-02$ & $1.43 \mathrm{E}-02$ & $7.55 \mathrm{E}-03$ & $2.92 \mathrm{E}-03$ & $-5.40 \mathrm{E}-03$ \\
\hline & Northern Asia & $6.30 \mathrm{E}-03$ & $-2.94 \mathrm{E}-02$ & 9.09E-04 & $-1.67 \mathrm{E}-02$ & $3.47 \mathrm{E}-02$ & $4.36 \mathrm{E}-02$ & $-6.50 \mathrm{E}-04$ \\
\hline & Europe & $-8.36 \mathrm{E}-03$ & $7.63 E-03$ & 3.07E-04 & $2.20 \mathrm{E}-02$ & $-1.83 \mathrm{E}-01$ & $2.07 \mathrm{E}-02$ & $3.20 \mathrm{E}-04$ \\
\hline & North America & $-2.02 \mathrm{E}-03$ & $6.86 \mathrm{E}-02$ & $9.67 \mathrm{E}-04$ & $-1.72 \mathrm{E}-01$ & $1.21 \mathrm{E}-02$ & $-1.82 \mathrm{E}-01$ & $8.11 \mathrm{E}-04$ \\
\hline & Oceania & 7.92E-04 & $-9.97 \mathrm{E}-04$ & $-1.85 \mathrm{E}-03$ & \begin{tabular}{|l|}
$-6.29 E-03$ \\
\end{tabular} & $8.18 \mathrm{E}-04$ & $-2.47 \mathrm{E}-04$ & $-4.67 \mathrm{E}-03$ \\
\hline \multirow{6}{*}{ Ocean } & North Pacific & $-1.82 \mathrm{E}-03$ & $-5.34 \mathrm{E}-02$ & $5.74 \mathrm{E}-04$ & $2.40 \mathrm{E}-02$ & $-1.32 \mathrm{E}-02$ & $-2.27 \mathrm{E}-02$ & $5.85 \mathrm{E}-04$ \\
\hline & \begin{tabular}{|l} 
South Pacific \\
\end{tabular} & $-2.77 \mathrm{E}-03$ & $2.40 \mathrm{E}-02$ & $-4.44 \mathrm{E}-03$ & \begin{tabular}{|c|} 
\\
\end{tabular} & $6.44 \mathrm{E}-03$ & $9.83 \mathrm{E}-04$ & $1.25 \mathrm{E}-03$ \\
\hline & Northern Ocean & $-4.93 \mathrm{E}-04$ & $-1.27 \mathrm{E}-02$ & $9.82 \mathrm{E}-05$ & $\begin{array}{ll}1.42 \mathrm{E}-02 \\
\end{array}$ & $1.93 \mathrm{E}-02$ & $2.01 \mathrm{E}-02$ & $2.53 \mathrm{E}-04$ \\
\hline & Atlantic & $-1.39 \mathrm{E}-02$ & $-2.10 \mathrm{E}-02$ & $-2.19 \mathrm{E}-03$ & \begin{tabular}{|l|l|} 
& $-1.66 \mathrm{E}-01$ \\
\end{tabular} & $-1.45 \mathrm{E}-02$ & $-9.08 \mathrm{E}-02$ & $1.17 \mathrm{E}-03$ \\
\hline & Southern Ocean & $2.28 \mathrm{E}-03$ & $-2.58 \mathrm{E}-02$ & $-1.20 \mathrm{E}-02$ & $-1.23 \mathrm{E}-02$ & $-7.27 E-04$ & $7.20 \mathrm{E}-03$ & $-3.76 \mathrm{E}-03$ \\
\hline & Indian Ocean & $-2.30 \mathrm{E}-03$ & $2.56 \mathrm{E}-02$ & $-7.38 \mathrm{E}-03$ & \begin{tabular}{|l|l|} 
& $9.67 \mathrm{E}-04$ \\
\end{tabular} & $2.41 \mathrm{E}-03$ & $-2.79 \mathrm{E}-03$ & $-2.54 \mathrm{E}-03$ \\
\hline \multirow{7}{*}{$\begin{array}{l}\text { Biomass } \\
\text { burning }\end{array}$} & Africa/Middle East & $-1.96 \mathrm{E}-03$ & $1.35 \mathrm{E}-02$ & $-2.40 \mathrm{E}-03$ & \begin{tabular}{|l|}
$7.78 \mathrm{E}-03$ \\
\end{tabular} & $1.81 \mathrm{E}-03$ & $2.31 \mathrm{E}-03$ & $2.36 \mathrm{E}-04$ \\
\hline & Southern Asia & $-5.24 \mathrm{E}-05$ & $-3.57 \mathrm{E}-03$ & $-5.57 \mathrm{E}-05$ & $-2.15 \mathrm{E}-03$ & $3.21 \mathrm{E}-04$ & $7.65 \mathrm{E}-04$ & $7.75 \mathrm{E}-05$ \\
\hline & Central/South America & $-1.34 \mathrm{E}-04$ & $1.11 \mathrm{E}-03$ & $-4.23 \mathrm{E}-04$ & $\begin{array}{l}4.57 \mathrm{E}-04 \\
\end{array}$ & $2.06 \mathrm{E}-04$ & $2.70 \mathrm{E}-04$ & $-2.63 \mathrm{E}-05$ \\
\hline & Northern Asia & $5.60 \mathrm{E}-04$ & $-6.67 \mathrm{E}-03$ & $2.84 \mathrm{E}-05$ & $\begin{array}{l}1.11 \mathrm{E}-02 \\
\end{array}$ & $1.52 \mathrm{E}-03$ & $7.51 \mathrm{E}-03$ & $4.93 \mathrm{E}-05$ \\
\hline & Europe & $-2.38 \mathrm{E}-04$ & $6.21 \mathrm{E}-05$ & $-3.10 \mathrm{E}-06$ & $-3.41 E-05$ & $6.67 E-04$ & $1.54 \mathrm{E}-04$ & $2.70 \mathrm{E}-06$ \\
\hline & North America & $1.32 \mathrm{E}-05$ & $3.07 E-04$ & $8.02 \mathrm{E}-06$ & \begin{tabular}{|l|}
$7.25 \mathrm{E}-04$ \\
\end{tabular} & $4.44 \mathrm{E}-04$ & $3.62 \mathrm{E}-05$ & $1.46 \mathrm{E}-05$ \\
\hline & Oceania & $4.64 \mathrm{E}-05$ & $1.55 \mathrm{E}-03$ & $-8.10 \mathrm{E}-04$ & $-1.62 \mathrm{E}-03$ & $4.17 \mathrm{E}-04$ & $2.20 \mathrm{E}-04$ & $-4.15 \mathrm{E}-04$ \\
\hline
\end{tabular}

Fig. S2ae. Correlation of the emissions in 2001 for industrial. 


\begin{tabular}{|c|c|c|c|c|c|c|c|c|}
\hline \multirow[b]{3}{*}{ Source sector } & \multirow[b]{3}{*}{ Region } & \multirow{2}{*}{\multicolumn{7}{|c|}{ Natural Soil }} \\
\hline & & & & & & & & \\
\hline & & $\begin{array}{l}\text { Africa/M. } \\
\text { East }\end{array}$ & $\begin{array}{l}\text { Southern } \\
\text { Asia }\end{array}$ & $\begin{array}{l}\text { C. /S. } \\
\text { America }\end{array}$ & $\begin{array}{l}\text { Northern } \\
\text { Asia }\end{array}$ & Europe & $\begin{array}{l}\text { North } \\
\text { America }\end{array}$ & Oceania \\
\hline \multirow{7}{*}{$\begin{array}{l}\text { Agricultural } \\
\text { Soil }\end{array}$} & Africa/Middle East & $-1.58 \mathrm{E}-01$ & $-8.46 \mathrm{E}-02$ & $-2.31 \mathrm{E}-02$ & $2.64 \mathrm{E}-02$ & $-2.26 \mathrm{E}-02$ & $-3.29 \mathrm{E}-02$ & $3.65 \mathrm{E}-03$ \\
\hline & Southern Asia & $-3.39 \mathrm{E}-03$ & $-4.07 \mathrm{E}-01$ & $6.65 \mathrm{E}-02$ & $-4.10 \mathrm{E}-02$ & $-4.94 \mathrm{E}-03$ & $-1.09 \mathrm{E}-02$ & $2.02 \mathrm{E}-02$ \\
\hline & Central/South America & $-6.81 \mathrm{E}-02$ & $-1.59 \mathrm{E}-02$ & $-1.89 \mathrm{E}-01$ & $1.16 \mathrm{E}-02$ & $4.15 \mathrm{E}-03$ & $1.38 \mathrm{E}-02$ & $-2.14 \mathrm{E}-02$ \\
\hline & Northern Asia & $8.62 \mathrm{E}-03$ & $-4.69 \mathrm{E}-02$ & $2.03 \mathrm{E}-03$ & $-1.70 \mathrm{E}-01$ & $-1.87 \mathrm{E}-02$ & 4.17E-02 & $-1.19 \mathrm{E}-02$ \\
\hline & \begin{tabular}{|l|} 
Europe \\
\end{tabular} & $-4.32 \mathrm{E}-03$ & $-1.39 \mathrm{E}-02$ & $2.17 \mathrm{E}-02$ & $3.27 \mathrm{E}-02$ & $-2.69 \mathrm{E}-01$ & $-2.34 \mathrm{E}-02$ & $4.39 \mathrm{E}-03$ \\
\hline & \begin{tabular}{|l|} 
North America \\
\end{tabular} & $1.28 \mathrm{E}-03$ & $-4.37 \mathrm{E}-02$ & $2.12 \mathrm{E}-02$ & $6.89 \mathrm{E}-02$ & $1.74 \mathrm{E}-02$ & $-4.39 \mathrm{E}-01$ & $6.58 \mathrm{E}-03$ \\
\hline & Oceania & $2.22 \mathrm{E}-03$ & $3.53 \mathrm{E}-02$ & $-8.07 \mathrm{E}-02$ & $-6.87 \mathrm{E}-03$ & $4.23 \mathrm{E}-03$ & $1.22 \mathrm{E}-02$ & $-6.07 \mathrm{E}-02$ \\
\hline \multirow{7}{*}{ Industrial } & Africa/Middle East & $-2.65 \mathrm{E}-02$ & $-9.57 \mathrm{E}-03$ & $1.48 \mathrm{E}-03$ & $6.30 \mathrm{E}-03$ & $-8.36 \mathrm{E}-03$ & $-2.02 \mathrm{E}-03$ & $7.92 \mathrm{E}-04$ \\
\hline & \begin{tabular}{|l|} 
Southern Asia \\
\end{tabular} & $5.16 \mathrm{E}-02$ & $-1.34 \mathrm{E}-01$ & $5.39 \mathrm{E}-03$ & $-2.94 \mathrm{E}-02$ & $7.63 \mathrm{E}-03$ & $6.86 \mathrm{E}-02$ & $-9.97 \mathrm{E}-04$ \\
\hline & Central/South America & $-6.26 \mathrm{E}-03$ & $-1.68 \mathrm{E}-03$ & $-1.60 \mathrm{E}-02$ & $9.09 \mathrm{E}-04$ & $3.07 E-04$ & $9.67 \mathrm{E}-04$ & $-1.85 \mathrm{E}-03$ \\
\hline & Northern Asia & $4.24 \mathrm{E}-02$ & $2.16 \mathrm{E}-02$ & $1.43 \mathrm{E}-02$ & $-1.67 \mathrm{E}-02$ & $2.20 \mathrm{E}-02$ & $-1.72 \mathrm{E}-01$ & $-6.29 \mathrm{E}-03$ \\
\hline & Europe & $-4.59 \mathrm{E}-04$ & $-1.74 \mathrm{E}-02$ & $7.55 \mathrm{E}-03$ & $3.47 \mathrm{E}-02$ & $-1.83 \mathrm{E}-01$ & $1.21 \mathrm{E}-02$ & $8.18 \mathrm{E}-04$ \\
\hline & North America & $1.40 \mathrm{E}-02$ & $9.76 \mathrm{E}-03$ & $2.92 \mathrm{E}-03$ & $4.36 \mathrm{E}-02$ & 2.07E-02 & $-1.82 \mathrm{E}-01$ & $-2.47 \mathrm{E}-04$ \\
\hline & Oceania & $2.55 \mathrm{E}-04$ & $2.59 \mathrm{E}-03$ & $-5.40 \mathrm{E}-03$ & $-6.50 \mathrm{E}-04$ & $3.20 \mathrm{E}-04$ & $8.11 \mathrm{E}-04$ & $-4.67 \mathrm{E}-03$ \\
\hline \multirow{7}{*}{ Natural Soil } & Africa/Middle East & 1 & $-2.79 \mathrm{E}-01$ & $-3.37 \mathrm{E}-01$ & $6.95 \mathrm{E}-02$ & $-1.57 \mathrm{E}-02$ & $-8.76 \mathrm{E}-02$ & $-1.21 \mathrm{E}-02$ \\
\hline & Southern Asia & $-2.79 \mathrm{E}-01$ & 1 & $-9.00 \mathrm{E}-02$ & $-1.96 \mathrm{E}-01$ & $4.48 \mathrm{E}-02$ & $-1.21 \mathrm{E}-01$ & $1.04 \mathrm{E}-02$ \\
\hline & Central/South America & $-3.37 \mathrm{E}-01$ & $-9.00 \mathrm{E}-02$ & 1 & $-9.13 \mathrm{E}-02$ & $-4.38 \mathrm{E}-03$ & $-2.66 \mathrm{E}-02$ & $-1.08 \mathrm{E}-01$ \\
\hline & Northern Asia & $6.95 \mathrm{E}-02$ & $-1.96 \mathrm{E}-01$ & $-9.13 E-02$ & 1 & $-1.88 \mathrm{E}-01$ & $-3.23 \mathrm{E}-01$ & $-1.69 \mathrm{E}-02$ \\
\hline & Europe & $-1.57 \mathrm{E}-02$ & $4.48 \mathrm{E}-02$ & $-4.38 \mathrm{E}-03$ & $-1.88 \mathrm{E}-01$ & 1 & $-1.29 \mathrm{E}-01$ & $3.34 \mathrm{E}-03$ \\
\hline & North America & $-8.76 \mathrm{E}-02$ & $-1.21 \mathrm{E}-01$ & $-2.66 \mathrm{E}-02$ & $-3.23 \mathrm{E}-01$ & $-1.29 \mathrm{E}-01$ & 1 & $1.86 \mathrm{E}-02$ \\
\hline & Oceania & $-1.21 \mathrm{E}-02$ & $1.04 \mathrm{E}-02$ & $-1.08 \mathrm{E}-01$ & $-1.69 \mathrm{E}-02$ & $3.34 \mathrm{E}-03$ & $1.86 \mathrm{E}-02$ & 1 \\
\hline \multirow{6}{*}{ Ocean } & North Pacific & $4.41 \mathrm{E}-02$ & $1.51 \mathrm{E}-01$ & $1.13 \mathrm{E}-02$ & $-2.18 \mathrm{E}-01$ & $4.05 E-02$ & $8.35 \mathrm{E}-02$ & $4.47 \mathrm{E}-03$ \\
\hline & \begin{tabular}{|l} 
South Pacific \\
\end{tabular} & $-8.45 \mathrm{E}-02$ & $-1.22 \mathrm{E}-01$ & $-4.14 \mathrm{E}-01$ & $4.90 \mathrm{E}-04$ & $3.22 \mathrm{E}-03$ & $5.22 \mathrm{E}-02$ & $-2.02 \mathrm{E}-02$ \\
\hline & \begin{tabular}{|l|} 
Northern Ocean \\
\end{tabular} & $2.62 \mathrm{E}-02$ & $1.33 \mathrm{E}-01$ & $4.07 \mathrm{E}-02$ & $-9.56 \mathrm{E}-02$ & $2.68 \mathrm{E}-03$ & $-1.00 \mathrm{E}-02$ & $1.32 \mathrm{E}-03$ \\
\hline & Atlantic & $-1.30 \mathrm{E}-01$ & $1.27 \mathrm{E}-01$ & $2.33 \mathrm{E}-03$ & $1.72 \mathrm{E}-02$ & $1.56 \mathrm{E}-02$ & \begin{tabular}{|l|}
$8.56 \mathrm{E}-02$ \\
\end{tabular} & $4.42 \mathrm{E}-03$ \\
\hline & Southern Ocean & $6.64 \mathrm{E}-02$ & $2.30 \mathrm{E}-01$ & $-1.41 \mathrm{E}-01$ & $-2.03 \mathrm{E}-02$ & $8.78 \mathrm{E}-03$ & \begin{tabular}{|l|l|} 
& $4.21 \mathrm{E}-02$ \\
\end{tabular} & $-3.19 \mathrm{E}-02$ \\
\hline & Indian Ocean & $-1.21 \mathrm{E}-01$ & $-1.15 \mathrm{E}-01$ & $-2.12 \mathrm{E}-01$ & $4.18 \mathrm{E}-02$ & $8.70 \mathrm{E}-03$ & $4.89 \mathrm{E}-02$ & $-4.00 \mathrm{E}-02$ \\
\hline \multirow{7}{*}{$\begin{array}{l}\text { Biomass } \\
\text { burning }\end{array}$} & Africa/Middle East & $-8.49 \mathrm{E}-02$ & $-5.11 \mathrm{E}-02$ & $2.31 \mathrm{E}-04$ & $2.60 \mathrm{E}-02$ & $3.52 \mathrm{E}-03$ & $1.10 \mathrm{E}-02$ & $-3.75 \mathrm{E}-03$ \\
\hline & Southern Asia & $-1.24 \mathrm{E}-03$ & $3.62 \mathrm{E}-03$ & $-3.45 \mathrm{E}-03$ & $-5.59 \mathrm{E}-04$ & $-7.58 \mathrm{E}-05$ & \begin{tabular}{|l|}
$2.73 \mathrm{E}-03$ \\
\end{tabular} & $-3.02 \mathrm{E}-04$ \\
\hline & Central/South America & $-6.80 \mathrm{E}-03$ & $-3.81 \mathrm{E}-03$ & $7.51 \mathrm{E}-04$ & $2.17 \mathrm{E}-03$ & $2.84 \mathrm{E}-04$ & $2.34 \mathrm{E}-03$ & $-1.62 \mathrm{E}-03$ \\
\hline & Northern Asia & $5.82 \mathrm{E}-03$ & $-3.18 \mathrm{E}-03$ & $-1.97 \mathrm{E}-03$ & $-9.24 \mathrm{E}-02$ & $-1.77 \mathrm{E}-02$ & $-6.06 \mathrm{E}-02$ & $1.17 \mathrm{E}-03$ \\
\hline & Europe & $-6.00 \mathrm{E}-04$ & $1.92 \mathrm{E}-04$ & $-5.39 \mathrm{E}-05$ & $4.50 \mathrm{E}-04$ & $-1.43 \mathrm{E}-02$ & \begin{tabular}{|l|} 
\\
\end{tabular} & $-1.47 \mathrm{E}-06$ \\
\hline & North America & $2.90 \mathrm{E}-05$ & $1.90 \mathrm{E}-03$ & $-5.91 \mathrm{E}-04$ & $-7.41 \mathrm{E}-03$ & $-1.75 \mathrm{E}-03$ & \begin{tabular}{|l|}
$-9.93 E-03$ \\
\end{tabular} & $1.31 \mathrm{E}-04$ \\
\hline & Oceania & $-9.03 E-03$ & $-7.73 \mathrm{E}-03$ & $-1.89 \mathrm{E}-03$ & $3.27 \mathrm{E}-03$ & $1.20 \mathrm{E}-03$ & \begin{tabular}{|l|l|}
$3.74 \mathrm{E}-03$ \\
\end{tabular} & $-1.02 \mathrm{E}-02$ \\
\hline
\end{tabular}

Fig. S2af. Correlation of the emissions in 2001 for natural soil. 


\begin{tabular}{|c|c|c|c|c|c|c|c|}
\hline & \multirow{2}{*}{\multicolumn{6}{|c|}{ Ocean }} \\
\hline & & & & & & & \\
\hline Source sector & Region & $\begin{array}{l}\text { North } \\
\text { Pacific }\end{array}$ & $\begin{array}{l}\text { South } \\
\text { Pacific }\end{array}$ & $\begin{array}{l}\text { Northern } \\
\text { Ocean }\end{array}$ & Atlantic & $\begin{array}{l}\text { Southern } \\
\text { Ocean }\end{array}$ & $\begin{array}{l}\text { Indian } \\
\text { Ocean }\end{array}$ \\
\hline \multirow{7}{*}{$\begin{array}{l}\text { Agricultural } \\
\text { Soil }\end{array}$} & Africa/Middle East & $-4.62 \mathrm{E}-03$ & $-3.43 \mathrm{E}-02$ & $7.38 \mathrm{E}-04$ & $-8.37 \mathrm{E}-02$ & $2.10 \mathrm{E}-02$ & $-2.91 \mathrm{E}-02$ \\
\hline & \begin{tabular}{|l} 
Southern Asia \\
\end{tabular} & $-1.63 \mathrm{E}-01$ & $2.75 \mathrm{E}-03$ & $-2.84 \mathrm{E}-02$ & $-1.15 \mathrm{E}-01$ & $7.49 \mathrm{E}-02$ & $-4.98 \mathrm{E}-02$ \\
\hline & Central/South America & $8.02 \mathrm{E}-03$ & $-3.51 \mathrm{E}-02$ & $1.22 \mathrm{E}-03$ & $-2.12 \mathrm{E}-02$ & $-1.53 \mathrm{E}-01$ & $-7.96 \mathrm{E}-02$ \\
\hline & \begin{tabular}{|l} 
Northern Asia \\
\end{tabular} & $-2.42 \mathrm{E}-02$ & $1.86 \mathrm{E}-02$ & $-6.97 \mathrm{E}-02$ & $-5.35 \mathrm{E}-02$ & $-2.59 \mathrm{E}-02$ & $1.90 \mathrm{E}-02$ \\
\hline & Europe & $6.51 \mathrm{E}-03$ & $1.46 \mathrm{E}-02$ & \begin{tabular}{|l|}
$-8.33 \mathrm{E}-02$ \\
\end{tabular} & $-4.99 \mathrm{E}-02$ & $6.19 \mathrm{E}-03$ & $1.62 \mathrm{E}-03$ \\
\hline & North America & $-5.39 \mathrm{E}-02$ & $2.04 \mathrm{E}-03$ & $-1.23 \mathrm{E}-04$ & $-1.80 \mathrm{E}-01$ & $2.45 \mathrm{E}-02$ & $2.00 \mathrm{E}-02$ \\
\hline & Oceania & $8.08 \mathrm{E}-03$ & $1.01 \mathrm{E}-02$ & $2.78 \mathrm{E}-03$ & $1.64 \mathrm{E}-02$ & $-5.27 \mathrm{E}-02$ & $-2.66 \mathrm{E}-02$ \\
\hline \multirow{7}{*}{ Industrial } & Africa/Middle East & $-1.82 \mathrm{E}-03$ & $-2.77 \mathrm{E}-03$ & $\mid-4.93 \mathrm{E}-04$ & $-1.39 \mathrm{E}-02$ & $2.28 \mathrm{E}-03$ & $-2.30 \mathrm{E}-03$ \\
\hline & Southern Asia & $-5.34 \mathrm{E}-02$ & $2.40 \mathrm{E}-02$ & \begin{tabular}{|l|}
$-1.27 \mathrm{E}-02$ \\
\end{tabular} & $-2.10 \mathrm{E}-02$ & $-2.58 \mathrm{E}-02$ & $2.56 \mathrm{E}-02$ \\
\hline & Central/South America & $5.74 \mathrm{E}-04$ & $-4.44 \mathrm{E}-03$ & $9.82 \mathrm{E}-05$ & $-2.19 \mathrm{E}-03$ & $-1.20 \mathrm{E}-02$ & $-7.38 \mathrm{E}-03$ \\
\hline & Northern Asia & $2.40 \mathrm{E}-02$ & $1.67 \mathrm{E}-02$ & $1.42 \mathrm{E}-02$ & $-1.66 \mathrm{E}-01$ & $-1.23 \mathrm{E}-02$ & $9.67 \mathrm{E}-04$ \\
\hline & \begin{tabular}{|l|} 
Europe \\
\end{tabular} & $-1.32 \mathrm{E}-02$ & $6.44 \mathrm{E}-03$ & $1.93 \mathrm{E}-02$ & $-1.45 \mathrm{E}-02$ & $-7.27 E-04$ & $2.41 \mathrm{E}-03$ \\
\hline & North America & $-2.27 \mathrm{E}-02$ & $9.83 \mathrm{E}-04$ & $2.01 \mathrm{E}-02$ & $-9.08 \mathrm{E}-02$ & $7.20 \mathrm{E}-03$ & $-2.79 \mathrm{E}-03$ \\
\hline & Oceania & 5.85E-04 & $1.25 \mathrm{E}-03$ & $2.53 E-04$ & 1.17E-03 & $-3.76 \mathrm{E}-03$ & $-2.54 \mathrm{E}-03$ \\
\hline \multirow{7}{*}{ Natural Soil } & Africa/Middle East & $4.41 \mathrm{E}-02$ & $-8.45 \mathrm{E}-02$ & $2.62 \mathrm{E}-02$ & $-1.30 \mathrm{E}-01$ & $6.64 \mathrm{E}-02$ & $-1.21 \mathrm{E}-01$ \\
\hline & Southern Asia & $1.51 \mathrm{E}-01$ & $-1.22 \mathrm{E}-01$ & $1.33 \mathrm{E}-01$ & $1.27 \mathrm{E}-01$ & $2.30 \mathrm{E}-01$ & $-1.15 \mathrm{E}-01$ \\
\hline & Central/South America & $1.13 \mathrm{E}-02$ & $-4.14 \mathrm{E}-01$ & $4.07 \mathrm{E}-02$ & $2.33 \mathrm{E}-03$ & $-1.41 \mathrm{E}-01$ & $-2.12 \mathrm{E}-01$ \\
\hline & Northern Asia & $-2.18 \mathrm{E}-01$ & $4.90 \mathrm{E}-04$ & \begin{tabular}{|l|}
$-9.56 \mathrm{E}-02$ \\
\end{tabular} & $1.72 \mathrm{E}-02$ & $-2.03 \mathrm{E}-02$ & $4.18 \mathrm{E}-02$ \\
\hline & Europe & $4.05 \mathrm{E}-02$ & $3.22 \mathrm{E}-03$ & $2.68 \mathrm{E}-03$ & $1.56 \mathrm{E}-02$ & $8.78 \mathrm{E}-03$ & $8.70 \mathrm{E}-03$ \\
\hline & North America & $8.35 \mathrm{E}-02$ & $5.22 \mathrm{E}-02$ & \begin{tabular}{|l|}
$-1.00 \mathrm{E}-02$ \\
\end{tabular} & $8.56 \mathrm{E}-02$ & $4.21 \mathrm{E}-02$ & $4.89 \mathrm{E}-02$ \\
\hline & Oceania & 4.47E-03 & $-2.02 \mathrm{E}-02$ & $1.32 \mathrm{E}-03$ & $4.42 \mathrm{E}-03$ & $-3.19 \mathrm{E}-02$ & $-4.00 \mathrm{E}-02$ \\
\hline \multirow{6}{*}{ Ocean } & North Pacific & 1 & $-2.05 \mathrm{E}-02$ & $-7.78 \mathrm{E}-02$ & $-2.02 \mathrm{E}-01$ & $2.63 \mathrm{E}-02$ & $4.38 \mathrm{E}-02$ \\
\hline & South Pacific & $-2.05 \mathrm{E}-02$ & 1 & \begin{tabular}{|l|}
$-3.74 \mathrm{E}-03$ \\
\end{tabular} & $-9.92 \mathrm{E}-02$ & $-1.52 \mathrm{E}-02$ & $-3.41 \mathrm{E}-02$ \\
\hline & Northern Ocean & $-7.78 \mathrm{E}-02$ & $-3.74 \mathrm{E}-03$ & 1 & $-2.80 \mathrm{E}-01$ & $2.05 \mathrm{E}-02$ & $4.46 \mathrm{E}-03$ \\
\hline & Atlantic & $-2.02 \mathrm{E}-01$ & $-9.92 \mathrm{E}-02$ & $-2.80 \mathrm{E}-01$ & 1 & $-5.25 \mathrm{E}-02$ & $-2.35 \mathrm{E}-02$ \\
\hline & Southern Ocean & $2.63 \mathrm{E}-02$ & $-1.52 \mathrm{E}-02$ & $2.05 \mathrm{E}-02$ & $-5.25 \mathrm{E}-02$ & 1 & $-5.48 \mathrm{E}-01$ \\
\hline & Indian Ocean & $4.38 \mathrm{E}-02$ & $-3.41 \mathrm{E}-02$ & $4.46 \mathrm{E}-03$ & $-2.35 \mathrm{E}-02$ & $-5.48 \mathrm{E}-01$ & \\
\hline \multirow{7}{*}{$\begin{array}{l}\text { Biomass } \\
\text { burning }\end{array}$} & Africa/Middle East & $7.81 \mathrm{E}-03$ & $-7.10 \mathrm{E}-02$ & \begin{tabular}{|l|}
$-9.23 \mathrm{E}-04$ \\
\end{tabular} & $-5.13 E-02$ & $-3.30 \mathrm{E}-02$ & $-5.85 \mathrm{E}-02$ \\
\hline & Southern Asia & $-2.29 \mathrm{E}-03$ & $-9.18 \mathrm{E}-03$ & \begin{tabular}{|l|}
$-4.43 \mathrm{E}-04$ \\
\end{tabular} & $-5.24 \mathrm{E}-03$ & $3.84 \mathrm{E}-03$ & $-3.99 \mathrm{E}-03$ \\
\hline & Central/South America & $3.32 \mathrm{E}-04$ & $-1.51 \mathrm{E}-02$ & $-2.65 \mathrm{E}-04$ & $-6.08 \mathrm{E}-03$ & $-1.95 \mathrm{E}-02$ & $-9.78 \mathrm{E}-03$ \\
\hline & Northern Asia & $-5.36 \mathrm{E}-02$ & $6.17 \mathrm{E}-03$ & $\mid-9.89 \mathrm{E}-03$ & $1.03 \mathrm{E}-02$ & $-2.01 \mathrm{E}-03$ & $5.64 \mathrm{E}-03$ \\
\hline & Europe & $1.03 \mathrm{E}-03$ & $-1.46 \mathrm{E}-05$ & $8.61 \mathrm{E}-04$ & $7.48 \mathrm{E}-04$ & $9.62 \mathrm{E}-05$ & $6.56 \mathrm{E}-06$ \\
\hline & North America & $-5.17 \mathrm{E}-05$ & $-2.33 \mathrm{E}-04$ & \begin{tabular}{|l|}
$-1.94 \mathrm{E}-03$ \\
\end{tabular} & $-1.45 \mathrm{E}-04$ & $5.30 \mathrm{E}-04$ & $3.04 \mathrm{E}-04$ \\
\hline & Oceania & $1.23 \mathrm{E}-03$ & $-1.50 \mathrm{E}-02$ & \begin{tabular}{|l|}
$-1.30 \mathrm{E}-03$ \\
\end{tabular} & $-4.92 \mathrm{E}-03$ & $-1.00 \mathrm{E}-02$ & $-1.45 \mathrm{E}-02$ \\
\hline
\end{tabular}

Fig. S2ag. Correlation of the emissions in 2001 for ocean. 


\begin{tabular}{|c|c|c|c|c|c|c|c|c|}
\hline \multirow[b]{3}{*}{ Source sector } & \multirow[b]{3}{*}{ Region } & \multirow{2}{*}{\multicolumn{7}{|c|}{ Biomass burning }} \\
\hline & & & & & & & & \\
\hline & & $\begin{array}{l}\text { Africa/M. } \\
\text { East }\end{array}$ & $\begin{array}{l}\text { Southern } \\
\text { Asia }\end{array}$ & $\begin{array}{l}\text { C. /S. } \\
\text { America }\end{array}$ & $\begin{array}{l}\text { Northern } \\
\text { Asia }\end{array}$ & Europe & $\begin{array}{l}\text { North } \\
\text { America }\end{array}$ & Oceania \\
\hline \multirow{7}{*}{$\begin{array}{l}\text { Agricultural } \\
\text { Soil }\end{array}$} & Africa/Middle East & $-2.19 \mathrm{E}-02$ & $-7.75 \mathrm{E}-04$ & $-1.69 \mathrm{E}-03$ & $3.11 \mathrm{E}-03$ & $-8.51 \mathrm{E}-04$ & $-6.58 \mathrm{E}-05$ & $-9.79 \mathrm{E}-04$ \\
\hline & Southern Asia & $-2.35 \mathrm{E}-03$ & $-1.77 \mathrm{E}-02$ & $1.92 \mathrm{E}-03$ & $-4.37 \mathrm{E}-03$ & $1.62 \mathrm{E}-04$ & $-2.60 \mathrm{E}-04$ & $7.84 \mathrm{E}-03$ \\
\hline & Central/South America & $-2.60 \mathrm{E}-02$ & $-4.88 \mathrm{E}-04$ & $-4.62 \mathrm{E}-03$ & $3.09 \mathrm{E}-04$ & $-2.85 \mathrm{E}-05$ & $1.23 \mathrm{E}-04$ & $-9.02 \mathrm{E}-03$ \\
\hline & Northern Asia & $8.63 \mathrm{E}-03$ & $-7.61 \mathrm{E}-03$ & $3.57 \mathrm{E}-04$ & $-2.45 \mathrm{E}-03$ & $2.67 \mathrm{E}-04$ & $8.45 \mathrm{E}-04$ & $-8.44 \mathrm{E}-04$ \\
\hline & \begin{tabular}{|l} 
Europe \\
\end{tabular} & $2.32 \mathrm{E}-03$ & $2.02 \mathrm{E}-03$ & $4.67 \mathrm{E}-04$ & $2.18 \mathrm{E}-03$ & $8.98 \mathrm{E}-04$ & $1.12 \mathrm{E}-04$ & $1.10 \mathrm{E}-03$ \\
\hline & \begin{tabular}{|l} 
North America \\
\end{tabular} & $3.73 \mathrm{E}-03$ & $-1.12 \mathrm{E}-03$ & $7.83 \mathrm{E}-04$ & $1.43 \mathrm{E}-02$ & $4.18 \mathrm{E}-04$ & $-6.92 \mathrm{E}-04$ & $3.25 \mathrm{E}-03$ \\
\hline & Oceania & $3.03 \mathrm{E}-03$ & $9.99 \mathrm{E}-04$ & $-4.52 \mathrm{E}-04$ & $5.36 \mathrm{E}-04$ & $2.89 \mathrm{E}-05$ & $1.91 \mathrm{E}-04$ & $-5.59 \mathrm{E}-03$ \\
\hline \multirow{7}{*}{ Industrial } & Africa/Middle East & $-1.96 \mathrm{E}-03$ & $-5.24 \mathrm{E}-05$ & $-1.34 \mathrm{E}-04$ & $5.60 \mathrm{E}-04$ & $-2.38 \mathrm{E}-04$ & $1.32 \mathrm{E}-05$ & $4.64 \mathrm{E}-05$ \\
\hline & Southern Asia & $1.35 \mathrm{E}-02$ & $-3.57 \mathrm{E}-03$ & $1.11 \mathrm{E}-03$ & $-6.67 \mathrm{E}-03$ & $6.21 \mathrm{E}-05$ & $3.07 \mathrm{E}-04$ & $1.55 \mathrm{E}-03$ \\
\hline & Central/South America & $-2.40 \mathrm{E}-03$ & $-5.57 \mathrm{E}-05$ & $-4.23 \mathrm{E}-04$ & $2.84 \mathrm{E}-05$ & $-3.10 \mathrm{E}-06$ & $8.02 \mathrm{E}-06$ & $-8.10 \mathrm{E}-04$ \\
\hline & Northern Asia & $7.78 \mathrm{E}-03$ & $-2.15 \mathrm{E}-03$ & $4.57 E-04$ & $1.11 \mathrm{E}-02$ & $-3.41 \mathrm{E}-05$ & $7.25 \mathrm{E}-04$ & $-1.62 \mathrm{E}-03$ \\
\hline & Europe & $1.81 \mathrm{E}-03$ & $3.21 \mathrm{E}-04$ & $2.06 \mathrm{E}-04$ & $1.52 \mathrm{E}-03$ & $6.67 \mathrm{E}-04$ & $4.44 \mathrm{E}-04$ & $4.17 \mathrm{E}-04$ \\
\hline & North America & $2.31 \mathrm{E}-03$ & $7.65 \mathrm{E}-04$ & $2.70 \mathrm{E}-04$ & $7.51 \mathrm{E}-03$ & $1.54 \mathrm{E}-04$ & $3.62 \mathrm{E}-05$ & $2.20 \mathrm{E}-04$ \\
\hline & Oceania & $2.36 \mathrm{E}-04$ & $7.75 \mathrm{E}-05$ & $-2.63 \mathrm{E}-05$ & $4.93 \mathrm{E}-05$ & $2.70 \mathrm{E}-06$ & $1.46 \mathrm{E}-05$ & $-4.15 E-04$ \\
\hline \multirow{7}{*}{ Natural Soil } & Africa/Middle East & $-8.49 \mathrm{E}-02$ & $-1.24 \mathrm{E}-03$ & $-6.80 \mathrm{E}-03$ & $5.82 \mathrm{E}-03$ & $-6.00 \mathrm{E}-04$ & $2.90 \mathrm{E}-05$ & $-9.03 \mathrm{E}-03$ \\
\hline & Southern Asia & $-5.11 \mathrm{E}-02$ & $3.62 \mathrm{E}-03$ & $-3.81 \mathrm{E}-03$ & $-3.18 \mathrm{E}-03$ & $1.92 \mathrm{E}-04$ & $1.90 \mathrm{E}-03$ & $-7.73 \mathrm{E}-03$ \\
\hline & Central/South America & $2.31 \mathrm{E}-04$ & $-3.45 E-03$ & $7.51 \mathrm{E}-04$ & $-1.97 \mathrm{E}-03$ & $-5.39 \mathrm{E}-05$ & $-5.91 \mathrm{E}-04$ & $-1.89 \mathrm{E}-03$ \\
\hline & Northern Asia & $2.60 \mathrm{E}-02$ & $-5.59 \mathrm{E}-04$ & 2.17E-03 & $-9.24 \mathrm{E}-02$ & $4.50 \mathrm{E}-04$ & $-7.41 \mathrm{E}-03$ & $3.27 \mathrm{E}-03$ \\
\hline & Europe & $3.52 \mathrm{E}-03$ & $-7.58 \mathrm{E}-05$ & $2.84 \mathrm{E}-04$ & $-1.77 \mathrm{E}-02$ & $-1.43 \mathrm{E}-02$ & $-1.75 \mathrm{E}-03$ & $1.20 \mathrm{E}-03$ \\
\hline & North America & $1.10 \mathrm{E}-02$ & $2.73 \mathrm{E}-03$ & $2.34 \mathrm{E}-03$ & $-6.06 \mathrm{E}-02$ & $-8.22 \mathrm{E}-04$ & $-9.93 \mathrm{E}-03$ & $7.74 \mathrm{E}-03$ \\
\hline & Oceania & $-3.75 \mathrm{E}-03$ & $-3.02 \mathrm{E}-04$ & $-1.62 \mathrm{E}-03$ & $1.17 \mathrm{E}-03$ & $-1.47 \mathrm{E}-06$ & $1.31 \mathrm{E}-04$ & $-1.02 \mathrm{E}-02$ \\
\hline \multirow{6}{*}{ Ocean } & North Pacific & $7.81 \mathrm{E}-03$ & $-2.29 \mathrm{E}-03$ & $3.32 \mathrm{E}-04$ & $-5.36 \mathrm{E}-02$ & $1.03 E-03$ & $-5.17 \mathrm{E}-05$ & $1.23 \mathrm{E}-03$ \\
\hline & South Pacific & $-7.10 \mathrm{E}-02$ & $-9.18 \mathrm{E}-03$ & $-1.51 \mathrm{E}-02$ & $6.17 \mathrm{E}-03$ & $-1.46 \mathrm{E}-05$ & $-2.33 \mathrm{E}-04$ & $-1.50 \mathrm{E}-02$ \\
\hline & Northern Ocean & $-9.23 \mathrm{E}-04$ & $-4.43 \mathrm{E}-04$ & $-2.65 \mathrm{E}-04$ & $-9.89 \mathrm{E}-03$ & $8.61 \mathrm{E}-04$ & $-1.94 \mathrm{E}-03$ & $-1.30 \mathrm{E}-03$ \\
\hline & Atlantic & $-5.13 E-02$ & $-5.24 \mathrm{E}-03$ & $-6.08 \mathrm{E}-03$ & $1.03 \mathrm{E}-02$ & $7.48 \mathrm{E}-04$ & $-1.45 E-04$ & $-4.92 \mathrm{E}-03$ \\
\hline & Southern Ocean & $-3.30 \mathrm{E}-02$ & $3.84 \mathrm{E}-03$ & $-1.95 \mathrm{E}-02$ & $-2.01 \mathrm{E}-03$ & $9.62 \mathrm{E}-05$ & \begin{tabular}{|l|}
$5.30 \mathrm{E}-04$ \\
\end{tabular} & $-1.00 \mathrm{E}-02$ \\
\hline & Indian Ocean & $-5.85 \mathrm{E}-02$ & $-3.99 \mathrm{E}-03$ & $-9.78 \mathrm{E}-03$ & $5.64 \mathrm{E}-03$ & $6.56 \mathrm{E}-06$ & $3.04 \mathrm{E}-04$ & $-1.45 \mathrm{E}-02$ \\
\hline \multirow{7}{*}{$\begin{array}{l}\text { Biomass } \\
\text { burning }\end{array}$} & Africa/Middle East & 1 & $-1.31 \mathrm{E}-03$ & $-5.72 \mathrm{E}-03$ & $2.84 \mathrm{E}-03$ & $-5.47 \mathrm{E}-05$ & \begin{tabular}{|l|}
$1.66 \mathrm{E}-04$ \\
\end{tabular} & $-8.07 \mathrm{E}-03$ \\
\hline & Southern Asia & $-1.31 \mathrm{E}-03$ & 1 & $-2.36 \mathrm{E}-04$ & $-1.79 \mathrm{E}-03$ & $1.63 \mathrm{E}-05$ & \begin{tabular}{|l|}
$-8.63 E-06$ \\
\end{tabular} & $-4.08 \mathrm{E}-04$ \\
\hline & Central/South America & $-5.72 \mathrm{E}-03$ & $-2.36 \mathrm{E}-04$ & 1 & $1.98 \mathrm{E}-04$ & $-2.81 \mathrm{E}-06$ & $1.14 \mathrm{E}-05$ & $-1.84 \mathrm{E}-03$ \\
\hline & Northern Asia & $2.84 \mathrm{E}-03$ & $-1.79 \mathrm{E}-03$ & $1.98 \mathrm{E}-04$ & 1 & $-7.98 \mathrm{E}-04$ & $-1.07 \mathrm{E}-03$ & $2.70 \mathrm{E}-04$ \\
\hline & Europe & $-5.47 \mathrm{E}-05$ & $1.63 \mathrm{E}-05$ & $-2.81 \mathrm{E}-06$ & $-7.98 \mathrm{E}-04$ & 1 & $-2.81 \mathrm{E}-05$ & $-1.38 \mathrm{E}-05$ \\
\hline & North America & $1.66 \mathrm{E}-04$ & $-8.63 \mathrm{E}-06$ & $1.14 \mathrm{E}-05$ & $-1.07 \mathrm{E}-03$ & $-2.81 \mathrm{E}-05$ & 1 & $4.29 \mathrm{E}-05$ \\
\hline & Oceania & -8.07E-03 & $-4.08 \mathrm{E}-04$ & $-1.84 \mathrm{E}-03$ & $2.70 \mathrm{E}-04$ & $-1.38 \mathrm{E}-05$ & \begin{tabular}{|l|l|} 
& $4.29 \mathrm{E}-05$ \\
\end{tabular} & 1 \\
\hline
\end{tabular}

Fig. S2ah. Correlation of the emissions in 2001 for biomass burning. 


\begin{tabular}{|c|c|c|c|c|c|c|c|c|}
\hline \multirow[b]{2}{*}{ Source sector } & \multirow[b]{2}{*}{ Region } & \multicolumn{7}{|c|}{ Agricultural Soil } \\
\hline & & $\begin{array}{l}\text { Africa/M. } \\
\text { East }\end{array}$ & $\begin{array}{l}\text { Southern } \\
\text { Asia }\end{array}$ & $\begin{array}{l}\text { C. /S. } \\
\text { America }\end{array}$ & $\begin{array}{l}\text { Northern } \\
\text { Asia }\end{array}$ & Europe & $\begin{array}{l}\text { North } \\
\text { America }\end{array}$ & Oceania \\
\hline \multirow{7}{*}{$\begin{array}{c}\text { Agricultural } \\
\text { Soil }\end{array}$} & Africa/Middle East & 1 & $-7.41 \mathrm{E}-02$ & $-7.05 \mathrm{E}-03$ & $-3.54 \mathrm{E}-02$ & $-2.05 \mathrm{E}-02$ & $-1.28 \mathrm{E}-02$ & $6.39 \mathrm{E}-03$ \\
\hline & Southern Asia & $-7.41 \mathrm{E}-02$ & 1 & $2.36 \mathrm{E}-02$ & $-2.01 \mathrm{E}-01$ & $1.98 \mathrm{E}-02$ & $-5.42 \mathrm{E}-02$ & $1.33 \mathrm{E}-02$ \\
\hline & Central/South America & $-7.05 E-03$ & $2.36 \mathrm{E}-02$ & 1 & $-8.38 \mathrm{E}-04$ & $5.41 \mathrm{E}-03$ & $6.89 \mathrm{E}-03$ & $-1.34 \mathrm{E}-02$ \\
\hline & Northern Asia & $-3.54 \mathrm{E}-02$ & $-2.01 \mathrm{E}-01$ & $-8.38 \mathrm{E}-04$ & 1 & $-1.60 \mathrm{E}-01$ & $-4.38 \mathrm{E}-02$ & $-1.69 \mathrm{E}-02$ \\
\hline & Europe & $-2.05 \mathrm{E}-02$ & $1.98 \mathrm{E}-02$ & $5.41 \mathrm{E}-03$ & $-1.60 \mathrm{E}-01$ & 1 & $-9.40 \mathrm{E}-03$ & $1.82 \mathrm{E}-03$ \\
\hline & North America & $-1.28 \mathrm{E}-02$ & $-5.42 \mathrm{E}-02$ & $6.89 \mathrm{E}-03$ & $-4.38 \mathrm{E}-02$ & $-9.40 \mathrm{E}-03$ & 1 & $3.93 \mathrm{E}-04$ \\
\hline & Oceania & $6.39 \mathrm{E}-03$ & $1.33 \mathrm{E}-02$ & $-1.34 \mathrm{E}-02$ & $-1.69 \mathrm{E}-02$ & $1.82 \mathrm{E}-03$ & $3.93 \mathrm{E}-04$ & 1 \\
\hline \multirow{7}{*}{ Industrial } & Africa/Middle East & $-5.98 \mathrm{E}-02$ & $-1.16 \mathrm{E}-02$ & $5.74 \mathrm{E}-04$ & $-8.97 \mathrm{E}-03$ & $\mid-7.23 \mathrm{E}-03$ & $-3.45 \mathrm{E}-03$ & $8.67 \mathrm{E}-04$ \\
\hline & \begin{tabular}{|l} 
Southern Asia \\
\end{tabular} & $1.08 \mathrm{E}-02$ & $-3.55 \mathrm{E}-01$ & $1.75 \mathrm{E}-03$ & $-4.08 \mathrm{E}-02$ & $1.29 \mathrm{E}-02$ & $6.93 \mathrm{E}-04$ & $-7.55 \mathrm{E}-04$ \\
\hline & Central/South America & $-7.91 \mathrm{E}-04$ & $1.97 \mathrm{E}-03$ & $-5.15 \mathrm{E}-03$ & $3.05 \mathrm{E}-05$ & $4.99 \mathrm{E}-04$ & $6.60 \mathrm{E}-04$ & $-1.08 \mathrm{E}-03$ \\
\hline & Northern Asia & $2.21 \mathrm{E}-02$ & $-2.21 \mathrm{E}-02$ & $3.45 \mathrm{E}-03$ & $-1.08 \mathrm{E}-01$ & $-4.58 \mathrm{E}-02$ & $1.85 \mathrm{E}-01$ & $-6.23 E-03$ \\
\hline & Europe & $-1.25 \mathrm{E}-02$ & 3.09E-03 & $1.58 \mathrm{E}-03$ & $-3.49 E-02$ & $-6.99 \mathrm{E}-01$ & $-1.19 \mathrm{E}-02$ & $5.15 \mathrm{E}-04$ \\
\hline & North America & $8.69 \mathrm{E}-03$ & $1.37 \mathrm{E}-02$ & $2.03 E-03$ & $2.37 \mathrm{E}-02$ & $2.05 \mathrm{E}-02$ & $-3.63 \mathrm{E}-01$ & $-1.14 \mathrm{E}-03$ \\
\hline & Oceania & $4.79 \mathrm{E}-04$ & $8.74 \mathrm{E}-04$ & $-8.71 \mathrm{E}-04$ & $-1.19 \mathrm{E}-03$ & $1.08 \mathrm{E}-04$ & $-4.52 \mathrm{E}-06$ & $-5.26 \mathrm{E}-03$ \\
\hline \multirow{7}{*}{ Natural Soil } & Africa/Middle East & $-1.87 \mathrm{E}-01$ & $-5.15 \mathrm{E}-02$ & $-6.86 \mathrm{E}-02$ & $6.24 \mathrm{E}-03$ & $-1.08 \mathrm{E}-02$ & $1.94 \mathrm{E}-02$ & $6.36 \mathrm{E}-03$ \\
\hline & Southern Asia & $-7.12 \mathrm{E}-02$ & $-4.33 \mathrm{E}-01$ & $-4.95 \mathrm{E}-03$ & $-7.06 \mathrm{E}-02$ & $-1.48 \mathrm{E}-02$ & $-1.21 \mathrm{E}-02$ & $2.24 \mathrm{E}-02$ \\
\hline & Central/South America & $-5.83 E-02$ & $8.43 \mathrm{E}-02$ & $-3.08 \mathrm{E}-01$ & $4.40 \mathrm{E}-03$ & $2.76 \mathrm{E}-02$ & $3.41 \mathrm{E}-02$ & $-7.85 \mathrm{E}-02$ \\
\hline & Northern Asia & $2.81 \mathrm{E}-02$ & $3.52 \mathrm{E}-02$ & $1.00 \mathrm{E}-02$ & $-1.51 \mathrm{E}-01$ & $3.26 \mathrm{E}-02$ & $7.04 \mathrm{E}-02$ & $-1.21 \mathrm{E}-02$ \\
\hline & Europe & $-2.76 \mathrm{E}-02$ & $-7.97 \mathrm{E}-03$ & $8.10 \mathrm{E}-03$ & $-4.09 \mathrm{E}-02$ & $-2.89 \mathrm{E}-01$ & $2.17 \mathrm{E}-02$ & $4.18 \mathrm{E}-03$ \\
\hline & North America & $-2.91 \mathrm{E}-02$ & $-2.38 \mathrm{E}-02$ & $1.85 \mathrm{E}-02$ & $6.14 \mathrm{E}-02$ & $-1.19 \mathrm{E}-02$ & $-4.98 \mathrm{E}-01$ & $8.78 \mathrm{E}-03$ \\
\hline & Oceania & $4.95 \mathrm{E}-03$ & $1.20 \mathrm{E}-02$ & $-1.43 \mathrm{E}-02$ & $-1.37 \mathrm{E}-02$ & $4.13 \mathrm{E}-03$ & $1.62 \mathrm{E}-03$ & $-5.50 \mathrm{E}-02$ \\
\hline \multirow{6}{*}{ Ocean } & North Pacific & $1.70 \mathrm{E}-03$ & $9.53 \mathrm{E}-03$ & $8.74 \mathrm{E}-04$ & $2.90 \mathrm{E}-03$ & $2.69 \mathrm{E}-03$ & $5.30 \mathrm{E}-03$ & $-6.94 \mathrm{E}-04$ \\
\hline & South Pacific & $-1.23 \mathrm{E}-03$ & $-9.43 \mathrm{E}-03$ & $5.53 E-04$ & $1.29 \mathrm{E}-03$ & $1.05 \mathrm{E}-03$ & $-1.85 \mathrm{E}-03$ & $1.50 \mathrm{E}-03$ \\
\hline & Northern Ocean & $7.37 \mathrm{E}-05$ & $-2.46 \mathrm{E}-03$ & $7.41 \mathrm{E}-04$ & $1.13 \mathrm{E}-03$ & $2.92 \mathrm{E}-03$ & $2.05 \mathrm{E}-04$ & $-1.19 \mathrm{E}-03$ \\
\hline & Atlantic & $7.03 E-04$ & $-1.12 \mathrm{E}-02$ & $2.73 \mathrm{E}-03$ & $-1.61 \mathrm{E}-03$ & $1.14 \mathrm{E}-03$ & $-4.59 \mathrm{E}-03$ & $-4.98 \mathrm{E}-03$ \\
\hline & Southern Ocean & $4.31 \mathrm{E}-03$ & $2.78 \mathrm{E}-02$ & $5.52 \mathrm{E}-03$ & $2.34 \mathrm{E}-03$ & $5.73 \mathrm{E}-04$ & $1.10 \mathrm{E}-02$ & $-7.78 \mathrm{E}-04$ \\
\hline & Indian Ocean & $3.03 \mathrm{E}-04$ & $-5.27 \mathrm{E}-03$ & $-5.01 \mathrm{E}-05$ & $-1.67 \mathrm{E}-04$ & $7.68 \mathrm{E}-04$ & $-1.16 \mathrm{E}-03$ & $-2.50 \mathrm{E}-03$ \\
\hline \multirow{7}{*}{$\begin{array}{l}\text { Biomass } \\
\text { burning }\end{array}$} & Africa/Middle East & $-2.70 \mathrm{E}-02$ & $-1.77 \mathrm{E}-02$ & $-3.53 \mathrm{E}-02$ & $1.23 \mathrm{E}-02$ & $5.36 \mathrm{E}-03$ & $7.24 \mathrm{E}-03$ & $5.75 \mathrm{E}-03$ \\
\hline & Southern Asia & $-6.07 E-03$ & $-3.16 \mathrm{E}-02$ & $-3.39 \mathrm{E}-03$ & $4.31 \mathrm{E}-03$ & $2.59 \mathrm{E}-03$ & $-2.07 \mathrm{E}-03$ & $1.90 \mathrm{E}-03$ \\
\hline & Central/South America & $-5.15 E-03$ & $7.11 \mathrm{E}-03$ & $-1.56 \mathrm{E}-02$ & $2.41 \mathrm{E}-03$ & $1.92 \mathrm{E}-03$ & $4.38 \mathrm{E}-03$ & $2.06 \mathrm{E}-03$ \\
\hline & \begin{tabular}{|l} 
Northern Asia \\
\end{tabular} & $7.92 \mathrm{E}-03$ & $-1.18 \mathrm{E}-02$ & $6.29 \mathrm{E}-03$ & $1.59 \mathrm{E}-02$ & $-7.03 E-03$ & $2.63 \mathrm{E}-02$ & $1.24 \mathrm{E}-03$ \\
\hline & Europe & $-2.46 \mathrm{E}-05$ & $1.57 \mathrm{E}-04$ & $5.54 \mathrm{E}-05$ & $-3.52 \mathrm{E}-04$ & $9.44 \mathrm{E}-05$ & $3.44 \mathrm{E}-04$ & $5.55 \mathrm{E}-05$ \\
\hline & North America & $4.65 \mathrm{E}-03$ & $1.62 \mathrm{E}-02$ & $2.87 \mathrm{E}-03$ & $1.82 \mathrm{E}-02$ & $1.21 \mathrm{E}-03$ & $2.21 \mathrm{E}-02$ & $1.05 \mathrm{E}-03$ \\
\hline & Oceania & $-7.53 \mathrm{E}-04$ & $6.23 \mathrm{E}-03$ & $-7.36 \mathrm{E}-03$ & $-2.05 \mathrm{E}-03$ & $1.56 \mathrm{E}-03$ & $1.94 \mathrm{E}-03$ & $-1.46 \mathrm{E}-03$ \\
\hline
\end{tabular}

Fig. S2ai. Correlation of the emissions in 2002 for agricultural soil. 


\begin{tabular}{|c|c|c|c|c|c|c|c|c|}
\hline & \multirow{2}{*}{\multicolumn{7}{|c|}{ Industrial }} \\
\hline & & & & & & & & \\
\hline Source sector & Region & $\begin{array}{l}\text { Africa/M. } \\
\text { East }\end{array}$ & $\begin{array}{l}\text { Southern } \\
\text { Asia }\end{array}$ & $\begin{array}{l}\text { C. /S. } \\
\text { America }\end{array}$ & $\begin{array}{l}\text { Northern } \\
\text { Asia }\end{array}$ & Europe & $\begin{array}{l}\text { North } \\
\text { America }\end{array}$ & Oceania \\
\hline \multirow{7}{*}{$\begin{array}{l}\text { Agricultural } \\
\text { Soil }\end{array}$} & Africa/Middle East & $-5.98 \mathrm{E}-02$ & $1.08 \mathrm{E}-02$ & $-7.91 \mathrm{E}-04$ & $2.21 \mathrm{E}-02$ & $-1.25 \mathrm{E}-02$ & $8.69 \mathrm{E}-03$ & $4.79 \mathrm{E}-04$ \\
\hline & Southern Asia & $-1.16 \mathrm{E}-02$ & $-3.55 \mathrm{E}-01$ & $1.97 \mathrm{E}-03$ & \begin{tabular}{|l|} 
\\
\end{tabular} & $3.09 \mathrm{E}-03$ & $1.37 \mathrm{E}-02$ & $8.74 \mathrm{E}-04$ \\
\hline & Central/South America & $5.74 \mathrm{E}-04$ & $1.75 \mathrm{E}-03$ & $-5.15 \mathrm{E}-03$ & $3.45 \mathrm{E}-03$ & $1.58 \mathrm{E}-03$ & $2.03 \mathrm{E}-03$ & $-8.71 \mathrm{E}-04$ \\
\hline & Northern Asia & $-8.97 \mathrm{E}-03$ & $-4.08 \mathrm{E}-02$ & $3.05 \mathrm{E}-05$ & \begin{tabular}{|l|} 
\\
\end{tabular} & $-3.49 \mathrm{E}-02$ & $2.37 \mathrm{E}-02$ & $-1.19 \mathrm{E}-03$ \\
\hline & Europe & $-7.23 \mathrm{E}-03$ & $1.29 \mathrm{E}-02$ & $4.99 \mathrm{E}-04$ & $-4.58 \mathrm{E}-02$ & $-6.99 \mathrm{E}-01$ & $2.05 \mathrm{E}-02$ & $1.08 \mathrm{E}-04$ \\
\hline & \begin{tabular}{|l|} 
North America \\
\end{tabular} & $-3.45 \mathrm{E}-03$ & $6.93 \mathrm{E}-04$ & $6.60 \mathrm{E}-04$ & $1.85 \mathrm{E}-01$ & $-1.19 \mathrm{E}-02$ & $-3.63 \mathrm{E}-01$ & $-4.52 \mathrm{E}-06$ \\
\hline & Oceania & $8.67 \mathrm{E}-04$ & $-7.55 \mathrm{E}-04$ & $-1.08 \mathrm{E}-03$ & 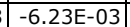 & $5.15 \mathrm{E}-04$ & $-1.14 \mathrm{E}-03$ & $-5.26 \mathrm{E}-03$ \\
\hline \multirow{7}{*}{ Industrial } & Africa/Middle East & 1 & $1.64 \mathrm{E}-03$ & $3.36 \mathrm{E}-05$ & $1.55 \mathrm{E}-03$ & $-5.17 \mathrm{E}-03$ & $1.43 \mathrm{E}-03$ & $6.12 \mathrm{E}-05$ \\
\hline & \begin{tabular}{|l|} 
Southern Asia \\
\end{tabular} & $1.64 \mathrm{E}-03$ & 1 & $2.10 \mathrm{E}-04$ & $-2.48 \mathrm{E}-02$ & $2.99 \mathrm{E}-03$ & $6.14 \mathrm{E}-03$ & $-7.36 \mathrm{E}-05$ \\
\hline & Central/South America & $3.36 \mathrm{E}-05$ & $2.10 \mathrm{E}-04$ & 1 & $4.53 \mathrm{E}-04$ & $1.56 \mathrm{E}-04$ & $2.16 \mathrm{E}-04$ & $-6.95 \mathrm{E}-05$ \\
\hline & Northern Asia & $1.55 \mathrm{E}-03$ & $-2.48 \mathrm{E}-02$ & $4.53 \mathrm{E}-04$ & 1 & $-2.34 \mathrm{E}-02$ & $-2.13 \mathrm{E}-01$ & $-4.47 \mathrm{E}-04$ \\
\hline & Europe & $-5.17 E-03$ & $2.99 \mathrm{E}-03$ & $1.56 \mathrm{E}-04$ & $-2.34 \mathrm{E}-02$ & 1 & $6.03 \mathrm{E}-03$ & $2.79 \mathrm{E}-05$ \\
\hline & North America & $1.43 \mathrm{E}-03$ & $6.14 \mathrm{E}-03$ & $2.16 \mathrm{E}-04$ & $-2.13 \mathrm{E}-01$ & $6.03 E-03$ & 1 & $-8.23 \mathrm{E}-05$ \\
\hline & Oceania & $6.12 \mathrm{E}-05$ & $-7.36 \mathrm{E}-05$ & $-6.95 E-05$ & $-4.47 E-04$ & $2.79 E-05$ & $-8.23 \mathrm{E}-05$ & 1 \\
\hline \multirow{7}{*}{ Natural Soil } & Africa/Middle East & $-3.00 \mathrm{E}-02$ & $2.50 \mathrm{E}-02$ & $-6.41 \mathrm{E}-03$ & 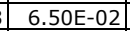 & $-1.83 \mathrm{E}-03$ & $1.92 \mathrm{E}-02$ & $5.29 \mathrm{E}-04$ \\
\hline & Southern Asia & $-9.28 \mathrm{E}-03$ & $-9.23 \mathrm{E}-02$ & $-9.29 \mathrm{E}-04$ & $3.71 \mathrm{E}-02$ & $-5.02 \mathrm{E}-03$ & $1.62 \mathrm{E}-02$ & $1.89 \mathrm{E}-03$ \\
\hline & Central/South America & $4.75 \mathrm{E}-04$ & $1.49 \mathrm{E}-02$ & $-2.64 \mathrm{E}-02$ & $4.51 \mathrm{E}-02$ & $1.75 \mathrm{E}-02$ & $1.56 \mathrm{E}-02$ & $-5.34 \mathrm{E}-03$ \\
\hline & Northern Asia & $5.22 \mathrm{E}-03$ & $5.28 \mathrm{E}-03$ & 9.99E-04 & $-6.95 \mathrm{E}-02$ & $1.71 \mathrm{E}-02$ & $1.42 \mathrm{E}-02$ & $-1.08 \mathrm{E}-03$ \\
\hline & Europe & $-9.74 \mathrm{E}-03$ & $5.03 E-03$ & $6.71 \mathrm{E}-04$ & $1.00 \mathrm{E}-02$ & $-1.69 \mathrm{E}-01$ & $2.02 \mathrm{E}-02$ & $3.18 \mathrm{E}-04$ \\
\hline & North America & $-2.56 \mathrm{E}-03$ & $2.42 \mathrm{E}-02$ & $1.41 \mathrm{E}-03$ & \begin{tabular}{|l|}
$-2.45 \mathrm{E}-01$ \\
\end{tabular} & $1.77 \mathrm{E}-02$ & $-1.78 \mathrm{E}-01$ & $5.79 E-04$ \\
\hline & Oceania & $8.92 \mathrm{E}-04$ & $3.54 \mathrm{E}-04$ & $-1.21 \mathrm{E}-03$ & \begin{tabular}{|l|}
$-4.85 E-03$ \\
\end{tabular} & $1.89 \mathrm{E}-03$ & $-5.91 \mathrm{E}-04$ & $-4.22 \mathrm{E}-03$ \\
\hline \multirow{6}{*}{ Ocean } & North Pacific & $3.97 \mathrm{E}-04$ & $8.27 \mathrm{E}-04$ & $8.19 \mathrm{E}-05$ & $4.94 \mathrm{E}-04$ & $3.35 \mathrm{E}-03$ & $1.81 \mathrm{E}-03$ & $-5.53 \mathrm{E}-05$ \\
\hline & South Pacific & $-2.86 \mathrm{E}-04$ & $-6.05 \mathrm{E}-04$ & $2.65 \mathrm{E}-05$ & $2.15 \mathrm{E}-03$ & $1.53 \mathrm{E}-04$ & $-2.14 \mathrm{E}-04$ & $1.09 \mathrm{E}-04$ \\
\hline & Northern Ocean & $-5.90 \mathrm{E}-05$ & $-1.93 \mathrm{E}-04$ & $7.45 \mathrm{E}-05$ & $1.27 \mathrm{E}-03$ & $2.34 \mathrm{E}-03$ & $4.81 \mathrm{E}-04$ & $-9.27 \mathrm{E}-05$ \\
\hline & Atlantic & $-2.23 \mathrm{E}-04$ & $-1.69 \mathrm{E}-03$ & $2.80 \mathrm{E}-04$ & $1.69 \mathrm{E}-03$ & $-3.11 \mathrm{E}-04$ & $-2.98 \mathrm{E}-04$ & $-3.73 \mathrm{E}-04$ \\
\hline & Southern Ocean & $1.11 \mathrm{E}-03$ & $3.18 \mathrm{E}-03$ & $4.83 \mathrm{E}-04$ & $-2.98 \mathrm{E}-03$ & $2.61 \mathrm{E}-03$ & $1.44 \mathrm{E}-03$ & $-4.19 \mathrm{E}-05$ \\
\hline & Indian Ocean & $-5.51 \mathrm{E}-05$ & $-8.97 E-04$ & $1.47 \mathrm{E}-05$ & $4.55 \mathrm{E}-04$ & $5.98 \mathrm{E}-04$ & $1.15 \mathrm{E}-04$ & $-1.86 \mathrm{E}-04$ \\
\hline \multirow{7}{*}{$\begin{array}{l}\text { Biomass } \\
\text { burning }\end{array}$} & Africa/Middle East & $-1.95 \mathrm{E}-03$ & 4.07E-03 & $-3.39 \mathrm{E}-03$ & \begin{tabular}{|l|} 
\\
\end{tabular} & $2.11 \mathrm{E}-03$ & $3.76 \mathrm{E}-03$ & $4.80 \mathrm{E}-04$ \\
\hline & Southern Asia & $-7.48 \mathrm{E}-04$ & $-6.29 \mathrm{E}-04$ & $-3.49 \mathrm{E}-04$ & \begin{tabular}{|l|}
$4.60 \mathrm{E}-03$ \\
\end{tabular} & $1.15 \mathrm{E}-03$ & $-9.99 \mathrm{E}-05$ & $1.84 \mathrm{E}-04$ \\
\hline & Central/South America & $-1.82 \mathrm{E}-04$ & $1.78 \mathrm{E}-03$ & $-1.48 \mathrm{E}-03$ & 4.19E-03 & $2.70 \mathrm{E}-04$ & $1.37 \mathrm{E}-03$ & $2.08 \mathrm{E}-04$ \\
\hline & Northern Asia & $1.01 \mathrm{E}-03$ & $9.00 \mathrm{E}-03$ & $5.82 \mathrm{E}-04$ & $3.04 \mathrm{E}-02$ & $-3.67 \mathrm{E}-04$ & $1.40 \mathrm{E}-02$ & $1.00 \mathrm{E}-04$ \\
\hline & Europe & $-2.18 \mathrm{E}-05$ & $7.25 \mathrm{E}-05$ & $4.52 \mathrm{E}-06$ & 3.95E-04 & $2.59 \mathrm{E}-04$ & $2.71 \mathrm{E}-04$ & $4.50 \mathrm{E}-06$ \\
\hline & North America & $1.00 \mathrm{E}-03$ & $3.94 \mathrm{E}-03$ & $2.31 \mathrm{E}-04$ & 2.93E-02 & $1.99 \mathrm{E}-03$ & $-8.68 \mathrm{E}-02$ & $7.35 \mathrm{E}-05$ \\
\hline & Oceania & $1.47 \mathrm{E}-04$ & $1.40 \mathrm{E}-03$ & $-6.61 \mathrm{E}-04$ & $3.60 \mathrm{E}-04$ & $2.33 \mathrm{E}-04$ & $5.67 \mathrm{E}-05$ & $-5.36 \mathrm{E}-05$ \\
\hline
\end{tabular}

Fig. S2aj. Correlation of the emissions in 2002 for industrial. 


\begin{tabular}{|c|c|c|c|c|c|c|c|c|}
\hline & \multirow{2}{*}{\multicolumn{7}{|c|}{ Natural Soil }} \\
\hline & & & & & & & & \\
\hline Source sector & Region & $\begin{array}{l}\text { Africa/M. } \\
\text { East }\end{array}$ & $\begin{array}{l}\text { Southern } \\
\text { Asia }\end{array}$ & $\begin{array}{l}\text { C. /S. } \\
\text { America }\end{array}$ & $\begin{array}{l}\text { Northern } \\
\text { Asia }\end{array}$ & Europe & $\begin{array}{l}\text { North } \\
\text { America }\end{array}$ & Oceania \\
\hline \multirow{7}{*}{$\begin{array}{l}\text { Agricultural } \\
\text { Soil }\end{array}$} & Africa/Middle East & $-1.87 \mathrm{E}-01$ & $-7.12 \mathrm{E}-02$ & $-5.83 \mathrm{E}-02$ & \begin{tabular}{|r|}
$2.81 \mathrm{E}-02$ \\
\end{tabular} & $-2.76 \mathrm{E}-02$ & $-2.91 \mathrm{E}-02$ & $4.95 \mathrm{E}-03$ \\
\hline & Southern Asia & $-5.15 \mathrm{E}-02$ & $-4.33 \mathrm{E}-01$ & $8.43 \mathrm{E}-02$ & $3.52 \mathrm{E}-02$ & $-7.97 \mathrm{E}-03$ & $-2.38 \mathrm{E}-02$ & $1.20 \mathrm{E}-02$ \\
\hline & Central/South America & $-6.86 \mathrm{E}-02$ & $-4.95 \mathrm{E}-03$ & $-3.08 \mathrm{E}-01$ & $1.00 \mathrm{E}-02$ & $8.10 \mathrm{E}-03$ & $1.85 \mathrm{E}-02$ & $-1.43 \mathrm{E}-02$ \\
\hline & Northern Asia & $6.24 \mathrm{E}-03$ & $-7.06 \mathrm{E}-02$ & $4.40 \mathrm{E}-03$ & \begin{tabular}{|l|}
$-1.51 \mathrm{E}-01$ \\
\end{tabular} & $-4.09 \mathrm{E}-02$ & $6.14 \mathrm{E}-02$ & $-1.37 E-02$ \\
\hline & Europe & $-1.08 \mathrm{E}-02$ & $-1.48 \mathrm{E}-02$ & $2.76 \mathrm{E}-02$ & $3.26 \mathrm{E}-02$ & $-2.89 \mathrm{E}-01$ & $-1.19 \mathrm{E}-02$ & $4.13 \mathrm{E}-03$ \\
\hline & \begin{tabular}{|l|} 
North America \\
\end{tabular} & $1.94 \mathrm{E}-02$ & $-1.21 \mathrm{E}-02$ & $3.41 \mathrm{E}-02$ & $7.04 \mathrm{E}-02$ & $2.17 \mathrm{E}-02$ & $-4.98 \mathrm{E}-01$ & $1.62 \mathrm{E}-03$ \\
\hline & Oceania & $6.36 \mathrm{E}-03$ & $2.24 \mathrm{E}-02$ & $-7.85 \mathrm{E}-02$ & $-1.21 \mathrm{E}-02$ & $4.18 \mathrm{E}-03$ & $8.78 \mathrm{E}-03$ & $-5.50 \mathrm{E}-02$ \\
\hline \multirow{7}{*}{ Industrial } & Africa/Middle East & $-3.00 \mathrm{E}-02$ & $-9.28 \mathrm{E}-03$ & $4.75 \mathrm{E}-04$ & $5.22 \mathrm{E}-03$ & $-9.74 \mathrm{E}-03$ & $-2.56 \mathrm{E}-03$ & $8.92 \mathrm{E}-04$ \\
\hline & \begin{tabular}{|l|} 
Southern Asia \\
\end{tabular} & $2.50 \mathrm{E}-02$ & $-9.23 \mathrm{E}-02$ & $1.49 \mathrm{E}-02$ & $5.28 \mathrm{E}-03$ & $5.03 \mathrm{E}-03$ & $2.42 \mathrm{E}-02$ & $3.54 \mathrm{E}-04$ \\
\hline & Central/South America & $-6.41 \mathrm{E}-03$ & $-9.29 \mathrm{E}-04$ & $-2.64 \mathrm{E}-02$ & $9.99 \mathrm{E}-04$ & $6.71 \mathrm{E}-04$ & $1.41 \mathrm{E}-03$ & $-1.21 \mathrm{E}-03$ \\
\hline & Northern Asia & $6.50 \mathrm{E}-02$ & $3.71 \mathrm{E}-02$ & $4.51 \mathrm{E}-02$ & \begin{tabular}{|l|}
$-6.95 \mathrm{E}-02$ \\
\end{tabular} & $1.00 \mathrm{E}-02$ & $-2.45 \mathrm{E}-01$ & $-4.85 \mathrm{E}-03$ \\
\hline & Europe & $-1.83 \mathrm{E}-03$ & $-5.02 \mathrm{E}-03$ & $1.75 \mathrm{E}-02$ & $1.71 \mathrm{E}-02$ & $-1.69 \mathrm{E}-01$ & $1.77 \mathrm{E}-02$ & $1.89 \mathrm{E}-03$ \\
\hline & North America & $1.92 \mathrm{E}-02$ & $1.62 \mathrm{E}-02$ & $1.56 \mathrm{E}-02$ & $1.42 \mathrm{E}-02$ & $2.02 \mathrm{E}-02$ & $-1.78 \mathrm{E}-01$ & $-5.91 E-04$ \\
\hline & Oceania & 5.29E-04 & $1.89 \mathrm{E}-03$ & $-5.34 \mathrm{E}-03$ & $-1.08 \mathrm{E}-03$ & $3.18 \mathrm{E}-04$ & 5.79E-04 & $-4.22 \mathrm{E}-03$ \\
\hline \multirow{7}{*}{ Natural Soil } & Africa/Middle East & 1 & $-2.40 \mathrm{E}-01$ & $-4.60 \mathrm{E}-01$ & $8.25 \mathrm{E}-02$ & $-2.58 \mathrm{E}-02$ & $-9.29 \mathrm{E}-02$ & 8.97E-04 \\
\hline & Southern Asia & $-2.40 \mathrm{E}-01$ & 1 & $-1.38 \mathrm{E}-01$ & $-3.88 \mathrm{E}-01$ & $4.13 \mathrm{E}-02$ & $-9.82 \mathrm{E}-02$ & $6.28 \mathrm{E}-03$ \\
\hline & Central/South America & $-4.60 \mathrm{E}-01$ & $-1.38 \mathrm{E}-01$ & 1 & \begin{tabular}{|l|}
$-4.67 E-02$ \\
\end{tabular} & $1.33 \mathrm{E}-03$ & $-1.73 \mathrm{E}-03$ & $-1.49 \mathrm{E}-01$ \\
\hline & Northern Asia & $8.25 \mathrm{E}-02$ & $-3.88 \mathrm{E}-01$ & $-4.67 \mathrm{E}-02$ & 1 & $-1.62 \mathrm{E}-01$ & $-1.91 \mathrm{E}-01$ & $-1.82 \mathrm{E}-02$ \\
\hline & Europe & $-2.58 \mathrm{E}-02$ & $4.13 \mathrm{E}-02$ & $1.33 \mathrm{E}-03$ & \begin{tabular}{|l|}
$-1.62 \mathrm{E}-01$ \\
\end{tabular} & 1 & $-9.00 \mathrm{E}-02$ & $2.10 \mathrm{E}-03$ \\
\hline & North America & $-9.29 \mathrm{E}-02$ & $-9.82 \mathrm{E}-02$ & $-1.73 \mathrm{E}-03$ & \begin{tabular}{|l|}
$-1.91 \mathrm{E}-01$ \\
\end{tabular} & $-9.00 \mathrm{E}-02$ & 1 & 1.17E-02 \\
\hline & Oceania & 8.97E-04 & $6.28 \mathrm{E}-03$ & $-1.49 \mathrm{E}-01$ & $-1.82 \mathrm{E}-02$ & $2.10 \mathrm{E}-03$ & $1.17 \mathrm{E}-02$ & 1 \\
\hline \multirow{6}{*}{ Ocean } & North Pacific & $-4.82 \mathrm{E}-04$ & $-2.08 \mathrm{E}-03$ & $2.21 \mathrm{E}-03$ & $-3.65 \mathrm{E}-03$ & $-5.49 \mathrm{E}-03$ & $-5.10 \mathrm{E}-03$ & $-6.30 \mathrm{E}-04$ \\
\hline & \begin{tabular}{|l} 
South Pacific \\
\end{tabular} & $3.76 \mathrm{E}-03$ & $7.40 \mathrm{E}-03$ & $1.55 \mathrm{E}-03$ & $-1.30 \mathrm{E}-03$ & $-7.84 \mathrm{E}-04$ & 2.27E-03 & $1.19 \mathrm{E}-03$ \\
\hline & Northern Ocean & $2.54 \mathrm{E}-03$ & $5.58 \mathrm{E}-03$ & $-1.04 \mathrm{E}-02$ & $-4.48 \mathrm{E}-03$ & $-4.70 \mathrm{E}-03$ & $-1.11 \mathrm{E}-03$ & $-1.93 \mathrm{E}-03$ \\
\hline & Atlantic & $1.02 \mathrm{E}-02$ & $1.81 \mathrm{E}-02$ & $-3.19 \mathrm{E}-02$ & $-5.69 E-03$ & $-1.33 \mathrm{E}-04$ & $4.32 \mathrm{E}-03$ & $-6.88 \mathrm{E}-03$ \\
\hline & Southern Ocean & $-1.10 \mathrm{E}-02$ & $-2.98 \mathrm{E}-02$ & $5.59 \mathrm{E}-02$ & $7.86 \mathrm{E}-03$ & $-2.18 \mathrm{E}-03$ & $-1.07 \mathrm{E}-02$ & $2.75 \mathrm{E}-03$ \\
\hline & Indian Ocean & $4.76 \mathrm{E}-03$ & $1.05 \mathrm{E}-02$ & $-2.28 \mathrm{E}-02$ & $-5.46 \mathrm{E}-03$ & $-1.35 \mathrm{E}-03$ & $9.31 \mathrm{E}-04$ & $-3.97 \mathrm{E}-03$ \\
\hline \multirow{7}{*}{$\begin{array}{l}\text { Biomass } \\
\text { burning }\end{array}$} & Africa/Middle East & $-1.03 E-01$ & $-2.61 \mathrm{E}-02$ & $-8.60 \mathrm{E}-02$ & $2.18 \mathrm{E}-02$ & $5.19 \mathrm{E}-03$ & $1.32 \mathrm{E}-02$ & $2.83 \mathrm{E}-03$ \\
\hline & Southern Asia & $-6.17 \mathrm{E}-03$ & $-3.42 \mathrm{E}-03$ & $-2.24 \mathrm{E}-02$ & $-3.48 \mathrm{E}-03$ & $-2.20 \mathrm{E}-03$ & $5.73 \mathrm{E}-03$ & $-2.29 \mathrm{E}-03$ \\
\hline & Central/South America & $-2.29 \mathrm{E}-02$ & $-1.20 \mathrm{E}-02$ & $-2.45 \mathrm{E}-02$ & $9.34 \mathrm{E}-03$ & $2.93 \mathrm{E}-03$ & $7.19 \mathrm{E}-03$ & $2.35 \mathrm{E}-03$ \\
\hline & Northern Asia & $1.48 \mathrm{E}-02$ & $-4.35 \mathrm{E}-02$ & $9.33 \mathrm{E}-03$ & $-2.32 \mathrm{E}-01$ & $-3.53 \mathrm{E}-02$ & $-1.12 \mathrm{E}-01$ & $4.19 \mathrm{E}-03$ \\
\hline & Europe & $-1.73 \mathrm{E}-04$ & $5.22 \mathrm{E}-04$ & $1.01 \mathrm{E}-04$ & $-3.20 \mathrm{E}-03$ & $-7.44 \mathrm{E}-03$ & $-1.08 \mathrm{E}-03$ & $7.00 \mathrm{E}-05$ \\
\hline & North America & $4.16 \mathrm{E}-03$ & $-1.56 \mathrm{E}-03$ & $-2.62 \mathrm{E}-02$ & $-1.30 \mathrm{E}-02$ & $-7.97 \mathrm{E}-03$ & $-4.80 \mathrm{E}-02$ & $-1.67 \mathrm{E}-03$ \\
\hline & Oceania & $-7.12 \mathrm{E}-03$ & $-9.94 \mathrm{E}-03$ & $-1.44 \mathrm{E}-02$ & $2.88 \mathrm{E}-03$ & $2.16 \mathrm{E}-03$ & $6.22 \mathrm{E}-03$ & $-3.60 \mathrm{E}-03$ \\
\hline
\end{tabular}

Fig. S2ak. Correlation of the emissions in 2002 for natural soil. 


\begin{tabular}{|c|c|c|c|c|c|c|c|}
\hline \multirow[b]{2}{*}{ Source sector } & \multirow[b]{2}{*}{ Region } & \multicolumn{6}{|c|}{ Ocean } \\
\hline & & $\begin{array}{l}\text { North } \\
\text { Pacific }\end{array}$ & $\begin{array}{l}\text { South } \\
\text { Pacific }\end{array}$ & $\begin{array}{l}\text { Northern } \\
\text { Ocean }\end{array}$ & Atlantic & $\begin{array}{l}\text { Southern } \\
\text { Ocean }\end{array}$ & $\begin{array}{l}\text { Indian } \\
\text { Ocean }\end{array}$ \\
\hline \multirow{7}{*}{$\begin{array}{l}\text { Agricultural } \\
\text { Soil }\end{array}$} & Africa/Middle East & $1.70 \mathrm{E}-03$ & $-1.23 \mathrm{E}-03$ & 7.37E-05 & 7.03E-04 & $4.31 \mathrm{E}-03$ & 3.03E-04 \\
\hline & Southern Asia & $9.53 \mathrm{E}-03$ & $-9.43 E-03$ & $-2.46 \mathrm{E}-03$ & $-1.12 \mathrm{E}-02$ & $2.78 \mathrm{E}-02$ & $-5.27 \mathrm{E}-03$ \\
\hline & Central/South America & $8.74 \mathrm{E}-04$ & $5.53 \mathrm{E}-04$ & $7.41 \mathrm{E}-04$ & $2.73 \mathrm{E}-03$ & $5.52 \mathrm{E}-03$ & $-5.01 \mathrm{E}-05$ \\
\hline & \begin{tabular}{|l} 
Northern Asia \\
\end{tabular} & $2.90 \mathrm{E}-03$ & $1.29 \mathrm{E}-03$ & $1.13 \mathrm{E}-03$ & $-1.61 \mathrm{E}-03$ & $2.34 \mathrm{E}-03$ & $-1.67 \mathrm{E}-04$ \\
\hline & Europe & $2.69 E-03$ & $1.05 \mathrm{E}-03$ & $2.92 \mathrm{E}-03$ & $1.14 \mathrm{E}-03$ & $5.73 \mathrm{E}-04$ & $7.68 \mathrm{E}-04$ \\
\hline & \begin{tabular}{|l|} 
North America \\
\end{tabular} & $5.30 \mathrm{E}-03$ & $-1.85 \mathrm{E}-03$ & $2.05 \mathrm{E}-04$ & $-4.59 E-03$ & $1.10 \mathrm{E}-02$ & $-1.16 \mathrm{E}-03$ \\
\hline & Oceania & $-6.94 \mathrm{E}-04$ & $1.50 \mathrm{E}-03$ & $-1.19 \mathrm{E}-03$ & $-4.98 \mathrm{E}-03$ & $-7.78 \mathrm{E}-04$ & $-2.50 \mathrm{E}-03$ \\
\hline \multirow{7}{*}{ Industrial } & Africa/Middle East & 3.97E-04 & $-2.86 \mathrm{E}-04$ & $-5.90 \mathrm{E}-05$ & $-2.23 \mathrm{E}-04$ & $1.11 \mathrm{E}-03$ & $-5.51 \mathrm{E}-05$ \\
\hline & Southern Asia & $8.27 \mathrm{E}-04$ & $-6.05 \mathrm{E}-04$ & $-1.93 \mathrm{E}-04$ & $-1.69 \mathrm{E}-03$ & $3.18 \mathrm{E}-03$ & $-8.97 \mathrm{E}-04$ \\
\hline & Central/South America & $8.19 \mathrm{E}-05$ & $2.65 \mathrm{E}-05$ & $7.45 \mathrm{E}-05$ & $2.80 \mathrm{E}-04$ & $4.83 \mathrm{E}-04$ & $1.47 \mathrm{E}-05$ \\
\hline & \begin{tabular}{|l|} 
Northern Asia \\
\end{tabular} & $4.94 \mathrm{E}-04$ & $2.15 \mathrm{E}-03$ & $1.27 \mathrm{E}-03$ & $1.69 \mathrm{E}-03$ & $-2.98 \mathrm{E}-03$ & $4.55 \mathrm{E}-04$ \\
\hline & Europe & $3.35 \mathrm{E}-03$ & $1.53 \mathrm{E}-04$ & $2.34 \mathrm{E}-03$ & $-3.11 \mathrm{E}-04$ & E-03 & $8 \mathrm{E}-04$ \\
\hline & North America & $1.81 \mathrm{E}-03$ & $-2.14 \mathrm{E}-04$ & $4.81 \mathrm{E}-04$ & $-2.98 \mathrm{E}-04$ & $1.44 \mathrm{E}-03$ & $1.15 \mathrm{E}-04$ \\
\hline & Oceania & $-5.53 \mathrm{E}-05$ & $1.09 \mathrm{E}-04$ & $-9.27 \mathrm{E}-05$ & $-3.73 \mathrm{E}-04$ & $-4.19 \mathrm{E}-05$ & $-1.86 \mathrm{E}-04$ \\
\hline \multirow{7}{*}{ Natural Soil } & Africa/Middle East & $-4.82 \mathrm{E}-04$ & $3.76 \mathrm{E}-03$ & $2.54 \mathrm{E}-03$ & $1.02 \mathrm{E}-02$ & $-1.10 \mathrm{E}-02$ & $4.76 \mathrm{E}-03$ \\
\hline & Southern Asia & $-2.08 \mathrm{E}-03$ & $7.40 \mathrm{E}-03$ & $5.58 \mathrm{E}-03$ & $1.81 \mathrm{E}-02$ & $-2.98 \mathrm{E}-02$ & $1.05 \mathrm{E}-02$ \\
\hline & \begin{tabular}{|l|} 
Central/South America \\
\end{tabular} & $2.21 \mathrm{E}-03$ & $1.55 \mathrm{E}-03$ & $-1.04 \mathrm{E}-02$ & $-3.19 E-02$ & $5.59 \mathrm{E}-02$ & $-2.28 \mathrm{E}-02$ \\
\hline & \begin{tabular}{|l|} 
Northern Asia \\
\end{tabular} & $-3.65 \mathrm{E}-03$ & $-1.30 \mathrm{E}-03$ & $-4.48 \mathrm{E}-03$ & $-5.69 E-03$ & $7.86 \mathrm{E}-03$ & $-5.46 \mathrm{E}-03$ \\
\hline & Europe & $9 \mathrm{E}-03$ & $-7.84 \mathrm{E}-04$ & $-4.70 \mathrm{E}-03$ & $-1.33 \mathrm{E}-04$ & $8 \mathrm{E}-03$ & $5 \mathrm{E}-03$ \\
\hline & \begin{tabular}{|l} 
North America \\
\end{tabular} & $-5.10 \mathrm{E}-03$ & $2.27 \mathrm{E}-03$ & $-1.11 \mathrm{E}-03$ & $4.32 \mathrm{E}-03$ & $-1.07 E-02$ & $9.31 \mathrm{E}-04$ \\
\hline & Oceania & $-6.30 \mathrm{E}-04$ & $1.19 \mathrm{E}-03$ & $-1.93 \mathrm{E}-03$ & $-6.88 \mathrm{E}-03$ & $2.75 \mathrm{E}-03$ & $-3.97 E-03$ \\
\hline \multirow{6}{*}{ Ocean } & North Pacific & 1 & $-2.07 E-02$ & $-7.79 \mathrm{E}-02$ & $-2.02 \mathrm{E}-01$ & $2.67 \mathrm{E}-02$ & $4.37 \mathrm{E}-02$ \\
\hline & South Pacific & $-2.07 \mathrm{E}-02$ & 1 & $-3.92 \mathrm{E}-03$ & $-9.97 E-02$ & $-1.45 \mathrm{E}-02$ & $-3.44 \mathrm{E}-02$ \\
\hline & \begin{tabular}{|l|} 
Northern Ocean \\
\end{tabular} & $-7.79 \mathrm{E}-02$ & $-3.92 \mathrm{E}-03$ & 1 & $-2.80 \mathrm{E}-01$ & $2.07 \mathrm{E}-02$ & $4.46 \mathrm{E}-03$ \\
\hline & Atlantic & $-2.02 \mathrm{E}-01$ & $-9.97 \mathrm{E}-02$ & $-2.80 \mathrm{E}-01$ & 1 & $-5.24 \mathrm{E}-02$ & $-2.35 \mathrm{E}-02$ \\
\hline & Southern Ocean & $2.67 \mathrm{E}-02$ & $-1.45 \mathrm{E}-02$ & $2.07 E-02$ & $-5.24 \mathrm{E}-02$ & 1 & $-5.48 \mathrm{E}-01$ \\
\hline & Indian Ocean & $4.37 \mathrm{E}-02$ & $-3.44 \mathrm{E}-02$ & $4.46 \mathrm{E}-03$ & $-2.35 \mathrm{E}-02$ & $-5.48 \mathrm{E}-01$ & 1 \\
\hline \multirow{7}{*}{$\begin{array}{l}\text { Biomass } \\
\text { burning }\end{array}$} & Africa/Middle East & $1.08 \mathrm{E}-03$ & $-9.66 \mathrm{E}-04$ & $1.36 \mathrm{E}-03$ & $4.86 \mathrm{E}-03$ & $1.18 \mathrm{E}-03$ & $1.90 \mathrm{E}-03$ \\
\hline & Southern Asia & $2.24 \mathrm{E}-04$ & $-1.14 \mathrm{E}-03$ & $-5.20 \mathrm{E}-04$ & $-9.23 E-04$ & $3.05 \mathrm{E}-03$ & $-8.45 \mathrm{E}-04$ \\
\hline & Central/South America & $5.37 \mathrm{E}-04$ & $-1.57 \mathrm{E}-04$ & $1.22 \mathrm{E}-03$ & $4.31 \mathrm{E}-03$ & $-4.06 \mathrm{E}-04$ & $1.76 \mathrm{E}-03$ \\
\hline & \begin{tabular}{|l} 
Northern Asia \\
\end{tabular} & $2.79 \mathrm{E}-05$ & $1.27 \mathrm{E}-03$ & 5.91E-04 & $1.33 \mathrm{E}-03$ & $-2.58 \mathrm{E}-03$ & $7.72 \mathrm{E}-04$ \\
\hline & Europe & $4.46 \mathrm{E}-06$ & $4.22 \mathrm{E}-06$ & $5.29 \mathrm{E}-06$ & $1.45 \mathrm{E}-05$ & $-2.27 \mathrm{E}-05$ & $1.24 \mathrm{E}-05$ \\
\hline & North America & $-1.05 \mathrm{E}-03$ & $7.49 \mathrm{E}-04$ & $-1.90 \mathrm{E}-05$ & $8.57 \mathrm{E}-04$ & $-2.27 \mathrm{E}-03$ & $-7.00 \mathrm{E}-05$ \\
\hline & Oceania & $2.69 \mathrm{E}-04$ & $2.49 \mathrm{E}-05$ & $4.99 \mathrm{E}-04$ & $1.54 \mathrm{E}-03$ & $-7.40 \mathrm{E}-04$ & $7.62 \mathrm{E}-04$ \\
\hline
\end{tabular}

Fig. S2al. Correlation of the emissions in 2002 for ocean. 


\begin{tabular}{|c|c|c|c|c|c|c|c|c|}
\hline \multirow[b]{3}{*}{ Source sector } & \multirow[b]{3}{*}{ Region } & \multirow{2}{*}{\multicolumn{7}{|c|}{ Biomass burning }} \\
\hline & & & & & & & & \\
\hline & & $\begin{array}{l}\text { Africa/M. } \\
\text { East }\end{array}$ & $\begin{array}{l}\text { Southern } \\
\text { Asia }\end{array}$ & $\begin{array}{l}\text { C. /S. } \\
\text { America }\end{array}$ & $\begin{array}{l}\text { Northern } \\
\text { Asia }\end{array}$ & Europe & $\begin{array}{l}\text { North } \\
\text { America }\end{array}$ & Oceania \\
\hline \multirow{7}{*}{$\begin{array}{l}\text { Agricultural } \\
\text { Soil }\end{array}$} & Africa/Middle East & $-2.70 \mathrm{E}-02$ & $-6.07 E-03$ & $-5.15 \mathrm{E}-03$ & $7.92 \mathrm{E}-03$ & $-2.46 \mathrm{E}-05$ & $4.65 \mathrm{E}-03$ & $-7.53 \mathrm{E}-04$ \\
\hline & Southern Asia & $-1.77 \mathrm{E}-02$ & $-3.16 \mathrm{E}-02$ & $7.11 \mathrm{E}-03$ & $-1.18 \mathrm{E}-02$ & $1.57 \mathrm{E}-04$ & $1.62 \mathrm{E}-02$ & $6.23 \mathrm{E}-03$ \\
\hline & Central/South America & $-3.53 \mathrm{E}-02$ & $-3.39 \mathrm{E}-03$ & $-1.56 \mathrm{E}-02$ & $6.29 \mathrm{E}-03$ & $5.54 \mathrm{E}-05$ & $2.87 \mathrm{E}-03$ & $-7.36 \mathrm{E}-03$ \\
\hline & \begin{tabular}{|l} 
Northern Asia \\
\end{tabular} & $1.23 \mathrm{E}-02$ & $4.31 \mathrm{E}-03$ & $2.41 \mathrm{E}-03$ & $1.59 \mathrm{E}-02$ & $-3.52 \mathrm{E}-04$ & $1.82 \mathrm{E}-02$ & $-2.05 \mathrm{E}-03$ \\
\hline & \begin{tabular}{|l} 
Europe \\
\end{tabular} & $5.36 \mathrm{E}-03$ & $2.59 \mathrm{E}-03$ & $1.92 \mathrm{E}-03$ & $-7.03 \mathrm{E}-03$ & $9.44 \mathrm{E}-05$ & $1.21 \mathrm{E}-03$ & $1.56 \mathrm{E}-03$ \\
\hline & \begin{tabular}{|l} 
North America \\
\end{tabular} & $7.24 \mathrm{E}-03$ & $-2.07 \mathrm{E}-03$ & $4.38 \mathrm{E}-03$ & $2.63 \mathrm{E}-02$ & $3.44 \mathrm{E}-04$ & $2.21 \mathrm{E}-02$ & $1.94 \mathrm{E}-03$ \\
\hline & Oceania & $5.75 \mathrm{E}-03$ & $1.90 \mathrm{E}-03$ & $2.06 \mathrm{E}-03$ & $1.24 \mathrm{E}-03$ & $5.55 \mathrm{E}-05$ & $1.05 \mathrm{E}-03$ & $-1.46 \mathrm{E}-03$ \\
\hline \multirow{7}{*}{ Industrial } & Africa/Middle East & $-1.95 \mathrm{E}-03$ & $-7.48 \mathrm{E}-04$ & $-1.82 \mathrm{E}-04$ & $1.01 \mathrm{E}-03$ & $-2.18 \mathrm{E}-05$ & $1.00 \mathrm{E}-03$ & $1.47 \mathrm{E}-04$ \\
\hline & Southern Asia & $4.07 \mathrm{E}-03$ & $-6.29 \mathrm{E}-04$ & $1.78 \mathrm{E}-03$ & $9.00 \mathrm{E}-03$ & $7.25 \mathrm{E}-05$ & $3.94 \mathrm{E}-03$ & $1.40 \mathrm{E}-03$ \\
\hline & Central/South America & $-3.39 \mathrm{E}-03$ & $-3.49 \mathrm{E}-04$ & $-1.48 \mathrm{E}-03$ & $5.82 \mathrm{E}-04$ & $4.52 \mathrm{E}-06$ & $2.31 \mathrm{E}-04$ & $-6.61 \mathrm{E}-04$ \\
\hline & Northern Asia & $1.64 \mathrm{E}-02$ & $4.60 \mathrm{E}-03$ & $4.19 \mathrm{E}-03$ & $3.04 \mathrm{E}-02$ & $3.95 E-04$ & $2.93 \mathrm{E}-02$ & $3.60 \mathrm{E}-04$ \\
\hline & Europe & $2.11 \mathrm{E}-03$ & $1.15 \mathrm{E}-03$ & $2.70 \mathrm{E}-04$ & $-3.67 \mathrm{E}-04$ & $2.59 \mathrm{E}-04$ & $1.99 \mathrm{E}-03$ & $2.33 \mathrm{E}-04$ \\
\hline & North America & $3.76 \mathrm{E}-03$ & $-9.99 \mathrm{E}-05$ & $1.37 \mathrm{E}-03$ & $1.40 \mathrm{E}-02$ & $2.71 \mathrm{E}-04$ & $-8.68 \mathrm{E}-02$ & $5.67 \mathrm{E}-05$ \\
\hline & Oceania & $4.80 \mathrm{E}-04$ & $1.84 \mathrm{E}-04$ & $2.08 \mathrm{E}-04$ & $1.00 \mathrm{E}-04$ & $4.50 \mathrm{E}-06$ & $7.35 \mathrm{E}-05$ & $-5.36 \mathrm{E}-05$ \\
\hline \multirow{7}{*}{ Natural Soil } & Africa/Middle East & $-1.03 \mathrm{E}-01$ & $-6.17 \mathrm{E}-03$ & $-2.29 \mathrm{E}-02$ & $1.48 \mathrm{E}-02$ & $-1.73 \mathrm{E}-04$ & $4.16 \mathrm{E}-03$ & $-7.12 \mathrm{E}-03$ \\
\hline & Southern Asia & $-2.61 \mathrm{E}-02$ & $-3.42 \mathrm{E}-03$ & $-1.20 \mathrm{E}-02$ & $-4.35 \mathrm{E}-02$ & $5.22 \mathrm{E}-04$ & $-1.56 \mathrm{E}-03$ & $-9.94 \mathrm{E}-03$ \\
\hline & Central/South America & $-8.60 \mathrm{E}-02$ & $-2.24 \mathrm{E}-02$ & $-2.45 \mathrm{E}-02$ & $9.33 \mathrm{E}-03$ & $1.01 \mathrm{E}-04$ & $-2.62 \mathrm{E}-02$ & $-1.44 \mathrm{E}-02$ \\
\hline & Northern Asia & $2.18 \mathrm{E}-02$ & $-3.48 \mathrm{E}-03$ & $9.34 \mathrm{E}-03$ & $-2.32 \mathrm{E}-01$ & $-3.20 \mathrm{E}-03$ & $-1.30 \mathrm{E}-02$ & $2.88 \mathrm{E}-03$ \\
\hline & Europe & $5.19 \mathrm{E}-03$ & $-2.20 \mathrm{E}-03$ & $2.93 \mathrm{E}-03$ & $-3.53 \mathrm{E}-02$ & $-7.44 \mathrm{E}-03$ & $-7.97 \mathrm{E}-03$ & $2.16 \mathrm{E}-03$ \\
\hline & North America & $1.32 \mathrm{E}-02$ & $5.73 \mathrm{E}-03$ & $7.19 \mathrm{E}-03$ & $-1.12 \mathrm{E}-01$ & $-1.08 \mathrm{E}-03$ & $-4.80 \mathrm{E}-02$ & $6.22 \mathrm{E}-03$ \\
\hline & Oceania & $2.83 \mathrm{E}-03$ & $-2.29 \mathrm{E}-03$ & $2.35 \mathrm{E}-03$ & $4.19 \mathrm{E}-03$ & $7.00 \mathrm{E}-05$ & $-1.67 \mathrm{E}-03$ & $-3.60 \mathrm{E}-03$ \\
\hline \multirow{6}{*}{ Ocean } & North Pacific & $1.08 \mathrm{E}-03$ & $2.24 \mathrm{E}-04$ & $5.37 \mathrm{E}-04$ & $2.79 \mathrm{E}-05$ & $4.46 \mathrm{E}-06$ & $-1.05 \mathrm{E}-03$ & $2.69 \mathrm{E}-04$ \\
\hline & South Pacific & $-9.66 \mathrm{E}-04$ & $-1.14 \mathrm{E}-03$ & $-1.57 \mathrm{E}-04$ & $1.27 \mathrm{E}-03$ & $4.22 \mathrm{E}-06$ & $7.49 \mathrm{E}-04$ & $2.49 \mathrm{E}-05$ \\
\hline & Northern Ocean & $1.36 \mathrm{E}-03$ & $-5.20 \mathrm{E}-04$ & $1.22 \mathrm{E}-03$ & $5.91 \mathrm{E}-04$ & $5.29 \mathrm{E}-06$ & $-1.90 \mathrm{E}-05$ & $4.99 \mathrm{E}-04$ \\
\hline & Atlantic & $4.86 \mathrm{E}-03$ & $-9.23 \mathrm{E}-04$ & $4.31 \mathrm{E}-03$ & $1.33 \mathrm{E}-03$ & $1.45 \mathrm{E}-05$ & 8.57E-04 & $1.54 \mathrm{E}-03$ \\
\hline & Southern Ocean & $1.18 \mathrm{E}-03$ & $3.05 \mathrm{E}-03$ & $-4.06 \mathrm{E}-04$ & $-2.58 \mathrm{E}-03$ & $-2.27 \mathrm{E}-05$ & \begin{tabular}{|l|}
$-2.27 \mathrm{E}-03$ \\
\end{tabular} & $-7.40 \mathrm{E}-04$ \\
\hline & Indian Ocean & $1.90 \mathrm{E}-03$ & $-8.45 \mathrm{E}-04$ & $1.76 \mathrm{E}-03$ & $7.72 \mathrm{E}-04$ & $1.24 \mathrm{E}-05$ & \begin{tabular}{|l|}
$-7.00 \mathrm{E}-05$ \\
\end{tabular} & $7.62 \mathrm{E}-04$ \\
\hline \multirow{7}{*}{$\begin{array}{l}\text { Biomass } \\
\text { burning }\end{array}$} & Africa/Middle East & 1 & $-6.97 \mathrm{E}-03$ & $-1.91 \mathrm{E}-02$ & $1.24 \mathrm{E}-02$ & $6.99 \mathrm{E}-05$ & 3.99E-03 & $-6.29 \mathrm{E}-03$ \\
\hline & Southern Asia & $-6.97 E-03$ & 1 & $-2.92 \mathrm{E}-03$ & $5.40 \mathrm{E}-03$ & $5.81 \mathrm{E}-05$ & \begin{tabular}{|l|}
$6.30 \mathrm{E}-04$ \\
\end{tabular} & $-3.10 \mathrm{E}-03$ \\
\hline & Central/South America & $-1.91 \mathrm{E}-02$ & $-2.92 \mathrm{E}-03$ & 1 & $3.70 \mathrm{E}-03$ & $1.96 \mathrm{E}-05$ & $1.12 \mathrm{E}-03$ & $-3.94 \mathrm{E}-03$ \\
\hline & Northern Asia & $1.24 \mathrm{E}-02$ & $5.40 \mathrm{E}-03$ & $3.70 \mathrm{E}-03$ & 1 & $-1.26 \mathrm{E}-03$ & \begin{tabular}{|l|} 
\\
\end{tabular} & $1.95 \mathrm{E}-03$ \\
\hline & Europe & $6.99 \mathrm{E}-05$ & $5.81 \mathrm{E}-05$ & $1.96 \mathrm{E}-05$ & $-1.26 \mathrm{E}-03$ & 1 & $1.03 \mathrm{E}-04$ & $1.74 \mathrm{E}-05$ \\
\hline & North America & $3.99 \mathrm{E}-03$ & $6.30 \mathrm{E}-04$ & $1.12 \mathrm{E}-03$ & $-7.31 \mathrm{E}-03$ & $1.03 \mathrm{E}-04$ & 1 & $4.83 \mathrm{E}-04$ \\
\hline & Oceania & $-6.29 \mathrm{E}-03$ & $-3.10 \mathrm{E}-03$ & $-3.94 E-03$ & $1.95 \mathrm{E}-03$ & $1.74 \mathrm{E}-05$ & \begin{tabular}{|l|l|} 
& $4.83 \mathrm{E}-04$ \\
\end{tabular} & 1 \\
\hline
\end{tabular}

Fig. S2am. Correlation of the emissions in 2002 for biomass burning. 


\begin{tabular}{|c|c|c|c|c|c|c|c|c|}
\hline \multirow[b]{2}{*}{ Source sector } & \multirow[b]{2}{*}{ Region } & \multicolumn{7}{|c|}{ Agricultural Soil } \\
\hline & & $\begin{array}{l}\text { Africa/M. } \\
\text { East }\end{array}$ & $\begin{array}{l}\text { Southern } \\
\text { Asia }\end{array}$ & $\begin{array}{l}\text { C. /S. } \\
\text { America }\end{array}$ & $\begin{array}{l}\text { Northern } \\
\text { Asia }\end{array}$ & Europe & $\begin{array}{l}\text { North } \\
\text { America }\end{array}$ & Oceania \\
\hline \multirow{7}{*}{$\begin{array}{l}\text { Agricultural } \\
\text { Soil }\end{array}$} & Africa/Middle East & 1 & $-1.05 \mathrm{E}-01$ & $-3.13 \mathrm{E}-03$ & $-3.83 \mathrm{E}-02$ & $-1.74 \mathrm{E}-02$ & $-1.48 \mathrm{E}-02$ & $5.40 \mathrm{E}-03$ \\
\hline & Southern Asia & $-1.05 \mathrm{E}-01$ & 1 & $3.51 \mathrm{E}-02$ & $-2.94 \mathrm{E}-01$ & $4.27 \mathrm{E}-02$ & $-7.31 \mathrm{E}-02$ & $1.72 \mathrm{E}-02$ \\
\hline & Central/South America & $-3.13 E-03$ & $3.51 \mathrm{E}-02$ & 1 & $-5.78 \mathrm{E}-03$ & $7.86 \mathrm{E}-03$ & $2.62 \mathrm{E}-03$ & $-1.66 \mathrm{E}-02$ \\
\hline & Northern Asia & $-3.83 E-02$ & $-2.94 \mathrm{E}-01$ & $-5.78 \mathrm{E}-03$ & 1 & $-1.88 \mathrm{E}-01$ & $-4.57 \mathrm{E}-02$ & $-1.48 \mathrm{E}-02$ \\
\hline & Europe & $-1.74 \mathrm{E}-02$ & $4.27 \mathrm{E}-02$ & $7.86 \mathrm{E}-03$ & $-1.88 \mathrm{E}-01$ & 1 & $-9.85 \mathrm{E}-03$ & $3.20 \mathrm{E}-03$ \\
\hline & North America & $-1.48 \mathrm{E}-02$ & $-7.31 \mathrm{E}-02$ & $2.62 \mathrm{E}-03$ & $-4.57 \mathrm{E}-02$ & $-9.85 \mathrm{E}-03$ & 1 & $5.43 \mathrm{E}-04$ \\
\hline & Oceania & $5.40 \mathrm{E}-03$ & $1.72 \mathrm{E}-02$ & $-1.66 \mathrm{E}-02$ & $-1.48 \mathrm{E}-02$ & $3.20 \mathrm{E}-03$ & $5.43 \mathrm{E}-04$ & 1 \\
\hline \multirow{7}{*}{ Industrial } & Africa/Middle East & $-6.88 \mathrm{E}-02$ & $-1.64 \mathrm{E}-02$ & $9.34 \mathrm{E}-04$ & $-9.55 E-03$ & $-6.37 E-03$ & $-3.82 \mathrm{E}-03$ & $7.39 \mathrm{E}-04$ \\
\hline & \begin{tabular}{|l} 
Southern Asia \\
\end{tabular} & $1.95 \mathrm{E}-02$ & $-3.91 \mathrm{E}-01$ & $-5.02 E-04$ & $-4.84 \mathrm{E}-02$ & $2.06 \mathrm{E}-02$ & $-3.48 \mathrm{E}-03$ & $-2.03 \mathrm{E}-03$ \\
\hline & Central/South America & $-4.54 \mathrm{E}-04$ & $2.48 \mathrm{E}-03$ & $-6.17 \mathrm{E}-03$ & $-3.64 \mathrm{E}-04$ & $6.22 \mathrm{E}-04$ & $1.98 \mathrm{E}-04$ & $-1.21 \mathrm{E}-03$ \\
\hline & Northern Asia & $1.21 \mathrm{E}-02$ & $-1.32 \mathrm{E}-02$ & $3.75 \mathrm{E}-04$ & $-1.10 \mathrm{E}-01$ & $-3.76 \mathrm{E}-02$ & $2.02 \mathrm{E}-01$ & $-2.98 \mathrm{E}-03$ \\
\hline & Europe & $-2.28 \mathrm{E}-02$ & $-2.98 \mathrm{E}-03$ & $3.23 \mathrm{E}-03$ & $-3.69 E-02$ & $-7.50 \mathrm{E}-01$ & $-1.28 \mathrm{E}-02$ & $4.87 \mathrm{E}-04$ \\
\hline & North America & $9.08 \mathrm{E}-03$ & $2.73 \mathrm{E}-02$ & $1.74 \mathrm{E}-03$ & $4.12 \mathrm{E}-02$ & $2.99 \mathrm{E}-02$ & $-3.83 \mathrm{E}-01$ & $-5.71 \mathrm{E}-04$ \\
\hline & Oceania & $4.35 \mathrm{E}-04$ & $1.28 \mathrm{E}-03$ & $-1.20 \mathrm{E}-03$ & $-9.88 \mathrm{E}-04$ & $2.08 \mathrm{E}-04$ & $4.00 \mathrm{E}-05$ & $-5.66 \mathrm{E}-03$ \\
\hline \multirow{7}{*}{ Natural Soil } & Africa/Middle East & $-2.24 \mathrm{E}-01$ & $-1.52 \mathrm{E}-01$ & $-7.31 \mathrm{E}-02$ & $-9.90 \mathrm{E}-03$ & $-8.60 \mathrm{E}-03$ & $1.39 \mathrm{E}-02$ & $2.36 \mathrm{E}-03$ \\
\hline & Southern Asia & $-4.09 \mathrm{E}-02$ & $-2.89 \mathrm{E}-01$ & $4.46 \mathrm{E}-03$ & $9.35 \mathrm{E}-02$ & $-9.74 \mathrm{E}-03$ & $-2.29 \mathrm{E}-02$ & $1.77 \mathrm{E}-02$ \\
\hline & Central/South America & $-1.79 E-02$ & $8.44 \mathrm{E}-02$ & $-2.48 \mathrm{E}-01$ & $9.21 \mathrm{E}-03$ & $2.00 \mathrm{E}-02$ & $2.87 \mathrm{E}-02$ & $-5.35 \mathrm{E}-02$ \\
\hline & Northern Asia & $3.64 \mathrm{E}-02$ & $-2.51 \mathrm{E}-02$ & $-5.35 \mathrm{E}-03$ & $-2.05 \mathrm{E}-01$ & $2.28 \mathrm{E}-02$ & $7.02 \mathrm{E}-02$ & $-1.54 \mathrm{E}-02$ \\
\hline & Europe & $-2.46 \mathrm{E}-02$ & $-1.81 \mathrm{E}-02$ & $5.85 \mathrm{E}-03$ & $7.35 \mathrm{E}-04$ & $-2.19 \mathrm{E}-01$ & $2.43 \mathrm{E}-02$ & $2.80 \mathrm{E}-03$ \\
\hline & North America & $-3.69 \mathrm{E}-02$ & $-8.24 \mathrm{E}-02$ & $2.37 \mathrm{E}-02$ & $6.72 \mathrm{E}-02$ & $-1.68 \mathrm{E}-02$ & $-4.21 \mathrm{E}-01$ & $9.57 \mathrm{E}-03$ \\
\hline & Oceania & $2.05 \mathrm{E}-03$ & $1.54 \mathrm{E}-02$ & $-2.58 \mathrm{E}-02$ & $-7.02 \mathrm{E}-03$ & $4.80 \mathrm{E}-03$ & $1.40 \mathrm{E}-03$ & $-5.27 \mathrm{E}-02$ \\
\hline \multirow{6}{*}{ Ocean } & North Pacific & $-1.50 \mathrm{E}-02$ & $-1.55 \mathrm{E}-01$ & $2.38 \mathrm{E}-03$ & $3.17 \mathrm{E}-02$ & $8.67 \mathrm{E}-03$ & $-5.61 \mathrm{E}-02$ & $3.71 \mathrm{E}-03$ \\
\hline & South Pacific & $-1.39 \mathrm{E}-02$ & $-5.63 \mathrm{E}-03$ & $-3.94 \mathrm{E}-02$ & $1.65 \mathrm{E}-02$ & $1.34 \mathrm{E}-02$ & $1.49 \mathrm{E}-03$ & $2.65 \mathrm{E}-02$ \\
\hline & Northern Ocean & $4.72 \mathrm{E}-03$ & $-5.59 \mathrm{E}-03$ & $-5.81 \mathrm{E}-05$ & $-6.93 \mathrm{E}-02$ & $-8.71 \mathrm{E}-02$ & $-9.66 \mathrm{E}-03$ & $1.93 \mathrm{E}-03$ \\
\hline & Atlantic & $-7.68 \mathrm{E}-02$ & $-1.35 \mathrm{E}-01$ & $-3.04 \mathrm{E}-02$ & $-2.78 \mathrm{E}-02$ & $-5.66 \mathrm{E}-02$ & $-8.74 \mathrm{E}-02$ & $9.03 \mathrm{E}-03$ \\
\hline & Southern Ocean & $2.43 \mathrm{E}-02$ & $1.20 \mathrm{E}-01$ & $-1.83 \mathrm{E}-01$ & $-2.26 \mathrm{E}-02$ & $1.27 \mathrm{E}-02$ & $1.25 \mathrm{E}-03$ & $-9.08 \mathrm{E}-02$ \\
\hline & Indian Ocean & $-3.70 \mathrm{E}-02$ & $-5.31 \mathrm{E}-02$ & $-9.33 \mathrm{E}-02$ & $1.30 \mathrm{E}-03$ & $2.67 \mathrm{E}-03$ & $8.54 \mathrm{E}-03$ & $-2.24 \mathrm{E}-02$ \\
\hline \multirow{7}{*}{$\begin{array}{l}\text { Biomass } \\
\text { burning }\end{array}$} & Africa/Middle East & $-2.83 \mathrm{E}-02$ & $-2.20 \mathrm{E}-02$ & $-3.42 \mathrm{E}-02$ & $5.32 \mathrm{E}-03$ & $4.51 \mathrm{E}-03$ & $-8.16 \mathrm{E}-04$ & $4.18 \mathrm{E}-03$ \\
\hline & Southern Asia & $-2.14 \mathrm{E}-03$ & $-3.54 \mathrm{E}-02$ & $-2.11 \mathrm{E}-03$ & $-1.09 \mathrm{E}-03$ & $4.18 \mathrm{E}-03$ & $-3.57 \mathrm{E}-03$ & $3.02 \mathrm{E}-04$ \\
\hline & Central/South America & $-3.32 \mathrm{E}-03$ & $2.34 \mathrm{E}-03$ & $-1.16 \mathrm{E}-02$ & $-6.35 \mathrm{E}-04$ & $1.56 \mathrm{E}-03$ & $1.47 \mathrm{E}-04$ & $1.06 \mathrm{E}-03$ \\
\hline & \begin{tabular}{|l} 
Northern Asia \\
\end{tabular} & $1.37 \mathrm{E}-02$ & $-7.92 \mathrm{E}-02$ & $3.75 \mathrm{E}-03$ & $1.42 \mathrm{E}-02$ & $4.37 \mathrm{E}-03$ & $5.48 \mathrm{E}-02$ & $2.47 \mathrm{E}-03$ \\
\hline & Europe & $-1.97 \mathrm{E}-04$ & $-2.50 \mathrm{E}-04$ & $6.43 \mathrm{E}-05$ & $1.36 \mathrm{E}-04$ & $-2.67 \mathrm{E}-04$ & $2.98 \mathrm{E}-04$ & $7.39 \mathrm{E}-06$ \\
\hline & North America & $1.31 \mathrm{E}-03$ & $-1.85 \mathrm{E}-03$ & $1.22 \mathrm{E}-03$ & $5.67 \mathrm{E}-04$ & $-4.54 \mathrm{E}-03$ & $1.50 \mathrm{E}-02$ & $3.20 \mathrm{E}-04$ \\
\hline & Oceania & $1.60 \mathrm{E}-04$ & $2.81 \mathrm{E}-03$ & $-3.84 \mathrm{E}-03$ & $-9.29 \mathrm{E}-04$ & $7.02 \mathrm{E}-04$ & $8.22 \mathrm{E}-04$ & $-1.83 \mathrm{E}-03$ \\
\hline
\end{tabular}

Fig. S2an. Correlation of the emissions in 2003 for agricultural soil. 


\begin{tabular}{|c|c|c|c|c|c|c|c|c|}
\hline & \multirow{2}{*}{\multicolumn{7}{|c|}{ Industrial }} \\
\hline & & & & & & & & \\
\hline Source sector & Region & $\begin{array}{l}\text { Africa/M. } \\
\text { East }\end{array}$ & $\begin{array}{l}\text { Southern } \\
\text { Asia }\end{array}$ & $\begin{array}{l}\text { C. /S. } \\
\text { America }\end{array}$ & $\begin{array}{l}\text { Northern } \\
\text { Asia }\end{array}$ & Europe & $\begin{array}{l}\text { North } \\
\text { America }\end{array}$ & Oceania \\
\hline \multirow{7}{*}{$\begin{array}{l}\text { Agricultural } \\
\text { Soil }\end{array}$} & Africa/Middle East & $-6.88 \mathrm{E}-02$ & $1.95 \mathrm{E}-02$ & $-4.54 \mathrm{E}-04$ & $1.21 \mathrm{E}-02$ & $-2.28 \mathrm{E}-02$ & $9.08 \mathrm{E}-03$ & $4.35 \mathrm{E}-04$ \\
\hline & Southern Asia & $-1.64 \mathrm{E}-02$ & $-3.91 \mathrm{E}-01$ & $2.48 \mathrm{E}-03$ & $-1.32 \mathrm{E}-02$ & $-2.98 \mathrm{E}-03$ & $2.73 \mathrm{E}-02$ & $1.28 \mathrm{E}-03$ \\
\hline & Central/South America & $9.34 \mathrm{E}-04$ & $-5.02 \mathrm{E}-04$ & $-6.17 \mathrm{E}-03$ & $3.75 \mathrm{E}-04$ & $3.23 \mathrm{E}-03$ & $1.74 \mathrm{E}-03$ & $-1.20 \mathrm{E}-03$ \\
\hline & Northern Asia & $-9.55 \mathrm{E}-03$ & $-4.84 \mathrm{E}-02$ & $-3.64 \mathrm{E}-04$ & \begin{tabular}{|l|} 
\\
\end{tabular} & $-3.69 \mathrm{E}-02$ & $4.12 \mathrm{E}-02$ & $-9.88 \mathrm{E}-04$ \\
\hline & Europe & $-6.37 \mathrm{E}-03$ & $2.06 \mathrm{E}-02$ & $6.22 \mathrm{E}-04$ & $-3.76 \mathrm{E}-02$ & $-7.50 \mathrm{E}-01$ & $2.99 \mathrm{E}-02$ & $2.08 \mathrm{E}-04$ \\
\hline & \begin{tabular}{|l|} 
North America \\
\end{tabular} & $-3.82 \mathrm{E}-03$ & $-3.48 \mathrm{E}-03$ & $1.98 \mathrm{E}-04$ & $2.02 \mathrm{E}-01$ & $-1.28 \mathrm{E}-02$ & $-3.83 \mathrm{E}-01$ & $4.00 \mathrm{E}-05$ \\
\hline & Oceania & $7.39 \mathrm{E}-04$ & $-2.03 \mathrm{E}-03$ & $-1.21 \mathrm{E}-03$ & 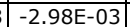 & $4.87 \mathrm{E}-04$ & $-5.71 \mathrm{E}-04$ & $-5.66 \mathrm{E}-03$ \\
\hline \multirow{7}{*}{ Industrial } & Africa/Middle East & 1 & $2.63 \mathrm{E}-03$ & $5.16 \mathrm{E}-05$ & $-1.23 \mathrm{E}-03$ & $-7.71 \mathrm{E}-03$ & $1.75 \mathrm{E}-03$ & $5.68 \mathrm{E}-05$ \\
\hline & \begin{tabular}{|l|} 
Southern Asia \\
\end{tabular} & $2.63 \mathrm{E}-03$ & 1 & $4.55 \mathrm{E}-05$ & $-4.10 \mathrm{E}-02$ & $3.59 \mathrm{E}-03$ & $1.10 \mathrm{E}-02$ & $-1.76 \mathrm{E}-04$ \\
\hline & Central/South America & $5.16 \mathrm{E}-05$ & $4.55 \mathrm{E}-05$ & 1 & $2.19 \mathrm{E}-04$ & $2.65 \mathrm{E}-04$ & $1.61 \mathrm{E}-04$ & $-8.74 \mathrm{E}-05$ \\
\hline & Northern Asia & $-1.23 \mathrm{E}-03$ & $-4.10 \mathrm{E}-02$ & $2.19 \mathrm{E}-04$ & 1 & $-3.06 \mathrm{E}-02$ & $-3.10 \mathrm{E}-01$ & $-2.44 \mathrm{E}-04$ \\
\hline & Europe & $-7.71 \mathrm{E}-03$ & $3.59 \mathrm{E}-03$ & $2.65 \mathrm{E}-04$ & $-3.06 \mathrm{E}-02$ & 1 & $8.34 \mathrm{E}-03$ & $2.91 \mathrm{E}-05$ \\
\hline & North America & $1.75 \mathrm{E}-03$ & $1.10 \mathrm{E}-02$ & $1.61 \mathrm{E}-04$ & $-3.10 \mathrm{E}-01$ & $8.34 \mathrm{E}-03$ & 1 & $-5.35 \mathrm{E}-05$ \\
\hline & Oceania & $5.68 \mathrm{E}-05$ & $-1.76 \mathrm{E}-04$ & $-8.74 \mathrm{E}-05$ & $-2.44 \mathrm{E}-04$ & $2.91 \mathrm{E}-05$ & $-5.35 \mathrm{E}-05$ & 1 \\
\hline \multirow{7}{*}{ Natural Soil } & Africa/Middle East & $-3.73 E-02$ & $5.84 \mathrm{E}-02$ & $-6.21 \mathrm{E}-03$ & \begin{tabular}{|l|} 
\\
\end{tabular} & $-6.81 \mathrm{E}-03$ & $1.60 \mathrm{E}-02$ & $3.47 \mathrm{E}-04$ \\
\hline & Southern Asia & $-4.61 \mathrm{E}-03$ & $-1.39 \mathrm{E}-01$ & $-1.48 \mathrm{E}-06$ & \begin{tabular}{|l|}
$2.73 E-02$ \\
\end{tabular} & $-1.34 \mathrm{E}-02$ & $5.50 \mathrm{E}-03$ & $1.47 \mathrm{E}-03$ \\
\hline & Central/South America & $4.78 \mathrm{E}-03$ & 7.87E-03 & $-1.87 \mathrm{E}-02$ & $\begin{array}{l}1.89 \mathrm{E}-02 \\
\end{array}$ & $1.09 \mathrm{E}-02$ & $8.92 \mathrm{E}-03$ & $-4.50 \mathrm{E}-03$ \\
\hline & Northern Asia & $7.23 \mathrm{E}-03$ & $-8.22 \mathrm{E}-03$ & $-3.20 \mathrm{E}-04$ & $-7.46 \mathrm{E}-02$ & $2.72 \mathrm{E}-02$ & $2.01 \mathrm{E}-02$ & $-1.18 \mathrm{E}-03$ \\
\hline & Europe & $-8.00 \mathrm{E}-03$ & $1.55 \mathrm{E}-02$ & 3.84E-04 & $2.81 \mathrm{E}-02$ & $-1.55 \mathrm{E}-01$ & $2.69 \mathrm{E}-02$ & $2.17 \mathrm{E}-04$ \\
\hline & North America & $-2.10 \mathrm{E}-03$ & 7.07E-02 & $1.43 \mathrm{E}-03$ & \begin{tabular}{|l|} 
\\
\end{tabular} & $1.68 \mathrm{E}-02$ & $-2.11 \mathrm{E}-01$ & $7.25 \mathrm{E}-04$ \\
\hline & Oceania & $6.16 \mathrm{E}-04$ & $-1.69 \mathrm{E}-03$ & $-2.01 \mathrm{E}-03$ & \begin{tabular}{|l|} 
\\
\end{tabular} & $1.51 \mathrm{E}-03$ & $1.28 \mathrm{E}-04$ & $-3.71 \mathrm{E}-03$ \\
\hline \multirow{6}{*}{ Ocean } & North Pacific & $-4.64 \mathrm{E}-03$ & $-4.13 \mathrm{E}-03$ & $1.53 \mathrm{E}-04$ & $4.31 \mathrm{E}-02$ & $-1.37 \mathrm{E}-02$ & $-5.26 \mathrm{E}-02$ & $2.61 \mathrm{E}-04$ \\
\hline & \begin{tabular}{|l} 
South Pacific \\
\end{tabular} & $-6.60 \mathrm{E}-04$ & $7.21 \mathrm{E}-03$ & $-5.17 \mathrm{E}-03$ & \begin{tabular}{|c|} 
\\
\end{tabular} & $6.44 \mathrm{E}-03$ & $1.24 \mathrm{E}-03$ & $2.71 \mathrm{E}-03$ \\
\hline & Northern Ocean & $9.61 \mathrm{E}-04$ & $-1.15 \mathrm{E}-02$ & $5.66 \mathrm{E}-05$ & $3.38 \mathrm{E}-02$ & $1.64 \mathrm{E}-02$ & $3.70 \mathrm{E}-02$ & $1.13 \mathrm{E}-04$ \\
\hline & Atlantic & $-1.17 \mathrm{E}-02$ & $-7.48 \mathrm{E}-03$ & $-2.72 E-03$ & \begin{tabular}{|l|l|} 
& $-2.30 \mathrm{E}-01$ \\
\end{tabular} & $-1.09 \mathrm{E}-02$ & $-1.10 \mathrm{E}-01$ & $6.87 \mathrm{E}-04$ \\
\hline & Southern Ocean & $2.25 \mathrm{E}-03$ & $-2.57 \mathrm{E}-02$ & $-1.26 \mathrm{E}-02$ & $-7.06 \mathrm{E}-03$ & $9.58 \mathrm{E}-04$ & $8.88 \mathrm{E}-03$ & $-6.99 \mathrm{E}-03$ \\
\hline & Indian Ocean & $-2.70 \mathrm{E}-03$ & $2.25 \mathrm{E}-02$ & $-7.75 \mathrm{E}-03$ & \begin{tabular}{|l|l|} 
& $9.47 \mathrm{E}-03$ \\
\end{tabular} & $3.70 \mathrm{E}-03$ & $-3.04 \mathrm{E}-03$ & $-2.14 \mathrm{E}-03$ \\
\hline \multirow{7}{*}{$\begin{array}{l}\text { Biomass } \\
\text { burning }\end{array}$} & Africa/Middle East & $-2.21 \mathrm{E}-03$ & $9.44 \mathrm{E}-03$ & $-2.94 \mathrm{E}-03$ & \begin{tabular}{|l|} 
\\
\end{tabular} & $3.25 \mathrm{E}-03$ & $1.45 \mathrm{E}-03$ & $4.40 \mathrm{E}-04$ \\
\hline & Southern Asia & $-3.92 \mathrm{E}-04$ & $-1.32 \mathrm{E}-02$ & $-1.78 \mathrm{E}-04$ & \begin{tabular}{|l|} 
\\
\end{tabular} & $1.08 \mathrm{E}-03$ & $1.98 \mathrm{E}-03$ & $2.85 \mathrm{E}-05$ \\
\hline & Central/South America & $-2.52 \mathrm{E}-04$ & $1.21 \mathrm{E}-03$ & $-9.58 \mathrm{E}-04$ & $\begin{array}{ll}1.64 \mathrm{E}-03 \\
\end{array}$ & $4.95 \mathrm{E}-04$ & $-7.07 E-05$ & $1.39 \mathrm{E}-04$ \\
\hline & Northern Asia & $3.09 \mathrm{E}-03$ & $-1.92 \mathrm{E}-02$ & $2.53 \mathrm{E}-04$ & $6.08 \mathrm{E}-02$ & $9.44 \mathrm{E}-03$ & $3.13 \mathrm{E}-02$ & $1.91 \mathrm{E}-04$ \\
\hline & Europe & $-1.59 \mathrm{E}-05$ & $1.48 \mathrm{E}-04$ & $5.19 \mathrm{E}-06$ & \begin{tabular}{|l|}
$4.69 E-04$ \\
\end{tabular} & $-3.49 \mathrm{E}-04$ & $1.58 \mathrm{E}-04$ & $8.05 \mathrm{E}-07$ \\
\hline & North America & $3.94 \mathrm{E}-04$ & $5.00 \mathrm{E}-03$ & $9.40 \mathrm{E}-05$ & \begin{tabular}{|l|} 
\\
$2.58 \mathrm{E}-02$ \\
\end{tabular} & $2.66 \mathrm{E}-03$ & $9.13 \mathrm{E}-03$ & $2.08 \mathrm{E}-05$ \\
\hline & Oceania & $1.32 \mathrm{E}-04$ & $5.38 \mathrm{E}-04$ & $-2.91 \mathrm{E}-04$ & $-2.47 \mathrm{E}-05$ & $3.10 \mathrm{E}-04$ & $5.71 \mathrm{E}-05$ & $-1.64 \mathrm{E}-04$ \\
\hline
\end{tabular}

Fig. S2ao. Correlation of the emissions in 2003 for industrial. 


\begin{tabular}{|c|c|c|c|c|c|c|c|c|}
\hline & \multirow{2}{*}{\multicolumn{7}{|c|}{ Natural Soil }} \\
\hline & & & & & & & & \\
\hline Source sector & Region & $\begin{array}{l}\text { Africa/M. } \\
\text { East }\end{array}$ & $\begin{array}{l}\text { Southern } \\
\text { Asia }\end{array}$ & $\begin{array}{l}\text { C. /S. } \\
\text { America }\end{array}$ & $\begin{array}{l}\text { Northern } \\
\text { Asia }\end{array}$ & Europe & $\begin{array}{l}\text { North } \\
\text { America }\end{array}$ & Oceania \\
\hline \multirow{7}{*}{$\begin{array}{l}\text { Agricultural } \\
\text { Soil }\end{array}$} & Africa/Middle East & $-2.24 \mathrm{E}-01$ & $-4.09 \mathrm{E}-02$ & $-1.79 \mathrm{E}-02$ & 3.64E-02 & $-2.46 \mathrm{E}-02$ & $-3.69 \mathrm{E}-02$ & $2.05 \mathrm{E}-03$ \\
\hline & Southern Asia & $-1.52 \mathrm{E}-01$ & $-2.89 \mathrm{E}-01$ & $8.44 \mathrm{E}-02$ & $\mid-2.51 \mathrm{E}-02$ & $-1.81 \mathrm{E}-02$ & $-8.24 \mathrm{E}-02$ & $1.54 \mathrm{E}-02$ \\
\hline & Central/South America & $-7.31 \mathrm{E}-02$ & $4.46 \mathrm{E}-03$ & $-2.48 \mathrm{E}-01$ & \begin{tabular}{|l|}
$-5.35 \mathrm{E}-03$ \\
\end{tabular} & $5.85 \mathrm{E}-03$ & $2.37 \mathrm{E}-02$ & $-2.58 \mathrm{E}-02$ \\
\hline & Northern Asia & $-9.90 \mathrm{E}-03$ & $9.35 \mathrm{E}-02$ & $9.21 \mathrm{E}-03$ & \begin{tabular}{|l|}
$-2.05 \mathrm{E}-01$ \\
\end{tabular} & $7.35 \mathrm{E}-04$ & $6.72 \mathrm{E}-02$ & $-7.02 \mathrm{E}-03$ \\
\hline & Europe & $-8.60 \mathrm{E}-03$ & $-9.74 \mathrm{E}-03$ & $2.00 \mathrm{E}-02$ & $2.28 \mathrm{E}-02$ & $-2.19 \mathrm{E}-01$ & $-1.68 \mathrm{E}-02$ & $4.80 \mathrm{E}-03$ \\
\hline & \begin{tabular}{|l|} 
North America \\
\end{tabular} & $1.39 \mathrm{E}-02$ & $-2.29 \mathrm{E}-02$ & $2.87 \mathrm{E}-02$ & $7.02 \mathrm{E}-02$ & $2.43 \mathrm{E}-02$ & $-4.21 \mathrm{E}-01$ & $1.40 \mathrm{E}-03$ \\
\hline & Oceania & $2.36 \mathrm{E}-03$ & $1.77 \mathrm{E}-02$ & $-5.35 \mathrm{E}-02$ & $-1.54 \mathrm{E}-02$ & $2.80 \mathrm{E}-03$ & $9.57 \mathrm{E}-03$ & $-5.27 \mathrm{E}-02$ \\
\hline \multirow{7}{*}{ Industrial } & Africa/Middle East & $-3.73 \mathrm{E}-02$ & $-4.61 \mathrm{E}-03$ & $4.78 \mathrm{E}-03$ & $7.23 \mathrm{E}-03$ & $-8.00 \mathrm{E}-03$ & $-2.10 \mathrm{E}-03$ & $6.16 \mathrm{E}-04$ \\
\hline & \begin{tabular}{|l|} 
Southern Asia \\
\end{tabular} & $5.84 \mathrm{E}-02$ & $-1.39 \mathrm{E}-01$ & $7.87 \mathrm{E}-03$ & $-8.22 \mathrm{E}-03$ & $1.55 \mathrm{E}-02$ & $7.07 \mathrm{E}-02$ & $-1.69 \mathrm{E}-03$ \\
\hline & Central/South America & $-6.21 \mathrm{E}-03$ & $-1.48 \mathrm{E}-06$ & $-1.87 \mathrm{E}-02$ & $-3.20 \mathrm{E}-04$ & $3.84 \mathrm{E}-04$ & $1.43 \mathrm{E}-03$ & $-2.01 \mathrm{E}-03$ \\
\hline & Northern Asia & $6.26 \mathrm{E}-02$ & $2.73 \mathrm{E}-02$ & $1.89 \mathrm{E}-02$ & \begin{tabular}{|l|}
$-7.46 \mathrm{E}-02$ \\
\end{tabular} & $2.81 \mathrm{E}-02$ & $-2.27 \mathrm{E}-01$ & $-7.30 \mathrm{E}-04$ \\
\hline & Europe & $-6.81 \mathrm{E}-03$ & $-1.34 \mathrm{E}-02$ & $1.09 \mathrm{E}-02$ & $2.72 \mathrm{E}-02$ & $-1.55 \mathrm{E}-01$ & $1.68 \mathrm{E}-02$ & $1.51 \mathrm{E}-03$ \\
\hline & North America & $1.60 \mathrm{E}-02$ & $5.50 \mathrm{E}-03$ & 8.92E-03 & $2.01 \mathrm{E}-02$ & $2.69 \mathrm{E}-02$ & $-2.11 \mathrm{E}-01$ & $1.28 \mathrm{E}-04$ \\
\hline & Oceania & $3.47 \mathrm{E}-04$ & $1.47 \mathrm{E}-03$ & $-4.50 \mathrm{E}-03$ & $-1.18 \mathrm{E}-03$ & $2.17 E-04$ & $7.25 \mathrm{E}-04$ & $-3.71 \mathrm{E}-03$ \\
\hline \multirow{7}{*}{ Natural Soil } & Africa/Middle East & 1 & $-1.73 \mathrm{E}-01$ & $-4.17 \mathrm{E}-01$ & $1.05 \mathrm{E}-01$ & $-3.78 \mathrm{E}-02$ & $-1.57 \mathrm{E}-01$ & $-1.03 \mathrm{E}-02$ \\
\hline & Southern Asia & $-1.73 \mathrm{E}-01$ & 1 & $-7.13 \mathrm{E}-02$ & $-5.42 \mathrm{E}-01$ & $6.76 \mathrm{E}-02$ & $2.78 \mathrm{E}-02$ & $2.32 \mathrm{E}-03$ \\
\hline & Central/South America & $-4.17 \mathrm{E}-01$ & $-7.13 \mathrm{E}-02$ & 1 & \begin{tabular}{|l|}
$-5.48 \mathrm{E}-02$ \\
\end{tabular} & $7.63 E-03$ & $-4.24 \mathrm{E}-03$ & $-1.06 \mathrm{E}-01$ \\
\hline & Northern Asia & $1.05 \mathrm{E}-01$ & $-5.42 \mathrm{E}-01$ & $-5.48 \mathrm{E}-02$ & 1 & $-1.44 \mathrm{E}-01$ & $-1.95 \mathrm{E}-01$ & $-2.16 \mathrm{E}-02$ \\
\hline & Europe & $-3.78 \mathrm{E}-02$ & $6.76 \mathrm{E}-02$ & $7.63 \mathrm{E}-03$ & $-1.44 \mathrm{E}-01$ & 1 & $-1.35 \mathrm{E}-01$ & $8.05 E-04$ \\
\hline & North America & $-1.57 \mathrm{E}-01$ & $2.78 \mathrm{E}-02$ & $-4.24 \mathrm{E}-03$ & \begin{tabular}{|l|}
$-1.95 \mathrm{E}-01$ \\
\end{tabular} & $-1.35 \mathrm{E}-01$ & 1 & $1.41 \mathrm{E}-02$ \\
\hline & Oceania & $-1.03 E-02$ & $2.32 \mathrm{E}-03$ & $-1.06 \mathrm{E}-01$ & $-2.16 \mathrm{E}-02$ & $8.05 \mathrm{E}-04$ & $1.41 \mathrm{E}-02$ & 1 \\
\hline \multirow{6}{*}{ Ocean } & North Pacific & $7.36 \mathrm{E}-02$ & $1.41 \mathrm{E}-01$ & $2.41 \mathrm{E}-02$ & $-1.65 \mathrm{E}-01$ & $6.19 \mathrm{E}-02$ & $1.31 \mathrm{E}-01$ & $-2.81 \mathrm{E}-03$ \\
\hline & \begin{tabular}{|l} 
South Pacific \\
\end{tabular} & $-1.17 \mathrm{E}-02$ & $1.58 \mathrm{E}-02$ & $-5.00 \mathrm{E}-01$ & $-1.65 \mathrm{E}-02$ & $3.64 \mathrm{E}-03$ & $4.14 \mathrm{E}-02$ & $1.43 \mathrm{E}-02$ \\
\hline & Northern Ocean & $6.95 \mathrm{E}-02$ & $1.05 \mathrm{E}-01$ & $3.14 \mathrm{E}-02$ & $-7.07 \mathrm{E}-02$ & $9.55 \mathrm{E}-03$ & $-7.01 \mathrm{E}-03$ & $9.92 \mathrm{E}-05$ \\
\hline & Atlantic & $-8.93 \mathrm{E}-02$ & $8.96 \mathrm{E}-02$ & $-2.42 \mathrm{E}-02$ & $1.18 \mathrm{E}-02$ & $3.78 \mathrm{E}-02$ & \begin{tabular}{|l|} 
\\
\end{tabular} & $-4.21 \mathrm{E}-03$ \\
\hline & Southern Ocean & $1.22 \mathrm{E}-01$ & $1.53 \mathrm{E}-01$ & $-4.03 \mathrm{E}-02$ & $-7.77 \mathrm{E}-02$ & $9.37 \mathrm{E}-03$ & $6.15 \mathrm{E}-02$ & $-7.98 \mathrm{E}-02$ \\
\hline & Indian Ocean & $-1.81 \mathrm{E}-01$ & $-9.18 \mathrm{E}-02$ & $-1.48 \mathrm{E}-01$ & $3.21 \mathrm{E}-02$ & $8.10 \mathrm{E}-03$ & $2.09 \mathrm{E}-02$ & $-4.58 \mathrm{E}-02$ \\
\hline \multirow{7}{*}{$\begin{array}{l}\text { Biomass } \\
\text { burning }\end{array}$} & Africa/Middle East & $-1.27 \mathrm{E}-01$ & $-1.63 \mathrm{E}-02$ & $-1.45 \mathrm{E}-02$ & $1.91 \mathrm{E}-02$ & $6.33 \mathrm{E}-03$ & \begin{tabular}{|l|l|}
$1.35 \mathrm{E}-02$ \\
\end{tabular} & $-3.85 \mathrm{E}-03$ \\
\hline & Southern Asia & $4.34 \mathrm{E}-03$ & $1.97 \mathrm{E}-02$ & $-4.32 \mathrm{E}-03$ & $-4.62 \mathrm{E}-03$ & $-3.16 \mathrm{E}-03$ & \begin{tabular}{|l|l|} 
& $9.48 \mathrm{E}-03$ \\
\end{tabular} & $-2.15 \mathrm{E}-03$ \\
\hline & Central/South America & $-1.79 \mathrm{E}-02$ & $2.16 \mathrm{E}-05$ & $-9.93 \mathrm{E}-03$ & $6.98 \mathrm{E}-04$ & 5.37E-04 & $4.86 \mathrm{E}-03$ & $-2.14 \mathrm{E}-03$ \\
\hline & Northern Asia & $2.83 \mathrm{E}-02$ & $8.48 \mathrm{E}-02$ & $-1.14 \mathrm{E}-02$ & $-2.74 \mathrm{E}-01$ & $-5.07 \mathrm{E}-02$ & $-1.84 \mathrm{E}-01$ & $-2.75 \mathrm{E}-03$ \\
\hline & Europe & $-8.32 \mathrm{E}-04$ & $-2.93 \mathrm{E}-04$ & $3.06 \mathrm{E}-04$ & $-2.65 \mathrm{E}-04$ & $-2.99 \mathrm{E}-03$ & \begin{tabular}{|l|}
$-1.39 \mathrm{E}-03$ \\
\end{tabular} & 4.69E-05 \\
\hline & North America & $2.56 \mathrm{E}-03$ & $7.41 \mathrm{E}-03$ & $-2.58 \mathrm{E}-03$ & $-3.28 \mathrm{E}-02$ & $-2.39 \mathrm{E}-02$ & \begin{tabular}{|l|}
$-1.21 \mathrm{E}-01$ \\
\end{tabular} & $3.59 \mathrm{E}-04$ \\
\hline & Oceania & $-4.50 \mathrm{E}-03$ & $-2.88 \mathrm{E}-03$ & $-6.48 \mathrm{E}-03$ & $-5.16 E-04$ & $7.62 \mathrm{E}-04$ & $2.43 \mathrm{E}-03$ & $-2.66 \mathrm{E}-03$ \\
\hline
\end{tabular}

Fig. S2ap. Correlation of the emissions in 2003 for natural soil. 


\begin{tabular}{|c|c|c|c|c|c|c|c|}
\hline & \multirow{2}{*}{\multicolumn{6}{|c|}{ Ocean }} \\
\hline & & & & & & & \\
\hline Source sector & Region & $\begin{array}{l}\text { North } \\
\text { Pacific }\end{array}$ & $\begin{array}{l}\text { South } \\
\text { Pacific }\end{array}$ & $\begin{array}{l}\text { Northern } \\
\text { Ocean }\end{array}$ & Atlantic & $\begin{array}{l}\text { Southern } \\
\text { Ocean }\end{array}$ & $\begin{array}{l}\text { Indian } \\
\text { Ocean }\end{array}$ \\
\hline \multirow{7}{*}{$\begin{array}{l}\text { Agricultural } \\
\text { Soil }\end{array}$} & Africa/Middle East & $-1.50 \mathrm{E}-02$ & $-1.39 \mathrm{E}-02$ & $4.72 \mathrm{E}-03$ & $-7.68 \mathrm{E}-02$ & $2.43 \mathrm{E}-02$ & $-3.70 \mathrm{E}-02$ \\
\hline & Southern Asia & $-1.55 \mathrm{E}-01$ & $-5.63 \mathrm{E}-03$ & $-5.59 \mathrm{E}-03$ & $-1.35 \mathrm{E}-01$ & $1.20 \mathrm{E}-01$ & $-5.31 \mathrm{E}-02$ \\
\hline & Central/South America & $2.38 \mathrm{E}-03$ & $-3.94 \mathrm{E}-02$ & $-5.81 \mathrm{E}-05$ & $-3.04 \mathrm{E}-02$ & $-1.83 \mathrm{E}-01$ & $-9.33 \mathrm{E}-02$ \\
\hline & \begin{tabular}{|l} 
Northern Asia \\
\end{tabular} & $3.17 \mathrm{E}-02$ & $1.65 \mathrm{E}-02$ & $-6.93 \mathrm{E}-02$ & $-2.78 \mathrm{E}-02$ & $-2.26 \mathrm{E}-02$ & $1.30 \mathrm{E}-03$ \\
\hline & Europe & $8.67 \mathrm{E}-03$ & $1.34 \mathrm{E}-02$ & $\mid-8.71 \mathrm{E}-02$ & $-5.66 \mathrm{E}-02$ & $1.27 \mathrm{E}-02$ & $2.67 \mathrm{E}-03$ \\
\hline & North America & $-5.61 \mathrm{E}-02$ & $1.49 \mathrm{E}-03$ & $\mid-9.66 \mathrm{E}-03$ & $-8.74 \mathrm{E}-02$ & $1.25 \mathrm{E}-03$ & $8.54 \mathrm{E}-03$ \\
\hline & Oceania & $3.71 \mathrm{E}-03$ & $2.65 \mathrm{E}-02$ & $1.93 \mathrm{E}-03$ & $9.03 \mathrm{E}-03$ & $-9.08 \mathrm{E}-02$ & $-2.24 \mathrm{E}-02$ \\
\hline \multirow{7}{*}{ Industrial } & Africa/Middle East & $-4.64 \mathrm{E}-03$ & $-6.60 \mathrm{E}-04$ & $9.61 \mathrm{E}-04$ & $-1.17 \mathrm{E}-02$ & $2.25 \mathrm{E}-03$ & $-2.70 \mathrm{E}-03$ \\
\hline & Southern Asia & $-4.13 \mathrm{E}-03$ & $7.21 \mathrm{E}-03$ & $\mid-1.15 \mathrm{E}-02$ & $-7.48 \mathrm{E}-03$ & $-2.57 \mathrm{E}-02$ & $2.25 \mathrm{E}-02$ \\
\hline & Central/South America & $1.53 \mathrm{E}-04$ & $-5.17 \mathrm{E}-03$ & $5.66 \mathrm{E}-05$ & $-2.72 \mathrm{E}-03$ & $-1.26 \mathrm{E}-02$ & $-7.75 \mathrm{E}-03$ \\
\hline & Northern Asia & $4.31 \mathrm{E}-02$ & $1.53 \mathrm{E}-02$ & $3.38 \mathrm{E}-02$ & $-2.30 \mathrm{E}-01$ & $-7.06 \mathrm{E}-03$ & 9.47E-03 \\
\hline & Europe & $-1.37 \mathrm{E}-02$ & $6.44 \mathrm{E}-03$ & $1.64 \mathrm{E}-02$ & $-1.09 \mathrm{E}-02$ & $9.58 \mathrm{E}-04$ & $3.70 \mathrm{E}-03$ \\
\hline & North America & $-5.26 \mathrm{E}-02$ & $1.24 \mathrm{E}-03$ & $3.70 \mathrm{E}-02$ & $-1.10 \mathrm{E}-01$ & $8.88 \mathrm{E}-03$ & $-3.04 \mathrm{E}-03$ \\
\hline & Oceania & $2.61 \mathrm{E}-04$ & $2.71 \mathrm{E}-03$ & $1.13 \mathrm{E}-04$ & 6.87E-04 & $-6.99 \mathrm{E}-03$ & $-2.14 \mathrm{E}-03$ \\
\hline \multirow{7}{*}{ Natural Soil } & Africa/Middle East & $7.36 \mathrm{E}-02$ & $-1.17 \mathrm{E}-02$ & $6.95 \mathrm{E}-02$ & $-8.93 E-02$ & $1.22 \mathrm{E}-01$ & $-1.81 \mathrm{E}-01$ \\
\hline & Southern Asia & $1.41 \mathrm{E}-01$ & $1.58 \mathrm{E}-02$ & $1.05 \mathrm{E}-01$ & $8.96 \mathrm{E}-02$ & $1.53 \mathrm{E}-01$ & $-9.18 \mathrm{E}-02$ \\
\hline & Central/South America & $2.41 \mathrm{E}-02$ & $-5.00 \mathrm{E}-01$ & $3.14 \mathrm{E}-02$ & $-2.42 \mathrm{E}-02$ & $-4.03 \mathrm{E}-02$ & $-1.48 \mathrm{E}-01$ \\
\hline & Northern Asia & $-1.65 \mathrm{E}-01$ & $-1.65 \mathrm{E}-02$ & \begin{tabular}{|l|}
$-7.07 E-02$ \\
\end{tabular} & $1.18 \mathrm{E}-02$ & $-7.77 \mathrm{E}-02$ & $3.21 \mathrm{E}-02$ \\
\hline & Europe & $6.19 \mathrm{E}-02$ & $3.64 \mathrm{E}-03$ & $9.55 \mathrm{E}-03$ & $3.78 \mathrm{E}-02$ & $9.37 \mathrm{E}-03$ & $8.10 \mathrm{E}-03$ \\
\hline & North America & $1.31 \mathrm{E}-01$ & $4.14 \mathrm{E}-02$ & \begin{tabular}{|l|}
$-7.01 \mathrm{E}-03$ \\
\end{tabular} & $8.33 \mathrm{E}-02$ & $6.15 \mathrm{E}-02$ & $2.09 \mathrm{E}-02$ \\
\hline & Oceania & $-2.81 \mathrm{E}-03$ & $1.43 \mathrm{E}-02$ & 9.92E-05 & $-4.21 \mathrm{E}-03$ & $-7.98 \mathrm{E}-02$ & $-4.58 \mathrm{E}-02$ \\
\hline \multirow{6}{*}{ Ocean } & North Pacific & 1 & $-2.18 \mathrm{E}-02$ & $-8.19 \mathrm{E}-02$ & $-2.01 \mathrm{E}-01$ & $1.96 \mathrm{E}-02$ & $2.73 \mathrm{E}-02$ \\
\hline & South Pacific & $-2.18 \mathrm{E}-02$ & 1 & \begin{tabular}{|l|}
$-7.97 E-04$ \\
\end{tabular} & $-6.17 \mathrm{E}-02$ & $6.46 \mathrm{E}-02$ & $2.35 \mathrm{E}-03$ \\
\hline & Northern Ocean & $-8.19 \mathrm{E}-02$ & $-7.97 \mathrm{E}-04$ & 1 & $-3.18 \mathrm{E}-01$ & $1.78 \mathrm{E}-02$ & $1.22 \mathrm{E}-02$ \\
\hline & Atlantic & $-2.01 \mathrm{E}-01$ & $-6.17 \mathrm{E}-02$ & $-3.18 \mathrm{E}-01$ & 1 & $-6.46 \mathrm{E}-02$ & $-2.34 \mathrm{E}-02$ \\
\hline & Southern Ocean & $1.96 \mathrm{E}-02$ & $6.46 \mathrm{E}-02$ & $1.78 \mathrm{E}-02$ & $-6.46 \mathrm{E}-02$ & 1 & $-6.55 \mathrm{E}-01$ \\
\hline & Indian Ocean & $2.73 \mathrm{E}-02$ & $2.35 \mathrm{E}-03$ & $1.22 \mathrm{E}-02$ & $-2.34 \mathrm{E}-02$ & $-6.55 \mathrm{E}-01$ & 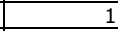 \\
\hline \multirow{7}{*}{$\begin{array}{l}\text { Biomass } \\
\text { burning }\end{array}$} & Africa/Middle East & $2.81 \mathrm{E}-03$ & $-4.97 \mathrm{E}-02$ & \begin{tabular}{|l|}
$-5.02 \mathrm{E}-04$ \\
\end{tabular} & $-5.63 E-02$ & $1.66 \mathrm{E}-03$ & $-7.44 \mathrm{E}-02$ \\
\hline & Southern Asia & $-4.20 \mathrm{E}-02$ & $-1.09 \mathrm{E}-02$ & \begin{tabular}{|l|}
$-1.03 \mathrm{E}-02$ \\
\end{tabular} & $-2.27 \mathrm{E}-02$ & 4.19E-03 & $-6.11 \mathrm{E}-03$ \\
\hline & Central/South America & $-1.19 \mathrm{E}-03$ & $-5.17 \mathrm{E}-02$ & $-1.52 \mathrm{E}-04$ & $-1.07 \mathrm{E}-02$ & $-1.52 \mathrm{E}-02$ & $-2.02 \mathrm{E}-02$ \\
\hline & Northern Asia & $-2.85 \mathrm{E}-01$ & $2.08 \mathrm{E}-02$ & $-4.01 \mathrm{E}-02$ & $3.46 \mathrm{E}-02$ & $6.88 \mathrm{E}-04$ & $2.24 \mathrm{E}-02$ \\
\hline & Europe & $5.86 \mathrm{E}-04$ & $1.65 \mathrm{E}-04$ & $1.18 \mathrm{E}-04$ & $1.40 \mathrm{E}-04$ & $1.30 \mathrm{E}-04$ & $3.28 \mathrm{E}-05$ \\
\hline & North America & $1.98 \mathrm{E}-02$ & $5.21 \mathrm{E}-03$ & \begin{tabular}{|l|}
$-1.32 \mathrm{E}-02$ \\
\end{tabular} & $5.53 \mathrm{E}-03$ & $6.12 \mathrm{E}-04$ & $1.66 \mathrm{E}-03$ \\
\hline & Oceania & 8.87E-04 & $1.50 \mathrm{E}-03$ & $6.94 \mathrm{E}-05$ & $-7.52 \mathrm{E}-04$ & $-2.90 \mathrm{E}-03$ & $-4.98 \mathrm{E}-03$ \\
\hline
\end{tabular}

Fig. S2aq. Correlation of the emissions in 2003 for ocean. 


\begin{tabular}{|c|c|c|c|c|c|c|c|c|}
\hline \multirow[b]{3}{*}{ Source sector } & \multirow[b]{3}{*}{ Region } & \multirow{2}{*}{\multicolumn{7}{|c|}{ Biomass burning }} \\
\hline & & & & & & & & \\
\hline & & $\begin{array}{l}\text { Africa/M. } \\
\text { East }\end{array}$ & $\begin{array}{l}\text { Southern } \\
\text { Asia }\end{array}$ & $\begin{array}{l}\text { C. /S. } \\
\text { America }\end{array}$ & $\begin{array}{l}\text { Northern } \\
\text { Asia }\end{array}$ & Europe & $\begin{array}{l}\text { North } \\
\text { America }\end{array}$ & Oceania \\
\hline \multirow{7}{*}{$\begin{array}{l}\text { Agricultural } \\
\text { Soil }\end{array}$} & Africa/Middle East & $-2.83 \mathrm{E}-02$ & $-2.14 \mathrm{E}-03$ & $-3.32 \mathrm{E}-03$ & $1.37 \mathrm{E}-02$ & $-1.97 \mathrm{E}-04$ & $1.31 \mathrm{E}-03$ & $1.60 \mathrm{E}-04$ \\
\hline & Southern Asia & $-2.20 \mathrm{E}-02$ & $-3.54 \mathrm{E}-02$ & $2.34 \mathrm{E}-03$ & $-7.92 \mathrm{E}-02$ & $-2.50 \mathrm{E}-04$ & $-1.85 \mathrm{E}-03$ & $2.81 \mathrm{E}-03$ \\
\hline & Central/South America & $-3.42 \mathrm{E}-02$ & $-2.11 \mathrm{E}-03$ & $-1.16 \mathrm{E}-02$ & $3.75 \mathrm{E}-03$ & $6.43 \mathrm{E}-05$ & $1.22 \mathrm{E}-03$ & $-3.84 \mathrm{E}-03$ \\
\hline & Northern Asia & $5.32 \mathrm{E}-03$ & $-1.09 \mathrm{E}-03$ & $-6.35 \mathrm{E}-04$ & $1.42 \mathrm{E}-02$ & $1.36 \mathrm{E}-04$ & $5.67 \mathrm{E}-04$ & $-9.29 \mathrm{E}-04$ \\
\hline & \begin{tabular}{|l} 
Europe \\
\end{tabular} & $4.51 \mathrm{E}-03$ & $4.18 \mathrm{E}-03$ & $1.56 \mathrm{E}-03$ & $4.37 \mathrm{E}-03$ & $-2.67 \mathrm{E}-04$ & $-4.54 \mathrm{E}-03$ & $7.02 \mathrm{E}-04$ \\
\hline & \begin{tabular}{|l} 
North America \\
\end{tabular} & $-8.16 \mathrm{E}-04$ & $-3.57 \mathrm{E}-03$ & $1.47 \mathrm{E}-04$ & $5.48 \mathrm{E}-02$ & $2.98 \mathrm{E}-04$ & $1.50 \mathrm{E}-02$ & $8.22 \mathrm{E}-04$ \\
\hline & Oceania & $4.18 \mathrm{E}-03$ & $3.02 \mathrm{E}-04$ & $1.06 \mathrm{E}-03$ & $2.47 \mathrm{E}-03$ & $7.39 \mathrm{E}-06$ & $3.20 \mathrm{E}-04$ & $-1.83 \mathrm{E}-03$ \\
\hline \multirow{7}{*}{ Industrial } & Africa/Middle East & $-2.21 \mathrm{E}-03$ & $-3.92 \mathrm{E}-04$ & $-2.52 \mathrm{E}-04$ & $3.09 E-03$ & $-1.59 \mathrm{E}-05$ & $3.94 \mathrm{E}-04$ & $1.32 \mathrm{E}-04$ \\
\hline & Southern Asia & $9.44 \mathrm{E}-03$ & $-1.32 \mathrm{E}-02$ & $1.21 \mathrm{E}-03$ & $-1.92 \mathrm{E}-02$ & $1.48 \mathrm{E}-04$ & $5.00 \mathrm{E}-03$ & $5.38 \mathrm{E}-04$ \\
\hline & Central/South America & $-2.94 \mathrm{E}-03$ & $-1.78 \mathrm{E}-04$ & $-9.58 \mathrm{E}-04$ & $2.53 \mathrm{E}-04$ & $5.19 \mathrm{E}-06$ & $9.40 \mathrm{E}-05$ & $-2.91 \mathrm{E}-04$ \\
\hline & Northern Asia & $1.18 \mathrm{E}-02$ & $-1.75 \mathrm{E}-03$ & $1.64 \mathrm{E}-03$ & $6.08 \mathrm{E}-02$ & $4.69 E-04$ & $2.58 \mathrm{E}-02$ & $-2.47 \mathrm{E}-05$ \\
\hline & Europe & $3.25 \mathrm{E}-03$ & $1.08 \mathrm{E}-03$ & $4.95 \mathrm{E}-04$ & $9.44 \mathrm{E}-03$ & $-3.49 \mathrm{E}-04$ & $2.66 \mathrm{E}-03$ & $3.10 \mathrm{E}-04$ \\
\hline & North America & $1.45 \mathrm{E}-03$ & $1.98 \mathrm{E}-03$ & $-7.07 E-05$ & $3.13 \mathrm{E}-02$ & $1.58 \mathrm{E}-04$ & $9.13 \mathrm{E}-03$ & $5.71 \mathrm{E}-05$ \\
\hline & Oceania & $4.40 \mathrm{E}-04$ & $2.85 \mathrm{E}-05$ & $1.39 \mathrm{E}-04$ & $1.91 \mathrm{E}-04$ & $8.05 E-07$ & $2.08 \mathrm{E}-05$ & $-1.64 \mathrm{E}-04$ \\
\hline \multirow{7}{*}{ Natural Soil } & Africa/Middle East & $-1.27 \mathrm{E}-01$ & $4.34 \mathrm{E}-03$ & $-1.79 \mathrm{E}-02$ & $2.83 \mathrm{E}-02$ & $-8.32 \mathrm{E}-04$ & $2.56 \mathrm{E}-03$ & $-4.50 \mathrm{E}-03$ \\
\hline & Southern Asia & $-1.63 \mathrm{E}-02$ & $1.97 \mathrm{E}-02$ & $2.16 \mathrm{E}-05$ & $8.48 \mathrm{E}-02$ & $-2.93 \mathrm{E}-04$ & $7.41 \mathrm{E}-03$ & $-2.88 \mathrm{E}-03$ \\
\hline & Central/South America & $-1.45 \mathrm{E}-02$ & $-4.32 \mathrm{E}-03$ & $-9.93 \mathrm{E}-03$ & $-1.14 \mathrm{E}-02$ & $3.06 \mathrm{E}-04$ & $-2.58 \mathrm{E}-03$ & $-6.48 \mathrm{E}-03$ \\
\hline & Northern Asia & $1.91 \mathrm{E}-02$ & $-4.62 \mathrm{E}-03$ & $6.98 \mathrm{E}-04$ & $-2.74 \mathrm{E}-01$ & $-2.65 \mathrm{E}-04$ & $-3.28 \mathrm{E}-02$ & $-5.16 \mathrm{E}-04$ \\
\hline & Europe & $6.33 \mathrm{E}-03$ & $-3.16 \mathrm{E}-03$ & 5.37E-04 & $-5.07 E-02$ & $-2.99 \mathrm{E}-03$ & $-2.39 \mathrm{E}-02$ & $7.62 \mathrm{E}-04$ \\
\hline & North America & $1.35 \mathrm{E}-02$ & $9.48 \mathrm{E}-03$ & $4.86 \mathrm{E}-03$ & $-1.84 \mathrm{E}-01$ & $-1.39 \mathrm{E}-03$ & $-1.21 \mathrm{E}-01$ & $2.43 \mathrm{E}-03$ \\
\hline & Oceania & $-3.85 \mathrm{E}-03$ & $-2.15 \mathrm{E}-03$ & $-2.14 \mathrm{E}-03$ & $-2.75 \mathrm{E}-03$ & $4.69 \mathrm{E}-05$ & $3.59 \mathrm{E}-04$ & $-2.66 \mathrm{E}-03$ \\
\hline \multirow{6}{*}{ Ocean } & North Pacific & $2.81 \mathrm{E}-03$ & $-4.20 \mathrm{E}-02$ & $-1.19 \mathrm{E}-03$ & $-2.85 \mathrm{E}-01$ & $5.86 \mathrm{E}-04$ & $1.98 \mathrm{E}-02$ & $8.87 \mathrm{E}-04$ \\
\hline & South Pacific & $-4.97 \mathrm{E}-02$ & $-1.09 \mathrm{E}-02$ & $-5.17 \mathrm{E}-02$ & $2.08 \mathrm{E}-02$ & $1.65 \mathrm{E}-04$ & $5.21 \mathrm{E}-03$ & $1.50 \mathrm{E}-03$ \\
\hline & Northern Ocean & $-5.02 \mathrm{E}-04$ & $-1.03 \mathrm{E}-02$ & $-1.52 \mathrm{E}-04$ & $-4.01 \mathrm{E}-02$ & $1.18 \mathrm{E}-04$ & $-1.32 \mathrm{E}-02$ & $6.94 \mathrm{E}-05$ \\
\hline & Atlantic & $-5.63 \mathrm{E}-02$ & $-2.27 \mathrm{E}-02$ & $-1.07 \mathrm{E}-02$ & $3.46 \mathrm{E}-02$ & $1.40 \mathrm{E}-04$ & $5.53 \mathrm{E}-03$ & $-7.52 \mathrm{E}-04$ \\
\hline & Southern Ocean & $1.66 \mathrm{E}-03$ & $4.19 \mathrm{E}-03$ & $-1.52 \mathrm{E}-02$ & $6.88 \mathrm{E}-04$ & $1.30 \mathrm{E}-04$ & $6.12 \mathrm{E}-04$ & $-2.90 \mathrm{E}-03$ \\
\hline & Indian Ocean & $-7.44 \mathrm{E}-02$ & $-6.11 \mathrm{E}-03$ & $-2.02 \mathrm{E}-02$ & $2.24 \mathrm{E}-02$ & $3.28 \mathrm{E}-05$ & $1.66 \mathrm{E}-03$ & $-4.98 \mathrm{E}-03$ \\
\hline \multirow{7}{*}{$\begin{array}{l}\text { Biomass } \\
\text { burning }\end{array}$} & Africa/Middle East & 1 & $-2.36 \mathrm{E}-03$ & $-1.12 \mathrm{E}-02$ & $1.37 \mathrm{E}-02$ & $2.21 \mathrm{E}-05$ & $2.88 \mathrm{E}-03$ & $-2.76 \mathrm{E}-03$ \\
\hline & Southern Asia & $-2.36 \mathrm{E}-03$ & 1 & $-1.04 \mathrm{E}-03$ & $-1.18 \mathrm{E}-02$ & $6.69 \mathrm{E}-05$ & $1.48 \mathrm{E}-03$ & $-1.29 \mathrm{E}-04$ \\
\hline & Central/South America & $-1.12 \mathrm{E}-02$ & $-1.04 \mathrm{E}-03$ & 1 & $1.39 \mathrm{E}-03$ & $2.53 \mathrm{E}-05$ & $5.87 \mathrm{E}-04$ & $-7.50 \mathrm{E}-04$ \\
\hline & Northern Asia & $1.37 \mathrm{E}-02$ & $-1.18 \mathrm{E}-02$ & $1.39 \mathrm{E}-03$ & 1 & $-3.80 \mathrm{E}-04$ & $-3.69 \mathrm{E}-02$ & $5.73 \mathrm{E}-04$ \\
\hline & Europe & $2.21 \mathrm{E}-05$ & $6.69 \mathrm{E}-05$ & $2.53 \mathrm{E}-05$ & $-3.80 \mathrm{E}-04$ & 1 & $-2.47 \mathrm{E}-04$ & $1.86 \mathrm{E}-06$ \\
\hline & North America & $2.88 \mathrm{E}-03$ & $1.48 \mathrm{E}-03$ & $5.87 \mathrm{E}-04$ & $-3.69 \mathrm{E}-02$ & $-2.47 \mathrm{E}-04$ & 1 & $2.03 E-04$ \\
\hline & Oceania & $-2.76 \mathrm{E}-03$ & $-1.29 \mathrm{E}-04$ & $-7.50 \mathrm{E}-04$ & $5.73 \mathrm{E}-04$ & $1.86 \mathrm{E}-06$ & \begin{tabular}{|l|} 
\\
\end{tabular} & 1 \\
\hline
\end{tabular}

Fig. S2ar. Correlation of the emissions in 2003 for biomass burning. 


\begin{tabular}{|c|c|c|c|c|c|c|c|c|}
\hline \multirow[b]{2}{*}{ Source sector } & \multirow[b]{2}{*}{ Region } & \multicolumn{7}{|c|}{ Agricultural Soil } \\
\hline & & $\begin{array}{l}\text { Africa/M. } \\
\text { East }\end{array}$ & $\begin{array}{l}\text { Southern } \\
\text { Asia }\end{array}$ & $\begin{array}{l}\text { C. /S. } \\
\text { America }\end{array}$ & $\begin{array}{l}\text { Northern } \\
\text { Asia }\end{array}$ & Europe & $\begin{array}{l}\text { North } \\
\text { America }\end{array}$ & Oceania \\
\hline \multirow{7}{*}{$\begin{array}{c}\text { Agricultural } \\
\text { Soil }\end{array}$} & Africa/Middle East & 1 & $-1.12 \mathrm{E}-01$ & $-6.00 \mathrm{E}-04$ & $-3.26 \mathrm{E}-02$ & $-2.65 \mathrm{E}-02$ & $-2.11 \mathrm{E}-02$ & $8.54 \mathrm{E}-03$ \\
\hline & Southern Asia & $-1.12 \mathrm{E}-01$ & 1 & $4.20 \mathrm{E}-02$ & $-2.93 \mathrm{E}-01$ & $1.89 \mathrm{E}-02$ & $-7.89 \mathrm{E}-02$ & $2.59 \mathrm{E}-02$ \\
\hline & Central/South America & $-6.00 \mathrm{E}-04$ & $4.20 \mathrm{E}-02$ & 1 & $-8.09 \mathrm{E}-03$ & $7.82 \mathrm{E}-03$ & $6.84 \mathrm{E}-03$ & $-2.42 \mathrm{E}-02$ \\
\hline & Northern Asia & $-3.26 \mathrm{E}-02$ & $-2.93 \mathrm{E}-01$ & $-8.09 \mathrm{E}-03$ & 1 & $-1.65 \mathrm{E}-01$ & $-4.82 \mathrm{E}-02$ & $-2.37 \mathrm{E}-02$ \\
\hline & Europe & $-2.65 \mathrm{E}-02$ & $1.89 \mathrm{E}-02$ & $7.82 \mathrm{E}-03$ & $-1.65 \mathrm{E}-01$ & 1 & $-1.56 \mathrm{E}-02$ & $4.34 \mathrm{E}-03$ \\
\hline & North America & $-2.11 \mathrm{E}-02$ & $-7.89 \mathrm{E}-02$ & $6.84 \mathrm{E}-03$ & $-4.82 \mathrm{E}-02$ & $-1.56 \mathrm{E}-02$ & 1 & $3.03 \mathrm{E}-03$ \\
\hline & Oceania & $8.54 \mathrm{E}-03$ & $2.59 \mathrm{E}-02$ & $-2.42 \mathrm{E}-02$ & $-2.37 \mathrm{E}-02$ & $4.34 \mathrm{E}-03$ & $3.03 \mathrm{E}-03$ & 1 \\
\hline \multirow{7}{*}{ Industrial } & Africa/Middle East & $-7.07 E-02$ & $-1.73 \mathrm{E}-02$ & $1.52 \mathrm{E}-03$ & $-8.48 \mathrm{E}-03$ & \begin{tabular}{|l|}
$-8.75 E-03$ \\
\end{tabular} & $-4.66 \mathrm{E}-03$ & $1.28 \mathrm{E}-03$ \\
\hline & \begin{tabular}{|l} 
Southern Asia \\
\end{tabular} & $1.63 \mathrm{E}-02$ & $-4.40 \mathrm{E}-01$ & $-1.34 \mathrm{E}-03$ & $-4.40 \mathrm{E}-02$ & $1.90 \mathrm{E}-02$ & $4.35 \mathrm{E}-03$ & $-9.84 \mathrm{E}-04$ \\
\hline & Central/South America & $-1.86 \mathrm{E}-04$ & $3.16 \mathrm{E}-03$ & $-7.92 \mathrm{E}-03$ & $-5.86 \mathrm{E}-04$ & $6.12 \mathrm{E}-04$ & $5.70 \mathrm{E}-04$ & $-1.98 \mathrm{E}-03$ \\
\hline & Northern Asia & $1.66 \mathrm{E}-02$ & $-3.33 E-02$ & $-1.78 \mathrm{E}-03$ & $-9.93 E-02$ & $-4.99 \mathrm{E}-02$ & $1.74 \mathrm{E}-01$ & $-4.51 \mathrm{E}-03$ \\
\hline & Europe & $-1.65 E-02$ & $-4.62 \mathrm{E}-03$ & $2.75 \mathrm{E}-03$ & $-3.49 E-02$ & \begin{tabular}{|l|}
$-7.13 E-01$ \\
\end{tabular} & $-9.18 \mathrm{E}-03$ & $8.17 \mathrm{E}-04$ \\
\hline & North America & $9.24 \mathrm{E}-03$ & $3.06 \mathrm{E}-02$ & $1.70 \mathrm{E}-03$ & $3.86 \mathrm{E}-02$ & $2.94 \mathrm{E}-02$ & $-3.64 \mathrm{E}-01$ & $-9.79 \mathrm{E}-04$ \\
\hline & Oceania & $6.66 \mathrm{E}-04$ & $1.88 \mathrm{E}-03$ & $-1.87 \mathrm{E}-03$ & $-1.40 \mathrm{E}-03$ & $2.66 \mathrm{E}-04$ & $2.01 \mathrm{E}-04$ & $-7.91 \mathrm{E}-03$ \\
\hline \multirow{7}{*}{ Natural Soil } & Africa/Middle East & $-2.16 \mathrm{E}-01$ & $-1.03 \mathrm{E}-01$ & $-8.72 \mathrm{E}-02$ & $2.48 \mathrm{E}-03$ & $\mid-1.22 \mathrm{E}-02$ & $5.34 \mathrm{E}-03$ & $-2.43 \mathrm{E}-03$ \\
\hline & Southern Asia & $-3.17 \mathrm{E}-02$ & $-3.50 \mathrm{E}-01$ & $-2.08 \mathrm{E}-03$ & $4.70 \mathrm{E}-02$ & $-6.25 \mathrm{E}-04$ & $2.12 \mathrm{E}-03$ & $1.33 \mathrm{E}-02$ \\
\hline & Central/South America & $-5.15 E-02$ & $2.92 \mathrm{E}-02$ & $-2.52 \mathrm{E}-01$ & $2.09 \mathrm{E}-02$ & $2.04 \mathrm{E}-02$ & $1.97 \mathrm{E}-02$ & $-3.81 \mathrm{E}-02$ \\
\hline & Northern Asia & $3.23 \mathrm{E}-02$ & $-2.55 \mathrm{E}-02$ & $6.20 \mathrm{E}-03$ & $-2.73 \mathrm{E}-01$ & $3.25 \mathrm{E}-02$ & $6.60 \mathrm{E}-02$ & $-8.12 \mathrm{E}-03$ \\
\hline & Europe & $-3.11 \mathrm{E}-02$ & $-7.91 \mathrm{E}-03$ & $9.26 \mathrm{E}-03$ & $9.49 \mathrm{E}-04$ & $-2.70 \mathrm{E}-01$ & $1.85 \mathrm{E}-02$ & $3.22 \mathrm{E}-03$ \\
\hline & North America & $-3.39 \mathrm{E}-02$ & $-8.28 \mathrm{E}-02$ & $2.63 \mathrm{E}-02$ & $7.27 \mathrm{E}-02$ & $-1.27 \mathrm{E}-02$ & $-4.46 \mathrm{E}-01$ & $7.23 \mathrm{E}-03$ \\
\hline & Oceania & $6.66 \mathrm{E}-03$ & $2.78 \mathrm{E}-02$ & $-3.61 \mathrm{E}-02$ & $-1.30 \mathrm{E}-02$ & $6.79 \mathrm{E}-03$ & $6.66 \mathrm{E}-03$ & $-7.29 \mathrm{E}-02$ \\
\hline \multirow{6}{*}{ Ocean } & North Pacific & $-4.09 \mathrm{E}-03$ & $-2.12 \mathrm{E}-01$ & $1.16 \mathrm{E}-02$ & $4.52 \mathrm{E}-02$ & $-2.09 \mathrm{E}-04$ & $-4.47 \mathrm{E}-02$ & $6.34 \mathrm{E}-03$ \\
\hline & South Pacific & $-4.91 \mathrm{E}-03$ & $-1.12 \mathrm{E}-02$ & $-9.16 \mathrm{E}-03$ & $1.33 \mathrm{E}-02$ & $9.96 \mathrm{E}-03$ & $2.32 \mathrm{E}-03$ & $1.85 \mathrm{E}-02$ \\
\hline & Northern Ocean & $6.57 \mathrm{E}-03$ & $-8.30 \mathrm{E}-03$ & $3.68 \mathrm{E}-03$ & $-5.57 \mathrm{E}-02$ & $-8.12 \mathrm{E}-02$ & $-6.93 \mathrm{E}-03$ & $3.26 \mathrm{E}-03$ \\
\hline & Atlantic & $-8.03 E-02$ & $-1.17 \mathrm{E}-01$ & $-2.03 \mathrm{E}-02$ & $-4.41 \mathrm{E}-02$ & $-4.28 \mathrm{E}-02$ & $-9.28 \mathrm{E}-02$ & $1.50 \mathrm{E}-02$ \\
\hline & Southern Ocean & $4.02 \mathrm{E}-02$ & $1.20 \mathrm{E}-01$ & $-2.13 \mathrm{E}-01$ & $-2.50 \mathrm{E}-02$ & $1.03 \mathrm{E}-02$ & $1.02 \mathrm{E}-02$ & $-9.42 \mathrm{E}-02$ \\
\hline & Indian Ocean & $-4.48 \mathrm{E}-02$ & $-3.96 \mathrm{E}-02$ & $-9.87 \mathrm{E}-02$ & $-1.59 \mathrm{E}-03$ & $5.02 \mathrm{E}-03$ & $7.31 \mathrm{E}-03$ & $-2.72 \mathrm{E}-02$ \\
\hline \multirow{7}{*}{$\begin{array}{l}\text { Biomass } \\
\text { burning }\end{array}$} & Africa/Middle East & $-3.34 \mathrm{E}-02$ & $-1.52 \mathrm{E}-02$ & $-3.34 \mathrm{E}-02$ & $5.34 \mathrm{E}-03$ & $3.05 \mathrm{E}-03$ & $8.49 \mathrm{E}-04$ & $8.49 \mathrm{E}-03$ \\
\hline & Southern Asia & $-6.82 \mathrm{E}-03$ & $-4.38 \mathrm{E}-02$ & $4.05 \mathrm{E}-03$ & $8.29 \mathrm{E}-03$ & $3.36 \mathrm{E}-03$ & $-8.38 \mathrm{E}-03$ & $3.42 \mathrm{E}-03$ \\
\hline & Central/South America & $-6.54 \mathrm{E}-03$ & $6.21 \mathrm{E}-03$ & $-2.51 \mathrm{E}-02$ & $-1.91 \mathrm{E}-03$ & $2.26 \mathrm{E}-03$ & $1.20 \mathrm{E}-03$ & $2.85 \mathrm{E}-03$ \\
\hline & Northern Asia & $-6.51 E-04$ & $-8.60 \mathrm{E}-03$ & $1.26 \mathrm{E}-04$ & $1.04 \mathrm{E}-03$ & $-4.32 \mathrm{E}-04$ & $2.36 \mathrm{E}-03$ & $4.92 \mathrm{E}-04$ \\
\hline & Europe & $-1.62 \mathrm{E}-04$ & $1.40 \mathrm{E}-04$ & $-1.15 \mathrm{E}-05$ & $5.48 \mathrm{E}-05$ & $1.21 \mathrm{E}-04$ & $8.30 \mathrm{E}-05$ & $-1.08 \mathrm{E}-06$ \\
\hline & North America & $4.24 \mathrm{E}-03$ & $-5.66 \mathrm{E}-03$ & $3.54 \mathrm{E}-03$ & $1.20 \mathrm{E}-02$ & $-8.48 \mathrm{E}-03$ & $2.99 \mathrm{E}-02$ & $6.59 \mathrm{E}-04$ \\
\hline & Oceania & $-1.54 \mathrm{E}-03$ & $5.57 \mathrm{E}-03$ & $-1.30 \mathrm{E}-02$ & $-2.28 \mathrm{E}-03$ & $2.21 \mathrm{E}-03$ & $2.13 \mathrm{E}-03$ & $1.25 \mathrm{E}-04$ \\
\hline
\end{tabular}

Fig. S2as. Correlation of the emissions in 2004 for agricultural soil. 


\begin{tabular}{|c|c|c|c|c|c|c|c|c|}
\hline \multirow[b]{2}{*}{ Source sector } & \multirow[b]{2}{*}{ Region } & \multicolumn{7}{|c|}{ Industrial } \\
\hline & & $\begin{array}{l}\text { Africa/M. } \\
\text { East }\end{array}$ & $\begin{array}{l}\text { Southern } \\
\text { Asia }\end{array}$ & $\begin{array}{l}\text { C. /S. } \\
\text { America }\end{array}$ & $\begin{array}{l}\text { Northern } \\
\text { Asia }\end{array}$ & Europe & $\begin{array}{l}\text { North } \\
\text { America }\end{array}$ & Oceania \\
\hline \multirow{7}{*}{$\begin{array}{l}\text { Agricultural } \\
\text { Soil }\end{array}$} & Africa/Middle East & $-7.07 \mathrm{E}-02$ & $1.63 \mathrm{E}-02$ & $-1.86 \mathrm{E}-04$ & $1.66 \mathrm{E}-02$ & $-1.65 \mathrm{E}-02$ & $9.24 \mathrm{E}-03$ & $6.66 \mathrm{E}-04$ \\
\hline & Southern Asia & $-1.73 \mathrm{E}-02$ & $-4.40 \mathrm{E}-01$ & $3.16 \mathrm{E}-03$ & $-3.33 \mathrm{E}-02$ & $-4.62 \mathrm{E}-03$ & $3.06 \mathrm{E}-02$ & $1.88 \mathrm{E}-03$ \\
\hline & Central/South America & $1.52 \mathrm{E}-03$ & $-1.34 \mathrm{E}-03$ & $-7.92 \mathrm{E}-03$ & $-1.78 \mathrm{E}-03$ & $2.75 \mathrm{E}-03$ & $1.70 \mathrm{E}-03$ & $-1.87 \mathrm{E}-03$ \\
\hline & Northern Asia & $-8.48 \mathrm{E}-03$ & $-4.40 \mathrm{E}-02$ & $-5.86 \mathrm{E}-04$ & $-9.93 \mathrm{E}-02$ & $-3.49 \mathrm{E}-02$ & $3.86 \mathrm{E}-02$ & $-1.40 \mathrm{E}-03$ \\
\hline & Europe & $-8.75 \mathrm{E}-03$ & $1.90 \mathrm{E}-02$ & $6.12 \mathrm{E}-04$ & $-4.99 \mathrm{E}-02$ & $-7.13 \mathrm{E}-01$ & $2.94 \mathrm{E}-02$ & $2.66 \mathrm{E}-04$ \\
\hline & North America & $-4.66 \mathrm{E}-03$ & $4.35 \mathrm{E}-03$ & $5.70 \mathrm{E}-04$ & $1.74 \mathrm{E}-01$ & $-9.18 \mathrm{E}-03$ & $-3.64 \mathrm{E}-01$ & $2.01 \mathrm{E}-04$ \\
\hline & Oceania & $1.28 \mathrm{E}-03$ & $-9.84 \mathrm{E}-04$ & $-1.98 \mathrm{E}-03$ & $-4.51 \mathrm{E}-03$ & $8.17 \mathrm{E}-04$ & $-9.79 E-04$ & $-7.91 \mathrm{E}-03$ \\
\hline \multirow{7}{*}{ Industrial } & Africa/Middle East & 1 & $2.22 \mathrm{E}-03$ & $1.08 \mathrm{E}-04$ & $-4.30 \mathrm{E}-04$ & $-5.11 \mathrm{E}-03$ & $1.65 \mathrm{E}-03$ & $9.57 \mathrm{E}-05$ \\
\hline & Southern Asia & $2.22 \mathrm{E}-03$ & 1 & $-4.92 \mathrm{E}-05$ & $-4.17 \mathrm{E}-02$ & $3.10 \mathrm{E}-03$ & $1.24 \mathrm{E}-02$ & $-1.15 \mathrm{E}-04$ \\
\hline & Central/South America & $1.08 \mathrm{E}-04$ & $-4.92 \mathrm{E}-05$ & 1 & $-1.64 \mathrm{E}-05$ & $2.12 \mathrm{E}-04$ & $1.74 \mathrm{E}-04$ & $-1.52 \mathrm{E}-04$ \\
\hline & Northern Asia & $-4.30 \mathrm{E}-04$ & $-4.17 \mathrm{E}-02$ & $-1.64 \mathrm{E}-05$ & 1 & $-2.49 \mathrm{E}-02$ & $-2.58 \mathrm{E}-01$ & $-3.59 \mathrm{E}-04$ \\
\hline & Europe & $-5.11 \mathrm{E}-03$ & $3.10 \mathrm{E}-03$ & $2.12 \mathrm{E}-04$ & $-2.49 \mathrm{E}-02$ & 1 & $5.90 \mathrm{E}-03$ & $4.58 \mathrm{E}-05$ \\
\hline & North America & $1.65 \mathrm{E}-03$ & $1.24 \mathrm{E}-02$ & $1.74 \mathrm{E}-04$ & $-2.58 \mathrm{E}-01$ & 5.90E-03 & 1 & $-7.19 E-05$ \\
\hline & Oceania & $9.57 \mathrm{E}-05$ & $-1.15 \mathrm{E}-04$ & $-1.52 \mathrm{E}-04$ & $-3.59 \mathrm{E}-04$ & $4.58 \mathrm{E}-05$ & $-7.19 \mathrm{E}-05$ & 1 \\
\hline \multirow{7}{*}{ Natural Soil } & Africa/Middle East & $-3.36 \mathrm{E}-02$ & $3.78 \mathrm{E}-02$ & $-7.35 \mathrm{E}-03$ & $5.44 \mathrm{E}-02$ & $-6.54 \mathrm{E}-03$ & $1.85 \mathrm{E}-02$ & $-2.07 \mathrm{E}-05$ \\
\hline & Southern Asia & $-2.73 \mathrm{E}-03$ & $-6.15 \mathrm{E}-02$ & $-3.94 \mathrm{E}-04$ & $2.35 \mathrm{E}-02$ & $-6.41 \mathrm{E}-03$ & $1.42 \mathrm{E}-02$ & $1.25 \mathrm{E}-03$ \\
\hline & Central/South America & $-1.74 \mathrm{E}-04$ & $3.32 \mathrm{E}-03$ & $-1.99 \mathrm{E}-02$ & $3.45 \mathrm{E}-02$ & $9.16 \mathrm{E}-03$ & $1.05 \mathrm{E}-02$ & $-3.56 \mathrm{E}-03$ \\
\hline & Northern Asia & $7.14 \mathrm{E}-03$ & $-2.07 \mathrm{E}-02$ & $5.79 \mathrm{E}-04$ & $-6.98 \mathrm{E}-02$ & $2.80 \mathrm{E}-02$ & $7.74 \mathrm{E}-03$ & $-7.09 E-04$ \\
\hline & Europe & $-1.13 \mathrm{E}-02$ & $1.59 \mathrm{E}-02$ & $7.24 \mathrm{E}-04$ & $4.24 \mathrm{E}-03$ & $-1.75 \mathrm{E}-01$ & $3.13 \mathrm{E}-02$ & $2.58 \mathrm{E}-04$ \\
\hline & North America & $-1.91 \mathrm{E}-03$ & 5.97E-02 & $1.77 \mathrm{E}-03$ & $-2.12 \mathrm{E}-01$ & $1.07 \mathrm{E}-02$ & $-2.42 \mathrm{E}-01$ & $6.48 \mathrm{E}-04$ \\
\hline & Oceania & $1.44 \mathrm{E}-03$ & $1.02 \mathrm{E}-03$ & $-2.91 \mathrm{E}-03$ & $-2.86 \mathrm{E}-03$ & $2.27 \mathrm{E}-03$ & $-8.52 \mathrm{E}-04$ & $-4.49 \mathrm{E}-03$ \\
\hline \multirow{6}{*}{ Ocean } & North Pacific & $-2.62 \mathrm{E}-03$ & $-1.40 \mathrm{E}-02$ & $1.04 \mathrm{E}-03$ & $5.25 \mathrm{E}-02$ & $-9.06 \mathrm{E}-03$ & $-3.08 \mathrm{E}-02$ & $4.16 \mathrm{E}-04$ \\
\hline & South Pacific & $-1.20 \mathrm{E}-04$ & $1.22 \mathrm{E}-02$ & $-2.32 \mathrm{E}-03$ & $1.18 \mathrm{E}-02$ & $4.08 \mathrm{E}-03$ & $-5.95 E-03$ & $2.32 \mathrm{E}-03$ \\
\hline & Northern Ocean & $6.11 \mathrm{E}-04$ & $-6.25 \mathrm{E}-03$ & $3.78 \mathrm{E}-04$ & $4.06 \mathrm{E}-02$ & $5.81 \mathrm{E}-03$ & $2.91 \mathrm{E}-02$ & $1.64 \mathrm{E}-04$ \\
\hline & Atlantic & $-1.24 \mathrm{E}-02$ & $-2.49 \mathrm{E}-04$ & $-1.72 \mathrm{E}-03$ & $-2.63 \mathrm{E}-01$ & $-1.15 \mathrm{E}-02$ & $-1.25 \mathrm{E}-01$ & $1.00 \mathrm{E}-03$ \\
\hline & Southern Ocean & $4.21 \mathrm{E}-03$ & $-2.06 \mathrm{E}-02$ & $-1.50 \mathrm{E}-02$ & $-1.84 \mathrm{E}-03$ & $1.49 \mathrm{E}-03$ & $1.22 \mathrm{E}-02$ & $-6.48 \mathrm{E}-03$ \\
\hline & Indian Ocean & $-3.37 \mathrm{E}-03$ & $1.71 \mathrm{E}-02$ & $-8.48 \mathrm{E}-03$ & $8.35 \mathrm{E}-03$ & $2.42 \mathrm{E}-03$ & $-3.42 \mathrm{E}-03$ & $-3.11 \mathrm{E}-03$ \\
\hline \multirow{7}{*}{$\begin{array}{l}\text { Biomass } \\
\text { burning }\end{array}$} & Africa/Middle East & $-2.77 \mathrm{E}-03$ & $8.70 \mathrm{E}-03$ & $-2.79 \mathrm{E}-03$ & $1.37 \mathrm{E}-02$ & $1.40 \mathrm{E}-03$ & $3.20 \mathrm{E}-03$ & $6.42 \mathrm{E}-04$ \\
\hline & Southern Asia & $-1.24 \mathrm{E}-03$ & $-1.29 \mathrm{E}-03$ & $3.57 E-04$ & $6.98 \mathrm{E}-03$ & $1.84 \mathrm{E}-03$ & $-2.13 \mathrm{E}-03$ & $2.47 \mathrm{E}-04$ \\
\hline & Central/South America & $-3.91 \mathrm{E}-04$ & $2.90 \mathrm{E}-03$ & $-2.03 \mathrm{E}-03$ & $2.83 E-03$ & $6.23 \mathrm{E}-04$ & $1.17 \mathrm{E}-03$ & $2.00 \mathrm{E}-04$ \\
\hline & Northern Asia & $-4.93 E-05$ & $1.14 \mathrm{E}-03$ & $5.64 \mathrm{E}-06$ & $3.58 \mathrm{E}-03$ & $4.51 \mathrm{E}-04$ & $2.45 \mathrm{E}-03$ & $4.57 \mathrm{E}-05$ \\
\hline & Europe & $-9.58 \mathrm{E}-05$ & $7.31 \mathrm{E}-06$ & $-7.51 \mathrm{E}-07$ & $-1.13 \mathrm{E}-04$ & $8.11 \mathrm{E}-05$ & $6.61 \mathrm{E}-05$ & $2.48 \mathrm{E}-07$ \\
\hline & North America & $1.11 \mathrm{E}-03$ & $9.52 \mathrm{E}-03$ & $2.64 \mathrm{E}-04$ & $3.01 \mathrm{E}-02$ & $6.41 \mathrm{E}-03$ & $1.87 \mathrm{E}-02$ & $6.13 \mathrm{E}-05$ \\
\hline & Oceania & $1.06 \mathrm{E}-04$ & $1.95 \mathrm{E}-03$ & $-1.05 \mathrm{E}-03$ & $7.04 \mathrm{E}-04$ & $8.02 \mathrm{E}-04$ & $2.72 \mathrm{E}-04$ & $2.01 \mathrm{E}-06$ \\
\hline
\end{tabular}

Fig. S2at. Correlation of the emissions in 2004 for industrial. 


\begin{tabular}{|c|c|c|c|c|c|c|c|c|}
\hline & \multirow{2}{*}{\multicolumn{7}{|c|}{ Natural Soil }} \\
\hline & & & & & & & & \\
\hline Source sector & Region & $\begin{array}{l}\text { Africa/M. } \\
\text { East }\end{array}$ & $\begin{array}{l}\text { Southern } \\
\text { Asia }\end{array}$ & $\begin{array}{l}\text { C. /S. } \\
\text { America }\end{array}$ & $\begin{array}{l}\text { Northern } \\
\text { Asia }\end{array}$ & Europe & $\begin{array}{l}\text { North } \\
\text { America }\end{array}$ & Oceania \\
\hline \multirow{7}{*}{$\begin{array}{l}\text { Agricultural } \\
\text { Soil }\end{array}$} & Africa/Middle East & $-2.16 \mathrm{E}-01$ & $-3.17 \mathrm{E}-02$ & $-5.15 \mathrm{E}-02$ & 3.23E-02 & $-3.11 \mathrm{E}-02$ & $-3.39 \mathrm{E}-02$ & $6.66 \mathrm{E}-03$ \\
\hline & Southern Asia & $-1.03 \mathrm{E}-01$ & $-3.50 \mathrm{E}-01$ & $2.92 \mathrm{E}-02$ & $\mid-2.55 \mathrm{E}-02$ & $-7.91 \mathrm{E}-03$ & $-8.28 \mathrm{E}-02$ & $2.78 \mathrm{E}-02$ \\
\hline & Central/South America & $-8.72 \mathrm{E}-02$ & $-2.08 \mathrm{E}-03$ & $-2.52 \mathrm{E}-01$ & $6.20 \mathrm{E}-03$ & $9.26 \mathrm{E}-03$ & $2.63 \mathrm{E}-02$ & $-3.61 \mathrm{E}-02$ \\
\hline & Northern Asia & $2.48 \mathrm{E}-03$ & $4.70 \mathrm{E}-02$ & $2.09 \mathrm{E}-02$ & \begin{tabular}{|l|}
$-2.73 \mathrm{E}-01$ \\
\end{tabular} & $9.49 \mathrm{E}-04$ & $7.27 \mathrm{E}-02$ & $-1.30 \mathrm{E}-02$ \\
\hline & Europe & $-1.22 \mathrm{E}-02$ & $-6.25 \mathrm{E}-04$ & $2.04 \mathrm{E}-02$ & $3.25 \mathrm{E}-02$ & $\mid-2.70 \mathrm{E}-01$ & $-1.27 \mathrm{E}-02$ & $6.79 \mathrm{E}-03$ \\
\hline & North America & $5.34 \mathrm{E}-03$ & $2.12 \mathrm{E}-03$ & $1.97 \mathrm{E}-02$ & $6.60 \mathrm{E}-02$ & $1.85 \mathrm{E}-02$ & $-4.46 \mathrm{E}-01$ & $6.66 \mathrm{E}-03$ \\
\hline & Oceania & $-2.43 \mathrm{E}-03$ & $1.33 \mathrm{E}-02$ & $-3.81 \mathrm{E}-02$ & $-8.12 \mathrm{E}-03$ & $3.22 \mathrm{E}-03$ & $7.23 \mathrm{E}-03$ & $-7.29 \mathrm{E}-02$ \\
\hline \multirow{7}{*}{ Industrial } & Africa/Middle East & $-3.36 \mathrm{E}-02$ & $-2.73 \mathrm{E}-03$ & $-1.74 \mathrm{E}-04$ & $7.14 \mathrm{E}-03$ & $\mid-1.13 \mathrm{E}-02$ & $-1.91 \mathrm{E}-03$ & $1.44 \mathrm{E}-03$ \\
\hline & Southern Asia & $3.78 \mathrm{E}-02$ & $-6.15 \mathrm{E}-02$ & $3.32 \mathrm{E}-03$ & $-2.07 \mathrm{E}-02$ & $1.59 \mathrm{E}-02$ & $5.97 \mathrm{E}-02$ & $1.02 \mathrm{E}-03$ \\
\hline & Central/South America & $-7.35 \mathrm{E}-03$ & $-3.94 \mathrm{E}-04$ & $-1.99 \mathrm{E}-02$ & $5.79 \mathrm{E}-04$ & $7.24 \mathrm{E}-04$ & $1.77 \mathrm{E}-03$ & $-2.91 \mathrm{E}-03$ \\
\hline & Northern Asia & $5.44 \mathrm{E}-02$ & $2.35 \mathrm{E}-02$ & $3.45 \mathrm{E}-02$ & \begin{tabular}{|l|}
$-6.98 \mathrm{E}-02$ \\
\end{tabular} & $4.24 \mathrm{E}-03$ & $-2.12 \mathrm{E}-01$ & $-2.86 \mathrm{E}-03$ \\
\hline & Europe & $-6.54 \mathrm{E}-03$ & $-6.41 \mathrm{E}-03$ & $9.16 \mathrm{E}-03$ & $2.80 \mathrm{E}-02$ & \begin{tabular}{|l|}
$-1.75 \mathrm{E}-01$ \\
\end{tabular} & $1.07 \mathrm{E}-02$ & 2.27E-03 \\
\hline & North America & $1.85 \mathrm{E}-02$ & $1.42 \mathrm{E}-02$ & $1.05 \mathrm{E}-02$ & 7.74E-03 & $3.13 \mathrm{E}-02$ & $-2.42 \mathrm{E}-01$ & $-8.52 \mathrm{E}-04$ \\
\hline & Oceania & $-2.07 E-05$ & $1.25 \mathrm{E}-03$ & $-3.56 \mathrm{E}-03$ & \begin{tabular}{|l|}
$-7.09 E-04$ \\
\end{tabular} & $2.58 \mathrm{E}-04$ & $6.48 \mathrm{E}-04$ & $-4.49 \mathrm{E}-03$ \\
\hline \multirow{7}{*}{ Natural Soil } & Africa/Middle East & 1 & $-1.33 \mathrm{E}-01$ & $-3.92 \mathrm{E}-01$ & $5.61 \mathrm{E}-02$ & \begin{tabular}{|l|}
$-1.66 \mathrm{E}-02$ \\
\end{tabular} & $-1.01 \mathrm{E}-01$ & $-1.56 \mathrm{E}-02$ \\
\hline & Southern Asia & $-1.33 \mathrm{E}-01$ & 1 & $-5.26 \mathrm{E}-02$ & $-3.56 \mathrm{E}-01$ & $1.88 \mathrm{E}-02$ & $-4.39 \mathrm{E}-02$ & $-1.00 \mathrm{E}-02$ \\
\hline & Central/South America & $-3.92 \mathrm{E}-01$ & $-5.26 \mathrm{E}-02$ & 1 & $-9.53 E-02$ & \begin{tabular}{|l|}
$-1.04 \mathrm{E}-02$ \\
\end{tabular} & $-4.02 \mathrm{E}-02$ & $-1.35 \mathrm{E}-01$ \\
\hline & Northern Asia & $5.61 \mathrm{E}-02$ & $-3.56 \mathrm{E}-01$ & $-9.53 \mathrm{E}-02$ & 1 & \begin{tabular}{|l|}
$-1.30 \mathrm{E}-01$ \\
\end{tabular} & $-1.80 \mathrm{E}-01$ & $-1.65 \mathrm{E}-02$ \\
\hline & Europe & $-1.66 \mathrm{E}-02$ & $1.88 \mathrm{E}-02$ & $-1.04 \mathrm{E}-02$ & $-1.30 \mathrm{E}-01$ & \begin{tabular}{r|}
1 \\
\end{tabular} & $-1.17 \mathrm{E}-01$ & $2.24 \mathrm{E}-03$ \\
\hline & North America & $-1.01 \mathrm{E}-01$ & $-4.39 \mathrm{E}-02$ & $-4.02 \mathrm{E}-02$ & $-1.80 \mathrm{E}-01$ & \begin{tabular}{|l|}
$-1.17 \mathrm{E}-01$ \\
\end{tabular} & 1 & $1.70 \mathrm{E}-02$ \\
\hline & Oceania & $-1.56 \mathrm{E}-02$ & $-1.00 \mathrm{E}-02$ & $-1.35 \mathrm{E}-01$ & $\begin{array}{ll}-1.65 \mathrm{E}-02 \\
\end{array}$ & $2.24 \mathrm{E}-03$ & $1.70 \mathrm{E}-02$ & 1 \\
\hline \multirow{6}{*}{ Ocean } & North Pacific & $8.83 \mathrm{E}-02$ & $1.57 \mathrm{E}-01$ & $-1.26 \mathrm{E}-03$ & $-2.32 \mathrm{E}-01$ & $2.70 \mathrm{E}-02$ & $7.99 \mathrm{E}-02$ & $1.77 \mathrm{E}-03$ \\
\hline & \begin{tabular}{|l} 
South Pacific \\
\end{tabular} & $2.12 \mathrm{E}-02$ & $1.40 \mathrm{E}-02$ & $-5.68 \mathrm{E}-01$ & $1.49 \mathrm{E}-02$ & $1.23 \mathrm{E}-02$ & $6.11 \mathrm{E}-02$ & $2.60 \mathrm{E}-02$ \\
\hline & Northern Ocean & $6.12 \mathrm{E}-02$ & $7.94 \mathrm{E}-02$ & $3.00 \mathrm{E}-02$ & $-5.47 \mathrm{E}-02$ & $-4.24 \mathrm{E}-03$ & $-1.59 \mathrm{E}-03$ & $5.61 \mathrm{E}-04$ \\
\hline & Atlantic & $-8.49 \mathrm{E}-02$ & $9.52 \mathrm{E}-02$ & $-4.90 \mathrm{E}-02$ & $6.75 \mathrm{E}-02$ & $4.82 \mathrm{E}-02$ & $\begin{array}{ll} & 8.30 \mathrm{E}-02 \\
\end{array}$ & $2.54 \mathrm{E}-03$ \\
\hline & Southern Ocean & $1.15 \mathrm{E}-01$ & $1.41 \mathrm{E}-01$ & $1.03 \mathrm{E}-02$ & $-4.36 \mathrm{E}-02$ & $1.71 \mathrm{E}-03$ & $3.32 \mathrm{E}-02$ & $-5.71 \mathrm{E}-02$ \\
\hline & Indian Ocean & $-1.52 \mathrm{E}-01$ & $-1.22 \mathrm{E}-01$ & $-1.70 \mathrm{E}-01$ & $3.09 \mathrm{E}-02$ & $1.31 \mathrm{E}-02$ & $3.35 \mathrm{E}-02$ & $-4.66 \mathrm{E}-02$ \\
\hline \multirow{7}{*}{$\begin{array}{l}\text { Biomass } \\
\text { burning }\end{array}$} & Africa/Middle East & $-1.30 \mathrm{E}-01$ & $-2.75 \mathrm{E}-02$ & $-1.78 \mathrm{E}-02$ & $2.58 \mathrm{E}-02$ & $7.20 \mathrm{E}-03$ & \begin{tabular}{|l|}
$6.00 \mathrm{E}-03$ \\
\end{tabular} & $2.75 \mathrm{E}-03$ \\
\hline & Southern Asia & $1.65 \mathrm{E}-02$ & $7.77 \mathrm{E}-03$ & $-2.09 \mathrm{E}-02$ & $-1.09 \mathrm{E}-02$ & \begin{tabular}{|l|}
$-7.73 \mathrm{E}-03$ \\
\end{tabular} & \begin{tabular}{|l|l|} 
& $9.92 \mathrm{E}-03$ \\
\end{tabular} & $-3.88 \mathrm{E}-03$ \\
\hline & Central/South America & $-3.64 \mathrm{E}-02$ & $-1.33 \mathrm{E}-02$ & $1.37 \mathrm{E}-02$ & $9.47 \mathrm{E}-03$ & 4.87E-03 & $1.07 \mathrm{E}-02$ & $-1.40 \mathrm{E}-03$ \\
\hline & Northern Asia & $-5.32 \mathrm{E}-04$ & $-2.12 \mathrm{E}-03$ & $-1.03 \mathrm{E}-03$ & $-2.10 \mathrm{E}-02$ & $-8.28 \mathrm{E}-03$ & \begin{tabular}{|l|}
$-1.29 \mathrm{E}-02$ \\
\end{tabular} & $1.53 \mathrm{E}-05$ \\
\hline & \begin{tabular}{|l|} 
Europe \\
\end{tabular} & $-7.77 \mathrm{E}-05$ & $-1.70 \mathrm{E}-04$ & $2.59 \mathrm{E}-05$ & $-3.49 \mathrm{E}-05$ & \begin{tabular}{|l|}
$-3.62 \mathrm{E}-03$ \\
\end{tabular} & \begin{tabular}{|l|}
$-1.83 \mathrm{E}-04$ \\
\end{tabular} & $-1.62 \mathrm{E}-06$ \\
\hline & North America & $4.54 \mathrm{E}-03$ & $-1.21 \mathrm{E}-02$ & $-1.01 \mathrm{E}-02$ & $-7.07 E-02$ & \begin{tabular}{|l|}
$-5.21 \mathrm{E}-02$ \\
\end{tabular} & \begin{tabular}{|l|}
$-1.57 \mathrm{E}-01$ \\
\end{tabular} & $1.07 \mathrm{E}-03$ \\
\hline & Oceania & $-1.41 \mathrm{E}-02$ & $-1.27 \mathrm{E}-02$ & $9.02 \mathrm{E}-03$ & $5.07 \mathrm{E}-03$ & $2.39 \mathrm{E}-03$ & $\begin{array}{ll}7.74 \mathrm{E}-03 \\
\end{array}$ & $-5.58 \mathrm{E}-03$ \\
\hline
\end{tabular}

Fig. S2au. Correlation of the emissions in 2004 for natural soil. 


\begin{tabular}{|c|c|c|c|c|c|c|c|}
\hline & \multirow{2}{*}{\multicolumn{6}{|c|}{ Ocean }} \\
\hline & & & & & & & \\
\hline Source sector & Region & $\begin{array}{l}\text { North } \\
\text { Pacific }\end{array}$ & $\begin{array}{l}\text { South } \\
\text { Pacific }\end{array}$ & $\begin{array}{l}\text { Northern } \\
\text { Ocean }\end{array}$ & Atlantic & $\begin{array}{l}\text { Southern } \\
\text { Ocean }\end{array}$ & $\begin{array}{l}\text { Indian } \\
\text { Ocean }\end{array}$ \\
\hline \multirow{7}{*}{$\begin{array}{l}\text { Agricultural } \\
\text { Soil }\end{array}$} & Africa/Middle East & $-4.09 \mathrm{E}-03$ & $-4.91 \mathrm{E}-03$ & $6.57 \mathrm{E}-03$ & $-8.03 E-02$ & $4.02 \mathrm{E}-02$ & $-4.48 \mathrm{E}-02$ \\
\hline & \begin{tabular}{|l} 
Southern Asia \\
\end{tabular} & $-2.12 \mathrm{E}-01$ & $-1.12 \mathrm{E}-02$ & $-8.30 \mathrm{E}-03$ & $-1.17 \mathrm{E}-01$ & $1.20 \mathrm{E}-01$ & $-3.96 \mathrm{E}-02$ \\
\hline & Central/South America & $1.16 \mathrm{E}-02$ & $-9.16 \mathrm{E}-03$ & $3.68 \mathrm{E}-03$ & $-2.03 E-02$ & $-2.13 \mathrm{E}-01$ & $-9.87 \mathrm{E}-02$ \\
\hline & \begin{tabular}{|l} 
Northern Asia \\
\end{tabular} & $4.52 \mathrm{E}-02$ & $1.33 \mathrm{E}-02$ & $-5.57 \mathrm{E}-02$ & $-4.41 \mathrm{E}-02$ & $-2.50 \mathrm{E}-02$ & $-1.59 \mathrm{E}-03$ \\
\hline & Europe & $-2.09 \mathrm{E}-04$ & $9.96 \mathrm{E}-03$ & $\mid-8.12 \mathrm{E}-02$ & $-4.28 \mathrm{E}-02$ & $1.03 \mathrm{E}-02$ & $5.02 \mathrm{E}-03$ \\
\hline & North America & $-4.47 \mathrm{E}-02$ & $2.32 \mathrm{E}-03$ & $\mid-6.93 \mathrm{E}-03$ & $-9.28 \mathrm{E}-02$ & $1.02 \mathrm{E}-02$ & $7.31 \mathrm{E}-03$ \\
\hline & Oceania & $6.34 \mathrm{E}-03$ & $1.85 \mathrm{E}-02$ & $3.26 \mathrm{E}-03$ & $1.50 \mathrm{E}-02$ & $-9.42 \mathrm{E}-02$ & $-2.72 \mathrm{E}-02$ \\
\hline \multirow{7}{*}{ Industrial } & Africa/Middle East & $-2.62 \mathrm{E}-03$ & $-1.20 \mathrm{E}-04$ & $6.11 \mathrm{E}-04$ & $-1.24 \mathrm{E}-02$ & $4.21 \mathrm{E}-03$ & $-3.37 \mathrm{E}-03$ \\
\hline & Southern Asia & $-1.40 \mathrm{E}-02$ & $1.22 \mathrm{E}-02$ & $\mid-6.25 \mathrm{E}-03$ & $-2.49 \mathrm{E}-04$ & $-2.06 \mathrm{E}-02$ & $1.71 \mathrm{E}-02$ \\
\hline & Central/South America & $1.04 \mathrm{E}-03$ & $-2.32 \mathrm{E}-03$ & $3.78 \mathrm{E}-04$ & $-1.72 \mathrm{E}-03$ & $-1.50 \mathrm{E}-02$ & $-8.48 \mathrm{E}-03$ \\
\hline & Northern Asia & $5.25 \mathrm{E}-02$ & $1.18 \mathrm{E}-02$ & $4.06 \mathrm{E}-02$ & $-2.63 \mathrm{E}-01$ & $-1.84 \mathrm{E}-03$ & $8.35 \mathrm{E}-03$ \\
\hline & \begin{tabular}{|l|} 
Europe \\
\end{tabular} & $-9.06 \mathrm{E}-03$ & $4.08 \mathrm{E}-03$ & $5.81 \mathrm{E}-03$ & $-1.15 \mathrm{E}-02$ & $1.49 \mathrm{E}-03$ & $2.42 \mathrm{E}-03$ \\
\hline & North America & $-3.08 \mathrm{E}-02$ & $-5.95 \mathrm{E}-03$ & $2.91 \mathrm{E}-02$ & $-1.25 \mathrm{E}-01$ & $1.22 \mathrm{E}-02$ & $-3.42 E-03$ \\
\hline & Oceania & $4.16 \mathrm{E}-04$ & $2.32 \mathrm{E}-03$ & $1.64 \mathrm{E}-04$ & $1.00 \mathrm{E}-03$ & $-6.48 \mathrm{E}-03$ & $-3.11 \mathrm{E}-03$ \\
\hline \multirow{7}{*}{ Natural Soil } & Africa/Middle East & $8.83 \mathrm{E}-02$ & $2.12 \mathrm{E}-02$ & $6.12 \mathrm{E}-02$ & $-8.49 \mathrm{E}-02$ & $1.15 \mathrm{E}-01$ & $-1.52 \mathrm{E}-01$ \\
\hline & Southern Asia & $1.57 \mathrm{E}-01$ & $1.40 \mathrm{E}-02$ & 7.94E-02 & $9.52 \mathrm{E}-02$ & $1.41 \mathrm{E}-01$ & $-1.22 \mathrm{E}-01$ \\
\hline & Central/South America & $-1.26 \mathrm{E}-03$ & $-5.68 \mathrm{E}-01$ & $3.00 \mathrm{E}-02$ & $-4.90 \mathrm{E}-02$ & $1.03 \mathrm{E}-02$ & $-1.70 \mathrm{E}-01$ \\
\hline & Northern Asia & $-2.32 \mathrm{E}-01$ & $1.49 \mathrm{E}-02$ & \begin{tabular}{|l|}
$-5.47 \mathrm{E}-02$ \\
\end{tabular} & $6.75 \mathrm{E}-02$ & $-4.36 \mathrm{E}-02$ & 3.09E-02 \\
\hline & Europe & $2.70 \mathrm{E}-02$ & $1.23 \mathrm{E}-02$ & \begin{tabular}{|l|}
$-4.24 \mathrm{E}-03$ \\
\end{tabular} & $4.82 \mathrm{E}-02$ & $1.71 \mathrm{E}-03$ & $1.31 \mathrm{E}-02$ \\
\hline & North America & 7.99E-02 & $6.11 \mathrm{E}-02$ & \begin{tabular}{|l|}
$-1.59 \mathrm{E}-03$ \\
\end{tabular} & $8.30 \mathrm{E}-02$ & $3.32 \mathrm{E}-02$ & $3.35 \mathrm{E}-02$ \\
\hline & Oceania & $1.77 \mathrm{E}-03$ & $2.60 \mathrm{E}-02$ & $5.61 \mathrm{E}-04$ & $2.54 \mathrm{E}-03$ & $-5.71 \mathrm{E}-02$ & $-4.66 \mathrm{E}-02$ \\
\hline \multirow{6}{*}{ Ocean } & North Pacific & 1 & $1.44 \mathrm{E}-02$ & $-9.69 \mathrm{E}-02$ & $-2.02 \mathrm{E}-01$ & $3.73 \mathrm{E}-03$ & $4.70 \mathrm{E}-02$ \\
\hline & South Pacific & $1.44 \mathrm{E}-02$ & 1 & $9.11 \mathrm{E}-03$ & $-5.12 \mathrm{E}-02$ & $2.37 \mathrm{E}-02$ & $3.54 \mathrm{E}-02$ \\
\hline & Northern Ocean & $-9.69 \mathrm{E}-02$ & $9.11 \mathrm{E}-03$ & 1 & $-3.18 \mathrm{E}-01$ & $1.26 \mathrm{E}-02$ & $2.18 \mathrm{E}-02$ \\
\hline & Atlantic & $-2.02 \mathrm{E}-01$ & $-5.12 \mathrm{E}-02$ & $-3.18 \mathrm{E}-01$ & 1 & $-7.36 \mathrm{E}-02$ & $-2.15 \mathrm{E}-02$ \\
\hline & Southern Ocean & $3.73 \mathrm{E}-03$ & $2.37 \mathrm{E}-02$ & $1.26 \mathrm{E}-02$ & $-7.36 \mathrm{E}-02$ & 1 & $-6.38 \mathrm{E}-01$ \\
\hline & Indian Ocean & $4.70 \mathrm{E}-02$ & $3.54 \mathrm{E}-02$ & $2.18 \mathrm{E}-02$ & $-2.15 \mathrm{E}-02$ & $-6.38 \mathrm{E}-01$ & \\
\hline \multirow{7}{*}{$\begin{array}{l}\text { Biomass } \\
\text { burning }\end{array}$} & Africa/Middle East & $1.59 \mathrm{E}-02$ & $-4.58 \mathrm{E}-02$ & $8.45 \mathrm{E}-03$ & $-5.29 \mathrm{E}-02$ & $-8.72 \mathrm{E}-03$ & $-6.87 E-02$ \\
\hline & Southern Asia & $-3.35 \mathrm{E}-02$ & $-1.83 \mathrm{E}-02$ & \begin{tabular}{|l|}
$-1.22 \mathrm{E}-02$ \\
\end{tabular} & $-4.22 \mathrm{E}-02$ & $2.04 \mathrm{E}-02$ & $-1.51 \mathrm{E}-02$ \\
\hline & Central/South America & $6.06 \mathrm{E}-03$ & $-5.18 \mathrm{E}-02$ & $1.74 \mathrm{E}-03$ & $-1.96 \mathrm{E}-02$ & $-4.33 \mathrm{E}-02$ & $-4.12 \mathrm{E}-02$ \\
\hline & Northern Asia & $-4.73 \mathrm{E}-03$ & $2.00 \mathrm{E}-03$ & $\mid-2.46 \mathrm{E}-03$ & $2.16 \mathrm{E}-03$ & $2.12 \mathrm{E}-03$ & $-1.07 \mathrm{E}-03$ \\
\hline & Europe & 1.17E-04 & $-7.30 \mathrm{E}-07$ & $2.02 \mathrm{E}-04$ & $2.60 \mathrm{E}-04$ & $-1.79 \mathrm{E}-05$ & $9.12 \mathrm{E}-06$ \\
\hline & North America & $-2.41 \mathrm{E}-02$ & $1.18 \mathrm{E}-02$ & \begin{tabular}{|l|}
$-3.02 \mathrm{E}-02$ \\
\end{tabular} & $2.63 \mathrm{E}-02$ & $-3.01 \mathrm{E}-04$ & $7.37 \mathrm{E}-03$ \\
\hline & Oceania & $2.38 \mathrm{E}-03$ & $-8.79 \mathrm{E}-03$ & \begin{tabular}{|l|}
$-7.90 \mathrm{E}-04$ \\
\end{tabular} & $-8.04 \mathrm{E}-03$ & $9.91 \mathrm{E}-04$ & $-2.41 \mathrm{E}-02$ \\
\hline
\end{tabular}

Fig. S2av. Correlation of the emissions in 2004 for ocean. 


\begin{tabular}{|c|c|c|c|c|c|c|c|c|}
\hline \multirow[b]{3}{*}{ Source sector } & \multirow[b]{3}{*}{ Region } & \multirow{2}{*}{\multicolumn{7}{|c|}{ Biomass burning }} \\
\hline & & & & & & & & \\
\hline & & $\begin{array}{l}\text { Africa/M. } \\
\text { East }\end{array}$ & $\begin{array}{l}\text { Southern } \\
\text { Asia }\end{array}$ & $\begin{array}{l}\text { C. /S. } \\
\text { America }\end{array}$ & $\begin{array}{l}\text { Northern } \\
\text { Asia }\end{array}$ & Europe & $\begin{array}{l}\text { North } \\
\text { America }\end{array}$ & Oceania \\
\hline \multirow{7}{*}{$\begin{array}{l}\text { Agricultural } \\
\text { Soil }\end{array}$} & Africa/Middle East & $-3.34 \mathrm{E}-02$ & $-6.82 \mathrm{E}-03$ & $-6.54 \mathrm{E}-03$ & $-6.51 \mathrm{E}-04$ & $-1.62 \mathrm{E}-04$ & $4.24 \mathrm{E}-03$ & $-1.54 \mathrm{E}-03$ \\
\hline & Southern Asia & $-1.52 \mathrm{E}-02$ & $-4.38 \mathrm{E}-02$ & $6.21 \mathrm{E}-03$ & $-8.60 \mathrm{E}-03$ & $1.40 \mathrm{E}-04$ & $-5.66 \mathrm{E}-03$ & $5.57 \mathrm{E}-03$ \\
\hline & Central/South America & $-3.34 \mathrm{E}-02$ & $4.05 E-03$ & $-2.51 \mathrm{E}-02$ & $1.26 \mathrm{E}-04$ & $-1.15 \mathrm{E}-05$ & $3.54 \mathrm{E}-03$ & $-1.30 \mathrm{E}-02$ \\
\hline & Northern Asia & $5.34 \mathrm{E}-03$ & $8.29 \mathrm{E}-03$ & $-1.91 \mathrm{E}-03$ & $1.04 \mathrm{E}-03$ & $5.48 \mathrm{E}-05$ & $1.20 \mathrm{E}-02$ & $-2.28 \mathrm{E}-03$ \\
\hline & \begin{tabular}{|l} 
Europe \\
\end{tabular} & $3.05 \mathrm{E}-03$ & $3.36 \mathrm{E}-03$ & $2.26 \mathrm{E}-03$ & $-4.32 \mathrm{E}-04$ & $1.21 \mathrm{E}-04$ & $-8.48 \mathrm{E}-03$ & $2.21 \mathrm{E}-03$ \\
\hline & \begin{tabular}{|l} 
North America \\
\end{tabular} & $8.49 \mathrm{E}-04$ & $-8.38 \mathrm{E}-03$ & $1.20 \mathrm{E}-03$ & $2.36 \mathrm{E}-03$ & $8.30 \mathrm{E}-05$ & $2.99 \mathrm{E}-02$ & $2.13 \mathrm{E}-03$ \\
\hline & Oceania & $8.49 \mathrm{E}-03$ & $3.42 \mathrm{E}-03$ & $2.85 \mathrm{E}-03$ & $4.92 \mathrm{E}-04$ & $-1.08 \mathrm{E}-06$ & $6.59 \mathrm{E}-04$ & $1.25 \mathrm{E}-04$ \\
\hline \multirow{7}{*}{ Industrial } & Africa/Middle East & $-2.77 \mathrm{E}-03$ & $-1.24 \mathrm{E}-03$ & $-3.91 \mathrm{E}-04$ & $-4.93 \mathrm{E}-05$ & $-9.58 \mathrm{E}-05$ & $1.11 \mathrm{E}-03$ & $1.06 \mathrm{E}-04$ \\
\hline & Southern Asia & $8.70 \mathrm{E}-03$ & $-1.29 \mathrm{E}-03$ & $2.90 \mathrm{E}-03$ & $1.14 \mathrm{E}-03$ & $7.31 \mathrm{E}-06$ & $9.52 \mathrm{E}-03$ & $1.95 \mathrm{E}-03$ \\
\hline & Central/South America & $-2.79 \mathrm{E}-03$ & $3.57 \mathrm{E}-04$ & $-2.03 \mathrm{E}-03$ & $5.64 \mathrm{E}-06$ & $-7.51 \mathrm{E}-07$ & $2.64 \mathrm{E}-04$ & $-1.05 \mathrm{E}-03$ \\
\hline & Northern Asia & $1.37 \mathrm{E}-02$ & $6.98 \mathrm{E}-03$ & $2.83 \mathrm{E}-03$ & $3.58 \mathrm{E}-03$ & $-1.13 \mathrm{E}-04$ & $3.01 \mathrm{E}-02$ & $7.04 \mathrm{E}-04$ \\
\hline & Europe & $1.40 \mathrm{E}-03$ & $1.84 \mathrm{E}-03$ & $6.23 \mathrm{E}-04$ & $4.51 \mathrm{E}-04$ & $8.11 \mathrm{E}-05$ & $6.41 \mathrm{E}-03$ & $8.02 \mathrm{E}-04$ \\
\hline & North America & $3.20 \mathrm{E}-03$ & $-2.13 \mathrm{E}-03$ & $1.17 \mathrm{E}-03$ & $2.45 \mathrm{E}-03$ & $6.61 \mathrm{E}-05$ & $1.87 \mathrm{E}-02$ & $2.72 \mathrm{E}-04$ \\
\hline & Oceania & $6.42 \mathrm{E}-04$ & $2.47 \mathrm{E}-04$ & $2.00 \mathrm{E}-04$ & $4.57 \mathrm{E}-05$ & $2.48 \mathrm{E}-07$ & $6.13 \mathrm{E}-05$ & $2.01 \mathrm{E}-06$ \\
\hline \multirow{7}{*}{ Natural Soil } & Africa/Middle East & $-1.30 \mathrm{E}-01$ & $1.65 \mathrm{E}-02$ & $-3.64 \mathrm{E}-02$ & $-5.32 E-04$ & $-7.77 \mathrm{E}-05$ & $4.54 \mathrm{E}-03$ & $-1.41 \mathrm{E}-02$ \\
\hline & Southern Asia & $-2.75 \mathrm{E}-02$ & $7.77 \mathrm{E}-03$ & $-1.33 \mathrm{E}-02$ & $-2.12 \mathrm{E}-03$ & $-1.70 \mathrm{E}-04$ & $-1.21 \mathrm{E}-02$ & $-1.27 \mathrm{E}-02$ \\
\hline & Central/South America & $-1.78 \mathrm{E}-02$ & $-2.09 \mathrm{E}-02$ & $1.37 \mathrm{E}-02$ & $-1.03 E-03$ & \begin{tabular}{|l|}
$2.59 \mathrm{E}-05$ \\
\end{tabular} & $-1.01 \mathrm{E}-02$ & $9.02 \mathrm{E}-03$ \\
\hline & Northern Asia & $2.58 \mathrm{E}-02$ & $-1.09 \mathrm{E}-02$ & 9.47E-03 & $-2.10 \mathrm{E}-02$ & $-3.49 \mathrm{E}-05$ & $-7.07 \mathrm{E}-02$ & 5.07E-03 \\
\hline & Europe & $7.20 \mathrm{E}-03$ & $-7.73 \mathrm{E}-03$ & 4.87E-03 & $-8.28 \mathrm{E}-03$ & $-3.62 \mathrm{E}-03$ & $-5.21 \mathrm{E}-02$ & $2.39 \mathrm{E}-03$ \\
\hline & North America & $6.00 \mathrm{E}-03$ & $9.92 \mathrm{E}-03$ & $1.07 \mathrm{E}-02$ & $-1.29 \mathrm{E}-02$ & $-1.83 \mathrm{E}-04$ & $-1.57 \mathrm{E}-01$ & $7.74 \mathrm{E}-03$ \\
\hline & Oceania & $2.75 \mathrm{E}-03$ & $-3.88 \mathrm{E}-03$ & $-1.40 \mathrm{E}-03$ & $1.53 \mathrm{E}-05$ & $-1.62 \mathrm{E}-06$ & $1.07 \mathrm{E}-03$ & $-5.58 \mathrm{E}-03$ \\
\hline \multirow{6}{*}{ Ocean } & North Pacific & $1.59 \mathrm{E}-02$ & $-3.35 \mathrm{E}-02$ & $6.06 \mathrm{E}-03$ & $-4.73 \mathrm{E}-03$ & $1.17 \mathrm{E}-04$ & $-2.41 \mathrm{E}-02$ & $2.38 \mathrm{E}-03$ \\
\hline & South Pacific & $-4.58 \mathrm{E}-02$ & $-1.83 \mathrm{E}-02$ & $-5.18 \mathrm{E}-02$ & $2.00 \mathrm{E}-03$ & $-7.30 \mathrm{E}-07$ & $1.18 \mathrm{E}-02$ & $-8.79 \mathrm{E}-03$ \\
\hline & Northern Ocean & $8.45 \mathrm{E}-03$ & $-1.22 \mathrm{E}-02$ & $1.74 \mathrm{E}-03$ & $-2.46 \mathrm{E}-03$ & $2.02 \mathrm{E}-04$ & $-3.02 \mathrm{E}-02$ & $-7.90 \mathrm{E}-04$ \\
\hline & Atlantic & $-5.29 \mathrm{E}-02$ & $-4.22 \mathrm{E}-02$ & $-1.96 \mathrm{E}-02$ & $2.16 \mathrm{E}-03$ & $2.60 \mathrm{E}-04$ & $2.63 \mathrm{E}-02$ & $-8.04 \mathrm{E}-03$ \\
\hline & Southern Ocean & $-8.72 \mathrm{E}-03$ & $2.04 \mathrm{E}-02$ & $-4.33 \mathrm{E}-02$ & $2.12 \mathrm{E}-03$ & $-1.79 \mathrm{E}-05$ & $-3.01 \mathrm{E}-04$ & $9.91 \mathrm{E}-04$ \\
\hline & Indian Ocean & $-6.87 \mathrm{E}-02$ & $-1.51 \mathrm{E}-02$ & $-4.12 E-02$ & $-1.07 \mathrm{E}-03$ & $9.12 \mathrm{E}-06$ & 7.37E-03 & $-2.41 \mathrm{E}-02$ \\
\hline \multirow{7}{*}{$\begin{array}{l}\text { Biomass } \\
\text { burning }\end{array}$} & Africa/Middle East & 1 & $7.69 \mathrm{E}-04$ & $-3.07 \mathrm{E}-02$ & $1.09 \mathrm{E}-04$ & $6.97 \mathrm{E}-07$ & $5.20 \mathrm{E}-03$ & $-1.22 \mathrm{E}-02$ \\
\hline & Southern Asia & $7.69 \mathrm{E}-04$ & 1 & $2.44 \mathrm{E}-03$ & $-4.86 \mathrm{E}-04$ & $3.35 \mathrm{E}-05$ & $3.56 \mathrm{E}-03$ & $-2.12 \mathrm{E}-03$ \\
\hline & Central/South America & $-3.07 \mathrm{E}-02$ & $2.44 \mathrm{E}-03$ & 1 & $-3.11 \mathrm{E}-04$ & $-7.68 \mathrm{E}-07$ & $3.27 \mathrm{E}-03$ & $-1.04 \mathrm{E}-02$ \\
\hline & Northern Asia & $1.09 \mathrm{E}-04$ & $-4.86 \mathrm{E}-04$ & $-3.11 \mathrm{E}-04$ & 1 & $-2.45 \mathrm{E}-05$ & $-5.77 \mathrm{E}-03$ & $-7.72 \mathrm{E}-04$ \\
\hline & Europe & $6.97 \mathrm{E}-07$ & $3.35 \mathrm{E}-05$ & $-7.68 \mathrm{E}-07$ & $-2.45 \mathrm{E}-05$ & 1 & $-1.76 \mathrm{E}-04$ & $-3.12 \mathrm{E}-06$ \\
\hline & North America & $5.20 \mathrm{E}-03$ & $3.56 \mathrm{E}-03$ & $3.27 \mathrm{E}-03$ & $-5.77 \mathrm{E}-03$ & \begin{tabular}{|l|}
$-1.76 \mathrm{E}-04$ \\
\end{tabular} & 1 & $1.54 \mathrm{E}-03$ \\
\hline & Oceania & $-1.22 \mathrm{E}-02$ & $-2.12 \mathrm{E}-03$ & $-1.04 \mathrm{E}-02$ & $-7.72 \mathrm{E}-04$ & $-3.12 \mathrm{E}-06$ & $1.54 \mathrm{E}-03$ & 1 \\
\hline
\end{tabular}

Fig. S2aw. Correlation of the emissions in 2004 for biomass burning. 


\begin{tabular}{|c|c|c|c|c|c|c|c|c|}
\hline \multirow[b]{2}{*}{ Source sector } & \multirow[b]{2}{*}{ Region } & \multicolumn{7}{|c|}{ Agricultural Soil } \\
\hline & & $\begin{array}{l}\text { Africa/M. } \\
\text { East }\end{array}$ & $\begin{array}{l}\text { Southern } \\
\text { Asia }\end{array}$ & $\begin{array}{l}\text { C. /S. } \\
\text { America }\end{array}$ & $\begin{array}{l}\text { Northern } \\
\text { Asia }\end{array}$ & Europe & $\begin{array}{l}\text { North } \\
\text { America }\end{array}$ & Oceania \\
\hline \multirow{7}{*}{$\begin{array}{c}\text { Agricultural } \\
\text { Soil }\end{array}$} & Africa/Middle East & 1 & $-8.17 \mathrm{E}-02$ & $2.54 \mathrm{E}-04$ & $-3.64 \mathrm{E}-02$ & $-3.44 \mathrm{E}-02$ & $-2.13 \mathrm{E}-02$ & $6.54 \mathrm{E}-03$ \\
\hline & Southern Asia & $-8.17 \mathrm{E}-02$ & 1 & $5.10 \mathrm{E}-02$ & $-2.52 \mathrm{E}-01$ & $2.90 \mathrm{E}-02$ & $-8.48 \mathrm{E}-02$ & $2.50 \mathrm{E}-02$ \\
\hline & Central/South America & $2.54 \mathrm{E}-04$ & $5.10 \mathrm{E}-02$ & 1 & $-7.63 \mathrm{E}-03$ & $8.63 E-03$ & $9.40 \mathrm{E}-03$ & $-2.71 \mathrm{E}-02$ \\
\hline & Northern Asia & $-3.64 \mathrm{E}-02$ & $-2.52 \mathrm{E}-01$ & $-7.63 \mathrm{E}-03$ & 1 & $-1.58 \mathrm{E}-01$ & $-4.29 \mathrm{E}-02$ & $-2.75 \mathrm{E}-02$ \\
\hline & Europe & $-3.44 \mathrm{E}-02$ & $2.90 \mathrm{E}-02$ & $8.63 \mathrm{E}-03$ & $-1.58 \mathrm{E}-01$ & 1 & $-1.98 \mathrm{E}-02$ & $4.38 \mathrm{E}-03$ \\
\hline & North America & $-2.13 \mathrm{E}-02$ & $-8.48 \mathrm{E}-02$ & $9.40 \mathrm{E}-03$ & $-4.29 \mathrm{E}-02$ & $-1.98 \mathrm{E}-02$ & 1 & $-1.13 \mathrm{E}-03$ \\
\hline & Oceania & $6.54 \mathrm{E}-03$ & $2.50 \mathrm{E}-02$ & $-2.71 \mathrm{E}-02$ & $-2.75 \mathrm{E}-02$ & $4.38 \mathrm{E}-03$ & $-1.13 \mathrm{E}-03$ & 1 \\
\hline \multirow{7}{*}{ Industrial } & Africa/Middle East & $-7.78 \mathrm{E}-02$ & $-1.17 \mathrm{E}-02$ & $1.63 \mathrm{E}-03$ & $-9.57 \mathrm{E}-03$ & $-1.36 \mathrm{E}-02$ & $-4.40 \mathrm{E}-03$ & $8.91 \mathrm{E}-04$ \\
\hline & \begin{tabular}{|l} 
Southern Asia \\
\end{tabular} & $2.39 \mathrm{E}-02$ & $-4.14 \mathrm{E}-01$ & $-1.09 \mathrm{E}-03$ & $-5.09 \mathrm{E}-02$ & $2.12 \mathrm{E}-02$ & $8.12 \mathrm{E}-03$ & $-1.56 \mathrm{E}-03$ \\
\hline & Central/South America & $-1.78 \mathrm{E}-04$ & $3.82 \mathrm{E}-03$ & $-8.30 \mathrm{E}-03$ & $-4.69 \mathrm{E}-04$ & $7.05 \mathrm{E}-04$ & $7.62 \mathrm{E}-04$ & $-2.07 \mathrm{E}-03$ \\
\hline & Northern Asia & $8.73 \mathrm{E}-03$ & $-3.04 \mathrm{E}-02$ & $-4.50 \mathrm{E}-04$ & $-1.12 \mathrm{E}-01$ & $-5.36 \mathrm{E}-02$ & $1.21 \mathrm{E}-01$ & $-3.51 \mathrm{E}-03$ \\
\hline & Europe & $-2.22 \mathrm{E}-02$ & $2.23 \mathrm{E}-03$ & $3.39 \mathrm{E}-03$ & $-4.23 \mathrm{E}-02$ & $-7.24 \mathrm{E}-01$ & $-1.34 \mathrm{E}-02$ & $2.76 \mathrm{E}-04$ \\
\hline & North America & $8.73 \mathrm{E}-03$ & $3.10 \mathrm{E}-02$ & $1.26 \mathrm{E}-03$ & $3.82 \mathrm{E}-02$ & $3.05 \mathrm{E}-02$ & $-3.57 \mathrm{E}-01$ & $-9.40 \mathrm{E}-04$ \\
\hline & Oceania & $5.27 \mathrm{E}-04$ & $1.84 \mathrm{E}-03$ & $-2.15 \mathrm{E}-03$ & $-1.94 \mathrm{E}-03$ & $3.13 \mathrm{E}-04$ & $-1.26 \mathrm{E}-04$ & $-1.18 \mathrm{E}-02$ \\
\hline \multirow{7}{*}{ Natural Soil } & Africa/Middle East & $-2.01 \mathrm{E}-01$ & $-2.00 \mathrm{E}-02$ & $-8.48 \mathrm{E}-02$ & $2.76 \mathrm{E}-03$ & $-1.07 \mathrm{E}-02$ & $4.97 \mathrm{E}-03$ & $3.72 \mathrm{E}-03$ \\
\hline & Southern Asia & $-7.09 \mathrm{E}-02$ & $-4.07 \mathrm{E}-01$ & $-1.78 \mathrm{E}-02$ & $2.99 \mathrm{E}-02$ & $-4.97 \mathrm{E}-03$ & $-3.81 \mathrm{E}-02$ & $3.30 \mathrm{E}-02$ \\
\hline & Central/South America & $-5.07 E-02$ & $4.37 \mathrm{E}-02$ & $-2.81 \mathrm{E}-01$ & $2.80 \mathrm{E}-03$ & $2.75 \mathrm{E}-02$ & $1.61 \mathrm{E}-02$ & $-4.91 \mathrm{E}-02$ \\
\hline & Northern Asia & $4.45 \mathrm{E}-02$ & $-9.47 \mathrm{E}-02$ & $8.41 \mathrm{E}-03$ & $-1.95 \mathrm{E}-01$ & $2.21 \mathrm{E}-03$ & $1.11 \mathrm{E}-01$ & $-2.67 \mathrm{E}-02$ \\
\hline & Europe & $-1.21 \mathrm{E}-02$ & $-4.28 \mathrm{E}-04$ & $4.92 \mathrm{E}-03$ & $4.12 \mathrm{E}-03$ & $-2.60 \mathrm{E}-01$ & $1.68 \mathrm{E}-02$ & $6.28 \mathrm{E}-03$ \\
\hline & North America & $-2.89 \mathrm{E}-02$ & $-2.68 \mathrm{E}-02$ & $2.26 \mathrm{E}-02$ & $8.50 \mathrm{E}-02$ & $-5.02 \mathrm{E}-03$ & $-4.37 \mathrm{E}-01$ & $1.37 \mathrm{E}-02$ \\
\hline & Oceania & $2.94 \mathrm{E}-03$ & $2.16 \mathrm{E}-02$ & $-3.42 \mathrm{E}-02$ & $-1.44 \mathrm{E}-02$ & $7.28 \mathrm{E}-03$ & $4.42 \mathrm{E}-03$ & $-7.78 \mathrm{E}-02$ \\
\hline \multirow{6}{*}{ Ocean } & North Pacific & $-1.85 \mathrm{E}-02$ & $-2.44 \mathrm{E}-01$ & $2.77 \mathrm{E}-03$ & $3.08 \mathrm{E}-02$ & $1.71 \mathrm{E}-03$ & $-5.13 \mathrm{E}-02$ & $1.21 \mathrm{E}-02$ \\
\hline & South Pacific & $-2.46 \mathrm{E}-03$ & $-1.73 \mathrm{E}-02$ & $-7.89 \mathrm{E}-03$ & $1.97 \mathrm{E}-02$ & $1.25 \mathrm{E}-02$ & $9.24 \mathrm{E}-03$ & $1.51 \mathrm{E}-02$ \\
\hline & Northern Ocean & $-1.59 \mathrm{E}-03$ & $-3.38 \mathrm{E}-02$ & $4.55 \mathrm{E}-04$ & $-5.33 \mathrm{E}-02$ & $-7.71 \mathrm{E}-02$ & $-7.06 \mathrm{E}-03$ & $5.43 \mathrm{E}-03$ \\
\hline & Atlantic & $-8.81 \mathrm{E}-02$ & $-1.66 \mathrm{E}-01$ & $-2.34 \mathrm{E}-02$ & $-3.36 \mathrm{E}-02$ & $-5.30 \mathrm{E}-02$ & $-1.00 \mathrm{E}-01$ & $1.69 \mathrm{E}-02$ \\
\hline & Southern Ocean & $2.25 \mathrm{E}-02$ & $8.01 \mathrm{E}-02$ & $-1.90 \mathrm{E}-01$ & $-1.44 \mathrm{E}-02$ & $6.00 \mathrm{E}-03$ & $3.82 \mathrm{E}-03$ & $-8.31 \mathrm{E}-02$ \\
\hline & Indian Ocean & $-2.85 \mathrm{E}-02$ & $3.41 \mathrm{E}-03$ & $-9.17 \mathrm{E}-02$ & $-6.89 \mathrm{E}-03$ & $8.98 \mathrm{E}-03$ & $1.42 \mathrm{E}-02$ & $-3.98 \mathrm{E}-02$ \\
\hline \multirow{7}{*}{$\begin{array}{l}\text { Biomass } \\
\text { burning }\end{array}$} & Africa/Middle East & $-4.00 \mathrm{E}-02$ & $-2.13 \mathrm{E}-02$ & $-3.32 \mathrm{E}-02$ & $8.10 \mathrm{E}-03$ & $3.70 \mathrm{E}-03$ & $-9.86 \mathrm{E}-04$ & $7.11 \mathrm{E}-03$ \\
\hline & Southern Asia & $-4.75 \mathrm{E}-03$ & $-3.64 \mathrm{E}-02$ & $2.63 \mathrm{E}-03$ & $3.29 \mathrm{E}-03$ & $1.22 \mathrm{E}-03$ & $-3.32 \mathrm{E}-03$ & $2.05 \mathrm{E}-03$ \\
\hline & Central/South America & $-3.89 \mathrm{E}-03$ & $2.75 \mathrm{E}-02$ & $-3.28 \mathrm{E}-02$ & $6.97 \mathrm{E}-04$ & $2.97 \mathrm{E}-03$ & $7.74 \mathrm{E}-03$ & $5.69 \mathrm{E}-03$ \\
\hline & \begin{tabular}{|l} 
Northern Asia \\
\end{tabular} & $2.43 \mathrm{E}-04$ & $-4.61 \mathrm{E}-03$ & $1.87 \mathrm{E}-03$ & $6.61 \mathrm{E}-03$ & $-3.42 \mathrm{E}-04$ & $4.14 \mathrm{E}-03$ & $1.74 \mathrm{E}-03$ \\
\hline & Europe & $-2.40 \mathrm{E}-04$ & $-6.55 \mathrm{E}-07$ & $6.49 \mathrm{E}-05$ & $-2.81 \mathrm{E}-04$ & $-6.19 \mathrm{E}-05$ & $-7.35 \mathrm{E}-07$ & $6.42 \mathrm{E}-06$ \\
\hline & North America & $1.06 \mathrm{E}-05$ & $-1.19 \mathrm{E}-03$ & $2.69 \mathrm{E}-03$ & $8.21 \mathrm{E}-03$ & $-1.51 \mathrm{E}-03$ & $8.82 \mathrm{E}-03$ & $1.96 \mathrm{E}-03$ \\
\hline & Oceania & $4.76 \mathrm{E}-04$ & $5.74 \mathrm{E}-03$ & $-5.70 \mathrm{E}-03$ & $-1.00 \mathrm{E}-03$ & $1.01 \mathrm{E}-03$ & $1.50 \mathrm{E}-03$ & $-5.55 \mathrm{E}-04$ \\
\hline
\end{tabular}

Fig. S2ax. Correlation of the emissions in 2005 for agricultural soil. 


\begin{tabular}{|c|c|c|c|c|c|c|c|c|}
\hline & \multirow{2}{*}{\multicolumn{7}{|c|}{ Industrial }} \\
\hline & & & & & & & & \\
\hline Source sector & Region & $\begin{array}{l}\text { Africa/M. } \\
\text { East }\end{array}$ & $\begin{array}{l}\text { Southern } \\
\text { Asia }\end{array}$ & $\begin{array}{l}\text { C. /S. } \\
\text { America }\end{array}$ & $\begin{array}{l}\text { Northern } \\
\text { Asia }\end{array}$ & Europe & $\begin{array}{l}\text { North } \\
\text { America }\end{array}$ & Oceania \\
\hline \multirow{7}{*}{$\begin{array}{l}\text { Agricultural } \\
\text { Soil }\end{array}$} & Africa/Middle East & $-7.78 \mathrm{E}-02$ & $2.39 \mathrm{E}-02$ & $-1.78 \mathrm{E}-04$ & $8.73 \mathrm{E}-03$ & $-2.22 \mathrm{E}-02$ & $8.73 \mathrm{E}-03$ & 5.27E-04 \\
\hline & Southern Asia & $-1.17 \mathrm{E}-02$ & $-4.14 \mathrm{E}-01$ & $3.82 \mathrm{E}-03$ & $-3.04 \mathrm{E}-02$ & $2.23 \mathrm{E}-03$ & $3.10 \mathrm{E}-02$ & $1.84 \mathrm{E}-03$ \\
\hline & Central/South America & $1.63 \mathrm{E}-03$ & $-1.09 \mathrm{E}-03$ & $-8.30 \mathrm{E}-03$ & \begin{tabular}{|l|}
$-4.50 E-04$ \\
\end{tabular} & $3.39 E-03$ & $1.26 \mathrm{E}-03$ & $-2.15 \mathrm{E}-03$ \\
\hline & \begin{tabular}{|l|} 
Northern Asia \\
\end{tabular} & $-9.57 \mathrm{E}-03$ & $-5.09 \mathrm{E}-02$ & $-4.69 \mathrm{E}-04$ & \begin{tabular}{|l|} 
\\
\end{tabular} & $-4.23 \mathrm{E}-02$ & $3.82 \mathrm{E}-02$ & $-1.94 \mathrm{E}-03$ \\
\hline & Europe & $-1.36 \mathrm{E}-02$ & $2.12 \mathrm{E}-02$ & $7.05 \mathrm{E}-04$ & $-5.36 \mathrm{E}-02$ & $-7.24 \mathrm{E}-01$ & $3.05 \mathrm{E}-02$ & $3.13 \mathrm{E}-04$ \\
\hline & \begin{tabular}{|l|} 
North America \\
\end{tabular} & $-4.40 \mathrm{E}-03$ & $8.12 \mathrm{E}-03$ & $7.62 \mathrm{E}-04$ & $1.21 \mathrm{E}-01$ & $-1.34 \mathrm{E}-02$ & $-3.57 \mathrm{E}-01$ & $-1.26 \mathrm{E}-04$ \\
\hline & Oceania & $8.91 \mathrm{E}-04$ & $-1.56 \mathrm{E}-03$ & $-2.07 \mathrm{E}-03$ & 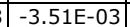 & $2.76 \mathrm{E}-04$ & $-9.40 \mathrm{E}-04$ & $-1.18 \mathrm{E}-02$ \\
\hline \multirow{7}{*}{ Industrial } & Africa/Middle East & 1 & $3.45 \mathrm{E}-03$ & $1.06 \mathrm{E}-04$ & $-2.38 \mathrm{E}-03$ & $-8.26 \mathrm{E}-03$ & $1.71 \mathrm{E}-03$ & $6.83 \mathrm{E}-05$ \\
\hline & \begin{tabular}{|l|} 
Southern Asia \\
\end{tabular} & $3.45 \mathrm{E}-03$ & 1 & $-1.24 \mathrm{E}-05$ & $\begin{array}{ll}-4.25 \mathrm{E}-02 \\
\end{array}$ & $4.14 \mathrm{E}-03$ & $1.26 \mathrm{E}-02$ & $-1.50 \mathrm{E}-04$ \\
\hline & Central/South America & $1.06 \mathrm{E}-04$ & $-1.24 \mathrm{E}-05$ & 1 & $1.44 \mathrm{E}-04$ & $2.81 \mathrm{E}-04$ & $1.46 \mathrm{E}-04$ & $-1.63 \mathrm{E}-04$ \\
\hline & Northern Asia & $-2.38 \mathrm{E}-03$ & $-4.25 \mathrm{E}-02$ & $1.44 \mathrm{E}-04$ & 1 & $-3.40 \mathrm{E}-02$ & $-2.51 \mathrm{E}-01$ & $-2.65 \mathrm{E}-04$ \\
\hline & Europe & $-8.26 \mathrm{E}-03$ & $4.14 \mathrm{E}-03$ & $2.81 \mathrm{E}-04$ & $-3.40 \mathrm{E}-02$ & 1 & $4.49 \mathrm{E}-03$ & $1.10 \mathrm{E}-05$ \\
\hline & North America & $1.71 \mathrm{E}-03$ & $1.26 \mathrm{E}-02$ & $1.46 \mathrm{E}-04$ & $-2.51 E-01$ & 4.49E-03 & 1 & $-6.79 \mathrm{E}-05$ \\
\hline & Oceania & $6.83 \mathrm{E}-05$ & $-1.50 \mathrm{E}-04$ & $-1.63 \mathrm{E}-04$ & $-2.65 \mathrm{E}-04$ & $1.10 \mathrm{E}-05$ & $-6.79 \mathrm{E}-05$ & 1 \\
\hline \multirow{7}{*}{ Natural Soil } & Africa/Middle East & $-3.25 \mathrm{E}-02$ & $5.68 \mathrm{E}-02$ & $-7.16 \mathrm{E}-03$ & \begin{tabular}{|l|} 
\\
\end{tabular} & $-2.79 \mathrm{E}-03$ & $1.46 \mathrm{E}-02$ & 3.77E-04 \\
\hline & Southern Asia & $-7.13 E-03$ & $-5.82 \mathrm{E}-02$ & $-1.81 \mathrm{E}-03$ & \begin{tabular}{|l|} 
\\
$2.52 E-02$ \\
\end{tabular} & $-1.52 \mathrm{E}-02$ & $6.87 \mathrm{E}-03$ & $2.87 \mathrm{E}-03$ \\
\hline & Central/South America & $1.68 \mathrm{E}-04$ & $-4.92 \mathrm{E}-04$ & $-2.13 \mathrm{E}-02$ & $2.76 \mathrm{E}-02$ & $8.34 \mathrm{E}-03$ & $1.03 \mathrm{E}-02$ & $-4.47 \mathrm{E}-03$ \\
\hline & Northern Asia & $9.18 \mathrm{E}-03$ & $-2.77 \mathrm{E}-02$ & $6.90 \mathrm{E}-04$ & $-7.35 \mathrm{E}-02$ & $3.28 \mathrm{E}-02$ & $1.46 \mathrm{E}-02$ & $-2.14 \mathrm{E}-03$ \\
\hline & Europe & $-1.30 \mathrm{E}-03$ & $1.54 \mathrm{E}-02$ & $2.92 \mathrm{E}-04$ & $1.80 \mathrm{E}-02$ & $-1.80 \mathrm{E}-01$ & $3.16 \mathrm{E}-02$ & 5.03E-04 \\
\hline & North America & $-1.68 \mathrm{E}-03$ & $6.28 \mathrm{E}-02$ & $1.37 \mathrm{E}-03$ & \begin{tabular}{|l|} 
\\
\end{tabular} & $1.60 \mathrm{E}-02$ & $-2.12 \mathrm{E}-01$ & $1.13 \mathrm{E}-03$ \\
\hline & Oceania & $8.90 \mathrm{E}-04$ & $-1.45 \mathrm{E}-03$ & $-2.74 \mathrm{E}-03$ & \begin{tabular}{|l|} 
\\
\end{tabular} & $1.88 \mathrm{E}-03$ & $4.23 \mathrm{E}-04$ & $-5.51 \mathrm{E}-03$ \\
\hline \multirow{6}{*}{ Ocean } & North Pacific & $-4.01 \mathrm{E}-03$ & $-1.13 \mathrm{E}-02$ & $1.22 \mathrm{E}-04$ & $6.32 \mathrm{E}-02$ & $-1.81 \mathrm{E}-02$ & $-3.25 \mathrm{E}-02$ & $9.29 \mathrm{E}-04$ \\
\hline & South Pacific & $4.03 \mathrm{E}-05$ & $8.77 \mathrm{E}-03$ & $-2.98 \mathrm{E}-03$ & \begin{tabular}{|c|} 
\\
\end{tabular} & $4.59 \mathrm{E}-03$ & $-1.77 \mathrm{E}-03$ & $2.43 \mathrm{E}-03$ \\
\hline & \begin{tabular}{|l} 
Northern Ocean \\
\end{tabular} & $2.68 \mathrm{E}-05$ & $-1.03 \mathrm{E}-02$ & $5.04 \mathrm{E}-05$ & $3.51 \mathrm{E}-02$ & $1.59 \mathrm{E}-02$ & $3.41 \mathrm{E}-02$ & 4.04E-04 \\
\hline & Atlantic & $-1.25 \mathrm{E}-02$ & $-1.38 \mathrm{E}-03$ & $-2.32 \mathrm{E}-03$ & \begin{tabular}{|l|} 
\\
\end{tabular} & $-8.86 \mathrm{E}-03$ & $-1.18 \mathrm{E}-01$ & $1.22 \mathrm{E}-03$ \\
\hline & Southern Ocean & $2.36 \mathrm{E}-03$ & $-2.36 \mathrm{E}-02$ & $-1.29 \mathrm{E}-02$ & $4.22 \mathrm{E}-03$ & $-1.84 \mathrm{E}-03$ & $1.21 \mathrm{E}-02$ & $-6.18 \mathrm{E}-03$ \\
\hline & Indian Ocean & $-1.95 \mathrm{E}-03$ & $2.08 \mathrm{E}-02$ & $-7.66 \mathrm{E}-03$ & 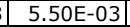 & 5.07E-03 & $-3.37 \mathrm{E}-03$ & $-3.85 \mathrm{E}-03$ \\
\hline \multirow{7}{*}{$\begin{array}{l}\text { Biomass } \\
\text { burning }\end{array}$} & Africa/Middle East & $-3.84 \mathrm{E}-03$ & $1.19 \mathrm{E}-02$ & $-2.84 \mathrm{E}-03$ & \begin{tabular}{|c|} 
\\
\end{tabular} & $2.35 \mathrm{E}-03$ & $5.18 \mathrm{E}-04$ & $6.27 \mathrm{E}-04$ \\
\hline & Southern Asia & $-8.08 \mathrm{E}-04$ & $-1.89 \mathrm{E}-03$ & $1.90 \mathrm{E}-04$ & 3.87E-03 & $3.15 \mathrm{E}-04$ & $-4.37 \mathrm{E}-04$ & $1.57 \mathrm{E}-04$ \\
\hline & Central/South America & $1.50 \mathrm{E}-04$ & $4.83 \mathrm{E}-03$ & $-2.76 \mathrm{E}-03$ & \begin{tabular}{|l|} 
\\
\end{tabular} & $1.51 \mathrm{E}-03$ & $8.99 \mathrm{E}-04$ & $3.98 \mathrm{E}-04$ \\
\hline & Northern Asia & $9.24 \mathrm{E}-05$ & $3.40 \mathrm{E}-03$ & $1.36 \mathrm{E}-04$ & $1.10 \mathrm{E}-02$ & $2.49 \mathrm{E}-04$ & $6.07 \mathrm{E}-03$ & $1.43 \mathrm{E}-04$ \\
\hline & Europe & $-4.47 \mathrm{E}-05$ & $1.34 \mathrm{E}-04$ & 5.19E-06 & $-1.06 \mathrm{E}-04$ & $1.98 \mathrm{E}-04$ & $1.57 \mathrm{E}-04$ & $4.81 \mathrm{E}-07$ \\
\hline & North America & $1.95 \mathrm{E}-04$ & $4.69 \mathrm{E}-03$ & $1.80 \mathrm{E}-04$ & $1.46 \mathrm{E}-02$ & $2.29 E-03$ & $2.22 \mathrm{E}-04$ & $1.58 \mathrm{E}-04$ \\
\hline & Oceania & $1.82 \mathrm{E}-04$ & $3.75 \mathrm{E}-04$ & $-4.66 \mathrm{E}-04$ & $6.64 \mathrm{E}-05$ & $3.99 \mathrm{E}-04$ & $3.89 \mathrm{E}-07$ & $-3.67 \mathrm{E}-05$ \\
\hline
\end{tabular}

Fig. S2ay. Correlation of the emissions in 2005 for industrial. 


\begin{tabular}{|c|c|c|c|c|c|c|c|c|}
\hline & \multirow{2}{*}{\multicolumn{7}{|c|}{ Natural Soil }} \\
\hline & & & & & & & & \\
\hline Source sector & Region & $\begin{array}{l}\text { Africa/M. } \\
\text { East }\end{array}$ & $\begin{array}{l}\text { Southern } \\
\text { Asia }\end{array}$ & $\begin{array}{l}\text { C. /S. } \\
\text { America }\end{array}$ & $\begin{array}{l}\text { Northern } \\
\text { Asia }\end{array}$ & Europe & $\begin{array}{l}\text { North } \\
\text { America }\end{array}$ & Oceania \\
\hline \multirow{7}{*}{$\begin{array}{l}\text { Agricultural } \\
\text { Soil }\end{array}$} & Africa/Middle East & $-2.01 \mathrm{E}-01$ & $-7.09 \mathrm{E}-02$ & $-5.07 \mathrm{E}-02$ & $4.45 \mathrm{E}-02$ & $-1.21 \mathrm{E}-02$ & $-2.89 \mathrm{E}-02$ & $2.94 \mathrm{E}-03$ \\
\hline & Southern Asia & $-2.00 \mathrm{E}-02$ & $-4.07 \mathrm{E}-01$ & $4.37 \mathrm{E}-02$ & $-9.47 \mathrm{E}-02$ & $-4.28 \mathrm{E}-04$ & $-2.68 \mathrm{E}-02$ & $2.16 \mathrm{E}-02$ \\
\hline & Central/South America & $-8.48 \mathrm{E}-02$ & $-1.78 \mathrm{E}-02$ & $-2.81 \mathrm{E}-01$ & $8.41 \mathrm{E}-03$ & 4.92E-03 & $2.26 \mathrm{E}-02$ & $-3.42 \mathrm{E}-02$ \\
\hline & Northern Asia & $2.76 \mathrm{E}-03$ & $2.99 \mathrm{E}-02$ & $2.80 \mathrm{E}-03$ & $-1.95 \mathrm{E}-01$ & $4.12 \mathrm{E}-03$ & $8.50 \mathrm{E}-02$ & $-1.44 \mathrm{E}-02$ \\
\hline & Europe & $-1.07 E-02$ & $-4.97 \mathrm{E}-03$ & $2.75 \mathrm{E}-02$ & $2.21 \mathrm{E}-03$ & $-2.60 \mathrm{E}-01$ & $-5.02 \mathrm{E}-03$ & $7.28 \mathrm{E}-03$ \\
\hline & North America & $4.97 \mathrm{E}-03$ & $-3.81 \mathrm{E}-02$ & $1.61 \mathrm{E}-02$ & $1.11 \mathrm{E}-01$ & $1.68 \mathrm{E}-02$ & $-4.37 \mathrm{E}-01$ & $4.42 \mathrm{E}-03$ \\
\hline & Oceania & $3.72 \mathrm{E}-03$ & $3.30 \mathrm{E}-02$ & $-4.91 \mathrm{E}-02$ & $-2.67 \mathrm{E}-02$ & $6.28 \mathrm{E}-03$ & $1.37 \mathrm{E}-02$ & $-7.78 \mathrm{E}-02$ \\
\hline \multirow{7}{*}{ Industrial } & Africa/Middle East & $-3.25 \mathrm{E}-02$ & $-7.13 \mathrm{E}-03$ & $1.68 \mathrm{E}-04$ & $9.18 \mathrm{E}-03$ & $-1.30 \mathrm{E}-03$ & $-1.68 \mathrm{E}-03$ & $8.90 \mathrm{E}-04$ \\
\hline & Southern Asia & $5.68 \mathrm{E}-02$ & $-5.82 \mathrm{E}-02$ & $-4.92 \mathrm{E}-04$ & $-2.77 \mathrm{E}-02$ & $1.54 \mathrm{E}-02$ & $6.28 \mathrm{E}-02$ & $-1.45 \mathrm{E}-03$ \\
\hline & Central/South America & $-7.16 \mathrm{E}-03$ & $-1.81 \mathrm{E}-03$ & $-2.13 \mathrm{E}-02$ & $6.90 \mathrm{E}-04$ & $2.92 \mathrm{E}-04$ & $1.37 \mathrm{E}-03$ & $-2.74 \mathrm{E}-03$ \\
\hline & Northern Asia & $4.42 \mathrm{E}-02$ & $2.52 \mathrm{E}-02$ & $2.76 \mathrm{E}-02$ & $-7.35 \mathrm{E}-02$ & $1.80 \mathrm{E}-02$ & $-1.40 \mathrm{E}-01$ & 8.17E-04 \\
\hline & Europe & $-2.79 \mathrm{E}-03$ & $-1.52 \mathrm{E}-02$ & $8.34 \mathrm{E}-03$ & $3.28 \mathrm{E}-02$ & $-1.80 \mathrm{E}-01$ & $1.60 \mathrm{E}-02$ & $1.88 \mathrm{E}-03$ \\
\hline & North America & $1.46 \mathrm{E}-02$ & 6.87E-03 & $1.03 E-02$ & $1.46 \mathrm{E}-02$ & $3.16 \mathrm{E}-02$ & $-2.12 \mathrm{E}-01$ & $4.23 E-04$ \\
\hline & Oceania & 3.77E-04 & $2.87 \mathrm{E}-03$ & $-4.47 \mathrm{E}-03$ & $-2.14 \mathrm{E}-03$ & 5.03E-04 & $1.13 \mathrm{E}-03$ & $-5.51 \mathrm{E}-03$ \\
\hline \multirow{7}{*}{ Natural Soil } & Africa/Middle East & 1 & $-3.32 \mathrm{E}-01$ & $-3.46 \mathrm{E}-01$ & $1.08 \mathrm{E}-01$ & $-3.24 \mathrm{E}-02$ & $-1.09 \mathrm{E}-01$ & $-1.23 \mathrm{E}-02$ \\
\hline & Southern Asia & $-3.32 \mathrm{E}-01$ & 1 & 7.97E-03 & $-3.61 \mathrm{E}-01$ & $6.24 \mathrm{E}-02$ & $2.24 \mathrm{E}-02$ & $1.71 \mathrm{E}-02$ \\
\hline & Central/South America & $-3.46 \mathrm{E}-01$ & 7.97E-03 & 1 & $-1.35 \mathrm{E}-01$ & $2.64 \mathrm{E}-03$ & $3.08 \mathrm{E}-03$ & $-1.02 \mathrm{E}-01$ \\
\hline & Northern Asia & $1.08 \mathrm{E}-01$ & $-3.61 \mathrm{E}-01$ & $-1.35 \mathrm{E}-01$ & 1 & $-1.85 \mathrm{E}-01$ & $-3.73 \mathrm{E}-01$ & $-2.98 \mathrm{E}-02$ \\
\hline & Europe & $-3.24 \mathrm{E}-02$ & $6.24 \mathrm{E}-02$ & $2.64 \mathrm{E}-03$ & $-1.85 \mathrm{E}-01$ & 1 & $-9.60 \mathrm{E}-02$ & 3.92E-03 \\
\hline & North America & $-1.09 \mathrm{E}-01$ & $2.24 \mathrm{E}-02$ & $3.08 \mathrm{E}-03$ & $-3.73 E-01$ & $-9.60 \mathrm{E}-02$ & 1 & $2.10 \mathrm{E}-02$ \\
\hline & Oceania & $-1.23 \mathrm{E}-02$ & $1.71 \mathrm{E}-02$ & $-1.02 \mathrm{E}-01$ & $-2.98 \mathrm{E}-02$ & $3.92 \mathrm{E}-03$ & $2.10 \mathrm{E}-02$ & 1 \\
\hline \multirow{6}{*}{ Ocean } & North Pacific & $3.47 \mathrm{E}-02$ & $2.61 \mathrm{E}-01$ & $3.22 \mathrm{E}-02$ & $-2.58 \mathrm{E}-01$ & $6.82 \mathrm{E}-02$ & $1.06 \mathrm{E}-01$ & $3.45 \mathrm{E}-03$ \\
\hline & \begin{tabular}{|l} 
South Pacific \\
\end{tabular} & $1.64 \mathrm{E}-02$ & $1.05 \mathrm{E}-03$ & $-5.30 \mathrm{E}-01$ & $1.40 \mathrm{E}-02$ & $6.72 \mathrm{E}-03$ & $4.62 \mathrm{E}-02$ & $5.92 \mathrm{E}-03$ \\
\hline & \begin{tabular}{|l|} 
Northern Ocean \\
\end{tabular} & $3.81 \mathrm{E}-02$ & $1.31 \mathrm{E}-01$ & $4.33 \mathrm{E}-02$ & $-7.35 \mathrm{E}-02$ & $2.23 \mathrm{E}-02$ & $-2.15 \mathrm{E}-02$ & $2.50 \mathrm{E}-03$ \\
\hline & Atlantic & $-1.14 \mathrm{E}-01$ & $1.44 \mathrm{E}-01$ & $-3.75 \mathrm{E}-02$ & $4.79 \mathrm{E}-02$ & $5.55 \mathrm{E}-02$ & $4.38 \mathrm{E}-02$ & $-5.44 \mathrm{E}-03$ \\
\hline & Southern Ocean & $6.45 \mathrm{E}-02$ & $1.90 \mathrm{E}-01$ & $2.10 \mathrm{E}-02$ & $-7.19 \mathrm{E}-02$ & $9.23 \mathrm{E}-03$ & $3.49 \mathrm{E}-02$ & $-6.12 \mathrm{E}-02$ \\
\hline & Indian Ocean & $-9.07 \mathrm{E}-02$ & $-1.75 \mathrm{E}-01$ & $-2.08 \mathrm{E}-01$ & $5.21 \mathrm{E}-02$ & $5.33 \mathrm{E}-04$ & $3.40 \mathrm{E}-02$ & $-4.97 \mathrm{E}-02$ \\
\hline \multirow{7}{*}{$\begin{array}{l}\text { Biomass } \\
\text { burning }\end{array}$} & Africa/Middle East & $-1.23 \mathrm{E}-01$ & $-6.03 \mathrm{E}-02$ & $-1.49 \mathrm{E}-02$ & $4.69 \mathrm{E}-02$ & $5.19 \mathrm{E}-04$ & $4.51 \mathrm{E}-03$ & $-5.05 \mathrm{E}-03$ \\
\hline & Southern Asia & $5.68 \mathrm{E}-03$ & $2.24 \mathrm{E}-02$ & $-1.32 \mathrm{E}-02$ & $-7.21 \mathrm{E}-03$ & $-1.69 \mathrm{E}-03$ & $4.18 \mathrm{E}-03$ & $-1.96 \mathrm{E}-03$ \\
\hline & Central/South America & $-5.37 \mathrm{E}-02$ & $-5.77 \mathrm{E}-02$ & $3.34 \mathrm{E}-02$ & $3.65 \mathrm{E}-02$ & $1.86 \mathrm{E}-03$ & $6.24 \mathrm{E}-03$ & $-9.31 \mathrm{E}-03$ \\
\hline & Northern Asia & $5.52 \mathrm{E}-04$ & $2.07 \mathrm{E}-03$ & $-1.43 \mathrm{E}-04$ & $-7.69 \mathrm{E}-02$ & $-1.07 \mathrm{E}-02$ & $-2.52 \mathrm{E}-02$ & $2.01 \mathrm{E}-03$ \\
\hline & Europe & $-3.93 E-04$ & $-4.85 \mathrm{E}-04$ & $1.94 \mathrm{E}-04$ & $-2.53 \mathrm{E}-04$ & $-3.89 \mathrm{E}-03$ & $-5.28 \mathrm{E}-04$ & $6.16 \mathrm{E}-05$ \\
\hline & North America & $-8.60 \mathrm{E}-04$ & $1.30 \mathrm{E}-02$ & $-7.48 \mathrm{E}-03$ & $-5.80 \mathrm{E}-02$ & $-1.24 \mathrm{E}-02$ & $-7.26 \mathrm{E}-02$ & $1.71 \mathrm{E}-03$ \\
\hline & Oceania & $-4.59 \mathrm{E}-03$ & $-6.88 \mathrm{E}-03$ & $-2.67 \mathrm{E}-03$ & $1.59 \mathrm{E}-03$ & $4.78 \mathrm{E}-04$ & $2.89 \mathrm{E}-03$ & $-3.21 \mathrm{E}-03$ \\
\hline
\end{tabular}

Fig. S2az. Correlation of the emissions in 2005 for natural soil. 


\begin{tabular}{|c|c|c|c|c|c|c|c|}
\hline & \multirow{2}{*}{\multicolumn{6}{|c|}{ Ocean }} \\
\hline & & & & & & & \\
\hline Source sector & Region & $\begin{array}{l}\text { North } \\
\text { Pacific }\end{array}$ & $\begin{array}{l}\text { South } \\
\text { Pacific }\end{array}$ & $\begin{array}{l}\text { Northern } \\
\text { Ocean }\end{array}$ & Atlantic & $\begin{array}{l}\text { Southern } \\
\text { Ocean }\end{array}$ & $\begin{array}{l}\text { Indian } \\
\text { Ocean }\end{array}$ \\
\hline \multirow{7}{*}{$\begin{array}{l}\text { Agricultural } \\
\text { Soil }\end{array}$} & Africa/Middle East & $-1.85 \mathrm{E}-02$ & $-2.46 \mathrm{E}-03$ & $-1.59 \mathrm{E}-03$ & $-8.81 \mathrm{E}-02$ & $2.25 \mathrm{E}-02$ & $-2.85 \mathrm{E}-02$ \\
\hline & Southern Asia & $-2.44 \mathrm{E}-01$ & $-1.73 \mathrm{E}-02$ & $-3.38 \mathrm{E}-02$ & $-1.66 \mathrm{E}-01$ & $8.01 \mathrm{E}-02$ & $3.41 \mathrm{E}-03$ \\
\hline & Central/South America & $2.77 \mathrm{E}-03$ & $-7.89 \mathrm{E}-03$ & $4.55 \mathrm{E}-04$ & $-2.34 \mathrm{E}-02$ & $-1.90 \mathrm{E}-01$ & $-9.17 \mathrm{E}-02$ \\
\hline & Northern Asia & $3.08 \mathrm{E}-02$ & $1.97 \mathrm{E}-02$ & $-5.33 E-02$ & $-3.36 \mathrm{E}-02$ & $-1.44 \mathrm{E}-02$ & $-6.89 \mathrm{E}-03$ \\
\hline & Europe & $1.71 \mathrm{E}-03$ & $1.25 \mathrm{E}-02$ & $-7.71 \mathrm{E}-02$ & $-5.30 \mathrm{E}-02$ & $6.00 \mathrm{E}-03$ & $8.98 \mathrm{E}-03$ \\
\hline & North America & $-5.13 E-02$ & $9.24 \mathrm{E}-03$ & $-7.06 \mathrm{E}-03$ & $-1.00 \mathrm{E}-01$ & $3.82 \mathrm{E}-03$ & $1.42 \mathrm{E}-02$ \\
\hline & Oceania & $1.21 \mathrm{E}-02$ & $1.51 \mathrm{E}-02$ & $5.43 \mathrm{E}-03$ & $1.69 \mathrm{E}-02$ & $-8.31 \mathrm{E}-02$ & $-3.98 \mathrm{E}-02$ \\
\hline \multirow{7}{*}{ Industrial } & Africa/Middle East & $-4.01 \mathrm{E}-03$ & $4.03 \mathrm{E}-05$ & $2.68 \mathrm{E}-05$ & $-1.25 \mathrm{E}-02$ & $2.36 \mathrm{E}-03$ & $-1.95 \mathrm{E}-03$ \\
\hline & Southern Asia & $-1.13 \mathrm{E}-02$ & $8.77 \mathrm{E}-03$ & $-1.03 \mathrm{E}-02$ & $-1.38 \mathrm{E}-03$ & $-2.36 \mathrm{E}-02$ & $2.08 \mathrm{E}-02$ \\
\hline & Central/South America & $1.22 \mathrm{E}-04$ & $-2.98 \mathrm{E}-03$ & $5.04 \mathrm{E}-05$ & $-2.32 \mathrm{E}-03$ & $-1.29 \mathrm{E}-02$ & $-7.66 \mathrm{E}-03$ \\
\hline & \begin{tabular}{|l|} 
Northern Asia \\
\end{tabular} & $6.32 \mathrm{E}-02$ & $1.78 \mathrm{E}-02$ & $3.51 \mathrm{E}-02$ & $-2.27 \mathrm{E}-01$ & $4.22 \mathrm{E}-03$ & $5.50 \mathrm{E}-03$ \\
\hline & Europe & $-1.81 \mathrm{E}-02$ & $4.59 \mathrm{E}-03$ & $1.59 \mathrm{E}-02$ & $-8.86 \mathrm{E}-03$ & $-1.84 \mathrm{E}-03$ & 5.07E-03 \\
\hline & North America & $-3.25 \mathrm{E}-02$ & $-1.77 \mathrm{E}-03$ & $3.41 \mathrm{E}-02$ & $-1.18 \mathrm{E}-01$ & $1.21 \mathrm{E}-02$ & $-3.37 \mathrm{E}-03$ \\
\hline & Oceania & $9.29 \mathrm{E}-04$ & $2.43 \mathrm{E}-03$ & 4.04E-04 & $1.22 \mathrm{E}-03$ & $-6.18 \mathrm{E}-03$ & $-3.85 \mathrm{E}-03$ \\
\hline \multirow{7}{*}{ Natural Soil } & Africa/Middle East & $3.47 \mathrm{E}-02$ & $1.64 \mathrm{E}-02$ & $3.81 \mathrm{E}-02$ & $-1.14 \mathrm{E}-01$ & $6.45 \mathrm{E}-02$ & $-9.07 E-02$ \\
\hline & Southern Asia & $2.61 \mathrm{E}-01$ & $1.05 \mathrm{E}-03$ & $1.31 \mathrm{E}-01$ & $1.44 \mathrm{E}-01$ & $1.90 \mathrm{E}-01$ & $-1.75 \mathrm{E}-01$ \\
\hline & Central/South America & $3.22 \mathrm{E}-02$ & $-5.30 \mathrm{E}-01$ & $4.33 \mathrm{E}-02$ & $-3.75 \mathrm{E}-02$ & $2.10 \mathrm{E}-02$ & $-2.08 \mathrm{E}-01$ \\
\hline & Northern Asia & $-2.58 \mathrm{E}-01$ & $1.40 \mathrm{E}-02$ & $-7.35 \mathrm{E}-02$ & $4.79 \mathrm{E}-02$ & $-7.19 \mathrm{E}-02$ & $5.21 \mathrm{E}-02$ \\
\hline & Europe & $6.82 \mathrm{E}-02$ & $6.72 \mathrm{E}-03$ & $2.23 \mathrm{E}-02$ & $5.55 \mathrm{E}-02$ & $9.23 \mathrm{E}-03$ & $5.33 \mathrm{E}-04$ \\
\hline & North America & $1.06 \mathrm{E}-01$ & $4.62 \mathrm{E}-02$ & $-2.15 \mathrm{E}-02$ & $4.38 \mathrm{E}-02$ & $3.49 E-02$ & $3.40 \mathrm{E}-02$ \\
\hline & Oceania & $3.45 \mathrm{E}-03$ & 5.92E-03 & $2.50 \mathrm{E}-03$ & $-5.44 \mathrm{E}-03$ & $-6.12 \mathrm{E}-02$ & $-4.97 \mathrm{E}-02$ \\
\hline \multirow{6}{*}{ Ocean } & North Pacific & 1 & $1.56 \mathrm{E}-03$ & $-6.28 \mathrm{E}-02$ & $-1.79 \mathrm{E}-01$ & $3.49 \mathrm{E}-02$ & $5.12 \mathrm{E}-03$ \\
\hline & South Pacific & $1.56 \mathrm{E}-03$ & 1 & $7.39 \mathrm{E}-03$ & $-5.40 \mathrm{E}-02$ & $3.83 \mathrm{E}-02$ & $3.29 \mathrm{E}-02$ \\
\hline & Northern Ocean & $-6.28 \mathrm{E}-02$ & $7.39 \mathrm{E}-03$ & 1 & $-3.19 \mathrm{E}-01$ & $3.22 \mathrm{E}-02$ & $-3.85 \mathrm{E}-04$ \\
\hline & Atlantic & $-1.79 \mathrm{E}-01$ & $-5.40 \mathrm{E}-02$ & $-3.19 \mathrm{E}-01$ & 1 & $-6.42 \mathrm{E}-02$ & $-4.06 \mathrm{E}-02$ \\
\hline & Southern Ocean & $3.49 \mathrm{E}-02$ & $3.83 \mathrm{E}-02$ & $3.22 \mathrm{E}-02$ & $-6.42 \mathrm{E}-02$ & 1 & $-6.51 \mathrm{E}-01$ \\
\hline & Indian Ocean & $5.12 \mathrm{E}-03$ & $3.29 \mathrm{E}-02$ & $-3.85 \mathrm{E}-04$ & $-4.06 \mathrm{E}-02$ & $-6.51 \mathrm{E}-01$ & \\
\hline \multirow{7}{*}{$\begin{array}{l}\text { Biomass } \\
\text { burning }\end{array}$} & Africa/Middle East & $-5.36 \mathrm{E}-03$ & $-5.95 \mathrm{E}-02$ & $-2.77 \mathrm{E}-04$ & $-6.92 \mathrm{E}-02$ & $-1.25 \mathrm{E}-02$ & $-7.30 \mathrm{E}-02$ \\
\hline & Southern Asia & $-1.04 \mathrm{E}-02$ & $-1.06 \mathrm{E}-02$ & $-2.91 \mathrm{E}-03$ & $-1.69 \mathrm{E}-02$ & $1.52 \mathrm{E}-02$ & $-1.38 \mathrm{E}-02$ \\
\hline & Central/South America & $-7.95 \mathrm{E}-03$ & $-6.72 \mathrm{E}-02$ & $-4.78 \mathrm{E}-03$ & $-3.14 \mathrm{E}-02$ & $-6.09 \mathrm{E}-02$ & $-4.75 \mathrm{E}-02$ \\
\hline & Northern Asia & 7.97E-04 & $6.45 \mathrm{E}-03$ & $-6.26 \mathrm{E}-04$ & 8.37E-03 & $2.08 \mathrm{E}-03$ & $1.09 \mathrm{E}-03$ \\
\hline & Europe & $2.68 \mathrm{E}-04$ & $1.36 \mathrm{E}-04$ & $2.86 \mathrm{E}-04$ & $2.80 \mathrm{E}-04$ & $-3.19 \mathrm{E}-05$ & $1.18 \mathrm{E}-04$ \\
\hline & North America & $-9.71 \mathrm{E}-03$ & $5.54 \mathrm{E}-03$ & $-8.05 \mathrm{E}-03$ & 9.34E-03 & $3.11 \mathrm{E}-03$ & $3.31 \mathrm{E}-03$ \\
\hline & Oceania & $-1.01 \mathrm{E}-03$ & $-1.10 \mathrm{E}-03$ & $-1.02 \mathrm{E}-03$ & $-2.90 \mathrm{E}-03$ & $-1.91 \mathrm{E}-03$ & $-8.09 \mathrm{E}-03$ \\
\hline
\end{tabular}

Fig. S2ba. Correlation of the emissions in 2005 for ocean. 


\begin{tabular}{|c|c|c|c|c|c|c|c|c|}
\hline & \multirow{2}{*}{\multicolumn{7}{|c|}{ Biomass burning }} \\
\hline & & & & & & & & \\
\hline Source sector & Region & $\begin{array}{l}\text { Africa/M. } \\
\text { East }\end{array}$ & $\begin{array}{l}\text { Southern } \\
\text { Asia }\end{array}$ & $\begin{array}{l}\text { C. /S. } \\
\text { America }\end{array}$ & $\begin{array}{l}\text { Northern } \\
\text { Asia }\end{array}$ & Europe & $\begin{array}{l}\text { North } \\
\text { America }\end{array}$ & Oceania \\
\hline \multirow{7}{*}{$\begin{array}{l}\text { Agricultural } \\
\text { Soil }\end{array}$} & Africa/Middle East & $-4.00 \mathrm{E}-02$ & $-4.75 \mathrm{E}-03$ & $-3.89 \mathrm{E}-03$ & $2.43 \mathrm{E}-04$ & $-2.40 \mathrm{E}-04$ & $1.06 \mathrm{E}-05$ & $4.76 \mathrm{E}-04$ \\
\hline & Southern Asia & $-2.13 \mathrm{E}-02$ & $-3.64 \mathrm{E}-02$ & $2.75 \mathrm{E}-02$ & $-4.61 \mathrm{E}-03$ & $-6.55 \mathrm{E}-07$ & $-1.19 \mathrm{E}-03$ & $5.74 \mathrm{E}-03$ \\
\hline & Central/South America & $-3.32 \mathrm{E}-02$ & $2.63 \mathrm{E}-03$ & $-3.28 \mathrm{E}-02$ & $1.87 \mathrm{E}-03$ & $6.49 \mathrm{E}-05$ & $2.69 \mathrm{E}-03$ & $-5.70 \mathrm{E}-03$ \\
\hline & Northern Asia & $8.10 \mathrm{E}-03$ & $3.29 \mathrm{E}-03$ & $6.97 \mathrm{E}-04$ & $6.61 \mathrm{E}-03$ & $-2.81 \mathrm{E}-04$ & $8.21 \mathrm{E}-03$ & $-1.00 \mathrm{E}-03$ \\
\hline & Europe & $3.70 \mathrm{E}-03$ & $1.22 \mathrm{E}-03$ & $2.97 \mathrm{E}-03$ & $-3.42 \mathrm{E}-04$ & $-6.19 \mathrm{E}-05$ & $-1.51 \mathrm{E}-03$ & $1.01 \mathrm{E}-03$ \\
\hline & North America & $-9.86 \mathrm{E}-04$ & $-3.32 \mathrm{E}-03$ & $7.74 \mathrm{E}-03$ & $4.14 \mathrm{E}-03$ & $-7.35 \mathrm{E}-07$ & $8.82 \mathrm{E}-03$ & $1.50 \mathrm{E}-03$ \\
\hline & Oceania & $7.11 \mathrm{E}-03$ & $2.05 \mathrm{E}-03$ & $5.69 \mathrm{E}-03$ & $1.74 \mathrm{E}-03$ & $6.42 \mathrm{E}-06$ & $1.96 \mathrm{E}-03$ & $-5.55 \mathrm{E}-04$ \\
\hline \multirow{7}{*}{ Industrial } & Africa/Middle East & $-3.84 \mathrm{E}-03$ & $-8.08 \mathrm{E}-04$ & $1.50 \mathrm{E}-04$ & $9.24 \mathrm{E}-05$ & $-4.47 \mathrm{E}-05$ & $1.95 \mathrm{E}-04$ & $1.82 \mathrm{E}-04$ \\
\hline & Southern Asia & $1.19 \mathrm{E}-02$ & $-1.89 \mathrm{E}-03$ & $4.83 \mathrm{E}-03$ & $3.40 \mathrm{E}-03$ & $1.34 \mathrm{E}-04$ & $4.69 \mathrm{E}-03$ & $3.75 \mathrm{E}-04$ \\
\hline & Central/South America & $-2.84 \mathrm{E}-03$ & $1.90 \mathrm{E}-04$ & $-2.76 \mathrm{E}-03$ & $1.36 \mathrm{E}-04$ & 5.19E-06 & $1.80 \mathrm{E}-04$ & $-4.66 \mathrm{E}-04$ \\
\hline & Northern Asia & $1.45 \mathrm{E}-02$ & $3.87 E-03$ & $3.55 \mathrm{E}-03$ & $1.10 \mathrm{E}-02$ & $-1.06 \mathrm{E}-04$ & $1.46 \mathrm{E}-02$ & $6.64 \mathrm{E}-05$ \\
\hline & Europe & $2.35 \mathrm{E}-03$ & $3.15 \mathrm{E}-04$ & $1.51 \mathrm{E}-03$ & $2.49 \mathrm{E}-04$ & $1.98 \mathrm{E}-04$ & $2.29 \mathrm{E}-03$ & 3.99E-04 \\
\hline & North America & $5.18 \mathrm{E}-04$ & $-4.37 \mathrm{E}-04$ & 8.99E-04 & 6.07E-03 & $1.57 \mathrm{E}-04$ & $2.22 \mathrm{E}-04$ & 3.89E-07 \\
\hline & Oceania & $6.27 \mathrm{E}-04$ & $1.57 \mathrm{E}-04$ & $3.98 \mathrm{E}-04$ & $1.43 \mathrm{E}-04$ & $4.81 \mathrm{E}-07$ & $1.58 \mathrm{E}-04$ & $-3.67 \mathrm{E}-05$ \\
\hline \multirow{7}{*}{ Natural Soil } & Africa/Middle East & $-1.23 \mathrm{E}-01$ & $5.68 \mathrm{E}-03$ & $-5.37 \mathrm{E}-02$ & 5.52E-04 & $-3.93 \mathrm{E}-04$ & $-8.60 \mathrm{E}-04$ & $-4.59 \mathrm{E}-03$ \\
\hline & Southern Asia & $-6.03 E-02$ & $2.24 \mathrm{E}-02$ & \begin{tabular}{|l|}
$-5.77 \mathrm{E}-02$ \\
\end{tabular} & $2.07 E-03$ & $-4.85 \mathrm{E}-04$ & $1.30 \mathrm{E}-02$ & $-6.88 \mathrm{E}-03$ \\
\hline & Central/South America & $-1.49 \mathrm{E}-02$ & $-1.32 \mathrm{E}-02$ & $3.34 \mathrm{E}-02$ & $-1.43 \mathrm{E}-04$ & $1.94 \mathrm{E}-04$ & $-7.48 \mathrm{E}-03$ & $-2.67 \mathrm{E}-03$ \\
\hline & Northern Asia & $4.69 \mathrm{E}-02$ & $-7.21 \mathrm{E}-03$ & $3.65 \mathrm{E}-02$ & \begin{tabular}{|l|}
$-7.69 \mathrm{E}-02$ \\
\end{tabular} & $-2.53 \mathrm{E}-04$ & $-5.80 \mathrm{E}-02$ & $1.59 \mathrm{E}-03$ \\
\hline & Europe & 5.19E-04 & $-1.69 \mathrm{E}-03$ & $1.86 \mathrm{E}-03$ & \begin{tabular}{|l|}
$-1.07 E-02$ \\
\end{tabular} & $-3.89 \mathrm{E}-03$ & $-1.24 \mathrm{E}-02$ & $4.78 \mathrm{E}-04$ \\
\hline & North America & $4.51 \mathrm{E}-03$ & $4.18 \mathrm{E}-03$ & $6.24 \mathrm{E}-03$ & \begin{tabular}{|l|}
$-2.52 \mathrm{E}-02$ \\
\end{tabular} & $-5.28 \mathrm{E}-04$ & $-7.26 \mathrm{E}-02$ & $2.89 \mathrm{E}-03$ \\
\hline & Oceania & $-5.05 E-03$ & $-1.96 \mathrm{E}-03$ & $-9.31 \mathrm{E}-03$ & $2.01 \mathrm{E}-03$ & $6.16 \mathrm{E}-05$ & $1.71 \mathrm{E}-03$ & $-3.21 \mathrm{E}-03$ \\
\hline \multirow{6}{*}{ Ocean } & North Pacific & $-5.36 \mathrm{E}-03$ & $-1.04 \mathrm{E}-02$ & $-7.95 \mathrm{E}-03$ & $7.97 \mathrm{E}-04$ & $2.68 \mathrm{E}-04$ & $-9.71 \mathrm{E}-03$ & $-1.01 \mathrm{E}-03$ \\
\hline & South Pacific & $-5.95 \mathrm{E}-02$ & $-1.06 \mathrm{E}-02$ & $-6.72 \mathrm{E}-02$ & $6.45 \mathrm{E}-03$ & $1.36 \mathrm{E}-04$ & $5.54 \mathrm{E}-03$ & $-1.10 \mathrm{E}-03$ \\
\hline & Northern Ocean & $-2.77 \mathrm{E}-04$ & $-2.91 \mathrm{E}-03$ & $-4.78 \mathrm{E}-03$ & $-6.26 \mathrm{E}-04$ & $2.86 \mathrm{E}-04$ & $-8.05 \mathrm{E}-03$ & $-1.02 \mathrm{E}-03$ \\
\hline & Atlantic & $-6.92 \mathrm{E}-02$ & $-1.69 \mathrm{E}-02$ & $-3.14 \mathrm{E}-02$ & $8.37 \mathrm{E}-03$ & $2.80 \mathrm{E}-04$ & $9.34 \mathrm{E}-03$ & $-2.90 \mathrm{E}-03$ \\
\hline & Southern Ocean & $-1.25 \mathrm{E}-02$ & $1.52 \mathrm{E}-02$ & $-6.09 \mathrm{E}-02$ & $2.08 \mathrm{E}-03$ & $-3.19 \mathrm{E}-05$ & \begin{tabular}{|l|}
$3.11 \mathrm{E}-03$ \\
\end{tabular} & $-1.91 \mathrm{E}-03$ \\
\hline & Indian Ocean & $-7.30 \mathrm{E}-02$ & $-1.38 \mathrm{E}-02$ & $-4.75 \mathrm{E}-02$ & $1.09 \mathrm{E}-03$ & $1.18 \mathrm{E}-04$ & $3.31 \mathrm{E}-03$ & $-8.09 \mathrm{E}-03$ \\
\hline \multirow{7}{*}{$\begin{array}{l}\text { Biomass } \\
\text { burning }\end{array}$} & Africa/Middle East & 1 & $-2.95 \mathrm{E}-03$ & $-3.91 \mathrm{E}-02$ & $2.07 \mathrm{E}-03$ & $2.42 \mathrm{E}-05$ & $2.29 \mathrm{E}-03$ & $-3.16 \mathrm{E}-03$ \\
\hline & Southern Asia & $-2.95 \mathrm{E}-03$ & 1 & $2.40 \mathrm{E}-03$ & 5.19E-06 & $3.56 \mathrm{E}-05$ & \begin{tabular}{|l|}
$2.81 \mathrm{E}-04$ \\
\end{tabular} & $1.30 \mathrm{E}-05$ \\
\hline & Central/South America & $-3.91 \mathrm{E}-02$ & $2.40 \mathrm{E}-03$ & 1 & $8.64 \mathrm{E}-04$ & $4.42 \mathrm{E}-05$ & $1.33 \mathrm{E}-03$ & $-4.70 \mathrm{E}-03$ \\
\hline & Northern Asia & $2.07 \mathrm{E}-03$ & $5.19 \mathrm{E}-06$ & $8.64 \mathrm{E}-04$ & 1 & $-9.91 \mathrm{E}-05$ & \begin{tabular}{|l|l|} 
& $-6.53 \mathrm{E}-03$ \\
\end{tabular} & $1.62 \mathrm{E}-04$ \\
\hline & Europe & $2.42 \mathrm{E}-05$ & $3.56 \mathrm{E}-05$ & $4.42 \mathrm{E}-05$ & $-9.91 \mathrm{E}-05$ & 1 & $-3.52 \mathrm{E}-05$ & $1.13 \mathrm{E}-05$ \\
\hline & North America & $2.29 \mathrm{E}-03$ & $2.81 \mathrm{E}-04$ & $1.33 \mathrm{E}-03$ & $-6.53 \mathrm{E}-03$ & $-3.52 \mathrm{E}-05$ & 1 & $2.67 \mathrm{E}-04$ \\
\hline & Oceania & $-3.16 \mathrm{E}-03$ & $1.30 \mathrm{E}-05$ & $-4.70 \mathrm{E}-03$ & 1.62E-04 & $1.13 \mathrm{E}-05$ & \begin{tabular}{|l|l|}
$2.67 \mathrm{E}-04$ \\
\end{tabular} & 1 \\
\hline
\end{tabular}

Fig. S2bb. Correlation of the emissions in 2005 for biomass burning. 


\begin{tabular}{|c|c|c|c|c|c|c|c|c|}
\hline \multirow[b]{2}{*}{ Source sector } & \multirow[b]{2}{*}{ Region } & \multicolumn{7}{|c|}{ Agricultural Soil } \\
\hline & & $\begin{array}{l}\text { Africa/M. } \\
\text { East }\end{array}$ & $\begin{array}{l}\text { Southern } \\
\text { Asia }\end{array}$ & $\begin{array}{l}\text { C. /S. } \\
\text { America }\end{array}$ & $\begin{array}{l}\text { Northern } \\
\text { Asia }\end{array}$ & Europe & $\begin{array}{l}\text { North } \\
\text { America }\end{array}$ & Oceania \\
\hline \multirow{7}{*}{$\begin{array}{c}\text { Agricultural } \\
\text { Soil }\end{array}$} & Africa/Middle East & 1 & $-1.03 \mathrm{E}-01$ & $5.33 \mathrm{E}-04$ & $-4.51 \mathrm{E}-02$ & $-1.52 \mathrm{E}-02$ & $-2.96 \mathrm{E}-02$ & $7.23 \mathrm{E}-03$ \\
\hline & Southern Asia & $-1.03 \mathrm{E}-01$ & 1 & $5.26 \mathrm{E}-02$ & $-3.24 \mathrm{E}-01$ & $3.97 E-02$ & $-8.40 \mathrm{E}-02$ & $2.47 \mathrm{E}-02$ \\
\hline & Central/South America & $5.33 E-04$ & $5.26 \mathrm{E}-02$ & 1 & $-5.35 \mathrm{E}-03$ & $7.55 \mathrm{E}-03$ & $1.72 \mathrm{E}-02$ & $-2.47 \mathrm{E}-02$ \\
\hline & Northern Asia & $-4.51 \mathrm{E}-02$ & $-3.24 \mathrm{E}-01$ & $-5.35 \mathrm{E}-03$ & 1 & $-1.72 \mathrm{E}-01$ & $-4.21 \mathrm{E}-02$ & $-2.25 \mathrm{E}-02$ \\
\hline & Europe & $-1.52 \mathrm{E}-02$ & $3.97 \mathrm{E}-02$ & $7.55 \mathrm{E}-03$ & $-1.72 \mathrm{E}-01$ & 1 & $-1.97 \mathrm{E}-02$ & $4.60 \mathrm{E}-03$ \\
\hline & North America & $-2.96 \mathrm{E}-02$ & $-8.40 \mathrm{E}-02$ & $1.72 \mathrm{E}-02$ & $-4.21 \mathrm{E}-02$ & $-1.97 \mathrm{E}-02$ & 1 & $4.11 \mathrm{E}-03$ \\
\hline & Oceania & $7.23 \mathrm{E}-03$ & $2.47 \mathrm{E}-02$ & $-2.47 \mathrm{E}-02$ & $-2.25 \mathrm{E}-02$ & $4.60 \mathrm{E}-03$ & $4.11 \mathrm{E}-03$ & 1 \\
\hline \multirow{7}{*}{ Industrial } & Africa/Middle East & $-8.66 \mathrm{E}-02$ & $-1.39 \mathrm{E}-02$ & $2.23 \mathrm{E}-03$ & $-9.44 \mathrm{E}-03$ & \begin{tabular}{|l|}
$-7.60 \mathrm{E}-03$ \\
\end{tabular} & $-5.63 \mathrm{E}-03$ & $1.14 \mathrm{E}-03$ \\
\hline & \begin{tabular}{|l} 
Southern Asia \\
\end{tabular} & $2.17 \mathrm{E}-02$ & $-4.78 \mathrm{E}-01$ & $-4.30 \mathrm{E}-03$ & $-4.38 \mathrm{E}-02$ & $1.79 \mathrm{E}-02$ & $1.61 \mathrm{E}-02$ & $-2.70 \mathrm{E}-03$ \\
\hline & Central/South America & $-1.12 \mathrm{E}-04$ & $4.17 \mathrm{E}-03$ & $-9.08 \mathrm{E}-03$ & $-3.76 \mathrm{E}-04$ & $6.25 \mathrm{E}-04$ & $1.32 \mathrm{E}-03$ & $-1.92 \mathrm{E}-03$ \\
\hline & Northern Asia & $1.28 \mathrm{E}-02$ & $-3.46 \mathrm{E}-02$ & $-3.84 \mathrm{E}-03$ & $-1.19 \mathrm{E}-01$ & $-5.40 \mathrm{E}-02$ & $1.57 \mathrm{E}-01$ & $-5.16 \mathrm{E}-03$ \\
\hline & Europe & $-1.63 E-02$ & $-3.15 E-04$ & $4.24 \mathrm{E}-03$ & $-2.00 \mathrm{E}-02$ & $-7.46 \mathrm{E}-01$ & $-1.52 \mathrm{E}-02$ & $4.12 \mathrm{E}-04$ \\
\hline & North America & $8.68 \mathrm{E}-03$ & $3.20 \mathrm{E}-02$ & $1.48 \mathrm{E}-03$ & $3.02 \mathrm{E}-02$ & $2.01 \mathrm{E}-02$ & $-3.65 \mathrm{E}-01$ & $-7.10 \mathrm{E}-04$ \\
\hline & Oceania & $5.80 \mathrm{E}-04$ & $1.85 \mathrm{E}-03$ & $-1.91 \mathrm{E}-03$ & $-1.48 \mathrm{E}-03$ & $3.05 \mathrm{E}-04$ & $2.92 \mathrm{E}-04$ & $-6.90 \mathrm{E}-03$ \\
\hline \multirow{7}{*}{ Natural Soil } & Africa/Middle East & $-2.24 \mathrm{E}-01$ & $-1.22 \mathrm{E}-01$ & $-1.22 \mathrm{E}-01$ & $-6.30 \mathrm{E}-03$ & \begin{tabular}{|l|}
$-1.18 \mathrm{E}-02$ \\
\end{tabular} & $-1.31 \mathrm{E}-02$ & $-2.09 \mathrm{E}-03$ \\
\hline & Southern Asia & $-3.59 E-02$ & $-3.33 \mathrm{E}-01$ & $6.39 \mathrm{E}-03$ & $6.60 \mathrm{E}-02$ & $1.80 \mathrm{E}-04$ & $-2.04 \mathrm{E}-02$ & $1.77 \mathrm{E}-02$ \\
\hline & Central/South America & $-5.09 E-02$ & $1.38 \mathrm{E}-02$ & $-2.48 \mathrm{E}-01$ & $6.46 \mathrm{E}-03$ & $1.84 \mathrm{E}-02$ & $1.43 \mathrm{E}-02$ & $-3.54 \mathrm{E}-02$ \\
\hline & Northern Asia & $4.28 \mathrm{E}-02$ & $2.11 \mathrm{E}-01$ & $2.27 \mathrm{E}-03$ & $-2.20 \mathrm{E}-01$ & $2.91 \mathrm{E}-02$ & $3.62 \mathrm{E}-02$ & $-1.23 \mathrm{E}-02$ \\
\hline & Europe & $-2.51 \mathrm{E}-02$ & $-4.34 \mathrm{E}-02$ & $1.14 \mathrm{E}-02$ & $-2.09 \mathrm{E}-02$ & $-2.67 \mathrm{E}-01$ & $1.80 \mathrm{E}-02$ & $3.44 \mathrm{E}-03$ \\
\hline & North America & $-2.07 \mathrm{E}-02$ & $-1.03 \mathrm{E}-01$ & $2.82 \mathrm{E}-02$ & $6.45 \mathrm{E}-02$ & $1.31 \mathrm{E}-02$ & $-4.16 \mathrm{E}-01$ & $7.84 \mathrm{E}-03$ \\
\hline & Oceania & $5.38 \mathrm{E}-03$ & $2.19 \mathrm{E}-02$ & $-2.72 \mathrm{E}-02$ & $-1.76 \mathrm{E}-02$ & $6.45 \mathrm{E}-03$ & $1.03 \mathrm{E}-02$ & $-5.62 \mathrm{E}-02$ \\
\hline \multirow{6}{*}{ Ocean } & North Pacific & $-3.92 \mathrm{E}-03$ & $-2.22 \mathrm{E}-01$ & $1.18 \mathrm{E}-02$ & $1.78 \mathrm{E}-02$ & $2.01 \mathrm{E}-03$ & $-3.63 \mathrm{E}-02$ & $6.64 \mathrm{E}-03$ \\
\hline & South Pacific & $-5.65 E-04$ & $1.81 \mathrm{E}-03$ & $-2.23 \mathrm{E}-02$ & $1.01 \mathrm{E}-02$ & $8.89 \mathrm{E}-03$ & $1.45 \mathrm{E}-02$ & $6.87 \mathrm{E}-03$ \\
\hline & Northern Ocean & $1.70 \mathrm{E}-03$ & $-1.37 \mathrm{E}-02$ & $2.39 \mathrm{E}-03$ & $-5.80 \mathrm{E}-02$ & $-6.52 \mathrm{E}-02$ & $-9.19 \mathrm{E}-03$ & $2.79 \mathrm{E}-03$ \\
\hline & Atlantic & $-8.79 \mathrm{E}-02$ & $-1.02 \mathrm{E}-01$ & $-2.53 \mathrm{E}-02$ & $-2.61 \mathrm{E}-02$ & $-3.96 \mathrm{E}-02$ & $-9.53 \mathrm{E}-02$ & $1.40 \mathrm{E}-02$ \\
\hline & Southern Ocean & $3.17 \mathrm{E}-02$ & $1.15 \mathrm{E}-01$ & $-2.17 \mathrm{E}-01$ & $-1.37 \mathrm{E}-02$ & $1.05 \mathrm{E}-02$ & $1.52 \mathrm{E}-02$ & $-7.09 \mathrm{E}-02$ \\
\hline & Indian Ocean & $-3.29 \mathrm{E}-02$ & $-2.57 \mathrm{E}-02$ & $-1.05 \mathrm{E}-01$ & $-9.05 \mathrm{E}-03$ & $4.61 \mathrm{E}-03$ & $2.04 \mathrm{E}-02$ & $-4.62 \mathrm{E}-02$ \\
\hline \multirow{7}{*}{$\begin{array}{l}\text { Biomass } \\
\text { burning }\end{array}$} & Africa/Middle East & $-3.16 \mathrm{E}-02$ & $-1.80 \mathrm{E}-02$ & $-4.29 \mathrm{E}-02$ & $1.01 \mathrm{E}-02$ & $2.09 \mathrm{E}-03$ & $3.31 \mathrm{E}-03$ & $5.95 \mathrm{E}-03$ \\
\hline & Southern Asia & $6.38 \mathrm{E}-03$ & $2.31 \mathrm{E}-02$ & $-1.20 \mathrm{E}-02$ & $-3.04 \mathrm{E}-02$ & $6.86 \mathrm{E}-03$ & $1.03 \mathrm{E}-02$ & $-2.82 \mathrm{E}-03$ \\
\hline & Central/South America & $-3.36 \mathrm{E}-03$ & $7.72 \mathrm{E}-03$ & $-1.67 \mathrm{E}-02$ & $3.17 \mathrm{E}-04$ & $1.05 \mathrm{E}-03$ & $3.61 \mathrm{E}-03$ & $7.02 \mathrm{E}-04$ \\
\hline & \begin{tabular}{|l} 
Northern Asia \\
\end{tabular} & $6.43 \mathrm{E}-04$ & $-4.69 \mathrm{E}-02$ & $4.91 \mathrm{E}-03$ & $-6.72 \mathrm{E}-03$ & $1.77 \mathrm{E}-03$ & $1.32 \mathrm{E}-02$ & $9.31 \mathrm{E}-04$ \\
\hline & Europe & $-1.11 \mathrm{E}-04$ & $-4.52 \mathrm{E}-05$ & $-1.55 \mathrm{E}-05$ & $2.09 \mathrm{E}-04$ & $6.36 \mathrm{E}-04$ & $6.31 \mathrm{E}-05$ & $5.92 \mathrm{E}-06$ \\
\hline & North America & $-1.23 \mathrm{E}-03$ & $-1.04 \mathrm{E}-02$ & $2.04 \mathrm{E}-03$ & $3.43 \mathrm{E}-03$ & $-1.27 \mathrm{E}-04$ & $1.56 \mathrm{E}-03$ & $9.85 \mathrm{E}-04$ \\
\hline & Oceania & $-1.73 \mathrm{E}-04$ & $6.79 \mathrm{E}-03$ & $-1.11 \mathrm{E}-02$ & $-3.89 \mathrm{E}-03$ & $1.83 \mathrm{E}-03$ & $3.82 \mathrm{E}-03$ & $-4.12 \mathrm{E}-03$ \\
\hline
\end{tabular}

Fig. S2bc. Correlation of the emissions in 2006 for agricultural soil. 


\begin{tabular}{|c|c|c|c|c|c|c|c|c|}
\hline & \multirow{2}{*}{\multicolumn{7}{|c|}{ Industrial }} \\
\hline & & & & & & & & \\
\hline Source sector & Region & $\begin{array}{l}\text { Africa/M. } \\
\text { East }\end{array}$ & $\begin{array}{l}\text { Southern } \\
\text { Asia }\end{array}$ & $\begin{array}{l}\text { C. /S. } \\
\text { America }\end{array}$ & $\begin{array}{l}\text { Northern } \\
\text { Asia }\end{array}$ & Europe & $\begin{array}{l}\text { North } \\
\text { America }\end{array}$ & Oceania \\
\hline \multirow{7}{*}{$\begin{array}{l}\text { Agricultural } \\
\text { Soil }\end{array}$} & Africa/Middle East & $-8.66 \mathrm{E}-02$ & $2.17 \mathrm{E}-02$ & $-1.12 \mathrm{E}-04$ & $1.28 \mathrm{E}-02$ & $-1.63 \mathrm{E}-02$ & $8.68 \mathrm{E}-03$ & $5.80 \mathrm{E}-04$ \\
\hline & Southern Asia & $-1.39 \mathrm{E}-02$ & $-4.78 \mathrm{E}-01$ & $4.17 \mathrm{E}-03$ & $-3.46 \mathrm{E}-02$ & $-3.15 \mathrm{E}-04$ & $3.20 \mathrm{E}-02$ & $1.85 \mathrm{E}-03$ \\
\hline & Central/South America & $2.23 \mathrm{E}-03$ & $-4.30 \mathrm{E}-03$ & $-9.08 \mathrm{E}-03$ & \begin{tabular}{|l|} 
\\
\end{tabular} & $4.24 \mathrm{E}-03$ & $1.48 \mathrm{E}-03$ & $-1.91 \mathrm{E}-03$ \\
\hline & Northern Asia & $-9.44 \mathrm{E}-03$ & $-4.38 \mathrm{E}-02$ & $-3.76 \mathrm{E}-04$ & \begin{tabular}{|l|} 
\\
\end{tabular} & \begin{tabular}{|l|}
$-2.00 \mathrm{E}-02$ \\
\end{tabular} & $3.02 \mathrm{E}-02$ & $-1.48 \mathrm{E}-03$ \\
\hline & Europe & $-7.60 \mathrm{E}-03$ & $1.79 \mathrm{E}-02$ & $6.25 \mathrm{E}-04$ & $-5.40 \mathrm{E}-02$ & $\mid-7.46 \mathrm{E}-01$ & $2.01 \mathrm{E}-02$ & $3.05 \mathrm{E}-04$ \\
\hline & \begin{tabular}{|l|} 
North America \\
\end{tabular} & $-5.63 \mathrm{E}-03$ & $1.61 \mathrm{E}-02$ & $1.32 \mathrm{E}-03$ & \begin{tabular}{|c|} 
\\
\end{tabular} & $\mid-1.52 \mathrm{E}-02$ & $-3.65 \mathrm{E}-01$ & $2.92 \mathrm{E}-04$ \\
\hline & Oceania & $1.14 \mathrm{E}-03$ & $-2.70 \mathrm{E}-03$ & $-1.92 \mathrm{E}-03$ & 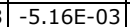 & $4.12 \mathrm{E}-04$ & $-7.10 \mathrm{E}-04$ & $-6.90 \mathrm{E}-03$ \\
\hline \multirow{7}{*}{ Industrial } & Africa/Middle East & 1 & $2.96 \mathrm{E}-03$ & $1.64 \mathrm{E}-04$ & $-1.20 \mathrm{E}-03$ & $\mid-6.33 \mathrm{E}-03$ & $1.45 \mathrm{E}-03$ & $8.72 \mathrm{E}-05$ \\
\hline & \begin{tabular}{|l|} 
Southern Asia \\
\end{tabular} & $2.96 \mathrm{E}-03$ & 1 & $-3.28 \mathrm{E}-04$ & $-4.49 \mathrm{E}-02$ & $4.40 \mathrm{E}-03$ & $1.02 \mathrm{E}-02$ & $-2.65 \mathrm{E}-04$ \\
\hline & Central/South America & $1.64 \mathrm{E}-04$ & $-3.28 \mathrm{E}-04$ & 1 & $-1.04 \mathrm{E}-04$ & $3.66 \mathrm{E}-04$ & $1.87 \mathrm{E}-04$ & $-1.49 \mathrm{E}-04$ \\
\hline & Northern Asia & $-1.20 \mathrm{E}-03$ & $-4.49 \mathrm{E}-02$ & $-1.04 \mathrm{E}-04$ & 1 & \begin{tabular}{|l|}
$-2.63 \mathrm{E}-02$ \\
\end{tabular} & $-1.96 \mathrm{E}-01$ & $-4.23 \mathrm{E}-04$ \\
\hline & Europe & $-6.33 E-03$ & $4.40 \mathrm{E}-03$ & $3.66 \mathrm{E}-04$ & $-2.63 \mathrm{E}-02$ & 1 & $3.89 \mathrm{E}-03$ & $2.05 \mathrm{E}-05$ \\
\hline & North America & $1.45 \mathrm{E}-03$ & $1.02 \mathrm{E}-02$ & $1.87 \mathrm{E}-04$ & $-1.96 \mathrm{E}-01$ & $3.89 \mathrm{E}-03$ & 1 & $-5.54 \mathrm{E}-05$ \\
\hline & Oceania & $8.72 \mathrm{E}-05$ & $-2.65 \mathrm{E}-04$ & $-1.49 \mathrm{E}-04$ & $-4.23 E-04$ & $2.05 E-05$ & $-5.54 \mathrm{E}-05$ & 1 \\
\hline \multirow{7}{*}{ Natural Soil } & Africa/Middle East & $-3.39 \mathrm{E}-02$ & $5.41 \mathrm{E}-02$ & $-1.05 \mathrm{E}-02$ & $5.72 \mathrm{E}-02$ & \begin{tabular}{|l|}
$-2.88 \mathrm{E}-03$ \\
\end{tabular} & $1.44 \mathrm{E}-02$ & $-2.08 \mathrm{E}-05$ \\
\hline & Southern Asia & $-3.59 \mathrm{E}-03$ & $-8.49 \mathrm{E}-02$ & $3.62 \mathrm{E}-04$ & $1.36 \mathrm{E}-02$ & \begin{tabular}{|l|}
$-1.27 \mathrm{E}-02$ \\
\end{tabular} & $8.60 \mathrm{E}-03$ & $1.78 \mathrm{E}-03$ \\
\hline & Central/South America & $-1.36 \mathrm{E}-04$ & $1.27 \mathrm{E}-03$ & $-1.92 \mathrm{E}-02$ & $4.16 \mathrm{E}-02$ & $9.32 \mathrm{E}-03$ & $1.43 \mathrm{E}-02$ & $-3.42 \mathrm{E}-03$ \\
\hline & Northern Asia & $5.92 \mathrm{E}-03$ & $3.95 \mathrm{E}-02$ & 2.67E-04 & $-4.62 \mathrm{E}-02$ & $2.14 \mathrm{E}-02$ & $-9.92 \mathrm{E}-04$ & $-1.22 \mathrm{E}-03$ \\
\hline & Europe & $-6.21 \mathrm{E}-03$ & $8.89 \mathrm{E}-03$ & 8.07E-04 & $2.61 \mathrm{E}-02$ & \begin{tabular}{|l|}
$-1.76 \mathrm{E}-01$ \\
\end{tabular} & $3.87 \mathrm{E}-02$ & $2.85 \mathrm{E}-04$ \\
\hline & North America & $9.31 \mathrm{E}-04$ & $4.72 \mathrm{E}-02$ & $1.95 \mathrm{E}-03$ & \begin{tabular}{|l|} 
\\
\end{tabular} & $2.38 \mathrm{E}-02$ & $-2.51 \mathrm{E}-01$ & $6.92 \mathrm{E}-04$ \\
\hline & Oceania & $1.29 \mathrm{E}-03$ & $6.55 \mathrm{E}-04$ & $-2.08 \mathrm{E}-03$ & \begin{tabular}{|l|}
$-2.80 \mathrm{E}-03$ \\
\end{tabular} & $2.26 \mathrm{E}-03$ & $3.81 \mathrm{E}-04$ & $-3.79 \mathrm{E}-03$ \\
\hline \multirow{6}{*}{ Ocean } & North Pacific & $-2.35 \mathrm{E}-03$ & $-1.15 \mathrm{E}-02$ & $8.38 \mathrm{E}-04$ & $\begin{array}{l}5.02 \mathrm{E}-02 \\
\end{array}$ & \begin{tabular}{|l|}
$-3.81 \mathrm{E}-03$ \\
\end{tabular} & $-2.80 \mathrm{E}-02$ & $4.51 \mathrm{E}-04$ \\
\hline & South Pacific & $1.00 \mathrm{E}-03$ & $1.17 \mathrm{E}-02$ & $-3.68 \mathrm{E}-03$ & \begin{tabular}{|c|} 
\\
\end{tabular} & $6.40 \mathrm{E}-03$ & $-4.27 \mathrm{E}-03$ & $1.55 \mathrm{E}-03$ \\
\hline & Northern Ocean & $3.58 \mathrm{E}-04$ & $-7.84 \mathrm{E}-03$ & $2.49 \mathrm{E}-04$ & $\begin{array}{ll} & 2.59 \mathrm{E}-02 \\
\end{array}$ & $1.60 \mathrm{E}-02$ & $2.73 \mathrm{E}-02$ & $1.99 \mathrm{E}-04$ \\
\hline & Atlantic & $-1.46 \mathrm{E}-02$ & $-2.18 \mathrm{E}-03$ & $-2.60 \mathrm{E}-03$ & 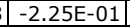 & $-2.49 \mathrm{E}-02$ & $-9.46 \mathrm{E}-02$ & $1.01 \mathrm{E}-03$ \\
\hline & Southern Ocean & $3.33 \mathrm{E}-03$ & $-2.78 \mathrm{E}-02$ & $-1.49 \mathrm{E}-02$ & $-2.81 \mathrm{E}-03$ & $8.47 \mathrm{E}-04$ & $9.56 \mathrm{E}-03$ & $-5.63 \mathrm{E}-03$ \\
\hline & Indian Ocean & $-7.74 \mathrm{E}-04$ & $2.12 \mathrm{E}-02$ & $-9.13 E-03$ & \begin{tabular}{|l|} 
\\
\end{tabular} & $5.32 \mathrm{E}-03$ & $-4.18 \mathrm{E}-03$ & $-4.60 \mathrm{E}-03$ \\
\hline \multirow{7}{*}{$\begin{array}{l}\text { Biomass } \\
\text { burning }\end{array}$} & Africa/Middle East & $-2.28 \mathrm{E}-03$ & $1.04 \mathrm{E}-02$ & $-3.75 \mathrm{E}-03$ & \begin{tabular}{|c|} 
\\
\end{tabular} & $3.28 \mathrm{E}-03$ & $2.77 \mathrm{E}-03$ & $5.76 \mathrm{E}-04$ \\
\hline & Southern Asia & $1.08 \mathrm{E}-03$ & $2.21 \mathrm{E}-02$ & $-9.22 \mathrm{E}-04$ & \begin{tabular}{|l|}
$1.29 E-03$ \\
\end{tabular} & $4.64 \mathrm{E}-03$ & $-3.22 \mathrm{E}-03$ & $-5.60 \mathrm{E}-05$ \\
\hline & Central/South America & $-1.68 \mathrm{E}-05$ & $1.94 \mathrm{E}-03$ & $-1.46 \mathrm{E}-03$ & $2.50 \mathrm{E}-03$ & $9.59 \mathrm{E}-04$ & $6.29 \mathrm{E}-04$ & $1.40 \mathrm{E}-04$ \\
\hline & Northern Asia & $6.47 \mathrm{E}-04$ & $8.82 \mathrm{E}-04$ & $3.45 \mathrm{E}-04$ & $2.41 \mathrm{E}-02$ & $2.35 \mathrm{E}-03$ & $1.64 \mathrm{E}-02$ & $1.04 \mathrm{E}-04$ \\
\hline & Europe & $-1.50 \mathrm{E}-05$ & $-1.88 \mathrm{E}-05$ & $-1.66 \mathrm{E}-06$ & -1.87E-06 & 5.62E-04 & $8.66 \mathrm{E}-05$ & $5.96 \mathrm{E}-07$ \\
\hline & North America & $1.01 \mathrm{E}-04$ & $1.37 \mathrm{E}-03$ & $1.28 \mathrm{E}-04$ & 1.47E-02 & 4.09E-03 & $9.60 \mathrm{E}-03$ & $8.32 \mathrm{E}-05$ \\
\hline & Oceania & $2.58 \mathrm{E}-04$ & $1.33 \mathrm{E}-03$ & $-9.11 \mathrm{E}-04$ & $6.05 \mathrm{E}-04$ & $9.33 \mathrm{E}-04$ & $1.52 \mathrm{E}-04$ & $-4.63 \mathrm{E}-04$ \\
\hline
\end{tabular}

Fig. S2bd. Correlation of the emissions in 2006 for industrial. 


\begin{tabular}{|c|c|c|c|c|c|c|c|c|}
\hline & \multirow{2}{*}{\multicolumn{7}{|c|}{ Natural Soil }} \\
\hline & & & & & & & & \\
\hline Source sector & Region & $\begin{array}{l}\text { Africa/M. } \\
\text { East }\end{array}$ & $\begin{array}{l}\text { Southern } \\
\text { Asia }\end{array}$ & $\begin{array}{l}\text { C. /S. } \\
\text { America }\end{array}$ & $\begin{array}{l}\text { Northern } \\
\text { Asia }\end{array}$ & Europe & $\begin{array}{l}\text { North } \\
\text { America }\end{array}$ & Oceania \\
\hline \multirow{7}{*}{$\begin{array}{l}\text { Agricultural } \\
\text { Soil }\end{array}$} & Africa/Middle East & $-2.24 \mathrm{E}-01$ & $-3.59 \mathrm{E}-02$ & $-5.09 \mathrm{E}-02$ & \begin{tabular}{|l|}
$4.28 \mathrm{E}-02$ \\
\end{tabular} & $-2.51 \mathrm{E}-02$ & $-2.07 \mathrm{E}-02$ & $5.38 \mathrm{E}-03$ \\
\hline & Southern Asia & $-1.22 \mathrm{E}-01$ & $-3.33 \mathrm{E}-01$ & $1.38 \mathrm{E}-02$ & $2.11 \mathrm{E}-01$ & $-4.34 \mathrm{E}-02$ & $-1.03 \mathrm{E}-01$ & $2.19 \mathrm{E}-02$ \\
\hline & Central/South America & $-1.22 \mathrm{E}-01$ & $6.39 \mathrm{E}-03$ & $-2.48 \mathrm{E}-01$ & $2.27 \mathrm{E}-03$ & $1.14 \mathrm{E}-02$ & $2.82 \mathrm{E}-02$ & $-2.72 \mathrm{E}-02$ \\
\hline & Northern Asia & $-6.30 \mathrm{E}-03$ & $6.60 \mathrm{E}-02$ & $6.46 \mathrm{E}-03$ & \begin{tabular}{|l|}
$-2.20 \mathrm{E}-01$ \\
\end{tabular} & $-2.09 \mathrm{E}-02$ & $6.45 \mathrm{E}-02$ & $-1.76 \mathrm{E}-02$ \\
\hline & Europe & $-1.18 \mathrm{E}-02$ & $1.80 \mathrm{E}-04$ & $1.84 \mathrm{E}-02$ & $2.91 \mathrm{E}-02$ & $-2.67 \mathrm{E}-01$ & $1.31 \mathrm{E}-02$ & $6.45 \mathrm{E}-03$ \\
\hline & \begin{tabular}{|l|} 
North America \\
\end{tabular} & $-1.31 \mathrm{E}-02$ & $-2.04 \mathrm{E}-02$ & $1.43 \mathrm{E}-02$ & $3.62 \mathrm{E}-02$ & $1.80 \mathrm{E}-02$ & $-4.16 \mathrm{E}-01$ & $1.03 \mathrm{E}-02$ \\
\hline & Oceania & $-2.09 \mathrm{E}-03$ & $1.77 \mathrm{E}-02$ & $-3.54 \mathrm{E}-02$ & $-1.23 \mathrm{E}-02$ & $3.44 \mathrm{E}-03$ & $7.84 \mathrm{E}-03$ & $-5.62 \mathrm{E}-02$ \\
\hline \multirow{7}{*}{ Industrial } & Africa/Middle East & $-3.39 \mathrm{E}-02$ & $-3.59 \mathrm{E}-03$ & $-1.36 \mathrm{E}-04$ & $5.92 \mathrm{E}-03$ & $-6.21 \mathrm{E}-03$ & $9.31 \mathrm{E}-04$ & $1.29 \mathrm{E}-03$ \\
\hline & \begin{tabular}{|l|} 
Southern Asia \\
\end{tabular} & $5.41 \mathrm{E}-02$ & $-8.49 \mathrm{E}-02$ & $1.27 \mathrm{E}-03$ & $3.95 \mathrm{E}-02$ & $8.89 \mathrm{E}-03$ & $4.72 \mathrm{E}-02$ & $6.55 \mathrm{E}-04$ \\
\hline & Central/South America & $-1.05 \mathrm{E}-02$ & $3.62 \mathrm{E}-04$ & $-1.92 \mathrm{E}-02$ & $2.67 \mathrm{E}-04$ & $8.07 \mathrm{E}-04$ & $1.95 \mathrm{E}-03$ & $-2.08 \mathrm{E}-03$ \\
\hline & Northern Asia & $5.72 \mathrm{E}-02$ & $1.36 \mathrm{E}-02$ & $4.16 \mathrm{E}-02$ & \begin{tabular}{|l|}
$-4.62 \mathrm{E}-02$ \\
\end{tabular} & $2.61 \mathrm{E}-02$ & $-3.00 \mathrm{E}-01$ & $-2.80 \mathrm{E}-03$ \\
\hline & Europe & $-2.88 \mathrm{E}-03$ & $-1.27 \mathrm{E}-02$ & $9.32 \mathrm{E}-03$ & $2.14 \mathrm{E}-02$ & $-1.76 \mathrm{E}-01$ & $2.38 \mathrm{E}-02$ & $2.26 \mathrm{E}-03$ \\
\hline & North America & $1.44 \mathrm{E}-02$ & $8.60 \mathrm{E}-03$ & $1.43 \mathrm{E}-02$ & \begin{tabular}{|l|}
$-9.92 E-04$ \\
\end{tabular} & $3.87 \mathrm{E}-02$ & $-2.51 \mathrm{E}-01$ & $3.81 \mathrm{E}-04$ \\
\hline & Oceania & $-2.08 \mathrm{E}-05$ & $1.78 \mathrm{E}-03$ & $-3.42 \mathrm{E}-03$ & $\mid-1.22 \mathrm{E}-03$ & $2.85 E-04$ & $6.92 \mathrm{E}-04$ & $-3.79 \mathrm{E}-03$ \\
\hline \multirow{7}{*}{ Natural Soil } & Africa/Middle East & 1 & $-1.74 \mathrm{E}-01$ & $-3.43 \mathrm{E}-01$ & $1.55 \mathrm{E}-01$ & $-2.39 \mathrm{E}-02$ & $-1.02 \mathrm{E}-01$ & $1.41 \mathrm{E}-04$ \\
\hline & Southern Asia & $-1.74 \mathrm{E}-01$ & 1 & $-5.87 \mathrm{E}-02$ & $-7.52 \mathrm{E}-01$ & $3.87 \mathrm{E}-02$ & $2.72 \mathrm{E}-02$ & $-2.24 \mathrm{E}-02$ \\
\hline & Central/South America & $-3.43 E-01$ & $-5.87 \mathrm{E}-02$ & 1 & \begin{tabular}{|l|}
$-3.56 \mathrm{E}-02$ \\
\end{tabular} & $-3.22 \mathrm{E}-02$ & $-6.75 \mathrm{E}-02$ & $-1.76 \mathrm{E}-01$ \\
\hline & Northern Asia & $1.55 \mathrm{E}-01$ & $-7.52 \mathrm{E}-01$ & $-3.56 \mathrm{E}-02$ & 1 & $-6.11 \mathrm{E}-02$ & $-7.48 \mathrm{E}-02$ & $7.43 \mathrm{E}-03$ \\
\hline & Europe & $-2.39 \mathrm{E}-02$ & $3.87 \mathrm{E}-02$ & $-3.22 \mathrm{E}-02$ & \begin{tabular}{|l|}
$-6.11 \mathrm{E}-02$ \\
\end{tabular} & 1 & $-1.63 \mathrm{E}-01$ & $-2.31 \mathrm{E}-03$ \\
\hline & North America & $-1.02 \mathrm{E}-01$ & $2.72 \mathrm{E}-02$ & $-6.75 \mathrm{E}-02$ & \begin{tabular}{|l|}
$-7.48 \mathrm{E}-02$ \\
\end{tabular} & $-1.63 \mathrm{E}-01$ & 1 & 5.91E-03 \\
\hline & Oceania & $1.41 \mathrm{E}-04$ & $-2.24 \mathrm{E}-02$ & $-1.76 \mathrm{E}-01$ & $7.43 \mathrm{E}-03$ & $-2.31 \mathrm{E}-03$ & $5.91 \mathrm{E}-03$ & 1 \\
\hline \multirow{6}{*}{ Ocean } & North Pacific & $1.19 \mathrm{E}-01$ & $1.35 \mathrm{E}-01$ & $-3.73 \mathrm{E}-02$ & $-1.47 \mathrm{E}-01$ & $1.81 \mathrm{E}-02$ & $4.35 \mathrm{E}-02$ & $-3.62 \mathrm{E}-03$ \\
\hline & \begin{tabular}{|l} 
South Pacific \\
\end{tabular} & $1.92 \mathrm{E}-02$ & $1.02 \mathrm{E}-03$ & $-5.28 \mathrm{E}-01$ & $1.97 \mathrm{E}-02$ & $1.84 \mathrm{E}-02$ & $5.82 \mathrm{E}-02$ & $-6.44 \mathrm{E}-04$ \\
\hline & Northern Ocean & $6.76 \mathrm{E}-02$ & $8.86 \mathrm{E}-02$ & $2.98 \mathrm{E}-02$ & $-7.22 \mathrm{E}-02$ & $-1.48 \mathrm{E}-02$ & $-1.25 \mathrm{E}-02$ & $-1.23 \mathrm{E}-03$ \\
\hline & Atlantic & $-7.77 \mathrm{E}-02$ & $9.99 \mathrm{E}-02$ & $-5.34 \mathrm{E}-02$ & $-2.95 \mathrm{E}-02$ & $6.60 \mathrm{E}-02$ & \begin{tabular}{|l|}
$3.35 \mathrm{E}-02$ \\
\end{tabular} & $1.68 \mathrm{E}-03$ \\
\hline & Southern Ocean & $1.11 \mathrm{E}-01$ & $1.58 \mathrm{E}-01$ & $1.71 \mathrm{E}-03$ & $-1.11 \mathrm{E}-01$ & $4.06 \mathrm{E}-03$ & $4.35 \mathrm{E}-02$ & $-3.70 \mathrm{E}-02$ \\
\hline & Indian Ocean & $-1.84 \mathrm{E}-01$ & $-1.26 \mathrm{E}-01$ & $-1.68 \mathrm{E}-01$ & $9.14 \mathrm{E}-02$ & $1.44 \mathrm{E}-02$ & $3.33 \mathrm{E}-02$ & $-5.56 \mathrm{E}-02$ \\
\hline \multirow{7}{*}{$\begin{array}{l}\text { Biomass } \\
\text { burning }\end{array}$} & Africa/Middle East & $-1.63 \mathrm{E}-01$ & $-2.37 \mathrm{E}-02$ & $3.23 \mathrm{E}-02$ & $2.73 \mathrm{E}-02$ & $9.34 \mathrm{E}-03$ & \begin{tabular}{|l|l|}
$1.12 \mathrm{E}-02$ \\
\end{tabular} & $9.85 \mathrm{E}-03$ \\
\hline & Southern Asia & $1.35 \mathrm{E}-02$ & $-1.65 \mathrm{E}-01$ & $2.16 \mathrm{E}-02$ & $1.30 \mathrm{E}-01$ & $-8.96 \mathrm{E}-04$ & \begin{tabular}{|l|}
$1.07 \mathrm{E}-02$ \\
\end{tabular} & $-1.04 \mathrm{E}-02$ \\
\hline & Central/South America & $-3.59 \mathrm{E}-02$ & $-1.08 \mathrm{E}-02$ & $2.40 \mathrm{E}-02$ & $1.03 \mathrm{E}-02$ & $3.46 \mathrm{E}-03$ & \begin{tabular}{|l|}
$5.54 \mathrm{E}-03$ \\
\end{tabular} & $2.37 \mathrm{E}-03$ \\
\hline & Northern Asia & $-7.01 \mathrm{E}-04$ & $2.42 \mathrm{E}-04$ & $-2.06 \mathrm{E}-02$ & $-3.42 \mathrm{E}-02$ & $-4.96 \mathrm{E}-02$ & $-1.05 \mathrm{E}-01$ & $-1.20 \mathrm{E}-03$ \\
\hline & Europe & $-1.18 \mathrm{E}-04$ & $4.63 \mathrm{E}-05$ & $4.23 \mathrm{E}-05$ & \begin{tabular}{|l|}
$-7.60 \mathrm{E}-05$ \\
\end{tabular} & $-6.02 \mathrm{E}-03$ & \begin{tabular}{|l|}
$-2.26 \mathrm{E}-04$ \\
\end{tabular} & $-1.57 \mathrm{E}-05$ \\
\hline & North America & $-1.77 \mathrm{E}-04$ & $1.24 \mathrm{E}-02$ & $-1.01 \mathrm{E}-02$ & $-1.99 \mathrm{E}-02$ & $-2.57 \mathrm{E}-02$ & \begin{tabular}{|l|}
$-6.87 E-02$ \\
\end{tabular} & $-1.27 \mathrm{E}-03$ \\
\hline & Oceania & $-1.40 \mathrm{E}-02$ & $-1.10 \mathrm{E}-02$ & $1.26 \mathrm{E}-02$ & $7.89 \mathrm{E}-03$ & $1.90 \mathrm{E}-03$ & \begin{tabular}{l|l}
$3.49 \mathrm{E}-03$ \\
\end{tabular} & $-7.46 \mathrm{E}-03$ \\
\hline
\end{tabular}

Fig. S2be. Correlation of the emissions in 2006 for natural soil. 


\begin{tabular}{|c|c|c|c|c|c|c|c|}
\hline & \multirow{2}{*}{\multicolumn{6}{|c|}{ Ocean }} \\
\hline & & & & & & & \\
\hline Source sector & Region & $\begin{array}{l}\text { North } \\
\text { Pacific }\end{array}$ & $\begin{array}{l}\text { South } \\
\text { Pacific }\end{array}$ & $\begin{array}{l}\text { Northern } \\
\text { Ocean }\end{array}$ & Atlantic & $\begin{array}{l}\text { Southern } \\
\text { Ocean }\end{array}$ & $\begin{array}{l}\text { Indian } \\
\text { Ocean }\end{array}$ \\
\hline \multirow{7}{*}{$\begin{array}{l}\text { Agricultural } \\
\text { Soil }\end{array}$} & Africa/Middle East & $-3.92 \mathrm{E}-03$ & $-5.65 \mathrm{E}-04$ & $1.70 \mathrm{E}-03$ & $-8.79 \mathrm{E}-02$ & $3.17 \mathrm{E}-02$ & $-3.29 \mathrm{E}-02$ \\
\hline & Southern Asia & $-2.22 \mathrm{E}-01$ & $1.81 \mathrm{E}-03$ & $-1.37 \mathrm{E}-02$ & $-1.02 \mathrm{E}-01$ & $1.15 \mathrm{E}-01$ & $-2.57 \mathrm{E}-02$ \\
\hline & Central/South America & $1.18 \mathrm{E}-02$ & $-2.23 \mathrm{E}-02$ & $2.39 \mathrm{E}-03$ & $-2.53 \mathrm{E}-02$ & $-2.17 \mathrm{E}-01$ & $-1.05 \mathrm{E}-01$ \\
\hline & \begin{tabular}{|l} 
Northern Asia \\
\end{tabular} & $1.78 \mathrm{E}-02$ & $1.01 \mathrm{E}-02$ & $-5.80 \mathrm{E}-02$ & $-2.61 \mathrm{E}-02$ & $-1.37 \mathrm{E}-02$ & $-9.05 E-03$ \\
\hline & Europe & $2.01 \mathrm{E}-03$ & $8.89 \mathrm{E}-03$ & $\mid-6.52 \mathrm{E}-02$ & $-3.96 \mathrm{E}-02$ & $1.05 \mathrm{E}-02$ & $4.61 \mathrm{E}-03$ \\
\hline & North America & $-3.63 E-02$ & $1.45 \mathrm{E}-02$ & \begin{tabular}{|l|}
$-9.19 \mathrm{E}-03$ \\
\end{tabular} & $-9.53 E-02$ & $1.52 \mathrm{E}-02$ & $2.04 \mathrm{E}-02$ \\
\hline & Oceania & $6.64 \mathrm{E}-03$ & $6.87 \mathrm{E}-03$ & $2.79 \mathrm{E}-03$ & $1.40 \mathrm{E}-02$ & $-7.09 \mathrm{E}-02$ & $-4.62 \mathrm{E}-02$ \\
\hline \multirow{7}{*}{ Industrial } & Africa/Middle East & $-2.35 \mathrm{E}-03$ & $1.00 \mathrm{E}-03$ & $3.58 \mathrm{E}-04$ & $-1.46 \mathrm{E}-02$ & $3.33 \mathrm{E}-03$ & $-7.74 \mathrm{E}-04$ \\
\hline & Southern Asia & $-1.15 \mathrm{E}-02$ & $1.17 \mathrm{E}-02$ & $-7.84 \mathrm{E}-03$ & $-2.18 \mathrm{E}-03$ & $-2.78 \mathrm{E}-02$ & $2.12 \mathrm{E}-02$ \\
\hline & Central/South America & $8.38 \mathrm{E}-04$ & $-3.68 \mathrm{E}-03$ & $2.49 \mathrm{E}-04$ & $-2.60 \mathrm{E}-03$ & $-1.49 \mathrm{E}-02$ & $-9.13 \mathrm{E}-03$ \\
\hline & Northern Asia & $5.02 \mathrm{E}-02$ & $7.65 \mathrm{E}-03$ & $2.59 \mathrm{E}-02$ & $-2.25 \mathrm{E}-01$ & $-2.81 \mathrm{E}-03$ & $2.97 \mathrm{E}-03$ \\
\hline & Europe & $-3.81 \mathrm{E}-03$ & $6.40 \mathrm{E}-03$ & $1.60 \mathrm{E}-02$ & $-2.49 \mathrm{E}-02$ & $8.47 \mathrm{E}-04$ & $5.32 \mathrm{E}-03$ \\
\hline & North America & $-2.80 \mathrm{E}-02$ & $-4.27 \mathrm{E}-03$ & $2.73 \mathrm{E}-02$ & $-9.46 \mathrm{E}-02$ & $9.56 \mathrm{E}-03$ & $-4.18 \mathrm{E}-03$ \\
\hline & Oceania & $4.51 \mathrm{E}-04$ & $1.55 \mathrm{E}-03$ & 1.99E-04 & $1.01 \mathrm{E}-03$ & $-5.63 E-03$ & $-4.60 \mathrm{E}-03$ \\
\hline \multirow{7}{*}{ Natural Soil } & Africa/Middle East & 1.19E-01 & $1.92 \mathrm{E}-02$ & $6.76 \mathrm{E}-02$ & $-7.77 \mathrm{E}-02$ & $1.11 \mathrm{E}-01$ & $-1.84 \mathrm{E}-01$ \\
\hline & Southern Asia & $1.35 \mathrm{E}-01$ & $1.02 \mathrm{E}-03$ & $8.86 \mathrm{E}-02$ & $9.99 \mathrm{E}-02$ & $1.58 \mathrm{E}-01$ & $-1.26 \mathrm{E}-01$ \\
\hline & Central/South America & $-3.73 \mathrm{E}-02$ & $-5.28 \mathrm{E}-01$ & $2.98 \mathrm{E}-02$ & $-5.34 \mathrm{E}-02$ & $1.71 \mathrm{E}-03$ & $-1.68 \mathrm{E}-01$ \\
\hline & Northern Asia & $-1.47 \mathrm{E}-01$ & $1.97 \mathrm{E}-02$ & \begin{tabular}{|l|}
$-7.22 \mathrm{E}-02$ \\
\end{tabular} & $-2.95 \mathrm{E}-02$ & $-1.11 \mathrm{E}-01$ & $9.14 \mathrm{E}-02$ \\
\hline & Europe & $1.81 \mathrm{E}-02$ & $1.84 \mathrm{E}-02$ & \begin{tabular}{|l|}
$-1.48 \mathrm{E}-02$ \\
\end{tabular} & $6.60 \mathrm{E}-02$ & $4.06 \mathrm{E}-03$ & $1.44 \mathrm{E}-02$ \\
\hline & North America & $4.35 \mathrm{E}-02$ & $5.82 \mathrm{E}-02$ & \begin{tabular}{|l|}
$-1.25 \mathrm{E}-02$ \\
\end{tabular} & $3.35 \mathrm{E}-02$ & $4.35 \mathrm{E}-02$ & $3.33 \mathrm{E}-02$ \\
\hline & Oceania & $-3.62 \mathrm{E}-03$ & $-6.44 \mathrm{E}-04$ & \begin{tabular}{|l|}
$-1.23 \mathrm{E}-03$ \\
\end{tabular} & $1.68 \mathrm{E}-03$ & $-3.70 \mathrm{E}-02$ & $-5.56 \mathrm{E}-02$ \\
\hline \multirow{6}{*}{ Ocean } & North Pacific & 1 & $2.29 \mathrm{E}-02$ & $-1.11 \mathrm{E}-01$ & $-2.18 \mathrm{E}-01$ & $1.37 \mathrm{E}-02$ & $4.90 \mathrm{E}-02$ \\
\hline & South Pacific & $2.29 \mathrm{E}-02$ & 1 & $1.23 \mathrm{E}-02$ & $-5.39 \mathrm{E}-02$ & $4.20 \mathrm{E}-02$ & $1.99 \mathrm{E}-02$ \\
\hline & Northern Ocean & $-1.11 \mathrm{E}-01$ & $1.23 \mathrm{E}-02$ & 1 & $-3.07 \mathrm{E}-01$ & $2.10 \mathrm{E}-02$ & $1.47 \mathrm{E}-02$ \\
\hline & Atlantic & $-2.18 \mathrm{E}-01$ & $-5.39 \mathrm{E}-02$ & $-3.07 E-01$ & 1 & $-8.81 \mathrm{E}-02$ & $-2.92 \mathrm{E}-02$ \\
\hline & Southern Ocean & $1.37 \mathrm{E}-02$ & $4.20 \mathrm{E}-02$ & $2.10 \mathrm{E}-02$ & $-8.81 \mathrm{E}-02$ & 1 & $-6.25 \mathrm{E}-01$ \\
\hline & Indian Ocean & $4.90 \mathrm{E}-02$ & $1.99 \mathrm{E}-02$ & $1.47 \mathrm{E}-02$ & $-2.92 \mathrm{E}-02$ & $-6.25 \mathrm{E}-01$ & \\
\hline \multirow{7}{*}{$\begin{array}{l}\text { Biomass } \\
\text { burning }\end{array}$} & Africa/Middle East & $1.51 \mathrm{E}-02$ & $-6.28 \mathrm{E}-02$ & $6.25 \mathrm{E}-03$ & $-7.15 \mathrm{E}-02$ & $-8.36 \mathrm{E}-04$ & $-7.00 \mathrm{E}-02$ \\
\hline & Southern Asia & $-1.93 \mathrm{E}-02$ & $-1.63 \mathrm{E}-02$ & \begin{tabular}{|l|}
$-1.70 \mathrm{E}-02$ \\
\end{tabular} & $-3.28 \mathrm{E}-02$ & $3.39 \mathrm{E}-02$ & $-5.17 \mathrm{E}-02$ \\
\hline & Central/South America & $2.13 \mathrm{E}-03$ & $-3.66 \mathrm{E}-02$ & $9.58 \mathrm{E}-05$ & $-1.75 \mathrm{E}-02$ & $-3.48 \mathrm{E}-02$ & $-2.77 \mathrm{E}-02$ \\
\hline & Northern Asia & $-5.89 \mathrm{E}-02$ & $1.73 \mathrm{E}-02$ & $\mid-1.62 \mathrm{E}-02$ & $3.12 \mathrm{E}-02$ & $5.07 \mathrm{E}-03$ & $6.40 \mathrm{E}-03$ \\
\hline & Europe & $1.23 \mathrm{E}-04$ & $-2.01 \mathrm{E}-05$ & \begin{tabular}{|l|}
$-5.10 \mathrm{E}-05$ \\
\end{tabular} & $-3.13 E-05$ & $1.08 \mathrm{E}-05$ & $1.26 \mathrm{E}-05$ \\
\hline & North America & $-1.04 \mathrm{E}-02$ & $4.43 \mathrm{E}-03$ & \begin{tabular}{|l|}
$-2.02 \mathrm{E}-02$ \\
\end{tabular} & $1.02 \mathrm{E}-03$ & $2.47 \mathrm{E}-03$ & $3.75 \mathrm{E}-03$ \\
\hline & Oceania & 1.07E-03 & $-6.40 \mathrm{E}-03$ & \begin{tabular}{|l|}
$-6.25 \mathrm{E}-04$ \\
\end{tabular} & $-7.54 \mathrm{E}-03$ & $-1.24 \mathrm{E}-03$ & $-1.98 \mathrm{E}-02$ \\
\hline
\end{tabular}

Fig. S2bf. Correlation of the emissions in 2006 for ocean. 


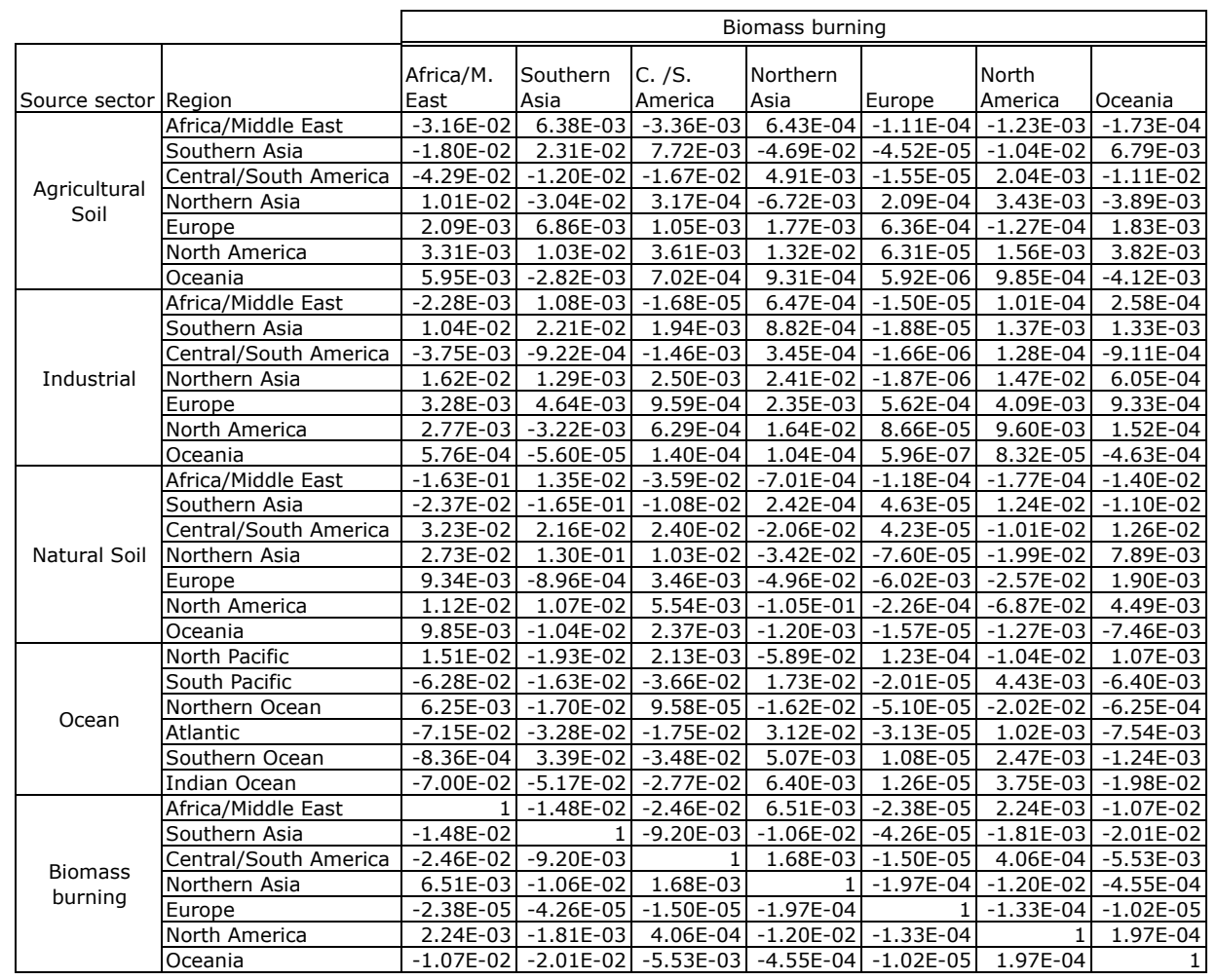

\section{Fig. S2bg. Correlation of the emissions in 2006 for biomass burning.}




\begin{tabular}{|c|c|c|c|c|c|c|c|c|}
\hline \multirow[b]{2}{*}{ Source sector } & \multirow[b]{2}{*}{ Region } & \multicolumn{7}{|c|}{ Agricultural Soil } \\
\hline & & $\begin{array}{l}\text { Africa/M. } \\
\text { East }\end{array}$ & $\begin{array}{l}\text { Southern } \\
\text { Asia }\end{array}$ & $\begin{array}{l}\text { C. /S. } \\
\text { America }\end{array}$ & $\begin{array}{l}\text { Northern } \\
\text { Asia }\end{array}$ & Europe & $\begin{array}{l}\text { North } \\
\text { America }\end{array}$ & Oceania \\
\hline \multirow{7}{*}{$\begin{array}{c}\text { Agricultural } \\
\text { Soil }\end{array}$} & Africa/Middle East & 1 & $-7.49 \mathrm{E}-02$ & $-4.04 \mathrm{E}-03$ & $-4.21 \mathrm{E}-02$ & $-2.63 \mathrm{E}-02$ & $-1.69 \mathrm{E}-02$ & $6.13 \mathrm{E}-03$ \\
\hline & Southern Asia & $-7.49 \mathrm{E}-02$ & 1 & $3.11 \mathrm{E}-02$ & $-2.80 \mathrm{E}-01$ & $4.85 \mathrm{E}-02$ & $-6.17 \mathrm{E}-02$ & $2.13 \mathrm{E}-02$ \\
\hline & Central/South America & $-4.04 \mathrm{E}-03$ & $3.11 \mathrm{E}-02$ & 1 & $-3.71 \mathrm{E}-03$ & $9.94 \mathrm{E}-03$ & $8.49 \mathrm{E}-03$ & $-2.21 \mathrm{E}-02$ \\
\hline & Northern Asia & $-4.21 \mathrm{E}-02$ & $-2.80 \mathrm{E}-01$ & $-3.71 \mathrm{E}-03$ & 1 & $-1.86 \mathrm{E}-01$ & $-5.01 \mathrm{E}-02$ & $-2.92 \mathrm{E}-02$ \\
\hline & Europe & $-2.63 \mathrm{E}-02$ & $4.85 \mathrm{E}-02$ & $9.94 \mathrm{E}-03$ & $-1.86 \mathrm{E}-01$ & 1 & $-2.11 \mathrm{E}-02$ & $5.14 \mathrm{E}-03$ \\
\hline & North America & $-1.69 \mathrm{E}-02$ & $-6.17 \mathrm{E}-02$ & $8.49 \mathrm{E}-03$ & $-5.01 \mathrm{E}-02$ & $-2.11 \mathrm{E}-02$ & 1 & $1.88 \mathrm{E}-03$ \\
\hline & Oceania & $6.13 \mathrm{E}-03$ & $2.13 \mathrm{E}-02$ & $-2.21 \mathrm{E}-02$ & $-2.92 \mathrm{E}-02$ & $5.14 \mathrm{E}-03$ & $1.88 \mathrm{E}-03$ & 1 \\
\hline \multirow{7}{*}{ Industrial } & Africa/Middle East & $-9.64 \mathrm{E}-02$ & $-1.08 \mathrm{E}-02$ & $1.69 \mathrm{E}-03$ & $-1.07 \mathrm{E}-02$ & $-9.02 \mathrm{E}-03$ & $-4.08 \mathrm{E}-03$ & $1.02 \mathrm{E}-03$ \\
\hline & \begin{tabular}{|l} 
Southern Asia \\
\end{tabular} & $2.88 \mathrm{E}-02$ & $-4.72 \mathrm{E}-01$ & $-4.81 \mathrm{E}-03$ & $-4.21 \mathrm{E}-02$ & $2.62 \mathrm{E}-02$ & $1.13 \mathrm{E}-02$ & $-2.13 \mathrm{E}-03$ \\
\hline & Central/South America & $-4.37 \mathrm{E}-04$ & $2.50 \mathrm{E}-03$ & $-9.05 \mathrm{E}-03$ & $-1.34 \mathrm{E}-04$ & $8.18 \mathrm{E}-04$ & $7.43 \mathrm{E}-04$ & $-1.58 \mathrm{E}-03$ \\
\hline & Northern Asia & $5.24 \mathrm{E}-03$ & $-5.02 E-02$ & $2.04 \mathrm{E}-03$ & $-1.30 \mathrm{E}-01$ & $-5.51 \mathrm{E}-02$ & $1.89 \mathrm{E}-01$ & $-2.43 \mathrm{E}-03$ \\
\hline & Europe & $-1.14 \mathrm{E}-02$ & $3.32 \mathrm{E}-03$ & $2.98 \mathrm{E}-03$ & $-1.52 \mathrm{E}-02$ & \begin{tabular}{|l|}
$-7.10 \mathrm{E}-01$ \\
\end{tabular} & $-1.13 \mathrm{E}-02$ & $9.05 \mathrm{E}-05$ \\
\hline & North America & $8.37 \mathrm{E}-03$ & $3.28 \mathrm{E}-02$ & $3.06 \mathrm{E}-03$ & $4.09 \mathrm{E}-02$ & $2.93 \mathrm{E}-02$ & $-3.82 \mathrm{E}-01$ & $-6.28 \mathrm{E}-04$ \\
\hline & Oceania & $4.61 \mathrm{E}-04$ & $1.48 \mathrm{E}-03$ & $-1.69 \mathrm{E}-03$ & $-2.04 \mathrm{E}-03$ & $3.37 \mathrm{E}-04$ & $6.82 \mathrm{E}-05$ & $-1.20 \mathrm{E}-02$ \\
\hline \multirow{7}{*}{ Natural Soil } & Africa/Middle East & $-1.92 \mathrm{E}-01$ & $-1.38 \mathrm{E}-02$ & $-9.76 \mathrm{E}-02$ & $-3.14 \mathrm{E}-03$ & $-8.90 \mathrm{E}-03$ & $5.96 \mathrm{E}-03$ & $3.40 \mathrm{E}-03$ \\
\hline & Southern Asia & $-7.83 E-02$ & $-4.21 \mathrm{E}-01$ & $1.45 \mathrm{E}-02$ & $3.04 \mathrm{E}-02$ & $-3.29 \mathrm{E}-02$ & $-5.66 \mathrm{E}-02$ & $2.25 \mathrm{E}-02$ \\
\hline & Central/South America & $-4.81 \mathrm{E}-02$ & $4.65 \mathrm{E}-02$ & $-2.81 \mathrm{E}-01$ & $-2.43 E-03$ & $2.08 \mathrm{E}-02$ & $2.34 \mathrm{E}-02$ & $-5.40 \mathrm{E}-02$ \\
\hline & Northern Asia & $3.45 \mathrm{E}-02$ & $-2.46 \mathrm{E}-02$ & $-5.38 \mathrm{E}-03$ & $-1.89 \mathrm{E}-01$ & $2.96 \mathrm{E}-02$ & $9.16 \mathrm{E}-02$ & $-1.22 \mathrm{E}-02$ \\
\hline & Europe & $-3.04 \mathrm{E}-02$ & $-1.96 \mathrm{E}-02$ & $4.78 \mathrm{E}-03$ & $7.89 \mathrm{E}-05$ & $-2.83 \mathrm{E}-01$ & $2.91 \mathrm{E}-02$ & $3.97 \mathrm{E}-03$ \\
\hline & North America & $-2.62 \mathrm{E}-02$ & $-2.66 \mathrm{E}-02$ & $1.86 \mathrm{E}-02$ & $7.03 \mathrm{E}-02$ & $6.75 \mathrm{E}-03$ & $-4.96 \mathrm{E}-01$ & $5.65 \mathrm{E}-03$ \\
\hline & Oceania & $2.45 \mathrm{E}-03$ & $1.44 \mathrm{E}-02$ & $-2.78 \mathrm{E}-02$ & $-1.53 \mathrm{E}-02$ & $6.88 \mathrm{E}-03$ & $6.02 \mathrm{E}-03$ & $-8.26 \mathrm{E}-02$ \\
\hline \multirow{6}{*}{ Ocean } & North Pacific & $-1.75 \mathrm{E}-02$ & $-2.25 \mathrm{E}-01$ & $7.49 \mathrm{E}-03$ & $2.34 \mathrm{E}-02$ & $5.92 \mathrm{E}-03$ & $-2.90 \mathrm{E}-02$ & $9.29 \mathrm{E}-03$ \\
\hline & South Pacific & $-1.62 \mathrm{E}-02$ & $-1.84 \mathrm{E}-02$ & $-2.24 \mathrm{E}-02$ & $2.81 \mathrm{E}-02$ & $1.62 \mathrm{E}-02$ & $9.28 \mathrm{E}-03$ & $2.38 \mathrm{E}-02$ \\
\hline & Northern Ocean & $3.96 \mathrm{E}-04$ & $-2.46 \mathrm{E}-02$ & $3.38 \mathrm{E}-03$ & $-4.28 \mathrm{E}-02$ & $-7.89 \mathrm{E}-02$ & $-1.26 \mathrm{E}-02$ & $4.77 \mathrm{E}-03$ \\
\hline & Atlantic & $-8.43 \mathrm{E}-02$ & $-1.46 \mathrm{E}-01$ & $-2.73 \mathrm{E}-02$ & $-2.66 \mathrm{E}-02$ & $-6.11 \mathrm{E}-02$ & $-1.01 \mathrm{E}-01$ & $1.65 \mathrm{E}-02$ \\
\hline & Southern Ocean & $1.85 \mathrm{E}-02$ & $6.29 \mathrm{E}-02$ & $-1.80 \mathrm{E}-01$ & $-1.96 \mathrm{E}-02$ & $6.64 \mathrm{E}-03$ & $3.91 \mathrm{E}-03$ & $-7.22 \mathrm{E}-02$ \\
\hline & Indian Ocean & $-2.13 \mathrm{E}-02$ & $3.77 \mathrm{E}-03$ & $-1.13 \mathrm{E}-01$ & $-1.25 \mathrm{E}-03$ & $1.25 \mathrm{E}-02$ & $1.52 \mathrm{E}-02$ & $-4.17 \mathrm{E}-02$ \\
\hline \multirow{7}{*}{$\begin{array}{l}\text { Biomass } \\
\text { burning }\end{array}$} & Africa/Middle East & $-2.16 \mathrm{E}-02$ & $-4.65 \mathrm{E}-03$ & $-3.28 \mathrm{E}-02$ & $7.59 \mathrm{E}-03$ & $4.41 \mathrm{E}-03$ & $2.43 \mathrm{E}-03$ & $5.64 \mathrm{E}-03$ \\
\hline & Southern Asia & $-1.18 \mathrm{E}-02$ & $-8.19 \mathrm{E}-02$ & $1.68 \mathrm{E}-03$ & $1.89 \mathrm{E}-02$ & $2.16 \mathrm{E}-03$ & $-1.80 \mathrm{E}-03$ & $2.99 \mathrm{E}-03$ \\
\hline & Central/South America & $8.17 \mathrm{E}-04$ & $2.98 \mathrm{E}-02$ & $-3.10 \mathrm{E}-02$ & $7.08 \mathrm{E}-04$ & $6.87 \mathrm{E}-03$ & $6.00 \mathrm{E}-03$ & $5.12 \mathrm{E}-03$ \\
\hline & \begin{tabular}{|l} 
Northern Asia \\
\end{tabular} & $-5.22 \mathrm{E}-04$ & $-1.63 \mathrm{E}-02$ & $5.07 \mathrm{E}-04$ & $-1.20 \mathrm{E}-03$ & $5.31 \mathrm{E}-04$ & $4.29 \mathrm{E}-03$ & $4.18 \mathrm{E}-04$ \\
\hline & Europe & $-9.63 \mathrm{E}-04$ & $1.02 \mathrm{E}-05$ & $6.60 \mathrm{E}-05$ & $-2.10 \mathrm{E}-04$ & $7.55 \mathrm{E}-05$ & $3.58 \mathrm{E}-04$ & $5.59 \mathrm{E}-05$ \\
\hline & North America & $-1.36 \mathrm{E}-03$ & $-2.70 \mathrm{E}-03$ & $6.40 \mathrm{E}-04$ & $3.51 \mathrm{E}-03$ & $3.27 \mathrm{E}-04$ & $5.82 \mathrm{E}-03$ & $5.01 \mathrm{E}-04$ \\
\hline & Oceania & $1.39 \mathrm{E}-03$ & $7.22 \mathrm{E}-03$ & $-5.15 \mathrm{E}-03$ & $-3.11 \mathrm{E}-03$ & $2.32 \mathrm{E}-03$ & $2.47 \mathrm{E}-03$ & $-4.47 \mathrm{E}-03$ \\
\hline
\end{tabular}

Fig. S2bh. Correlation of the emissions in 2007 for agricultural soil. 


\begin{tabular}{|c|c|c|c|c|c|c|c|c|}
\hline & \multirow{2}{*}{\multicolumn{7}{|c|}{ Industrial }} \\
\hline & & & & & & & & \\
\hline Source sector & Region & $\begin{array}{l}\text { Africa/M. } \\
\text { East }\end{array}$ & $\begin{array}{l}\text { Southern } \\
\text { Asia }\end{array}$ & $\begin{array}{l}\text { C. /S. } \\
\text { America }\end{array}$ & $\begin{array}{l}\text { Northern } \\
\text { Asia }\end{array}$ & Europe & $\begin{array}{l}\text { North } \\
\text { America }\end{array}$ & Oceania \\
\hline \multirow{7}{*}{$\begin{array}{l}\text { Agricultural } \\
\text { Soil }\end{array}$} & Africa/Middle East & $-9.64 \mathrm{E}-02$ & $2.88 \mathrm{E}-02$ & $-4.37 \mathrm{E}-04$ & $5.24 \mathrm{E}-03$ & $-1.14 \mathrm{E}-02$ & $8.37 \mathrm{E}-03$ & $4.61 \mathrm{E}-04$ \\
\hline & Southern Asia & $-1.08 \mathrm{E}-02$ & $-4.72 \mathrm{E}-01$ & $2.50 \mathrm{E}-03$ & $-5.02 E-02$ & $3.32 \mathrm{E}-03$ & $3.28 \mathrm{E}-02$ & $1.48 \mathrm{E}-03$ \\
\hline & Central/South America & $1.69 \mathrm{E}-03$ & $-4.81 \mathrm{E}-03$ & $-9.05 \mathrm{E}-03$ & \begin{tabular}{|l|} 
\\
\end{tabular} & $2.98 \mathrm{E}-03$ & $3.06 \mathrm{E}-03$ & $-1.69 \mathrm{E}-03$ \\
\hline & Northern Asia & $-1.07 \mathrm{E}-02$ & $-4.21 \mathrm{E}-02$ & $-1.34 \mathrm{E}-04$ & \begin{tabular}{|l|} 
\\
\end{tabular} & $-1.52 \mathrm{E}-02$ & 4.09E-02 & $-2.04 \mathrm{E}-03$ \\
\hline & Europe & $-9.02 \mathrm{E}-03$ & $2.62 \mathrm{E}-02$ & $8.18 \mathrm{E}-04$ & $-5.51 \mathrm{E}-02$ & $-7.10 \mathrm{E}-01$ & $2.93 \mathrm{E}-02$ & $3.37 \mathrm{E}-04$ \\
\hline & \begin{tabular}{|l|} 
North America \\
\end{tabular} & $-4.08 \mathrm{E}-03$ & $1.13 \mathrm{E}-02$ & $7.43 \mathrm{E}-04$ & $1.89 \mathrm{E}-01$ & $-1.13 \mathrm{E}-02$ & $-3.82 \mathrm{E}-01$ & $6.82 \mathrm{E}-05$ \\
\hline & Oceania & $1.02 \mathrm{E}-03$ & $-2.13 \mathrm{E}-03$ & $-1.58 \mathrm{E}-03$ & 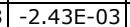 & $9.05 \mathrm{E}-05$ & $-6.28 \mathrm{E}-04$ & $-1.20 \mathrm{E}-02$ \\
\hline \multirow{7}{*}{ Industrial } & Africa/Middle East & 1 & $4.02 \mathrm{E}-03$ & $1.39 \mathrm{E}-04$ & $-1.89 \mathrm{E}-03$ & $-3.67 \mathrm{E}-03$ & $1.73 \mathrm{E}-03$ & $7.40 \mathrm{E}-05$ \\
\hline & \begin{tabular}{|l|} 
Southern Asia \\
\end{tabular} & $4.02 \mathrm{E}-03$ & 1 & $-4.13 \mathrm{E}-04$ & $-4.17 \mathrm{E}-02$ & $3.12 \mathrm{E}-03$ & $2.22 \mathrm{E}-02$ & $-2.06 \mathrm{E}-04$ \\
\hline & Central/South America & $1.39 \mathrm{E}-04$ & $-4.13 \mathrm{E}-04$ & 1 & $2.32 \mathrm{E}-04$ & $2.57 \mathrm{E}-04$ & $2.78 \mathrm{E}-04$ & $-1.21 \mathrm{E}-04$ \\
\hline & Northern Asia & $-1.89 \mathrm{E}-03$ & $-4.17 \mathrm{E}-02$ & $2.32 \mathrm{E}-04$ & 1 & $-2.43 \mathrm{E}-02$ & $-3.04 \mathrm{E}-01$ & $-2.11 \mathrm{E}-04$ \\
\hline & Europe & $-3.67 E-03$ & $3.12 \mathrm{E}-03$ & $2.57 \mathrm{E}-04$ & $-2.43 \mathrm{E}-02$ & 1 & $7.40 \mathrm{E}-03$ & $-1.26 \mathrm{E}-05$ \\
\hline & North America & $1.73 \mathrm{E}-03$ & $2.22 \mathrm{E}-02$ & $2.78 \mathrm{E}-04$ & $-3.04 \mathrm{E}-01$ & $7.40 \mathrm{E}-03$ & 1 & $-5.22 \mathrm{E}-05$ \\
\hline & Oceania & $7.40 \mathrm{E}-05$ & $-2.06 \mathrm{E}-04$ & $-1.21 \mathrm{E}-04$ & $-2.11 \mathrm{E}-04$ & $-1.26 \mathrm{E}-05$ & $-5.22 \mathrm{E}-05$ & 1 \\
\hline \multirow{7}{*}{ Natural Soil } & Africa/Middle East & $-3.15 \mathrm{E}-02$ & $6.49 \mathrm{E}-02$ & $-8.78 \mathrm{E}-03$ & \begin{tabular}{|l|}
$3.74 \mathrm{E}-02$ \\
\end{tabular} & $6.23 \mathrm{E}-04$ & $1.20 \mathrm{E}-02$ & 3.93E-04 \\
\hline & Southern Asia & $-7.40 \mathrm{E}-03$ & $-4.38 \mathrm{E}-02$ & $1.44 \mathrm{E}-03$ & \begin{tabular}{|l|}
$2.42 \mathrm{E}-02$ \\
\end{tabular} & $-1.89 \mathrm{E}-02$ & $-2.11 \mathrm{E}-02$ & $2.25 \mathrm{E}-03$ \\
\hline & Central/South America & $1.68 \mathrm{E}-03$ & $-5.59 \mathrm{E}-03$ & $-2.37 \mathrm{E}-02$ & $2.23 \mathrm{E}-02$ & $6.25 \mathrm{E}-03$ & $8.23 \mathrm{E}-03$ & $-4.71 \mathrm{E}-03$ \\
\hline & Northern Asia & $7.25 \mathrm{E}-03$ & $-1.40 \mathrm{E}-02$ & $-6.02 E-04$ & $-5.67 \mathrm{E}-02$ & $3.99 E-02$ & $2.60 \mathrm{E}-02$ & $-1.10 \mathrm{E}-03$ \\
\hline & Europe & $-7.74 \mathrm{E}-03$ & $1.37 \mathrm{E}-02$ & $3.36 \mathrm{E}-04$ & $1.22 \mathrm{E}-02$ & $-2.13 \mathrm{E}-01$ & $2.54 \mathrm{E}-02$ & $3.34 \mathrm{E}-04$ \\
\hline & North America & $-4.03 E-04$ & $5.14 \mathrm{E}-02$ & $1.30 \mathrm{E}-03$ & \begin{tabular}{|l|} 
\\
\end{tabular} & $1.72 \mathrm{E}-02$ & $-1.99 \mathrm{E}-01$ & $4.73 E-04$ \\
\hline & Oceania & $8.81 \mathrm{E}-04$ & $-1.34 \mathrm{E}-03$ & $-2.10 \mathrm{E}-03$ & \begin{tabular}{|l|}
$5.18 \mathrm{E}-04$ \\
\end{tabular} & $1.34 \mathrm{E}-03$ & $1.10 \mathrm{E}-03$ & $-6.04 \mathrm{E}-03$ \\
\hline \multirow{6}{*}{ Ocean } & North Pacific & $-3.51 \mathrm{E}-03$ & $-1.06 \mathrm{E}-02$ & $6.46 \mathrm{E}-04$ & 5.83E-02 & $-3.40 \mathrm{E}-03$ & $-6.42 \mathrm{E}-02$ & $7.20 \mathrm{E}-04$ \\
\hline & South Pacific & $5.85 \mathrm{E}-05$ & $1.13 \mathrm{E}-02$ & $-2.24 \mathrm{E}-03$ & \begin{tabular}{|l|}
$1.97 \mathrm{E}-02$ \\
\end{tabular} & $7.40 \mathrm{E}-03$ & $-5.63 \mathrm{E}-04$ & $2.68 \mathrm{E}-03$ \\
\hline & Northern Ocean & $-2.31 \mathrm{E}-04$ & $-9.46 \mathrm{E}-03$ & $3.94 \mathrm{E}-04$ & \begin{tabular}{l|}
$2.87 \mathrm{E}-02$ \\
\end{tabular} & $1.96 \mathrm{E}-02$ & $2.01 \mathrm{E}-02$ & $3.64 \mathrm{E}-04$ \\
\hline & Atlantic & $-1.32 \mathrm{E}-02$ & $-1.55 \mathrm{E}-03$ & $-2.37 E-03$ & \begin{tabular}{|l|l|} 
& $-1.97 \mathrm{E}-01$ \\
\end{tabular} & $-2.66 \mathrm{E}-02$ & $-9.01 \mathrm{E}-02$ & $1.27 \mathrm{E}-03$ \\
\hline & Southern Ocean & $2.13 \mathrm{E}-03$ & $-2.52 \mathrm{E}-02$ & $-1.24 \mathrm{E}-02$ & $2.54 \mathrm{E}-03$ & $-5.07 E-04$ & $8.89 \mathrm{E}-03$ & $-5.59 \mathrm{E}-03$ \\
\hline & Indian Ocean & $-1.04 \mathrm{E}-04$ & $1.82 \mathrm{E}-02$ & $-8.97 E-03$ & \begin{tabular}{l|l|} 
& $6.80 \mathrm{E}-03$ \\
\end{tabular} & $4.69 \mathrm{E}-03$ & $8.28 \mathrm{E}-05$ & $-4.33 \mathrm{E}-03$ \\
\hline \multirow{7}{*}{$\begin{array}{l}\text { Biomass } \\
\text { burning }\end{array}$} & Africa/Middle East & $-1.18 \mathrm{E}-03$ & $1.18 \mathrm{E}-02$ & $-2.87 \mathrm{E}-03$ & \begin{tabular}{|c|}
$1.12 \mathrm{E}-02$ \\
\end{tabular} & $2.82 \mathrm{E}-03$ & $1.95 \mathrm{E}-03$ & $4.65 \mathrm{E}-04$ \\
\hline & Southern Asia & $-2.02 \mathrm{E}-03$ & $-2.99 \mathrm{E}-03$ & $1.26 \mathrm{E}-04$ & $9.78 \mathrm{E}-03$ & $2.27 E-03$ & $8.64 \mathrm{E}-04$ & $2.23 \mathrm{E}-04$ \\
\hline & Central/South America & $1.27 \mathrm{E}-03$ & $3.83 \mathrm{E}-03$ & $-2.59 \mathrm{E}-03$ & \begin{tabular}{|l|} 
\\
\end{tabular} & $2.14 \mathrm{E}-03$ & $1.86 \mathrm{E}-03$ & $5.13 \mathrm{E}-04$ \\
\hline & Northern Asia & $-8.50 \mathrm{E}-05$ & $-2.24 \mathrm{E}-03$ & $3.23 \mathrm{E}-05$ & $2.77 \mathrm{E}-03$ & $1.27 \mathrm{E}-03$ & $3.48 \mathrm{E}-03$ & $4.67 \mathrm{E}-05$ \\
\hline & Europe & $-2.16 \mathrm{E}-04$ & $1.16 \mathrm{E}-04$ & $5.49 \mathrm{E}-06$ & \begin{tabular}{|l|}
$1.50 \mathrm{E}-04$ \\
\end{tabular} & $4.54 \mathrm{E}-05$ & $2.88 \mathrm{E}-04$ & 5.02E-06 \\
\hline & North America & $-1.08 \mathrm{E}-04$ & $2.13 \mathrm{E}-03$ & 4.59E-05 & 3.25E-04 & $1.15 \mathrm{E}-03$ & $-1.46 \mathrm{E}-03$ & $4.05 \mathrm{E}-05$ \\
\hline & Oceania & $4.23 \mathrm{E}-04$ & $1.06 \mathrm{E}-03$ & $-4.07 E-04$ & \begin{tabular}{l|}
$3.34 \mathrm{E}-04$ \\
\end{tabular} & $5.74 \mathrm{E}-04$ & $3.62 \mathrm{E}-04$ & $-4.75 \mathrm{E}-04$ \\
\hline
\end{tabular}

Fig. S2bi. Correlation of the emissions in 2007 for industrial. 


\begin{tabular}{|c|c|c|c|c|c|c|c|c|}
\hline & \multirow{2}{*}{\multicolumn{7}{|c|}{ Natural Soil }} \\
\hline & & & & & & & & \\
\hline Source sector & Region & $\begin{array}{l}\text { Africa/M. } \\
\text { East }\end{array}$ & $\begin{array}{l}\text { Southern } \\
\text { Asia }\end{array}$ & $\begin{array}{l}\text { C. /S. } \\
\text { America }\end{array}$ & $\begin{array}{l}\text { Northern } \\
\text { Asia }\end{array}$ & Europe & $\begin{array}{l}\text { North } \\
\text { America }\end{array}$ & Oceania \\
\hline \multirow{7}{*}{$\begin{array}{l}\text { Agricultural } \\
\text { Soil }\end{array}$} & Africa/Middle East & $-1.92 \mathrm{E}-01$ & $-7.83 \mathrm{E}-02$ & $-4.81 \mathrm{E}-02$ & 3.45E-02 & $-3.04 \mathrm{E}-02$ & $-2.62 \mathrm{E}-02$ & $2.45 \mathrm{E}-03$ \\
\hline & Southern Asia & $-1.38 \mathrm{E}-02$ & $-4.21 \mathrm{E}-01$ & $4.65 \mathrm{E}-02$ & $-2.46 \mathrm{E}-02$ & $-1.96 \mathrm{E}-02$ & $-2.66 \mathrm{E}-02$ & $1.44 \mathrm{E}-02$ \\
\hline & Central/South America & $-9.76 \mathrm{E}-02$ & $1.45 \mathrm{E}-02$ & $-2.81 \mathrm{E}-01$ & \begin{tabular}{|l|}
$-5.38 \mathrm{E}-03$ \\
\end{tabular} & $4.78 \mathrm{E}-03$ & $1.86 \mathrm{E}-02$ & $-2.78 \mathrm{E}-02$ \\
\hline & Northern Asia & $-3.14 \mathrm{E}-03$ & $3.04 \mathrm{E}-02$ & $-2.43 \mathrm{E}-03$ & \begin{tabular}{|l|}
$-1.89 \mathrm{E}-01$ \\
\end{tabular} & $7.89 \mathrm{E}-05$ & $7.03 \mathrm{E}-02$ & $-1.53 \mathrm{E}-02$ \\
\hline & Europe & $-8.90 \mathrm{E}-03$ & $-3.29 E-02$ & $2.08 \mathrm{E}-02$ & $2.96 \mathrm{E}-02$ & $-2.83 \mathrm{E}-01$ & $6.75 \mathrm{E}-03$ & $6.88 \mathrm{E}-03$ \\
\hline & \begin{tabular}{|l|} 
North America \\
\end{tabular} & $5.96 \mathrm{E}-03$ & $-5.66 \mathrm{E}-02$ & $2.34 \mathrm{E}-02$ & $9.16 \mathrm{E}-02$ & $2.91 \mathrm{E}-02$ & $-4.96 \mathrm{E}-01$ & $6.02 \mathrm{E}-03$ \\
\hline & Oceania & $3.40 \mathrm{E}-03$ & $2.25 \mathrm{E}-02$ & $-5.40 \mathrm{E}-02$ & $-1.22 \mathrm{E}-02$ & $3.97 \mathrm{E}-03$ & $5.65 \mathrm{E}-03$ & $-8.26 \mathrm{E}-02$ \\
\hline \multirow{7}{*}{ Industrial } & Africa/Middle East & $-3.15 \mathrm{E}-02$ & $-7.40 \mathrm{E}-03$ & $1.68 \mathrm{E}-03$ & $7.25 \mathrm{E}-03$ & $-7.74 \mathrm{E}-03$ & $-4.03 \mathrm{E}-04$ & $8.81 \mathrm{E}-04$ \\
\hline & \begin{tabular}{|l|} 
Southern Asia \\
\end{tabular} & $6.49 \mathrm{E}-02$ & $-4.38 \mathrm{E}-02$ & $-5.59 \mathrm{E}-03$ & $-1.40 \mathrm{E}-02$ & $1.37 \mathrm{E}-02$ & $5.14 \mathrm{E}-02$ & $-1.34 \mathrm{E}-03$ \\
\hline & Central/South America & $-8.78 \mathrm{E}-03$ & $1.44 \mathrm{E}-03$ & $-2.37 \mathrm{E}-02$ & $\mid-6.02 \mathrm{E}-04$ & $3.36 \mathrm{E}-04$ & $1.30 \mathrm{E}-03$ & $-2.10 \mathrm{E}-03$ \\
\hline & Northern Asia & $3.74 \mathrm{E}-02$ & $2.42 \mathrm{E}-02$ & $2.23 \mathrm{E}-02$ & \begin{tabular}{|l|}
$-5.67 E-02$ \\
\end{tabular} & $1.22 \mathrm{E}-02$ & $-1.75 \mathrm{E}-01$ & $5.18 \mathrm{E}-04$ \\
\hline & Europe & $6.23 \mathrm{E}-04$ & $-1.89 \mathrm{E}-02$ & $6.25 \mathrm{E}-03$ & 3.99E-02 & $-2.13 \mathrm{E}-01$ & $1.72 \mathrm{E}-02$ & $1.34 \mathrm{E}-03$ \\
\hline & North America & $1.20 \mathrm{E}-02$ & $-2.11 \mathrm{E}-02$ & $8.23 E-03$ & $2.60 \mathrm{E}-02$ & $2.54 \mathrm{E}-02$ & $-1.99 \mathrm{E}-01$ & $1.10 \mathrm{E}-03$ \\
\hline & Oceania & 3.93E-04 & $2.25 \mathrm{E}-03$ & $-4.71 \mathrm{E}-03$ & $\mid-1.10 \mathrm{E}-03$ & $3.34 \mathrm{E}-04$ & $4.73 \mathrm{E}-04$ & $-6.04 \mathrm{E}-03$ \\
\hline \multirow{7}{*}{ Natural Soil } & Africa/Middle East & 1 & $-3.63 \mathrm{E}-01$ & $-3.06 \mathrm{E}-01$ & $1.15 \mathrm{E}-01$ & $-2.98 \mathrm{E}-02$ & $-8.71 \mathrm{E}-02$ & $-7.73 \mathrm{E}-03$ \\
\hline & Southern Asia & $-3.63 E-01$ & 1 & $-8.63 \mathrm{E}-03$ & $-3.66 \mathrm{E}-01$ & $5.92 \mathrm{E}-02$ & $-8.61 \mathrm{E}-03$ & 7.01E-03 \\
\hline & Central/South America & $-3.06 \mathrm{E}-01$ & $-8.63 \mathrm{E}-03$ & 1 & \begin{tabular}{|l|}
$-6.93 E-02$ \\
\end{tabular} & $3.39 E-03$ & $-2.91 \mathrm{E}-02$ & $-9.45 \mathrm{E}-02$ \\
\hline & Northern Asia & $1.15 \mathrm{E}-01$ & $-3.66 \mathrm{E}-01$ & $-6.93 \mathrm{E}-02$ & 1 & $-1.73 \mathrm{E}-01$ & $-2.39 \mathrm{E}-01$ & $-1.52 \mathrm{E}-02$ \\
\hline & Europe & $-2.98 \mathrm{E}-02$ & $5.92 \mathrm{E}-02$ & $3.39 \mathrm{E}-03$ & \begin{tabular}{|l|}
$-1.73 \mathrm{E}-01$ \\
\end{tabular} & 1 & $-1.20 \mathrm{E}-01$ & 4.37E-04 \\
\hline & North America & $-8.71 \mathrm{E}-02$ & $-8.61 \mathrm{E}-03$ & $-2.91 \mathrm{E}-02$ & \begin{tabular}{|l|}
$-2.39 \mathrm{E}-01$ \\
\end{tabular} & $-1.20 \mathrm{E}-01$ & 1 & $6.40 \mathrm{E}-03$ \\
\hline & Oceania & $-7.73 E-03$ & $7.01 \mathrm{E}-03$ & $-9.45 \mathrm{E}-02$ & $-1.52 \mathrm{E}-02$ & $4.37 \mathrm{E}-04$ & $6.40 \mathrm{E}-03$ & 1 \\
\hline \multirow{6}{*}{ Ocean } & North Pacific & $4.63 \mathrm{E}-02$ & $1.91 \mathrm{E}-01$ & 2.27E-02 & $-2.34 \mathrm{E}-01$ & $2.47 \mathrm{E}-02$ & $4.08 \mathrm{E}-02$ & $2.82 \mathrm{E}-03$ \\
\hline & \begin{tabular}{|l} 
South Pacific \\
\end{tabular} & $-4.13 \mathrm{E}-02$ & $6.67 \mathrm{E}-03$ & $-4.99 \mathrm{E}-01$ & $-1.00 \mathrm{E}-02$ & $1.09 \mathrm{E}-03$ & $4.79 \mathrm{E}-02$ & $9.45 \mathrm{E}-03$ \\
\hline & Northern Ocean & $2.85 \mathrm{E}-02$ & $1.29 \mathrm{E}-01$ & $4.69 \mathrm{E}-02$ & $-8.73 \mathrm{E}-02$ & $-1.98 \mathrm{E}-02$ & $-2.23 \mathrm{E}-03$ & $2.30 \mathrm{E}-03$ \\
\hline & Atlantic & $-1.22 \mathrm{E}-01$ & $1.61 \mathrm{E}-01$ & $-1.52 \mathrm{E}-02$ & $2.69 \mathrm{E}-02$ & $4.58 \mathrm{E}-02$ & \begin{tabular}{|l|} 
\\
\end{tabular} & $1.14 \mathrm{E}-03$ \\
\hline & Southern Ocean & $6.49 \mathrm{E}-02$ & $2.02 \mathrm{E}-01$ & $-3.75 E-04$ & $-5.95 \mathrm{E}-02$ & $8.62 \mathrm{E}-03$ & $2.23 \mathrm{E}-02$ & $-3.66 \mathrm{E}-02$ \\
\hline & Indian Ocean & $-1.09 \mathrm{E}-01$ & $-1.80 \mathrm{E}-01$ & $-1.87 \mathrm{E}-01$ & $4.13 \mathrm{E}-02$ & $4.47 \mathrm{E}-03$ & $3.90 \mathrm{E}-02$ & $-5.51 \mathrm{E}-02$ \\
\hline \multirow{7}{*}{$\begin{array}{l}\text { Biomass } \\
\text { burning }\end{array}$} & Africa/Middle East & $-1.20 \mathrm{E}-01$ & $-5.58 \mathrm{E}-02$ & $-2.17 \mathrm{E}-02$ & $3.02 \mathrm{E}-02$ & $1.56 \mathrm{E}-03$ & \begin{tabular}{|l|}
$3.15 \mathrm{E}-03$ \\
\end{tabular} & $-5.98 \mathrm{E}-04$ \\
\hline & Southern Asia & $1.21 \mathrm{E}-02$ & $5.41 \mathrm{E}-02$ & $-1.22 \mathrm{E}-02$ & $-1.73 \mathrm{E}-02$ & $-1.16 \mathrm{E}-02$ & \begin{tabular}{|l|}
$-6.68 \mathrm{E}-03$ \\
\end{tabular} & $-2.47 \mathrm{E}-03$ \\
\hline & Central/South America & $-8.02 \mathrm{E}-02$ & $-4.04 \mathrm{E}-02$ & $5.09 \mathrm{E}-02$ & $2.49 \mathrm{E}-02$ & $5.63 \mathrm{E}-03$ & \begin{tabular}{|l|}
$2.58 \mathrm{E}-02$ \\
\end{tabular} & $-9.52 \mathrm{E}-04$ \\
\hline & Northern Asia & $-4.16 \mathrm{E}-04$ & $1.11 \mathrm{E}-03$ & $2.69 \mathrm{E}-04$ & $-4.06 \mathrm{E}-02$ & $-1.09 \mathrm{E}-02$ & $-2.06 \mathrm{E}-02$ & $6.92 \mathrm{E}-05$ \\
\hline & Europe & $-5.63 \mathrm{E}-04$ & $3.96 \mathrm{E}-04$ & $1.46 \mathrm{E}-04$ & $-1.13 \mathrm{E}-03$ & $-4.95 \mathrm{E}-03$ & \begin{tabular}{|l|}
$-1.38 \mathrm{E}-03$ \\
\end{tabular} & $2.59 \mathrm{E}-05$ \\
\hline & North America & $-1.34 \mathrm{E}-03$ & $4.30 \mathrm{E}-03$ & $-1.40 \mathrm{E}-03$ & $-1.86 \mathrm{E}-02$ & $-9.94 \mathrm{E}-03$ & \begin{tabular}{|l|}
$-3.77 E-02$ \\
\end{tabular} & $1.62 \mathrm{E}-04$ \\
\hline & Oceania & $-7.37 \mathrm{E}-03$ & $-1.21 \mathrm{E}-02$ & $3.96 \mathrm{E}-03$ & $3.16 \mathrm{E}-03$ & $8.78 \mathrm{E}-04$ & $4.64 \mathrm{E}-03$ & $-6.19 \mathrm{E}-03$ \\
\hline
\end{tabular}

Fig. S2bj. Correlation of the emissions in 2007 for natural soil. 


\begin{tabular}{|c|c|c|c|c|c|c|c|}
\hline & \multirow{2}{*}{\multicolumn{6}{|c|}{ Ocean }} \\
\hline & & & & & & & \\
\hline Source sector & Region & $\begin{array}{l}\text { North } \\
\text { Pacific }\end{array}$ & $\begin{array}{l}\text { South } \\
\text { Pacific }\end{array}$ & $\begin{array}{l}\text { Northern } \\
\text { Ocean }\end{array}$ & Atlantic & $\begin{array}{l}\text { Southern } \\
\text { Ocean }\end{array}$ & $\begin{array}{l}\text { Indian } \\
\text { Ocean }\end{array}$ \\
\hline \multirow{7}{*}{$\begin{array}{l}\text { Agricultural } \\
\text { Soil }\end{array}$} & Africa/Middle East & $-1.75 \mathrm{E}-02$ & $-1.62 \mathrm{E}-02$ & $3.96 \mathrm{E}-04$ & $-8.43 \mathrm{E}-02$ & $1.85 \mathrm{E}-02$ & $-2.13 \mathrm{E}-02$ \\
\hline & \begin{tabular}{|l} 
Southern Asia \\
\end{tabular} & $-2.25 \mathrm{E}-01$ & $-1.84 \mathrm{E}-02$ & $-2.46 \mathrm{E}-02$ & $-1.46 \mathrm{E}-01$ & $6.29 \mathrm{E}-02$ & $3.77 \mathrm{E}-03$ \\
\hline & Central/South America & $7.49 \mathrm{E}-03$ & $-2.24 \mathrm{E}-02$ & $3.38 \mathrm{E}-03$ & $-2.73 \mathrm{E}-02$ & $-1.80 \mathrm{E}-01$ & $-1.13 \mathrm{E}-01$ \\
\hline & \begin{tabular}{|l} 
Northern Asia \\
\end{tabular} & $2.34 \mathrm{E}-02$ & $2.81 \mathrm{E}-02$ & $-4.28 \mathrm{E}-02$ & $-2.66 \mathrm{E}-02$ & $-1.96 \mathrm{E}-02$ & $-1.25 \mathrm{E}-03$ \\
\hline & Europe & $5.92 \mathrm{E}-03$ & $1.62 \mathrm{E}-02$ & $\mid-7.89 \mathrm{E}-02$ & $-6.11 \mathrm{E}-02$ & $6.64 \mathrm{E}-03$ & $1.25 \mathrm{E}-02$ \\
\hline & North America & $-2.90 \mathrm{E}-02$ & $9.28 \mathrm{E}-03$ & $-1.26 \mathrm{E}-02$ & $-1.01 \mathrm{E}-01$ & $3.91 \mathrm{E}-03$ & $1.52 \mathrm{E}-02$ \\
\hline & Oceania & $9.29 \mathrm{E}-03$ & $2.38 \mathrm{E}-02$ & $4.77 \mathrm{E}-03$ & $1.65 \mathrm{E}-02$ & $-7.22 \mathrm{E}-02$ & $-4.17 \mathrm{E}-02$ \\
\hline \multirow{7}{*}{ Industrial } & Africa/Middle East & $-3.51 \mathrm{E}-03$ & $5.85 \mathrm{E}-05$ & $-2.31 \mathrm{E}-04$ & $-1.32 \mathrm{E}-02$ & $2.13 \mathrm{E}-03$ & $-1.04 \mathrm{E}-04$ \\
\hline & Southern Asia & $-1.06 \mathrm{E}-02$ & $1.13 \mathrm{E}-02$ & $-9.46 \mathrm{E}-03$ & $-1.55 \mathrm{E}-03$ & $-2.52 \mathrm{E}-02$ & $1.82 \mathrm{E}-02$ \\
\hline & Central/South America & $6.46 \mathrm{E}-04$ & $-2.24 \mathrm{E}-03$ & $3.94 \mathrm{E}-04$ & $-2.37 \mathrm{E}-03$ & $-1.24 \mathrm{E}-02$ & $-8.97 \mathrm{E}-03$ \\
\hline & Northern Asia & $5.83 \mathrm{E}-02$ & $1.97 \mathrm{E}-02$ & $2.87 \mathrm{E}-02$ & $-1.97 \mathrm{E}-01$ & $2.54 \mathrm{E}-03$ & $6.80 \mathrm{E}-03$ \\
\hline & Europe & $-3.40 \mathrm{E}-03$ & $7.40 \mathrm{E}-03$ & $1.96 \mathrm{E}-02$ & $-2.66 \mathrm{E}-02$ & $-5.07 E-04$ & $4.69 \mathrm{E}-03$ \\
\hline & North America & $-6.42 \mathrm{E}-02$ & $-5.63 E-04$ & $2.01 \mathrm{E}-02$ & $-9.01 \mathrm{E}-02$ & $8.89 \mathrm{E}-03$ & $8.28 \mathrm{E}-05$ \\
\hline & Oceania & $7.20 \mathrm{E}-04$ & $2.68 \mathrm{E}-03$ & $3.64 \mathrm{E}-04$ & $1.27 \mathrm{E}-03$ & $-5.59 \mathrm{E}-03$ & $-4.33 \mathrm{E}-03$ \\
\hline \multirow{7}{*}{ Natural Soil } & Africa/Middle East & $4.63 \mathrm{E}-02$ & $-4.13 \mathrm{E}-02$ & $2.85 \mathrm{E}-02$ & $-1.22 \mathrm{E}-01$ & 6.49E-02 & $-1.09 \mathrm{E}-01$ \\
\hline & Southern Asia & $1.91 \mathrm{E}-01$ & $6.67 \mathrm{E}-03$ & $1.29 \mathrm{E}-01$ & $1.61 \mathrm{E}-01$ & $2.02 \mathrm{E}-01$ & $-1.80 \mathrm{E}-01$ \\
\hline & Central/South America & $2.27 \mathrm{E}-02$ & $-4.99 \mathrm{E}-01$ & $4.69 \mathrm{E}-02$ & $-1.52 \mathrm{E}-02$ & $-3.75 \mathrm{E}-04$ & $-1.87 \mathrm{E}-01$ \\
\hline & Northern Asia & $-2.34 \mathrm{E}-01$ & $-1.00 \mathrm{E}-02$ & \begin{tabular}{|l|}
$-8.73 E-02$ \\
\end{tabular} & $2.69 \mathrm{E}-02$ & $-5.95 \mathrm{E}-02$ & $4.13 \mathrm{E}-02$ \\
\hline & Europe & $2.47 \mathrm{E}-02$ & $1.09 \mathrm{E}-03$ & \begin{tabular}{|l|}
$-1.98 \mathrm{E}-02$ \\
\end{tabular} & $4.58 \mathrm{E}-02$ & $8.62 \mathrm{E}-03$ & 4.47E-03 \\
\hline & North America & $4.08 \mathrm{E}-02$ & $4.79 \mathrm{E}-02$ & \begin{tabular}{|l|}
$-2.23 \mathrm{E}-03$ \\
\end{tabular} & $5.85 \mathrm{E}-02$ & $2.23 \mathrm{E}-02$ & $3.90 \mathrm{E}-02$ \\
\hline & Oceania & $2.82 \mathrm{E}-03$ & $9.45 \mathrm{E}-03$ & $2.30 \mathrm{E}-03$ & $1.14 \mathrm{E}-03$ & $-3.66 \mathrm{E}-02$ & $-5.51 \mathrm{E}-02$ \\
\hline \multirow{6}{*}{ Ocean } & North Pacific & 1 & $-2.54 \mathrm{E}-03$ & $-7.22 \mathrm{E}-02$ & $-1.87 \mathrm{E}-01$ & $3.04 \mathrm{E}-02$ & $1.85 \mathrm{E}-02$ \\
\hline & South Pacific & $-2.54 \mathrm{E}-03$ & 1 & $1.13 \mathrm{E}-02$ & $-6.63 E-02$ & $5.11 \mathrm{E}-02$ & $5.39 \mathrm{E}-03$ \\
\hline & Northern Ocean & $-7.22 \mathrm{E}-02$ & $1.13 \mathrm{E}-02$ & 1 & $-3.36 \mathrm{E}-01$ & $3.43 \mathrm{E}-02$ & $-1.55 \mathrm{E}-04$ \\
\hline & Atlantic & $-1.87 \mathrm{E}-01$ & $-6.63 \mathrm{E}-02$ & $-3.36 \mathrm{E}-01$ & 1 & $-6.10 \mathrm{E}-02$ & $-4.86 \mathrm{E}-02$ \\
\hline & Southern Ocean & $3.04 \mathrm{E}-02$ & $5.11 \mathrm{E}-02$ & $3.43 \mathrm{E}-02$ & $-6.10 \mathrm{E}-02$ & 1 & $-6.42 \mathrm{E}-01$ \\
\hline & Indian Ocean & $1.85 \mathrm{E}-02$ & $5.39 \mathrm{E}-03$ & \begin{tabular}{|l|}
$-1.55 \mathrm{E}-04$ \\
\end{tabular} & $-4.86 \mathrm{E}-02$ & $-6.42 \mathrm{E}-01$ & \\
\hline \multirow{7}{*}{$\begin{array}{l}\text { Biomass } \\
\text { burning }\end{array}$} & Africa/Middle East & $6.49 \mathrm{E}-03$ & $-4.92 \mathrm{E}-02$ & $1.23 \mathrm{E}-03$ & $-5.40 \mathrm{E}-02$ & $2.13 \mathrm{E}-03$ & $-4.75 \mathrm{E}-02$ \\
\hline & Southern Asia & $-2.35 \mathrm{E}-02$ & $-2.56 \mathrm{E}-02$ & \begin{tabular}{|l|}
$-4.00 \mathrm{E}-03$ \\
\end{tabular} & $-3.26 \mathrm{E}-02$ & $2.10 \mathrm{E}-02$ & $-1.80 \mathrm{E}-02$ \\
\hline & Central/South America & $7.08 \mathrm{E}-04$ & $-8.64 \mathrm{E}-02$ & $-6.06 \mathrm{E}-03$ & $-4.13 \mathrm{E}-02$ & $-6.07 \mathrm{E}-02$ & $-5.55 \mathrm{E}-02$ \\
\hline & Northern Asia & $-1.62 \mathrm{E}-02$ & $2.09 \mathrm{E}-03$ & $-1.08 \mathrm{E}-03$ & $6.95 \mathrm{E}-03$ & $8.83 \mathrm{E}-04$ & $-6.18 \mathrm{E}-04$ \\
\hline & Europe & $3.54 \mathrm{E}-04$ & $6.45 \mathrm{E}-05$ & \begin{tabular}{|l|}
$-2.20 \mathrm{E}-04$ \\
\end{tabular} & $-1.34 \mathrm{E}-05$ & $1.38 \mathrm{E}-04$ & $1.08 \mathrm{E}-04$ \\
\hline & North America & $-3.46 \mathrm{E}-03$ & $-8.42 \mathrm{E}-05$ & \begin{tabular}{|l|}
$-5.07 E-03$ \\
\end{tabular} & $1.08 \mathrm{E}-04$ & $1.57 \mathrm{E}-03$ & 7.97E-04 \\
\hline & Oceania & $8.62 \mathrm{E}-04$ & $-3.58 \mathrm{E}-03$ & \begin{tabular}{|l|}
$-1.22 \mathrm{E}-03$ \\
\end{tabular} & $-4.30 \mathrm{E}-03$ & $-4.89 \mathrm{E}-04$ & $-1.54 \mathrm{E}-02$ \\
\hline
\end{tabular}

Fig. S2bk. Correlation of the emissions in 2007 for ocean. 


\begin{tabular}{|c|c|c|c|c|c|c|c|c|}
\hline \multirow[b]{3}{*}{ Source sector } & \multirow[b]{3}{*}{ Region } & \multirow{2}{*}{\multicolumn{7}{|c|}{ Biomass burning }} \\
\hline & & & & & & & & \\
\hline & & $\begin{array}{l}\text { Africa/M. } \\
\text { East }\end{array}$ & $\begin{array}{l}\text { Southern } \\
\text { Asia }\end{array}$ & $\begin{array}{l}\text { C. /S. } \\
\text { America }\end{array}$ & $\begin{array}{l}\text { Northern } \\
\text { Asia }\end{array}$ & Europe & $\begin{array}{l}\text { North } \\
\text { America }\end{array}$ & Oceania \\
\hline \multirow{7}{*}{$\begin{array}{l}\text { Agricultural } \\
\text { Soil }\end{array}$} & Africa/Middle East & $-2.16 \mathrm{E}-02$ & $-1.18 \mathrm{E}-02$ & 8.17E-04 & $-5.22 \mathrm{E}-04$ & $-9.63 \mathrm{E}-04$ & $-1.36 \mathrm{E}-03$ & $1.39 \mathrm{E}-03$ \\
\hline & Southern Asia & $-4.65 \mathrm{E}-03$ & $-8.19 \mathrm{E}-02$ & $2.98 \mathrm{E}-02$ & $-1.63 \mathrm{E}-02$ & $1.02 \mathrm{E}-05$ & $-2.70 \mathrm{E}-03$ & $7.22 \mathrm{E}-03$ \\
\hline & Central/South America & $-3.28 \mathrm{E}-02$ & $1.68 \mathrm{E}-03$ & $-3.10 \mathrm{E}-02$ & $5.07 \mathrm{E}-04$ & $6.60 \mathrm{E}-05$ & $6.40 \mathrm{E}-04$ & $-5.15 E-03$ \\
\hline & Northern Asia & $7.59 \mathrm{E}-03$ & $1.89 \mathrm{E}-02$ & $7.08 \mathrm{E}-04$ & $-1.20 \mathrm{E}-03$ & $-2.10 \mathrm{E}-04$ & $3.51 \mathrm{E}-03$ & $-3.11 \mathrm{E}-03$ \\
\hline & \begin{tabular}{|l} 
Europe \\
\end{tabular} & $4.41 \mathrm{E}-03$ & $2.16 \mathrm{E}-03$ & $6.87 \mathrm{E}-03$ & $5.31 \mathrm{E}-04$ & $7.55 \mathrm{E}-05$ & $3.27 \mathrm{E}-04$ & $2.32 \mathrm{E}-03$ \\
\hline & \begin{tabular}{|l} 
North America \\
\end{tabular} & $2.43 \mathrm{E}-03$ & $-1.80 \mathrm{E}-03$ & $6.00 \mathrm{E}-03$ & $4.29 \mathrm{E}-03$ & $3.58 \mathrm{E}-04$ & $5.82 \mathrm{E}-03$ & $2.47 \mathrm{E}-03$ \\
\hline & Oceania & $5.64 \mathrm{E}-03$ & $2.99 \mathrm{E}-03$ & $5.12 \mathrm{E}-03$ & $4.18 \mathrm{E}-04$ & $5.59 \mathrm{E}-05$ & $5.01 \mathrm{E}-04$ & $-4.47 \mathrm{E}-03$ \\
\hline \multirow{7}{*}{ Industrial } & Africa/Middle East & $-1.18 \mathrm{E}-03$ & $-2.02 \mathrm{E}-03$ & $1.27 \mathrm{E}-03$ & $-8.50 \mathrm{E}-05$ & $-2.16 \mathrm{E}-04$ & $-1.08 \mathrm{E}-04$ & $4.23 \mathrm{E}-04$ \\
\hline & Southern Asia & $1.18 \mathrm{E}-02$ & $-2.99 \mathrm{E}-03$ & $3.83 \mathrm{E}-03$ & $-2.24 \mathrm{E}-03$ & $1.16 \mathrm{E}-04$ & $2.13 \mathrm{E}-03$ & $1.06 \mathrm{E}-03$ \\
\hline & Central/South America & $-2.87 \mathrm{E}-03$ & $1.26 \mathrm{E}-04$ & $-2.59 \mathrm{E}-03$ & $3.23 \mathrm{E}-05$ & $5.49 \mathrm{E}-06$ & $4.59 \mathrm{E}-05$ & $-4.07 E-04$ \\
\hline & Northern Asia & $1.12 \mathrm{E}-02$ & $9.78 \mathrm{E}-03$ & $2.63 \mathrm{E}-03$ & $2.77 \mathrm{E}-03$ & $1.50 \mathrm{E}-04$ & $3.25 \mathrm{E}-04$ & $3.34 \mathrm{E}-04$ \\
\hline & Europe & $2.82 \mathrm{E}-03$ & $2.27 \mathrm{E}-03$ & $2.14 \mathrm{E}-03$ & $1.27 \mathrm{E}-03$ & $4.54 \mathrm{E}-05$ & $1.15 \mathrm{E}-03$ & $5.74 \mathrm{E}-04$ \\
\hline & North America & $1.95 \mathrm{E}-03$ & $8.64 \mathrm{E}-04$ & $1.86 \mathrm{E}-03$ & $3.48 \mathrm{E}-03$ & $2.88 \mathrm{E}-04$ & $-1.46 \mathrm{E}-03$ & $3.62 \mathrm{E}-04$ \\
\hline & Oceania & $4.65 \mathrm{E}-04$ & $2.23 \mathrm{E}-04$ & $5.13 \mathrm{E}-04$ & $4.67 \mathrm{E}-05$ & $5.02 \mathrm{E}-06$ & $4.05 \mathrm{E}-05$ & $-4.75 E-04$ \\
\hline \multirow{7}{*}{ Natural Soil } & Africa/Middle East & $-1.20 \mathrm{E}-01$ & $1.21 \mathrm{E}-02$ & $-8.02 \mathrm{E}-02$ & $-4.16 \mathrm{E}-04$ & $-5.63 \mathrm{E}-04$ & $-1.34 \mathrm{E}-03$ & $-7.37 \mathrm{E}-03$ \\
\hline & Southern Asia & $-5.58 \mathrm{E}-02$ & $5.41 \mathrm{E}-02$ & $-4.04 \mathrm{E}-02$ & $1.11 \mathrm{E}-03$ & $3.96 \mathrm{E}-04$ & $4.30 \mathrm{E}-03$ & $-1.21 \mathrm{E}-02$ \\
\hline & Central/South America & $-2.17 \mathrm{E}-02$ & $-1.22 \mathrm{E}-02$ & $5.09 E-02$ & $2.69 \mathrm{E}-04$ & $1.46 \mathrm{E}-04$ & $-1.40 \mathrm{E}-03$ & $3.96 \mathrm{E}-03$ \\
\hline & Northern Asia & $3.02 \mathrm{E}-02$ & $-1.73 \mathrm{E}-02$ & $2.49 \mathrm{E}-02$ & $-4.06 \mathrm{E}-02$ & $-1.13 \mathrm{E}-03$ & $-1.86 \mathrm{E}-02$ & $3.16 \mathrm{E}-03$ \\
\hline & Europe & $1.56 \mathrm{E}-03$ & $-1.16 \mathrm{E}-02$ & $5.63 \mathrm{E}-03$ & $-1.09 \mathrm{E}-02$ & $-4.95 \mathrm{E}-03$ & $-9.94 \mathrm{E}-03$ & $8.78 \mathrm{E}-04$ \\
\hline & North America & $3.15 \mathrm{E}-03$ & $-6.68 \mathrm{E}-03$ & $2.58 \mathrm{E}-02$ & $-2.06 \mathrm{E}-02$ & $-1.38 \mathrm{E}-03$ & $-3.77 \mathrm{E}-02$ & 4.64E-03 \\
\hline & Oceania & $-5.98 \mathrm{E}-04$ & $-2.47 \mathrm{E}-03$ & $-9.52 \mathrm{E}-04$ & $6.92 \mathrm{E}-05$ & $2.59 \mathrm{E}-05$ & $1.62 \mathrm{E}-04$ & $-6.19 \mathrm{E}-03$ \\
\hline \multirow{6}{*}{ Ocean } & North Pacific & $6.49 \mathrm{E}-03$ & $-2.35 \mathrm{E}-02$ & $7.08 \mathrm{E}-04$ & $-1.62 \mathrm{E}-02$ & $3.54 \mathrm{E}-04$ & $-3.46 \mathrm{E}-03$ & $8.62 \mathrm{E}-04$ \\
\hline & South Pacific & $-4.92 \mathrm{E}-02$ & $-2.56 \mathrm{E}-02$ & $-8.64 \mathrm{E}-02$ & $2.09 \mathrm{E}-03$ & $6.45 E-05$ & $-8.42 \mathrm{E}-05$ & $-3.58 \mathrm{E}-03$ \\
\hline & Northern Ocean & $1.23 \mathrm{E}-03$ & $-4.00 \mathrm{E}-03$ & $-6.06 \mathrm{E}-03$ & $-1.08 \mathrm{E}-03$ & $-2.20 \mathrm{E}-04$ & $-5.07 \mathrm{E}-03$ & $-1.22 \mathrm{E}-03$ \\
\hline & Atlantic & $-5.40 \mathrm{E}-02$ & $-3.26 \mathrm{E}-02$ & $-4.13 \mathrm{E}-02$ & $6.95 \mathrm{E}-03$ & $-1.34 \mathrm{E}-05$ & $1.08 \mathrm{E}-04$ & $-4.30 \mathrm{E}-03$ \\
\hline & Southern Ocean & $2.13 \mathrm{E}-03$ & $2.10 \mathrm{E}-02$ & $-6.07 \mathrm{E}-02$ & $8.83 \mathrm{E}-04$ & $1.38 \mathrm{E}-04$ & $1.57 \mathrm{E}-03$ & $-4.89 \mathrm{E}-04$ \\
\hline & Indian Ocean & $-4.75 \mathrm{E}-02$ & $-1.80 \mathrm{E}-02$ & $-5.55 \mathrm{E}-02$ & $-6.18 \mathrm{E}-04$ & $1.08 \mathrm{E}-04$ & $7.97 \mathrm{E}-04$ & $-1.54 \mathrm{E}-02$ \\
\hline \multirow{7}{*}{$\begin{array}{l}\text { Biomass } \\
\text { burning }\end{array}$} & Africa/Middle East & 1 & $-9.07 \mathrm{E}-04$ & $-4.12 \mathrm{E}-02$ & $9.23 \mathrm{E}-04$ & $-3.46 \mathrm{E}-05$ & \begin{tabular}{|l|}
$5.40 \mathrm{E}-04$ \\
\end{tabular} & $-4.05 \mathrm{E}-03$ \\
\hline & Southern Asia & $-9.07 E-04$ & 1 & 5.91E-03 & $3.59 \mathrm{E}-04$ & $-4.23 \mathrm{E}-05$ & \begin{tabular}{|l|}
$-7.22 \mathrm{E}-04$ \\
\end{tabular} & $8.25 \mathrm{E}-04$ \\
\hline & Central/South America & $-4.12 \mathrm{E}-02$ & $5.91 \mathrm{E}-03$ & 1 & $7.11 \mathrm{E}-05$ & $-4.32 \mathrm{E}-05$ & \begin{tabular}{|l|}
$5.65 \mathrm{E}-04$ \\
\end{tabular} & $-8.01 \mathrm{E}-03$ \\
\hline & Northern Asia & $9.23 \mathrm{E}-04$ & $3.59 \mathrm{E}-04$ & $7.11 \mathrm{E}-05$ & 1 & $-1.79 \mathrm{E}-04$ & $-1.14 \mathrm{E}-03$ & $-3.65 \mathrm{E}-04$ \\
\hline & Europe & $-3.46 \mathrm{E}-05$ & $-4.23 \mathrm{E}-05$ & $-4.32 \mathrm{E}-05$ & $-1.79 \mathrm{E}-04$ & 1 & $-1.69 \mathrm{E}-04$ & $3.30 \mathrm{E}-06$ \\
\hline & North America & $5.40 \mathrm{E}-04$ & $-7.22 \mathrm{E}-04$ & $5.65 \mathrm{E}-04$ & $-1.14 \mathrm{E}-03$ & $-1.69 \mathrm{E}-04$ & 1 & $1.18 \mathrm{E}-04$ \\
\hline & Oceania & $-4.05 \mathrm{E}-03$ & $8.25 \mathrm{E}-04$ & $-8.01 E-03$ & $-3.65 E-04$ & $3.30 \mathrm{E}-06$ & \begin{tabular}{|l|l|} 
& $1.18 \mathrm{E}-04$ \\
\end{tabular} & 1 \\
\hline
\end{tabular}

Fig. S2bl. Correlation of the emissions in 2007 for biomass burning. 


\begin{tabular}{|c|c|c|c|c|c|c|c|c|}
\hline \multirow[b]{2}{*}{ Source sector } & \multirow[b]{2}{*}{ Region } & \multicolumn{7}{|c|}{ Agricultural Soil } \\
\hline & & $\begin{array}{l}\text { Africa/M. } \\
\text { East }\end{array}$ & $\begin{array}{l}\text { Southern } \\
\text { Asia }\end{array}$ & $\begin{array}{l}\text { C. /S. } \\
\text { America }\end{array}$ & $\begin{array}{l}\text { Northern } \\
\text { Asia }\end{array}$ & Europe & $\begin{array}{l}\text { North } \\
\text { America }\end{array}$ & Oceania \\
\hline \multirow{7}{*}{$\begin{array}{c}\text { Agricultural } \\
\text { Soil }\end{array}$} & Africa/Middle East & 1 & $-6.79 \mathrm{E}-02$ & $-3.61 \mathrm{E}-03$ & $-2.86 \mathrm{E}-02$ & $-2.53 \mathrm{E}-02$ & $-1.71 \mathrm{E}-02$ & $6.92 \mathrm{E}-03$ \\
\hline & Southern Asia & $-6.79 \mathrm{E}-02$ & 1 & $3.29 \mathrm{E}-02$ & $-2.43 \mathrm{E}-01$ & $3.79 E-02$ & $-6.53 \mathrm{E}-02$ & $2.34 \mathrm{E}-02$ \\
\hline & Central/South America & $-3.61 \mathrm{E}-03$ & $3.29 \mathrm{E}-02$ & 1 & $-8.22 \mathrm{E}-03$ & $7.53 \mathrm{E}-03$ & $5.11 \mathrm{E}-03$ & $-2.44 \mathrm{E}-02$ \\
\hline & Northern Asia & $-2.86 \mathrm{E}-02$ & $-2.43 \mathrm{E}-01$ & $-8.22 \mathrm{E}-03$ & 1 & $-1.67 \mathrm{E}-01$ & $-5.24 \mathrm{E}-02$ & $-2.58 \mathrm{E}-02$ \\
\hline & Europe & $-2.53 \mathrm{E}-02$ & $3.79 \mathrm{E}-02$ & $7.53 \mathrm{E}-03$ & $-1.67 \mathrm{E}-01$ & 1 & $-2.21 \mathrm{E}-02$ & $5.45 \mathrm{E}-03$ \\
\hline & North America & $-1.71 \mathrm{E}-02$ & $-6.53 \mathrm{E}-02$ & $5.11 \mathrm{E}-03$ & $-5.24 \mathrm{E}-02$ & $-2.21 \mathrm{E}-02$ & 1 & $3.65 \mathrm{E}-03$ \\
\hline & Oceania & $6.92 \mathrm{E}-03$ & $2.34 \mathrm{E}-02$ & $-2.44 \mathrm{E}-02$ & $-2.58 \mathrm{E}-02$ & $5.45 \mathrm{E}-03$ & $3.65 \mathrm{E}-03$ & 1 \\
\hline \multirow{7}{*}{ Industrial } & Africa/Middle East & $-9.01 \mathrm{E}-02$ & $-9.16 \mathrm{E}-03$ & $1.32 \mathrm{E}-03$ & $-9.01 E-03$ & \begin{tabular}{|l|}
$-1.09 \mathrm{E}-02$ \\
\end{tabular} & $-3.13 \mathrm{E}-03$ & $1.21 \mathrm{E}-03$ \\
\hline & \begin{tabular}{|l} 
Southern Asia \\
\end{tabular} & $2.21 \mathrm{E}-02$ & $-4.46 \mathrm{E}-01$ & $-2.04 \mathrm{E}-03$ & $-4.26 \mathrm{E}-02$ & $2.19 \mathrm{E}-02$ & $9.86 \mathrm{E}-03$ & $-1.69 \mathrm{E}-03$ \\
\hline & Central/South America & $-3.39 E-04$ & $3.07 \mathrm{E}-03$ & $-8.69 \mathrm{E}-03$ & $-6.44 \mathrm{E}-04$ & $7.16 \mathrm{E}-04$ & $5.26 \mathrm{E}-04$ & $-1.92 \mathrm{E}-03$ \\
\hline & Northern Asia & $2.95 \mathrm{E}-03$ & $-4.05 \mathrm{E}-02$ & 1.07E-03 & $-1.51 \mathrm{E}-01$ & $-6.41 \mathrm{E}-02$ & $1.43 \mathrm{E}-01$ & $-6.24 \mathrm{E}-04$ \\
\hline & Europe & $-1.85 E-02$ & $3.28 \mathrm{E}-03$ & $2.90 \mathrm{E}-03$ & $-3.16 \mathrm{E}-02$ & \begin{tabular}{|l|}
$-7.18 \mathrm{E}-01$ \\
\end{tabular} & $-1.07 \mathrm{E}-02$ & $1.64 \mathrm{E}-04$ \\
\hline & North America & $8.20 \mathrm{E}-03$ & $2.29 \mathrm{E}-02$ & $3.18 \mathrm{E}-03$ & $3.89 \mathrm{E}-02$ & $3.05 \mathrm{E}-02$ & $-4.22 \mathrm{E}-01$ & $-2.98 \mathrm{E}-04$ \\
\hline & Oceania & $5.89 \mathrm{E}-04$ & $1.70 \mathrm{E}-03$ & $-2.07 \mathrm{E}-03$ & $-1.80 \mathrm{E}-03$ & $3.69 \mathrm{E}-04$ & $2.68 \mathrm{E}-04$ & $-1.02 \mathrm{E}-02$ \\
\hline \multirow{7}{*}{ Natural Soil } & Africa/Middle East & $-1.83 E-01$ & $-2.09 \mathrm{E}-02$ & $-8.04 \mathrm{E}-02$ & $4.84 \mathrm{E}-03$ & $-1.38 \mathrm{E}-02$ & $-2.48 \mathrm{E}-03$ & $-3.49 \mathrm{E}-03$ \\
\hline & Southern Asia & $-7.17 \mathrm{E}-02$ & $-4.16 \mathrm{E}-01$ & $9.36 \mathrm{E}-03$ & $1.85 \mathrm{E}-02$ & $-1.44 \mathrm{E}-02$ & $-3.92 \mathrm{E}-02$ & $1.89 \mathrm{E}-02$ \\
\hline & Central/South America & $-6.88 \mathrm{E}-02$ & $3.15 \mathrm{E}-03$ & $-2.65 \mathrm{E}-01$ & $1.97 \mathrm{E}-02$ & $2.73 \mathrm{E}-02$ & $1.18 \mathrm{E}-02$ & $-3.12 \mathrm{E}-02$ \\
\hline & Northern Asia & $1.45 \mathrm{E}-02$ & $-1.02 \mathrm{E}-01$ & $-5.54 \mathrm{E}-03$ & $-1.85 \mathrm{E}-01$ & $2.21 \mathrm{E}-03$ & $1.08 \mathrm{E}-01$ & $-1.61 \mathrm{E}-02$ \\
\hline & Europe & $-2.88 \mathrm{E}-02$ & $-5.50 \mathrm{E}-03$ & $7.84 \mathrm{E}-03$ & $1.12 \mathrm{E}-02$ & $-2.52 \mathrm{E}-01$ & $1.60 \mathrm{E}-02$ & $5.43 \mathrm{E}-03$ \\
\hline & North America & $-2.49 \mathrm{E}-02$ & $-1.95 \mathrm{E}-02$ & $1.80 \mathrm{E}-02$ & $9.43 \mathrm{E}-02$ & $2.49 \mathrm{E}-03$ & $-4.21 \mathrm{E}-01$ & $7.75 \mathrm{E}-03$ \\
\hline & Oceania & $3.53 \mathrm{E}-03$ & $1.69 \mathrm{E}-02$ & $-2.50 \mathrm{E}-02$ & $-1.47 \mathrm{E}-02$ & $6.05 \mathrm{E}-03$ & $5.31 \mathrm{E}-03$ & $-6.49 \mathrm{E}-02$ \\
\hline \multirow{6}{*}{ Ocean } & North Pacific & $-1.34 \mathrm{E}-02$ & $-1.89 \mathrm{E}-01$ & $1.10 \mathrm{E}-02$ & $1.85 \mathrm{E}-02$ & $2.39 \mathrm{E}-03$ & $-4.51 \mathrm{E}-02$ & $8.57 \mathrm{E}-03$ \\
\hline & South Pacific & $-2.08 \mathrm{E}-03$ & $1.51 \mathrm{E}-02$ & $-3.13 \mathrm{E}-02$ & $9.24 \mathrm{E}-03$ & $1.33 \mathrm{E}-02$ & $1.38 \mathrm{E}-02$ & $3.32 \mathrm{E}-03$ \\
\hline & Northern Ocean & $2.23 \mathrm{E}-03$ & $-3.66 \mathrm{E}-02$ & $6.80 \mathrm{E}-03$ & $-5.15 \mathrm{E}-02$ & $-7.98 \mathrm{E}-02$ & $-2.41 \mathrm{E}-02$ & $7.44 \mathrm{E}-03$ \\
\hline & Atlantic & $-8.03 \mathrm{E}-02$ & $-1.46 \mathrm{E}-01$ & $-1.49 \mathrm{E}-02$ & $-2.05 \mathrm{E}-02$ & $-5.48 \mathrm{E}-02$ & $-9.25 \mathrm{E}-02$ & $1.38 \mathrm{E}-02$ \\
\hline & Southern Ocean & $2.58 \mathrm{E}-02$ & $6.16 \mathrm{E}-02$ & $-1.88 \mathrm{E}-01$ & $-2.66 \mathrm{E}-02$ & $6.17 \mathrm{E}-03$ & $6.81 \mathrm{E}-03$ & $-9.68 \mathrm{E}-02$ \\
\hline & Indian Ocean & $-3.33 E-02$ & $1.51 \mathrm{E}-02$ & $-9.79 \mathrm{E}-02$ & $-3.06 \mathrm{E}-03$ & $9.41 \mathrm{E}-03$ & $1.52 \mathrm{E}-02$ & $-3.51 \mathrm{E}-02$ \\
\hline \multirow{7}{*}{$\begin{array}{l}\text { Biomass } \\
\text { burning }\end{array}$} & Africa/Middle East & $-2.64 \mathrm{E}-02$ & $-1.02 \mathrm{E}-02$ & $-2.16 \mathrm{E}-02$ & $2.96 \mathrm{E}-03$ & $9.66 \mathrm{E}-05$ & $-5.04 \mathrm{E}-03$ & $4.42 \mathrm{E}-03$ \\
\hline & Southern Asia & $-3.05 E-03$ & $-3.10 \mathrm{E}-02$ & $8.55 \mathrm{E}-04$ & $4.97 \mathrm{E}-03$ & $1.38 \mathrm{E}-03$ & $-1.03 \mathrm{E}-04$ & $9.62 \mathrm{E}-04$ \\
\hline & Central/South America & $-2.24 \mathrm{E}-03$ & $7.99 \mathrm{E}-03$ & $-7.73 \mathrm{E}-03$ & $-2.43 \mathrm{E}-04$ & $1.00 \mathrm{E}-03$ & 7.07E-04 & $1.68 \mathrm{E}-03$ \\
\hline & \begin{tabular}{|l} 
Northern Asia \\
\end{tabular} & $-1.86 \mathrm{E}-03$ & $-6.19 \mathrm{E}-02$ & $2.04 \mathrm{E}-03$ & $-1.64 \mathrm{E}-02$ & $1.06 \mathrm{E}-02$ & $1.26 \mathrm{E}-02$ & $2.12 \mathrm{E}-03$ \\
\hline & Europe & $-2.50 \mathrm{E}-04$ & $2.85 \mathrm{E}-04$ & $1.04 \mathrm{E}-05$ & $8.93 \mathrm{E}-05$ & $-9.95 \mathrm{E}-05$ & $2.19 \mathrm{E}-04$ & $2.84 \mathrm{E}-06$ \\
\hline & North America & $-1.65 \mathrm{E}-03$ & $-4.55 \mathrm{E}-03$ & $1.99 \mathrm{E}-03$ & $1.33 \mathrm{E}-02$ & $7.16 \mathrm{E}-04$ & $1.62 \mathrm{E}-02$ & $1.76 \mathrm{E}-03$ \\
\hline & Oceania & $2.96 \mathrm{E}-04$ & $2.81 \mathrm{E}-03$ & $-2.13 \mathrm{E}-03$ & $-1.85 \mathrm{E}-03$ & $7.68 \mathrm{E}-04$ & $6.46 \mathrm{E}-04$ & $-2.37 \mathrm{E}-03$ \\
\hline
\end{tabular}

Fig. S2bm. Correlation of the emissions in 2008 for agricultural soil. 


\begin{tabular}{|c|c|c|c|c|c|c|c|c|}
\hline & \multirow{2}{*}{\multicolumn{7}{|c|}{ Industrial }} \\
\hline & & & & & & & & \\
\hline Source sector & Region & $\begin{array}{l}\text { Africa/M. } \\
\text { East }\end{array}$ & $\begin{array}{l}\text { Southern } \\
\text { Asia }\end{array}$ & $\begin{array}{l}\text { C. /S. } \\
\text { America }\end{array}$ & $\begin{array}{l}\text { Northern } \\
\text { Asia }\end{array}$ & Europe & $\begin{array}{l}\text { North } \\
\text { America }\end{array}$ & Oceania \\
\hline \multirow{7}{*}{$\begin{array}{l}\text { Agricultural } \\
\text { Soil }\end{array}$} & Africa/Middle East & $-9.01 \mathrm{E}-02$ & $2.21 \mathrm{E}-02$ & $-3.39 \mathrm{E}-04$ & $2.95 \mathrm{E}-03$ & $-1.85 \mathrm{E}-02$ & $8.20 \mathrm{E}-03$ & $5.89 \mathrm{E}-04$ \\
\hline & Southern Asia & $-9.16 \mathrm{E}-03$ & $-4.46 \mathrm{E}-01$ & $3.07 \mathrm{E}-03$ & $-4.05 E-02$ & $3.28 \mathrm{E}-03$ & $2.29 \mathrm{E}-02$ & $1.70 \mathrm{E}-03$ \\
\hline & Central/South America & $1.32 \mathrm{E}-03$ & $-2.04 \mathrm{E}-03$ & $-8.69 \mathrm{E}-03$ & \begin{tabular}{|c|} 
\\
\end{tabular} & $2.90 \mathrm{E}-03$ & $3.18 \mathrm{E}-03$ & $-2.07 \mathrm{E}-03$ \\
\hline & Northern Asia & $-9.01 \mathrm{E}-03$ & $-4.26 \mathrm{E}-02$ & $-6.44 \mathrm{E}-04$ & \begin{tabular}{|l|} 
\\
\end{tabular} & $-3.16 \mathrm{E}-02$ & $3.89 \mathrm{E}-02$ & $-1.80 \mathrm{E}-03$ \\
\hline & Europe & $-1.09 \mathrm{E}-02$ & $2.19 \mathrm{E}-02$ & $7.16 \mathrm{E}-04$ & $-6.41 \mathrm{E}-02$ & $-7.18 \mathrm{E}-01$ & $3.05 \mathrm{E}-02$ & $3.69 \mathrm{E}-04$ \\
\hline & \begin{tabular}{|l|} 
North America \\
\end{tabular} & $-3.13 \mathrm{E}-03$ & $9.86 \mathrm{E}-03$ & $5.26 \mathrm{E}-04$ & $1.43 \mathrm{E}-01$ & $-1.07 \mathrm{E}-02$ & $-4.22 \mathrm{E}-01$ & $2.68 \mathrm{E}-04$ \\
\hline & Oceania & $1.21 \mathrm{E}-03$ & $-1.69 \mathrm{E}-03$ & $-1.92 \mathrm{E}-03$ & 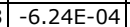 & $1.64 \mathrm{E}-04$ & $-2.98 \mathrm{E}-04$ & $-1.02 \mathrm{E}-02$ \\
\hline \multirow{7}{*}{ Industrial } & Africa/Middle East & 1 & $3.23 \mathrm{E}-03$ & $1.36 \mathrm{E}-04$ & $-4.08 \mathrm{E}-03$ & $-8.67 \mathrm{E}-03$ & $1.95 \mathrm{E}-03$ & $9.91 \mathrm{E}-05$ \\
\hline & \begin{tabular}{|l|} 
Southern Asia \\
\end{tabular} & $3.23 \mathrm{E}-03$ & 1 & $-2.46 \mathrm{E}-04$ & $-3.74 \mathrm{E}-02$ & $4.73 \mathrm{E}-03$ & $1.55 \mathrm{E}-02$ & $-1.97 \mathrm{E}-04$ \\
\hline & Central/South America & $1.36 \mathrm{E}-04$ & $-2.46 \mathrm{E}-04$ & 1 & $1.39 \mathrm{E}-04$ & $2.98 \mathrm{E}-04$ & $3.63 \mathrm{E}-04$ & $-1.60 \mathrm{E}-04$ \\
\hline & Northern Asia & $-4.08 \mathrm{E}-03$ & $-3.74 \mathrm{E}-02$ & $1.39 \mathrm{E}-04$ & 1 & $-2.71 \mathrm{E}-02$ & $-2.46 \mathrm{E}-01$ & $-1.27 \mathrm{E}-04$ \\
\hline & Europe & $-8.67 E-03$ & $4.73 \mathrm{E}-03$ & $2.98 \mathrm{E}-04$ & $-2.71 \mathrm{E}-02$ & 1 & $9.36 \mathrm{E}-03$ & $-4.37 \mathrm{E}-06$ \\
\hline & North America & $1.95 \mathrm{E}-03$ & $1.55 \mathrm{E}-02$ & $3.63 E-04$ & $-2.46 \mathrm{E}-01$ & $9.36 \mathrm{E}-03$ & 1 & $-1.04 \mathrm{E}-05$ \\
\hline & Oceania & $9.91 \mathrm{E}-05$ & $-1.97 \mathrm{E}-04$ & $-1.60 \mathrm{E}-04$ & $-1.27 E-04$ & $-4.37 E-06$ & $-1.04 \mathrm{E}-05$ & 1 \\
\hline \multirow{7}{*}{ Natural Soil } & Africa/Middle East & $-3.30 \mathrm{E}-02$ & $3.94 \mathrm{E}-02$ & $-8.54 \mathrm{E}-03$ & 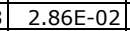 & $-4.66 \mathrm{E}-03$ & $1.05 \mathrm{E}-02$ & $-1.64 \mathrm{E}-04$ \\
\hline & Southern Asia & $-8.31 \mathrm{E}-03$ & $-5.88 \mathrm{E}-02$ & $1.07 \mathrm{E}-03$ & \begin{tabular}{|l|}
$2.33 E-02$ \\
\end{tabular} & $-2.65 \mathrm{E}-02$ & $-1.30 \mathrm{E}-02$ & $1.98 \mathrm{E}-03$ \\
\hline & Central/South America & $-2.26 \mathrm{E}-03$ & $1.06 \mathrm{E}-02$ & $-2.30 \mathrm{E}-02$ & $4.33 \mathrm{E}-02$ & $1.26 \mathrm{E}-02$ & $6.82 \mathrm{E}-03$ & $-3.52 \mathrm{E}-03$ \\
\hline & Northern Asia & $6.93 \mathrm{E}-03$ & $-1.33 \mathrm{E}-02$ & $-3.17 \mathrm{E}-04$ & $-6.54 \mathrm{E}-02$ & $4.32 \mathrm{E}-02$ & $3.39 \mathrm{E}-02$ & $-1.19 \mathrm{E}-03$ \\
\hline & Europe & $-6.65 \mathrm{E}-03$ & $1.42 \mathrm{E}-02$ & $6.47 \mathrm{E}-04$ & $6.06 \mathrm{E}-03$ & $-1.99 \mathrm{E}-01$ & $2.41 \mathrm{E}-02$ & $4.45 \mathrm{E}-04$ \\
\hline & North America & $-7.49 \mathrm{E}-04$ & $5.00 \mathrm{E}-02$ & $1.39 \mathrm{E}-03$ & \begin{tabular}{|l|} 
\\
\end{tabular} & $6.10 \mathrm{E}-03$ & $-2.06 \mathrm{E}-01$ & $6.41 \mathrm{E}-04$ \\
\hline & Oceania & $9.59 \mathrm{E}-04$ & $-4.51 \mathrm{E}-04$ & $-2.00 \mathrm{E}-03$ & \begin{tabular}{|l|}
$8.12 \mathrm{E}-04$ \\
\end{tabular} & $1.29 \mathrm{E}-03$ & $7.91 \mathrm{E}-04$ & $-4.43 \mathrm{E}-03$ \\
\hline \multirow{6}{*}{ Ocean } & North Pacific & $-3.94 \mathrm{E}-03$ & $-1.40 \mathrm{E}-02$ & $1.02 \mathrm{E}-03$ & \begin{tabular}{|l|} 
\\
\end{tabular} & $-1.47 \mathrm{E}-02$ & $-4.48 \mathrm{E}-02$ & $6.36 \mathrm{E}-04$ \\
\hline & South Pacific & $4.45 \mathrm{E}-04$ & $7.42 \mathrm{E}-03$ & $-2.47 \mathrm{E}-03$ & $\begin{array}{l}9.48 \mathrm{E}-03 \\
\end{array}$ & 7.07E-03 & $3.25 \mathrm{E}-04$ & $1.76 \mathrm{E}-03$ \\
\hline & \begin{tabular}{|l|} 
Northern Ocean \\
\end{tabular} & $1.81 \mathrm{E}-03$ & $-2.65 \mathrm{E}-03$ & $6.85 \mathrm{E}-04$ & \begin{tabular}{|l|}
$2.20 \mathrm{E}-02$ \\
\end{tabular} & $1.03 E-03$ & $2.53 \mathrm{E}-02$ & $5.40 \mathrm{E}-04$ \\
\hline & Atlantic & $-1.09 \mathrm{E}-02$ & $1.67 \mathrm{E}-03$ & $-1.59 \mathrm{E}-03$ & \begin{tabular}{|l|} 
\\
\end{tabular} & $-3.08 \mathrm{E}-02$ & $-1.20 \mathrm{E}-01$ & $1.15 \mathrm{E}-03$ \\
\hline & Southern Ocean & $3.25 \mathrm{E}-03$ & $-2.24 \mathrm{E}-02$ & $-1.23 \mathrm{E}-02$ & $-1.16 \mathrm{E}-03$ & $-2.21 \mathrm{E}-03$ & $9.86 \mathrm{E}-03$ & $-7.18 \mathrm{E}-03$ \\
\hline & Indian Ocean & $-1.67 \mathrm{E}-03$ & $1.73 \mathrm{E}-02$ & $-8.00 \mathrm{E}-03$ & \begin{tabular}{l|l|} 
& $6.94 \mathrm{E}-03$ \\
\end{tabular} & $6.20 \mathrm{E}-03$ & $1.47 \mathrm{E}-03$ & $-3.90 \mathrm{E}-03$ \\
\hline \multirow{7}{*}{$\begin{array}{l}\text { Biomass } \\
\text { burning }\end{array}$} & Africa/Middle East & $-2.91 \mathrm{E}-03$ & $9.24 \mathrm{E}-03$ & $-2.29 \mathrm{E}-03$ & \begin{tabular}{|c|}
$9.75 E-03$ \\
\end{tabular} & $1.89 \mathrm{E}-03$ & $1.27 \mathrm{E}-03$ & $4.31 \mathrm{E}-04$ \\
\hline & Southern Asia & $-5.27 \mathrm{E}-04$ & $-3.75 \mathrm{E}-03$ & $8.65 \mathrm{E}-05$ & 2.33E-03 & $1.11 \mathrm{E}-03$ & $8.24 \mathrm{E}-06$ & $8.09 \mathrm{E}-05$ \\
\hline & Central/South America & $-3.53 E-05$ & $1.87 \mathrm{E}-03$ & $-7.66 \mathrm{E}-04$ & $\begin{array}{l}8.16 \mathrm{E}-04 \\
\end{array}$ & $9.05 \mathrm{E}-04$ & $2.67 \mathrm{E}-04$ & $1.63 \mathrm{E}-04$ \\
\hline & Northern Asia & $-4.76 \mathrm{E}-04$ & $-3.20 \mathrm{E}-02$ & $1.53 \mathrm{E}-04$ & $-3.31 \mathrm{E}-03$ & $5.31 \mathrm{E}-03$ & $1.47 \mathrm{E}-02$ & $1.57 \mathrm{E}-04$ \\
\hline & Europe & $-2.13 \mathrm{E}-05$ & $1.55 \mathrm{E}-04$ & 5.37E-07 & $-2.20 \mathrm{E}-04$ & $4.69 \mathrm{E}-04$ & $2.06 \mathrm{E}-04$ & $3.24 \mathrm{E}-07$ \\
\hline & North America & $-1.20 \mathrm{E}-05$ & $4.17 \mathrm{E}-03$ & $1.37 \mathrm{E}-04$ & $1.95 \mathrm{E}-02$ & $3.10 \mathrm{E}-03$ & $-9.73 \mathrm{E}-03$ & $1.50 \mathrm{E}-04$ \\
\hline & Oceania & $1.12 \mathrm{E}-04$ & $5.50 \mathrm{E}-04$ & $-1.76 \mathrm{E}-04$ & $1.88 \mathrm{E}-04$ & $2.47 \mathrm{E}-04$ & $7.18 \mathrm{E}-05$ & $-1.83 \mathrm{E}-04$ \\
\hline
\end{tabular}

Fig. S2bn. Correlation of the emissions in 2008 for industrial. 


\begin{tabular}{|c|c|c|c|c|c|c|c|c|}
\hline & \multirow{2}{*}{\multicolumn{7}{|c|}{ Natural Soil }} \\
\hline & & & & & & & & \\
\hline Source sector & Region & $\begin{array}{l}\text { Africa/M. } \\
\text { East }\end{array}$ & $\begin{array}{l}\text { Southern } \\
\text { Asia }\end{array}$ & $\begin{array}{l}\text { C. /S. } \\
\text { America }\end{array}$ & $\begin{array}{l}\text { Northern } \\
\text { Asia }\end{array}$ & Europe & $\begin{array}{l}\text { North } \\
\text { America }\end{array}$ & Oceania \\
\hline \multirow{7}{*}{$\begin{array}{l}\text { Agricultural } \\
\text { Soil }\end{array}$} & Africa/Middle East & $-1.83 \mathrm{E}-01$ & $-7.17 \mathrm{E}-02$ & $-6.88 \mathrm{E}-02$ & \begin{tabular}{|l|}
$1.45 \mathrm{E}-02$ \\
\end{tabular} & $-2.88 \mathrm{E}-02$ & $-2.49 \mathrm{E}-02$ & $3.53 \mathrm{E}-03$ \\
\hline & Southern Asia & $-2.09 \mathrm{E}-02$ & $-4.16 \mathrm{E}-01$ & $3.15 \mathrm{E}-03$ & \begin{tabular}{|l|}
$-1.02 \mathrm{E}-01$ \\
\end{tabular} & $-5.50 \mathrm{E}-03$ & $-1.95 \mathrm{E}-02$ & $1.69 \mathrm{E}-02$ \\
\hline & Central/South America & $-8.04 \mathrm{E}-02$ & $9.36 \mathrm{E}-03$ & $-2.65 \mathrm{E}-01$ & \begin{tabular}{|l|}
$-5.54 \mathrm{E}-03$ \\
\end{tabular} & $7.84 \mathrm{E}-03$ & $1.80 \mathrm{E}-02$ & $-2.50 \mathrm{E}-02$ \\
\hline & Northern Asia & 4.84E-03 & $1.85 \mathrm{E}-02$ & $1.97 \mathrm{E}-02$ & \begin{tabular}{|l|}
$-1.85 \mathrm{E}-01$ \\
\end{tabular} & $1.12 \mathrm{E}-02$ & $9.43 \mathrm{E}-02$ & $-1.47 \mathrm{E}-02$ \\
\hline & Europe & $-1.38 \mathrm{E}-02$ & $-1.44 \mathrm{E}-02$ & $2.73 \mathrm{E}-02$ & $2.21 \mathrm{E}-03$ & $-2.52 \mathrm{E}-01$ & $2.49 \mathrm{E}-03$ & $6.05 \mathrm{E}-03$ \\
\hline & \begin{tabular}{|l|} 
North America \\
\end{tabular} & $-2.48 \mathrm{E}-03$ & $-3.92 \mathrm{E}-02$ & $1.18 \mathrm{E}-02$ & $1.08 \mathrm{E}-01$ & $1.60 \mathrm{E}-02$ & $-4.21 \mathrm{E}-01$ & $5.31 \mathrm{E}-03$ \\
\hline & Oceania & $-3.49 \mathrm{E}-03$ & $1.89 \mathrm{E}-02$ & $-3.12 \mathrm{E}-02$ & $-1.61 \mathrm{E}-02$ & $5.43 \mathrm{E}-03$ & $7.75 \mathrm{E}-03$ & $-6.49 \mathrm{E}-02$ \\
\hline \multirow{7}{*}{ Industrial } & Africa/Middle East & $-3.30 \mathrm{E}-02$ & $-8.31 \mathrm{E}-03$ & $-2.26 \mathrm{E}-03$ & $6.93 \mathrm{E}-03$ & $-6.65 \mathrm{E}-03$ & $-7.49 \mathrm{E}-04$ & $9.59 \mathrm{E}-04$ \\
\hline & \begin{tabular}{|l|} 
Southern Asia \\
\end{tabular} & $3.94 \mathrm{E}-02$ & $-5.88 \mathrm{E}-02$ & $1.06 \mathrm{E}-02$ & $-1.33 \mathrm{E}-02$ & $1.42 \mathrm{E}-02$ & $5.00 \mathrm{E}-02$ & $-4.51 \mathrm{E}-04$ \\
\hline & Central/South America & $-8.54 \mathrm{E}-03$ & $1.07 \mathrm{E}-03$ & $-2.30 \mathrm{E}-02$ & -3.17E-04 & $6.47 \mathrm{E}-04$ & $1.39 \mathrm{E}-03$ & $-2.00 \mathrm{E}-03$ \\
\hline & Northern Asia & $2.86 \mathrm{E}-02$ & $2.33 \mathrm{E}-02$ & $4.33 \mathrm{E}-02$ & \begin{tabular}{|l|}
$-6.54 \mathrm{E}-02$ \\
\end{tabular} & $6.06 \mathrm{E}-03$ & $-1.39 \mathrm{E}-01$ & $8.12 \mathrm{E}-04$ \\
\hline & Europe & $-4.66 \mathrm{E}-03$ & $-2.65 \mathrm{E}-02$ & $1.26 \mathrm{E}-02$ & $4.32 \mathrm{E}-02$ & $-1.99 \mathrm{E}-01$ & $6.10 \mathrm{E}-03$ & $1.29 \mathrm{E}-03$ \\
\hline & North America & $1.05 \mathrm{E}-02$ & $-1.30 \mathrm{E}-02$ & $6.82 \mathrm{E}-03$ & $3.39 \mathrm{E}-02$ & $2.41 \mathrm{E}-02$ & $-2.06 \mathrm{E}-01$ & $7.91 \mathrm{E}-04$ \\
\hline & Oceania & $-1.64 \mathrm{E}-04$ & $1.98 \mathrm{E}-03$ & $-3.52 \mathrm{E}-03$ & \begin{tabular}{|l|}
$-1.19 E-03$ \\
\end{tabular} & 4.45E-04 & $6.41 \mathrm{E}-04$ & $-4.43 \mathrm{E}-03$ \\
\hline \multirow{7}{*}{ Natural Soil } & Africa/Middle East & 1 & $-2.44 \mathrm{E}-01$ & $-3.65 \mathrm{E}-01$ & $3.64 \mathrm{E}-02$ & $-2.97 \mathrm{E}-02$ & $-7.47 \mathrm{E}-02$ & $-1.09 \mathrm{E}-02$ \\
\hline & Southern Asia & $-2.44 \mathrm{E}-01$ & 1 & $-3.03 E-02$ & $-3.27 \mathrm{E}-01$ & $6.34 \mathrm{E}-02$ & $1.10 \mathrm{E}-04$ & 4.43E-03 \\
\hline & Central/South America & $-3.65 \mathrm{E}-01$ & $-3.03 E-02$ & 1 & \begin{tabular}{|l|}
$-1.05 E-01$ \\
\end{tabular} & $2.62 \mathrm{E}-03$ & $-1.97 \mathrm{E}-02$ & $-8.49 \mathrm{E}-02$ \\
\hline & Northern Asia & $3.64 \mathrm{E}-02$ & $-3.27 \mathrm{E}-01$ & $-1.05 \mathrm{E}-01$ & 1 & $-1.99 \mathrm{E}-01$ & $-3.37 \mathrm{E}-01$ & $-1.89 \mathrm{E}-02$ \\
\hline & Europe & $-2.97 \mathrm{E}-02$ & $6.34 \mathrm{E}-02$ & $2.62 \mathrm{E}-03$ & \begin{tabular}{|l|}
$-1.99 \mathrm{E}-01$ \\
\end{tabular} & 1 & $-7.40 \mathrm{E}-02$ & $3.62 \mathrm{E}-03$ \\
\hline & North America & $-7.47 \mathrm{E}-02$ & $1.10 \mathrm{E}-04$ & $-1.97 \mathrm{E}-02$ & \begin{tabular}{|l|}
$-3.37 E-01$ \\
\end{tabular} & $-7.40 \mathrm{E}-02$ & 1 & 9.99E-03 \\
\hline & Oceania & $-1.09 \mathrm{E}-02$ & $4.43 \mathrm{E}-03$ & $-8.49 \mathrm{E}-02$ & $\begin{array}{ll}-1.89 E-02 \\
\end{array}$ & $3.62 \mathrm{E}-03$ & 9.99E-03 & 1 \\
\hline \multirow{6}{*}{ Ocean } & North Pacific & $4.45 \mathrm{E}-02$ & $1.80 \mathrm{E}-01$ & $2.78 \mathrm{E}-02$ & $-1.86 \mathrm{E}-01$ & $6.07 \mathrm{E}-02$ & $1.01 \mathrm{E}-01$ & $5.81 \mathrm{E}-03$ \\
\hline & South Pacific & $1.43 \mathrm{E}-02$ & $7.21 \mathrm{E}-04$ & $-5.06 \mathrm{E}-01$ & $-1.66 \mathrm{E}-02$ & $1.00 \mathrm{E}-02$ & $5.30 \mathrm{E}-02$ & $1.78 \mathrm{E}-03$ \\
\hline & Northern Ocean & $4.37 \mathrm{E}-02$ & $1.46 \mathrm{E}-01$ & $4.25 \mathrm{E}-02$ & $-9.90 \mathrm{E}-02$ & $2.16 \mathrm{E}-04$ & $3.84 \mathrm{E}-03$ & $4.94 \mathrm{E}-03$ \\
\hline & Atlantic & $-9.80 \mathrm{E}-02$ & $1.66 \mathrm{E}-01$ & $-7.40 \mathrm{E}-02$ & $1.75 \mathrm{E}-02$ & $3.29 E-02$ & $5.95 \mathrm{E}-02$ & $3.32 \mathrm{E}-03$ \\
\hline & Southern Ocean & $6.48 \mathrm{E}-02$ & $1.91 \mathrm{E}-01$ & $-1.29 \mathrm{E}-02$ & $-4.15 \mathrm{E}-02$ & $1.60 \mathrm{E}-02$ & $3.08 \mathrm{E}-02$ & $-4.73 \mathrm{E}-02$ \\
\hline & Indian Ocean & $-9.64 \mathrm{E}-02$ & $-1.65 \mathrm{E}-01$ & $-1.98 \mathrm{E}-01$ & $2.65 \mathrm{E}-02$ & $9.66 \mathrm{E}-03$ & $4.32 \mathrm{E}-02$ & $-4.37 \mathrm{E}-02$ \\
\hline \multirow{7}{*}{$\begin{array}{l}\text { Biomass } \\
\text { burning }\end{array}$} & Africa/Middle East & $-8.00 \mathrm{E}-02$ & $-4.78 \mathrm{E}-02$ & $-2.97 \mathrm{E}-02$ & $2.52 \mathrm{E}-02$ & $7.41 \mathrm{E}-04$ & $1.78 \mathrm{E}-03$ & $6.78 \mathrm{E}-04$ \\
\hline & Southern Asia & $1.80 \mathrm{E}-03$ & $1.97 \mathrm{E}-02$ & $-8.39 \mathrm{E}-03$ & $-8.33 \mathrm{E}-03$ & $-2.72 \mathrm{E}-03$ & $9.95 \mathrm{E}-04$ & $-8.34 \mathrm{E}-04$ \\
\hline & Central/South America & $-1.69 \mathrm{E}-02$ & $-1.43 \mathrm{E}-02$ & $-2.11 \mathrm{E}-03$ & $8.93 \mathrm{E}-03$ & $1.71 \mathrm{E}-03$ & $7.25 \mathrm{E}-03$ & $2.07 \mathrm{E}-04$ \\
\hline & Northern Asia & $5.20 \mathrm{E}-03$ & $6.67 \mathrm{E}-02$ & $5.87 \mathrm{E}-03$ & $-1.00 \mathrm{E}-01$ & $-1.68 \mathrm{E}-02$ & $-3.57 \mathrm{E}-02$ & $1.13 \mathrm{E}-03$ \\
\hline & Europe & $-1.61 \mathrm{E}-04$ & $-5.14 \mathrm{E}-04$ & $1.20 \mathrm{E}-04$ & $-1.13 \mathrm{E}-03$ & $-5.81 \mathrm{E}-03$ & $-7.88 \mathrm{E}-04$ & $1.50 \mathrm{E}-05$ \\
\hline & North America & $-6.20 \mathrm{E}-04$ & $3.40 \mathrm{E}-02$ & $-2.45 \mathrm{E}-03$ & $-7.60 \mathrm{E}-02$ & $-1.77 \mathrm{E}-02$ & $-1.09 \mathrm{E}-01$ & 4.49E-04 \\
\hline & Oceania & $-1.51 \mathrm{E}-03$ & $-5.08 \mathrm{E}-03$ & $-5.16 \mathrm{E}-03$ & $-7.83 \mathrm{E}-06$ & $6.02 \mathrm{E}-04$ & $2.12 \mathrm{E}-03$ & $-3.21 \mathrm{E}-03$ \\
\hline
\end{tabular}

Fig. S2bo. Correlation of the emissions in 2008 for natural soil. 


\begin{tabular}{|c|c|c|c|c|c|c|c|}
\hline & \multirow{2}{*}{\multicolumn{6}{|c|}{ Ocean }} \\
\hline & & & & & & & \\
\hline Source sector & Region & $\begin{array}{l}\text { North } \\
\text { Pacific }\end{array}$ & $\begin{array}{l}\text { South } \\
\text { Pacific }\end{array}$ & $\begin{array}{l}\text { Northern } \\
\text { Ocean }\end{array}$ & Atlantic & $\begin{array}{l}\text { Southern } \\
\text { Ocean }\end{array}$ & $\begin{array}{l}\text { Indian } \\
\text { Ocean }\end{array}$ \\
\hline \multirow{7}{*}{$\begin{array}{l}\text { Agricultural } \\
\text { Soil }\end{array}$} & Africa/Middle East & $-1.34 \mathrm{E}-02$ & $-2.08 \mathrm{E}-03$ & $2.23 \mathrm{E}-03$ & $-8.03 E-02$ & $2.58 \mathrm{E}-02$ & $-3.33 \mathrm{E}-02$ \\
\hline & Southern Asia & $-1.89 \mathrm{E}-01$ & $1.51 \mathrm{E}-02$ & $-3.66 \mathrm{E}-02$ & $-1.46 \mathrm{E}-01$ & $6.16 \mathrm{E}-02$ & $1.51 \mathrm{E}-02$ \\
\hline & Central/South America & $1.10 \mathrm{E}-02$ & $-3.13 E-02$ & $6.80 \mathrm{E}-03$ & $-1.49 \mathrm{E}-02$ & $-1.88 \mathrm{E}-01$ & $-9.79 \mathrm{E}-02$ \\
\hline & \begin{tabular}{|l|} 
Northern Asia \\
\end{tabular} & $1.85 \mathrm{E}-02$ & $9.24 \mathrm{E}-03$ & $-5.15 E-02$ & $-2.05 \mathrm{E}-02$ & $-2.66 \mathrm{E}-02$ & $-3.06 \mathrm{E}-03$ \\
\hline & Europe & $2.39 \mathrm{E}-03$ & $1.33 \mathrm{E}-02$ & $\mid-7.98 \mathrm{E}-02$ & $-5.48 \mathrm{E}-02$ & $6.17 \mathrm{E}-03$ & $9.41 \mathrm{E}-03$ \\
\hline & North America & $-4.51 \mathrm{E}-02$ & $1.38 \mathrm{E}-02$ & $-2.41 \mathrm{E}-02$ & $-9.25 \mathrm{E}-02$ & $6.81 \mathrm{E}-03$ & $1.52 \mathrm{E}-02$ \\
\hline & Oceania & $8.57 \mathrm{E}-03$ & $3.32 \mathrm{E}-03$ & $7.44 \mathrm{E}-03$ & $1.38 \mathrm{E}-02$ & $-9.68 \mathrm{E}-02$ & $-3.51 \mathrm{E}-02$ \\
\hline \multirow{7}{*}{ Industrial } & Africa/Middle East & $-3.94 \mathrm{E}-03$ & $4.45 \mathrm{E}-04$ & $1.81 \mathrm{E}-03$ & $-1.09 \mathrm{E}-02$ & $3.25 \mathrm{E}-03$ & $-1.67 \mathrm{E}-03$ \\
\hline & Southern Asia & $-1.40 \mathrm{E}-02$ & $7.42 \mathrm{E}-03$ & $-2.65 \mathrm{E}-03$ & $1.67 \mathrm{E}-03$ & $-2.24 \mathrm{E}-02$ & $1.73 \mathrm{E}-02$ \\
\hline & Central/South America & $1.02 \mathrm{E}-03$ & $-2.47 \mathrm{E}-03$ & $6.85 \mathrm{E}-04$ & $-1.59 \mathrm{E}-03$ & $-1.23 \mathrm{E}-02$ & $-8.00 \mathrm{E}-03$ \\
\hline & Northern Asia & $4.06 \mathrm{E}-02$ & $9.48 \mathrm{E}-03$ & $2.20 \mathrm{E}-02$ & $-1.98 \mathrm{E}-01$ & $-1.16 \mathrm{E}-03$ & $6.94 \mathrm{E}-03$ \\
\hline & Europe & $-1.47 \mathrm{E}-02$ & 7.07E-03 & $1.03 \mathrm{E}-03$ & $-3.08 \mathrm{E}-02$ & $-2.21 \mathrm{E}-03$ & $6.20 \mathrm{E}-03$ \\
\hline & North America & $-4.48 \mathrm{E}-02$ & $3.25 \mathrm{E}-04$ & $2.53 \mathrm{E}-02$ & $-1.20 \mathrm{E}-01$ & $9.86 \mathrm{E}-03$ & $1.47 \mathrm{E}-03$ \\
\hline & Oceania & $6.36 \mathrm{E}-04$ & $1.76 \mathrm{E}-03$ & $5.40 \mathrm{E}-04$ & $1.15 \mathrm{E}-03$ & $-7.18 \mathrm{E}-03$ & $-3.90 \mathrm{E}-03$ \\
\hline \multirow{7}{*}{ Natural Soil } & Africa/Middle East & $4.45 \mathrm{E}-02$ & $1.43 \mathrm{E}-02$ & $4.37 \mathrm{E}-02$ & $-9.80 \mathrm{E}-02$ & $6.48 \mathrm{E}-02$ & $-9.64 \mathrm{E}-02$ \\
\hline & Southern Asia & $1.80 \mathrm{E}-01$ & $7.21 \mathrm{E}-04$ & $1.46 \mathrm{E}-01$ & $1.66 \mathrm{E}-01$ & $1.91 \mathrm{E}-01$ & $-1.65 \mathrm{E}-01$ \\
\hline & Central/South America & $2.78 \mathrm{E}-02$ & $-5.06 \mathrm{E}-01$ & $4.25 \mathrm{E}-02$ & $-7.40 \mathrm{E}-02$ & $-1.29 \mathrm{E}-02$ & $-1.98 \mathrm{E}-01$ \\
\hline & Northern Asia & $-1.86 \mathrm{E}-01$ & $-1.66 \mathrm{E}-02$ & \begin{tabular}{|l|}
$-9.90 \mathrm{E}-02$ \\
\end{tabular} & $1.75 \mathrm{E}-02$ & $-4.15 \mathrm{E}-02$ & $2.65 \mathrm{E}-02$ \\
\hline & Europe & 6.07E-02 & $1.00 \mathrm{E}-02$ & $2.16 \mathrm{E}-04$ & $3.29 \mathrm{E}-02$ & $1.60 \mathrm{E}-02$ & $9.66 \mathrm{E}-03$ \\
\hline & North America & $1.01 \mathrm{E}-01$ & $5.30 \mathrm{E}-02$ & $3.84 \mathrm{E}-03$ & $5.95 \mathrm{E}-02$ & $3.08 \mathrm{E}-02$ & $4.32 \mathrm{E}-02$ \\
\hline & Oceania & $5.81 \mathrm{E}-03$ & $1.78 \mathrm{E}-03$ & $4.94 \mathrm{E}-03$ & $3.32 \mathrm{E}-03$ & $-4.73 \mathrm{E}-02$ & $-4.37 \mathrm{E}-02$ \\
\hline \multirow{6}{*}{ Ocean } & North Pacific & 1 & $3.81 \mathrm{E}-03$ & $-7.00 \mathrm{E}-02$ & $-1.60 \mathrm{E}-01$ & $2.93 \mathrm{E}-02$ & $1.78 \mathrm{E}-02$ \\
\hline & South Pacific & $3.81 \mathrm{E}-03$ & 1 & $5.09 \mathrm{E}-03$ & $-3.33 E-02$ & $1.75 \mathrm{E}-02$ & $1.84 \mathrm{E}-02$ \\
\hline & Northern Ocean & $-7.00 \mathrm{E}-02$ & $5.09 \mathrm{E}-03$ & 1 & $-2.97 \mathrm{E}-01$ & $3.47 \mathrm{E}-02$ & $-1.06 \mathrm{E}-03$ \\
\hline & Atlantic & $-1.60 \mathrm{E}-01$ & $-3.33 \mathrm{E}-02$ & \begin{tabular}{|l|}
$-2.97 \mathrm{E}-01$ \\
\end{tabular} & 1 & $-3.92 \mathrm{E}-02$ & $-3.95 \mathrm{E}-02$ \\
\hline & Southern Ocean & $2.93 \mathrm{E}-02$ & $1.75 \mathrm{E}-02$ & $3.47 \mathrm{E}-02$ & $-3.92 \mathrm{E}-02$ & 1 & $-6.12 \mathrm{E}-01$ \\
\hline & Indian Ocean & $1.78 \mathrm{E}-02$ & $1.84 \mathrm{E}-02$ & \begin{tabular}{|l|}
$-1.06 \mathrm{E}-03$ \\
\end{tabular} & $-3.95 \mathrm{E}-02$ & $-6.12 \mathrm{E}-01$ & \\
\hline \multirow{7}{*}{$\begin{array}{l}\text { Biomass } \\
\text { burning }\end{array}$} & Africa/Middle East & $-1.26 \mathrm{E}-03$ & $-4.47 \mathrm{E}-02$ & \begin{tabular}{|l|}
$-2.01 \mathrm{E}-03$ \\
\end{tabular} & $-5.22 \mathrm{E}-02$ & $-5.51 \mathrm{E}-03$ & $-5.36 \mathrm{E}-02$ \\
\hline & Southern Asia & $-7.23 \mathrm{E}-03$ & $-3.37 \mathrm{E}-03$ & \begin{tabular}{|l|}
$-1.74 \mathrm{E}-03$ \\
\end{tabular} & $-9.21 \mathrm{E}-03$ & $7.10 \mathrm{E}-03$ & $-4.74 \mathrm{E}-03$ \\
\hline & Central/South America & $-5.59 \mathrm{E}-04$ & $-2.93 \mathrm{E}-02$ & $-2.33 \mathrm{E}-03$ & $-1.09 \mathrm{E}-02$ & $-7.86 \mathrm{E}-03$ & $-1.74 \mathrm{E}-02$ \\
\hline & Northern Asia & $-1.94 \mathrm{E}-01$ & $5.64 \mathrm{E}-03$ & $\mid-1.65 \mathrm{E}-02$ & $-6.42 \mathrm{E}-03$ & $3.67 \mathrm{E}-03$ & $7.47 \mathrm{E}-03$ \\
\hline & Europe & $6.52 \mathrm{E}-04$ & $1.27 \mathrm{E}-04$ & $4.96 \mathrm{E}-04$ & $8.50 \mathrm{E}-04$ & $-5.26 \mathrm{E}-05$ & $8.79 E-05$ \\
\hline & North America & $-1.48 \mathrm{E}-02$ & $3.43 \mathrm{E}-03$ & \begin{tabular}{|l|}
$-1.59 \mathrm{E}-02$ \\
\end{tabular} & $1.04 \mathrm{E}-02$ & $6.57 \mathrm{E}-03$ & $1.17 \mathrm{E}-03$ \\
\hline & Oceania & $3.21 \mathrm{E}-04$ & $-9.88 \mathrm{E}-04$ & \begin{tabular}{|l|}
$-1.77 E-04$ \\
\end{tabular} & $-9.89 E-04$ & $7.22 \mathrm{E}-04$ & $-6.95 \mathrm{E}-03$ \\
\hline
\end{tabular}

Fig. S2bp. Correlation of the emissions in 2008 for ocean. 


\begin{tabular}{|c|c|c|c|c|c|c|c|c|}
\hline & \multirow{2}{*}{\multicolumn{7}{|c|}{ Biomass burning }} \\
\hline & & & & & & & & \\
\hline Source sector & Region & $\begin{array}{l}\text { Africa/M. } \\
\text { East }\end{array}$ & $\begin{array}{l}\text { Southern } \\
\text { Asia }\end{array}$ & $\begin{array}{l}\text { C. /S. } \\
\text { America }\end{array}$ & $\begin{array}{l}\text { Northern } \\
\text { Asia }\end{array}$ & Europe & $\begin{array}{l}\text { North } \\
\text { America }\end{array}$ & Oceania \\
\hline \multirow{7}{*}{$\begin{array}{l}\text { Agricultural } \\
\text { Soil }\end{array}$} & Africa/Middle East & $-2.64 \mathrm{E}-02$ & $-3.05 \mathrm{E}-03$ & $-2.24 \mathrm{E}-03$ & $-1.86 \mathrm{E}-03$ & $-2.50 \mathrm{E}-04$ & $-1.65 \mathrm{E}-03$ & $2.96 \mathrm{E}-04$ \\
\hline & Southern Asia & $-1.02 \mathrm{E}-02$ & $-3.10 \mathrm{E}-02$ & $7.99 \mathrm{E}-03$ & $-6.19 \mathrm{E}-02$ & $2.85 \mathrm{E}-04$ & $-4.55 \mathrm{E}-03$ & $2.81 \mathrm{E}-03$ \\
\hline & Central/South America & $-2.16 \mathrm{E}-02$ & $8.55 \mathrm{E}-04$ & $-7.73 \mathrm{E}-03$ & $2.04 \mathrm{E}-03$ & $1.04 \mathrm{E}-05$ & $1.99 \mathrm{E}-03$ & $-2.13 \mathrm{E}-03$ \\
\hline & Northern Asia & $2.96 \mathrm{E}-03$ & $4.97 \mathrm{E}-03$ & $-2.43 \mathrm{E}-04$ & $-1.64 \mathrm{E}-02$ & $8.93 E-05$ & $1.33 \mathrm{E}-02$ & $-1.85 \mathrm{E}-03$ \\
\hline & Europe & $9.66 \mathrm{E}-05$ & $1.38 \mathrm{E}-03$ & $1.00 \mathrm{E}-03$ & $1.06 \mathrm{E}-02$ & $-9.95 \mathrm{E}-05$ & $7.16 \mathrm{E}-04$ & $7.68 \mathrm{E}-04$ \\
\hline & North America & $-5.04 \mathrm{E}-03$ & $-1.03 \mathrm{E}-04$ & $7.07 \mathrm{E}-04$ & $1.26 \mathrm{E}-02$ & $2.19 \mathrm{E}-04$ & $1.62 \mathrm{E}-02$ & $6.46 \mathrm{E}-04$ \\
\hline & Oceania & $4.42 \mathrm{E}-03$ & $9.62 \mathrm{E}-04$ & $1.68 \mathrm{E}-03$ & $2.12 \mathrm{E}-03$ & $2.84 \mathrm{E}-06$ & $1.76 \mathrm{E}-03$ & $-2.37 \mathrm{E}-03$ \\
\hline \multirow{7}{*}{ Industrial } & Africa/Middle East & $-2.91 \mathrm{E}-03$ & $-5.27 \mathrm{E}-04$ & $-3.53 \mathrm{E}-05$ & $-4.76 \mathrm{E}-04$ & $-2.13 \mathrm{E}-05$ & $-1.20 \mathrm{E}-05$ & $1.12 \mathrm{E}-04$ \\
\hline & Southern Asia & $9.24 \mathrm{E}-03$ & $-3.75 \mathrm{E}-03$ & $1.87 \mathrm{E}-03$ & $-3.20 \mathrm{E}-02$ & $1.55 \mathrm{E}-04$ & $4.17 \mathrm{E}-03$ & $5.50 \mathrm{E}-04$ \\
\hline & Central/South America & $-2.29 \mathrm{E}-03$ & $8.65 \mathrm{E}-05$ & $-7.66 \mathrm{E}-04$ & $1.53 \mathrm{E}-04$ & $5.37 \mathrm{E}-07$ & $1.37 \mathrm{E}-04$ & $-1.76 \mathrm{E}-04$ \\
\hline & Northern Asia & $9.75 \mathrm{E}-03$ & $2.33 \mathrm{E}-03$ & $8.16 \mathrm{E}-04$ & $\mid-3.31 \mathrm{E}-03$ & $-2.20 \mathrm{E}-04$ & $1.95 \mathrm{E}-02$ & $1.88 \mathrm{E}-04$ \\
\hline & Europe & 1.89E-03 & $1.11 \mathrm{E}-03$ & $9.05 \mathrm{E}-04$ & $5.31 \mathrm{E}-03$ & 4.69E-04 & $3.10 \mathrm{E}-03$ & $2.47 \mathrm{E}-04$ \\
\hline & North America & $1.27 \mathrm{E}-03$ & $8.24 \mathrm{E}-06$ & $2.67 E-04$ & $1.47 \mathrm{E}-02$ & $2.06 \mathrm{E}-04$ & $-9.73 \mathrm{E}-03$ & $7.18 \mathrm{E}-05$ \\
\hline & Oceania & $4.31 \mathrm{E}-04$ & $8.09 E-05$ & $1.63 \mathrm{E}-04$ & 1.57E-04 & $3.24 \mathrm{E}-07$ & $1.50 \mathrm{E}-04$ & $-1.83 \mathrm{E}-04$ \\
\hline \multirow{7}{*}{ Natural Soil } & Africa/Middle East & $-8.00 \mathrm{E}-02$ & $1.80 \mathrm{E}-03$ & $-1.69 \mathrm{E}-02$ & $5.20 \mathrm{E}-03$ & $-1.61 \mathrm{E}-04$ & $-6.20 \mathrm{E}-04$ & $-1.51 \mathrm{E}-03$ \\
\hline & Southern Asia & $-4.78 \mathrm{E}-02$ & $1.97 \mathrm{E}-02$ & $-1.43 \mathrm{E}-02$ & $6.67 \mathrm{E}-02$ & $-5.14 \mathrm{E}-04$ & $3.40 \mathrm{E}-02$ & $-5.08 \mathrm{E}-03$ \\
\hline & Central/South America & $-2.97 \mathrm{E}-02$ & $-8.39 \mathrm{E}-03$ & $-2.11 \mathrm{E}-03$ & $5.87 \mathrm{E}-03$ & $1.20 \mathrm{E}-04$ & $-2.45 \mathrm{E}-03$ & $-5.16 \mathrm{E}-03$ \\
\hline & Northern Asia & $2.52 \mathrm{E}-02$ & $-8.33 \mathrm{E}-03$ & $8.93 \mathrm{E}-03$ & $-1.00 \mathrm{E}-01$ & $-1.13 \mathrm{E}-03$ & $-7.60 \mathrm{E}-02$ & $-7.83 \mathrm{E}-06$ \\
\hline & Europe & $7.41 \mathrm{E}-04$ & $-2.72 \mathrm{E}-03$ & $1.71 \mathrm{E}-03$ & \begin{tabular}{|l|}
$-1.68 \mathrm{E}-02$ \\
\end{tabular} & $-5.81 \mathrm{E}-03$ & $-1.77 \mathrm{E}-02$ & $6.02 \mathrm{E}-04$ \\
\hline & North America & $1.78 \mathrm{E}-03$ & $9.95 \mathrm{E}-04$ & $7.25 \mathrm{E}-03$ & \begin{tabular}{|l|}
$-3.57 \mathrm{E}-02$ \\
\end{tabular} & $-7.88 \mathrm{E}-04$ & $-1.09 \mathrm{E}-01$ & $2.12 \mathrm{E}-03$ \\
\hline & Oceania & $6.78 \mathrm{E}-04$ & $-8.34 \mathrm{E}-04$ & $2.07 \mathrm{E}-04$ & $1.13 \mathrm{E}-03$ & $1.50 \mathrm{E}-05$ & $4.49 \mathrm{E}-04$ & $-3.21 \mathrm{E}-03$ \\
\hline \multirow{6}{*}{ Ocean } & North Pacific & $-1.26 \mathrm{E}-03$ & $-7.23 \mathrm{E}-03$ & $-5.59 \mathrm{E}-04$ & $-1.94 \mathrm{E}-01$ & $6.52 \mathrm{E}-04$ & $-1.48 \mathrm{E}-02$ & $3.21 \mathrm{E}-04$ \\
\hline & \begin{tabular}{|l} 
South Pacific \\
\end{tabular} & $-4.47 \mathrm{E}-02$ & $-3.37 \mathrm{E}-03$ & $-2.93 \mathrm{E}-02$ & $5.64 \mathrm{E}-03$ & $1.27 \mathrm{E}-04$ & $3.43 \mathrm{E}-03$ & $-9.88 \mathrm{E}-04$ \\
\hline & \begin{tabular}{|l|} 
Northern Ocean \\
\end{tabular} & $-2.01 \mathrm{E}-03$ & $-1.74 \mathrm{E}-03$ & $-2.33 E-03$ & $-1.65 \mathrm{E}-02$ & $4.96 \mathrm{E}-04$ & $-1.59 \mathrm{E}-02$ & $-1.77 \mathrm{E}-04$ \\
\hline & Atlantic & $-5.22 \mathrm{E}-02$ & $-9.21 \mathrm{E}-03$ & $-1.09 \mathrm{E}-02$ & $-6.42 \mathrm{E}-03$ & $8.50 \mathrm{E}-04$ & $\begin{array}{ll}1.04 \mathrm{E}-02 \\
\end{array}$ & $-9.89 \mathrm{E}-04$ \\
\hline & Southern Ocean & $-5.51 \mathrm{E}-03$ & $7.10 \mathrm{E}-03$ & $-7.86 \mathrm{E}-03$ & $3.67 \mathrm{E}-03$ & $-5.26 \mathrm{E}-05$ & \begin{tabular}{|l|l|}
5 & $6.57 \mathrm{E}-03$ \\
\end{tabular} & $7.22 \mathrm{E}-04$ \\
\hline & Indian Ocean & $-5.36 \mathrm{E}-02$ & $-4.74 \mathrm{E}-03$ & $-1.74 \mathrm{E}-02$ & $7.47 \mathrm{E}-03$ & $8.79 \mathrm{E}-05$ & 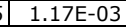 & $-6.95 \mathrm{E}-03$ \\
\hline \multirow{7}{*}{$\begin{array}{l}\text { Biomass } \\
\text { burning }\end{array}$} & Africa/Middle East & 1 & $-9.07 E-04$ & $-1.41 \mathrm{E}-02$ & $5.66 \mathrm{E}-04$ & $2.12 \mathrm{E}-05$ & $1.89 \mathrm{E}-03$ & $-1.16 \mathrm{E}-03$ \\
\hline & Southern Asia & $-9.07 E-04$ & 1 & $4.76 \mathrm{E}-04$ & $-3.85 \mathrm{E}-03$ & $4.05 E-05$ & \begin{tabular}{|l|}
$-5.54 \mathrm{E}-04$ \\
\end{tabular} & $-1.50 \mathrm{E}-04$ \\
\hline & Central/South America & $-1.41 \mathrm{E}-02$ & $4.76 \mathrm{E}-04$ & 1 & $-2.33 \mathrm{E}-04$ & $2.62 \mathrm{E}-05$ & \begin{tabular}{|l|l|}
$3.93 \mathrm{E}-04$ \\
\end{tabular} & $-7.06 \mathrm{E}-04$ \\
\hline & Northern Asia & $5.66 \mathrm{E}-04$ & $-3.85 \mathrm{E}-03$ & $-2.33 \mathrm{E}-04$ & 1 & $-3.52 \mathrm{E}-04$ & $-6.84 \mathrm{E}-03$ & $6.51 \mathrm{E}-05$ \\
\hline & \begin{tabular}{|l} 
Europe \\
\end{tabular} & $2.12 \mathrm{E}-05$ & $4.05 \mathrm{E}-05$ & $2.62 \mathrm{E}-05$ & $-3.52 \mathrm{E}-04$ & 1 & $-2.56 \mathrm{E}-04$ & 4.99E-06 \\
\hline & North America & $1.89 \mathrm{E}-03$ & $-5.54 \mathrm{E}-04$ & 3.93E-04 & $-6.84 \mathrm{E}-03$ & $-2.56 \mathrm{E}-04$ & 1 & $8.16 \mathrm{E}-05$ \\
\hline & Oceania & $-1.16 \mathrm{E}-03$ & $-1.50 \mathrm{E}-04$ & $-7.06 \mathrm{E}-04$ & $6.51 \mathrm{E}-05$ & $4.99 \mathrm{E}-06$ & \begin{tabular}{|l|}
$8.16 \mathrm{E}-05$ \\
\end{tabular} & 1 \\
\hline
\end{tabular}

\section{Fig. S2bq. Correlation of the emissions in 2008 for biomass burning.}


Table S1a. Root-mean-square deviations of the differences between measurements and modeled mixing ratios. The first column shows the model results using prior emissions and the second column shows the same with posterior emissions.

\begin{tabular}{ccc}
\hline Station & prior mixing ratio RMSD & posterior mixing ratio RMSD \\
\hline HAT & 9.24 & 2.72 \\
OCI & 11.38 & 7.83 \\
BRW & 17.86 & 6.49 \\
MLO & 19.96 & 4.31 \\
NWR & 31.18 & 11.87 \\
SMO & 18.28 & 3.33 \\
SPO & 16.56 & 4.16 \\
SUM & 11.42 & 1.34 \\
ALT & 18.55 & 6.36 \\
ASC & 15.02 & 0.99 \\
ASK & 18.69 & 2.70 \\
AZR & 16.43 & 1.91 \\
BAL & 15.05 & 3.82 \\
BHD & 2.77 & 2.69 \\
BME & 17.10 & 2.36 \\
BSC & 17.05 & 4.34 \\
CBA & 16.58 & 2.21 \\
CGO & 17.89 & 2.60 \\
CHR & 14.11 & 2.10 \\
CMO & 5.69 & 1.19 \\
CRZ & 11.54 & 3.18 \\
DRP2 & 12.92 & 2.65 \\
EIC & 14.27 & 1.37 \\
GMI & 17.36 & 1.81 \\
GOZ & 7.38 & 1.86 \\
HBA & 12.77 & 5.22 \\
HSU & 26.11 & 5.97 \\
HUN & 18.29 & 4.74 \\
ICE1 & 15.45 & 2.30 \\
ITN & 12.15 & 3.24 \\
IZO & 19.11 & 3.45 \\
KEY & 16.48 & 2.43 \\
KUM & 17.86 & 4.35 \\
KZD1 & 16.03 & 2.19 \\
KZM & 17.33 & 3.03 \\
LEF2 & 13.85 & 3.97 \\
\hline & & \\
\hline
\end{tabular}


Table S1b. Root-mean-square deviations of the differences between measurements and modeled mixing ratios. The first column shows the model results using prior emissions and the second column shows the same with posterior emissions.

\begin{tabular}{ccc}
\hline Station & prior mixing ratio RMSD & posterior mixing ratio RMSD \\
\hline MBC & 4.11 & 0.66 \\
MHD & 22.56 & 3.33 \\
MID & 16.10 & 1.69 \\
MVY & 10.30 & 6.29 \\
NWR & 18.85 & 6.67 \\
POC0003 & 2.82 & 0.23 \\
POC0005 & 4.52 & 0.93 \\
POC0007 & 3.92 & 0.41 \\
POC0008 & 3.89 & 0.71 \\
POC0009 & 3.20 & 0.86 \\
POC00010 & 8.59 & 1.35 \\
POC00013 & 2.77 & 0.15 \\
POC00014 & 4.04 & 0.50 \\
POC00015 & 3.91 & 0.43 \\
POC00016 & 7.11 & 1.14 \\
POC00017 & 2.68 & 0.13 \\
POC00019 & 7.18 & 2.03 \\
POC00020 & 4.67 & 1.17 \\
POCN053 & 2.92 & 0.33 \\
POCN054 & 4.78 & 0.58 \\
POCN056 & 8.23 & 1.51 \\
POCN057 & 9.99 & 1.43 \\
POCN058 & 2.89 & 0.55 \\
POCN0512 & 5.69 & 0.70 \\
POCN0513 & 7.82 & 1.31 \\
POCN0514 & 6.85 & 1.06 \\
POCN0515 & 2.47 & 0.15 \\
POCN102 & 2.78 & 0.18 \\
POCN103 & 5.54 & 0.52 \\
POCN107 & 3.04 & 0.59 \\
POCN108 & 7.19 & 1.51 \\
POCN109 & 8.70 & 1.09 \\
POCN1012 & 4.27 & 0.63 \\
POCN1014 & 8.20 & 1.43 \\
POCN1015 & 7.49 & 1.31 \\
POCN1016 & 4.02 & 0.53 \\
\hline
\end{tabular}


Table S1c. Root-mean-square deviations of the differences between measurements and modeled mixing ratios. The first column shows the model results using prior emissions and the second column shows the same with posterior emissions.

\begin{tabular}{ccc}
\hline Station & prior mixing ratio RMSD & posterior mixing ratio RMSD \\
\hline POCN153 & 2.44 & 0.16 \\
POCN154 & 4.91 & 0.35 \\
POCN158 & 6.77 & 1.08 \\
POCN159 & 8.73 & 2.25 \\
POCN1510 & 3.40 & 0.57 \\
POCN1512 & 2.08 & 0.15 \\
POCN1515 & 8.95 & 1.43 \\
POCN1518 & 3.79 & 0.29 \\
POCN202 & 4.65 & 0.67 \\
POCN207 & 6.43 & 1.00 \\
POCN208 & 6.37 & 1.55 \\
POCN209 & 3.02 & 2.73 \\
POCN2010 & 2.12 & 0.16 \\
POCN2012 & 8.22 & 1.34 \\
POCN2013 & 7.26 & 1.18 \\
POCN2015 & 2.56 & 0.04 \\
POCN252 & 2.86 & 0.26 \\
POCN253 & 2.97 & 0.56 \\
POCN255 & 3.44 & 0.68 \\
POCN257 & 3.34 & 0.83 \\
POCN258 & 5.86 & 0.71 \\
POCN2510 & 7.29 & 1.27 \\
POCN2511 & 6.70 & 1.10 \\
POCN2512 & 3.67 & 0.76 \\
POCN2513 & 3.96 & 0.69 \\
POCN2514 & 7.48 & 1.08 \\
POCN2515 & 2.64 & 0.02 \\
POCN304 & 3.09 & 0.47 \\
POCN305 & 2.16 & 0.45 \\
POCN307 & 6.69 & 1.24 \\
POCN3010 & 5.94 & 0.70 \\
POCN3012 & 7.89 & 1.18 \\
POCN3013 & 2.46 & 0.82 \\
POCN3014 & 7.29 & 0.89 \\
POCN352 & 1.51 & 0.70 \\
POCN353 & 3.36 & 0.55 \\
\hline
\end{tabular}


Table S1d. Root-mean-square deviations of the differences between measurements and modeled mixing ratios. The first column shows the model results using prior emissions and the second column shows the same with posterior emissions.

\begin{tabular}{|c|c|c|}
\hline Station & prior mixing ratio RMSD & posterior mixing ratio RMSD \\
\hline POCN354 & 5.03 & 0.55 \\
\hline POCN355 & 7.10 & 0.75 \\
\hline POCS051 & 2.91 & 0.54 \\
\hline POCS052 & 2.85 & 0.22 \\
\hline POCS054 & 3.08 & 0.51 \\
\hline POCS055 & 4.27 & 0.71 \\
\hline POCS056 & 2.73 & 0.25 \\
\hline POCS057 & 7.56 & 0.88 \\
\hline POCS058 & 6.41 & 0.66 \\
\hline POCS0512 & 2.62 & 0.04 \\
\hline POCS0513 & 7.56 & 0.89 \\
\hline POCS0514 & 2.82 & 0.28 \\
\hline POCS0515 & 7.52 & 1.12 \\
\hline POCS0516 & 2.84 & 0.26 \\
\hline POCS103 & 2.16 & 0.38 \\
\hline POCS104 & 8.13 & 0.89 \\
\hline POCS105 & 3.61 & 1.31 \\
\hline POCS1010 & 7.56 & 1.25 \\
\hline POCS1011 & 2.93 & 0.41 \\
\hline POCS1012 & 2.45 & 0.07 \\
\hline POCS1013 & 6.60 & 0.75 \\
\hline POCS154 & 8.47 & 1.14 \\
\hline POCS155 & 5.25 & 1.18 \\
\hline POCS156 & 2.81 & 0.34 \\
\hline POCS1514 & 7.47 & 0.83 \\
\hline POCS1515 & 3.98 & 0.52 \\
\hline POCS1516 & 4.93 & 1.02 \\
\hline POCS204 & 9.84 & 1.41 \\
\hline POCS206 & 2.16 & 0.38 \\
\hline POCS2010 & 2.39 & 0.14 \\
\hline POCS2011 & 7.12 & 1.17 \\
\hline POCS2014 & 4.87 & 0.28 \\
\hline POCS2015 & 4.73 & 0.38 \\
\hline POCS251 & 13.46 & 2.51 \\
\hline POCS257 & 2.57 & 0.04 \\
\hline POCS258 & 9.21 & 1.68 \\
\hline POCS259 & 1.30 & 1.03 \\
\hline
\end{tabular}


Table S1e. Root-mean-square deviations of the differences between measurements and modeled mixing ratios. The first column shows the model results using prior emissions and the second column shows the same with posterior emissions.

\begin{tabular}{ccc}
\hline Station & prior mixing ratio RMSD & posterior mixing ratio RMSD \\
\hline POCS2510 & 2.27 & 0.27 \\
POCS2513 & 2.31 & 0.21 \\
POCS2514 & 6.59 & 1.04 \\
POCS2516 & 5.48 & 0.67 \\
POCS2517 & 4.20 & 0.80 \\
POCS301 & 13.90 & 3.54 \\
POCS307 & 0.91 & 1.33 \\
POCS308 & 8.53 & 1.87 \\
POCS309 & 2.45 & 0.08 \\
POCS3012 & 2.19 & 0.34 \\
POCS3013 & 6.68 & 1.05 \\
POCS3014 & 5.52 & 0.65 \\
POCS3016 & 1.95 & 0.43 \\
POCS351 & 12.53 & 3.62 \\
POCS352 & 5.91 & 0.79 \\
POCS353 & 6.63 & 1.30 \\
POCS355 & 2.92 & 0.52 \\
POCS356 & 4.18 & 0.78 \\
POCS357 & 8.69 & 1.68 \\
POCS358 & 3.38 & 0.14 \\
POCS359 & 8.24 & 1.11 \\
POCS3510 & 3.24 & 0.29 \\
PTA & 11.26 & 2.54 \\
RPB & 25.02 & 4.15 \\
SCSN03 & 8.17 & 2.07 \\
SCSN06 & 9.75 & 0.99 \\
SCSN09 & 10.32 & 1.75 \\
SCSN12 & 9.28 & 1.47 \\
SCSN15 & 10.76 & 1.48 \\
SCSN18 & 10.03 & 1.56 \\
SCSN21 & 9.65 & 1.75 \\
SEY & 14.95 & 1.26 \\
SHM & 15.09 & 2.51 \\
STM & 14.69 & 2.73 \\
SYO & 13.63 & 3.18 \\
TAP & 12.66 & 4.71 \\
TDF & 10.66 & 2.59 \\
\hline
\end{tabular}


Table S1f. Root-mean-square deviations of the differences between measurements and modeled mixing ratios. The first column shows the model results using prior emissions and the second column shows the same with posterior emissions.

\begin{tabular}{|c|c|c|}
\hline Station & prior mixing ratio RMSD & posterior mixing ratio RMSD \\
\hline UTA & 16.87 & 2.20 \\
\hline UUM & 16.92 & 2.08 \\
\hline WIS & 16.90 & 2.55 \\
\hline WPC3 & 18.40 & 5.89 \\
\hline WPC5 & 18.79 & 3.07 \\
\hline WPC7 & 16.47 & 5.77 \\
\hline WPC8 & 16.84 & 4.33 \\
\hline WPC12 & 16.54 & 3.41 \\
\hline WPC15 & 13.74 & 3.73 \\
\hline WPC20 & 15.89 & 4.57 \\
\hline WPC35 & 8.11 & 1.35 \\
\hline WPC38 & 6.34 & 2.60 \\
\hline ZEP & 15.93 & 2.35 \\
\hline CGO & 16.62 & 3.71 \\
\hline SURGUT1 & 29.22 & 12.87 \\
\hline SURGUT2 & 28.39 & 12.15 \\
\hline SURGUT3 & 28.25 & 11.05 \\
\hline SURGUT4 & 29.75 & 12.58 \\
\hline SURGUT5 & 28.42 & 11.86 \\
\hline SURGUT6 & 28.43 & 10.57 \\
\hline SURGUT7 & 27.41 & 9.85 \\
\hline SURGUT8 & 27.97 & 10.97 \\
\hline TOHOKU4 & 15.74 & 5.91 \\
\hline TOHOKU5 & 14.66 & 4.80 \\
\hline TOHOKU6 & 17.83 & 4.64 \\
\hline TOHOKU7 & 22.46 & 5.60 \\
\hline TOHOKU8 & 20.70 & 5.01 \\
\hline TOHOKU9 & 21.05 & 6.11 \\
\hline TOHOKU10 & 16.90 & 4.87 \\
\hline TOHOKU11 & 19.06 & 4.82 \\
\hline TOHOKU12 & 21.21 & 5.20 \\
\hline TOHOKU13 & 19.25 & 4.45 \\
\hline TOHOKU14 & 17.77 & 5.83 \\
\hline TOHOKU15 & 15.11 & 9.16 \\
\hline TOHOKU17 & 13.24 & 5.10 \\
\hline TOHOKU18 & 6.20 & 2.02 \\
\hline TOHOKU19 & 8.68 & 1.39 \\
\hline
\end{tabular}


Table S1g. Root-mean-square deviations of the differences between measurements and modeled mixing ratios. The first column shows the model results using prior emissions and the second column shows the same with posterior emissions.

\begin{tabular}{ccc}
\hline Station & prior mixing ratio RMSD & posterior mixing ratio RMSD \\
\hline TOHOKU20 & 8.02 & 1.25 \\
TOHOKU21 & 6.62 & 3.63 \\
TOHOKU22 & 7.69 & 0.81 \\
TOHOKU23 & 8.47 & 2.78 \\
TOHOKU24 & 5.00 & 2.01 \\
TOHOKU25 & 4.48 & 2.42 \\
TOHOKU26 & 6.85 & 1.71 \\
TOHOKU27 & 6.17 & 1.71 \\
TOHOKU28 & 4.79 & 2.27 \\
TOHOKU29 & 6.45 & 1.03 \\
TOHOKU30 & 3.98 & 2.24 \\
TOHOKU31 & 7.00 & 3.00 \\
TOHOKU32 & 9.33 & 2.44 \\
TOHOKU33 & 5.09 & 1.53 \\
TOHOKU34 & 3.50 & 3.83 \\
TOHOKU35 & 4.83 & 0.76 \\
TOHOKU36 & 5.77 & 0.94 \\
TOHOKU37 & 7.23 & 1.92 \\
TOHOKU38 & 6.21 & 0.60 \\
TOHOKU39 & 6.52 & 2.60 \\
TOHOKU40 & 10.92 & 4.49 \\
TOHOKU41 & 12.88 & 6.26 \\
TOHOKU42 & 14.40 & 3.03 \\
TOHOKU43 & 14.29 & 4.10 \\
TOHOKU44 & 17.19 & 5.07 \\
TOHOKU45 & 17.19 & 4.83 \\
TOHOKU46 & 13.97 & 4.20 \\
TOHOKU47 & 12.17 & 4.03 \\
CSIRO1 & 24.40 & 4.66 \\
CSIRO2 & 19.05 & 2.87 \\
CSIRO3 & 25.56 & 7.93 \\
CSIRO4 & 16.54 & 3.08 \\
CSIRO5 & 23.99 & 5.37 \\
CSIRO6 & 19.91 & 2.92 \\
CSIRO7 & 19.83 & 3.26 \\
CSIRO8 & 21.83 & 4.19 \\
\hline
\end{tabular}

